\title{
PISA 2009 Ergebnisse: Lernfortschritte im globalen Wettbewerb
}

\section{VERÄNDERUNGEN BEI DEN}

SCHÜLERLEISTUNGEN SEIT 2000

\section{BAND V}

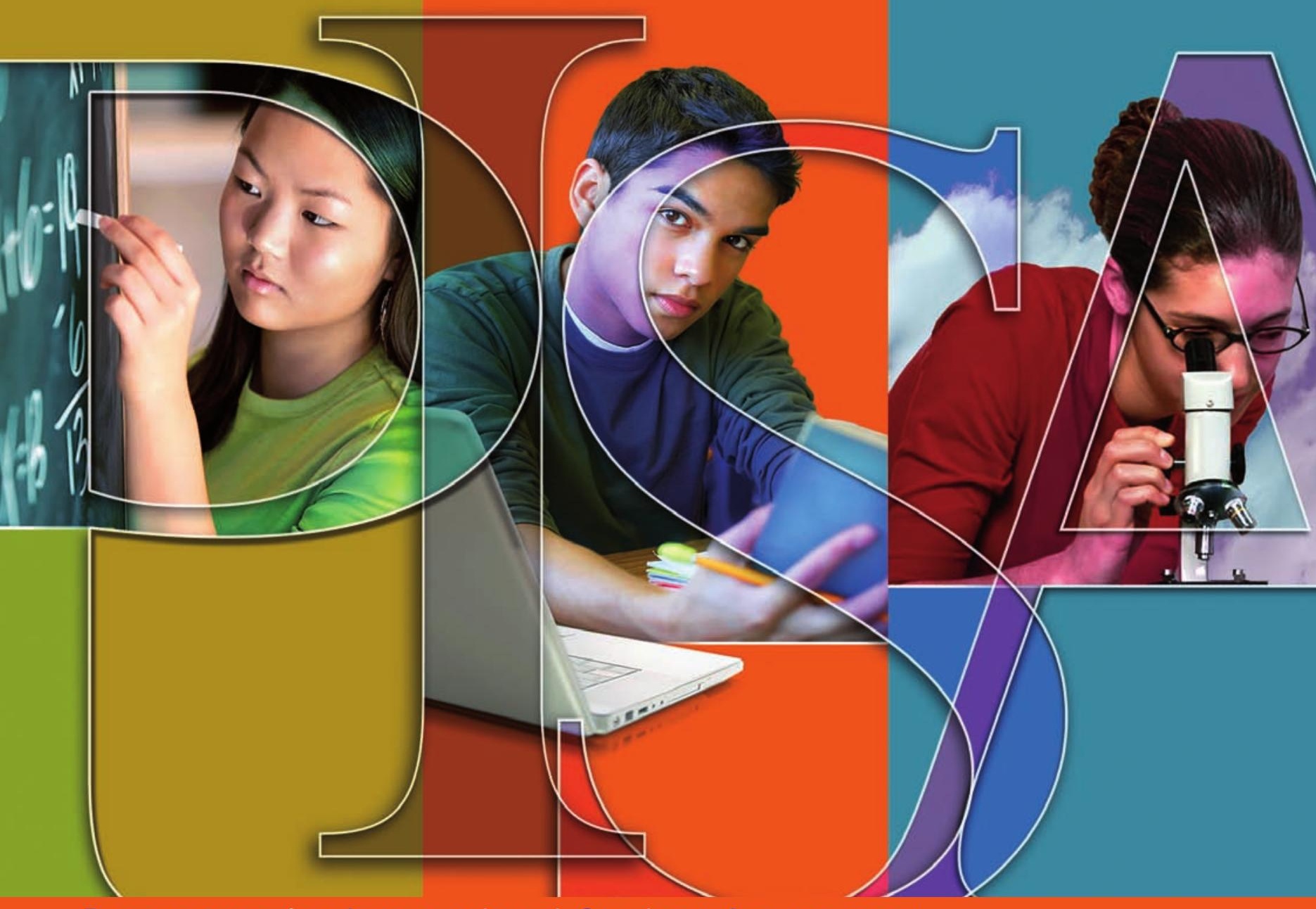

Programme for International Student Assessment

OECD $|\mathbb{H}| \mathrm{H} \mid$ 



\section{PISA 2009 Ergebnisse: Lernfortschritte im globalen Wettbewerb VERÄNDERUNGEN BEI DEN SCHÜLERLEISTUNGEN SEIT 2000 (BANDV)}


Das vorliegende Dokument wird unter der Verantwortung des Generalsekretärs der OECD veröffentlicht. Die darin zum Ausdruck gebrachten Meinungen und Argumente spiegeln nicht zwangsläufig die offizielle Einstellung der Organisation oder der Regierungen ihrer Mitgliedstaaten wider.

Bitte zitieren Sie diese Publikation wie folgt:

OECD (2011), PISA 2009 Ergebnisse: Lernfortschritte im globalen Wettbewerb - Veränderungen bei den Schülerleistungen seit 2000 (Band V), PISA, OECD Publishing.

http://dx.doi.org/10.1787/9789264098077-de

ISBN 978-92-64-09807-7 (PDF)

Originaltitel: PISA 2009 Results: Learning Trends: Changes in Student Performance Since 2000 (Volume V)

Übersetzung durch den Deutschen Übersetzungsdienst der OECD

Die statistischen Daten für Israel wurden von den zuständigen israelischen Stellen bereitgestellt, die für sie verantwortlich zeichnen. Die Verwendung dieser Daten durch die OECD erfolgt unbeschadet des völkerrechtlichen Status der Golanhöhen, von Ost-Jerusalem und der israelischen Siedlungen im Westjordanland.

\section{Fotos:}

Getty Images @ Ariel Skelley

Getty Images @ Geostock

Getty Images @ Jack Hollingsworth

Stocklib Image Bank @ Yuri Arcurs

Korrigenda zu OECD-Veröffentlichungen sind verfügbar unter: www.oecd.org/publishing/corrigenda.

Die Bezeichnungen PISA, OECD/PISA und das PISA-Logo sind geschützte Markenzeichen der Organisation für wirtschaftliche Zusammenarbeit und Entwicklung (OECD). Jegliche Verwendung von OECD-Markenzeichen ohne schriftliche Genehmigung der OECD ist unzulässig.

(C) OECD 2011

Die OECD gestattet das Kopieren, Herunterladen und Abdrucken von OECD-Inhalten für den eigenen Gebrauch sowie das Einfügen von Auszügen aus OECD-Veröffentlichungen, -Datenbanken und -Multimediaprodukten in eigene Dokumente, Präsentationen, Blogs, Websites und Lehrmaterialien, vorausgesetzt die OECD wird in geeigneter Weise als Quelle und Urheberrechtsinhaber genannt. Sämtliche Anfragen bezüglich Verwendung für öffentliche oder kommerzielle Zwecke bzw. Übersetzungsrechte sind zu richten an: rights@oecd.org. Die Genehmigung zur Kopie von Teilen dieser Publikation für den öffentlichen oder kommerziellen Gebrauch ist direkt einzuholen beim Copyright Clearance Center (CCC) unter info@copyright. com oder beim Centre français d'exploitation du droit de copie (CFC) unter contact@cfcopies.com. 


\section{Vorwort}

Eines der obersten Ziele politischer Entscheidungsträger besteht darin, die Bürger in die Lage zu versetzen, die Vorteile einer globalisierten Weltwirtschaft zu nutzen. Daher richten sie ihr Augenmerk auf die Verbesserung der Bildungspolitik, um die Qualität der Leistungserbringung, eine gerechtere Verteilung der Bildungschancen und stärkere Anreize für eine größere Effizienz der Schulen zu gewährleisten.

Entscheidend für entsprechende Politikmaßnahmen sind verlässliche Informationen darüber, wie gut die Bildungssysteme die Schülerinnen und Schüler für ihr weiteres Leben vorbereiten. Die meisten Länder beobachten den Lernprozess der Schülerinnen und Schüler und die Leistungen der Schulen. In einer globalisierten Wirtschaft wird Erfolg indessen nicht mehr nur an der Verbesserung nach nationalen Standards gemessen, sondern daran, wie gut die Bildungssysteme auf internationaler Ebene abschneiden. Die OECD hat sich der daraus resultierenden Herausforderung gestellt, indem sie die Internationale Schulleistungsstudie PISA (Programme for International Student Assessment) entwickelt hat, in der die Qualität, die Gerechtigkeit und die Effizienz von Schulsystemen in etwa siebzig Ländern bewertet werden, die insgesamt neun Zehntel der Weltwirtschaft auf sich vereinen. PISA steht für das Engagement der Regierungen, die Ergebnisse ihrer Bildungssysteme in regelmäßigen Abständen innerhalb eines international vereinbarten Rahmens zu bewerten, und bietet eine Basis für die internationale Zusammenarbeit bei der Festlegung und Umsetzung bildungspolitischer Maßnahmen.

Die Ergebnisse der PISA-Erhebung 2009 machen deutlich, dass bei den Bildungsergebnissen sowohl innerhalb als auch zwischen den einzelnen Ländern große Unterschiede bestehen. Bildungssysteme, denen es gelungen ist, hohe und ausgewogene Lernerträge zu gewährleisten und rasche Verbesserungen zu erzielen, zeigen anderen, was effektiv erreicht werden kann. Natürlich hängt der Bildungserfolg auch von der Höhe des Pro-Kopf-BIP ab, dies erklärt aber nur 6\% der Unterschiede bei den durchschnittlichen Schülerleistungen. In den restlichen 94\% drückt sich das Potenzial der staatlichen Politik aus, auf die Ergebnisse Einfluss zu nehmen. Der beeindruckende Erfolg von Shanghai (China), das bei dieser Erhebung in allen Ranglisten mit deutlichem Abstand an der Spitze steht, zeigt, was mit relativ geringen wirtschaftlichen Ressourcen in einem vielfältigen sozialen Kontext erreicht werden kann. In Shanghai (China) ist mehr als ein Viertel der 15-Jährigen in Mathematik in der Lage, aus der Untersuchung und Modellierung komplexer Problemsituationen abgeleitete Informationen zu konzeptualisieren, zu verallgemeinern und kreativ auf neue Situationen anzuwenden. Diese Schüler können ihr mathematisches Verständnis nutzen, um Ansätze und Strategien zur Lösung neuartiger Problemsituationen zu entwickeln. Im OECD-Raum erreichen nur 3\% der Schülerinnen und Schüler diese Kompetenzstufe.

Bessere Bildungsergebnisse sind zwar ein starker Prädiktor fürWirtschaftswachstum; Wohlstand und Bildungsausgaben allein sind aber noch keine Garantie für bessere Bildungsergebnisse. PISA macht deutlich, dass das Bild einer Welt, die sich klar in reiche Länder mit hohem Bildungsniveau und arme Länder mit niedrigem Bildungsniveau einteilen lässt, nicht mehr zeitgemäß ist.

Diese Feststellung muss zugleich als Warnung und als Chance begriffen werden. Sie ist eine Warnung für fortgeschritteneVolkswirtschaften, die es nicht mehr als selbstverständlich betrachten können, dass ihr "Humankapital“ auf ewig dem anderer Teile der Welt überlegen sein wird. In einer Zeit intensiven globalen Wettbewerbs müssen sie große Anstrengungen unternehmen, um eine Wissens- und Qualifikationsbasis aufrechtzuerhalten, die mit sich verändernden Anforderungen Schritt halten kann.

PISA unterstreicht insbesondere, dass sich viele fortgeschrittene Volkswirtschaften mit dem Problem der unzureichenden Bildungsergebnisse auseinandersetzen müssen, damit ein möglichst großer Anteil ihrer künftigen Erwerbsbevölkerung zumindest mit den Grundkompetenzen ausgestattet ist, die es ermöglichen, an der sozialen und wirtschaften Entwicklung teilzuhaben. Andernfalls könnten die hohen sozialen und wirtschaftlichen Kosten, 
die schlechte Bildungsergebnisse in fortgeschrittenen Volkswirtschaften nach sich ziehen, eine erhebliche Bremswirkung auf die wirtschaftliche Entwicklung ausüben. Gleichzeitig zeigen die Ergebnisse, dass ein niedriges Nationaleinkommen nicht zwangsläufig zu einem geringen Qualifikationsniveau führen muss - was eine wichtige Erkenntnis für die Länder darstellt, die mit weniger Mitteln mehr erreichen müssen.

Aus den PISA-Ergebnissen geht aber auch hervor, dass kein Grund zur Verzweiflung besteht. Länder mit sehr unterschiedlicher Ausgangssituation haben gezeigt, dass sie das Potenzial besitzen, die Qualität der Bildungserträge beträchtlich zu steigern. Koreas Durchschnittsergebnisse waren bereits im Jahr 2000 hoch, die koreanischen Politikverantwortlichen waren allerdings darüber besorgt, dass nur eine kleine Elite die obersten Kompetenzstufen von PISA erreichte. In weniger als zehn Jahren ist es Korea gelungen, den Anteil der Schülerinnen und Schüler zu verdoppeln, die in Lesekompetenz hervorragende Leistungen erzielen. In Polen hat eine grundlegende Reform des Schulsystems in hohem Maße dazu beigetragen, die Leistungsvarianz zwischen den einzelnen Schulen zu reduzieren, den Anteil der schlecht abschneidenden Schülerinnen und Schüler zu verringern und die Gesamtleistung in einem Umfang zu erhöhen, der dem Lernfortschritt eines halben Schuljahrs entspricht. Deutschland sah sich durch die Schockwirkung der unterdurchschnittlichen Ergebnisse bei PISA 2000 und die dabei zu Tage getretenen großen sozialen Disparitäten bei den Lernerträgen zum Handeln veranlasst und konnte an beiden Fronten Fortschritte erzielen. Israel, Italien und Portugal haben sich dem OECD-Durchschnitt angenähert, und Brasilien, Chile, Mexiko und die Türkei gehören zu den Ländern, in denen eine beeindruckende Verbesserung gegenüber einem zuvor sehr niedrigen Leistungsniveau festzustellen ist.

Der größte Nutzen der PISA-Studie liegt aber darin, dass sie den Anstoß zu Anstrengungen auf nationaler Ebene gibt, damit die Schülerinnen und Schüler besser lernen, die Lehrerinnen und Lehrer besser unterrichten und die Schulsysteme effektiver werden können.

Bei genauerer Betrachtung der Bildungssysteme, die sehr gut abschneiden oder rasche Verbesserungen erzielt haben, fällt auf, dass diese ungeachtet der Unterschiede in ihrer Geschichte, Kultur und wirtschaftlichen Entwicklung viele Gemeinsamkeiten aufweisen.

Erstens bekunden zwar die meisten Länder ihr Engagement für die Bildung, dieses Engagement wird jedoch auf die Probe gestellt, wenn es mit anderen Zielen in Konkurrenz tritt. Wie werden die Lehrkräfte im Vergleich zu anderen hochqualifizierten Arbeitskräften bezahlt? Welches Gewicht haben Bildungsabschlüsse gegenüber anderen Qualifikationen bei der Auswahl von Bewerbern für eine Stelle? Würden Sie es wünschen, dass Ihr Kind Lehrerin bzw. Lehrer wird? Wie viel Aufmerksamkeit wird Schulen und Schulbildung in den Medien beigemessen? Was ist wichtiger: der Erfolg eines Landes bei Sportwettkämpfen oder sein Platz auf einer Rangliste der schulischen Leistungen seiner Schülerinnen und Schüler? Ermutigen Eltern ihre Kinder eher, länger und mehr zu lernen oder mehr Zeit mit ihren Freunden oder sportlichen Aktivitäten zu verbringen?

In den erfolgreichsten Bildungssystemen ist es den politischen und sozialen Entscheidungsträgern gelungen, die Bürger zu überzeugen, Bildung in ihren Entscheidungen mehr Wert beizumessen als anderen Zielen. Es reicht jedoch nicht aus, der Bildung einen hohen Stellenwert einzuräumen, wenn die Lehrkräfte, Eltern und Bürger eines Landes noch der Ansicht sind, dass nur ein Teil der Kinder Weltklasseniveau erreichen kann bzw. muss. Dieser Bericht zeigt ganz deutlich, dass Bildungssysteme, die auf der Überzeugung aufbauen, dass die Schülerinnen und Schüler für unterschiedliche berufliche Laufbahnen "vorherbestimmt" sind und folglich unterschiedliche Schultypen mit unterschiedlich hohen Anforderungen besuchen sollten, in der Regel große soziale Disparitäten aufweisen. Im Gegensatz dazu tragen die am besten abschneidenden Bildungssysteme der Diversität der Fähigkeiten und Interessen sowie des sozialen Hintergrunds der Schülerinnen und Schüler Rechnung, indem sie individualisierte Lernansätze anbieten.

Zweitens zeichnen sich sehr gut abschneidende Bildungssysteme durch klare und ambitionierte Standards aus, die dem ganzen System gemeinsam sind, wobei der Unterricht auf die Vermittlung komplexer, hoch entwickelter Denkfähigkeiten ausgerichtet und auf die zu erfüllenden Anforderungen für den Übergang in höhere Bildungsbereiche abgestimmt ist. In diesen Bildungssystemen ist sich jeder darüber im Klaren, was sowohl im Hinblick auf die Unterrichtsinhalte als auch auf das nötige Leistungsniveau erforderlich ist, um eine bestimmte Qualifikation zu erwerben. Die Schülerinnen und Schüler können nicht den nächsten Abschnitt ihrer Laufbahn - sei es die Erwerbstätigkeit oder die weiterführende Bildung - beginnen, ohne bewiesen zu haben, dass sie dafür ausreichend qualifiziert sind. Sie wissen, was sie tun müssen, um ihren Traum zu verwirklichen, und sie unternehmen die dafür notwendigen Anstrengungen. 
Drittens kann die Qualität eines Bildungssystems nicht höher sein als die Qualität seiner Lehrkräfte und Schulleitungen, denn schließlich ist der Lernerfolg der Schülerinnen und Schüler das Ergebnis dessen, was im Unterricht geschieht. Unternehmen, Berufsverbände und Regierungen wissen, dass sie darauf achten müssen, wie das Reservoir der potenziellen Mitarbeiter, das ihnen zur Verfügung steht, herangebildet wird. Sie müssen darauf achten, wie sie bei der Anwerbung von Personal vorgehen, welche Art von Erstausbildung ihre potenziellen Mitarbeiter erhalten haben, wie sie neu eingestellte Kräfte betreuen und in ihren Betrieb einführen, wie sie sie weiterbilden können, wie ihr Verdienst strukturiert ist, wie sie die Leistungsstärksten belohnen und denen helfen können, die vor Schwierigkeiten stehen, und wie sie besonders Leistungsstarken die Möglichkeit geben können, mehr Ansehen und Verantwortung zu erlangen. Viele der weltweit am besten abschneidenden Bildungssysteme sind von einem bürokratischen "Auflageninstrumentarium“ abgegangen und setzen stattdessen auf Strukturen, in denen die Akteure an vorderster Front viel mehr Kontrolle darüber haben, wie die Ressourcen verwendet und die Mitarbeiter eingesetzt werden und wie die Arbeit organisiert und erledigt wird. Sie lassen den Schulleitungen und den Schulen erheblichen Freiraum bei der Festlegung der Unterrichtsinhalte und Lehrpläne, was ein Faktor ist, der in Kombination mit wirksamen Rechenschaftsstrukturen großen Einfluss auf die Ergebnisse der einzelnen Schulen hat, wie dieser Bericht zeigt. Darüber hinaus bieten solche Bildungssysteme ein Umfeld, in dem die Lehrkräfte zusammenarbeiten, um das zu formulieren, was sie als gute Praxis betrachten, um Feldstudien zur Bestätigung oder Widerlegung der von ihnen entwickelten Ansätze durchzuführen und um ihre Kollegen danach zu beurteilen, wie sie Praktiken einsetzen, die sich im Unterricht als effizient erwiesen haben.

Last but not least ist das wohl beeindruckendste Merkmal weltweit erstklassiger Bildungssysteme, dass sie in allen Schulen durchgehend qualitativ hochwertige Bildungsleistungen anbieten, so dass alle Schülerinnen und Schüler von hervorragenden Lernmöglichkeiten profitieren können. Um dies zu erreichen, investieren sie die Bildungsressourcen dort, wo sie mit ihnen am meisten bewirken können, schicken sie die talentiertesten Lehrkräfte in die schwierigsten Klassen und räumen sie bei ihren Ausgabenentscheidungen der Qualität der Lehrkräfte Priorität ein.

Dabei handelt es sich natürlich nicht um unabhängig voneinander konzipierte und ausgeführte Politikmaßnahmen. Sie müssen mit sämtlichen Aspekten des Systems im Einklang stehen, über einen längeren Zeitraum kohärent sein und konsistent umgesetzt werden. Der Reformprozess kann durch politische und praktische Hindernisse erschwert werden. Die Umstellung von einer administrativen und bürokratischen Kontrolle auf professionelle Kontrollregeln kann kontraproduktiv wirken, wenn die Lehrkräfte und Schulen des Landes noch nicht über die erforderlichen Kapazitäten verfügen, um diese Politiken und Praktiken umzusetzen. Die Delegierung von Entscheidungsbefugnissen an niedrigere Ebenen kann ebenso problematisch sein, wenn keine Vereinbarungen darüber getroffen wurden, was die Schülerinnen und Schüler wissen müssen und wozu sie in der Lage sein sollten. Auch bringt es nicht viel, ausgezeichnete Kräfte für den Lehrerberuf zu gewinnen, wenn diese durch ein System der Lehrererstausbildung, das sie als sinnlos betrachten, entmutigt werden und sich letztlich für einen anderen Beruf entscheiden. Der Erfolg eines Landes bei der Durchführung der erforderlichen Umstellungen hängt folglich in hohem Maß davon ab, inwieweit es ihm gelingt, Pläne aufzustellen und umzusetzen, die zum jeweiligen Zeitpunkt für die größtmögliche Kohärenz im System sorgen.

Dies sind gewaltige Herausforderungen, und daher wird es immer schwieriger werden, effektive bildungspolitische Maßnahmen zu konzipieren, die es den Schulen ermöglichen, die Schülerinnen und Schüler darauf vorzubereiten, sich an einen rascher denn je voranschreitenden Wandel anzupassen, den Anforderungen von Berufen gerecht zu werden, die bislang noch nicht einmal existieren, Technologien anzuwenden, die noch gar nicht erfunden wurden, und wirtschaftliche und soziale Probleme zu bewältigen, von denen wir uns heute noch keine Vorstellungen machen können. Die Schulsysteme, die heute gute Ergebnisse erzielen, ebenso wie die, die rasche Verbesserungen vorweisen können, zeigen aber, dass dies machbar ist. Die Welt misst Traditionen und vergangenem Ansehen keine große Bedeutung bei, sie verzeiht weder Schwäche noch Selbstgefälligkeit, und sie interessiert sich nicht für überlieferte Vorgehensweisen und Praktiken. Erfolgreich werden jene Menschen und Länder sein, die sich schnell anpassen, nicht voreilig klagen und offen gegenüber Änderungen sind. Die Aufgabe der Regierungen wird es sein, sicherzustellen, dass ihre Länder diese Herausforderungen bewältigen können. Die OECD wird ihre Anstrengungen dabei weiterhin unterstützen.

Dieser Bericht ist das Ergebnis eines Kooperationsprojekts der PISA-Teilnehmerländer, der im PISA-Konsortium vertretenen Experten und Einrichtungen sowie des OECD-Sekretariats. Der Bericht wurde von Andreas Schleicher, Francesca Borgonovi, Michael Davidson, Miyako Ikeda, Maciej Jakubowski, Guillermo Montt, Sophie Vayssettes 
und Pablo Zoido von der OECD-Direktion Bildung verfasst; beraten und in analytischer und redaktioneller Hinsicht unterstützt wurden sie von Marilyn Achiron, Simone Bloem, Marika Boiron, Henry Braun, Nihad Bunar, Niccolina Clements, Jude Cosgrove, John Cresswell, Aletta Grisay, Donald Hirsch, David Kaplan, Henry Levin, Juliette Mendelovitz, Christian Monseur, Soojin Park, Pasi Reinikainen, Mebrak Tareke, Elisabeth Villoutreix und Allan Wigfield. Band II stützt sich zudem auf von Jaap Scheerens und Douglas Willms im Zusammenhang mit PISA 2000 durchgeführte Analysen. Für die administrative Seite waren Juliet Evans und Diana Morales zuständig.

Die PISA-Erhebungsinstrumente und das Datenmaterial für den Bericht wurden vom PISA-Konsortium unter der Leitung von Raymond Adams vom Australian Council for Educational Research (ACER) und Henk Moelands vom niederländischen National Institute for Educational Measurement (CITO) vorbereitet. Die Expertengruppe, die die Orientierungen für die Ausarbeitung des Rahmenkonzepts und der Erhebungsinstrumente für den Lesekompetenztest vorgab, wurde von Irwin Kirsch geleitet.

Die Orientierungen für die Gestaltung des Berichts insgesamt kamen vom PISA-Verwaltungsrat unter dem Vorsitz von Lorna Bertrand (Vereinigtes Königreich) und dem stellvertretenden Vorsitz von Beno Csapo (Ungarn), Daniel McGrath (Vereinigte Staaten) und Ryo Watanabe (Japan). In Anhang C sind die Mitglieder der verschiedenen PISAOrgane wie auch die Fachleute und Consultants aufgeführt, die an diesem Bericht und der PISA-Erhebung allgemein mitgewirkt haben.

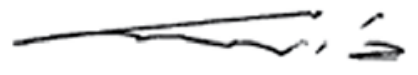

Angel Gurría

Generalsekretär der OECD 


\section{Inhaltsverzeichnis}

ZUSAMMENFASSUNG.

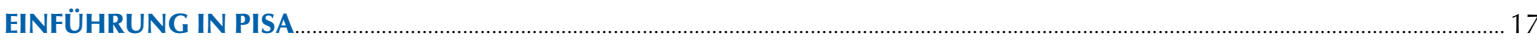

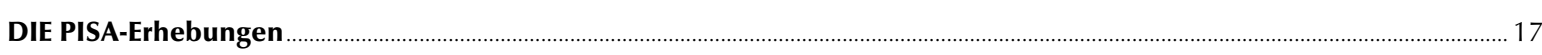

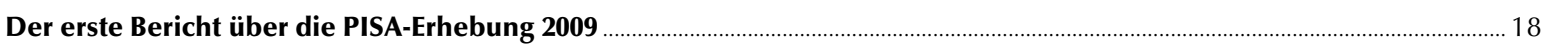

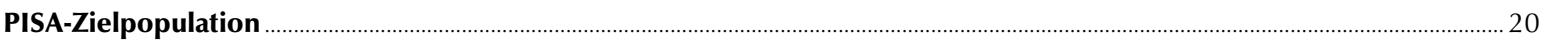

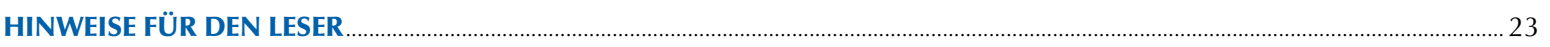

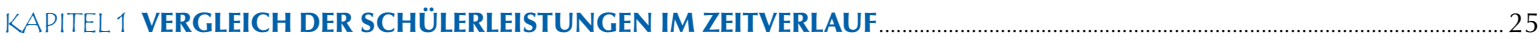

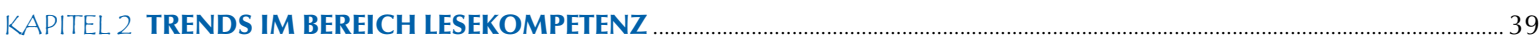

Kontinuität und Wandel beim Rahmenkonzept und bei den Erhebungsinstrumenten im Bereich Lesekompetenz ............ 40

Wie sich die Schülerleistungen im Bereich Lesekompetenz seit dem Jahr 2000 verändert haben .............................................. 40

Wie sich die geschlechtsspezifischen Unterschiede im Bereich Lesekompetenz entwickelt haben .......................................49

Leistungsveränderungen und Veränderungen der Schülerpopulationen …….................................................................................... 52

Der Effekt von Veränderungen der sozioökonomischen Zusammensetzung der Schülerpopulation auf die

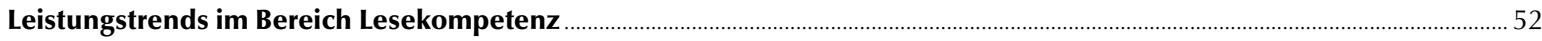

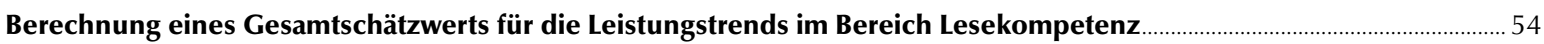

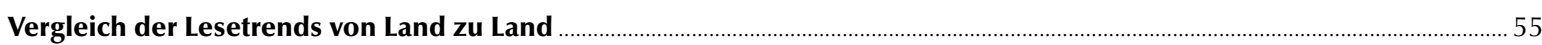

KAPITEL 3 TRENDS IN DEN BEREICHEN MATHEMATIK UND NATURWISSENSCHAFTEN ...................................................... 63

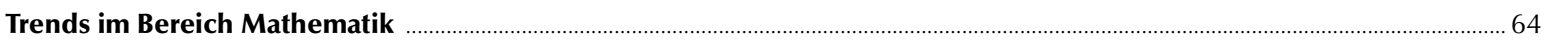

- Wie sich die Schülerleistungen im Bereich Mathematik seit 2003 verändert haben .......................................................................... 64

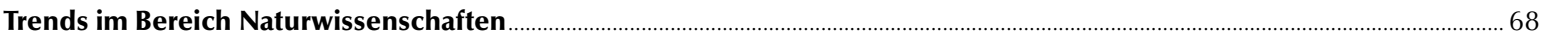

- Wie sich die Schülerleistungen im Bereich Naturwissenschaften seit 2006 verändert haben .................................................................. 68

KAPITEL 4 ENTWICKLUNGEN IM BEREICH DER CHANCENGERECHTIGKEIT .......................................................................... 77

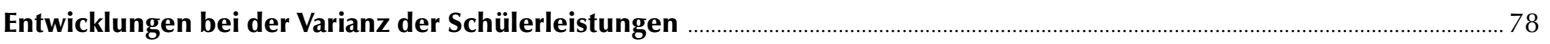

Entwicklungen bei den Hintergrundmerkmalen der Schüler und dem Zusammenhang mit den Leseleistungen................. 82

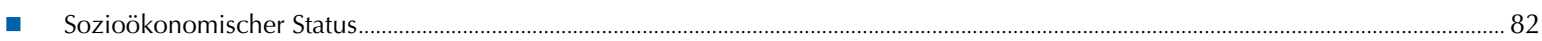

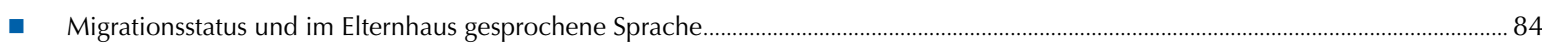

\section{KAPITEL 5 TRENDS BEI DEN EINSTELLUNGEN DER SCHÜLER UND DEN BEZIEHUNGEN ZWISCHEN SCHÜLERN}

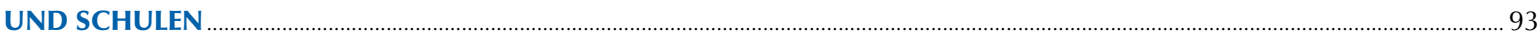

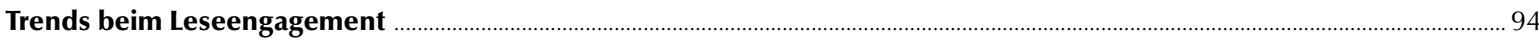

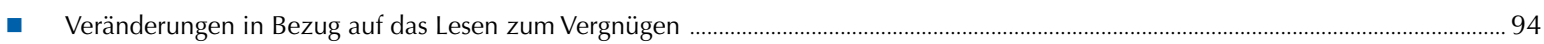

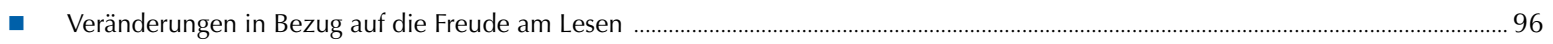

- Veränderungen bei den Inhalten, die Schülerinnen und Schüler zum Vergnügen lesen ......................................................................... 99

- Veränderungen des Leseengagements von in sozioökonomischer Hinsicht benachteiligten Schülerinnen und Schülern ...................103

- Veränderungen der Leistungen im Bereich Lesekompetenz der Schülerinnen und Schüler, die Romane und Erzählungen lesen ....104

Trends hinsichtlich der Schülereinstellungen zu Schule und Lehrern ……...................................................................................... 105

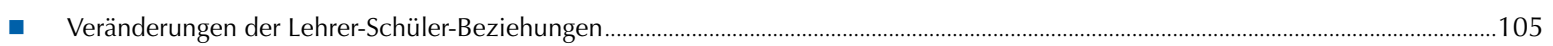

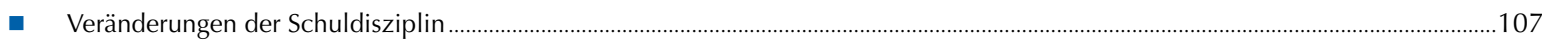


Anhang A1: Konstruktion der auf den Kontextfragebogen für Schüler, Schulen und Eltern beruhenden Lesekompetenzskalen und -indizes

Anhang A2: PISA-Zielpopulation, PISA-Stichproben und Definition der Schulen...

Anhang A3: Standardfehler, Signifikanztests und Vergleiche zwischen Untergruppen

Anhang A4: Qualitätssicherung

Anhang A5: Teilnahme der Länder an den PISA-Erhebungen ..

Anhang A6: Lineare und bereinigte Trends

Anhang B1: Ergebnisse für Länder und Volkswirtschaften

Anhang B2: Ergebnisse für einzelne Regionen innerhalb der Länder (nur auf Englisch verfügbar)

\section{Dieser Bericht enthält ...}

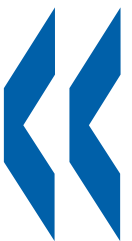

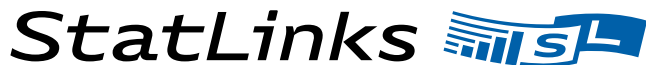

Ein Service für OECD-Veröffentlichungen, der es ermöglicht, Dateien im Excel-Format herunterzuladen.

Suchen Sie die StatLinks rechts unter den in diesem Bericht wiedergegebenen Tabellen oder Abbildungen. Um die entsprechende Datei im Excel-Format herunterzuladen, genügt es, den jeweiligen Link, beginnend mit $\boldsymbol{h t t p : / / d x . d o i . o r g , ~ i n ~ d e n ~ I n t e r n e t b r o w s e r ~ e i n z u g e b e n . ~}$ Wenn Sie die elektronische PDF-Version online lesen, dann brauchen Sie nur den Link anzuklicken. Sie finden StatLinks in weiteren OECD-Publikationen. 


\section{KÄSTEN}

Kasten V.A

Hauptmerkmale von PISA 2009

Kasten V.1.1

Bei der Interpretation von Trends ist Vorsicht geboten

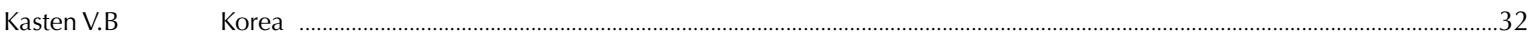

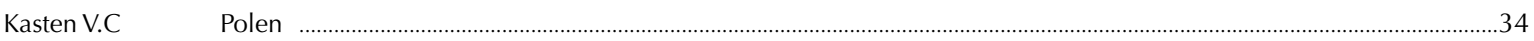

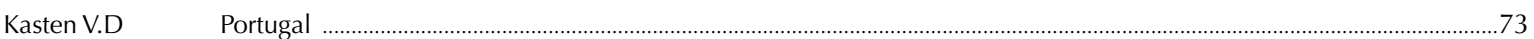

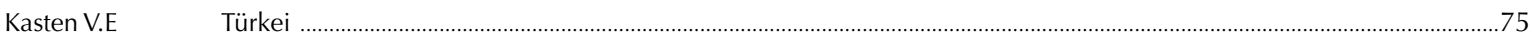

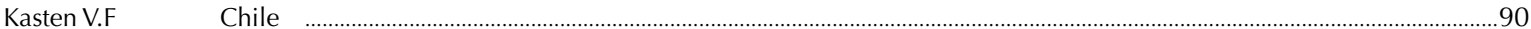

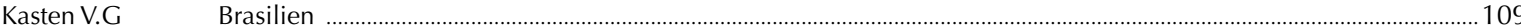

\section{ABBILDUNGEN}

Abbildung V.A

Abbildung V.1.1

Abbildung V.1.2

Abbildung V.2.1

Abbildung V.2.2

Abbildung V.2.3

Abbildung V.2.4

Abbildung V.2.5

Abbildung V.2.6

Abbildung V.2.7

Abbildung V.2.8

Abbildung V.2.9

Abbildung V.2.10

Abbildung V.2.11

Abbildung V.2.12

Abbildung V.2.13

Abbildung V.3.1

Abbildung V.3.2

Abbildung V.3.3

Abbildung V.3.4

Abbildung V.3.5

Abbildung V.3.6

Abbildung V.3.7

Abbildung V.3.8

Abbildung V.4.1

Abbildung V.4.2

Abbildung V.4.3

Abbildung V.4.4

Abbildung V.4.5

Abbildung V.4.6

Abbildung V.4.7

Abbildung V.4.8

Abbildung V.4.9
Ein Überblick über die PISA-Teilnehmerländer und -volkswirtschaften

Überblick über die Veränderungen der Schülerleistungen im Bereich Lesekompetenz

Überblick über die annualisierten Leistungstrends in den Bereichen Lesekompetenz, Mathematik und Naturwissenschaften.

Veränderungen der Schülerleistungen im Bereich Lesekompetenz zwischen 2000 und 2009.

Wie die Länder im Bereich Lesekompetenz abschneiden und wie sich die Leseleistungen seit dem Jahr 2000 verändert haben...

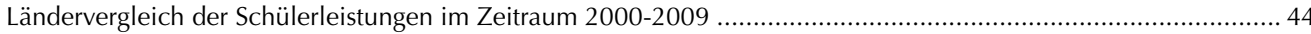

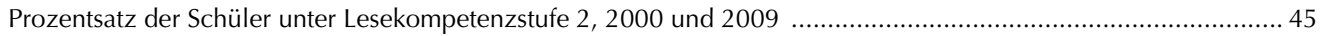

Prozentsatz der leistungsstärksten Schüler im Bereich Lesekompetenz, 2000-2009 ........................................ 47

Leistungsveränderungen bei den leistungsschwächsten und leistungsstärksten Schülern im Bereich Lesekompetenz zwischen 2000 und 2009

Vergleich des Leistungsabstands zwischen Jungen und Mädchen im Bereich Lesekompetenz, 2000-2009 .............. 50

Veränderung beim Anteil der Jungen und Mädchen, die im Zeitraum 2000-2009 im Bereich Lesekompetenz

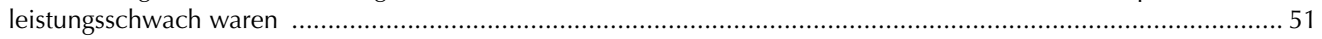

Veränderungen bei den Leistungen im Bereich Lesekompetenz zwischen 2000 und 2009 ................................... 53

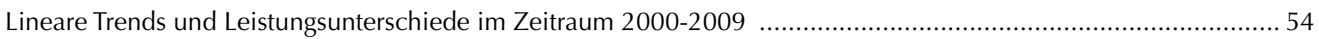

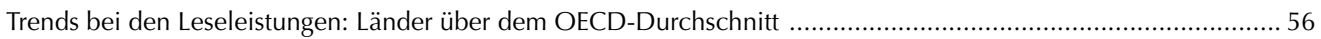

Trends bei den Leseleistungen: Länder im OECD-Durchschnitt .................................................................... 58

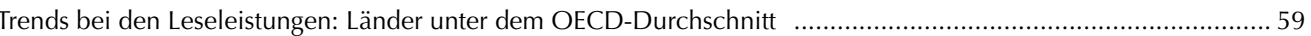

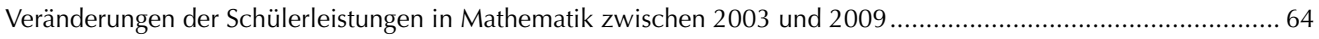

Wie die Länder in Mathematik abschneiden und wie sich die Mathematikleistungen seit 2003 verändert haben .... 65

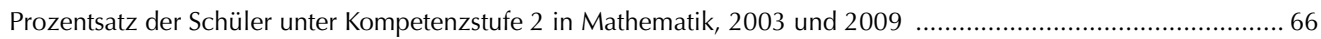

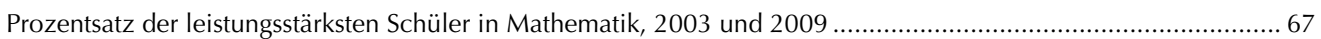

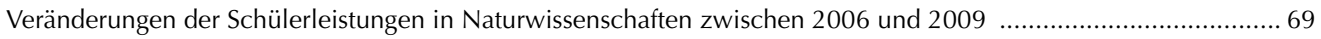

Wie die Länder in Naturwissenschaften abschneiden und wie sich die Leistungen in Naturwissenschaften

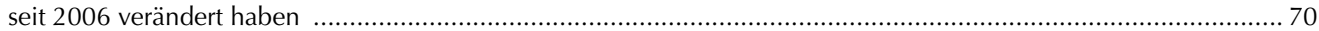

Prozentsatz der Schüler unter Kompetenzstufe 2 in Naturwissenschaften, 2006 und 2009 .................................. 71

Prozentsatz der leistungsstärksten Schüler in Naturwissenschaften, 2006 und 2009......................................... 72

Vergleich der Varianz der Schülerleistungen im Bereich Lesekompetenz, 2000 und 2009..................................... 78

Veränderung der Varianz und der Leistungen im Bereich Lesekompetenz zwischen 2000 und 2009...................... 80

Varianz der Leistungen im Bereich Lesekompetenz innerhalb und zwischen den Schulen, 2000 und 2009 ............. 81

Zusammenhang zwischen dem sozioökonomischen Hintergrund der Schüler und ihren Leistungen im Bereich Lesekompetenz, 2000 und 2009.

Zusammenhang zwischen dem sozioökonomischen Hintergrund der Schüler und ihren Leistungen im Bereich

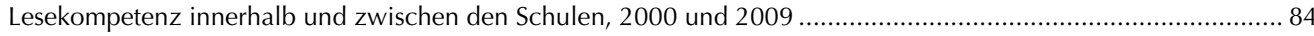

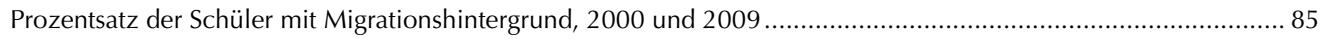

Migrationshintergrund und Leistungen im Bereich Lesekompetenz, 2000 und 2009 ........................................ 86

Prozentsatz der Schüler, die im Elternhaus eine andere Sprache als die Testsprache sprechen, 2000 und 2009 ........ 87

Im Elternhaus gesprochene Sprache und Leistungen im Bereich Lesekompetenz, 2000 und 2009......................... 87 
Abbildung V.5.1 Prozentsatz der Schüler, die zum Vergnügen lesen, 2000 und 2009.............................................................. 94

Abbildung V.5.2 Veränderung des Prozentsatzes der Jungen und Mädchen, die zum Vergnügen lesen, zwischen 2000 und 2009 ..... 95

Abbildung V.5.3 Prozentsatz der Schüler, die nur lesen, wenn sie müssen, und der Schüler, die gerne in Buchhandlungen oder

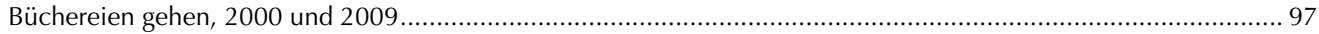

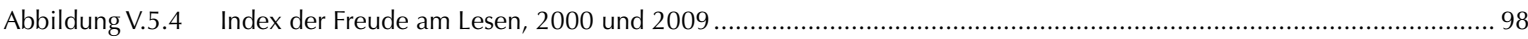

Abbildung V.5.5 Veränderung des Index der Freude am Lesen für Jungen und Mädchen zwischen 2000 und 2009 ......................... 99

Abbildung V.5.6 Veränderung des Index der Freude am Lesen und des Prozentsatzes der Schüler, die zum Vergnügen lesen,

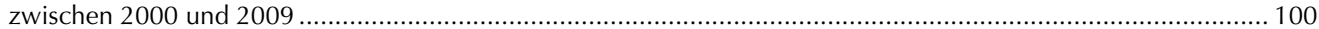

Abbildung V.5.7 Prozentsatz der Schüler, die fiktionale Literatur lesen, 2000 und 2009............................................................ 101

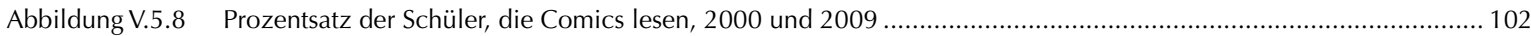

Abbildung V.5.9 Prozentsatz der Schüler, die zum Vergnügen lesen, 2000 und 2009, nach sozioökonomischem Hintergrund ........ 103

Abbildung V.5.10 Veränderung des Prozentsatzes der Jungen und Mädchen, die zum Vergnügen lesen, zwischen 2000 und 2009 ,

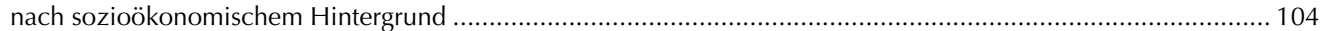

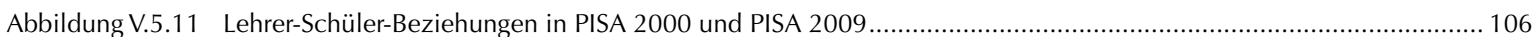

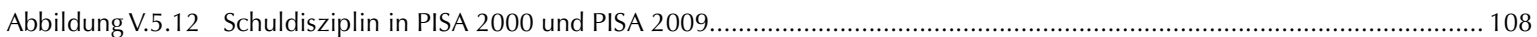

Abbildung A.6.1 Beobachtete Punktzahlveränderungen und um Stichprobenunterschiede zwischen 2000 und 2009 bereinigte Punktzahlveränderungen

\section{TABELLEN}

Tabelle A1.1

Schätzungen der Linking-Fehler

Tabelle A1.3 Mehr-Ebenen-Modell zur Schätzung von Klasseneffekten im Bereich Lesekompetenz nach Berücksichtigung mehrerer Hintergrundvariablen

Tabelle A2.1

PISA-Zielpopulationen und -Stichproben.

Tabelle A2.2

Ausschlüsse

Tabelle A5.1 Teilnahme der Länder an unterschiedlichen PISA-Erhebungen.

Tabelle A6.1 Hintergrundmerkmale der Schülerinnen und Schüler in PISA 2000 und PISA 2009 ..................................................... 149

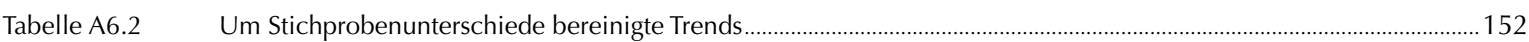

Tabelle V.2.1 Mittlere Punktzahlen im Bereich Lesekompetenz aus den PISA-Erhebungen 2000, 2003, 2006 und 2009 ................154

Tabelle V.2.2 Prozentsatz der Schüler unter Stufe 2 sowie auf Stufe 5 oder höher auf der Gesamtskala Lesekompetenz

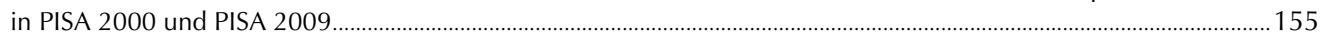

Tabelle V.2.3 Perzentile auf der Gesamtskala Lesekompetenz in PISA 2000 und PISA 2009.........................................................156

Tabelle V.2.4 Leistungsabstand zwischen Jungen und Mädchen im Bereich Lesekompetenz in PISA 2000 und PISA 2009 ............158

Tabelle V.2.5 Prozentsatz der Jungen unter Stufe 2 und auf Stufe 5 oder höher auf der Gesamtskala Lesekompetenz in PISA 2000 und PISA 2009

Tabelle V.2.6 Prozentsatz der Mädchen unter Stufe 2 und auf Stufe 5 oder höher auf der Gesamtskala Lesekompetenz

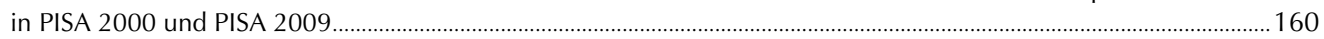

Tabelle V.2.7 Leistungstrends im Bereich Lesekompetenz, nach Berücksichtigung demografischer Veränderungen .......................... 161

Tabelle V.2.8 Lineare Trends und jährliche Veränderungen bei den Leseleistungen auf der Grundlage aller PISA-Erhebungen.......162

Tabelle V.2.9 Veränderung der durchschnittlichen Punktzahl in Lesekompetenz zwischen 2003 und 2009 sowie

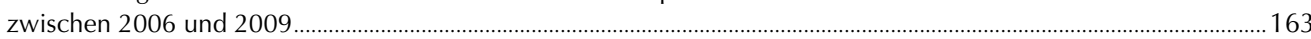

Tabelle V.3.1 Mittlere Punktzahlen in Mathematik in PISA 2003, PISA 2006 und PISA 2009 
Tabelle V.3.4
Tabelle V.3.5

Mittlere Punktzahlen in Naturwissenschaften in PISA 2006 und PISA 2009 167

Prozentsatz der Schüler unter Stufe 2 und auf Stufe 5 oder höher auf der Gesamtskala Naturwissenschaften in PISA 2006 und PISA 2009

Tabelle V.4.1 Varianz der Schülerleistungen im Bereich Lesekompetenz innerhalb und zwischen den Schulen PISA 2000 und PISA 2009

Tabelle V.4.2 Sozioökonomischer Hintergrund der Schüler, PISA 2000 und PISA 2009

Tabelle V.4.3

Zusammenhang zwischen Schülerleistungen im Bereich Lesekompetenz und PISA-Index des wirtschaftlichen, sozialen und kulturellen Status (ESCS), PISA 2000 und PISA 2009

Tabelle V.4.4

Schülerleistungen im Bereich Lesekompetenz nach Migrationsstatus, PISA 2000 und PISA 2009

Tabelle V.4.5 Schülerleistungen im Bereich Lesekompetenz, nach im Elternhaus gesprochener Sprache, PISA 2000 und PISA 2009

Tabelle V.5.1 Prozentsatz der Schüler in PISA 2000 und PISA 2009, die zum Vergnügen lesen, nach Geschlecht Index der Freude am Lesen in PISA 2000 und PISA 2009, nach Geschlecht Prozentsatz der Schüler für verschiedene Items auf dem Index der Freude am Lesen in PISA 2000 und PISA 2009.....176

Tabelle V.5.4 Prozentsatz der Schüler in PISA 2000 und PISA 2009, die zum Vergnügen lesen, nach sozioökonomischem Hintergrund und Geschlecht

Tabelle V.5.5 Index der Freude am Lesen in PISA 2000 und PISA 2009, nach soziökonomischem Hintergrund und Geschlecht .....182

Tabelle V.5.6

Tabelle V.5.7 Prozentsatz der Schüler, die verschiedene Arten von Texten lesen, in PISA 2000 und PISA 2009

Prozentsatz der Schüler, die verschiedene Arten von Texten lesen, in PISA 2000 und PISA 2009, nach Geschlecht

Tabelle V.5.8

Leistung auf PISA 2009

Tabelle V.5.9

Leistung auf der Gesamtskala Lesekompetenz von Schülern, die fiktionale Texte lesen, in PISA 2000 und PISA 2009, nach Geschlecht

Tabelle V.5.10

Vielseitigkeit des Lesestoffs in PISA 2000 und PISA 2009, nach Geschlecht 194

Tabelle V.5.11 Lehrer-Schüler-Beziehungen in PISA 2000 und PISA 2009 195

Tabelle V.5.12 



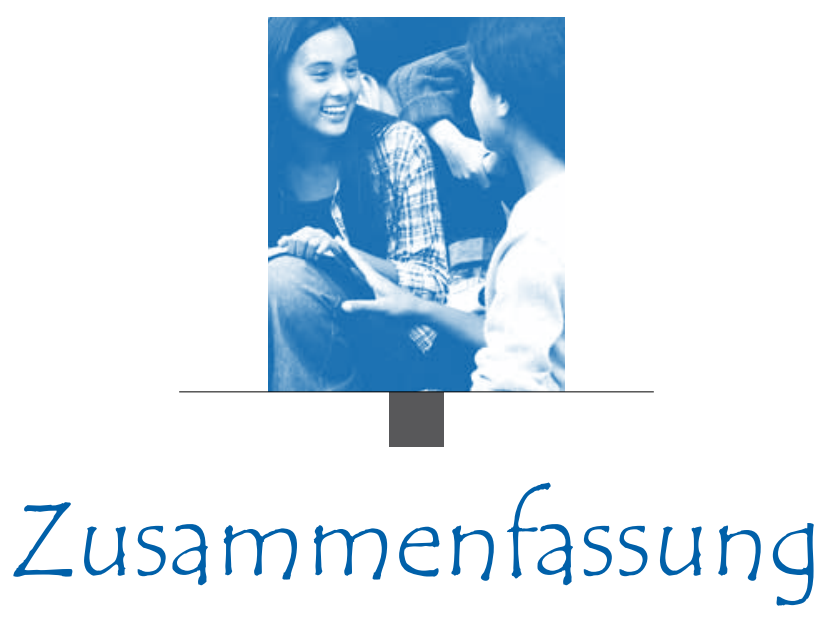

Die Gestaltung von PISA gestattet nicht nur einen Vergleich der jeweiligen Position der einzelnen Länder in Bezug auf die Lernergebnisse, sondern bietet zugleich jedem Land die Möglichkeit, die Veränderungen dieser Ergebnisse im Zeitverlauf zu beobachten. An diesen Veränderungen ist abzulesen, wie erfolgreich die Bildungssysteme im Hinblick auf die Weiterentwicklung der Kenntnisse und Fertigkeiten der 15-jährigen Schülerinnen und Schüler waren.

In der Tat konnten einige Länder in den letzten zehn Jahren eine beeindruckende Verbesserung der Lernergebnisse verzeichnen, die zuweilen dem Lernfortschritt während eines durchschnittlichen Schuljahrs, bezogen auf die gesamte Population der 15-jährigen Schülerinnen und Schüler, entsprach. Einige dieser Länder haben gegenüber einem vergleichsweise niedrigen Leistungsniveau aufgeholt, während andere von einem bereits hohen Niveau ausgehend weitere Fortschritte erzielt haben. Allen Ländern, die bestrebt sind, ihre Ergebnisse zu verbessern, kann das Beispiel der Länder, denen dies in einer relativ kurzen Zeitspanne gelungen ist, als Ansporn und als Chance dienen, aus ihren Erfahrungen zu lernen.

Die Veränderungen der Schülerleistungen im Zeitverlauf beweisen, dass die Ergebnisse eines Landes im Bereich Lesekompetenz zu einem gegebenen Zeitpunkt nichts Definitives haben. Bildungsergebnisse können sich sowohl in absoluten als auch in relativen Zahlen verbessern, und sie können weder als Teil unabänderlicher "kultureller" Unterschiede zwischen den Ländern noch als unweigerliche Konsequenz des wirtschaftlichen Entwicklungsstand des jeweiligen Landes betrachtet werden.

Da die Lesekompetenz sowohl 2000 als auch 2009 der Schwerpunktbereich der PISA-Erhebung war, lässt sich verfolgen, wie sich die Leistungen der Schülerinnen und Schüler in diesem Bereich während dieses Zeitraums verändert haben. Unter den 26 OECD-Ländern mit vergleichbaren Ergebnissen aus beiden Erhebungen konnten Chile, Israel, Polen, Portugal, Korea, Ungarn und Deutschland im Zeitraum 2000-2009 eine Verbesserung der Lesekompetenz verzeichnen, was auch für die Partnerländer Peru, Albanien, Indonesien, Lettland, Liechtenstein und Brasilien der Fall war, während in Irland, Schweden, der Tschechischen Republik und Australien eine Verschlechterung zu beobachten war.

Im Zeitraum 2000-2009 sank der Anteil der leistungsschwachen Schülerinnen und Schüler in Chile um über 17 Prozentpunkte, während sich der Anteil der leistungsstärksten Schülerinnen und Schüler in Korea um über 7 Prozentpunkte erhöhte.

In vielen Ländern waren die Verbesserungen der Gesamtergebnisse großenteils auf Verbesserungen im unteren Teil der Leistungsverteilung zurückzuführen, was als ein Zeichen für Fortschritte bei der Erzielung ausgewogenerer Lernerträge zu werten ist. Die Varianz der Schülerleistungen verringerte sich in den OECD-Ländern um 3\%. In den 26 OECD-Ländern mit vergleichbaren Daten aus beiden Erhebungen lagen 2009 durchschnittlich 18\% der Schülerinnen und Schüler mit ihren Ergebnissen unterhalb der als Basisniveau geltenden Lesekompetenzstufe 2, während dies 2000 für 19\% der Fall war. In der Gruppe der Länder, in denen im Jahr 2000 zwischen 40\% und 60\% der Schülerinnen und Schüler unterhalb von Stufe 2 lagen, verringerte sich dieser Anteil in Chile am stärksten, und auch 
Mexiko und das Partnerland Brasilien konnten einen bedeutenden Rückgang des Anteils der leistungsschwachen Schülerinnen und Schüler verbuchen. In der Gruppe der Länder, in denen der Anteil der unterhalb von Stufe 2 liegenden Schüler weniger als 40\%, aber mehr als den OECD-Durchschnitt von 19\% betrug, gelang Lettland eine Reduzierung dieses Anteils um 13 Prozentpunkte, während der Rückgang in Portugal, Polen, Ungarn, Deutschland und der Schweiz sowie im Partnerland Liechtenstein geringer ausfiel. In Dänemark reduzierte sich der Anteil der unter Stufe 2 liegenden Schülerinnen und Schüler gegenüber einem bereits unterdurchschnittlichen Ausgangsniveau.

Der Anteil der leistungsstärksten Schülerinnen und Schüler - d.h. derjenigen, die Lesekompetenzstufe 5 oder 6 erreichen - erhöhte sich in Japan, Korea und der Partnervolkswirtschaft Hongkong (China), womit diese Länder unter den Teilnehmerländern von PISA 2009 jetzt den höchsten Anteil leistungsstarker Schülerinnen und Schüler vorzuweisen haben. In mehreren Ländern, die 2000 einen überdurchschnittlich hohen Anteil sehr leistungsstarker Schülerinnen und Schüler hatten, ist dieser Anteil 2009 zurückgegangen. Besonders deutlich war dies in Irland, wo der Anteil der leistungsstärksten Schülerinnen und Schüler von 14\% auf 7\% - und somit unter den OECDDurchschnitt - gesunken ist.

Zwischen 2000 und 2009 gelang es Polen, Portugal, Deutschland, der Schweiz und den Partnerländern Lettland und Liechtenstein, die Ergebnisse ihrer leistungsschwächsten Schülerinnen und Schüler zu verbessern und dabei das Leistungsniveau ihrer leistungsstärksten Schülerinnen und Schüler aufrechtzuerhalten. Korea, Island und das Partnerland Brasilien erzielten eine Anhebung des Leistungsniveaus ihrer leistungsstärksten Schülerinnen und Schüler unter Aufrechterhaltung des Leistungsniveaus der leistungsschwächsten Schüler. Chile und die Partnerländer Indonesien, Albanien und Peru erzielten eine Verbesserung der Lesekompetenz der Schülerinnen und Schüler über das gesamte Kompetenzspektrum.

Im Durchschnitt der OECD-Länder verringerte sich im Zeitraum 2000-2009 die Punktzahldifferenz zwischen den leistungsstärksten und den leistungsschwächsten Schülerinnen und Schülern; einige Länder verbesserten zudem ihr Gesamtleistungsniveau. In Chile, Deutschland, Ungarn, Polen, Portugal und den Partnerländern Indonesien, Lettland und Liechtenstein verbesserte sich das Gesamtleistungsniveau, während die Leistungsvarianz abnahm. In vielen Fällen war dies das Ergebnis von bei den leistungsschwächeren Schülerinnen und Schülern erzielten Verbesserungen.

Die Leistungsunterschiede zwischen Jungen und Mädchen im Bereich Lesekompetenz verringerten sich im Zeitraum 2000-2009 in keinem Land.

Der Leistungsabstand zwischen Jungen und Mädchen im Bereich Lesekompetenz vergrößerte sich im Zeitraum 2000-2009 in Israel, Korea, Portugal, Frankreich und Schweden sowie in den Partnerländern und -volkswirtschaften Rumänien, Hongkong (China), Indonesien und Brasilien. Die Tatsache, dass Mädchen im Bereich Lesekompetenz besser abschneiden als Jungen, ist am deutlichsten am jeweiligen Anteil der Jungen und der Mädchen zu erkennen, die das Basisniveau, Kompetenzstufe 2, nicht erreichen. Im OECD-Durchschnitt beträgt der Anteil der unter Stufe 2 liegenden Jungen 24\%, während er sich bei den Mädchen nur auf 12\% beläuft. Der Anteil der Mädchen, deren Leistungen den Anforderungen von Stufe 2 nicht genügen, verringerte sich im Zeitraum 20002009 um 2 Prozentpunkte, während der entsprechende Anteil der Jungen im selben Zeitraum unverändert blieb.

Im OECD-Raum erhöhte sich der Prozentsatz der Schülerinnen und Schüler mit Migrationshintergrund im Zeitraum 2000-2009 durchschnittlich um 2 Prozentpunkte. Der Leistungsabstand zwischen Schülerinnen und Schülern mit und ohne Migrationshintergrund blieb in diesem Zeitraum etwa gleich. In einigen Ländern war jedoch eine erhebliche Verringerung des Leistungsvorsprungs der Schülerinnen und Schüler ohne Migrationshintergrund zu beobachten. In Belgien, der Schweiz und Deutschland verringerte sich die Differenz infolge von Verbesserungen der Lesekompetenz in der Gruppe der Schülerinnen und Schüler mit Migrationshintergrund um 28-38 Punkte. Die Differenz ist in diesen Ländern aber immer noch relativ groß.

Im OECD-Durchschnitt blieben die Gesamtleistungen in Mathematik im Zeitraum 2003-2009 unverändert, ebenso wie in den Naturwissenschaften im Zeitraum 2006-2009.

In Mathematik verbesserten die Schülerinnen und Schüler ihre Leistungen in Mexiko, der Türkei, Griechenland, Portugal, Italien, Deutschland und den Partnerländern Brasilien und Tunesien ganz erheblich, während bei den Schülerinnen und Schülern in der Tschechischen Republik, Irland, Schweden, Frankreich, Belgien, den Niederlanden, Dänemark, Australien und Island ein Leistungsabfall zu verzeichnen war. In den 28 OECD-Ländern, deren Ergebnisse bei den Erhebungen der Jahre 2003 und 2009 vergleichbar sind, blieb der durchschnittliche Anteil der Schülerinnen und Schüler, die in Mathematik unterhalb von Kompetenzstufe 2 lagen, in diesem Zeitraum mehr oder minder gleich bzw. verringerte sich nur geringfügig von 21,6\% auf 20,8\%. In der Gruppe der OECD- 
Länder, in denen 2003 mehr als die Hälfte der Schülerinnen und Schüler mit ihren Mathematikleistungen unter Kompetenzstufe 2 lag, gelang Mexiko 2009 eine Verringerung dieses Anteils um 15 Prozentpunkte von 66\% auf $51 \%$, während sich dieser Anteil in der Türkei im selben Zeitraum von $52 \%$ auf $42 \%$ reduzierte. Der Prozentsatz der leistungsstärksten Schülerinnen und Schüler in Mathematik hat sich in diesen 28 OECD-Ländern unterdessen leicht von 14,7\% im Jahr 2003 auf 13,4\% im Jahr 2009 verringert. Den stärksten Anstieg des Anteils der leistungsstärksten Schülerinnen und Schüler - 4 Prozentpunkte - erzielte Portugal.

Im Bereich Naturwissenschaften sind in 11 der 56 Länder, die 2006 und 2009 an PISA teilgenommen haben, Verbesserungen der Schülerleistungen festzustellen. Die Türkei konnte z.B. in nur drei Jahren einen Anstieg um 30 Punkte verzeichnen, was fast der Hälfte einer Kompetenzstufe entspricht. In der Türkei ging zudem der Prozentsatz der Schülerinnen und Schüler, die in Naturwissenschaften unter Kompetenzstufe 2 lagen, um nahezu 17 Prozentpunkte - von $47 \%$ auf $30 \%$ - zurück. In Portugal, Chile, den Vereinigten Staaten, Norwegen, Korea und Italien verringerte sich der Anteil der leistungsschwächsten Schülerinnen und Schüler im Bereich Naturwissenschaften um mindestens rd. 5 Prozentpunkte, ebenso wie in den Partnerländern Katar, Tunesien, Brasilien und Kolumbien. In fünf Ländern sank das Leistungsniveau in den Naturwissenschaften ganz erheblich.

Im Durchschnitt der OECD-Länder ist der Anteil der Schülerinnen und Schüler, die eigenen Angaben zufolge täglich zum Vergnügen lesen, um 5 Prozentpunkte gesunken.

Hinsichtlich der Freude der Schüler am Lesen hat sich die Situation in der Regel verschlechtert, besonders bei den Jungen, was darauf hindeutet, dass es für die Schulen eine große Herausforderung darstellt, den Schülerinnen und Schülern Leseaktivitäten zu bieten, die 15-Jährige als zweckdienlich und interessant betrachten. Im OECD-Durchschnitt sank der Prozentsatz der Schülerinnen und Schüler, die eigenen Angaben zufolge täglich zum Vergnügen lesen, zwischen 2000 und 2009 von 69\% auf 64\%. Dagegen waren bei den Lehrer-Schüler-Beziehungen und beim Unterrichtsklima im Allgemeinen positive Veränderungen oder zumindest keine Verschlechterungen festzustellen, wie viele erwartet hatten. Ganz allgemein haben die Schülerinnen und Schüler größeres Vertrauen darin, dass ihnen von den Lehrkräften geholfen wird. In den 26 OECD-Ländern, die an beiden Erhebungen teilgenommen haben, stimmten im Jahr 2000 74\% der Schülerinnen und Schüler der Aussage "wenn ich zusätzliche Hilfe brauche, bekomme ich sie von meinen Lehrern/Lehrerinnen" oder "die meisten Lehrer/Lehrerinnen behandeln mich fair" eher oder ganz zu, während dies 2009 für 79\% der Schülerinnen und Schüler der Fall war. Auch bei den verschiedenen Aspekten der Unterrichtsdisziplin war insgesamt eine Verbesserung zu verzeichnen. Es gibt somit keine Befunde, die die Auffassung rechtfertigen würden, dass die Schülerinnen und Schüler immer weniger Interesse an der Schule zeigen. 



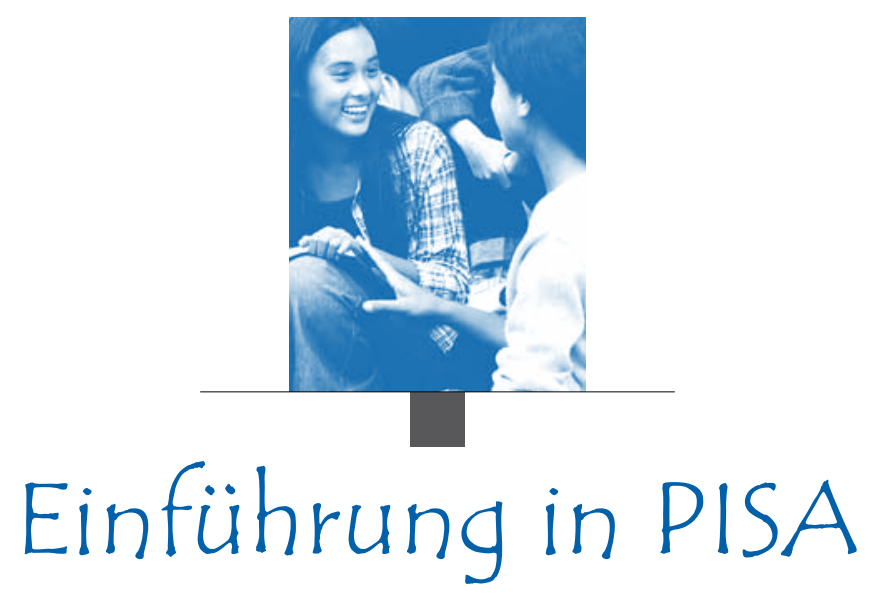

\section{DIE PISA-ERHEBUNGEN}

Sind die Schülerinnen und Schüler gut auf die Herausforderungen der Zukunft vorbereitet? Können sie analysieren, logisch denken und ihre Ideen effektiv kommunizieren? Haben sie Interessen entwickelt, die sie ihr ganzes Leben hindurch als produktive Mitglieder von Wirtschaft und Gesellschaft weiterverfolgen können? Die Internationale Schulleistungsstudie der OECD (PISA) sucht durch ihre im Dreijahresturnus durchgeführten Erhebungen der Kenntnisse und Fähigkeiten 15-jähriger Schülerinnen und Schüler aus OECD-Ländern und Partnerländern/-volkswirtschaften in wichtigen Grundbildungsbereichen Antworten auf diese Fragen zu liefern. Die an PISA teilnehmenden Länder vereinen fast $90 \%$ der Weltwirtschaft auf sich¹.

PISA evaluiert, inwieweit Schülerinnen und Schüler gegen Ende ihrer Pflichtschulzeit Kenntnisse und Fähigkeiten erworben haben, die für eine volle Teilhabe am Leben moderner Gesellschaften unerlässlich sind, wobei der Schwerpunkt auf Lesekompetenz, Mathematik und Naturwissenschaften liegt.

Inzwischen ist die vierte Erhebungsrunde von PISA abgeschlossen. Nach einer detaillierten Untersuchung von jedem der drei Schwerpunktbereiche von PISA - Lesekompetenz, Mathematik und Naturwissenschaften - in den Jahren 2000, 2003 und 2006 beginnt mit der Erhebung 2009 ein neuer Zyklus, wobei wieder die Lesekompetenz im Mittelpunkt steht, die diesmal allerdings anhand von Methoden beurteilt wird, die den Veränderungen Rechnung tragen, zu denen es in diesem Bereich seit dem Jahr 2000 gekommen ist, z.B. der zunehmenden Prävalenz digitaler Texte.

PISA 2009 bietet die bisher umfassendste und weitreichendste internationale Erhebung der Lesekompetenzen von Schülerinnen und Schülern. Die Studie untersucht nicht allein die Kenntnisse und Fähigkeiten der Schülerinnen und Schüler im Bereich Lesekompetenz, sondern auch ihre diesbezüglichen Einstellungen und Lernstrategien. PISA 2009 aktualisiert außerdem die Beurteilung der Schülerleistungen in Mathematik und Naturwissenschaften.

Die Erhebung konzentriert sich auf die Fähigkeit der Jugendlichen, ihre Kenntnisse und Fertigkeiten zur Bewältigung alltäglicher Herausforderungen einzusetzen. Diese Orientierung spiegelt eine Veränderung in den Zielen der Lehrpläne wider, in denen es zunehmend darum geht, wie die Schülerinnen und Schüler ihr Schulwissen konkret nutzen können, und nicht mehr nur um die Fähigkeit zur Wiedergabe des Gelernten. Zu den Besonderheiten von PISA gehören:

- Politikorientierung, wobei Daten über die Lernergebnisse der Schülerinnen und Schüler mit Informationen über deren Merkmale und über wichtige Faktoren verknüpft werden, die ihr Lernen innerhalb und außerhalb der Schule beeinflussen, um Unterschiede bei den Leistungsstrukturen hervorzuheben und zu untersuchen, wodurch sich Schülerinnen und Schüler, Schulen und Bildungssysteme, die hohe Leistungen erbringen, auszeichnen.

- Ein innovatives Konzept der Grundbildung, das sich auf die Kapazität der Schülerinnen und Schüler bezieht, Kenntnisse und Fertigkeiten in wichtigen Fächern zur Definition, Interpretation und Lösung von Problemen zu nutzen, sowie auf ihre Fähigkeit, zu analysieren, logisch zu denken und effektiv zu kommunizieren.

- Relevanz für das lebenslange Lernen, weil bei PISA nicht nur die Kompetenzen der Schülerinnen und Schüler in den Schulfächern erfasst, sondern auch Informationen über ihre Lernmotivation, Selbsteinschätzungen und Lernstrategien erhoben werden. 
- Regelmäßigkeit, dank der die Länder ihre Fortschritte bei der Verwirklichung entscheidender Lernziele beobachten können.

- Große geografische Reichweite und kooperativer Charakter, wie bei PISA 2009 durch die Teilnahme von allen 34 OECD-Mitgliedstaaten und 41 Partnerländern und -volkswirtschaften ${ }^{2}$ verdeutlicht wird.

Die Relevanz der von PISA gemessenen Kenntnisse und Fertigkeiten wird durch Untersuchungen bestätigt, die den Werdegang von Jugendlichen in den Jahren nach ihrer PISA-Teilnahme weiterverfolgten. Längsschnittstudien in Australien, Kanada und der Schweiz lassen einen engen Zusammenhang zwischen der Leistung im Bereich Lesekompetenz beim PISA-Test im Alter von 15 Jahren und dem späteren Bildungs- und Arbeitsmarkterfolg erkennen (vgl. Band I, Kapitel 2) ${ }^{3}$.

Die Rahmenkonzepte für die Evaluierung der Lesekompetenz sowie der mathematischen und naturwissenschaftlichen Grundbildung im Jahr 2009 sind in PISA 2009 Assessment Framework: Key Competencies in Reading, Mathematics and Science (OECD, 2009) ausführlich beschrieben.

Die Entscheidungen über Umfang und Art der Leistungsmessung und der Hintergrundbefragung in PISA werden von führenden Experten in den Teilnehmerstaaten getroffen. Die Regierungen geben Orientierungen für diese Entscheidungen auf der Basis der ihnen gemeinsamen bildungspolitischen Interessen. Es werden erhebliche Ressourcen und Anstrengungen darauf verwendet, bei dem Erhebungsmaterial kulturelle und sprachliche Breite sowie Ausgewogenheit zu gewährleisten. Für die Erstellung der Tests, Übersetzungsverfahren, Stichprobenauswahl und Datenerhebung werden strenge Mechanismen der Qualitätssicherung eingesetzt. Daher erreichen die Ergebnisse der PISA-Studie einen hohen Grad an Validität und Reliabilität.

Politische Entscheidungsträger in aller Welt nutzen mit PISA gewonnene Ergebnisse zur Beurteilung der Kenntnisse und Fähigkeiten der Schülerinnen und Schüler ihres Landes im Vergleich zu denen der Schüler in anderen Teilnehmerländern. PISA zeigt anhand dessen, was die Schüler in den Ländern mit den besten Ergebnissen in den Bereichen Lesekompetenz, Mathematik und Naturwissenschaften leisten können, was im Bildungswesen möglich ist. PISA wird außerdem zur Beurteilung des Tempos der Bildungsfortschritte verwendet, indem politische Entscheidungsträger die Möglichkeit erhalten, zu überprüfen, inwiefern im eigenen Land beobachtete Leistungsveränderungen denen anderer Länder entsprechen. In einer wachsenden Zahl von Ländern wird PISA auch genutzt, um politische Vorgaben festzulegen, die sich an in anderen Systemen erreichten, messbaren Zielen orientieren, um Forschung und gegenseitige Lernprozesse zur Identifizierung von Politikinstrumenten anzustoßen und die Bildungswege zur Verbesserung der Bildungsergebnisse zu reformieren. Die PISA-Studie kann zwar keine Kausalzusammenhänge zwischen Ressourcen, Prozessen und Bildungsergebnissen identifizieren, jedoch vermag das Programm die Kernelemente hervorzuheben, die Bildungssystemen gemeinsam sind oder sie unterscheiden, und diese Erkenntnisse Pädagogen, politischen Entscheidungsträgern und der breiten Öffentlichkeit mitzuteilen.

\section{DER ERSTE BERICHT ÜBER DIE PISA-ERHEBUNG 2009}

Dieser Band ist der fünfte von sechs Bänden, in denen die ersten internationalen Ergebnisse der PISA-Erhebung 2009 vorgestellt werden. Er liefert einen Überblick über Schülerleistungen in Lesekompetenz, Mathematik und Naturwissenschaften von PISA 2000 bis PISA 2009. Dieser Band verfolgt die Entwicklung der Bildungserträge im Zeitverlauf sowie Veränderungen bei Faktoren, die Einfluss auf die Leistungen von Schülern und Schulen ausüben, wie dem Hintergrund der Schüler sowie Schulmerkmalen und -praktiken.

Die anderen Bände befassen sich mit folgenden Themen:

- Band I, Was Schülerinnen und Schüler wissen und können: Schülerleistungen in Lesekompetenz, Mathematik und Naturwissenschaften, gibt einen Überblick über die Schülerleistungen bei der PISA-Erhebung 2009, wobei mit dem Schwerpunktthema Lesekompetenz begonnen wird und dann die Leistungen in Mathematik und Naturwissenschaften dargestellt werden. Dabei werden die Ergebnisse im Bereich Lesekompetenz zunächst in den Kontext der Definitions-, Messungs- und Darstellungsmethoden für die Schülerleistungen gestellt; anschließend wird untersucht, wozu die Schülerinnen und Schüler in diesem Bereich in der Lage sind. Nach einer Zusammenfassung der Leistungen in Lesekompetenz untersucht dieser Band, wie die Schülerleistungen auf Subskalen zu drei Aspekten der Lesekompetenz variieren. Dann werden die Ergebnisse nach unterschiedlichen Textformaten aufgeschlüsselt und geschlechtsspezifische Unterschiede im Bereich Lesekompetenz berücksichtigt, sowohl im Allgemeinen als auch für verschiedene Leseaspekte und Textformate. Bei allen Vergleichen der Ergebnisse unterschiedlicher Bildungssysteme müssen die sozialen und wirtschaftlichen Verhältnisse der jeweiligen Länder und die Ressourcen, die sie für die Bildung aufwenden, berücksichtigt werden. Zu diesem Zweck werden die 


\section{Kasten V.A Hauptmerkmale von PISA 2009}

Inhalt

- Der Schwerpunktbereich von PISA 2009 war die Lesekompetenz. Außerdem wurden in der Erhebung die Leistungsbewertungen in Mathematik und Naturwissenschaften aktualisiert. Bei PISA werden die Kenntnisse der Schülerinnen und Schüler in diesen Bereichen nicht isoliert untersucht, sondern im Zusammenhang mit ihrer Fähigkeit, über die eigenen Kenntnisse und Erfahrungen zu reflektieren und diese auf realitätsnahe Fragen anzuwenden. Das Hauptaugenmerk gilt der Beherrschung von Prozessen, dem Verständnis von Konzepten sowie der Fähigkeit, innerhalb des jeweiligen Erhebungsbereichs mit verschiedenen Kontextsituationen umzugehen.

- In PISA 2009 wurde zum ersten Mal auch die Fähigkeit der 15-jährigen Schülerinnen und Schüler untersucht, digitale Texte zu lesen und zu verstehen und die so gewonnenen Kenntnisse anzuwenden.

\section{Methoden}

Etwa 470000 Schülerinnen und Schüler absolvierten stellvertretend für die rund 26 Millionen 15-Jährigen in den Schulen der 65 teilnehmenden Länder und Volkswirtschaften die Testrunde 2009. Etwa 50000 Schülerinnen und Schüler nahmen an einer zweiten Runde dieser Erhebung im Jahr 2010 teil, stellvertretend für etwa zwei Millionen 15-Jährige aus 10 zusätzlichen Partnerländern und -volkswirtschaften.

- Die teilnehmenden Schülerinnen und Schüler verbrachten jeweils zwei Stunden mit der Lösung von Papier- und Bleistiftaufgaben in den Bereichen Lesekompetenz, Mathematik und Naturwissenschaften. In 20 Ländern wurden den Schülerinnen und Schülern zusätzliche, am Computer zu lösende Fragen gestellt, um ihre Fähigkeit zum Lesen elektronischer Texte zu testen.

- PISA enthielt Fragen, bei denen die Schülerinnen und Schüler eigene Antworten formulieren mussten, sowie Multiple-Choice-Fragen. Diese waren in der Regel in Testeinheiten zusammengefasst, die jeweils auf geschriebenen Texten oder Grafiken aufbauten, so wie viele Texte oder Abbildungen, denen die Schülerinnen und Schüler auch im wirklichen Leben begegnen.

- Die Schülerinnen und Schüler beantworteten zudem einen Fragebogen, dessen Bearbeitung etwa 30 Minuten in Anspruch nahm. Darin ging es um ihren persönlichen Hintergrund, ihre Lerngewohnheiten, ihre Einstellung zum Lesen sowie um ihr Engagement und ihre Motivation.

- Die Schulleitungen beantworteten einen Fragebogen zu ihrer Schule, in dem um Angaben zu deren demografischen Merkmalen sowie um eine Beurteilung der Qualität des Lernumfelds an ihrer Schule gebeten wurde.

\section{Ergebnisse}

Die Ergebnisse von PISA 2009 bieten:

- ein Profil der Kenntnisse und Fähigkeiten von 15-Jährigen im Jahr 2009, bestehend aus einer detaillierten Beurteilung im Bereich Lesekompetenz und einer aktualisierten Bewertung für die Bereiche Mathematik und Naturwissenschaften;

- kontextbezogene Indikatoren, mit denen ein Zusammenhang zwischen den Leistungsergebnissen und den Schüler- und Schulmerkmalen hergestellt wird;

- eine Beurteilung des Engagements der Schülerinnen und Schüler in Leseaktivitäten sowie ihrer Kenntnis und Nutzung unterschiedlicher Lernstrategien;

- eine Wissensbasis für Analysen und Forschung im Bildungsbereich und

- Trenddaten über Veränderungen bei den Kenntnissen und Fertigkeiten der Schülerinnen und Schüler in Lesekompetenz, Mathematik und Naturwissenschaften, in ihrer Einstellung und bei den sozioökonomischen Indikatoren sowie den Auswirkungen mancher Indikatoren auf die Leistungsergebnisse.

\section{Künftige Erhebungen}

- Bei PISA 2012 wird erneut die mathematische Grundbildung den Schwerpunktbereich der Erhebung bilden, während bei PISA 2015 wieder die naturwissenschaftliche Grundbildung im Mittelpunkt stehen wird. Danach beginnt ein neuer Zyklus mit der Lesekompetenz.

- Künftige Erhebungen werden mehr Betonung auf die Fähigkeit der Schülerinnen und Schüler legen, digitale Texte zu lesen und zu verstehen sowie in digitaler Form dargebotene Probleme zu lösen, womit der Bedeutung der Informations- und Kommunikationstechnologien in der modernen Gesellschaft Rechnung getragen wird. 
Ergebnisse in Band I auch im wirtschaftlichen und sozialen Kontext der einzelnen Länder betrachtet. Abschließend werden die Ergebnisse der Schülerinnen und Schüler in Mathematik und Naturwissenschaften beschrieben.

Band II, Potenziale nutzen und Chancengerechtigkeit sichern: Sozialer Hintergrund und Schülerleistungen, beginnt mit einer eingehenden Untersuchung der in Band I, Was Schülerinnen und Schüler wissen und können, aufgezeigten Leistungsvarianz und befasst sich insbesondere mit der Frage, wie die Gesamtvarianz der Schülerleistungen mit Unterschieden bei den Ergebnissen verschiedener Schulen in Zusammenhang steht. Anschließend wird untersucht, in welcher Weise Faktoren wie sozioökonomischer Hintergrund und Migrationsstatus die Schülerund die Schulleistungen beeinflussen und welche Rolle die Bildungspolitik dabei spielen kann, den Effekt dieser Faktoren abzumildern.

- Band III, Learning to learn: Student Engagement, Strategies und Practices (nur auf Englisch verfügbar), untersucht die gesammelten Informationen zum Leseengagement der Schülerinnen und Schüler sowie zu ihrer Einstellung zu Lesen und Lernen. Er beschreibt die Motivation, das Engagement und die Strategien der 15-Jährigen hinsichtlich des Lernens.

- Band IV, Was macht eine Schule erfolgreich? Lernumfeld und schulische Organisation in PISA, betrachtet die Zusammenhänge zwischen verschiedenen Merkmalen auf Schüler-, Schul- und Systemebene einerseits und Bildungsqualität sowie Chancengerechtigkeit andererseits. Hier wird versucht, Lösungsansätze aufzuzeigen, um den Schulen und den Bildungssystemen dabei zu helfen, das Leistungsniveau anzuheben und gleichzeitig die Auswirkungen des sozioökonomischen Hintergrunds auf die Schülerleistungen zu mindern und so für mehr Chancengerechtigkeit in der Bildung zu sorgen.

- Band VI, Students On Line: Reading and Using Digital Information (nur auf Englisch verfügbar), erklärt, wie die Schülerleistungen im Bereich der digitalen Lesekompetenz gemessen und dargestellt werden, und analysiert, wozu die Schülerinnen und Schüler in den 20 teilnehmenden Ländern in diesem Bereich in der Lage sind.

Alle in der Analyse zitierten Datentabellen sind am Ende des jeweiligen Bandes beigefügt. In jedem Band findet sich auch eine Anleitung für den Leser, die Hinweise darauf gibt, wie die Tabellen und Abbildungen zu diesem Bericht zu interpretieren sind.

Technische Anhänge erläutern die Konstruktion der Fragebogenindizes, befassen sich mit Fragen der Stichprobenauswahl, beschreiben die Qualitätssicherungsverfahren und die zur Entwicklung der Erhebungsinstrumente eingesetzten Methoden und liefern Informationen zur Reliabilität des Kodierungsprozesses. Sie sind auf der OECDPISA-Website veröffentlicht (www.pisa.oecd.org). Viele der in den technischen Anhängen behandelten Fragen werden im PISA 2009 Technical Report (OECD, erscheint demnächst) eingehender erörtert.

\section{PISA-ZIELPOPULATION}

Um die Vergleichbarkeit der Ergebnisse in den verschiedenen Ländern zu gewährleisten, wurde in PISA großer Wert daraufgelegt, vergleichbare Zielpopulationen zu erfassen. Auf Grund länderspezifischer Unterschiede bei Art und Verbreitung von Einrichtungen des Elementarbereichs, des regulären Einschulungsalters sowie der Struktur des Bildungssystems sind Definitionen der Zielpopulation, die sich auf bestimmte Klassenstufen beziehen, für internationale Vergleiche ungeeignet. Für valide internationale Vergleiche von Schulleistungen müssen die Populationen mithin in Bezug auf ein Zielalter definiert werden. PISA bezieht sich auf alle Schülerinnen und Schüler, die zum Zeitpunkt der Erhebung im Alter von 15 Jahren und 3 Monaten bis zu 16 Jahren und 2 Monaten sind und die mindestens sechs Jahre formaler Bildung abgeschlossen haben, ganz gleich in welcher Art von Bildungseinrichtung sie eingeschrieben sind und unabhängig davon, ob es sich um eine Ganztags- oder Halbtagsschule, eine allgemein- oder berufsbildende Einrichtung, eine öffentliche oder private Schule oder eine Auslandsschule im betreffenden Land handelt. (Wegen einer operationellen Definition dieser Zielpopulation vgl. PISA 2009 Technical Report, OECD, erscheint demnächst). Dank der Wahl dieser Altersgruppe können bei PISA die Leistungen von Schülerinnen und Schülern vor Ende der Pflichtschulzeit in allen Ländern und im Zeitverlauf auf einer einheitlichen Basis verglichen werden.

Daher kann dieser Bericht Aussagen über die Kenntnisse und Fähigkeiten von Personen machen, die im gleichen Jahr geboren sind und im Alter von 15 Jahren noch zur Schule gehen, die aber über unterschiedliche inner- wie außerschulische Bildungserfahrungen verfügen.

Für die Definition der nationalen PISA-Zielpopulation und die möglichen Ausschlüsse von dieser Definition wurden strenge technische Standards festgelegt (wegen näherer Einzelheiten vgl. die PISA-Website www.pisa.oecd.org). Die 
Gesamtausschlussrate für ein Land sollte unter 5\% bleiben, um sicherzustellen, dass unter normalen Umständen etwaige Verzerrungen bei den Mittelwerten für die einzelnen Länder innerhalb einer Spanne von plus/minus 5 Skalenpunkten bleiben, d.h. in der Regel in der Größenordnung von zwei Standardfehlern der Stichprobe (Anhang A2). Der Ausschluss konnte auf Ebene der teilnehmenden Schulen oder der teilnehmenden Schüler innerhalb der Schulen erfolgen. Bei PISA gibt es mehrere Gründe für den Ausschluss einer Schule bzw. einer Schülerin oder eines Schülers. Ausschlüsse auf Schulebene können sich dadurch erklären, dass die betreffenden Schulen in einer kleinen, abgelegenen Region liegen und daher schwer erreichbar sind, zu klein sind oder wegen organisatorischer oder operationeller Faktoren nicht berücksichtigt werden können. Zu Ausschlüssen auf Schülerebene kann es im Fall kognitiver Behinderungen oder einer beschränkten Beherrschung der Testsprache kommen.

In 29 der 65 an PISA 2009 teilnehmenden Länder belief sich die Ausschlussrate auf Schulebene auf weniger als $1 \%$, und in allen Ländern betrug sie weniger als 5\%. Bei Berücksichtigung des Ausschlusses von Schülerinnen und Schülern innerhalb der Schulen gemäß den international festgelegten Ausschlusskriterien erhöht sich die Ausschlussrate geringfügig. Dennoch liegt die Gesamtausschlussrate in 32 Teilnehmerländern noch unter $2 \%$, in 60 Teilnehmerländern unter $5 \%$ und in sämtlichen Ländern unter $7 \%$, mit Ausnahme Luxemburgs $(7,2 \%)$ und Dänemarks (8,6\%). In 15 von 34 OECD-Ländern belief sich die Ausschlussrate auf Schulebene auf weniger als 1\%, und in allen Ländern betrug sie weniger als 5\%. Bei zusätzlicher Berücksichtigung der Zahl der ausgeschlossenen Schüler innerhalb der Schulen lagen neun OECD-Länder unter 2\% und 25 unter 5\%. Die Beschränkungen in Bezug auf den Umfang der Ausklammerungen in PISA 2009 sind im Anhang A2 beschrieben.

\section{Ein Überblick über die PISA-Teilnehmerländer und -volkswirtschaften}

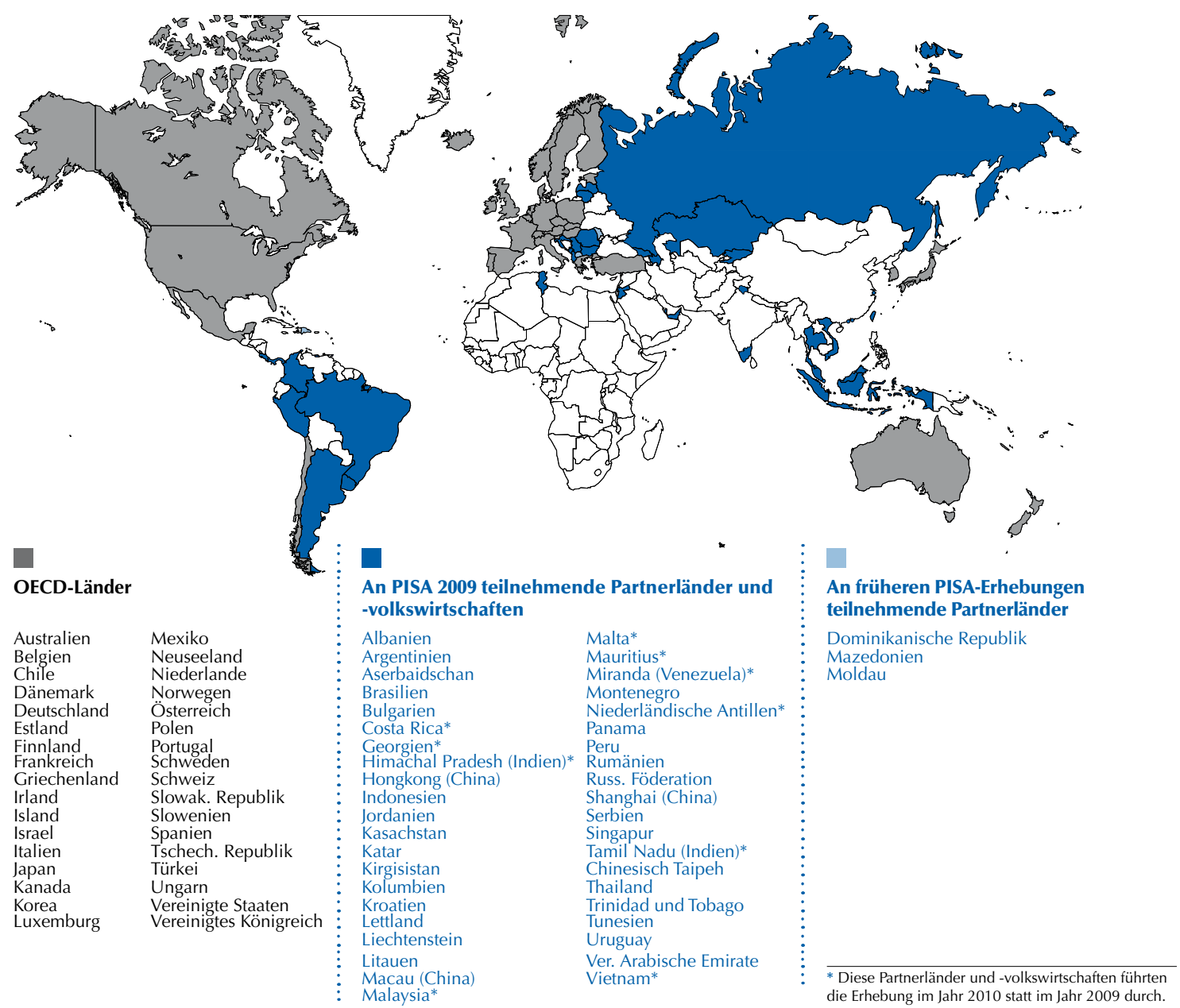


Aufbau und Umfang der Länderstichproben wurden jeweils so gewählt, dass eine größtmögliche Stichprobeneffizienz für die Schätzungen auf Schülerebene gewährleistet werden konnte. In den OECD-Ländern reichte der Stichprobenumfang von 4410 Schülern in Island bis zu 38250 Schülern in Mexiko. Länder mit umfangreichen Stichproben haben PISA häufig sowohl auf nationaler als auch auf regionaler Ebene durchgeführt (z.B. Australien, Belgien, Italien, Kanada, Mexiko, die Schweiz, Spanien und das Vereinigte Königreich). Die Stichprobenauswahl wurde auf internationaler Ebene überwacht und durch die Festlegung strenger Regeln in Bezug auf die Beteiligungsquote sowohl unter den von dem internationalen Kontraktor ausgewählten Schulen als auch unter den Schülerinnen und Schülern in diesen Schulen unterstützt, um sicherzustellen, dass die PISA-Ergebnisse die tatsächlichen Kompetenzen der 15-jährigen Schülerinnen und Schüler in den Teilnehmerländern widerspiegeln. Die Länder waren auch dazu verpflichtet, den Schülerinnen und Schülern den Test auf dieselbe Art und Weise darzulegen, um sicherzustellen, dass die Schülerinnen und Schüler dieselben Informationen vor dem Test und während seiner Bearbeitung erhalten (wegen näherer Einzelheiten, vgl. Anhang A4).

\section{Anmerkungen}

1. Das BIP der an PISA 2009 teilnehmenden Länder entspricht 87\% des weltweiten BIP von 2007. Einige der in diesem Bericht erwähnten Einheiten werden als Partnervolkswirtschaften bezeichnet, weil es sich genau genommen nicht um nationale Einheiten handelt.

2. An der PISA-Erhebung 2009 nahmen ursprünglich 31 Partnerländer und -volkswirtschaften teil, zehn weitere Partnerländer und -volkswirtschaften nahmen an einer zweiten Erhebungsrunde teil.

3. G.N. Marks (2007); K. Bertschy, M.A. Cattaneo und S.C. Wolter (2009); OECD (2010). 


\section{Hinweise für den Leser}

\section{Die den Abbildungen zu Grunde liegenden Daten}

Die Daten, auf die sich dieser Band bezieht, sind Anhang B zu entnehmen, zusätzliche Informationen finden sich auf der PISA-Website unter www.pisa.oecd.org.

Fehlende Daten werden durch fünf Symbole ausgedrückt:

a Die Kategorie ist für das betreffende Land nicht anwendbar. Es gibt diesbezüglich keine Daten.

c Die Zahl der Beobachtungen reicht nicht aus, um verlässliche Schätzungen zu liefern (d.h. es gibt weniger als 30 Schüler bzw. weniger als 5 Schulen mit validen Daten).

m Es stehen keine Daten zur Verfügung. Entsprechende Daten wurden von dem betreffenden Land nicht übermittelt oder wurden zwar erhoben, später jedoch aus technischen Gründen aus der Publikation herausgenommen.

w Die Daten wurden auf Ersuchen des betreffenden Landes zurückgezogen oder nicht erhoben.

$x$ Die Daten sind in einer anderen Kategorie oder einer anderen Spalte der Tabelle enthalten.

\section{Erfassung der einzelnen Länder}

Diese Publikation enthält Daten über 65 Länder und Volkswirtschaften, wozu alle 34 OECD-Länder und 31 Partnerländer und -volkswirtschaften zählen (vgl. Abb. IV.A). Die Daten von zehn weiteren Partnerländern wurden ein Jahr später erhoben und werden 2011 veröffentlicht.

Die statistischen Daten für Israel wurden von den zuständigen israelischen Stellen bereitgestellt, die für sie verantwortlich zeichnen. Die Verwendung dieser Daten durch die OECD erfolgt unbeschadet des völkerrechtlichen Status der Golanhöhen, von Ost-Jerusalem und der israelischen Siedlungen im Westjordanland.

\section{Berechnung der internationalen Durchschnittswerte}

Für die meisten Indikatoren in diesem Bericht wurde der OECD-Durchschnitt errechnet. Der OECD-Durchschnitt entspricht dem arithmetischen Mittel der jeweiligen Länderschätzungen.

Der Leser sollte daher beachten, dass sich der Begriff "OECD-Durchschnitt" auf die in die jeweiligen Vergleiche einbezogenen OECD-Länder bezieht.

\section{Runden von Zahlen}

Wegen des Auf- und Abrundens einiger Zahlen in den Tabellen stimmt die Summe der Zahlen möglicherweise nicht immer mit der Gesamtsumme überein. Summen, Differenzen und Durchschnittswerte werden stets auf der Grundlage der exakten Zahlenwerte berechnet und erst danach auf- bzw. abgerundet.

Alle Standardfehler in dieser Publikation wurden bis auf zwei Dezimalstellen auf- oder abgerundet. Wenn der Wert 0,00 angegeben ist, bedeutet dies nicht, dass der Standardfehler bei null liegt, sondern dass er geringer ist als 0,005 .

\section{Darstellung der Schülerdaten}

PISA bezieht sich auf alle Schülerinnen und Schüler, die zum Zeitpunkt der Erhebung im Alter von 15 Jahren und 3 Monaten bis zu 16 Jahren und 2 Monaten sind und die mindestens sechs Jahre formaler Bildung abgeschlossen haben, ganz gleich in welcher Art von Bildungseinrichtung sie eingeschrieben sind und unabhängig davon, ob es sich um eine Ganztags- oder Halbtagsschule, eine allgemein- oder berufsbildende Einrichtung, eine öffentliche oder private Schule oder eine Auslandsschule im betreffenden Teilnehmerland handelt. 


\section{Darstellung der Schuldaten}

Die Leiterinnen und Leiter der Schulen, in denen Schülerinnen und Schüler am Test teilnahmen, haben durch Ausfüllen eines Schulfragebogens Informationen über die Merkmale ihrer jeweiligen Schule geliefert. Bei der Darstellung der Antworten der Schulleiterinnen und Schulleiter in dieser Publikation wurde eine Gewichtung in der Weise vorgenommen, dass ihre Zahl im richtigen Verhältnis zur Anzahl der 15-Jährigen in der betreffenden Schule steht.

\section{Fokussierung auf statistisch signifikante Unterschiede}

In diesem Band werden nur statistisch signifikante Unterschiede oder Veränderungen erörtert. Diese sind in den Abbildungen mit dunkleren Farbtönen und in den Tabellen mit Fettdruck gekennzeichnet. Wegen weiterer Informationen vgl. Anhang A3.

\section{Im Bericht verwendete Abkürzungen}

ESCS PISA-Index des wirtschaftlichen, sozialen und kulturellen Status

BIP Bruttoinlandsprodukt

ISCED Internationale Standardklassifikation des Bildungswesens

KKP Kaufkraftparitäten

S.D. Standardabweichung

S.E. Standardfehler

\section{Weiterführende Dokumentation}

Für weitere Informationen zu den in PISA verwendeten Erhebungsinstrumenten und Methoden vgI. PISA 2009 Technical Report (OECD, erscheint demnächst) sowie die PISA-Website (www.pisa.oecd.org).

Dieser Bericht verwendet den StatLinks-Service für OECD-Veröffentlichungen. Unter jeder Tabelle und Abbildung befindet sich ein URL-Link, der zu einer Datei im Excel-Format führt, die die zu Grunde liegenden Daten enthält. Diese URL sind stabil und bleiben im Zeitverlauf unverändert. Darüber hinaus können Leser der E-Books diese Links direkt anklicken, die Excel-Datei öffnet sich dann in einem neuen Fenster, wenn der Internet-Browser aktiviert ist. 


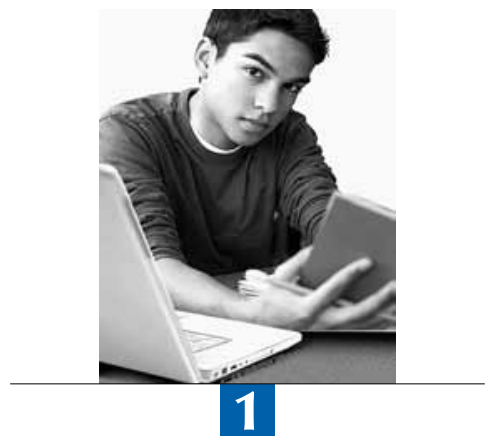

\section{Vergleich der Schülerleistungen im Zeitverlauf}

In diesem Kapitel wird beschrieben, wie bei PISA die Entwicklung der Schülerleistungen im Bereich Lesekompetenz zwischen der ersten Erhebung im Jahr 2000 und der jüngsten Erhebung im Jahr 2009 gemessen wurden. Da bei diesen beiden Erhebungen die Lesekompetenz im Mittelpunkt stand, ist es möglich, detaillierte Vergleiche in Bezug darauf anzustellen, wie sich die Schülerleistungen im Bereich Lesekompetenz im Zeitraum 2000-2009 verändert haben. In diesem Kapitel werden zudem die Methoden erörtert, die für die Erfassung der Trends bei den Schülerleistungen in den Bereichen Mathematik und Naturwissenschaften verwendet wurden. 
PISA 2009 ist die vierte umfassende Erhebung im Bereich Lesekompetenz seit dem Start der PISA-Studie im Jahr 2000, die dritte Erhebung im Bereich Mathematik seit dem ersten umfassenden Mathematiktest von 2003 und die zweite Erhebung im Bereich Naturwissenschaften seit dem ersten umfassenden Naturwissenschaftstest von 2006.

Sowohl bei PISA 2000 als auch bei PISA 2009 lag der Schwerpunkt auf der Lesekompetenz, daher ist es möglich, detaillierte Vergleiche in Bezug darauf anzustellen, wie sich die Schülerleistungen im Bereich Lesekompetenz im Zeitraum 2000-2009 verändert haben. Die Vergleiche im Zeitverlauf in den Bereichen Mathematik und Naturwissenschaften sind begrenzter, da in diesen beiden Bereichen in den neun Jahren seit dem Start der PISAStudie noch keine zwei umfassenden Tests durchgeführt wurden.

\section{Kasten V.1.1 Bei der Interpretation von Trends ist Vorsicht geboten}

- Die Methoden, die bei internationalen Bildungsstudien zur Bestimmung von Leistungstrends eingesetzt werden, sind komplex (Gebhardt und Adams, 2007). Um bei verschiedenen Studien die Vergleichbarkeit der Messung der Schülerleistungen im Bereich Lesekompetenz zu gewährleisten, wird in jeder Studie eine Reihe gemeinsamer Erhebungsitems verwendet. Die begrenzte Anzahl solcher Items erhöht jedoch das Risiko von Messfehlern. Daher ist das Konfidenzband für Vergleiche im Zeitverlauf weiter als für Daten eines einzigen Jahres und sollten nur solche Veränderungen als robust betrachtet werden, die in diesem Band als statistisch signifikant gekennzeichnet sind ${ }^{1}$.

- Einige Länder wurden aus methodischen Gründen nicht in den Vergleich zwischen PISA 2000, PISA 2003, PISA 2006 und PISA 2009 einbezogen. Die niederländische Stichprobe entsprach im Jahr 2000 wegen der zu geringen Beteiligungsquote nicht den PISA-Standards, weshalb für die Niederlande in PISA 2000 keine Mittelwerte angegeben wurden. In Luxemburg wurden die Testverfahren 2003 im Vergleich zu 2000 stark geändert, weshalb nur die Ergebnisse von PISA 2003, PISA 2006 und PISA 2009 vergleichbar sind². Die Stichproben für das Vereinigte Königreich wurden 2000 und 2003 den in Bezug auf die Beteiligungsquote geltenden Regeln von PISA nicht gerecht, weshalb die Daten für das Vereinigte Königreich nicht mit denen anderer Länder vergleichbar sind ${ }^{3}$. Aus den Vereinigten Staaten liegen für 2006 keine Ergebnisse zur Lesekompetenz vor ${ }^{4}$. Österreich hat Änderungen an der Gewichtung seiner Stichproben für PISA 2000 vorgenommen, um Vergleiche mit den darauffolgenden PISA-Erhebungen zu ermöglichen ${ }^{5}$. Bei der PISA-Erhebung 2009 kam es in Österreich zu einem Streit zwischen den Lehrergewerkschaften und der Bildungsministerin, der zu einem PISA-Boykott führte, welcher erst nach der ersten Testwoche aufgehoben wurde. Auf Grund des Boykotts musste die OECD identifizierbare Fälle aus der Datenreihe entfernen. Wenngleich die österreichischen Daten nach der Entfernung dieser Fälle die technischen Standards von PISA 2009 erfüllen, wurden die Testbedingungen durch eine negative Einstellung zu Bildungserhebungen beeinträchtigt, was die Motivation der Schüler bei der Beantwortung der PISA-Aufgaben ungünstig beeinflusst haben könnte. Die Vergleichbarkeit der Erhebungsdaten des Jahres 2009 mit denen früherer PISAErhebungen kann dementsprechend nicht gewährleistet werden, weshalb die österreichischen Daten aus den Trendvergleichen ausgeschlossen wurden.

Einige Länder haben nicht an allen PISA-Erhebungen teilgenommen. Beim Vergleich der Trends im Bereich Lesekompetenz sind in diesem Band die 38 Länder mit validen Ergebnissen aus den PISA-Erhebungen 2000 und 2009 berücksichtigt ${ }^{6}$. Beim Vergleich der Trends in Mathematik werden 39 Länder mit validen Ergebnissen aus den Erhebungen der Jahre 2003 und 2009 betrachtet. Die Mathematikergebnisse von PISA 2000 bleiben ausgeklammert, da die erste umfassende Erhebung im Bereich Mathematik erst 2003 erfolgte. Desgleichen können die Leistungen in Naturwissenschaften im Jahr 2009 nicht mit denen von PISA 2000 oder PISA 2003 verglichen werden, da der erste umfassende Naturwissenschaftstest erst 2006 stattfand. Folglich sind in den Vergleich der Trends in Naturwissenschaften die 56 Länder mit validen Ergebnissen aus den Erhebungen der Jahre 2006 und 2009 einbezogen. Anhang A5 enthält eine Liste der in dieser Trendanalyse berücksichtigten Länder.

In der Gruppe der OECD-Länder nehmen die Slowakische Republik und die Türkei erst seit 2003 an der PISA-Studie teil, Chile und Israel beteiligten sich nicht an der PISA-Erhebung 2003, und Estland und Slowenien haben bisher nur 2006 und 2009 teilgenommen. Der unterschiedlichen Anzahl der OECD-Länder, die an den aufeinanderfolgenden PISA-Erhebungen teilgenommen haben, wird durch separate OECD-Durchschnittswerte Rechnung getragen, die 
Bezugspunkte für Trendvergleiche liefern. Für die Lesekompetenz ist der wichtigste Bezugspunkt der OECD-Durchschnitt der 26 OECD-Länder, die sowohl an PISA 2000 als auch an PISA 2009 teilgenommen haben; für Vergleiche, in die alle vier Erhebungen einbezogen werden, wird aber auch der Durchschnitt der 23 OECD-Länder angegeben, die sich an allen Erhebungen beteiligt haben. Im Bereich Mathematik können die Trends für den Durchschnitt von 28 OECD-Ländern ermittelt werden, für die sowohl aus PISA 2003 als auch aus PISA 2009 valide Ergebnisse vorliegen. Für 33 OECD-Länder können valide Ergebnisse aus den Naturwissenschaftstests von 2006 und 2009 verglichen werden. Anhang A5 liefert nähere Einzelheiten darüber, wie der OECD-Durchschnitt für die verschiedenen in diesem Band enthaltenen Trendvergleiche berechnet wurde.

Abbildung V.1.1 liefert einen Überblick über die Leistungstrends im Bereich Lesekompetenz. Die erste Spalte enthält Informationen darüber, ob die Schülerleistungen im Bereich Lesekompetenz in PISA 2009 über (blau), bei (keine farbliche Markierung) oder unter (grau) dem Durchschnitt der OECD-Länder lagen. Die Länder sind nach dem Umfang der Veränderung der Leistungen im Bereich Lesekompetenz im Zeitraum zwischen PISA 2000 und PISA

Abbildung V.1.1

\section{Überblick über die Veränderungen der Schülerleistungen im Bereich Lesekompetenz}

\begin{tabular}{|c|}
\hline $\begin{array}{l}\text { Mittelwert im Bereich Lesekompetenz } 2009 \text { statistisch signifikant über dem OECD-Durchschnitt. Die Veränderungen bei den Leseleistungen und beim Anteil } \\
\text { der Schüler auf oder über Kompetenzstufe } 5 \text { sind statistisch signifikant positiv. Die Veränderungen beim Anteil der Schüler unter Kompetenzstufe } 2 \text { und beim } \\
\text { Zusammenhang zwischen dem sozioökonomischen Hintergrund und den Leseleistungen sind statistisch signifikant negativ. }\end{array}$ \\
\hline $\begin{array}{l}\text { Mittelwert im Bereich Lesekompetenz weicht } 2009 \text { nicht statistisch signifikant vom OECD-Durchschnitt ab. Die Veränderungen bei den Leseleistungen, } \\
\text { beim Anteil der Schüler auf oder über Kompetenzstufe 5, beim Anteil der Schüler unter Kompetenzstufe } 2 \text { und beim Zusammenhang zwischen dem } \\
\text { sozioökonomischen Hintergrund und den Leseleistungen sind nicht statistisch signifikant. }\end{array}$ \\
\hline $\begin{array}{l}\text { Mittelwert im Bereich Lesekompetenz } 2009 \text { statistisch signifikant unter dem OECD-Durchschnitt. Die Veränderungen bei den Leseleistungen und beim Anteil } \\
\text { der Schüler auf oder über Kompetenzstufe } 5 \text { sind statistisch signifikant negativ. Die Veränderungen beim Anteil der Schüler unter Kompetenzstufe } 2 \text { und beim } \\
\text { Zusammenhang zwischen dem sozioökonomischen Hintergrund und den Leseleistungen sind statistisch signifikant positiv. }\end{array}$ \\
\hline
\end{tabular}

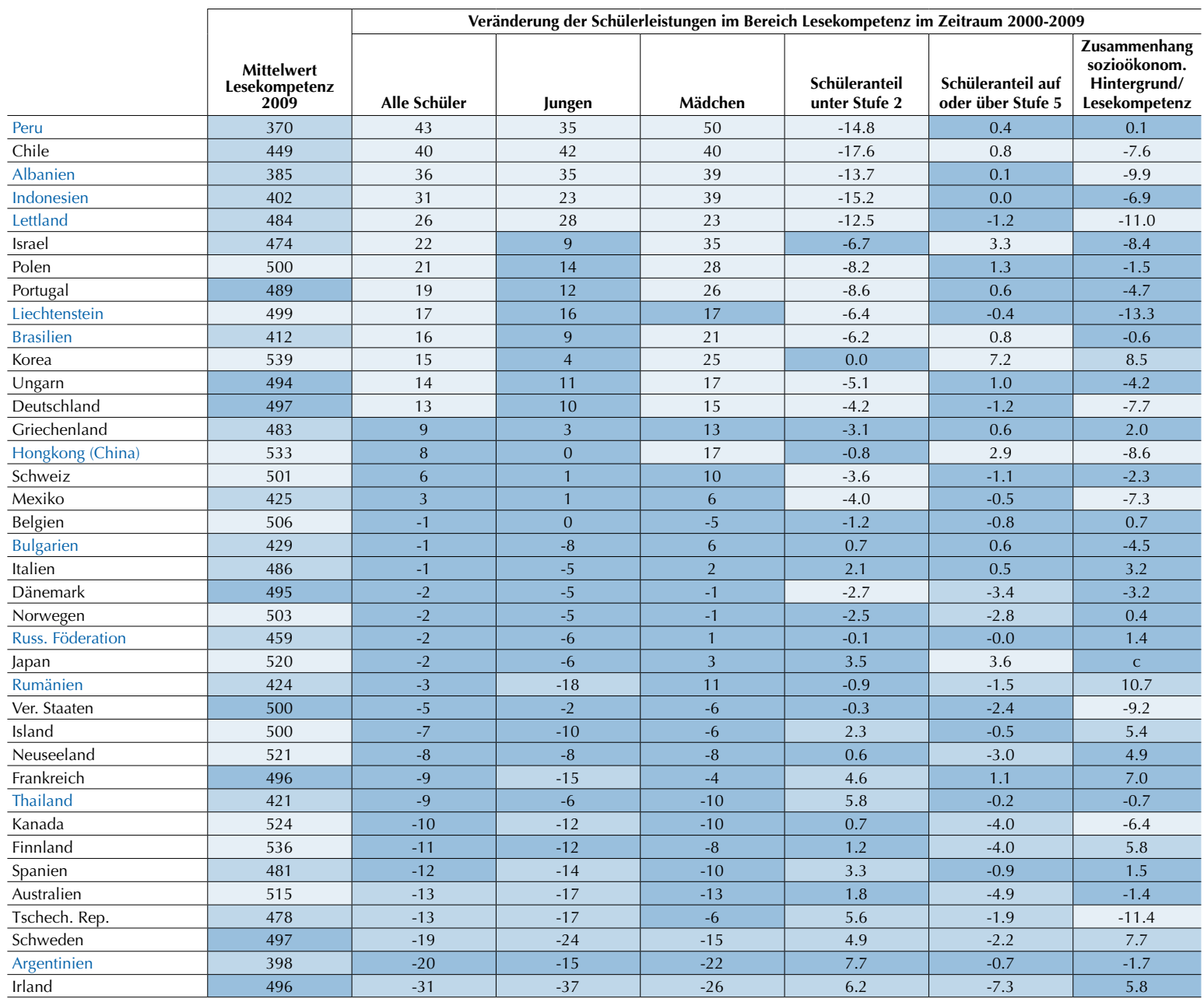

Die Länder sind in absteigender Reihenfolge nach dem Umfang der Veränderung der Leistungen im Bereich Lesekompetenz im Zeitraum 2000-2009 für alle Schüler angeordnet. Quelle: OECD, PISA-2009-Datenbank, Tabellen V.2.1, V.2.2, V.2.4 und V.4.3 
2009 angeordnet, der in der zweiten Spalte angegeben ist. Leistungssteigerungen sind blau, Leistungsrückgänge grau markiert. Keine farbliche Markierung bedeutet, dass keine statistisch signifikante Leistungsveränderung festzustellen war. Darüber hinaus werden in der Abbildung die Veränderungen der Schülerleistungen im Bereich Lesekompetenz für Jungen und Mädchen separat hervorgehoben und auch die Veränderungen des Anteils der leistungsschwächsten Schülerinnen und Schüler (unter Kompetenzstufe 2) und des Anteils der leistungsstärksten Schülerinnen und Schüler (auf Kompetenzstufe 5 oder 6) aufgezeigt. In der letzten Spalte werden die Veränderungen beim Zusammenhang zwischen dem sozioökonomischen Hintergrund der Schülerinnen und Schüler und ihren Leistungen angegeben, was Aufschluss darüber gibt, ob die Verteilungsgerechtigkeit der Bildungschancen zugenommen (wenn der Zusammenhang schwächer geworden ist) oder abgenommen hat (wenn der Zusammenhang stärker geworden ist) ${ }^{7}$. In allen Fällen steht eine blaue Markierung für eine positive Veränderung, eine graue Markierung für eine negative Veränderung, und keine farbliche Markierung bedeutet, dass keine statistisch signifikante Veränderung festzustellen war.

In mehreren Ländern haben sich die Schülerleistungen im Lauf der verschiedenen seit 2000 durchgeführten PISAErhebungen erheblich verbessert (Tabelle V.2.1). Alle diese Länder liefern Beispiele für Bildungssysteme, die bei der Verbesserung ihrer Ergebnisse erfolgreich waren (vgl. Kapitel 2). Dieser Band enthält kurze Beschreibungen einiger Bildungssysteme, die in PISA merkliche Verbesserungen der Schülerleistungen verzeichnen konnten. Die Artikel zu Korea (Kasten V.B) und Polen (Kasten V.C) finden sich zwischen Kapitel 1 und 2, die Artikel zu Portugal (Kasten V.D) und der Türkei (Kasten V.E) befinden sich zwischen Kapitel 3 und 4, der Artikel zu Chile (Kasten V.F) steht zwischen Kapitel 4 und 5, und der Artikel zu Brasilien (Kasten V.G) ist im Anschluss an Kapitel 5 zu finden.

Die einzelnen Schulsysteme unterscheiden sich in vielerlei Hinsicht, insbesondere im Hinblick auf ihr Gesamtleistungsniveau, den sozioökonomischen Hintergrund der Schüler und der Schulen, das Lernumfeld in den Schulen und die Organisation der Schulsysteme. Daher ist es wichtig, Veränderungen der Lernergebnisse im Zusammenhang mit den jeweiligen Merkmalen der Bildungssysteme zu interpretieren. In einigen Bildungssystemen, bei denen eine Verbesserung oder ein Rückgang ihrer Ergebnisse festzustellen war, können manche Veränderungen auf Veränderungen im demografischen Profil der Schülerinnen und Schüler zurückgeführt werden. So ist in manchen Ländern z.B. die Schülerpopulation während der letzten Jahre in sozioökonomischer Hinsicht heterogener geworden, was, wie in Band II, Potenziale nutzen und Chancengerechtigkeit sichern, aufgezeigt, mit Leistungsnachteilen verbunden sein kann, weshalb ein etwaiger Leistungsrückgang dort nicht zwangsläufig durch einen Rückgang der Qualität der erbrachten Bildungsdienstleistungen, sondern u.U. eher durch einen schwierigeren sozioökonomischen Kontext bedingt ist. Um derartigen Veränderungen Rechnung zu tragen, werden die beobachteten Veränderungen im Bereich Lesekompetenz zusammen mit Trendschätzungen erörtert, die um Veränderungen im demografischen und sozioökonomischen Profil der Schüler und Schulen berichtigt wurden. Genauere Beschreibungen der seit 2000 beobachteten Trends bei der Verteilungsgerechtigkeit der Bildungschancen und -erträge (Kapitel 4) und der Trends beim Lernumfeld (Kapitel 5) sind in diesem Band ebenfalls enthalten.

Anhang A1 liefert Einzelheiten darüber, wie die Leistungsskalen gleichgesetzt und die Trends ermittelt wurden. Anhang A6 liefert Einzelheiten darüber, wie die Leistungsskalen um den demografischen und sozioökonomischen Kontext berichtigt wurden. Insgesamt lässt das vorliegende Datenmaterial darauf schließen, dass die in diesem Band aufgezeigten Leistungstrends nicht durch methodische Entscheidungen beeinflusst sind und dass sie in den meisten Ländern nicht durch Veränderungen in der demografischen und sozioökonomischen Zusammensetzung der Schülerpopulation bedingt sind.

In diesem Band werden zudem die Trends in den Bereichen Mathematik und Naturwissenschaften erörtert, obwohl die Vergleiche im Zeitverlauf weitaus begrenzter sind (vgl. Kapitel 3). Die nachstehende Abbildung V.1.2 liefert einen Überblick über die Trends in allen drei Erhebungsbereichen. Die Länder sind nach ihren Ergebnissen im Bereich Lesekompetenz im Jahr 2009 angeordnet. Da die Ermittlung der Trends im Bereich Lesekompetenz für die meisten Länder über einen Neunjahreszeitraum, für einige aber über einen Sechs- bzw. Dreijahreszeitraum erfolgte, wurden die Trends auf Jahresbasis umgerechnet, um die Vergleichbarkeit zwischen den drei verschiedenen Bereichen zu gewährleisten ${ }^{8}$. Analog dazu wurden auch die Trends in den Bereichen Mathematik und Naturwissenschaften auf Jahresbasis umgerechnet, da sie für Mathematik über einen Sechs- bzw. Dreijahreszeitraum und für Naturwissenschaften über einen Dreijahreszeitraum ermittelt wurden. Obgleich die auf Jahresbasis umgerechneten Zahlen gewährleisten, dass der Umfang der Veränderungen zwischen den einzelnen Bereichen vergleichbar ist, steht bei den Trends in Lesekompetenz eine größere Variabilität zu erwarten, da sich Veränderungen in den Bildungssystemen über einen längeren Erhebungszeitraum stärker niederschlagen können. Dies war auch tatsächlich zu beobachten. 


\section{Überblick über die annualisierten Leistungstrends in den Bereichen Lesekompetenz, Mathematik und Naturwissenschaften}

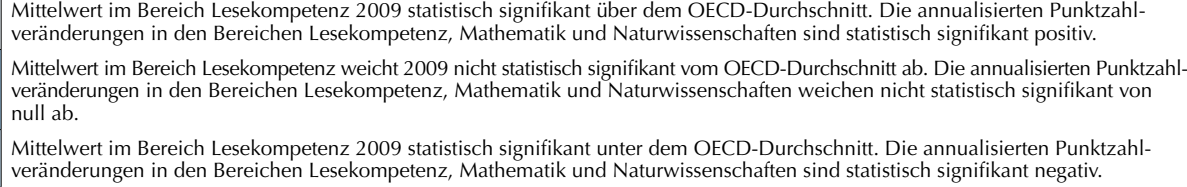

Mittelwert im Bereich Lesekompetenz 2009 statistisch signifikant über dem OECD-Durchschnitt. Die annualisierten Punktzahlveränderungen in den Bereichen Lesekompetenz, Mathematik und Naturwissenschaften sind statistisch signifikant positiv.

Mittelwert im Bereich Lesekompetenz weicht 2009 nicht statistisch signifikant vom OECD-Durchschnitt ab. Die annualisierten Punktzahlveränderungen in den Bereichen Lesekompetenz, Mathematik und Naturwissenschaften weichen nicht statistisch signifikant von null ab.

Mittelwert im Bereich Lesekompetenz 2009 statistisch signifikant unter dem OECD-Durchschnitt. Die annualisierten Punktzahlveränderungen in den Bereichen Lesekompetenz, Mathematik und Naturwissenschaften sind statistisch signifikant negativ.

\begin{tabular}{|c|c|c|c|c|c|}
\hline & \begin{tabular}{|c|} 
Mittelwert Lesekompetenz \\
2009
\end{tabular} & $\begin{array}{l}\text { Anzahl der Jahre, für die } \\
\text { PISA-Ergebnisse vorliegen }\end{array}$ & Lesekompetenz & Mathematik & Naturwissenschaften \\
\hline Korea & 539 & 9 & 1.6 & 0.7 & 5.3 \\
\hline Finnland & 536 & 9 & -1.2 & -0.6 & -3.1 \\
\hline Hongkong (China) & 533 & 8 & 1.0 & 0.7 & 2.3 \\
\hline Kanada & 524 & 9 & -1.1 & -0.9 & -1.9 \\
\hline Neuseeland & 521 & 9 & -0.9 & -0.7 & 0.5 \\
\hline Japan & 520 & 9 & -0.3 & -0.9 & 2.7 \\
\hline Australien & 515 & 9 & -1.5 & -1.7 & 0.1 \\
\hline Niederlande & 508 & 6 & -0.8 & -2.0 & -0.9 \\
\hline Belgien & 506 & 9 & -0.1 & -2.3 & -1.3 \\
\hline Norwegen & 503 & 9 & -0.2 & 0.5 & 4.4 \\
\hline Estland & 501 & 3 & 0.1 & -0.8 & -1.2 \\
\hline Schweiz & 501 & 9 & 0.7 & 1.2 & 1.7 \\
\hline Polen & 500 & 9 & 2.4 & 0.8 & 3.4 \\
\hline Island & 500 & 9 & -0.7 & -1.4 & 1.6 \\
\hline Ver. Staaten & 500 & 9 & -0.5 & 0.8 & 4.4 \\
\hline Liechtenstein & 499 & 9 & 1.9 & 0.0 & -0.7 \\
\hline Schweden & 497 & 9 & -2.1 & -2.5 & -2.7 \\
\hline Deutschland & 497 & 9 & 1.5 & 1.6 & 1.6 \\
\hline Irland & 496 & 9 & -3.4 & -2.6 & -0.1 \\
\hline Frankreich & 496 & 9 & -1.0 & -2.3 & 1.0 \\
\hline Chinesisch Taipeh & 495 & 3 & -0.3 & -2.1 & -4.0 \\
\hline Dänemark & 495 & 9 & -0.2 & -1.8 & 1.1 \\
\hline Ver. Königreich & 494 & 3 & -0.3 & -1.0 & -0.4 \\
\hline Ungarn & 494 & 9 & 1.6 & 0.0 & -0.4 \\
\hline Portugal & 489 & 9 & 2.1 & 3.5 & 6.2 \\
\hline Macau (China) & 487 & 6 & -1.8 & -0.3 & 0.1 \\
\hline Italien & 486 & 9 & -0.2 & 2.9 & 4.5 \\
\hline Lettland & 484 & 9 & 2.9 & -0.2 & 1.4 \\
\hline Slowenien & 483 & 3 & -3.8 & -1.0 & -2.4 \\
\hline Griechenland & 483 & 9 & 1.0 & 3.5 & -1.1 \\
\hline Spanien & 481 & 9 & -1.3 & -0.3 & -0.1 \\
\hline Tschech. Rep. & 478 & 9 & -1.5 & -3.9 & -4.1 \\
\hline Slowak. Rep. & 477 & 6 & 1.4 & -0.3 & 0.6 \\
\hline Kroatien & 476 & 3 & -0.5 & -2.4 & -2.3 \\
\hline Israel & 474 & 8 & 2.7 & 1.7 & 0.3 \\
\hline Luxemburg & 472 & 6 & -1.2 & -0.7 & -0.8 \\
\hline Litauen & 468 & 3 & -0.5 & -3.3 & 1.2 \\
\hline Türkei & 464 & 6 & 3.9 & 3.7 & 10.0 \\
\hline Russ. Föderation & 459 & 9 & -0.3 & -0.1 & -0.4 \\
\hline Chile & 449 & 8 & 5.0 & 3.2 & 3.1 \\
\hline Serbien & 442 & 6 & 5.0 & 0.9 & 2.4 \\
\hline Bulgarien & 429 & 8 & -0.2 & 4.9 & 1.7 \\
\hline Uruguay & 426 & 6 & -1.4 & 0.8 & -0.3 \\
\hline Mexiko & 425 & 9 & 0.4 & 5.5 & 2.1 \\
\hline Rumänien & 424 & 7 & -0.5 & 4.1 & 3.3 \\
\hline Thailand & 421 & 8 & -1.2 & 0.3 & 1.4 \\
\hline Kolumbien & 413 & 3 & 9.3 & 3.6 & 4.6 \\
\hline Brasilien & 412 & 9 & 1.7 & 5.0 & 5.0 \\
\hline Montenegro & 408 & 3 & 5.2 & 1.1 & -3.5 \\
\hline Jordanien & 405 & 3 & 1.5 & 0.9 & -2.2 \\
\hline Tunesien & 404 & 6 & 4.8 & 2.1 & 5.1 \\
\hline Indonesien & 402 & 8 & 3.9 & 1.9 & -3.6 \\
\hline Argentinien & 398 & 8 & -2.5 & 2.3 & 3.2 \\
\hline Albanien & 385 & 8 & 4.5 & $\mathrm{~m}$ & $\mathrm{~m}$ \\
\hline Katar & 372 & 3 & 19.8 & 16.7 & 10.0 \\
\hline Peru & 370 & 8 & 5.3 & $\mathrm{~m}$ & $\mathrm{~m}$ \\
\hline Aserbaidschan & 362 & 3 & 2.9 & -15.0 & -3.1 \\
\hline Kirgisistan & 314 & 3 & 9.8 & 6.9 & 2.5 \\
\hline
\end{tabular}

Die Länder sind in absteigender Reihenfolge nach dem Mittelwert im Bereich Lesekompetenz im Jahr 2009 angeordnet. Quelle: OECD, PISA-2009-Datenbank.

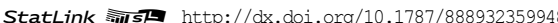


Die Ergebnisse sind für alle Länder aufgeführt, die an mindestens zwei Erhebungen teilgenommen haben. Die Anzahl der Jahre, für die Leistungstrends im Bereich Lesekompetenz ermittelt wurden, ist nach dem entsprechenden Mittelwert angegeben. Die Trends in Mathematik wurden über sechs Jahre ermittelt, wenn das betreffende Land seit mindestens 2003 teilgenommen hat, bzw. über drei Jahre, wenn es sich erst an den letzten beiden Erhebungen beteiligt hat. Alle Trends in Naturwissenschaften wurden für die drei Jahre zwischen 2006 und 2009 berechnet.

In der Gruppe der Länder, deren Ergebnisse bei oder über dem OECD-Durchschnitt lagen, war in Portugal eine Verbesserung in allen Erhebungsbereichen, in Korea und Polen sowohl in Lesekompetenz als auch in Naturwissenschaften, in Deutschland in Lesekompetenz und Mathematik, in Ungarn und Liechtenstein in Lesekompetenz und in Norwegen und in den Vereinigten Staaten in Naturwissenschaften festzustellen. 


\section{Anmerkungen}

1. Bei einem Vergleich von zwei verschiedenen Mittelwerten wird die Signifikanz ihrer Differenz in der Regel dadurch ermittelt, dass das Verhältnis der Differenz der Mittelwerte zum Standardfehler der Differenz der Mittelwerte berechnet wird. Ist der absolute Wert dieses Verhältnisses größer als 1,96, so liegt mit 95\%iger Wahrscheinlichkeit eine echte Differenz vor. Beim Vergleich von zwei Mittelwerten, die - wie bei den verschiedenen PISA-Studien - zu unterschiedlichen Zeitpunkten ermittelt wurden, wird ein zusätzlicher, auf Grund der begrenzten Anzahl gemeinsamer Items notwendiger Fehlerterm (Linking-Fehler) einbezogen, und ist die sich daraus ergebende Aussage zur Signifikanz der Differenz vorsichtiger.

2. Luxemburg hat die Testbedingungen zwischen PISA 2000 und PISA 2003 in organisatorischer und sprachlicher Hinsicht verändert, um die OECD-Standards genau einhalten zu können und den nationalen Besonderheiten des Schulsystems besser gerecht zu werden. Bei PISA 2000 erhielten die Schülerinnen und Schüler in Luxemburg ein Testheft in der Sprache, die sie eine Woche vor dem Test gewählt hatten. Im Nachhinein zeigte sich jedoch, dass ein großer Teil der luxemburgischen Schülerinnen und Schüler bei PISA 2000 durch eine mangelnde Vertrautheit mit der Testsprache behindert wurde. Bei PISA 2003 und PISA 2006 wurden den einzelnen Schülerinnen und Schülern daher zwei Testhefte ausgehändigt - eines in jeder der beiden Testsprachen -, so dass sie die von ihnen bevorzugte Sprache unmittelbar vor dem Test wählen konnten. Die so geschaffenen Testbedingungen waren besser vergleichbar mit jenen anderer Länder, die lediglich eine Testsprache haben, was zu einer gerechteren Bewertung der Schülerleistungen in Mathematik, Naturwissenschaften, Lesekompetenz und Problemlösen führte. Infolge dieser Verfahrensänderung können die Testbedingungen und mithin die Testergebnisse für Luxemburg in den Jahren 2000 und 2003 nicht miteinander verglichen werden. Da die Testbedingungen zwischen 2003 und 2006 nicht verändert wurden, sind die Ergebnisse dieser beiden Erhebungen vergleichbar.

3. Bei PISA 2000 lag die Beteiligungsquote im Vereinigten Königreich zunächst um 3,7\% unter dem erforderlichen Schwellenwert. Das Vereinigte Königreich hatte dem PISA-Konsortium damals Belege vorgelegt, die eine Evaluierung der erwarteten Leistungen der nicht teilnehmenden Schulen erlaubten. Auf der Grundlage dieser Belege gelangte das PISA-Konsortium zu der Auffassung, dass der Beteiligungsbias wahrscheinlich unerheblich sei, und die Ergebnisse wurden daher in den internationalen Bericht einbezogen. Bei PISA 2003 erfüllte die Beteiligungsquote des Vereinigten Königreichs nicht die Stichprobenstandards und mit einer weiteren Untersuchung des PISA-Konsortiums konnte auch nicht nachgewiesen werden, dass die durch Nichtbeteiligung bedingten Verzerrungen unerheblich waren. Daher wurden die entsprechenden Daten als nicht international vergleichbar eingestuft und in die meisten Ländervergleiche nicht einbezogen. Bei PISA 2006 und PISA 2009 wurden strengere Standards angewandt, weshalb die in PISA 2000 und PISA 2003 für das Vereinigte Königreich erhobenen Daten bei den Zeitreihenvergleichen unberücksichtigt bleiben.

4. In den Vereinigten Staaten enthielten einige der Lesekompetenz-Items auf Grund eines Fehlers beim Druck der Testhefte falsche Anweisungen, weshalb die durchschnittliche Leistung im Bereich Lesekompetenz nicht genau geschätzt werden konnte. Der Effekt dieses Fehlers bei den Schätzungen der Schülerleistungen dürfte über einem Standardstichprobenfehler von eins liegen. Bei den Aufgaben in Naturwissenschaften und Mathematik war dies nicht der Fall. Wegen Einzelheiten vgl. Anhang A3.

5. Wie in PISA 2000 Technical Report (OECD, 2002a) dargelegt, enthielt die österreichische Stichprobe für die PISA-Erhebung 2000 entgegen der technischen Standards für PISA keine Schülerinnen und Schüler, die an Bildungsprogrammen mit einer Kombination aus schulischer und beruflicher Ausbildung teilnahmen. Aus diesem Grund waren die im Rahmen der PISAErhebung 2000 veröffentlichten Schätzungen für Österreich verzerrt (OECD, 2001). Bei der PISA-Erhebung 2003 wurde diese Regelabweichung korrigiert. Um verlässliche Vergleiche zu ermöglichen, wurden Anpassungen vorgenommen und die Schülergewichtungen abgeändert, so dass die Schätzungen für PISA 2000 nun mit denen für PISA 2003 vergleichbar sind (Neuwirth, 2006; verfügbar unter http://www.oecd-ilibrary.org/education/oecd-education-working-papers_19939019).

6. Albanien, Argentinien, Bulgarien, Chile, Hongkong (China), Indonesien, Israel, Peru und Thailand führten die PISA-Erhebungsrunde 2000 erst 2001 durch, und Rumänien verlegte die Erhebung auf das Jahr 2002. Somit ist der Beobachtungszeitraum zwischen PISA 2000 und PISA 2009 für diese Länder kürzer.

7. Der Zusammenhang zwischen dem sozioökonomischen Hintergrund der Schülerinnen und Schüler und ihren Leistungen wird durch einen Steigungskoeffizienten des PISA-Index des wirtschaftlichen, sozialen und kulturellen Status (ESCS) in einer Regressionsgleichung erfasst, die die Schülerleistungen im Bereich Lesekompetenz erklärt (vgl. Kapitel 4).

8. Die hier auf Jahresbasis angegebenen Trends wurden berechnet, indem die Leistungsveränderung durch die Anzahl der zwischen den beiden Erhebungen liegenden Jahre dividiert wurde. So wurde z.B. eine Veränderung der Schülerleistungen im Bereich Lesekompetenz im Zeitraum 2000-2009 für die Länder, die an der ersten und der jüngsten Erhebung teilgenommen haben, durch neun dividiert. Für die Länder, die an PISA 2003 und PISA 2009, aber nicht an PISA 2000 teilgenommen haben, wurde die Veränderung der Schülerleistungen im Bereich Lesekompetenz im Zeitraum 2003-2009 durch sechs dividiert. Analog dazu wurde die Leistungsveränderung für Länder, die nur an PISA 2006 und PISA 2009 teilgenommen haben, durch drei dividiert. Für den Bereich Mathematik wurden zwar Trends auf Jahresbasis ermittelt, die Ergebnisse von PISA 2000 wurden dabei jedoch nicht berücksichtigt. Im Bereich Naturwissenschaften wurde die Leistungsveränderung im Zeitraum 2006-2009 durch drei geteilt. 


\section{Kasten V.B Korea}

Korea lag bereits im Jahr 2000 mit 525 Punkten im PISA-Erhebungsbereich Lesekompetenz über dem OECDDurchschnitt. Damals wiesen mehrere Länder ein ähnliches oder sogar höheres Leistungsniveau auf, darunter Australien, Kanada, Irland, Japan, Neuseeland und Finnland, das leistungsstärkste Land in jenem Jahr. Neun Jahre später steht Finnland zwar immer noch an der Spitze, Korea übertrifft aber inzwischen alle anderen oben genannten Länder. Koreas Erfahrung zeigt, dass selbst auf dem höchsten Leistungsniveau weitere Verbesserungen möglich sind.

Trotz des hohen Leistungsniveaus des Landes in PISA 2000 waren die koreanischen Politikverantwortlichen der Auffassung, dass die Leistungen der Schülerinnen und Schüler weiter gesteigert werden müssten, um den sich verändernden Anforderungen eines von starkem internationalem Wettbewerb geprägten Arbeitsmarkts gerecht zu werden. Um dies zu erreichen, wurde u.a. eine Umorientierung im Lehrplan für das Fach Koreanisch vorgenommen, um neben Kompetenzen in Grammatik und Literatur auch stärker Fähigkeiten und Strategien zu vermitteln, die für kritisches Verständnis und kreative Darstellung notwendig sind, was den Prinzipien des Ansatzes entspricht, der der PISA-Studie zu Grunde liegt. Es wurden verschiedene Unterrichtsmethoden und -materialien entwickelt, die diesen Veränderungen Rechnung trugen, wozu auch Investitionen in die diesbezügliche Computer- und Internet-Infrastruktur gehörten.

Da sie erkannt hatten, dass das Leseverständnis eine der Schlüsselkompetenzen des 21. Jahrhunderts ist, entwickelten und implementierten die zuständigen staatlichen Stellen auch spezifische Maßnahmen zur Förderung der Lesekompetenz. Die Schulen wurden aufgefordert, einen festen Anteil ihres Budgets für diesen Bereich aufzuwenden. Zudem wurden entsprechende Weiterbildungsprogramme für Lehrkräfte erstellt und verteilt. Die Eltern wurden ermutigt, mehr an den schulischen Aktivitäten teilzunehmen. Sie erhielten auch Informationen darüber, wie sie ihre Kinder bei den Schularbeiten unterstützen können. Darüber hinaus wurde sozioökonomisch benachteiligten Schülerinnen und Schülern durch verschiedene Kurse außerhalb der Schulzeit in den Bereichen Lesen, Schreiben und Rechnen, die Ende der 1990er Jahre eingeführt wurden, Unterstützung geboten.

Im Rahmen der neuen "National Human Resources Development Strategies for Korea“ wurden Politikziele und Umsetzungsstrategien festgelegt. In diesem Zusammenhang und unter dem Eindruck der Erfahrungen mit PISA und anderen Instrumenten entwickelten die zuständigen staatlichen Stellen das National Diagnostic Assessment of Basic Competency (NDABC) und bauten das National Assessment of Educational Achievement (NAEA) aus, um über verlässliche Messinstrumente zur Überwachung der Qualität der Bildungsergebnisse verfügen zu können. Diese Instrumente wurden eingesetzt, um sicherzustellen, dass alle Schülerinnen und Schüler grundlegende Kompetenzen erlangen. Das NDABC-Programm wurde 2002 als Diagnoseinstrument eingeführt, um die Grundkompetenzen von Drittklässlern in Lesen, Schreiben und Rechnen zu messen. Inzwischen wird dieses Messinstrument auf lokaler Ebene verwendet, um die Fortschritte von Grund- und Mittelstufenschülern in den verschiedenen Fächern zu beobachten. Das NAEA-Programm wurde 1998 eingeführt. Nach den 2002 erfolgten Änderungen in der Bildungspolitik wurde das Programm auf zusätzliche Fächer und Klassenstufen ausgeweitet. Mit ihm werden nun die Bildungsergebnisse und Leistungstrends der Sechs-, Neunt- und Zehntklässler in Koreanisch, Sozialkunde, Mathematik, Naturwissenschaften und Englisch bewertet. Mit Hilfe des NAEA-Programms beobachten die staatlichen Stellen die Leistungsniveaus der einzelnen Schülerinnen und Schüler sowie die Rechenschaftslegung der öffentlichen Bildungseinrichtungen.

Seit 2000 hat Korea sowohl im Bereich Lesekompetenz als auch im Bereich Naturwissenschaften beachtliche Verbesserungen erzielt (vgl. Abb. V.1.2 sowie Tabelle V.2.1 und V.3.4). Der Anteil der besonders leistungsstarken Schülerinnen und Schüler im Bereich Lesekompetenz stieg in Korea im Zeitraum 2000-2009 um mehr als 7 Prozentpunkte von 5,7\% auf 12,9\% (vgl. Abb. V.2.5 und Tabelle V.2.2). Dies entspricht der stärksten Veränderung, die unter den an PISA teilnehmenden Ländern zu beobachten war. Korea konnte auch seine Ergebnisse im Bereich Naturwissenschaften gegenüber 2006 verbessern, wo sie bereits hoch waren (vgl. Abb. V.3.5 und Tabelle V.3.4). Zudem sank der Anteil koreanischer Schülerinnen und Schüler unter Stufe 2, der 2006 im Bereich Naturwissenschaften $11 \%$ betrug, 2009 auf $6 \%$, womit er fast das niedrigste Niveau unter den OECD-Ländern erreichte (vgl. Abb. V.3.7 und Tabelle V.3.5). 
Korea gehört allerdings auch zu den Ländern, in denen der höchste Anstieg der Varianz der Schülerleistungen im Bereich Lesekompetenz festzustellen ist (vgl. Abb. V.4.1 und Tabelle V.4.1). Bei genauerer Betrachtung fällt auf, dass dieser Anstieg durch Verbesserungen unter den leistungsstärksten Schülerinnen und Schülern bedingt war, die in der Gruppe der leistungsschwächsten Schülerinnen und Schüler keine Entsprechung fanden (vgl. Abb. V.2.11 und Tabelle V.2.3). Aus den Ergebnissen von PISA 2009 für Korea lässt sich auch eine leichte Zunahme des Zusammenhangs zwischen dem sozioökonomischen Hintergrund und den Schülerleistungen ablesen.

Ein Faktor, der möglicherweise zu dem Anstieg der Zahl der besonders leistungsstarken Schülerinnen und Schüler im Bereich Lesekompetenz beigetragen hat, war die Einführung strengerer Standards, zu denen hohe Anforderungen in Bezug auf die Sprachbeherrschung hinzukamen. Im College Scholastic Ability Test (CSAt), den die Schülerinnen und Schüler bestehen müssen, um an einer Universität, insbesondere an einer mit hohem Ansehen, aufgenommen zu werden, wird Koreanisch als Testfach großes Gewicht beigemessen. Je nach angestrebtem Studienfach und Berufswunsch wählen die Aufnahmekandidaten bei dem Test im Allgemeinen fünf bis sieben Fächer aus. In fast allen Universitäten mit hohem Ranking liegt der Schwerpunkt allerdings auf Koreanisch, Mathematik und Englisch. Insbesondere der Aspekt Leseverständnis spielt im Koreanischtest eine große Rolle, während im Rahmen der NAEA/NDABC-Prüfungen in der Regel den sechs Bereichen des Lehrplans für das Fach Koreanisch - Zuhören, Sprechen, Lesen, Schreiben, Literatur und Grammatik - gleiches Gewicht beigemessen wird. Dies liefert leistungsstarken Schülerinnen und Schülern in Korea einen zusätzlichen Anreiz, mehr Zeit für das Lernen im Fach Koreanisch wie auch in Mathematik und Naturwissenschaften aufzuwenden.

Korea ist auch eines der Länder mit der höchsten Anzahl an Schülerinnen und Schülern, die Unterricht außerhalb der Schulzeit besuchen. Mehr als zwei Drittel der Schülerinnen und Schüler nehmen Nachhilfeunterricht, und nahezu die Hälfte der Schülerinnen und Schüler besucht in mindestens einem der Bereiche Naturwissenschaften, Mathematik und Lesekompetenz Neigungskurse bzw. Zusatzunterricht (vgl. Band IV, Was macht eine Schule erfolgreich? Lernumfeld und schulische Organisation in PISA, Tabelle IV.3.17a). Privatunterricht ist in Korea unter denjenigen, die es sich leisten können, sehr beliebt, außerhalb der Schulzeit besuchter Gruppenunterricht wird aber häufig bezuschusst, weshalb er selbst von sozioökonomisch benachteiligten Schülerinnen und Schülern oft in Anspruch genommen wird. So boten z.B. im Juni 2007 99,8\% aller Primar- und Sekundarschulen Programme außerhalb der Schulzeit an, und rd. 50\% aller Schüler des Primar- und Sekundarbereichs nahmen an Aktivitäten außerhalb der Schulzeit teil (MEHRD, 2007). Viele Beobachter vermuten, dass die hohen Teilnahmequoten an Unterricht außerhalb der Schulzeit auf kulturelle Faktoren und eine starke Ausrichtung auf die Vorbereitung der Aufnahmeprüfung fürs Studium zurückzuführen sind. Die Daten von PISA 2006 zeigen, dass koreanische Schülerinnen und Schüler, die Schulen mit einer sozioökonomisch besser gestellten Schülerschaft besuchen, mit größerer Wahrscheinlichkeit außerhalb der Schulzeit an Privatunterricht teilnehmen als Schülerinnen und Schüler in anderen Ländern. Sozioökonomisch benachteiligte Schülerinnen und Schüler besuchen ihrerseits in Korea häufiger Gruppenunterricht außerhalb der Schulzeit als Schülerinnen und Schüler in anderen Ländern. In beiden Fällen ist die Teilnahme an Zusatzunterricht außerhalb der Schulzeit mit höheren Leistungen in PISA verbunden (OECD, 2010d).

Der Leistungsabstand zwischen Jungen und Mädchen erhöhte sich in Korea um 20 Punkte, was hauptsächlich auf eine deutliche Leistungssteigerung der Mädchen zurückzuführen war, während unter den Jungen kein vergleichbarer Trend festzustellen war (vgl. Abb. V.2.7 und Tabelle V.2.4). Der Anteil der besonders leistungsstarken Schüler stieg unter den Mädchen um mehr als 9 Prozentpunkte, wohingegen er unter den Jungen um etwas weniger als 5 Prozentpunkte zunahm (vgl. Tabelle V.2.5 und V.2.6). Die Durchschnittsergebnisse im Bereich Lesekompetenz verbesserten sich nur unter den Mädchen, während sie unter den Jungen auf demselben Niveau verharrten. Die bemerkenswerte Verbesserung bei den Ergebnissen der Mädchen war nicht nur im Bereich Lesekompetenz, sondern auch in anderen in PISA sowie in sonstigen internationalen bzw. nationalen Studien erfassten Bereichen zu beobachten. Der Abstand zwischen Jungen und Mädchen in Mathematik und Naturwissenschaften hat sich seit mehreren Jahren verringert, und die Ergebnisse von PISA 2009 deuten darauf hin, dass der Leistungsvorsprung der Mädchen im Bereich Lesekompetenz sogar größer geworden ist. Aus nationalen Erhebungen geht hervor, dass die Anzahl der Mädchen, die die obersten Kompetenzstufen erreichen, seit 2002 stetig zugenommen hat. 
Mehrere Änderungen könnten mit dem positiveren Trend bei den Mädchen in Zusammenhang stehen. Seit 2000 wurden die Lehrpläne für die Fächer Naturwissenschaften und Mathematik nach und nach so umgestaltet, dass sie für Mädchen ansprechender wurden. Dies beinhaltete z.B., dass über Frauen berichtet wird, die als Wissenschaftlerinnen oder Ingenieurinnen tätig sind und folglich gute Rollenmodelle für Mädchen darstellen können, dass in Lehrbüchern eine geschlechtsneutralere Sprache verwendet wird und dass im Naturwissenschaftsunterricht Lehrmaterial eingesetzt wird, das für Mädchen interessanter sein dürfte. Gleichzeitig wurden nationale Evaluierungsprogramme, wie z.B. das NAEA, weiterentwickelt, um eine bessere Beobachtung der unterschiedlichen Formen der Kompetenzentwicklung bei Jungen und Mädchen zu ermöglichen und Formate einzuführen, die Mädchen vorziehen, beispielsweise Fragen mit offenem Antwortformat. Allerdings könnte sich diese Entwicklung z.T. auch aus gesellschaftlichen Veränderungen erklären. In den letzten Jahren hat sich die Familienstruktur in Korea verändert, da die Zahl der Kinder je Haushalt rasch gesunken und die Zahl der Einkindfamilien gestiegen ist. Während es in der Vergangenheit unwahrscheinlich war, dass Mädchen aus größeren Familien eine gute Ausbildung erhielten, haben Soziologen festgestellt, dass die Eltern in Korea heute unabhängig vom Geschlecht in der Regel großen Wert auf die Ausbildung ihrer Kinder legen. Kleinere Familien zusammen mit neuen Lernmöglichkeiten und -anreizen könnten ebenfalls eine Erklärung für diesen Trend sein.

Die im Vergleich zu 2003 niedrigeren Ergebnisse der koreanischen Schülerinnen und Schüler im Naturwissenschaftstest von PISA 2006 veranlassten die Politikverantwortlichen dazu, modernere wissenschaftliche Inhalte in die Schulprogramme aufzunehmen. Auch wenn die Anzahl der koreanischen Schülerinnen und Schüler, die unter Kompetenzstufe 2 lagen, sowohl in Mathematik als auch in Naturwissenschaften verglichen mit anderen Ländern sehr gering war, betrachteten die koreanischen Politikverantwortlichen das Gesamtleistungsniveau in Naturwissenschaften als zu niedrig. Im Jahr 2007 entschied die koreanische Regierung, das Ministerium für Forschung und Technologie und das Ministerium für Bildung zusammenzulegen, um den naturwissenschaftlichen Unterricht zu verbessern und auszubauen und so die Kreativität und die Problemlösefähigkeiten der Schüler zu erhöhen. Die ergriffenen Maßnahmen umfassten verschiedene Elemente, darunter nicht nur die Bereitstellung neuer Mathematik- und Naturwissenschaftslehrbücher, die für die Schüler verständlicher und interessanter sind, sondern auch den Einsatz von Unterrichtsmethoden, die die Experimentierfreude und naturwissenschaftliche Neugierde der Schüler fördern sollen. Die jüngsten Verbesserungen in Naturwissenschaften, insbesondere unter den leistungsstärksten Schülerinnen und Schülern, könnten mit diesen vor kurzem eingeführten Politikänderungen zusammenhängen. Auf allen Leistungsniveaus werden aber größere Verbesserungen erwartet, sobald die neue Politik vollständig zum Tragen gekommen ist.

\section{Kasten V.C Polen}

Im Jahr 2000 erzielten Polens 15-jährige Schülerinnen und Schüler im PISA-Lesekompetenztest im Durchschnitt 479 Punkte und lagen somit deutlich unter dem OECD-Durchschnitt von 500 Punkten. Besorgniserregender für die polnischen Politikverantwortlichen war aber die Tatsache, dass über 23\% der Schülerinnen und Schüler das Basisniveau an Lesekompetenz, d.h. Kompetenzstufe 2, nicht erreicht hatten. Die PISA-Ergebnisse deuteten auch auf große Disparitäten bei der Lesekompetenz zwischen Schülerinnen und Schülern in verschiedenen Sekundarschultypen hin. Die mittlere Punktzahl der Schülerinnen und Schüler, die einfache berufsbildende Schulen besuchten - zu diesem Zeitpunkt mehr als ein Fünftel aller Schülerinnen und Schüler -, belief sich auf 358 Punkte, wohingegen die mittlere Punktzahl der Schülerinnen und Schüler an allgemeinbildenden Schulen 543 Punkte betrug und die der Schülerinnen und Schüler an berufsbildenden Schulen des Sekundarbereichs bei rd. 480 Punkten lag.

Schon vor der Veröffentlichung der PISA-Ergebnisse des Jahrs 2000 existierten in Polen Pläne zur Verbesserung der Lernergebnisse der Schülerinnen und Schüler. 1998 legte das polnische Ministerium für Bildung ein Reformkonzept vor, um a) das Bildungsniveau in Polen durch eine Erhöhung der Zahl der Absolventen des Sekundar- und Tertiärbereichs zu steigern, b) Chancengerechtigkeit in der Bildung zu gewährleisten und c) Verbesserungen der Bildungsqualität zu fördern. Die Reform war zudem Teil eines größeren Katalogs von Umstellungen, der u.a. eine Übertragung von Zuständigkeiten im Bildungsbereich an lokale Behörden, Gesundheitsreformen und Reformen des Rentensystems umfasste. 
Die Bildungsreform sah Änderungen in der Struktur des Bildungssystems mit einer Reorganisation des Schulnetzes und des Schülertransports, Änderungen bei den Verwaltungs- und Aufsichtsmethoden, Änderungen der Lehrpläne, ein neues zentrales Prüfungssystem mit unabhängigen Schülerbeurteilungen, eine Umstellung der Schulfinanzierung durch Zuschüsse der nachgeordneten Gebietskörperschaften sowie neue Anreize für Lehrer, wie alternative Aufstiegsmöglichkeiten und ein überarbeitetes Besoldungssystem, vor. Obwohl nicht alle vorgeschlagenen Änderungen letztlich auch so umgesetzt wurden, veränderte die Reform ganz klar die Modalitäten der Verwaltung, Finanzierung und Evaluierung der Schulen, und sie hatte auch Auswirkungen auf die Unterrichtsinhalte und -methoden.

Die strukturellen Veränderungen resultierten in einem neuen Schultyp: der gymnasialen Sekundarstufe I, die ein gemeinsames allgemeinbildendes Programm für alle Schülerinnen und Schüler anbietet und zu einem Symbol der Reform wurde. Die frühere Struktur, die acht Jahre Primarschule umfasste, gefolgt von vier bzw. fünf Jahren Sekundarschule oder drei Jahren grundlegendem berufsbildendem Unterricht, wurde durch ein System nach dem Schema $6+3+3$ ersetzt. Dies bedeutete, dass die Primarschulzeit von acht auf sechs Jahre verkürzt wurde. Nach dem Abschluss der Primarschule setzen die Schüler ihren Bildungsweg in einem allgemeinbildenden dreijährigen Programm des Sekundarbereichs I fort. Damit wurde die Dauer des allgemeinbildenden Schulbesuchs, bei dem für alle Schülerinnen und Schüler dieselben Lehrpläne und Standards gelten, um ein Jahr verlängert. Erst nach Abschluss der drei Jahre in Sekundarstufe I können die Schüler in ein drei- bzw. vierjähriges Programm des Sekundarbereichs II, das den Zugang zur Hochschulbildung ermöglicht, oder in ein zwei- bzw. dreijähriges berufsbildendes Grundprogramm überwechseln. In dem neuen System endet jeder Bildungsabschnitt mit einer auf nationaler Ebene standardisierten Prüfung, dank der Schüler, Eltern und Lehrkräfte ein Feedback erhalten. Die Politikverantwortlichen können die Prüfungsergebnisse auch zur Überwachung des Schulsystems auf lokaler oder zentraler Ebene nutzen.

Die Reformverantwortlichen gingen davon aus, dass es Polen durch die gymnasiale Sekundarstufe I möglich sein würde, das Bildungsniveau zu steigern, insbesondere in ländlichen Gebieten, wo die Schulen relativ klein waren. Die neuen Schulen des Sekundarbereichs I sind größer, und sie sind zudem gut mit qualifizierten Lehrkräften ausgestattet. Da die Zahl der Schülerinnen und Schüler in den einzelnen Schulen je nach Einzugsgebiet variiert, implizierte die Einrichtung der gymnasialen Sekundarstufe I eine Reorganisation des Schulnetzes. Daher wurden seit 2000 mehrere kleine Primarschulen geschlossen, und wesentlich mehr Schülerinnen und Schüler müssen nun weitere Strecken zurücklegen, um die größeren Schulen der Sekundarstufe I zu erreichen.

Durch die Reform wurde die Wahl zwischen allgemein- oder berufsbildenden Unterrichtsinhalten der Sekundarstufe II um ein Jahr verschoben, womit alle Schülerinnen und Schüler ein Jahr länger am allgemeinbildenden Sekundarbereich I teilnehmen. Die Reform erstreckte sich weder auf dieVorschulbildung noch führte sie zu einer Herabsetzung des Alters, mit dem die Schulpflicht beginnt (sieben Jahre), stattdessen war sie auf Primarschulen und Schulen des Sekundarbereichs I ausgerichtet. Inzwischen hat der Hochschulbesuch deutlich zugenommen, und die Zahl der Studierenden erhöhte sich von ungefähr einer halben Million vor 1993 auf nahezu zwei Millionen 15 Jahre später (GUS, 2009). Das veränderte auch das Umfeld, in dem die neu eingerichteten Schulen tätig sind, da mehr Eltern daran interessiert sind, ihren Kindern eine bestmögliche Ausbildung zu bieten, und mehr Schülerinnen und Schüler die Schulen unter Berücksichtigung künftiger Berufsaussichten sorgfältig auswählen. Angesichts der zunehmenden wirtschaftlichen Erträge, die mit einer guten Ausbildung verbunden sind, nimmt in Polen die Bildung nunmehr einen hohen Stellenwert ein (OECD, 2006a).

Die Reformverantwortlichen hatten zwei Hauptargumente, mit denen sie die vorgeschlagenen Veränderungen verteidigten. Erstens sollte die Aufteilung der Ausbildung in Abschnitte es ermöglichen, die Unterrichtsmethoden und -inhalte besser an die spezifischen Bedürfnisse von Schülern unterschiedlichen Alters anzupassen. Zweitens würden die Lehrkräfte durch die Veränderung der Struktur des Bildungssystems dazu veranlasst, ihre Unterrichtsinhalte und -methoden anzupassen, d.h. nicht nur andere Stoffe, sondern auch auf andere Weise zu unterrichten.

Nach jahrelangen Beschwerden über überladene Lehrpläne und Streitigkeiten über die weitere Vorgehensweise entschloss man sich für das Konzept eines Kernlehrplans. Dadurch wurde den Schulen umfassende Autonomie eingeräumt, innerhalb eines vorgegebenen allgemeinen Rahmens die Unterrichtsinhalte selbst festzulegen, wobei die drei Bildungsziele miteinander in Einklang gebracht werden sollten: Vermittlung von Wissen, 
Entwicklung von Kompetenzen und Prägung positiver Einstellungen. Die Lehrplanreform sollte nicht nur die Inhalte der Schulbildung verändern und innovative Unterrichtsmethoden fördern, sondern sollte auch Änderungen in der Unterrichtsphilosophie und der Schulkultur herbeiführen. Anstatt nur passiv den Vorgaben der Bildungsbehörden zu folgen, wurde von den Lehrkräften erwartet, dass sie ihren eigenen, auf die Bedürfnisse ihrer Schüler abgestimmten Unterrichtsstil entwickeln.

Die Umsetzung der auf dem Prinzip der Dezentralisierung beruhenden Lehrplanreform machte die Errichtung eines Systems zur Sammlung von Informationen und gleichzeitigen Überwachung der Bildungseinrichtungen erforderlich. Die Reformverantwortlichen entschieden daher, obligatorische Prüfungen zur Evaluierung der Schülerleistungen am Ende der Primar- und der Sekundarstufe I zu organisieren. Die Ergebnisse der Primarschulprüfungen haben keinen Einfluss auf die schulische Laufbahn der Schülerinnen und Schüler, da der Abschluss dieses Bildungsabschnitts nicht von diesen Prüfungen abhängt. Für die Aufnahme an Schulen des Sekundarbereichs II wird indessen die in der Abschlussprüfung der gymnasialen Sekundarstufe I erzielte Punktzahl zusammen mit den Jahresendnoten der Schüler berücksichtigt. Beide Prüfungen wurden zum ersten Mal im Jahr 2002 durchgeführt. Die abschließende Prüfung ist die Matura, die erstmals 2005 durchgeführt wurde und am Ende von Sekundarstufe II abgelegt wird. Die Zuständigkeit für die Organisation, Durchführung und Korrektur all dieser Prüfungen obliegt einem zentralen und einem regionalen Prüfungsausschuss, zwei neuen Institutionen, die im Rahmen der Reform eingerichtet wurden.

Die Einführung des nationalen Prüfungssystems bot nicht nur eine Möglichkeit, die Ergebnisse der Schulen in einem teilweise dezentralisierten System auf zentraler Ebene zu bewerten, sondern veränderte auch die Anreizsituation für Schüler und Lehrer. Sie signalisierte den Schülerinnen und Schülern klar und deutlich, dass ihr Erfolg direkt von ihren extern evaluierten Ergebnissen abhängt. Darüber hinaus ermöglichte sie die Beurteilung von Lehrkräften und Schulen im ganzen Land anhand vergleichbarer Maßstäbe. Außerdem erhielten die nachgeordneten Gebietskörperschaften durch die Prüfungen Informationen über die Ergebnisse der Schulen, die nunmehr ihrer organisatorischen und finanziellen Verantwortung unterstanden.

Seit der Reform spielen die nachgeordneten Gebietskörperschaften eine noch viel wichtigere Rolle im polnischen Schulsystem. Zwar unterstanden bereits 1996 nahezu alle Primarschulen der Verantwortung der nachgeordneten Gebietskörperschaften, mit der Reform wurden aber auch Änderungen im Finanzierungssystem vorgenommen. Die erforderliche Reorganisation des Schulnetzes, um Platz für Schulen der Sekundarstufe I zu schaffen, lieferte den nachgeordneten Gebietskörperschaften zusätzliche Anreize, die Effizienz und Qualität ihrer Schulen zu erhöhen. Seit dem Jahr 2000 werden die Finanzmittel für Schulen nach einer auf der Schülerzahl basierenden Formel an die nachgeordneten Gebietskörperschaften übertragen. Diese Mittel machen nun einen Großteil ihres Haushalts aus. Nach 2002 begannen einige nachgeordnete Gebietskörperschaften zudem, die Ergebnisse der auf nationaler Ebene durchgeführten Prüfungen zu verwenden, um ihre Schulen zu evaluieren und die Bildungsangebote im Vorschulbereich und in Sekundarstufe II in ihren Gemeinden umzugestalten.

Mit der Reform wurde auch ein neues Weiterbildungs- und Beurteilungssystem für die Lehrkräfte eingeführt. Anfangs bemühten sich viele Lehrkräfte, ihr Bildungsniveau und ihre beruflichen Qualifikationen zu erhöhen, um den neuen Anforderungen gerecht zu werden. Die Veränderungen wirkten sich jedoch nur teilweise auf das Vergütungssystem aus, bei dem die nachgeordneten Gebietskörperschaften und die Schulleitungen über wenig Entscheidungsfreiheit verfügen. Zusammen mit der hohen Beschäftigungssicherheit und anderen Vorteilen, die die sogenannte Lehrercharta vorsieht, begrenzte dies den Einfluss der Änderungen auf die Lehrkräfte (OECD, 2006a).

Die Reform wirkte sich auf unterschiedliche Art auf die in den PISA-Erhebungen der Jahre 2000, 2003 und 2006 erfassten Alterskohorten aus. Die erste Gruppe, die im Jahr 2000 geprüft wurde, war nicht von der Reform betroffen. Die Gruppe der 15-Jährigen, die im Jahr 2003 in der zweiten PISA-Erhebung erfasst wurden, hatte die Primarschule im früheren System begonnen, dann aber die neue gymnasiale Sekundarstufe I besucht. Diese Schülerinnen und Schüler wurden alle nach demselben Lehrplan unterrichtet und wurden nicht auf verschiedene Schultypen verteilt. Die in PISA 2006 erfasste Gruppe hatte den Großteil ihrer schulischen Laufbahn im reformierten Bildungssystem absolviert, und diejenigen, die 2009 geprüft wurden, sogar ihre gesamte Schulzeit. 
Auch wenn es nicht möglich ist, zwischen der Reform und den in PISA gemessenen Ergebnissen einen Kausalzusammenhang nachzuweisen, konnte Polen seine Leistung im Bereich Lesekompetenz seit 2000 um 21 Punkte verbessern (vgl. Abb. V.2.1 und Tabelle V.2.1). Die größte Verbesserung war in PISA 2003, gleich nach der Reform, zu beobachten. Die Ergebnisse von PISA 2009 deuten darauf hin, dass die Reform den leistungsschwächsten Schülerinnen und Schülern offenbar am meisten zugute kam. Der Anteil der Schülerinnen und Schüler, die unter Kompetenzstufe 2 lagen, ging um 8 Prozentpunkte zurück, und die Ergebnisse der leistungsschwächsten Schülerinnen und Schüler stiegen um 40 Punkte, während sie für die leistungsstärksten Schülerinnen und Schüler in etwa unverändert blieben (vgl. Abb. V.2.4 sowie Tabelle V.2.2 und V.2.3).

Weitere Analysen lassen darauf schließen, dass die Schülerpopulation der berufsbildenden Schulen des früheren Systems am meisten von diesen Reformen profitierte (Jakubowski, Patrinos, Porta und Wisniewski, 2010). Die 2006 geprüften Schülerinnen und Schüler der Sekundarstufe I, die denselben Hintergrund aufwiesen wie die Schülerinnen und Schüler, die im Jahr 2000 einfache berufsbildende Schulen besuchten, erzielten auf der PISALesekompetenzskala in etwa um eine Standardabweichung höhere Leistungen. Verbesserungen, wenn auch in geringerem Umfang, waren auch unter den 2006 evaluierten Schülerinnen und Schülern der Sekundarstufe I zu erkennen, die einen ähnlichen Hintergrund aufwiesen wie jene, die im Jahr 2000 auf berufsbildende Schulen des Sekundarbereichs gingen; der Leistungsvorsprung derjenigen, die im Jahr 2000 einen vergleichbaren Hintergrund hatten wie die Schülerinnen und Schüler im allgemeinbildenden Sekundarbereich II, war allerdings unerheblich. Das deutet darauf hin, dass sich durch die Reform die Ergebnisse der Schülerinnen und Schüler verbesserten, die im alten System die früheren einfachen berufsbildenden Schulen besucht hätten und denen nun die Chance geboten wurde, in den neu geschaffenen Schulen des Sekundarbereichs I allgemeinere Kompetenzen zu erwerben.

Polen konnte seine Gesamtvarianz im Bereich Lesekompetenz um 20\% verringern (vgl. Abb. V.4.1 und Tabelle V.4.1). Dies wurde hauptsächlich erreicht, weil die Unterschiede zwischen den Leistungen der verschiedenen Schulen reduziert wurden und sich die Ergebnisse der leistungsschwächsten Schülerinnen und Schüler verbesserten. Die Leistungsvarianz zwischen den einzelnen Schulen sank gegenüber einem verhältnismäßig hohen Niveau im Jahr 2000 um drei Viertel auf ein Niveau, das weit unter dem OECD-Durchschnitt liegt. Darüber hinaus war der Zusammenhang zwischen dem sozioökonomischen Hintergrund der Schulen und ihren Durchschnittsergebnissen 2009 dreimal schwächer als im Jahr 2000, wenngleich der Gesamteffekt des sozioökonomischen Hintergrunds auf die Schülerleistungen unverändert blieb (vgl. Abb. V.4.3 und Tabelle V.4.3). Das lässt darauf schließen, dass Schülerinnen und Schüler mit unterschiedlichem Hintergrund durch die Schulreform in Polen gleichmäßiger auf die verschiedenen Schulen verteilt wurden. Die insgesamt erzielten Leistungssteigerungen, die größeren Verbesserungen unter den leistungsschwächsten Schülerinnen und Schülern und die Abnahme der Gesamtvarianz der Schülerleistungen legen aber auch den Schluss nahe, dass Polen sowohl im Hinblick auf seine mittlere Punktzahl als auch im Hinblick auf die Verteilungsgerechtigkeit im Bildungsbereich deutliche Fortschritte erzielt hat. 



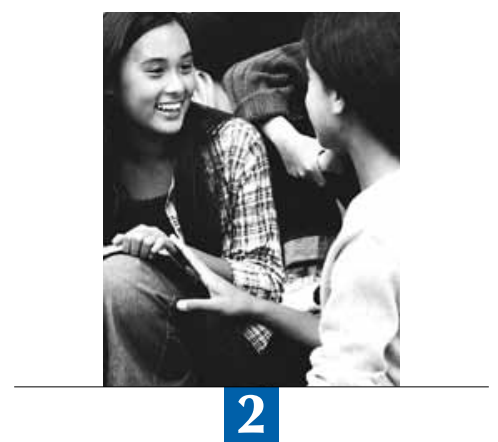

\section{Trends im Bereich Lesekompetenz}

In diesem Kapitel werden Trends im Bereich Lesekompetenz im Zeitraum 2000-2009 beschrieben. Dazu gehören Leistungsveränderungen bei den leistungsschwächsten und leistungsstärksten Schülern, bei Jungen und Mädchen, Schülern mit Migrationshintergrund, sozioökonomisch begünstigten bzw. benachteiligten Schülern sowie Veränderungen bei den Ergebnissen der Länder. 


\section{KONTINUITÄT UND WANDEL BEIM RAHMENKONZEPT UND BEI DEN ERHEBUNGSINSTRUMENTEN IM BEREICH LESEKOMPETENZ}

Lesekompetenz umfasst ein breites Spektrum kognitiver Fähigkeiten, von der Fähigkeit zum einfachen Entschlüsseln von Texten über dieVertrautheit mit Wörtern, grammatikalischen Regeln sowie sprachlichen und textuellen Strukturen und Merkmalen bis hin zur Kenntnis des Weltgeschehens. Zudem beinhaltet sie metakognitive Fähigkeiten, nämlich das Wissen um die Existenz einer Vielzahl verschiedener Strategien für den Umgang mit Texten und die Fähigkeit, diese Strategien einzusetzen. Konkret ist Lesekompetenz im Rahmen von PISA 2009 definiert als die Fähigkeit, geschriebene Texte zu verstehen, zu nutzen und über sie zu reflektieren, um eigene Ziele zu erreichen, Wissen zu erwerben, das eigene Potenzial weiterzuentwickeln und aktiv am gesellschaftlichen Leben teilzunehmen (OECD, 2006b). Eine ausführlichere Beschreibung des Rahmenkonzepts, das der Beurteilung der Lesekompetenz bei PISA zu Grunde liegt, findet sich in Band I des vorliegenden Berichts, Was Schülerinnen und Schüler wissen und können.

Das Rahmenkonzept und das Messinstrumentarium für den Bereich Lesekompetenz wurden für die PISA-Erhebung 2000 entwickelt. Der Mittelwert im Bereich Lesekompetenz wurde für die 28 OECD-Länder, die an PISA 2000 teilnahmen, mit 500 und die Standardabweichung mit 100 angesetzt, was die Skala ergibt, gegenüber der die Lesekompetenz in PISA 2009 beurteilt wurde. Zwei Länder, die an der PISA-Erhebung 2000 teilgenommen haben, sind der OECD seither beigetreten, während die Ergebnisse für vier OECD-Länder im Lauf der Zeit aus den Vergleichen ausgeschlossen wurden. Daher werden die Trends im Bereich Lesekompetenz für die 26 OECD-Länder erörtert, die sowohl an der Erhebung 2000 als auch an der Erhebung 2009 teilgenommen haben und für die vergleichbare Ergebnisse vorliegen. Der OECD-Durchschnitt in PISA 2000 beträgt für diese 26 OECD-Länder nunmehr 496, während die Gesamtskala Lesekompetenz unverändert geblieben ist ${ }^{1}$. In den PISA-Erhebungen 2003 und 2006, in denen sich der Schwerpunkt erst auf Mathematik und dann auf Naturwissenschaften verlagerte, wurde für den Bereich Lesekompetenz weniger Testzeit vorgesehen als im Jahr 2000, so dass lediglich eine aktualisierte Beurteilung der Gesamtleistungen, jedoch keine ähnlich detaillierte Analyse der Kenntnisse und Fähigkeiten wie in PISA 2000 und PISA 2009 vorgenommen werden konnte. Um Vergleichbarkeit zwischen den sukzessiven Lesekompetenztests zu gewährleisten, wurden 41 der 130 in der PISA-Erhebung 2009 verwendeten Leseaufgaben PISA 2000 entnommen. Diese Aufgaben wurden so ausgewählt, dass sie dem allgemeinen Verhältnis des Rahmenkonzepts Rechnung tragen, so dass der Anteil der Aufgaben bei jedem Aufgabentyp ähnlich war. 28 der 41 Aufgaben, die sowohl 2000 als auch 2009 im Rahmen der Erhebung verwendet wurden, kamen auch bei den PISA-Erhebungen 2003 und 2006 zum Einsatz, um die Vergleichbarkeit der Ergebnisse dieser Erhebungen sicherzustellen. Einzelheiten zu der für die Herausarbeitung von Trends im Bereich Lesekompetenz verwendeten Equating-Methode finden sich in Anhang A1.

Die Skala, auf der die Schülerleistungen dargestellt werden, ist daher dieselbe wie die im Jahr 2000 verwendete, so dass Vergleiche zwischen allen vier Erhebungsrunden möglich sind. Entsprechend werden nach wie vor dieselben Kompetenzstufen verwendet, wobei die Gesamtskala Lesekompetenz 2009 allerdings um neue Kompetenzstufen am oberen und am unteren Ende der Gesamtskala erweitert wurde; dies trägt der Tatsache Rechnung, dass im Rahmen von PISA 2009 detaillierte Daten über leistungsschwache und leistungsstarke Schülerinnen und Schüler gewonnen wurden.

\section{WIE SICH DIE SCHÜLERLEISTUNGEN IM BEREICH LESEKOMPETENZ SEIT DEM JAHR 2000 VERÄNDERT HABEN}

Die durchschnittlichen Schülerleistungen im Bereich Lesekompetenz sind in den OECD-Ländern seit dem Jahr 2000 im Hinblick auf die 26 OECD-Länder, für die aus den Erhebungsrunden 2000 und 2009 vergleichbare Ergebnisse vorliegen, ähnlich geblieben. Diese Entwicklung ist an sich bemerkenswert, da die meisten Länder ihre Bildungsinvestitionen in den vergangenen Jahren deutlich aufgestockt haben. Die Ausgaben pro Schüler im Primarund Sekundarbereich sind im Zeitraum 1995-2007 in den OECD-Ländern real um durchschnittlich 43\% gestiegen (OECD, 2010b, Tabelle B1 .5). In dem kurzen Zeitraum zwischen 2000, als die erste PISA-Studie durchgeführt wurde, und 2007 betrug der durchschnittliche Anstieg der Bildungsausgaben 25\%, und acht OECD-Länder erhöhten ihre Ausgaben um $35-71 \%$. Zwar zielten nicht alle diese Ausgaben darauf ab, die Leistungen der in PISA getesteten Schülerinnen und Schüler zu verbessern, es ist jedoch verblüffend, dass sich solch große finanzielle Anstrengungen in zahlreichen Ländern noch nicht in Leistungsverbesserungen niedergeschlagen haben.

In einigen Ländern wurden jedoch deutliche Verbesserungen bei den Bildungsergebnissen verzeichnet. In 13 der 38 Länder, die für den Zeitraum 2000-2009 miteinander verglichen werden können, haben sich die Leistungen in Lesekompetenz seit dem Jahr 2000 verbessert (Abb. V.2.1, vgl. auch Tabelle V.2.1). Sieben der 26 OECD-Länder, 
für die vergleichbare Ergebnisse aus den PISA-Erhebungen 2000 und 2009 vorliegen, erzielten bessere Ergebnisse: Chile, Israel und Polen verbesserten ihre Ergebnisse im Bereich Lesekompetenz um über 20 Punkte, und in Portugal, Korea, Ungarn und Deutschland betrugen die Verbesserungen 10-20 Punkte. Desgleichen verbesserten unter den Partnerländern Peru, Albanien, Indonesien und Lettland ihre Ergebnisse um über 20 Punkte, und Liechtenstein und Brasilien um 10-20 Punkte.

In vier Ländern wurde im Zeitraum 2000-2009 ein Leistungsrückgang im Bereich Lesekompetenz verzeichnet: In Irland verschlechterten sich die Schülerleistungen um 31 Punkte, in Schweden um 19 Punkte und in Australien sowie der Tschechischen Republik um jeweils 13 Punkte.

Im Rahmen der PISA-Erhebung werden nur diejenigen Ergebnisse als statistisch signifikant betrachtet und entsprechend gekennzeichnet, bei denen der Messfehler bei der Messung von Leistungsveränderungen darauf schließen lässt, dass eine Verbesserung oder Verschlechterung in weniger als fünf von 100 PISA-Wiederholungsmessungen ermittelt würde, wenn in der Realität gar keine Veränderung vorliegt. Es ist möglich, den genauen Prozentsatz an Wiederholungsmessungen zu berechnen, bei denen eine Veränderung ausgewiesen würde, wenn keine reale Veränderung stattgefunden hat. Dieser sogenannte "p-Wert" ist in Abbildung V.2.1 dargestellt (vgl. auch die letzte Spalte in Tabelle V.2.1). Je geringer dieser Prozentsatz ist, desto stärker kann man darauf vertrauen, dass die beobachteten Veränderungen real sind. Mittels des p-Werts können die Leser die Reliabilität der beobachteten Leistungsunterschiede anhand der oben beschriebenen strengen Kriterien prüfen, die bei PISA nicht als statistisch signifikant identifiziert wurden. Beispielsweise beträgt die beobachtete Verbesserung in Griechenland 9 Punkte und in Hongkong (China) 8 Punkte. Dies ist eine beträchtliche Verbesserung - allerdings lassen die p-Werte für diese Schätzwerte darauf schließen, dass PISA im Falle Griechenlands bei 28 von 100 Wiederholungsmessungen und im Falle Hongkongs (China) bei 21 von 100 Wiederholungen eine solche Veränderung selbst dann hätte identifizieren können, wenn in der Realität gar keine Veränderung stattgefunden hätte. In Anbetracht der Größenordnung des potenziellen Messfehlers werden diese Veränderungen bei PISA nicht als statistisch signifikant identifiziert. Leser, denen ein niedrigeres Konfidenzniveau genügt, können diese Werte aber dennoch berücksichtigen.

Zwischen den Ländern bestehen Unterschiede im Hinblick auf ihr absolutes Leistungsniveau, so dass einige Länder selbst im Falle von Verbesserungen ihrer Ergebnisse im Bereich Lesekompetenz nach wie vor weit unter dem OECD-Durchschnitt liegen, wohingegen manche Länder trotz einer Verschlechterung ihrer Ergebnisse im Bereich Lesekompetenz nach wie vor besser abschneiden als zahlreiche andere Länder. Es ist daher nützlich, sowohl zu untersuchen, wo die Länder stehen, als auch wie sich ihre Ergebnisse verändert haben.

Abbildung V.2.1

\section{Veränderungen der Schülerleistungen im Bereich Lesekompetenz zwischen 2000 und 2009}

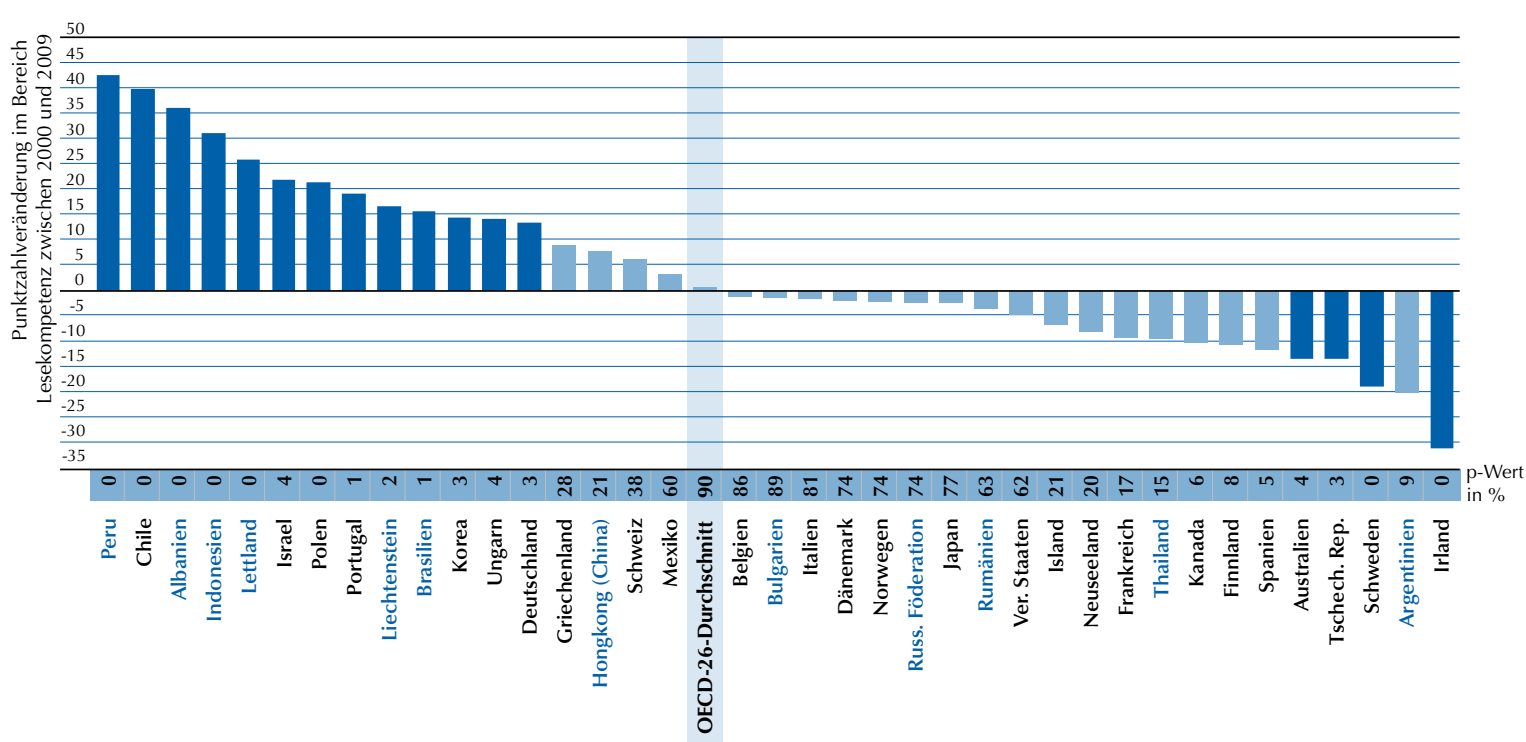

Anmerkung: Statistisch signifikante Punktzahlveränderungen sind durch einen dunkleren Farbton gekennzeichnet. 
Die Länder im rechten Teil von Abbildung V.2.2 verbesserten ihre Ergebnisse im Zeitraum 2000-2009, wohingegen sich die Schülerleistungen in den Ländern im linken Teil verschlechterten. Die Länder im oberen Teil erzielten 2009 über dem OECD-Durchschnitt liegende Ergebnisse, während diejenigen im unteren Teil Ergebnisse unter dem OECD-Durchschnitt erzielten. Die Länder, die ihre Ergebnisse im Zeitraum 2000-2009 verbessert haben, lassen sich - abhängig von ihrem Leistungsniveau im Jahr 2009 - in drei Gruppen einteilen. Die erste Gruppe umfasst Länder, deren Ergebnisse sich zwar verbessert haben, jedoch nach wie vor unter dem OECD-Durchschnitt liegen. Diese Länder sind im rechten unteren Teil von Abbildung V.2.2 dargestellt. Die zweite Gruppe enthält Länder, deren Ergebnisse sich verbessert haben und nunmehr nahe am OECD-Durchschnitt liegen. Diese Länder sind in Abbildung V.2.2 rechts in der Mitte dargestellt. Die dritte Gruppe umfasst die Länder, deren Leistungen bereits über denen der meisten PISA-Teilnehmerländer lagen, die ihre Leistungen jedoch noch weiter verbessert haben. Diese Länder sind in Abbildung V.2.2 oben rechts dargestellt. Bei Ländern, die weiß markiert sind, sind die Veränderungen statistisch nicht signifikant.

Unter den Ländern, die 2009 über dem OECD-Durchschnitt lagen, verbesserten drei Länder ihre Ergebnisse. Korea verbesserte seine Ergebnisse gegenüber dem bereits hohen Niveau im Jahr 2000 um 15 Punkte. Polen verbesserte seine Ergebnisse um 21 Punkte; nachdem es im Jahr 2000 noch unter dem OECD-Durchschnitt gelegen hatte, schnitt es bei PISA 2009 nunmehr überdurchschnittlich ab. Das Partnerland Liechtenstein verbesserte seine Ergebnisse um 17 Punkte. Die Schulsysteme Koreas und Polens werden in den zwischen Kapitel 1 und 2 eingefügten Kästen V.B und V.C jeweils eingehender analysiert.

Unter den Ländern, die 2009 durchschnittlich abgeschnitten haben, verbesserten sich die Leistungen in Lesekompetenz in Portugal, Ungarn und Deutschland. Kasten V.D, der zwischen Kapitel 3 und 4 eingefügt ist, enthält nähere Einzelheiten zu den in Portugal durchgeführten Reformen.

\section{Wie die Länder im Bereich Lesekompetenz abschneiden und wie sich die Leseleistungen seit dem Jahr 2000 verändert haben}

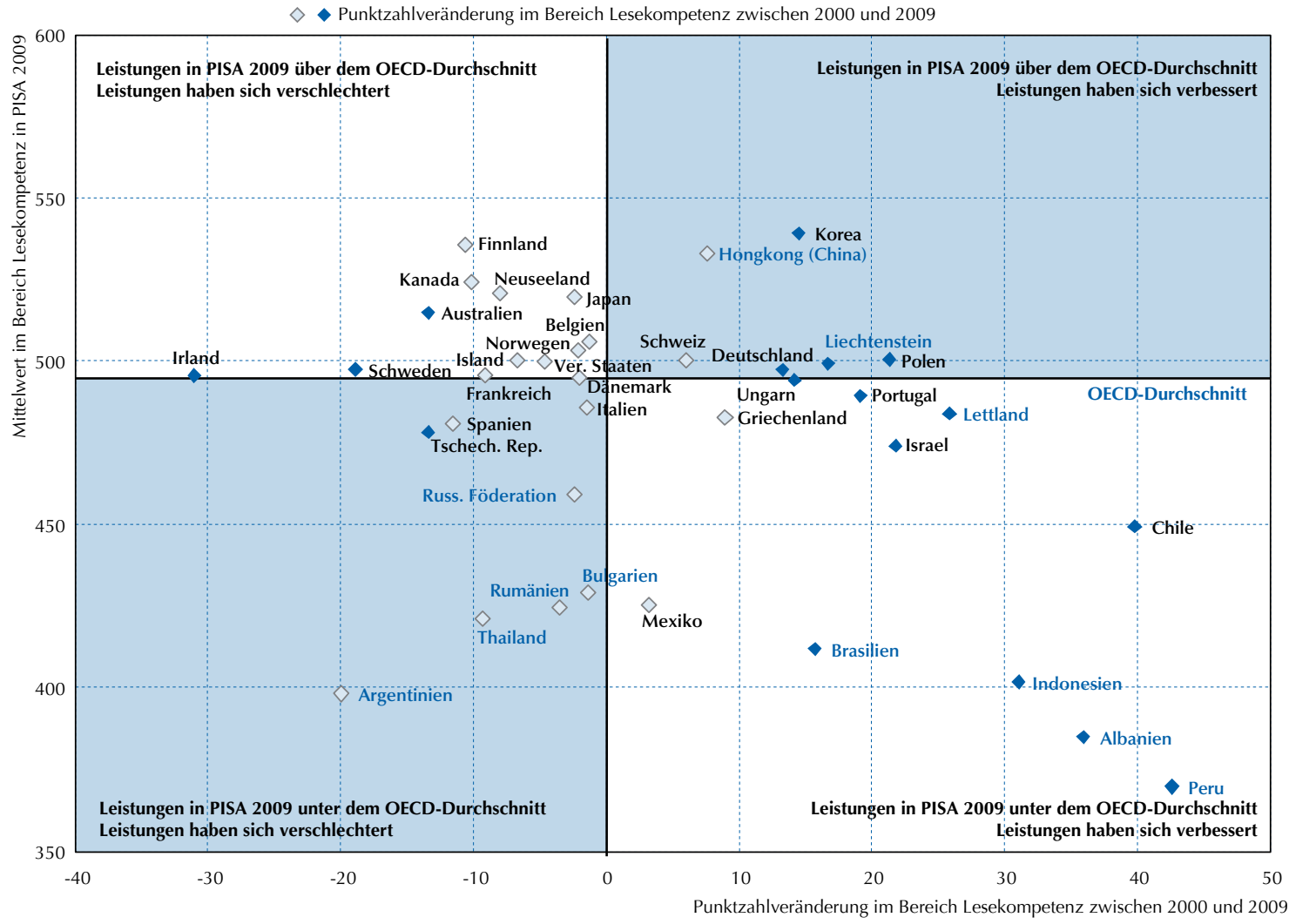

Anmerkung: Statistisch signifikante Punktzahlveränderungen zwischen 2003 und 2009 sind durch einen dunkleren Farbton gekennzeichnet Quelle: OECD, PISA-2009-Datenbank, Tabelle V.2.1.

StatLink 제피 $h t t p: / / d x . d o i . o r g / 10.1787 / 888932359967$ 
In mehreren Ländern, die 2009 unterdurchschnittlich abschnitten, wurden dennoch deutliche Verbesserungen verzeichnet. Unter den OECD-Ländern verbesserten sich die Schülerleistungen in Chile um 40 Punkte und liegen nunmehr nahe bei 450 Punkten, während sich die Schülerleistungen in Israel um 22 Punkte verbesserten und heute 474 Punkte betragen. Auf Chiles Schulsystem wird in Kasten V.F eingegangen, der im Anschluss an Kapitel $4 \mathrm{zu}$ finden ist. Das Partnerland Peru verzeichnete mit einem Anstieg um 43 Punkte die größte Verbesserung, auch wenn das Ergebnis des Landes insgesamt nach wie vor weniger als 400 Punkte beträgt. Albanien und Indonesien verbesserten ihre Ergebnisse um 30-40 Punkte, auch wenn beide Länder nach wie vor bei bzw. unter der 400-PunkteMarke liegen. Brasilien hat seine Leistungen um 16 Punkte verbessert und erreicht damit nunmehr über 400 Punkte (vgl. Kasten V.G im Anschluss an Kapitel 5). Lettland hat sein Ergebnis um 26 Punkte auf 484 Punkte verbessert.

In einer Reihe von Ländern, die überdurchschnittlich abgeschnitten haben, verschlechterten sich die Ergebnisse im Bereich Lesekompetenz. Australiens Ergebnisse verringerten sich um 13 Punkte, es zählt jedoch nach wie vor zu den leistungsstärksten Ländern im Bereich Lesekompetenz. Die Ergebnisse Irlands und Schwedens sanken um 31 bzw. 19 Punkte und entsprechen nunmehr jeweils in etwa dem OECD-Durchschnitt. Auch die Tschechische Republik verzeichnete rückläufige Ergebnisse; ihre Punktzahl sank unter den OECD-Durchschnitt.

Abbildung V.2.3 enthält Vergleiche der Veränderungen der jeweiligen Positionen der Länder im Hinblick auf die Ergebnisse im Bereich Lesekompetenz im Zeitraum 2000-2009. Die Länder sind nach ihren Ergebnissen in PISA 2009 angeordnet. In der Abbildung sind für jedes Land jeweils die Länder bzw. Volkswirtschaften aufgelistet, die ähnliche Ergebnisse erzielt haben. Die erste Gruppe enthält Vergleiche zwischen Ländern, die bei PISA 2000 niedrigere Punktzahlen erzielten, 2009 jedoch ein ähnliches Leistungsniveau wie das in der ersten Spalte angegebene Land aufwiesen. In der zweiten Gruppe sind Länder aufgeführt, die 2000 niedrigere oder ähnliche Punktzahlen erzielten, ihre Ergebnisse 2009 jedoch verbesserten. In der dritten Gruppe sind Länder erfasst, deren Ergebnisse 2000 und 2009 ähnlich waren. Die vierte Gruppe setzt sich aus Ländern zusammen, die 2000 ähnliche oder höhere Punktzahlen erzielten, deren Ergebnisse sich 2009 jedoch verschlechterten. Die fünfte Gruppe bilden Länder, die 2000 höhere und 2009 ähnliche Ergebnisse erzielten. In der Abbildung sind alle 38 Länder dargestellt, für die aus den PISA-Erhebungen 2000 und 2009 vergleichbare Ergebnisse vorliegen.

Aus der Abbildung lässt sich ablesen, wie sich die Position eines Landes im Vergleich zu anderen Ländern verändert hat, in denen ähnliche relative Ergebnisse erzielt werden.

Die Mittelwerte geben die Gesamtleistung der Schülerinnen und Schüler in PISA zusammenfassend wieder. Sie geben zwar eine allgemeine Vorstellung von den Ergebnissen eines Landes im Vergleich zu anderen Ländern, können jedoch auch erhebliche Unterschiede bei den Schülerleistungen verbergen. Für die Politikverantwortlichen sind Informationen über das Spektrum der Schülerleistungen jedoch wichtig. So sind für Leser, die sich für Maßnahmen und Praktiken im Hinblick auf die begabtesten Schülerinnen und Schüler interessieren, möglicherweise die Länder von besonderem Interesse, in denen die leistungsstärksten Schülerinnen und Schüler ihre Leistungen verbessert haben oder in denen der Anteil der leistungsstarken Schülerinnen und Schüler gestiegen ist. Ebenso befassen sich Leser, die sich für Maßnahmen und Praktiken im Hinblick auf leistungsschwächere Schülerinnen und Schüler interessieren, möglicherweise eingehender mit Ländern, die Verbesserungen bei den leistungsschwächsten Schülerinnen und Schülern verzeichnet haben bzw. in denen der Anteil der leistungsschwachen Schülerinnen und Schüler zurückgegangen ist.

Die Leistungstrends unter den leistungsschwachen und leistungsstarken Schülerinnen und Schülern lassen sich durch Betrachtung der Veränderungen des prozentualen Anteils der Schülerinnen und Schüler auf den einzelnen PISA-Kompetenzstufen untersuchen. Wie in Band I, Was Schülerinnen und Schüler wissen und können, erläutert, wurden die Ergebnisse im Bereich Lesekompetenz in PISA 2009 den verschiedenen Kompetenzstufen zugeordnet, die Aufgaben mit unterschiedlichem Schwierigkeitsgrad entsprechen. Die Festlegung von Kompetenzstufen im Bereich Lesekompetenz ermöglicht nicht nur eine Einstufung der Leistungen der Schülerinnen und Schüler, sondern auch eine Beschreibung der Fähigkeiten, über die sie auf den jeweiligen Kompetenzstufen verfügen.

Wie in Band I erklärt, gilt die Lesekompetenzstufe 2 als das Basisniveau, ab dem die Schülerinnen und Schüler lesen gelernt haben und die Kompetenzen unter Beweis zu stellen beginnen, die für das Lesen zum Zweck des Lernens erforderlich sind. Schülerinnen und Schüler unterhalb dieser Stufe sind u.U. dennoch in der Lage, explizit ausgedrückte, leicht sichtbare Informationen in einem Text zu finden und das Hauptthema eines Textes über ein vertrautes Thema oder den Zusammenhang zwischen in diesem Text enthaltenen Informationen und eigenen Alltagserfahrungen zu erkennen. Sie haben jedoch nicht das Lesekompetenzniveau erreicht, das erforderlich ist, um effektiv und produktiv am Leben teilzuhaben. Im Durchschnitt der 26 OECD-Länder, für die vergleichbare Ergebnisse aus beiden Erhebungen vorliegen, 
Abbildung V.2.3

Ländervergleich der Schülerleistungen im Zeitraum 2000-2009

\begin{tabular}{|c|c|c|c|c|c|c|c|}
\hline & 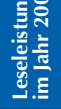 & 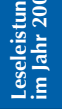 & $\begin{array}{l}\text { Länder, die } 2000 \\
\text { schlechter und } \\
2009 \text { ähnlich } \\
\text { abgeschnitten haben }\end{array}$ & $\begin{array}{l}\text { Länder, die } 2000 \\
\text { schlechter und } \\
2009 \text { besser } \\
\text { abgeschnitten haben }\end{array}$ & $\begin{array}{l}\text { Länder, die } 2000 \\
\text { und } 2009 \text { ähnlich } \\
\text { abgeschnitten haben }\end{array}$ & $\begin{array}{l}\text { Länder, die } 2000 \\
\text { ähnlich bzw. besser } \\
\text { und } 2009 \text { schlechter } \\
\text { abgeschnitten haben }\end{array}$ & $\begin{array}{l}\text { Länder, die } 2000 \text { besser } \\
\text { und } 2009 \text { ähnlich } \\
\text { abgeschnitten haben }\end{array}$ \\
\hline Korea & 525 & 539 & & & Hongkong (China) & $\begin{array}{l}\text { Japan, Kanada, Irland, } \\
\text { Neuseeland, Australien }\end{array}$ & Finnland \\
\hline Finnland & 546 & 536 & Korea, Hongkong (China) & & & & \\
\hline Hongkong (China) & 525 & 533 & & & Korea & $\begin{array}{l}\text { Japan, Kanada, Irland, } \\
\text { Neuseeland, Australien }\end{array}$ & Finnland \\
\hline Kanada & 534 & 524 & Japan & Korea, Hongkong (China) & Neuseeland & Australien & \\
\hline Neuseeland & 529 & 521 & & Korea, Hongkong (China) & Japan, Kanada, Australien & Irland & \\
\hline Japan & 522 & 520 & & Korea, Hongkong (China) & Neuseeland, Australien & Schweden, Irland & Kanada \\
\hline Australien & 528 & 515 & & $\begin{array}{l}\text { Kanada, Korea, } \\
\text { Hongkong (China) }\end{array}$ & Japan, Neuseeland & Irland & \\
\hline Belgien & 507 & 506 & $\begin{array}{l}\text { Liechtenstein, Schweiz, } \\
\text { Polen }\end{array}$ & & Norwegen, Ver. Staaten & $\begin{array}{l}\text { Island, Schweden, Irland, } \\
\text { Frankreich }\end{array}$ & \\
\hline Norwegen & 505 & 503 & $\begin{array}{l}\text { Liechtenstein, Deutschland, } \\
\text { Schweiz, Polen }\end{array}$ & & $\begin{array}{l}\text { Island, Belgien, Ver. Staaten, } \\
\text { Frankreich }\end{array}$ & & Schweden, Irland \\
\hline Schweiz & 494 & 501 & $\begin{array}{l}\text { Liechtenstein, Deutschland, } \\
\text { Polen, Ungarn }\end{array}$ & & Dänemark, Ver. Staaten & $\begin{array}{l}\text { Italien Spanien, } \\
\text { Tschech. Rep. }\end{array}$ & $\begin{array}{l}\text { Island, Norwegen, Belgien, } \\
\text { Schweden, Irland, Frankreich }\end{array}$ \\
\hline Polen & 479 & 500 & & & $\begin{array}{l}\text { Liechtenstein, Deutschland, } \\
\text { Ungarn }\end{array}$ & $\begin{array}{l}\text { Italien Portugal, } \\
\text { Spanien, Griechenland, } \\
\text { Tschech. Rep. }\end{array}$ & $\begin{array}{l}\text { Island, Norwegen, Schweiz, } \\
\text { Belgien, Dänemark, Schweden, } \\
\text { Irland, Ver. Staaten, Frankreich }\end{array}$ \\
\hline Island & 507 & 500 & $\begin{array}{l}\text { Liechtenstein, Deutschland, } \\
\text { Schweiz, Polen, Ungarn }\end{array}$ & Belgien & $\begin{array}{l}\text { Norwegen, Ver. Staaten, } \\
\text { Frankreich }\end{array}$ & & Schweden, Irland \\
\hline Ver. Staaten & 504 & 500 & $\begin{array}{l}\text { Liechtenstein, Deutschland, } \\
\text { Polen, Ungarn }\end{array}$ & & $\begin{array}{l}\text { Island, Norwegen, Schweiz, } \\
\text { Belgien, Dänemark, } \\
\text { Schweden, Frankreich }\end{array}$ & Spanien, Tschech. Rep. & Irland \\
\hline Liechtenstein & 483 & 499 & & & Deutschland, Polen, Ungarn & $\begin{array}{l}\text { Italien, Spanien, } \\
\text { Griechenland, } \\
\text { Tschech. Rep. }\end{array}$ & $\begin{array}{l}\text { Island, Norwegen, Schweiz, } \\
\text { Belgien, Dänemark, Schweden, } \\
\text { Irland, Ver. Staaten, Frankreich }\end{array}$ \\
\hline Schweden & 516 & 497 & $\begin{array}{l}\text { Island, Norwegen, Liechten- } \\
\text { stein, Deutschland, Schweiz, } \\
\text { Dänemark, Polen, Portugal, } \\
\text { Ungarn, Frankreich } \\
\end{array}$ & Japan, Belgien & Ver. Staaten & & 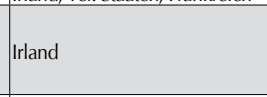 \\
\hline Deutschland & 484 & 497 & & & Liechtenstein, Polen, Ungarn & $\begin{array}{l}\text { Italien Spanien, } \\
\text { Griechenland, } \\
\text { Tschech. Rep. } \\
\end{array}$ & $\begin{array}{l}\text { Island, Norwegen, Schweiz, } \\
\text { Dänemark, Schweden, Irland, } \\
\text { Ver. Staaten, Frankreich }\end{array}$ \\
\hline Irland & 527 & 496 & \begin{tabular}{|l|} 
Island, Norwegen, Liechten- \\
stein, Deutschland, Schweiz, \\
Dänemark, Schweden, \\
Polen, Portugal, Ungarn, \\
Ver. Staaten, Frankreich \\
\end{tabular} & $\begin{array}{l}\text { Japan, Belgien, Korea, } \\
\text { Hongkong (China), } \\
\text { Neuseeland, Australien }\end{array}$ & & & \\
\hline Frankreich & 505 & 496 & $\begin{array}{l}\text { Liechtenstein, Deutschland, } \\
\text { Schweiz, Dänemark, Polen, } \\
\text { Portugal, Ungarn }\end{array}$ & Belgien & $\begin{array}{l}\text { Island, Norwegen, } \\
\text { Ver. Staaten }\end{array}$ & & Schweden, Irland \\
\hline Dänemark & 497 & 495 & $\begin{array}{l}\text { Liechtenstein, Deutschland, } \\
\text { Polen, Portugal, Ungarn }\end{array}$ & & Schweiz, Ver. Staaten & Spanien, Tschech. Rep. & Schweden, Irland, Frankreich \\
\hline Ungarn & 480 & 494 & & & $\begin{array}{l}\text { Liechtenstein, Deutschland, } \\
\text { Polen, Portugal }\end{array}$ & $\begin{array}{l}\text { Italien Spanien, } \\
\text { Griechenland, } \\
\text { Tschech. Rep. } \\
\end{array}$ & $\begin{array}{l}\text { Island, Schweiz, Dänemark, } \\
\text { Schweden, Irland, Ver. Staaten, } \\
\text { Frankreich }\end{array}$ \\
\hline Portugal & 470 & 489 & & Polen & $\begin{array}{l}\text { Lettland, Griechenland, } \\
\text { Ungarn }\end{array}$ & $\begin{array}{l}\text { Russ. Föderation, Israel, } \\
\text { Spanien, Tschech. Rep. }\end{array}$ & $\begin{array}{l}\text { Italien, Dänemark, Schweden, } \\
\text { Irland, Frankreich }\end{array}$ \\
\hline Italien & 487 & 486 & $\begin{array}{l}\text { Lettland, Portugal, } \\
\text { Griechenland }\end{array}$ & $\begin{array}{l}\text { Liechtenstein, Deutschland, } \\
\text { Schweiz, Polen, Ungarn }\end{array}$ & Spanien & Tschech. Rep. & \\
\hline Lettland & 458 & 484 & & & Portugal & Russ. Föderation, Israel & $\begin{array}{l}\text { Italien, Spanien, Griechenland, } \\
\text { Tschech. Rep. }\end{array}$ \\
\hline Griechenland & 474 & 483 & Lettland, Israel & $\begin{array}{l}\text { Liechtenstein, Deutschland, } \\
\text { Polen, Ungarn }\end{array}$ & Portugal & Russ. Föderation & Italien, Spanien, Tschech. Rep. \\
\hline Spanien & 493 & 481 & $\begin{array}{l}\text { Lettland, Israel, } \\
\text { Griechenland }\end{array}$ & $\begin{array}{l}\text { Liechtenstein, Deutschland, } \\
\text { Schweiz, Dänemark, } \\
\text { Polen, Portugal, Ungarn, } \\
\text { Ver. Staaten }\end{array}$ & Italien, Tschech. Rep. & & \\
\hline Tschech. Rep. & 492 & 478 & $\begin{array}{l}\text { Lettland, Israel, } \\
\text { Griechenland }\end{array}$ & \begin{tabular}{|l|} 
Italien, Liechtenstein, \\
Deutschland, Schweiz, \\
Dänemark, Polen, Portugal, \\
Ungarn, Ver. Staaten \\
\end{tabular} & Spanien & & \\
\hline Israel & 452 & 474 & & Lettland, Portugal & & Russ. Föderation & $\begin{array}{l}\text { Spanien, Griechenland, } \\
\text { Tschech. Rep. }\end{array}$ \\
\hline Russ. Föderation & 462 & 459 & & $\begin{array}{l}\text { Lettland, Israel, Portugal, } \\
\text { Griechenland }\end{array}$ & & & \\
\hline Chile & 410 & 449 & & & & $\begin{array}{l}\text { Argentinien, Thailand, } \\
\text { Bulgarien, Rumänien, } \\
\text { Mexiko }\end{array}$ & \\
\hline Bulgarien & 430 & 429 & & Chile & Thailand, Rumänien, Mexiko & Argentinien & \\
\hline Mexiko & 422 & 425 & & Chile & $\begin{array}{l}\text { Thailand, Bulgarien, } \\
\text { Rumänien }\end{array}$ & Argentinien & \\
\hline Rumänien & 428 & 424 & & Chile & Thailand, Bulgarien, Mexiko & Argentinien & \\
\hline Thailand & 431 & 421 & & Chile & $\begin{array}{l}\text { Bulgarien, Rumänien, } \\
\text { Mexiko }\end{array}$ & Argentinien & \\
\hline Brasilien & 396 & 412 & & & & Argentinien & \\
\hline Indonesien & 371 & 402 & & & & & Argentinien \\
\hline Argentinien & 418 & 398 & Indonesien & $\begin{array}{l}\text { Thailand, Bulgarien, } \\
\text { Rumänien, Brasilien, } \\
\text { Mexiko, Chile }\end{array}$ & & & \\
\hline Albanien & 349 & 385 & & & & & \\
\hline Peru & 327 & 370 & & & & & \\
\hline
\end{tabular}

Quelle: OECD, PISA-2009-Datenbank.

StatLink הत्राज http://dx.doi.org/10.1787/888932359967 
entsprachen die Leistungen von 18,1\% der Schülerinnen und Schüler 2009 nicht den Anforderungen von Stufe 2, während der entsprechende Prozentsatz 2000 19,3\% betrug (Tabelle V.2.2). Auch wenn sich dieser Prozentsatz zwischen den beiden Erhebungen nur leicht verändert hat, gab es von einem Land zum anderen deutliche Unterschiede.

Den prozentualen Anteil der schlecht abschneidenden Schülerinnen und Schüler zu verringern, wird in Anbetracht der hohen wirtschaftlichen und sozialen Kosten, die mit schlechten Schulleistungen verbunden sind, in vielen Ländern als eine der wichtigsten Aufgaben des Schulsystems betrachtet. Die Beobachtung der weiteren Entwicklung von Schülerinnen und Schülern, die in Kanada an der PISA-Erhebung 2000 teilgenommen hatten, im Rahmen des Canadian Youth in Transition Survey zeigt, dass für Schülerinnen und Schüler, deren Leistungen unter Stufe 2 liegen, ein unverhältnismäßig hohes Risiko besteht, dass sie nicht an postsekundärer Bildung teilnehmen oder im Alter von 19 Jahren schlechte Arbeitsmarktergebnisse erzielen, was sich im Alter von 21 Jahren, dem letzten Zeitpunkt, zu dem diese Daten vorliegen, noch verstärkt (OECD, 2010a).

In Abbildung V.2.4 sind die Veränderungen bei dem Anteil der Schülerinnen und Schüler dargestellt, deren Leistungen unter Stufe 2 lagen. Für jedes Land zeigt ein Balken den Prozentsatz der Schülerinnen und Schüler an, deren Leistungen bei PISA 2009 unter Stufe 2 lagen, während die rautenförmigen Symbole den entsprechenden Anteil bei PISA 2000 zeigen. Die Länder sind nach dem Prozentsatz der Schülerinnen und Schüler angeordnet, deren Leistungen 2009 unter Stufe 2 lagen, wobei auf der linken Seite diejenigen angegeben sind, in denen weniger Schülerinnen und Schüler lediglich diese niedrige Kompetenzstufe erreicht haben.

Um die Aussagefähigkeit von Vergleichen der Veränderungen bei den prozentualen Anteilen der Schülerinnen und Schüler auf den verschiedenen Kompetenzstufen zu erhöhen, können die Länder nach der Zahl der Schülerinnen und Schüler angeordnet werden, die bei PISA 2000 auf der jeweiligen Kompetenzstufe abgeschnitten hat. Bei PISA 2000 lagen die Leistungen von über $60 \%$ der Schülerinnen und Schüler in Peru, Albanien und Indonesien unter Stufe 2 (Tabelle V.2.2). In allen drei Ländern wurde eine Verringerung dieses Anteils um über 10 Prozentpunkte verzeichnet. Der Anteil der leistungsschwächeren Schülerinnen und Schüler liegt in diesen Ländern zwar nach wie vor auf einem relativ hohen Niveau, diese Entwicklung zeigt jedoch, dass in allen PISA-Teilnehmerländern, auf die die höchsten Anteile der 15-Jährigen entfallen, die eine begrenzte Lesekompetenz aufweisen, echte Fortschritte gemacht wurden.

Abbildung V.2.4

Prozentsatz der Schüler unter Lesekompetenzstufe 2, 2000 und 2009

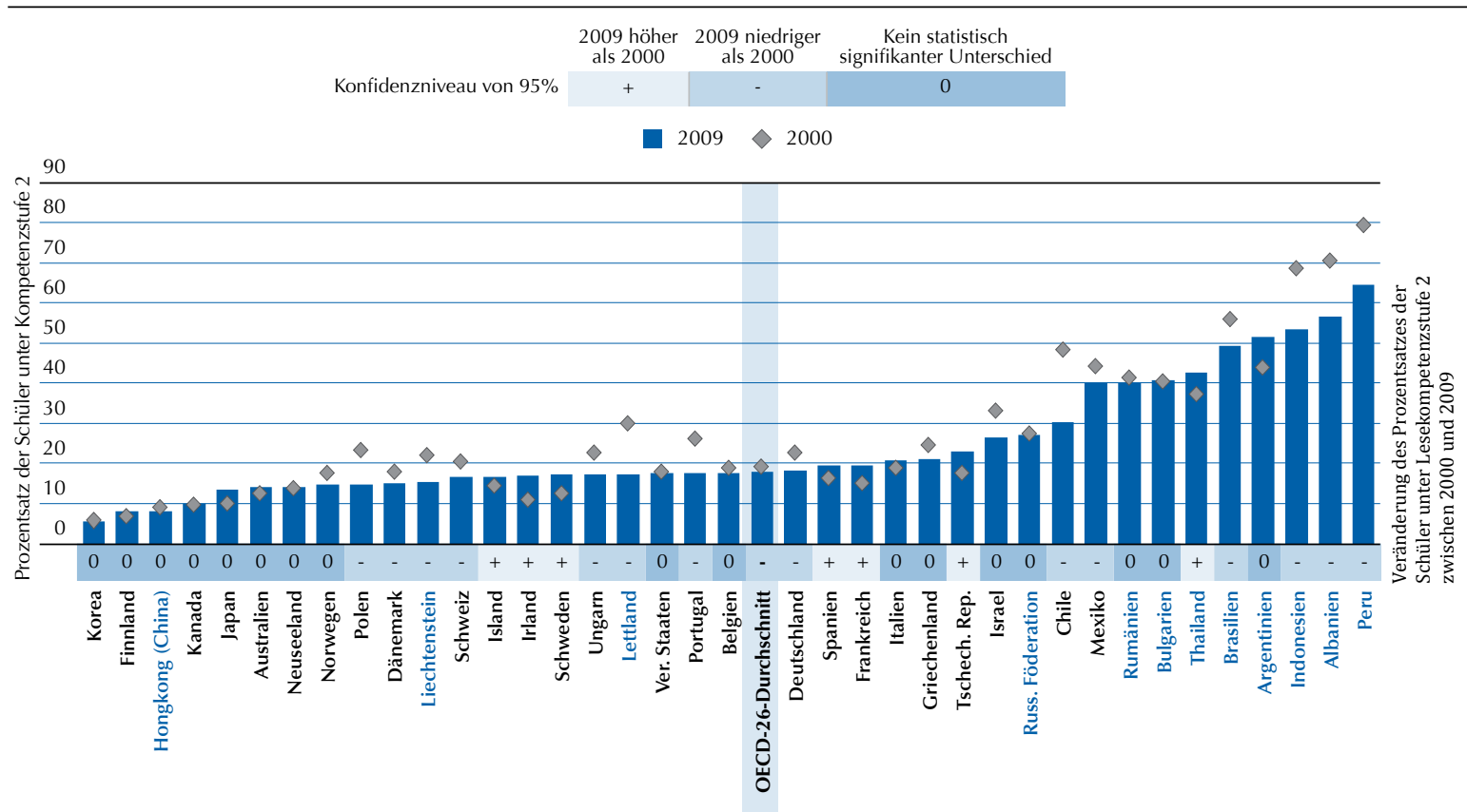

Die Länder sind in aufsteigender Reihenfolge des Prozentsatzes der Schülerinnen und Schüler unter Lesekompetenzstufe 2 angeordnet Quelle: OECD, PISA-2009-Datenbank, Tabelle V.2.2.

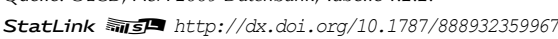


Unter den Ländern, in denen 40-60\% der Schülerinnen und Schüler bei PISA 2000 unter Stufe 2 lagen, verringerte sich dieser Anteil in Chile um 18 Prozentpunkte (vgl. Kasten V.F), während dieser Anteil in Mexiko sowie im Partnerland Brasilien langsamer abnahm (vgl. Kasten V.G).

Unter den Ländern, in denen der Anteil der Schülerinnen und Schüler, deren Leistungen unter Stufe 2 lagen, weniger als $40 \%$ betrug, aber immer noch über dem OECD-Durchschnitt von 19\% lag, verringerte das Partnerland Lettland den Anteil um 13\%, wohingegen der entsprechende Rückgang in Portugal, Polen, Ungarn, Deutschland, der Schweiz sowie dem Partnerland Liechtenstein geringer ausfiel (vgl. Kasten V.D für Portugal und V.C für Polen wegen Beispielen für Politikmaßnahmen, die mit diesen Entwicklungen im Zusammenhang stehen könnten). Im Partnerland Thailand erhöhte sich der Anteil der Schülerinnen und Schüler, deren Leistungen unter Stufe 2 lagen, gegenüber dem bereits relativ hohen Niveau von 37\% um weitere 6 Prozentpunkte. Unter den Ländern, in denen der Anteil der Schülerinnen und Schüler, deren Leistungen unter Stufe 2 lagen, bereits bei PISA 2000 unter dem Durchschnitt lag, senkte Dänemark diesen Anteil um weitere 3 Prozentpunkte, so dass dort nunmehr die Leistungen von 15\% der Schülerinnen und Schüler unter Stufe 2 liegen.

Der Anteil der Schülerinnen und Schüler, deren Leistungen unter Stufe 2 lagen, erhöhte sich in Irland, der Tschechischen Republik, Schweden, Frankreich, Spanien und Island. Dieser Anteil liegt in Island, Irland und Schweden zwar nach wie vor unter dem OECD-Durchschnitt, in Frankreich, Spanien und der Tschechischen Republik liegt er nun jedoch darüber.

Die Schülerinnen und Schüler, die die Anforderungen von Kompetenzstufe 5 oder 6 erfüllen, werden in diesem Bericht häufig als die „leistungsstärksten Schüler" bezeichnet. Diese Schülerinnen und Schüler sind in der Lage, mit von Inhalt oder Form her ungewohnten Texten umzugehen. Sie können Informationen in diesen Texten lokalisieren, ein genaues Verständnis dieser Texte nachweisen und herausfinden, welche der im Text enthaltenen Informationen für die Aufgabe von Belang sind. Anhand derartiger Texte sind sie auch in der Lage, kritische Bewertungen vorzunehmen und Hypothesen aufzustellen, dabei Fachwissen heranzuziehen und Konzepte zu begreifen, die u.U. im Gegensatz zum Erwarteten stehen. Aus einem Vergleich der Art von Aufgaben, die Schülerinnen und Schüler auf Stufe 5 und darüber lösen können, lässt sich schließen, dass diejenigen, die dieses Niveau erreichen, wohl als die potentiellen Weltklasse-Wissensarbeiter von morgen zu betrachten sind. Daher ist der Anteil der Schülerinnen und Schüler in den Teilnehmerländern, die den Anforderungen dieser Kompetenzstufe genügen, ein guter Indikator für die künftige wirtschaftliche Wettbewerbsfähigkeit der betreffenden Länder.

Im Durchschnitt der 26 OECD-Länder, für die vergleichbare Ergebnisse aus beiden PISA-Erhebungen vorliegen, betrug der kombinierte Prozentsatz der Schülerinnen und Schüler, deren Leistungen auf Stufe 5 oder 6 lagen, in PISA 2000 9,0\% und verringerte sich 2009 auf 8,2\% (vgl. Tabelle V.2.2). Auch wenn sich der Anteil der Schülerinnen und Schüler auf diesem Niveau zwischen den Erhebungen nur leicht verändert hat, bestehen zwischen den einzelnen Ländern erhebliche Unterschiede.

In Abbildung V.2.5 sind die Veränderungen bei den Anteilen der leistungsstärksten Schülerinnen und Schüler dargestellt. Die blauen Balken geben für jedes Land den Prozentsatz der Schülerinnen und Schüler an, deren Leistungen bei PISA 2009 auf Stufe 5 oder 6 lagen, wohingegen die rautenförmigen Symbole den jeweiligen Anteil bei PISA 2000 angeben. Die Länder sind nach dem Prozentsatz der Schülerinnen und Schüler angeordnet, deren Leistungen bei PISA 2009 auf Stufe 5 oder 6 lagen, wobei die Länder mit dem höchsten Anteil an besonders leistungsstarken Schülerinnen und Schülern auf der linken Seite stehen.

Der Anteil der besonders leistungsstarken Schülerinnen und Schüler erhöhte sich in Japan und Korea sowie in der Partnervolkswirtschaft Hongkong (China), auf die damit in dieser Hinsicht jeweils einer der höchsten Anteile unter den Teilnehmerländern der PISA-Erhebung 2009 entfällt (Tabelle V.2.2). In Japan stieg dieser Anteil von nahezu 10\% auf über $13 \%$. In Korea erhöhte er sich um über 7 Prozentpunkte von unter 6\% auf nahezu 13\%, was die größte beobachtete Veränderung unter den Teilnehmerländern war. Auf Grund dieser Verbesserung liegt Korea in Bezug auf den Anteil der leistungsstärksten Schülerinnen und Schüler nunmehr nicht mehr unter, sondern über dem OECDDurchschnitt (vgl. auch Kasten V.B). In Hongkong (China) erhöhte sich dieser Anteil um nahezu 3 Prozentpunkte auf leicht über $12 \%$. Unter den Ländern, in denen der Anteil der leistungsstärksten Schülerinnen und Schüler relativ gering ist, erhöhte sich der Prozentsatz der Schülerinnen und Schüler, deren Leistungen auf Stufe 5 oder 6 lagen, in Israel um 3 Prozentpunkte und in Chile sowie im Partnerland Brasilien um weniger als 1 Prozentpunkt.

In mehreren Ländern, in denen der Anteil der leistungsstärksten Schülerinnen und Schüler in PISA 2000 über dem Durchschnitt gelegen hatte, ging dieser Anteil zurück. Die deutlichste Veränderung war in Irland zu verzeichnen, 


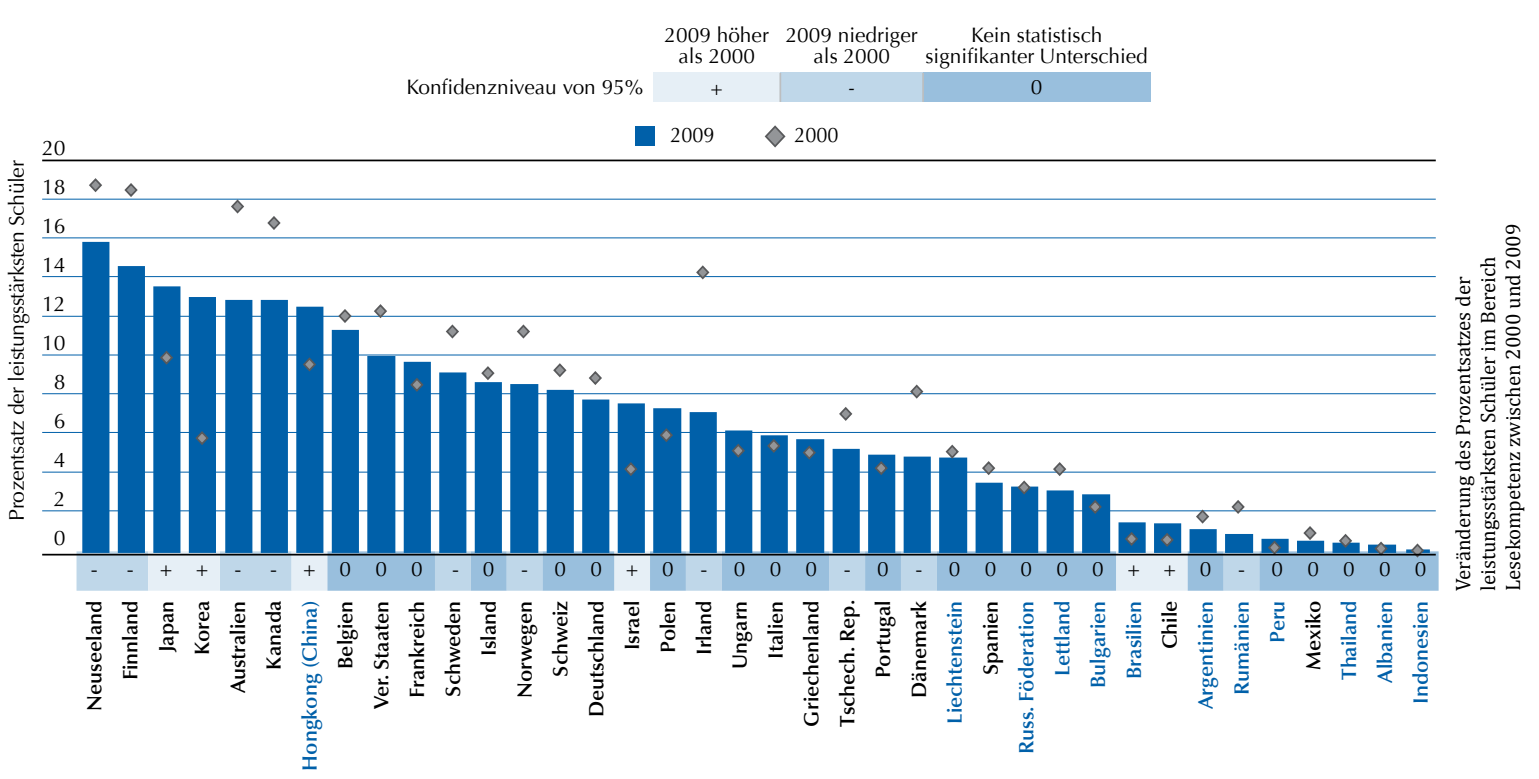

Anmerkung: Die Länder sind in absteigender Reihenfolge des Prozentsatzes der leistungsstärksten Schüler im Bereich Lesekompetenz im Jahr 2009 angeordnet. Quelle: OECD, PISA-2009-Datenbank, Tabelle V.2.2

StatLink त्राजम http://dx.doi.org/10.1787/888932359967

wo der Anteil der leistungsstärksten Schülerinnen und Schüler sich von $14 \%$ auf $7 \%$ verringerte, was unter dem Durchschnitt der 26 OECD-Länder liegt. In Australien, Kanada, Finnland und Neuseeland fiel der Rückgang geringer aus, und all diese Länder weisen immer noch mehr besonders leistungsstarke Schülerinnen und Schüler auf als der Durchschnitt der 26 OECD-Länder, für die aus beiden PISA-Erhebungen vergleichbare Ergebnisse vorliegen. Dieser Anteil sank in Norwegen und Schweden von einem ähnlichen Niveau von 11\% im Jahr 2000 auf 9\% in Schweden und $8 \%$ in Norwegen. Der Anteil der leistungsstärksten Schülerinnen und Schüler verringerte sich in Dänemark von $8 \%$ auf unter $5 \%$ und in der Tschechischen Republik von 7\% auf 5\%. Interessanterweise ging in Dänemark der Anteil der Schülerinnen und Schüler, deren Leistungen unter Stufe 2 lagen, ebenfalls zurück. Das Partnerland Rumänien ist das einzige Land, in dem der Anteil der leistungsstärksten Schülerinnen und Schüler von einem bereits niedrigen Niveau ausgehend weiter zurückging, von $2 \%$ auf unter $1 \%$.

Während für die Trends bei den Kompetenzstufen die leistungsstärksten und leistungsschwächsten Schülerinnen und Schüler anhand einer absoluten Messgröße verglichen werden, ist es auch möglich, die Leistungen am oberen und am unteren Ende der Verteilung auf der Leistungsskala im Verhältnis zu den durchschnittlichen Leistungen der Schülerinnen und Schüler innerhalb eines Landes zu vergleichen. Dies ist in Ländern, in denen das Leistungsniveau der Schülerinnen und Schüler insgesamt sehr niedrig bzw. sehr hoch ist und die internationalen Vergleichsgrößen für die leistungsstärksten und leistungsschwächsten Schülerinnen und Schüler u.U. weniger aussagefähig sind, besonders nützlich. Derartige Vergleiche innerhalb der Länder lassen sich durch die Analyse der Perzentile der Verteilung der Schülerleistungen innerhalb eines Landes erleichtern. Die Perzentile geben keinen Aufschluss darüber, wozu die Schülerinnen und Schüler in der Lage sind; sie liefern quantitative Informationen über die Leistungen der leistungsschwächsten bzw. leistungsstärksten Schülerinnen und Schüler im Vergleich zu anderen Schülerinnen und Schülern in dem betreffenden Land.

Das 90. Perzentil kennzeichnet den Punkt auf der PISA-Leistungsskala, unter dem die Leistungen von 90\% der Schülerinnen und Schüler in dem jeweiligen Land liegen bzw. den lediglich 10\% der Schülerinnen und Schüler übertreffen. DieVeränderungen des Werts des 90. Perzentils zeigen, ob in dem Land eine Anhebung oder Absenkung des Leistungsniveaus seiner leistungsstärksten Schülerinnen und Schüler erfolgt ist. Ebenso kennzeichnet das 10. Perzentil den Punkt auf der PISA-Leistungsskala, der lediglich von $10 \%$ der Schülerinnen und Schüler in dem betreffenden Land unterschritten wird. Eine Veränderung des Werts des 10. Perzentils zeigt, ob in dem Land eine Anhebung oder Absenkung des Leistungsniveaus seiner leistungsschwächsten Schülerinnen und Schüler erfolgt ist. 
Der Abstand zwischen dem 90. und dem 10. Perzentil kann als Messgröße des Leistungsspektrums in den einzelnen Ländern dienen. Die Entwicklung dieses Abstands zeigt, ob sich die Variationsbreite der Schülerleistungen in den einzelnen Ländern verändert.

Die auf den wichtigsten Perzentilrängen erzielten Ergebnisse können sich selbst dann verändern, wenn das Durchschnittsergebnis eines Landes unverändert bleibt.

In Abbildung V.2.6 sind die Länder in vier Gruppen klassifiziert (vgl. auch Tabelle V.2.3). Die Länder in der Ecke oben rechts weisen sowohl unter den leistungsstärksten als auch unter den leistungsschwächsten Schülerinnen und Schülern eine Leistungsverbesserung auf, wohingegen in den Ländern in der Ecke unten links für beide Schülergruppen ein Leistungsrückgang verzeichnet wurde. Die Länder in der Ecke oben links weisen Leistungsverbesserungen bei den leistungsstärksten Schülerinnen und Schülern sowie einen Leistungsrückgang bei den leistungsschwächsten Schülerinnen und Schülern auf. In diesen Ländern nahmen die Leistungsunterschiede auf Grund der sich vergrößernden Lücke zwischen den Schülerinnen und Schülern am oberen und am unteren Ende des Leistungsspektrums zu. Die Länder in der Ecke unten rechts weisen eine Leistungsverbesserung bei den leistungsschwächsten Schülerinnen und Schülern sowie einen Leistungsrückgang bei ihren leistungsfähigsten

\section{Leistungsveränderungen bei den leistungsschwächsten und leistungsstärksten Schülern im Bereich Lesekompetenz zwischen 2000 und 2009}

$\diamond \diamond$ Veränderungen bei den leistungsschwächsten und leistungsstärksten Schülern

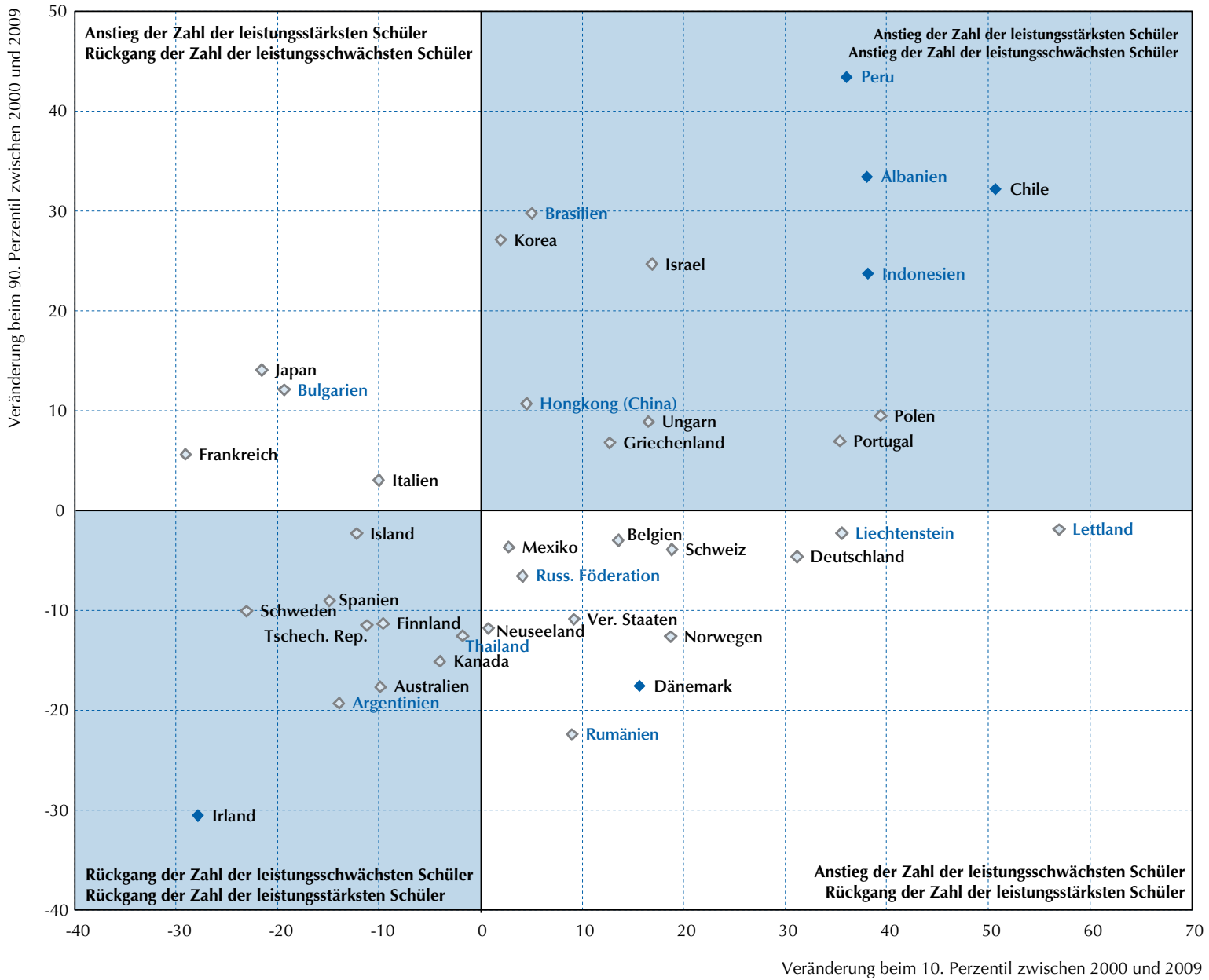

Anmerkung: Statistisch signifikante Veränderungen bei der Zahl der leistungsschwächsten und leistungsstärksten Schüler sind durch einen dunkleren Farbton gekennzeichnet. Quelle: OECD, PISA-2009-Datenbank, Tabelle V.2.3.

StatLink הत्ञाज http://dx.doi.org/10.1787/888932359967 
Schülerinnen und Schülern auf. In diesen Ländern hat sich der Leistungsabstand verringert. Die meisten Länder liegen jedoch in den Ecken oben rechts oder unten links, was darauf schließen lässt, dass die Leistungsentwicklung unter den leistungsschwächsten und leistungsstärksten Schülerinnen und Schülern in diesen Ländern ähnlich verlaufen ist. Die mit blauen Rautensymbolen gekennzeichneten Länder wiesen sowohl im Hinblick auf ihre leistungsstärksten als auch auf ihre leistungsschwächsten Schülerinnen und Schüler statistisch signifikante Leistungsveränderungen auf. Die mit weißen Rautensymbolen gekennzeichneten Länder wiesen entweder keine statistisch signifikanten Veränderungen auf bzw. verzeichneten diese lediglich für die leistungsstärksten oder leistungsschwächsten Schülerinnen und Schüler, aber nicht für beide zugleich.

Chile sowie drei Partnerländer, Indonesien, Albanien und Peru, weisen allesamt sowohl in Bezug auf die leistungsschwächsten als auch auf die leistungsstärksten Schülerinnen und Schüler deutlicheVerbesserungen bei den Leistungen im Bereich Lesekompetenz auf. Diese Länder zählen auch zu denjenigen, die die größte Verbesserung bei den Durchschnittsergebnissen verzeichnet haben und in denen der prozentuale Anteil der Schülerinnen und Schüler, deren Leistungen unter Stufe 2 liegen, abgenommen hat. Bei den leistungsschwächsten Schülerinnen und Schülern waren in Chile und Indonesien im Verhältnis größere Verbesserungen zu verzeichnen als bei den leistungsstärksten Schülerinnen und Schülern, wohingegen die Verbesserungen in Peru und Albanien bei beiden Schülergruppen ein ähnliches Niveau aufweisen. Kurz gesagt, haben sich die Leistungen der Schülerinnen und Schüler in diesen Ländern in Bezug auf die gesamte Leistungsskala verbessert.

Sechs Länder - Polen, Portugal, Deutschland, die Schweiz sowie die Partnerländer Lettland und Liechtenstein haben Leistungsverbesserungen bei ihren leistungsschwächsten Schülerinnen und Schülern verzeichnet, während das Leistungsniveau der leistungsstärksten Schülerinnen und Schüler unverändert geblieben ist.

In Korea, Israel und dem Partnerland Brasilien haben sich die Ergebnisse der leistungsstärksten Schülerinnen und Schüler verbessert, während das Leistungsniveau der leistungsschwächsten Schülerinnen und Schüler unverändert geblieben ist.

In Dänemark haben sich die Leistungen der leistungsschwächsten Schülerinnen und Schüler verbessert, während die Leistungen der leistungsstärksten Schülerinnen und Schüler zurückgegangen sind. Ebenso haben sich in Norwegen die Leistungen der leistungsschwächsten Schülerinnen und Schüler verbessert, und der Anteil der leistungsstärksten Schülerinnen und Schüler ist zurückgegangen. In der Folge hat sich der Leistungsabstand zwischen den leistungsschwächsten und den leistungsstärksten Schülerinnen und Schülern in diesen beiden Ländern deutlich verringert, während ihre Durchschnittsergebnisse unverändert geblieben sind.

In Australien und Kanada sowie im Partnerland Rumänien waren die Ergebnisse der leistungsstärksten Schülerinnen und Schüler rückläufig, während die Ergebnisse der leistungsschwächsten Schülerinnen und Schüler weitgehend unverändert blieben.

In Frankreich waren die Ergebnisse der leistungsschwächsten Schülerinnen und Schüler rückläufig, während die Ergebnisse der leistungsstärksten Schülerinnen und Schüler unverändert blieben.

In Irland und in gewissem Maße auch in Schweden gingen die Ergebnisse sowohl der leistungsschwächsten als auch der leistungsstärksten Schülerinnen und Schüler zurück. Diese Länder zählen auch zu denjenigen, deren Mittelwerte am stärksten gesunken sind und in denen der Prozentsatz der Schülerinnen und Schüler rückläufig war, die die höchste Kompetenzstufe erreichten, während der Prozentsatz derjenigen stieg, deren Leistungen unter Stufe 2 liegen.

Bei den restlichen Ländern fand keine messbare Veränderung der Leistungen der leistungsschwächsten und der leistungsstärksten Schülerinnen und Schüler statt.

\section{WIE SICH DIE GESCHLECHTSSPEZIFISCHEN UNTERSCHIEDE IM BEREICH LESEKOMPETENZ ENTWICKELT HABEN}

Der Abstand zwischen Jungen und Mädchen ist seit der ersten PISA-Erhebung im Jahr 2000 im Bereich Lesekompetenz weitaus größer als in Mathematik oder Naturwissenschaften. Die Mädchen schneiden in allen Ländern, die an PISA 2009 teilgenommen haben, besser ab als die Jungen, wobei der Leistungsvorsprung im Durchschnitt der OECD-Länder 39 Punkte beträgt (vgl. Tabelle V.2.4). Bei PISA 2000 betrug der entsprechende Leistungsvorsprung im Durchschnitt der OECD-Länder 32 Punkte. 


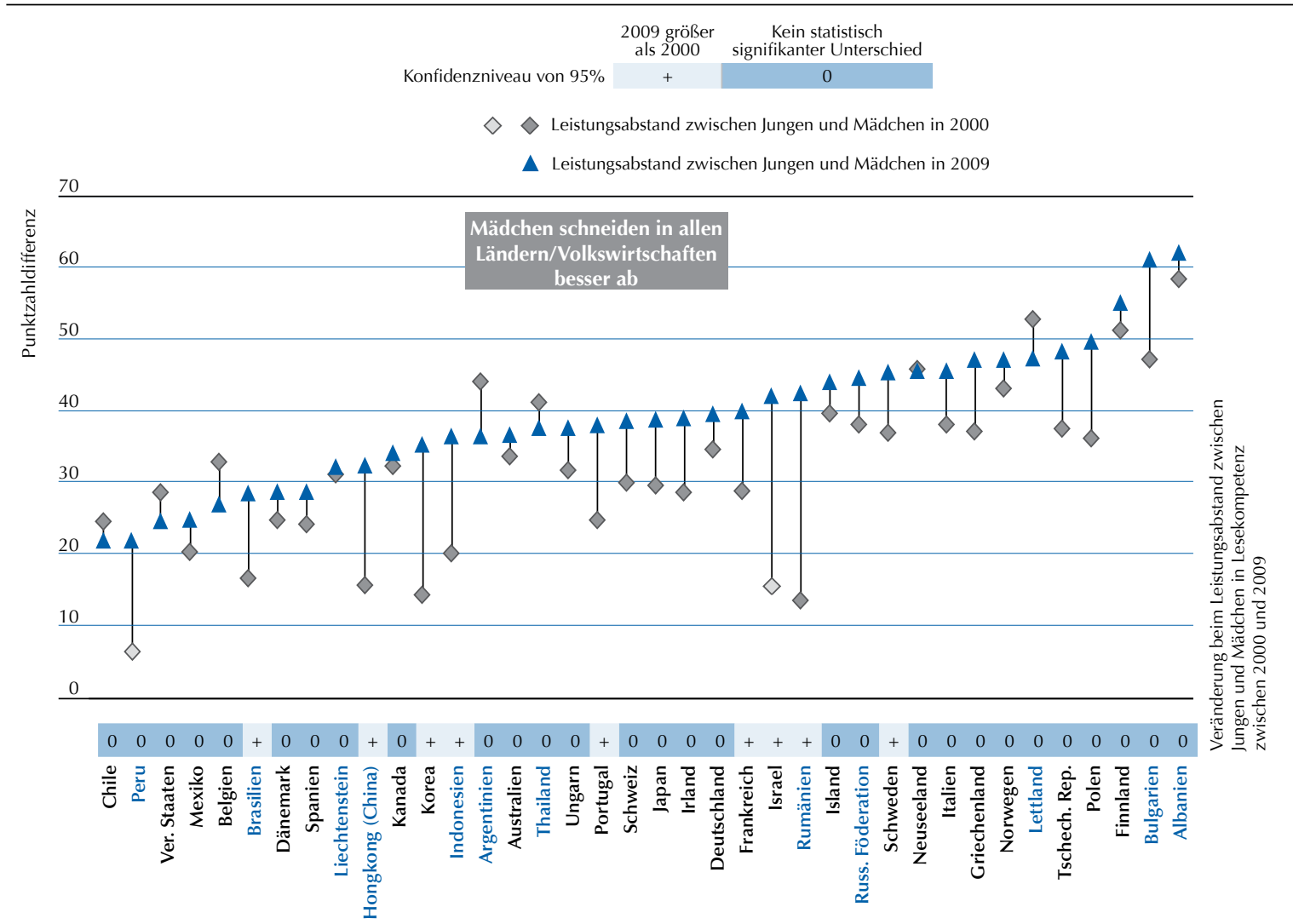

Anmerkung: Alle Unterschiede zwischen Jungen und Mädchen in PISA 2009 sind signifikant. Statistisch signifikante geschlechtsspezifische Unterschiede in PISA 2000 sind durch einen dunkleren Farbton gekennzeichnet.

Die Länder sind in aufsteigender Reihenfolge nach den geschlechtsspezifischen Unterschieden (Mädchen - Jungen) im lahr 2009 angeordnet. Quelle: OECD, PISA-2009-Datenbank, Tabelle V.2.4.

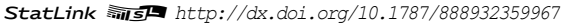

Die geschlechtsspezifischen Unterschiede vergrößerten sich in einigen Ländern, gingen jedoch in keinem einzigen Land zurück. Sie erhöhten sich in Israel und Korea sowie im Partnerland Rumänien um über 20 Punkte. In diesen drei Ländern stieg die Punktzahldifferenz zwischen Jungen und Mädchen mindestens auf das Doppelte. In Israel und Korea vergrößerte sich der Abstand, da sich die Leistungen der Mädchen deutlich verbesserten, während bei den Jungen keine ähnliche Entwicklung zu verzeichnen war (vgl. Kasten V.B, in dem die Veränderungen bei den Leistungen der Mädchen in Korea analysiert werden). Der Leistungsvorsprung der Mädchen vergrößerte sich auch in Portugal, der Partnervolkswirtschaft Hongkong (China) sowie den Partnerländern Indonesien und Brasilien, wo die insgesamt positive Entwicklung z.T. auf eine vergleichsweise stärkere Verbesserung bei den Mädchen als bei den Jungen zurückzuführen war. Der Leistungsabstand zwischen Jungen und Mädchen vergrößerte sich auch in Frankreich und Schweden, was insbesondere auf die Verschlechterung der Leistungen der Jungen zurückzuführen war.

Keines der Länder, in denen der Leistungsvorsprung der Mädchen sich erhöhte, zählt zu denjenigen, in denen die größten geschlechtsspezifischen Unterschiede bestehen. Nach den Veränderungen der relativen Leistungen der Jungen und Mädchen in Rumänien und Israel ist der Abstand in diesen Ländern jedoch größer geworden und liegt seither über dem OECD-Durchschnitt, während er zuvor darunter gelegen hatte.

Im Allgemeinen ist der Leistungsvorsprung der Mädchen im Hinblick auf den prozentualen Anteil der Schülerinnen und Schüler, deren Leistungen unter Stufe 2 liegen, besonders ausgeprägt (Tabelle V.2.5 und V.2.6). Im OECDDurchschnitt liegen die Leistungen von $24 \%$ der Jungen unter Stufe 2, gegenüber lediglich $12 \%$ der Mädchen. 
Die Politikverantwortlichen sind in vielen Ländern bereits besorgt über den hohen Anteil an Jungen, denen es an grundlegenden Lesefertigkeiten mangelt. Daher ist jeder Anstieg dieses Anteils erwähnenswert.

In Abbildung V.2.8 sind die Veränderungen der Anteile der Jungen und Mädchen dargestellt, deren Leistungen in Lesekompetenz unter Stufe 2 liegen. Die Länder sind nach der Grundtendenz bei den leistungsschwächeren Schülerinnen und Schülern angeordnet, wobei links diejenigen stehen, in denen ihre Zahl am stärksten zurückgegangen ist.

Im OECD-Durchschnitt ist der Anteil der Mädchen, deren Leistungen unter Stufe 2 liegen, um 2 Prozentpunkte gesunken, wohingegen der Anteil der leistungsschwächeren Jungen unverändert geblieben ist.

In fast allen Ländern, in denen der Anteil der Schülerinnen und Schüler zurückgegangen ist, deren Leistungen unter Stufe 2 liegen, ist dieser Trend bei den Mädchen in der Regel deutlicher zu Tage getreten. In Indonesien betrug der Rückgang des Anteils der Schülerinnen und Schüler, deren Leistungen unter Stufe 2 lagen, insgesamt rd. 15 Prozentpunkte; während der Anteil der Mädchen, deren Leistungen unter Stufe 2 lagen, jedoch um 21 Prozentpunkte zurückging, nahm der Anteil der Jungen, deren Leistungen auf demselben Niveau lagen, lediglich um 9 Prozentpunkte ab. Ebenso sank der Anteil der Mädchen, deren Leistungen unter Stufe 2 lagen, in Peru und Albanien um 19 bzw. 17 Prozentpunkte, wohingegen der entsprechende Anteil der Jungen jeweils nur um 11 bzw. 12 Prozentpunkte abnahm. In Israel und Brasilien war der insgesamt verzeichnete Rückgang des Anteils der Schülerinnen und Schüler, deren Leistungen unter Stufe 2 lagen, ebenfalls hauptsächlich das Ergebnis von Verbesserungen bei den Mädchen, wobei der Anteil der Mädchen, deren Leistungen unter Stufe 2 lagen, um 11 bzw. 9 Prozentpunkte sank. Die Abnahme des Anteils der Jungen, deren Leistungen unter Kompetenzstufe 2 lagen, fiel in diesen Ländern mit jeweils 2 bzw. 3 Prozentpunkten moderater aus.

In Chile und Polen verringerte sich der Anteil der Jungen und Mädchen, deren Leistungen unter Kompetenzstufe 2 lagen, etwa im selben Umfang.

Abbildung V.2.8

\section{Veränderung beim Anteil der Jungen und Mädchen, die im Zeitraum 2000-2009 im Bereich Lesekompetenz leistungsschwach waren}

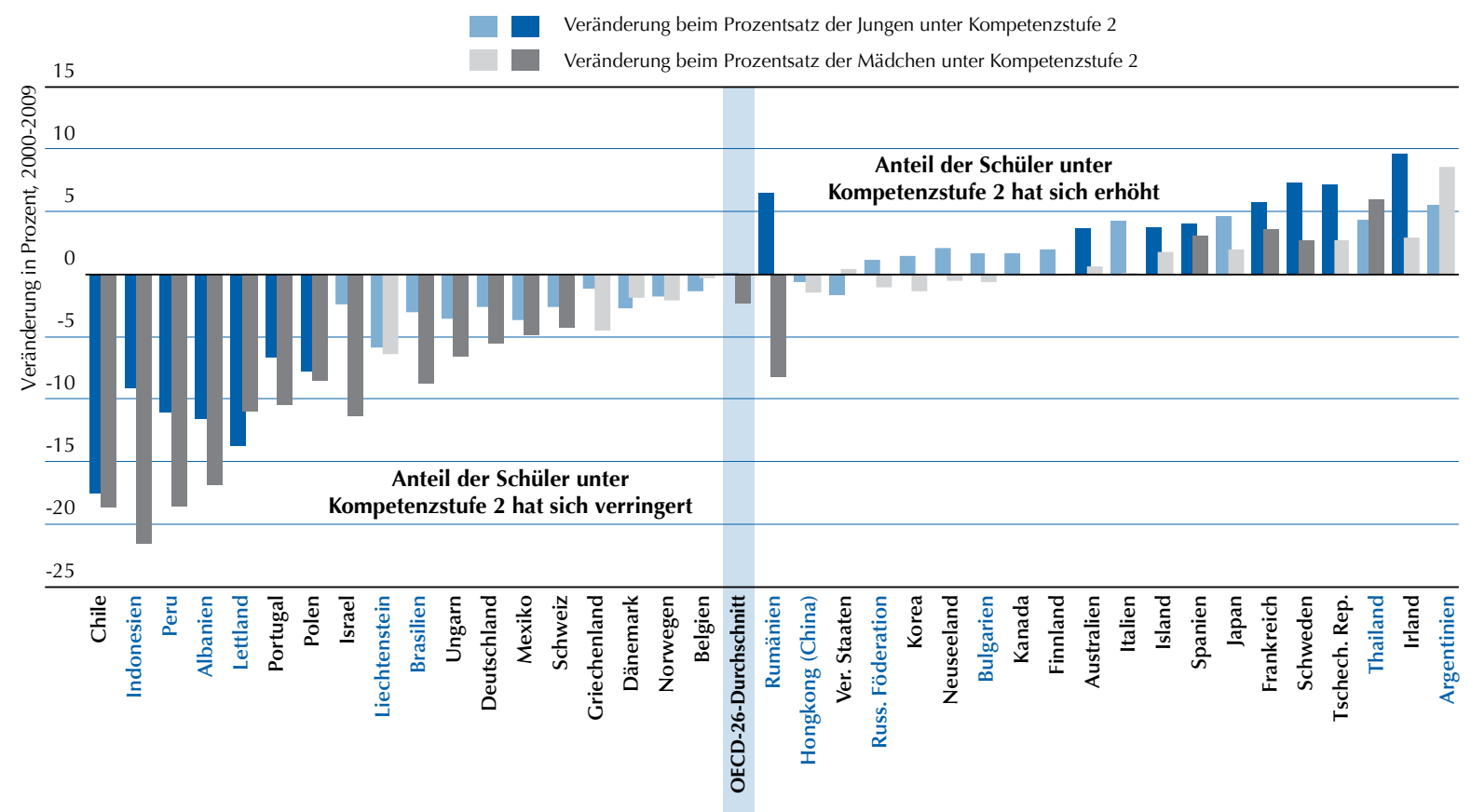

Anmerkung: Statistisch signifikante Veränderungen beim Anteil der Schüler unter Kompetenzstufe 2 sind durch einen dunkleren Farbton gekennzeichnet.

Die Länder sind in aufsteigender Reihenfolge nach der Veränderung des Prozentsatzes aller Schüler unter Stufe 2 auf der Gesamtskala Lesekompetenz im Zeitraum 2000-2009 angeordnet. Quelle: OECD, PISA-2009-Datenbank, Tabellen V.2.2, V.2.5 und V.2.6.

StatLink त्ताज $h t t p: / / d x . d o i . o r g / 10.1787 / 888932359967$ 
In einer anderen Ländergruppe ist der Anteil der Schülerinnen und Schüler gestiegen, deren Leistungen unter Kompetenzstufe 2 liegen. In Schweden, Frankreich und Spanien betraf dieser Anstieg sowohl die Jungen als auch die Mädchen, auch wenn er bei den Jungen stärker ausfiel. In Irland, der Tschechischen Republik und Island ist nur der Anteil der Jungen gestiegen, deren Leistungen in Lesekompetenz unter Stufe 2 liegen. In Thailand wiederum ist dieser Anteil bei den Mädchen leicht gestiegen, nicht jedoch bei den Jungen.

In den meisten Ländern verteilen sich die Veränderungen beim Anteil der leistungsstärksten Schülerinnen und Schüler, die die Lesekompetenzstufe 5 oder 6 erreichten, in etwa gleichermaßen auf Jungen und Mädchen, in einigen Ländern bestehen jedoch deutliche Unterschiede (Tabelle V.2.5 und V.2.6). Während etwa beim Anteil der leistungsstärksten Schülerinnen und Schüler in Dänemark und Rumänien für Jungen und Mädchen ein nahezu identischer Rückgang erfolgte, fiel die Entwicklung in Finnland, Australien, Kanada und Irland unterschiedlich aus. In Neuseeland nahm lediglich der Anteil der Mädchen unter den leistungsstärksten Schülerinnen und Schülern deutlich ab, wohingegen in der Tschechischen Republik und in Deutschland lediglich der Anteil der Jungen unter den leistungsstärksten Schülerinnen und Schülern signifikant abnahm.

Auch wenn der Anteil der besonders leistungsstarken Schülerinnen und Schüler in Japan und Korea sowie in der Partnervolkswirtschaft Hongkong (China) auf ein ähnlich hohes Niveau gestiegen ist, fiel der Anstieg bei Jungen und Mädchen sehr unterschiedlich aus. In Korea war der Anstieg bei Berücksichtigung aller Schülerinnen und Schüler zusammen am höchsten, aber auch bei gesonderter Betrachtung von Jungen und Mädchen. Indessen stieg der Anteil der leistungsstärksten Schülerinnen und Schüler bei den Mädchen um über 9 Prozentpunkte, bei den Jungen hingegen lediglich um knapp unter 5 Prozentpunkte. In Hongkong (China) stieg der Anteil der leistungsstärksten Schülerinnen und Schüler bei den Mädchen um über 6 Prozentpunkte, wohingegen er bei den Jungen unverändert blieb. Ebenso stieg dieser Anteil in Japan bei den Mädchen um nahezu 5 Prozentpunkte und übertraf damit den Anstieg bei den Jungen. Der Abstand hat sich im Hinblick auf den Anteil der leistungsstärksten Schülerinnen und Schüler bei den Jungen und Mädchen in diesen Ländern somit effektiv vergrößert.

\section{LEISTUNGSVERÄNDERUNGEN UND VERÄNDERUNGEN DER SCHÜLERPOPULATIONEN}

Die PISA-Erhebungen werden weiter fortentwickelt, um im Zuge der sich verändernden Lernziele und Unterrichtspraktiken in den Ländern, die methodische Fortschritte widerspiegeln, neu aufkommende Kenntnisse und Fähigkeiten zu erfassen. Gleichzeitig werden im Rahmen der aufeinanderfolgenden PISA-Erhebungen hohe technische Standards sowie eine kohärente Methodik umgesetzt, mit denen sichergestellt wird, dass die Leistungen im Zeitverlauf verlässlich beobachtet werden können und dass die Schülerstichproben jeweils für dieselbe Population repräsentativ sind.

In zahlreichen Ländern hat sich jedoch der demografische und sozioökonomische Kontext der Schülerpopulation gewandelt. Daher sind die beobachteten Veränderungen bei den Lernergebnissen u.U. nicht nur auf Veränderungen der Qualität der für die 15-Jährigen erbrachten Bildungsdienstleistungen zurückzuführen, sondern auch auf die Entwicklung in der Zusammensetzung der Schülerpopulation. Wenn ein Land etwa in den vergangenen zehn Jahren eine erhebliche Zuwanderung verzeichnet hat, wirkt sich dies u.U. auf die Lernergebnisse aus. Ebenso werden, wenn die Schülerpopulation in sozioökonomischer Hinsicht heterogener geworden ist, die Ergebnisse möglicherweise hierdurch beeinflusst.

In diesem Abschnitt wird untersucht, wie sich Veränderungen der Schülerpopulation auf die Trends auswirken. Er enthält ferner eine allgemeine Trendkurve, in der Daten aus allen PISA-Erhebungen zusammengefasst sind. Einzelheiten über die in diesem Abschnitt verwendeten Methoden finden sich in Anhang A6, in dem auch die Auswirkungen erörtert sind, die technische Veränderungen der nationalen Schülerstichproben im Lauf der Zeit u.U. auf die Vergleichbarkeit der Schülerleistungen haben.

\section{DER EFFEKT VON VERÄNDERUNGEN DER SOZIOÖKONOMISCHEN ZUSAMMENSETZUNG DER SCHÜLERPOPULATION AUF DIE LEISTUNGSTRENDS IM BEREICH LESEKOMPETENZ}

Im folgenden Abschnitt werden die Veränderungen bei der Zusammensetzung der Schülerpopulation in Bezug auf Alter und Geschlecht sowie beim sozioökonomischen Hintergrund der Schülerpopulation, Veränderungen des Anteils der Schülerinnen und Schüler, die die Testsprache immer oder fast immer zu Hause sprechen, sowie Veränderungen des Anteils der Schülerinnen und Schüler, deren Eltern im Ausland geboren sind, bei der Interpretation der Veränderungen der Schülerleistungen berücksichtigt. Die entsprechenden demografischen Daten für die Jahre 
2000 und 2009 sind in Anhang A6 dargestellt, wo auch die Anpassungsmethode detailliert erläutert ist. Die Daten über die Veränderungen beim sozioökonomischen Hintergrund finden sich in Tabelle V.4.2.

Abbildung V.2.9 zeigt sowohl die beobachtete Veränderung der Schülerleistungen als auch die vorhergesagte Leistungsveränderung, wenn die Zusammensetzung der Schülerpopulation im Jahr 2000 dieselbe gewesen wäre wie im Jahr 2009, d.h. wenn die Schülerpopulation 2000 dieselbe Zusammensetzung in Bezug auf Alter und Geschlecht, denselben sozioökonomischen Hintergrund und denselben Anteil von Schülerinnen und Schülern mit Migrationshintergrund wie 2009 aufgewiesen hätte (vgl. auch Tabelle V.2.7). Die beobachtete Veränderung wird durch Balken angezeigt, wohingegen die vorhergesagte Veränderung - bereinigt um Veränderungen der Zusammensetzung der Schülerschaft - durch rautenförmige Symbole gekennzeichnet ist. Die Länder sind nach der beobachteten Veränderung der Leistungen im Bereich Lesekompetenz angeordnet.

Abbildung V.2.9

Veränderungen bei den Leistungen im Bereich Lesekompetenz zwischen 2000 und 2009

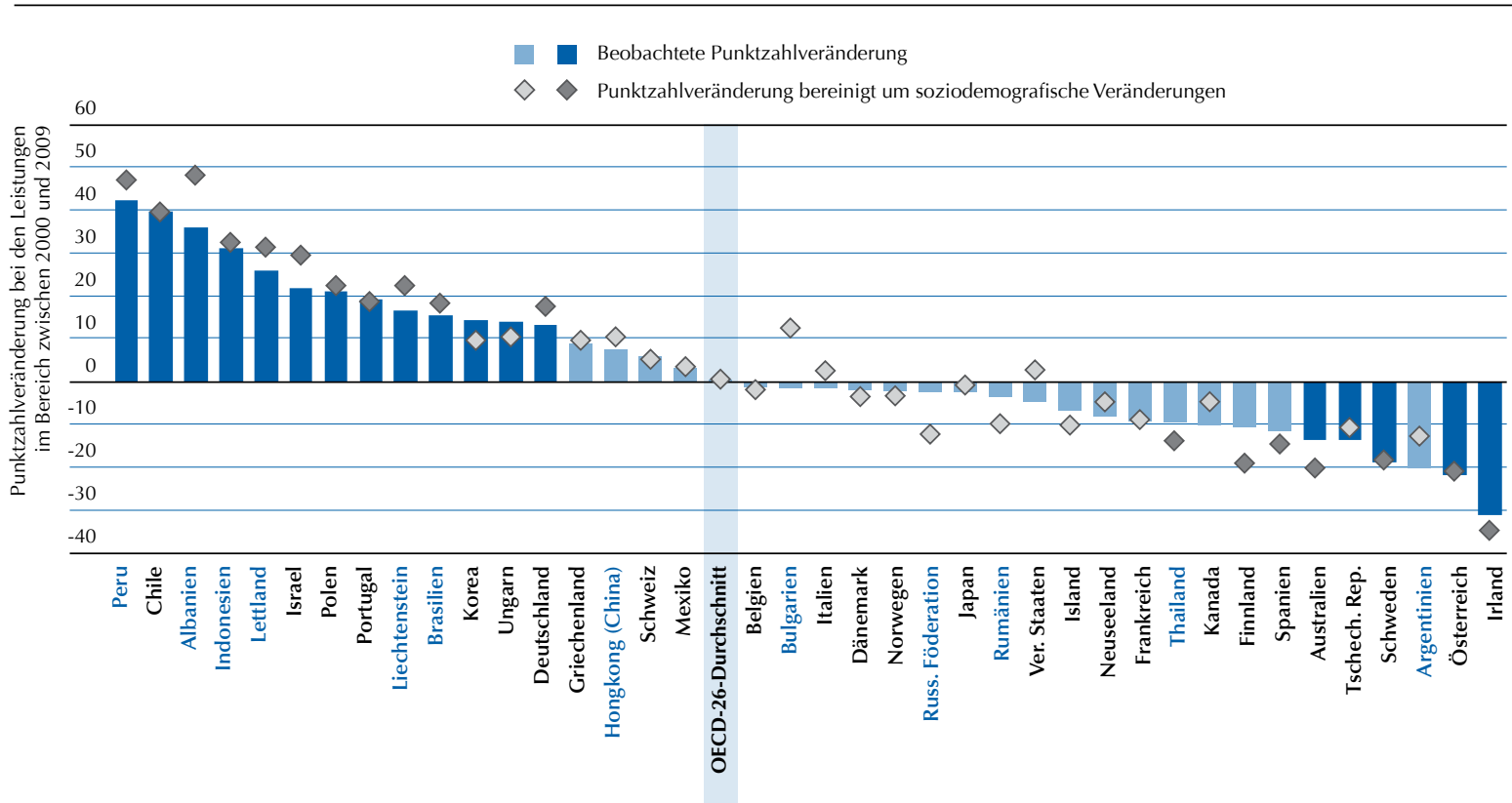

Anmerkung: Statistisch signifikante beobachtete Punktzahlveränderungen sind durch einen dunkleren Farbton gekennzeichnet. Die Länder sind in absteigender Reihenfolge nach der beobachteten Punktzahlveränderung im Zeitraum 2000-2009 angeordnet. Quelle: OECD, PISA-2009-Datenbank, Tabelle V.2.7.

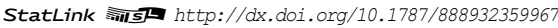

Unter den Ländern, deren Ergebnisse im Bereich Lesekompetenz sich verbessert haben, hatten Veränderungen der demografischen und sozioökonomischen Zusammensetzung der Schülerpopulation in Israel und im Partnerland Albanien die größten Auswirkungen, wo die Verbesserungen der Schülerleistungen jeweils um 7 bzw. 12 Punkte höher ausgefallen wären, wenn der demografische und sozioökonomische Kontext in den Jahren 2000 und 2009 derselbe gewesen wäre (vgl. Tabelle V.2.1 und V.2.7)2. In Deutschland sowie den Partnerländern Liechtenstein, Lettland und Peru fiel die bereinigte Leistungsverbesserung um rd. 5 Punkte höher aus. In diesen Ländern hätten sich die Schülerleistungen über die tatsächlich beobachteten Leistungssteigerungen hinaus noch rascher verbessert, wenn die Veränderungen der demografischen und sozioökonomischen Zusammensetzung der Schülerpopulation berücksichtigt worden wären. Dies ist auf die Tatsache zurückzuführen, dass die Schülerpopulation in diesen Ländern 2009 einen stärker benachteiligten sozioökonomischen Hintergrund hatte als im Jahr 2000 (vgl. Tabelle V.4.2 sowie Anhang A6).

In Australien und Irland hätten sich die Schülerleistungen rascher als tatsächlich beobachtet verschlechtert, wenn die Veränderungen des sozioökonomischen Hintergrunds der Schülerpopulation berücksichtigt worden wären. In Finnland lassen die Ergebnisse darauf schließen, dass sich die Leistungen bei Berücksichtigung der Veränderungen der demografischen und sozioökonomischen Schülermerkmale verschlechtert hätten. Dies erklärt sich aus der 
Tatsache, dass die Schülerpopulation in diesen Ländern 2009 in sozioökonomischer Hinsicht stärker begünstigt war als im Jahr 2000. In Ungarn und Korea verringert sich der Schätzwert für den positiven Trend durch die Berücksichtigung des sozioökonomischen Hintergrunds. In Thailand und Spanien erhält die Leistungsveränderung nach entsprechender Bereinigung ein negatives Vorzeichen, wohingegen ohne Bereinigung keine signifikante Veränderung stattgefunden hatte.

\section{BERECHNUNG EINES GESAMTSCHÄTZWERTS FÜR DIE LEISTUNGSTRENDS IM BEREICH LESEKOMPETENZ}

Die in den einzelnen PISA-Erhebungen erzielten Ergebnisse im Bereich Lesekompetenz lassen sich zu einem einzelnen Trendindikator zusammenfassen. Die Ergebnisse sind sowohl im Hinblick auf die beobachteten Schülerleistungen im Bereich Lesekompetenz als auch auf die Leistungen nach Berücksichtigung der oben beschriebenen demografischen und sozioökonomischen Merkmale in Tabelle V.2.8 dargestellt. Die daraus resultierenden Trends wurden auf Jahresrate hochgerechnet, so dass die Daten die Leistungsveränderungen in einem bestimmten Jahr widerspiegeln. In Anhang A6 finden sich Einzelheiten zu den Methoden, die angewandt wurden, um lineare Trends zu berechnen.

In Abbildung V.2.10 werden diese linearen Trends mit den annualisierten Leistungsunterschieden im Zeitraum 20002009 verglichen. Vier Länder haben ihre Ergebnisse im Bereich Lesekompetenz gesteigert, wenn die Ergebnisse aller Erhebungen berücksichtigt werden. In Korea lässt der lineare Trend auf größere Verbesserungen schließen, da der lineare Trend um 0,6 Punkte über dem auf Jahresrate berechneten Leistungsunterschied im Zeitraum 2000-2009 liegt. Dies ist auf rasche Leistungsverbesserungen bis 2006 zurückzuführen, an die sich ein leichter Rückgang im Jahr 2009 anschloss. In der Partnervolkswirtschaft Hongkong (China) steigt der lineare Trend zwischen 2000 und 2009 um 1 Punkt jährlich. Nachdem 2003 zunächst eine Verschlechterung verzeichnet wurde, verbesserten sich die

Lineare Trends und Leistungsunterschiede im Zeitraum 2000-2009

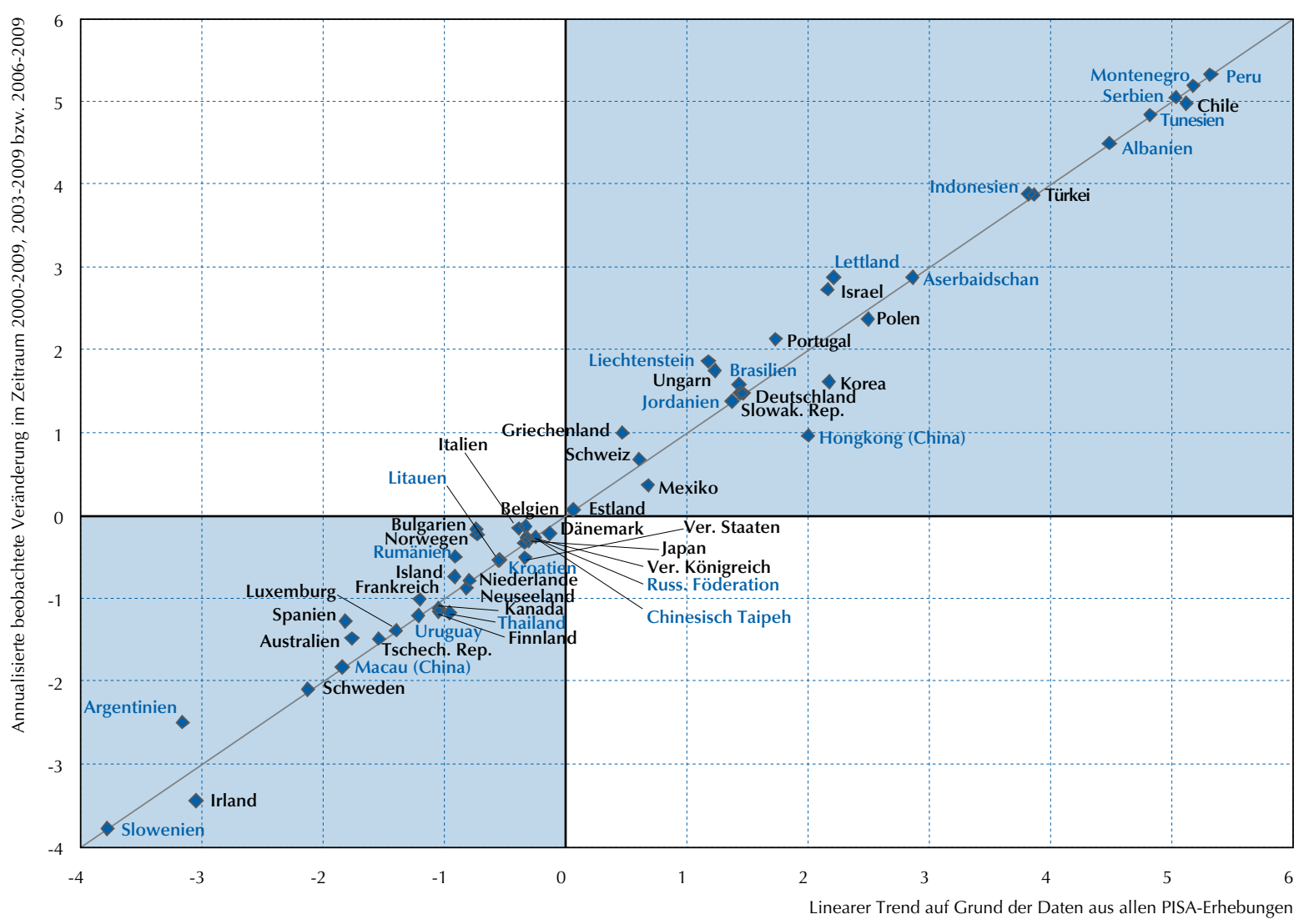


Leistungen in Hongkong (China) 2006 und noch weiter im Jahr 2009. In Polen und Chile zeigen die linearen Trends ebenfalls etwas größere Verbesserungen. In Polen spiegelt dies ein ähnliches Muster wie das in Korea beobachtete wider, wobei die Veränderungen jedoch geringer ausfielen. In Chile geht aus den Daten eine große Verbesserung im Zeitraum 2000-2006 hervor, der eine wesentlich geringere Veränderung zwischen den letzten beiden Erhebungen folgte.

Für Israel sowie die Partnerländer Liechtenstein, Lettland und Brasilien zeigen die linearen Trends im Vergleich zu den für den Zeitraum 2000-2009 beobachteten Leistungsunterschieden geringere oder sogar gar keine signifikanten Verbesserungen. Dies ist darauf zurückzuführen, dass die Leistungen in diesen Ländern von einer PISA-Erhebung zur nächsten erheblich variierten.

In Spanien und im Partnerland Argentinien ist der lineare Trend negativer als die Leistungsunterschiede zwischen 2000 und 2009. In Spanien ist dies auf eine stetige Verschlechterung der Leistungen zwischen 2000 und 2006 und jüngste Verbesserungen im Jahr 2009 zurückzuführen, wobei die Durchschnittsergebnisse nach wie vor unter den im Jahr 2000 erzielten lagen. In Argentinien liegt ein ähnliches Muster vor, da sich die Ergebnisse im Zeitraum 20002006 verschlechterten, bei der jüngsten Erhebung jedoch wiederum leicht verbesserten.

\section{VERGLEICH DER LESETRENDS VON LAND ZU LAND}

In Abbildung V.2.11, V.2.12 und V.2.13 sind die Veränderungen bei der Verteilung der Leistungen im Bereich Lesekompetenz zusammengefasst. Der Gesamttrend bei den Durchschnittsergebnissen im Zeitraum 2000-2009 ist durch die gestrichelte Linie dargestellt, die dem im vorstehenden Abschnitt beschriebenen linearen Trend entspricht. Wo die gestrichelte Linie fett gedruckt ist, haben sich die Durchschnittsergebnisse auf statistisch signifikante Weise verbessert bzw. verschlechtert. Die durchgezogene Linie zeigt Veränderungen bei den Gesamtergebnissen zwischen den aufeinanderfolgenden PISA-Erhebungen an.

Die Leistungstrends für die leistungsschwächsten Schülerinnen und Schüler sind jeweils im unteren Bereich der Abbildungen dargestellt. Der untere Rand dieser dunkelblauen Bereiche zeigt die Ergebnisse am 10. Perzentil an, und der untere dunkle Bereich insgesamt steht für das Leistungsspektrum zwischen dem 10. und dem 25. Perzentil. Der obere Rand des oberen dunkelblauen Bereichs gibt die Ergebnisse am 90. Perzentil wieder, und dieser zweite dunkle Bereich veranschaulicht das Leistungsspektrum zwischen dem 90. und dem 75. Perzentil. Der Bereich zwischen dem 10. und dem 90. Perzentil gibt die Varianz bei den Schülerleistungen an, d.h. je schmaler der blaue Bereich, desto weniger breit gestreut sind die Leistungen im Bereich Lesekompetenz in einer bestimmten Erhebung. Veränderungen des durch den blauen Bereich abgedeckten Spektrums zeigen die Leistungstrends an.

In Abbildung V.2.11, V.2.12 und V.2.13 sind die Länder nach Leistung angeordnet, so dass sich die Veränderungen in der Leistungsverteilung jedes Landes mit denen in den Ländern mit ähnlicher Gesamtleistung vergleichen lassen. In Abbildung V.2.11 sind die Länder dargestellt, deren Mittelwerte über dem OECD-Durchschnitt liegen, in Abbildung V.2.12 Länder, deren Mittelwerte in etwa dem OECD-Durchschnitt entsprechen, und in Abbildung V.2.13 Länder, deren Mittelwerte unter dem OECD-Durchschnitt liegen.

Aus diesen Abbildungen für die einzelnen Länder ist ersichtlich, dass sich die Länder nicht nur in Bezug auf die Entwicklung der Leistungen des durchschnittlichen Schülers in Lesekompetenz unterscheiden, sondern auch in Bezug darauf, wie sich die Leistungstrends bei den leistungsstärksten und den leistungsschwächsten Schülerinnen und Schülern unterscheiden. So unterscheiden sich etwa die beiden OECD-Länder Korea und Polen, deren Ergebnisse nach enormen Verbesserungen seit dem Jahr 2000 nunmehr überdurchschnittlich ausfallen, nicht nur in Bezug auf die durchschnittlichen Leistungen und die Stärke der Trends, sondern auch im Hinblick darauf, wie sich die verschiedenen Schülergruppen in diesen Ländern im Lauf der Zeit entwickelt haben. In beiden Ländern wurden im Zeitraum 2000-2006 Verbesserungen erzielt, wohingegen die Ergebnisse zwischen 2006 und 2009 leicht rückläufig waren. Indessen ist die Gesamttendenz in beiden Ländern jedoch signifikant und positiv. Während die Entwicklung in Korea weitgehend durch Verbesserungen unter den leistungsstärksten Schülerinnen und Schülern bestimmt war und bei den leistungsschwächsten keine Veränderung stattfand, verbesserten in Polen die leistungsschwächsten Schülerinnen und Schüler ihre Leistungen, wohingegen die Leistungen der leistungsstärksten sich nicht signifikant veränderten. Beim Vergleich der Abbildungen für diese beiden Länder wird darüber hinaus deutlich, dass sich der Abstand zwischen den leistungsstärksten und den leistungsschwächsten Schülerinnen und Schülern in Korea zwar vergrößerte, in Polen jedoch eine umgekehrte Entwicklung stattfand, wobei der daraus resultierende Leistungsabstand in Korea nach wie vor geringer ist als in Polen. 
Abbildung V.2.11 [Teil 1/2] $\square$

Trends bei den Leseleistungen: Länder über dem OECD-Durchschnitt
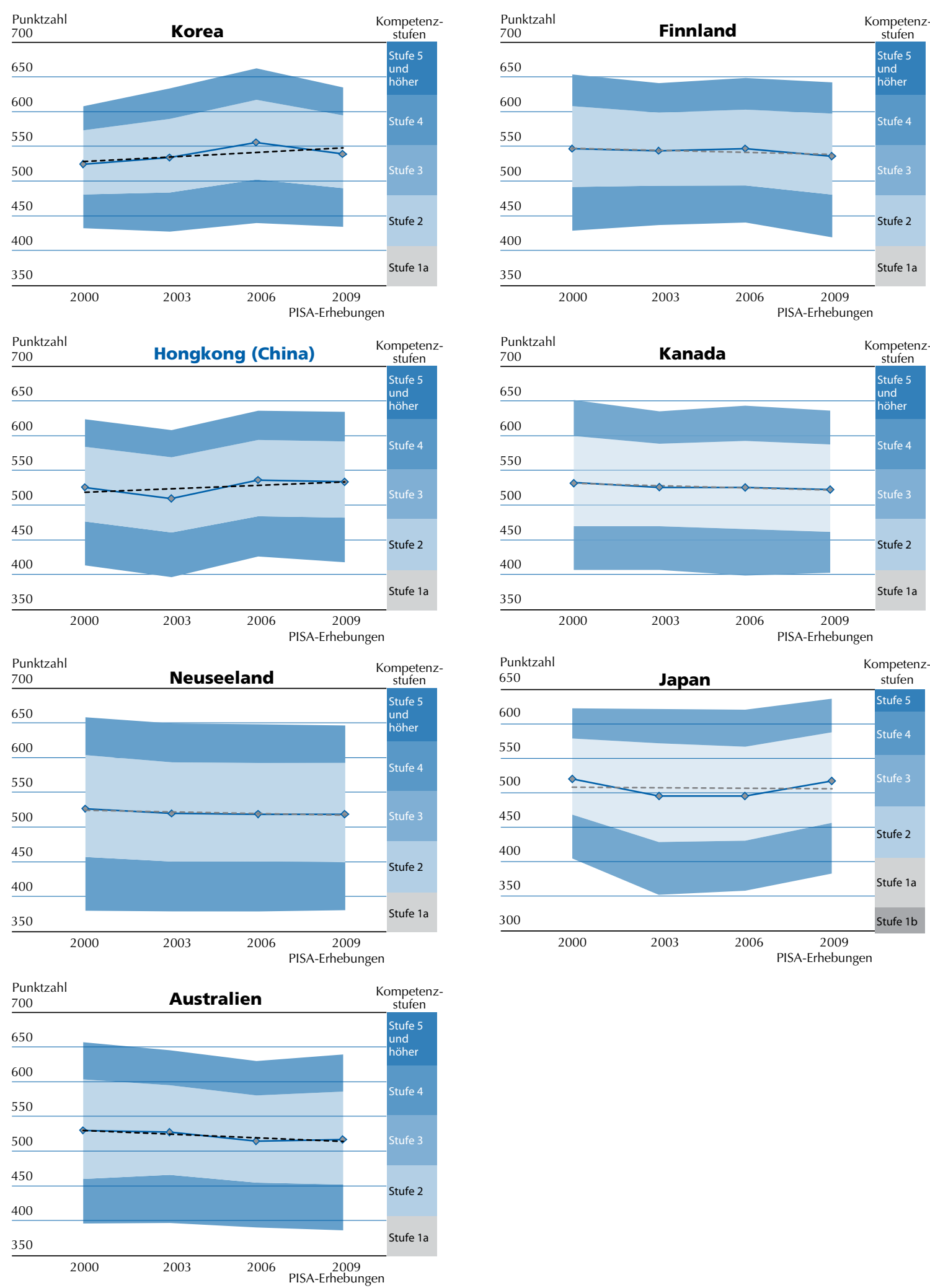

Quelle: OECD, PISA-2000-Datenbank, PISA-2003-Datenbank, PISA-2006-Datenbank und PISA-2009-Datenbank. StatLink 체내 http://dx.doi.org/10.1787/888932359967 
Abbildung V.2.11 [Teil 2/2]

Trends bei den Leseleistungen: Länder über dem OECD-Durchschnitt
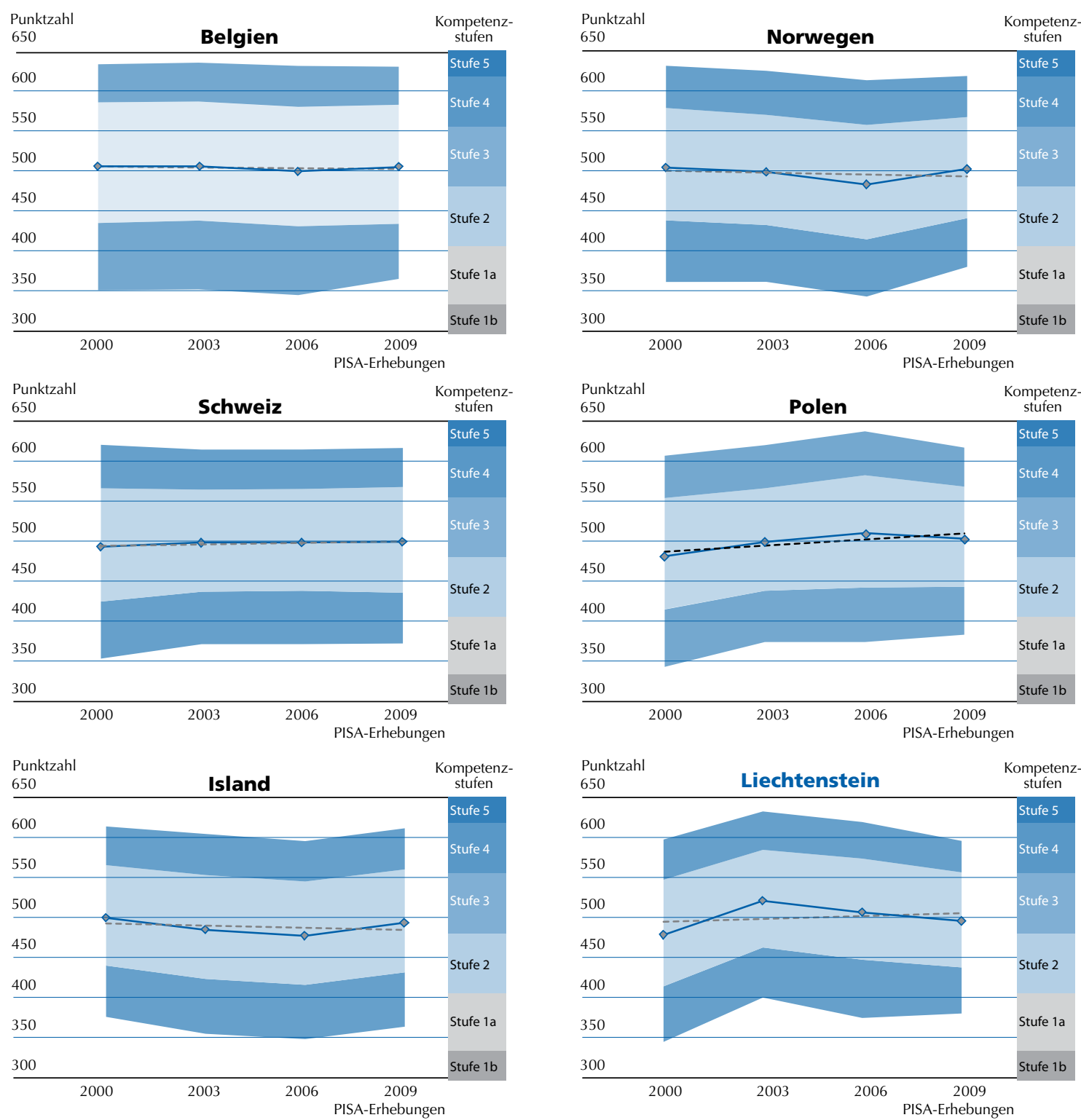

Quelle: OECD, PISA-2000-Datenbank, PISA-2003-Datenbank, PISA-2006-Datenbank und PISA-2009-Datenbank.

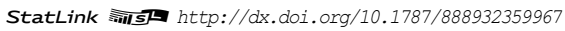


Abbildung V.2.12

Trends bei den Leseleistungen: Länder im OECD-Durchschnitt
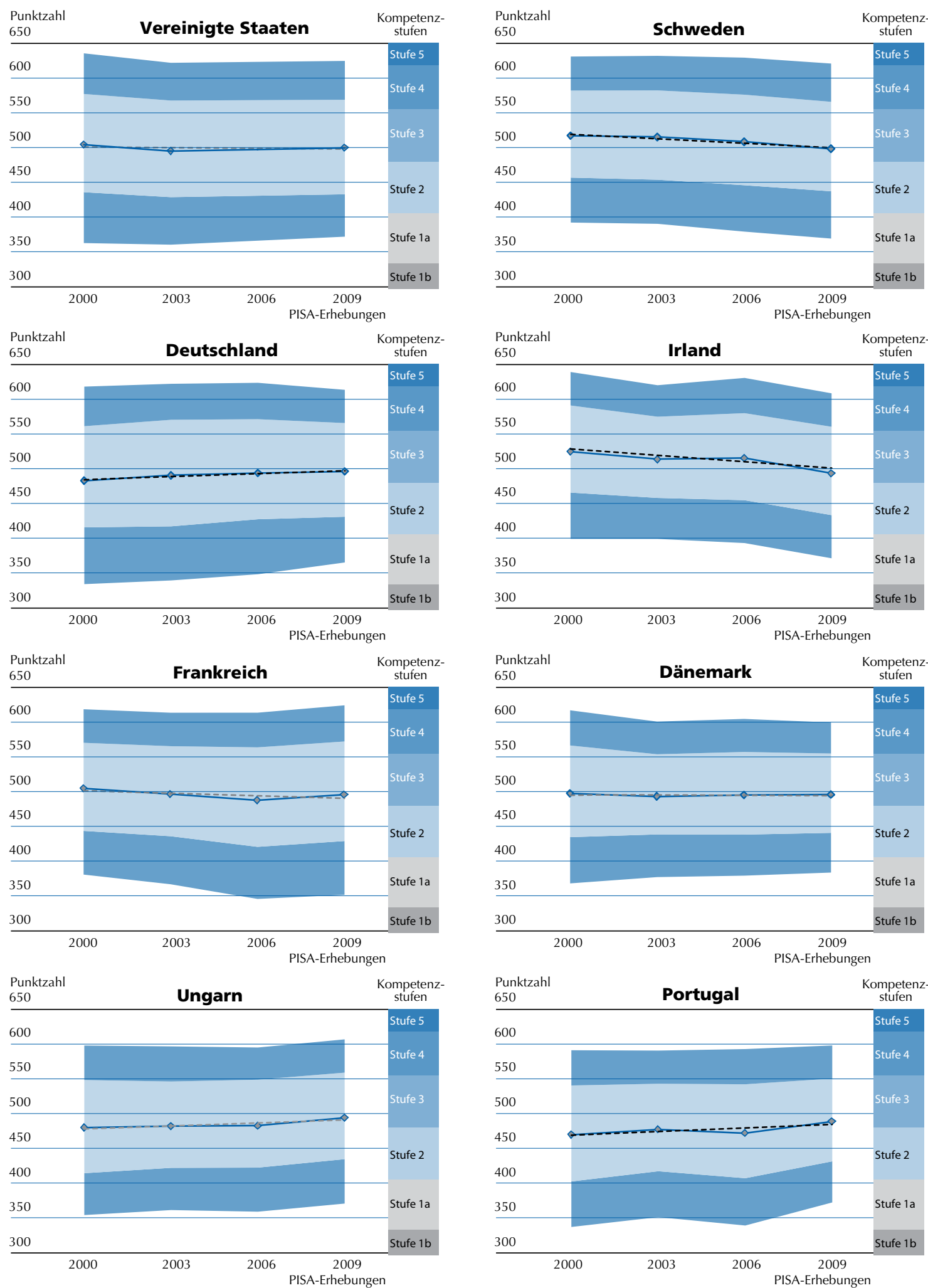

Quelle: OECD, PISA-2000-Datenbank, PISA-2003-Datenbank, PISA-2006-Datenbank und PISA-2009-Datenbank.

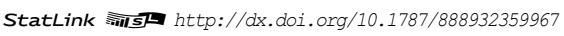


Abbildung V.2.13 [Teil 1/3]

Trends bei den Leseleistungen: Länder unter dem OECD-Durchschnitt
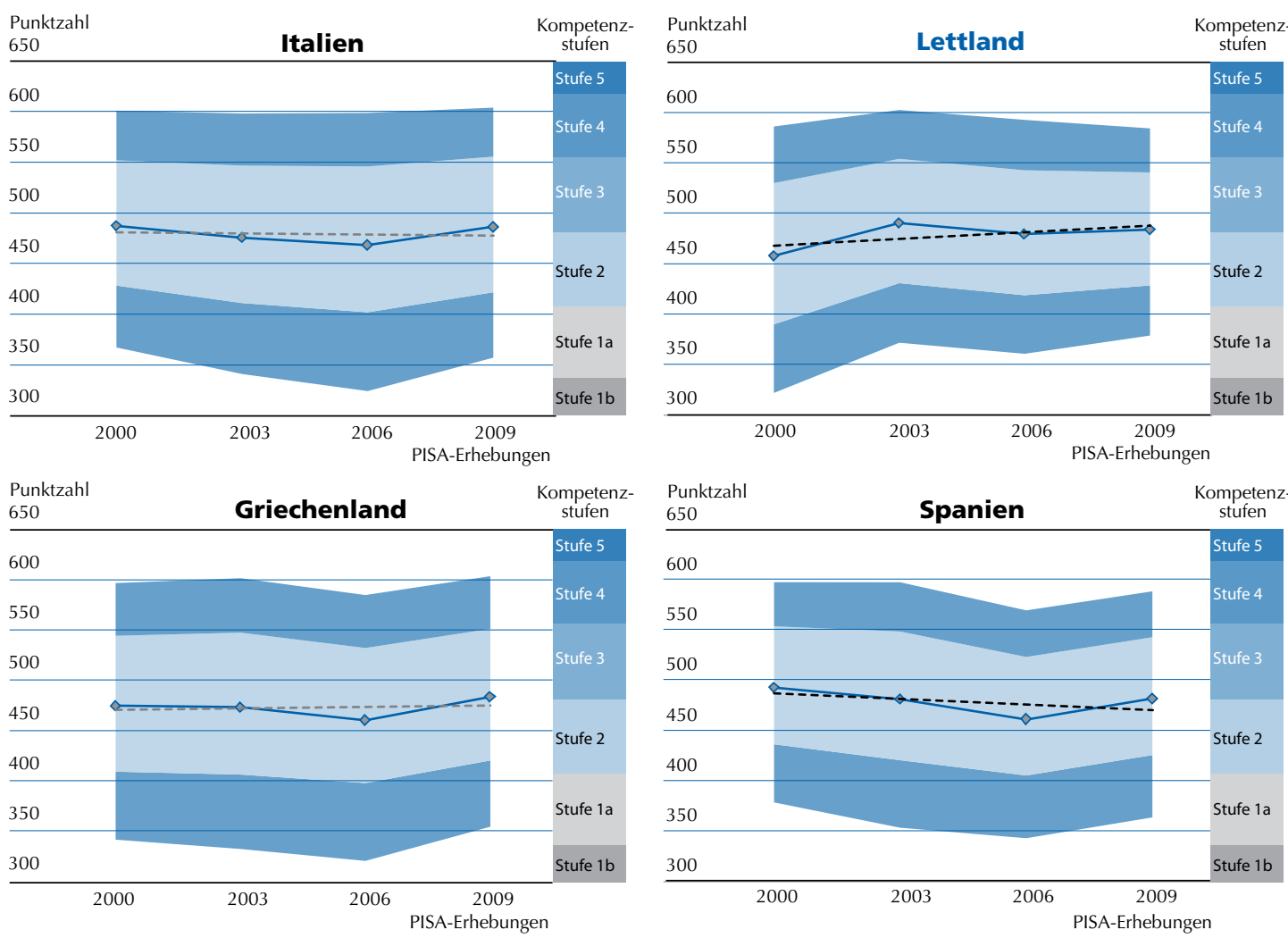

Kompeten
stufen

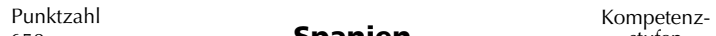

$\begin{array}{lll}650 & \text { Spanien stufen }\end{array}$
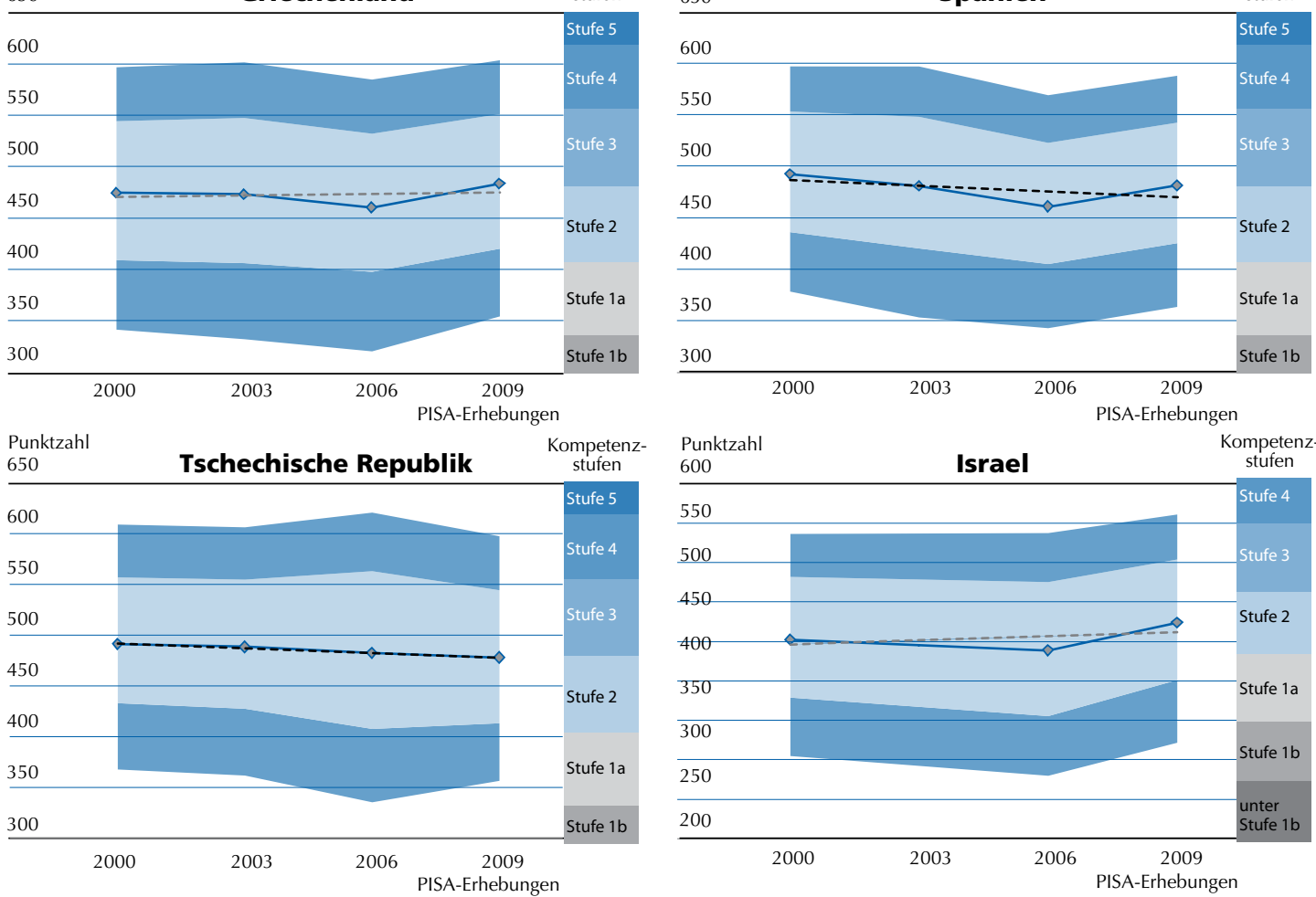

Quelle: OECD, PISA-2000-Datenbank, PISA-2003-Datenbank, PISA-2006-Datenbank und PISA-2009-Datenbank.

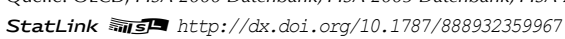


Abbildung V.2.13 [Teil 2/3]

Trends bei den Leseleistungen: Länder unter dem OECD-Durchschnitt
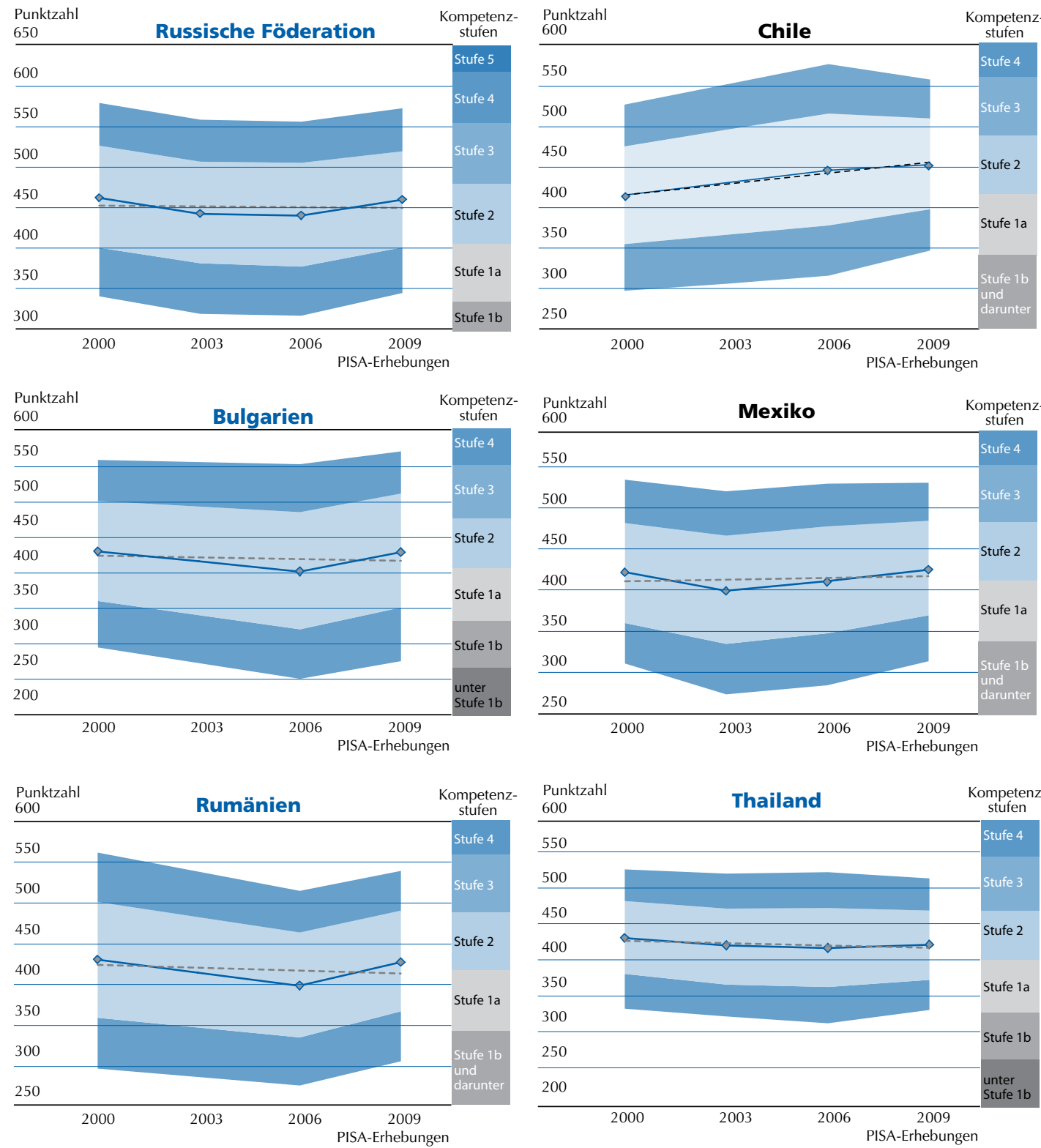

Quelle: OECD, PISA-2000-Datenbank, PISA-2003-Datenbank, PISA-2006-Datenbank und PISA-2009-Datenbank.

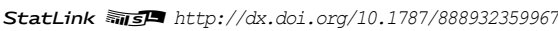


Abbildung V.2.13 [Teil 3/3]

Trends bei den Leseleistungen: Länder unter dem OECD-Durchschnitt
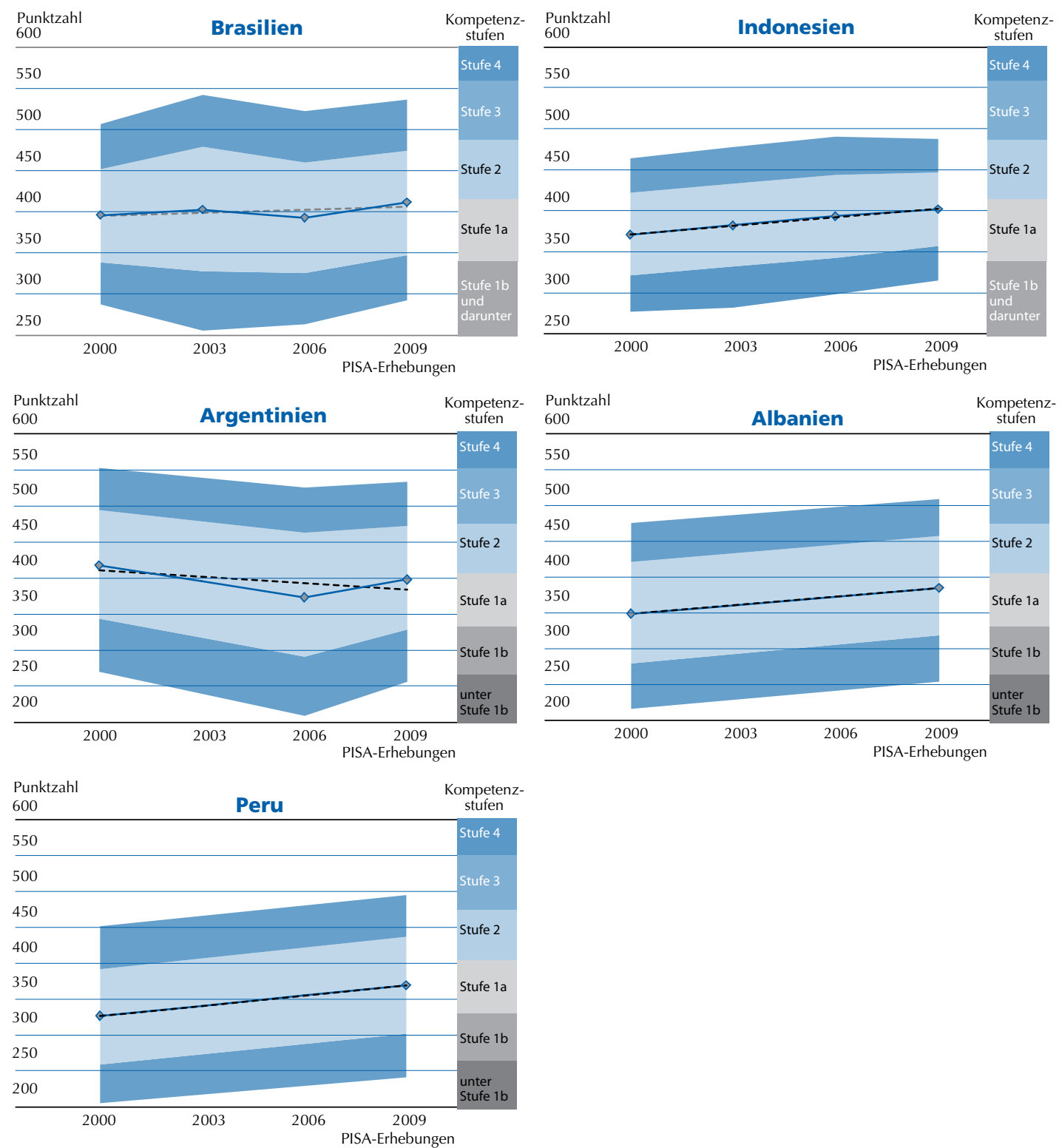

Quelle: OECD, PISA-2000-Datenbank, PISA-2003-Datenbank, PISA-2006-Datenbank und PISA-2009-Datenbank.

StatLink הत्ञाज $h t t p: / / d x . d o i . o r g / 10.1787 / 888932359967$ 


\section{Anmerkungen}

1. Die Ergebnisse für Luxemburg, die Niederlande und das Vereinigte Königreich aus der PISA-Erhebung 2000 sind hier nicht berücksichtigt, da die Validität der Vergleiche im Zeitverlauf auf Grund methodischer Probleme beeinträchtigt ist. Die österreichischen Daten wurden nach der Veröffentlichung des PISA-Berichts 2000 korrigiert; auf Grund von Schülerboykotts wurden die Daten für 2009 jedoch als nicht mit den Daten aus früheren Erhebungen vergleichbar erachtet, so dass die Trends für Österreich nicht erörtert werden. Chile und Israel nahmen an PISA 2000 teil. Diese Länder sind vor kurzem der OECD beigetreten und werden im Hinblick auf die in diesem Band angegebenen Ergebnisse aus PISA 2000 als OECD-Länder betrachtet. Die Slowakische Republik, die der OECD nach dem Jahr 2000 beigetreten ist, und die Türkei haben nicht an PISA 2000 teilgenommen, so dass sie in den OECD-Durchschnitten, die der Darstellung der Trends im Zeitraum 2000-2009 dienen, nicht berücksichtigt sind. Die Gesamtskala Lesekompetenz wurde zwar nicht verändert, der Mittelwert für die Gruppe der 26 Länder, für die vergleichbare Ergebnisse aus den Erhebungsrunden 2000 und 2009 vorliegen und die heute OECD-Mitglieder sind, beträgt nunmehr jedoch 496 und die Standardabweichung 96 Punkte. Ausführlichere Erläuterungen finden sich in Anhang A5 sowie in der Einführung zu diesem Band.

2. Israel verzeichnete einen Rückgang des gewichteten Prozentsatzes der in PISA getesteten Mädchen um 7 Prozentpunkte. Darüber hinaus war der sozioökonomische Hintergrund der Schülerinnen und Schüler im Jahr 2000 günstiger als 2009. Insgesamt fielen die bereinigten Ergebnisse aus dem Jahr 2000 niedriger aus als die ursprünglichen, was zu einer Stärkung der bereinigten Trends im Vergleich zu den beobachteten führte. Beim Stichprobenaufbau für Israel blieb die Zusammensetzung der Schulen nach Geschlecht in PISA 2000 unberücksichtigt, obwohl die Teilnahmequoten für Jungen und Mädchen in Israel unterschiedlich ausfielen, da die Jungen in einigen Schulen nicht an der Erhebung teilnehmen durften. Die geschlechtsspezifische Verteilung im Hinblick auf die in PISA 2000 für Israel erhobenen Daten unterlag auf Grund des ineffizienten Stichprobenaufbaus einer relativ großen Stichprobenvarianz. Dies wird in diesem Abschnitt durch Bereinigung der Ergebnisse für das Jahr 2000 berücksichtigt, so dass die geschlechtsspezifische Verteilung mit der im Jahr 2009 beobachteten vergleichbar ist. Indessen spielten auch die Trends beim sozioökonomischen Hintergrund der Schülerinnen und Schüler sowie beim Anteil an Schülerinnen und Schülern mit Migrationshintergrund bei den beobachteten Leistungsveränderungen in Israel eine wichtige Rolle. 


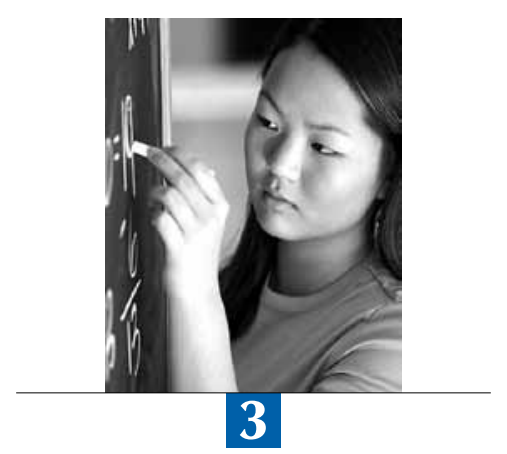

\section{Trends in den Bereichen Mathematik und Naturwissenschaften}

In Mathematik und Naturwissenschaften fallen die Leistungsveränderungen geringer aus als im Bereich Lesekompetenz, da die Ergebnisse in diesen beiden Bereichen über einen kürzeren Zeitraum gemessen wurden. Im vorliegenden Kapitel werden die Leistungstrends in Mathematik zwischen 2003 und 2009 und in Naturwissenschaften zwischen 2006 und 2009 beschrieben. 


\section{TRENDS IM BEREICH MATHEMATIK}

\section{Wie sich die Schülerleistungen im Bereich Mathematik seit 2003 verändert haben}

Die Leistungstrends in Mathematik beruhen auf Vergleichen der Ergebnisse von PISA 2009 mit denen der Erhebungen von 2003 und 2006. Da die Trends in Mathematik erst ab 2003 gemessen werden und nicht bereits ab dem Jahr 2000, wie im Bereich Lesekompetenz, ist davon auszugehen, dass die in Mathematik (seit 2003) beobachteten Leistungsveränderungen geringer ausfallen als die im Bereich Lesekompetenz (seit dem Jahr 2000) erfassten Veränderungen. In der PISA-Erhebung 2003 wurden die Mathematikleistungen genauer gemessen als in den PISA-Studien 2006 und 2009, da in den beiden letztgenannten Erhebungen für den Bereich Mathematik weniger Testzeit vorgesehen war. Aus diesem Grund werden die Trends in Mathematik nicht so ausführlich diskutiert wie die Ergebnisse im Bereich Lesekompetenz. Der Mittelwert im Bereich Mathematik wurde für die OECD-Länder in PISA 2003 auf 500 mit einer Standardabweichung von 100 gesetzt, woraus sich die Skala ergibt, die als Maßstab für Vergleiche mit den Mathematikleistungen in PISA 2009 verwendet wird. In diesem Abschnitt werden die Mathematikleistungen hauptsächlich zum Zweck der Erörterung der zwischen PISA 2003 und PISA 2009 festzustellenden Leistungsunterschiede untersucht.

Im OECD-Durchschnitt blieben die Mathematikleistungen zwischen 2003 und 2009 unverändert (Tabelle V.3.1). In einigen Ländern wurden allerdings deutliche Leistungsveränderungen im Bereich Mathematik beobachtet.

In 8 der 39 Länder mit vergleichbaren Ergebnissen aus den Jahren 2003 und 2009 haben sich die Schülerleistungen im Bereich Mathematik verbessert. Darunter fallen auch 6 der 28 OECD-Länder mit validen Daten für beide Erhebungen. Die Leistungen der Schülerinnen und Schüler stiegen in Mexiko um 33 Punkte, in der Türkei, Griechenland und Portugal um über 20 Punkte und in Italien und Deutschland um 17 bzw. 10 Punkte. Unter den Partnerländern und -volkswirtschaften verbesserten sich die Schülerleistungen in Brasilien um 30 Punkte, während in Tunesien ein Anstieg um 13 Punkte zu beobachten war (vgl. Kasten V.G zu Brasilien).

In neun Ländern waren die Mathematikergebnisse 2009 deutlich niedriger als 2003. In der Tschechischen Republik sanken die Schülerleistungen um 24 Punkte. In Irland, Schweden, Frankreich, Belgien, den Niederlanden und

Abbildung V.3.1

Veränderungen der Schülerleistungen in Mathematik zwischen 2003 und 2009

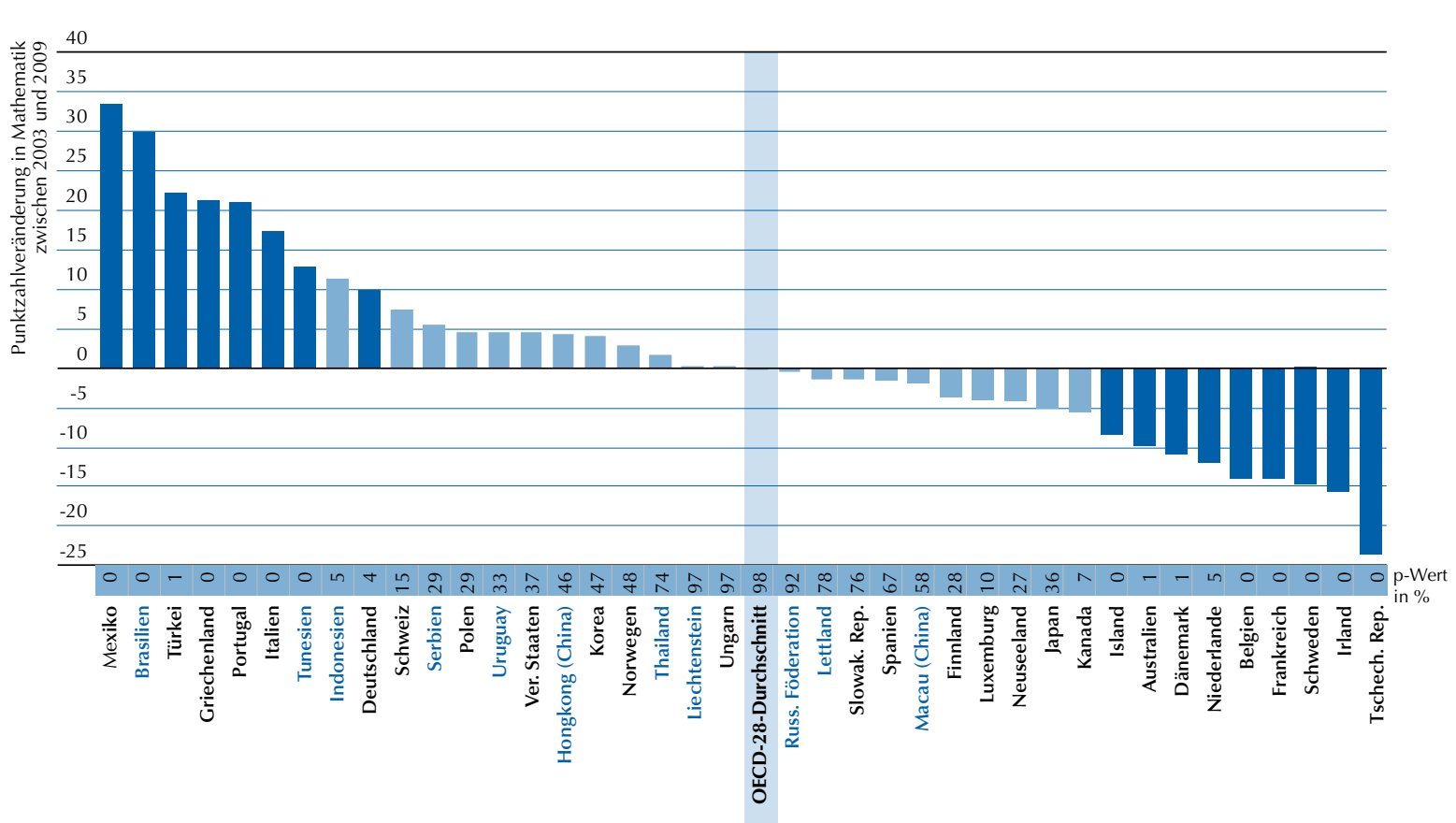

Anmerkung: Statistisch signifikante Punktzahlveränderungen sind durch einen dunkleren Farbton gekennzeichnet.

Die Länder sind in absteigender Reihenfolge der Punktzahlveränderung auf der Gesamtskala Mathematik zwischen 2003 und 2009 angeordnet.

Quelle: OECD, PISA-2009-Datenbank, Tabelle V.3.1

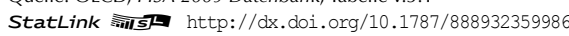


Dänemark gingen die Schülerleistungen in Mathematik um 11-16 Punkte zurück. In Australien verschlechterten sich die Schülerleistungen um 10 Punkte, in Island um 8 Punkte.

In 22 Ländern blieben die Mathematikleistungen zwischen 2003 und 2009 bei einem Konfidenzniveau von 95\% unverändert. Für jene Länder, in denen die Leistungsveränderungen statistisch nicht signifikant sind, ist in Abbildung V.3.1 der p-Wert angegeben, der dem Leser die Interpretation der Punktzahlveränderungen ermöglichen soll.

Selbst Länder, in denen sich die Mathematikleistungen verbessert haben, können mit ihren Ergebnissen weiterhin unter dem OECD-Durchschnitt liegen, während Länder, in denen die Leistungen gesunken sind, im Vergleich weiterhin besser abschneiden können. Zusammen betrachtet bieten Leistungsniveau und Leistungstrends ein vollständigeres Bild der Entwicklung der Schülerleistungen.

Abbildung V.3.2 gibt Aufschluss über die relative Stellung der Länder gemessen an ihrem Mittelwert in Mathematik und die in diesem Bereich beobachteten Leistungsveränderungen. Die Länder auf der rechten Seite haben ihre Leistungen seit 2003 verbessert, wohingegen die Länder auf der linken Seite eine Leistungsverschlechterung verzeichneten. Die Länder im oberen Teil der Abbildung erzielten 2009 über dem OECD-Durchschnitt liegende Ergebnisse, während die Leistungen der Länder im unteren Teil unter dem OECD-Durchschnitt lagen. Eine detailliertere Interpretation dieser Abbildung findet sich in Kapitel 2 (vgl. auch Abb. V.2.2).

Keines der am besten abschneidenden Länder hat seine Punktzahl in Mathematik erhöht, und in keinem der am schlechtesten abschneidenden Länder sind die Leistungen weiter gesunken. In sieben der acht Länder, die ihre Leistungen deutlich verbessert haben, lagen die Punktzahlen sowohl 2003 als auch 2009 effektiv unter dem OECD-

Abbildung V.3.2

Wie die Länder in Mathematik abschneiden und wie sich die Mathematikleistungen seit 2003 verändert haben

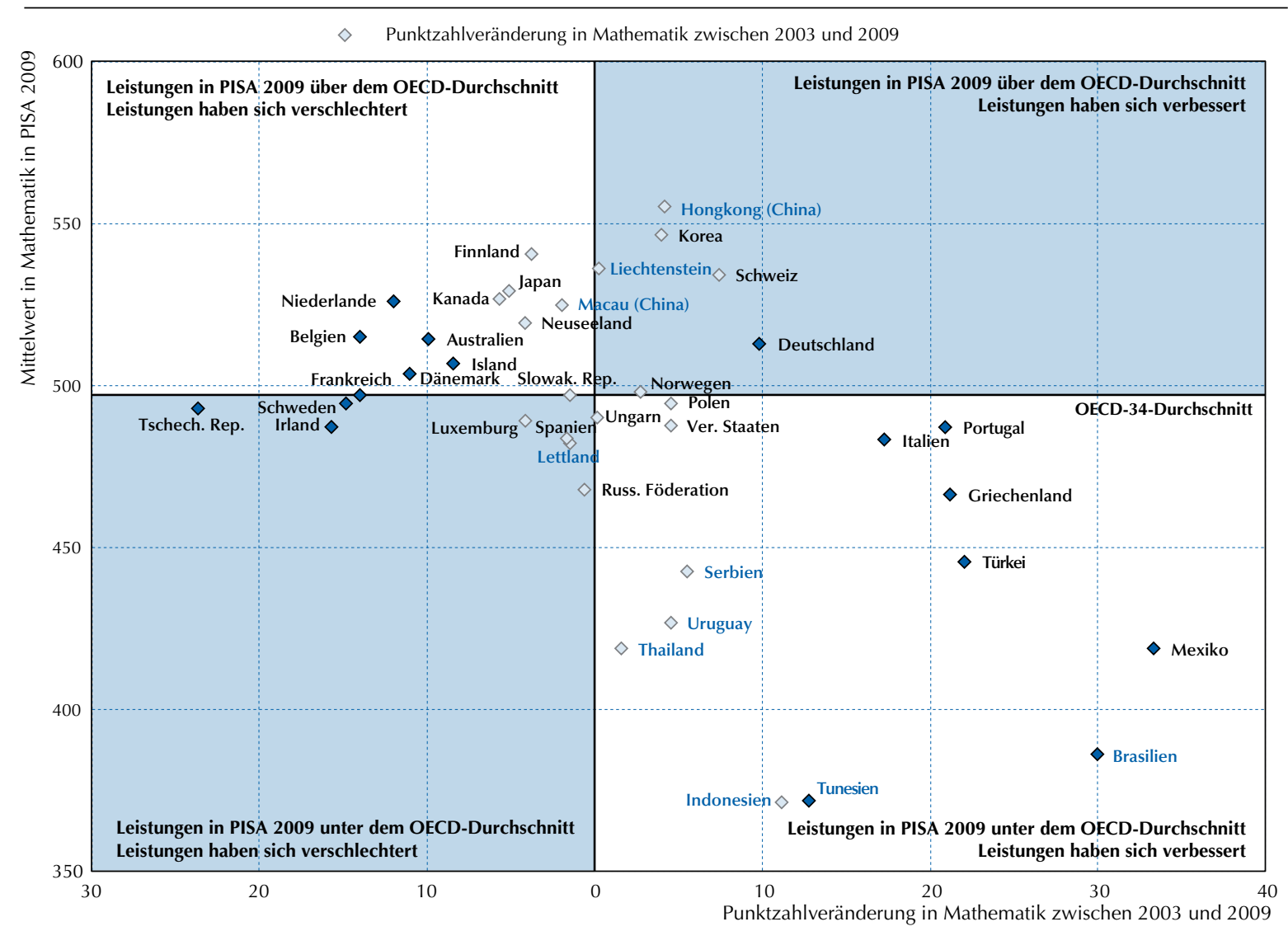

Anmerkung: Statistisch signifikante Punktzahlveränderungen in Mathematik zwischen 2003 und 2009 sind durch einen dunkleren Farbton gekennzeichnet.

Quelle: OECD, PISA-2009-Datenbank, Tabelle V.3.1

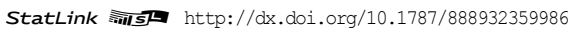


Durchschnitt, während die Länder, in denen eine Leistungsverschlechterung beobachtet wurde, zu Beginn alle durchschnittliche oder überdurchschnittliche Mittelwerte vorweisen konnten.

Deutschland erzielte 2003 nahe am OECD-Durchschnitt liegende Ergebnisse, verbesserte seine Leistungen zwischen 2003 und 2009 aber um 10 Punkte, so dass es inzwischen über dem OECD-Durchschnitt liegt. Portugal und Italien, die in der Erhebung von 2003 beide 466 Punkte erzielten, erhöhten ihre Leistungen um 21 bzw. 17 Punkte, so dass ihre Ergebnisse nun sehr viel näher am OECD-Durchschnitt liegen (vgl. Kasten V.D zu den in Portugal umgesetzten Maßnahmen).

Die Mittelwerte der verbleibenden fünf Länder, die im Jahr 2003 unter dem OECD-Durchschnitt liegende Ergebnisse erzielten und die ihre Leistungen bis 2009 verbessern konnten, erstrecken sich über ein breites Spektrum. In Mexiko, dem Land mit der größten Leistungsverbesserung, erhöhten sich die Schülerleistungen um 33 Punkte. Obwohl Mexiko jetzt mehr als 400 Punkte erzielt, liegt es nach wie vor deutlich unter dem OECD-Durchschnitt. Die Türkei und Griechenland haben ihre Leistungen um etwas mehr als 20 Punkte verbessert und erreichen nunmehr 445 bzw. 466 Punkte. Unter den Partnerländern steigerte Brasilien seine Leistungen im Bereich Mathematik um 30 Punkte, während Tunesien eine Verbesserung um mehr als 10 Punkte verzeichnen konnte. Dennoch liegen die Ergebnisse beider Länder nach wie vor unter 400 Punkten.

Einige Länder mit über dem OECD-Durchschnitt liegenden Ergebnissen verzeichnen im Bereich Mathematik einen Leistungsrückgang, schneiden aber nach wie vor besser ab als viele andere Länder. Obwohl die Schülerleistungen in den Niederlanden um 12 Punkte sanken, gehören die Niederlande weiterhin zur Gruppe der leistungsstärksten PISA-Länder. In Belgien, Dänemark, Australien und Island ließen die Schülerleistungen um etwa 10 Punkte nach, so dass diese Länder nun näher am - wenn auch weiterhin über - dem OECD-Durchschnitt liegen.

In mehreren Ländern, in denen die Ergebnisse im Bereich Mathematik über dem OECD-Durchschnitt lagen, war ein Rückgang der Leistungen festzustellen, die nun beim oder unter dem Durchschnitt angesiedelt sind. Die Tschechische Republik erzielte 2003 über dem OECD-Durchschnitt liegende Ergebnisse, befindet sich aber mittlerweile infolge eines Leistungseinbruchs um 24 Punkte leicht unter dem OECD-Durchschnitt. In Frankreich und Schweden hat sich das Leistungsniveau verschlechtert, was dazu führte, dass diese Länder von der Gruppe mit über dem OECD-Durchschnitt liegenden Leistungen in die Gruppe mit unter dem OECD-Durchschnitt liegenden Ergebnissen überwechselten.

Abbildung V.3.3

Prozentsatz der Schüler unter Kompetenzstufe 2 in Mathematik, 2003 und 2009

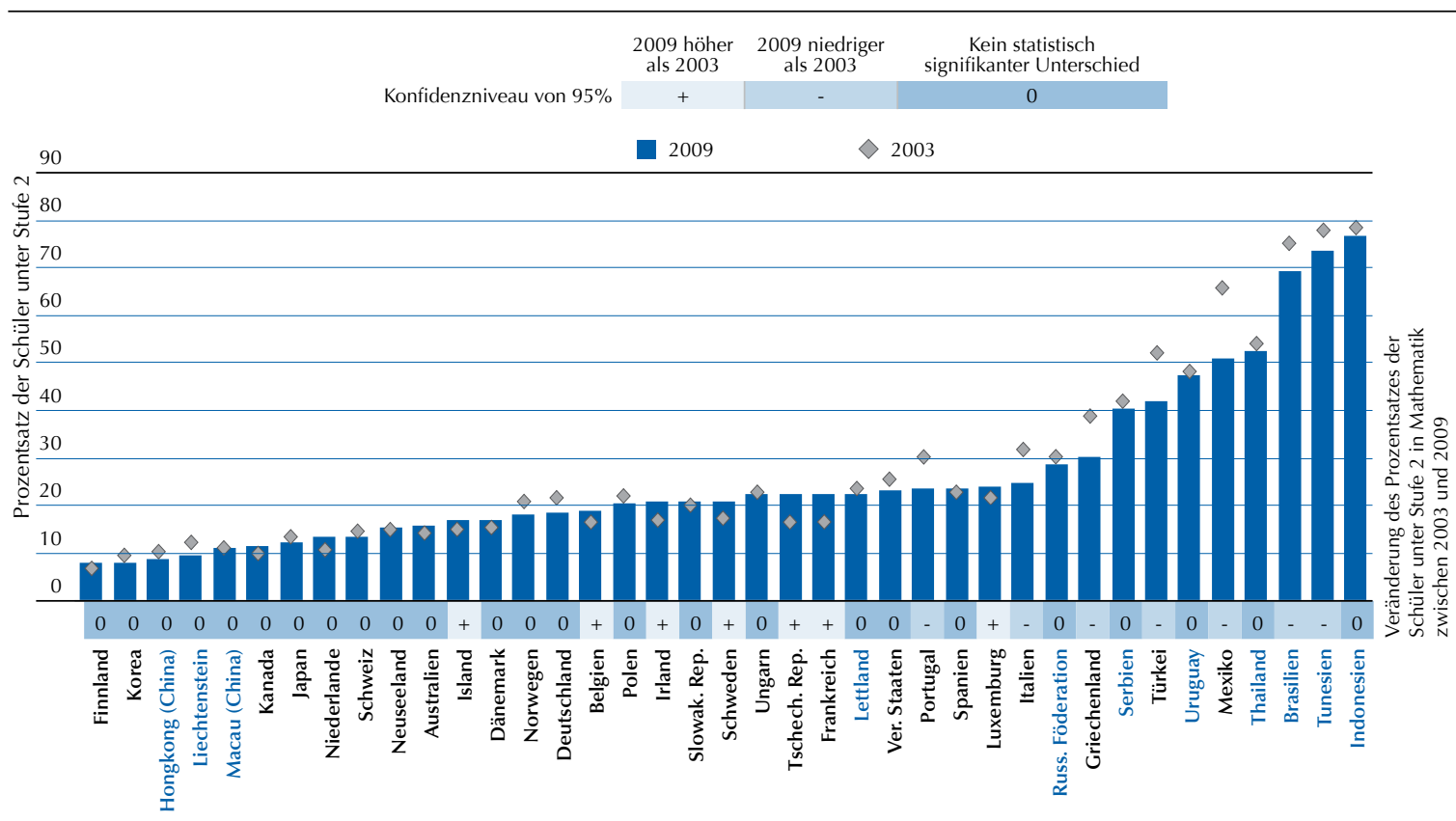

Die Länder sind in absteigender Reihenfolge des Prozentsatzes der Schüler unter Stufe 2 in Mathematik im Jahr 2009 angeordnet.

Quelle: OECD, PISA-2009-Datenbank, Tabelle V.3.2

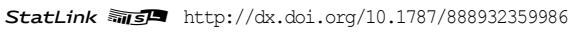


An den Veränderungen der Mittelwerte im Bereich Mathematik lassen sich allgemeine Trends ablesen, hinter denen sich aber Veränderungen unter den leistungsschwächsten und den leistungsstärksten Schülern verbergen können. Diese lassen sich anhand einer näheren Betrachtung der Veränderungen beim Anteil der Schülerinnen und Schüler analysieren, die bestimmte Kompetenzstufen erreichen. Wie in Kapitel 2 für den Bereich Lesekompetenz beschrieben, wurden die Schülerinnen und Schüler unterhalb von Stufe 2 für die Zwecke dieser Analysen in der Kategorie der leistungsschwächsten Schüler zusammengefasst, während die Schülerinnen und Schüler, die mindestens Stufe 5 erreichten, in der Kategorie der leistungsstärksten Schüler zusammengefasst wurden. Auf dieser Grundlage wurde die Veränderung der prozentualen Anteile dieser beiden Kategorien zwischen den Jahren 2003 und 2009 untersucht.

Die in der PISA-Erhebung 2009 für die Bewertung der mathematischen Grundbildung verwendeten Kompetenzstufen sind dieselben wie in PISA 2003, als dieser Bereich den Schwerpunkt bildete. Das zur Erstellung der Kompetenzstufen in Mathematik eingesetzte Verfahren ähnelt dem, das im Bereich Lesekompetenz verwendet wird und das in Band I, Was Schülerinnen und Schüler wissen und können, ausführlich beschrieben ist.

Im Durchschnitt der 28 OECD-Länder mit vergleichbaren Daten aus PISA 2003 und PISA 2009 blieb der Schüleranteil unterhalb von Kompetenzstufe 2 recht ähnlich und ging nur geringfügig von 21,6\% auf 20,8\% zurück (Tabelle V.3.2).

Unter den OECD-Ländern, in denen über die Hälfte der Schülerinnen und Schüler 2003 unter Stufe 2 lag, ging dieser Anteil in Mexiko um 15 Prozentpunkte von $66 \%$ auf 51\% und in der Türkei um 10 Prozentpunkte von $52 \%$ auf $42 \%$ zurück (vgl. Kasten V.E über Maßnahmen für leistungsschwache Schüler in der Türkei). In Griechenland, Italien und Portugal nahm der Prozentsatz der am schlechtesten abschneidenden Schülerinnen und Schüler deutlich weniger ab als in den oben genannten Ländern, in all diesen Ländern liegt der Anteil dieser Schülerinnen und Schüler aber inzwischen bei höchstens 30\%. In Griechenland ging der Prozentsatz dieser Schüler von 39\% auf 30\%, in Italien von $32 \%$ auf $25 \%$ und in Portugal von $30 \%$ auf $24 \%$ zurück. In zwei Partnerländern mit einem großen Anteil an besonders leistungsschwachen Schülern - Brasilien und Tunesien - ist dieser Anteil zwar um 4-6 Prozentpunkte gesunken, doch erfüllen dort nach wie vor etwa 69\% bzw. 74\% der Schülerinnen und Schüler in Mathematik nicht die Anforderungen von Stufe 2. Keines der Länder mit einem unterdurchschnittlichen Anteil an besonders leistungsschwachen Schülerinnen und Schülern verzeichnete einen weiteren Rückgang dieses Prozentsatzes.

Abbildung V.3.4

Prozentsatz der leistungsstärksten Schüler in Mathematik, 2003 und 2009

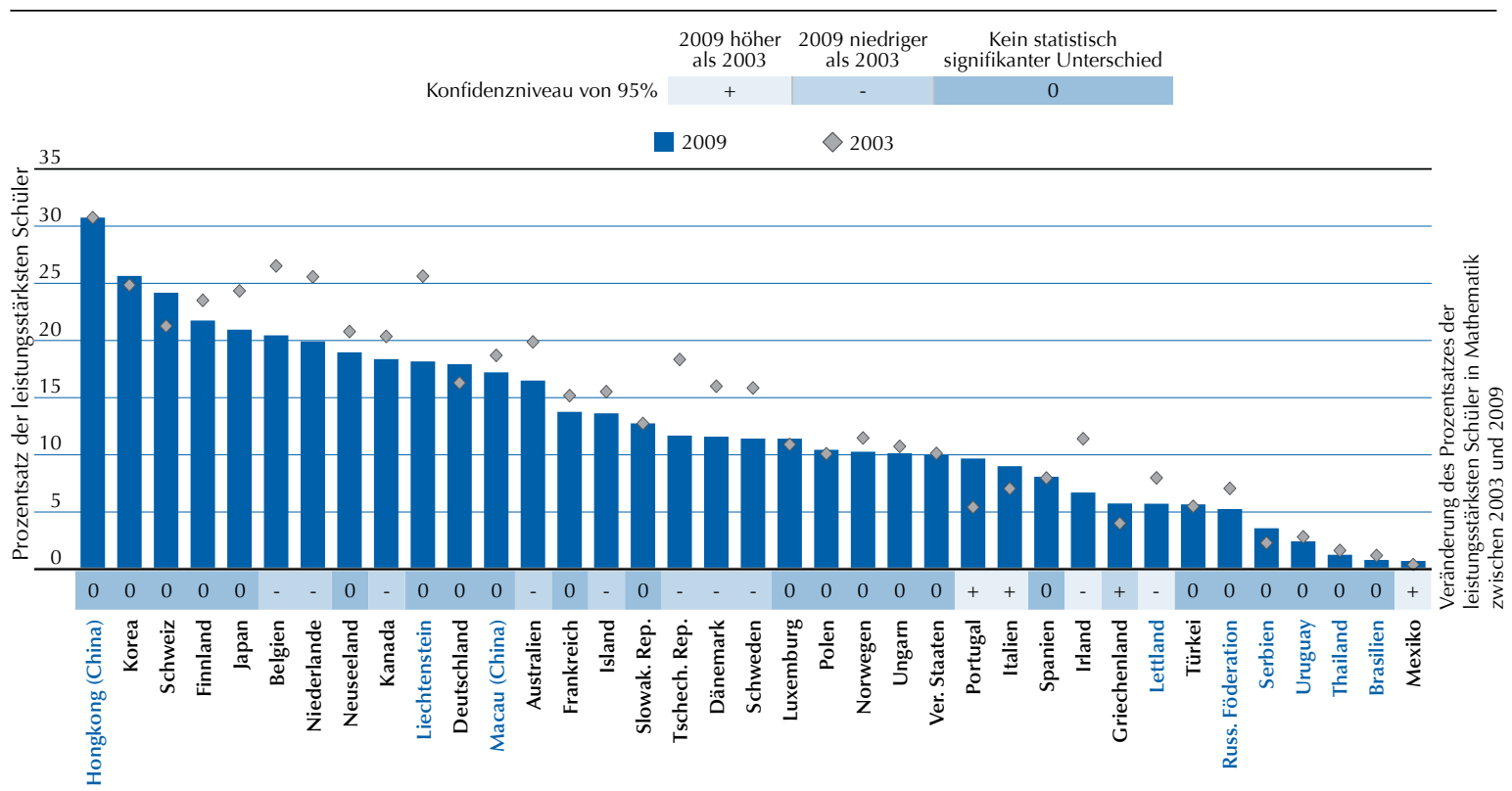

Die Länder sind in absteigender Reihenfolge des Prozentsatzes der Schüler auf Stufe 5 oder darüber in Mathematik im Jahr 2009 angeordnet. 
Der Anteil der Schülerinnen und Schüler, deren Leistungen unter Stufe 2 angesiedelt sind, erhöhte sich in Frankreich, der Tschechischen Republik, Irland, Schweden, Belgien, Luxemburg und Island (Abb. V.3.3).

Im Durchschnitt der 28 OECD-Länder, für die vergleichbare Daten aus den PISA-Erhebungen 2003 und 2009 zur Verfügung stehen, ging der Prozentsatz der besonders leistungsstarken Schüler zwischen 2003 und 2009 geringfügig von $14,7 \%$ auf $13,4 \%$ zurück (Tabelle V.3.2).

Unter den Ländern, in denen der Prozentsatz der Schülerinnen und Schüler, die mindestens Stufe 5 erreichten, unter dem Durchschnitt lag, stieg dieser Anteil in Portugal um über 4 Prozentpunkte auf nahezu 10\%, in Italien um nahezu 2 Prozentpunkte auf 9\% und in Griechenland um weniger als 2 Prozentpunkte auf nahezu 6\%. In Mexiko erhöhte sich der prozentuale Anteil der besonders leistungsstarken Schüler um 0,3 Prozentpunkte, verharrte aber auf einem sehr niedrigen Stand (0,7\%). Im Jahr 2003 lag der Anteil der besonders leistungsstarken Schülerinnen und Schüler in Irland und dem Partnerland Lettland unter dem OECD-Durchschnitt. In beiden Ländern ist er inzwischen zurückgegangen: in Irland um nahezu 5 Prozentpunkte auf weniger als $7 \%$ und in Lettland um 2 Prozentpunkte auf weniger als $6 \%$.

Unter den Ländern, in denen der Anteil der besonders leistungsstarken Schülerinnen und Schüler 2003 über dem Durchschnitt lag, verzeichnete keines einen weiteren Anstieg. In der Tschechischen Republik ging dieser Anteil um nahezu 7 Prozentpunkte, in Belgien und den Niederlanden um 6 Prozentpunkte, in Dänemark und Schweden um 4 Prozentpunkte, in Australien um 3 Prozentpunkte, in Kanada um 2 Prozentpunkte und in Island um nahezu 2 Prozentpunkte zurück (Abb. V.3.4).

Die Mathematikleistungen können auf dieselbe Weise auf Jahresbasis umgerechnet werden wie die Leistungen im Bereich Lesekompetenz. Die sich hieraus ergebenden Ergebnisse lassen sich mit den annualisierten Ergebnissen in den Bereichen Lesekompetenz und Naturwissenschaften vergleichen, um zu analysieren, inwieweit sich das Ausmaß der Leistungsveränderungen in den verschiedenen Erhebungsbereichen unterscheidet. Die auf Jahresbasis umgerechneten Ergebnisse finden sich in Tabelle V.3.3, zusammen mit zusätzlichen Vergleichen der Veränderungen bei den Mathematikleistungen zwischen den Erhebungen der Jahre 2006 und 2009.

\section{TRENDS IM BEREICH NATURWISSENSCHAFTEN}

\section{Wie sich die Schülerleistungen im Bereich Naturwissenschaften seit 2006 verändert haben}

Die Leistungstrends in Naturwissenschaften beruhen auf Vergleichen der Ergebnisse von PISA 2009 mit denen von PISA 2006. Da die Trends im Bereich Naturwissenschaften erst ab 2006 gemessen werden und nicht bereits ab dem Jahr 2000, wie im Bereich Lesekompetenz, ist davon auszugehen, dass die im Bereich Naturwissenschaften (seit 2006) beobachteten Leistungsunterschiede geringer ausfallen als die in den Bereichen Lesekompetenz und Mathematik (seit dem Jahr 2000 bzw. 2003) erfassten Unterschiede. Die 56 Teilnehmerländer und -volkswirtschaften von PISA 2006, darunter auch die 33 OECD-Länder, hatten auch an PISA 2009 teilgenommen, und es liegen vergleichbare Ergebnisse für sie vor. In PISA 2006 wurde der Mittelwert für die OECD-Länder auf 500 mit einer Standardabweichung von 100 gesetzt, woraus sich die Skala ergibt, die als Maßstab für Vergleiche mit den Schülerleistungen in Naturwissenschaften in PISA 2009 verwendet wird.

Der OECD-Durchschnitt der Leistungen in Naturwissenschaften blieb zwischen 2006 und 2009 unverändert. Wie aus Abbildung V.3.5. hervorgeht, haben sich die Ergebnisse in Naturwissenschaften in mehreren Ländern aber deutlich verändert (Tabelle V.3.4).

In elf der 56 Länder mit vergleichbaren Ergebnissen aus PISA 2006 und PISA 2009 haben sich die Schülerleistungen verbessert. Darunter befinden sich auch sieben der 33 OECD-Länder. Innerhalb von drei Jahren hat die Türkei ihre Leistungen um 30 Punkte gesteigert, was durchschnittlich nahezu einer halben Kompetenzstufe entspricht (vgl. Kasten V.E), und Portugal, Korea, Italien, Norwegen, die Vereinigten Staaten und Polen erzielten eine Verbesserung um 10-19 Punkte. Unter den Partnerländern erhöhte Katar seine Leistungen um 30 Punkte, während in Tunesien, Brasilien und Kolumbien eine Steigerung um 14 bzw. 15 Punkte zu beobachten war (vgl. Kasten V.G über Brasilien).

In fünf Ländern waren die Ergebnisse in Naturwissenschaften 2009 deutlich niedriger als 2006. In der Tschechischen Republik ließen die Leistungen um 12 Punkte nach, und in Finnland und Slowenien um 9 bzw. 7 Punkte. Unter den Partnerländern und -volkswirtschaften sind die Schülerleistungen in Chinesisch Taipeh um 12 Punkte und in Montenegro um 11 Punkte gesunken. 
In 40 Ländern blieben die Leistungen in Naturwissenschaften zwischen 2006 und 2009 bei einem Konfidenzniveau von 95\% unverändert. Für jene Länder, in denen die Leistungsveränderungen statistisch nicht signifikant sind, ist in Abbildung V.3.5 der p-Wert angegeben, der dem Leser die Interpretation der Punktzahlveränderungen ermöglichen soll.

Abbildung V.3.6 gibt Aufschluss über die relative Stellung der Länder gemessen an ihrem Mittelwert in Naturwissenschaften sowie die zwischen den Erhebungen in diesem Bereich beobachteten Veränderungen. Die Länder auf der rechten Seite der Abbildung konnten ihre Leistungen in Naturwissenschaften seit 2006 verbessern, wohingegen die Länder auf der linken Seite eine Leistungsverschlechterung verzeichneten. Die Länder im oberen Teil der Abbildung erzielten 2009 über dem OECD-Durchschnitt liegende Ergebnisse, während die Leistungen der Länder im unteren Teil unter dem OECD-Durchschnitt lagen. Diese Abbildung kann auf dieselbe Weise interpretiert werden wie die Abbildungen V.2.2 und V.3.2 (vgl. Kapitel 2).

Die Abbildung zeigt, dass das Spektrum der durchschnittlichen Leistungen der Länder, in denen sich die Ergebnisse in Naturwissenschaften verbessert oder verschlechtert haben, sehr viel weiter gestreut ist als in den Bereichen Mathematik und Lesekompetenz. Zu den Ländern, in denen sich die Leistungen im Bereich Naturwissenschaften verbessert haben, zählen Korea, dessen Ergebnisse 2006 weit über dem OECD-Durchschnitt lagen, Polen, wo die Leistungen in etwa beim OECD-Durchschnitt angesiedelt waren, und Katar, das 2006 zu den leistungsschwächsten Ländern zählte (vgl. Kasten V.B über Korea und Kasten V.C über Polen). Ein Rückgang der Punktzahlen in Naturwissenschaften war zwischen 2006 und 2009 in Finnland, einem sehr leistungsstarken Land, ebenso wie in Montenegro festzustellen, einem Land mit unterdurchschnittlichen Ergebnissen. Obwohl die Leistungen in Naturwissenschaften in Finnland nachgelassen haben, rangiert das Land in diesem Bereich der PISA-Erhebung nach wie vor auf Platz 2. Auch Chinesisch Taipeh schnitt 2006 sehr gut ab. Trotz rückläufiger Schülerleistungen ist Chinesisch Taipeh nach wie vor besser platziert als die meisten PISA-Teilnehmerländer und -volkswirtschaften.

Vier Länder, deren Ergebnisse im PISA-Naturwissenschaftstest 2006 unter dem OECD-Durchschnitt lagen, erzielten 2009 überdurchschnittliche oder durchschnittliche Leistungen. Die Vereinigten Staaten und Norwegen verzeichneten einen Leistungsanstieg um 13 Punkte, und ihre Ergebnisse entsprechen nun dem OECD-Durchschnitt. Portugal

Abbildung V.3.5

Veränderungen der Schülerleistungen in Naturwissenschaften zwischen 2006 und 2009

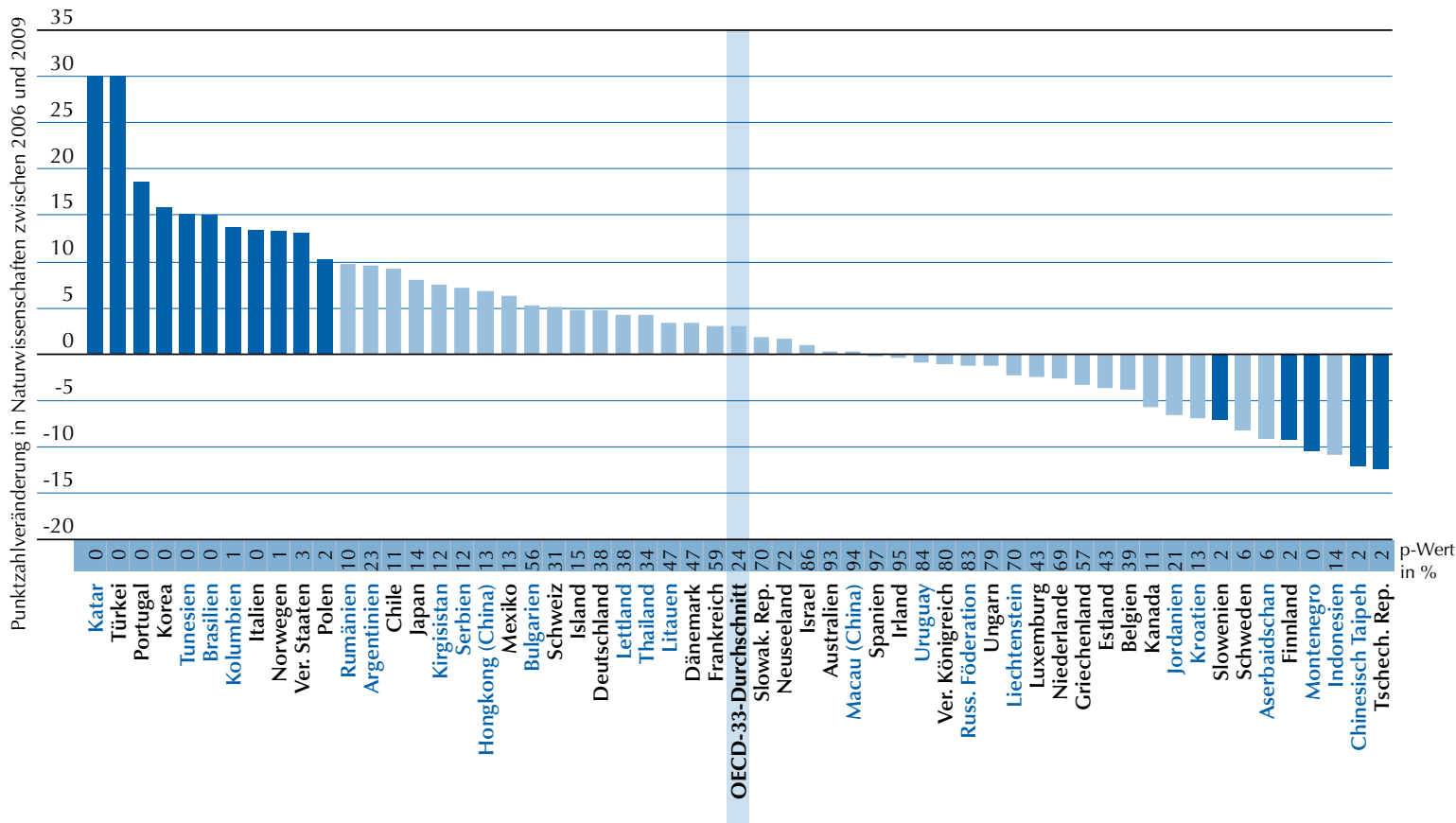

Anmerkung: Statistisch signifikante Punktzahlveränderungen sind durch einen dunkleren Farbton gekennzeichnet.

Die Länder sind in absteigender Reihenfolge der Punktzah/veränderung auf der Gesamtskala Naturwissenschaften zwischen 2006 und 2009 angeordnet.

Quelle: OECD, PISA-2009-Datenbank, Tabelle V.3.4

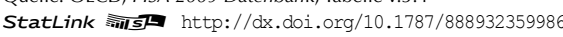


und Italien erzielten Leistungssteigerungen um 19 bzw. 13 Punkte und liegen mit ihren Leistungen nun knapp unter dem OECD-Durchschnitt (vgl. Kasten V.D über in Portugal umgesetzte Maßnahmen).

Slowenien und dieTschechische Republik erzielten 2006 über dem Durchschnitt liegende Leistungen, verzeichneten 2009 aber einen Leistungsrückgang. Obgleich die Leistungen in Slowenien um 7 Punkte nachließen, liegen sie immer noch über dem OECD-Durchschnitt; die Tschechische Republik hingegen fiel mit einem Leistungsrückgang um 12 Punkte auf den OECD-Durchschnitt zurück.

Unter den Ländern, die ihre Leistungen im Bereich Naturwissenschaften verbessert haben, gehören fünf zur Gruppe der leistungsschwachen Länder. Unter diesen erzielt die Türkei nun 454 Punkte, d.h. 30 Punkte mehr als 2006. In anderen Ländern dieser Gruppe liegen die Leistungen weiterhin auf einem sehr niedrigen Niveau, bei etwa 400 Punkten oder darunter. Katar verbesserte seine Leistungen um 30 Punkte, liegt im Gesamtergebnis aber weiterhin unter 400 Punkten, während Brasilien, Kolumbien und Tunesien ihre Leistungen um etwa 15 Punkte steigerten und nun knapp über 400 Punkte erreichen. Unter den leistungsschwachen Ländern verzeichnete Montenegro in Naturwissenschaften einen weiteren Leistungsrückgang um 11 Punkte. Andere leistungsschwache Länder verharrten auf ihrem Niveau von 2006.

In einigen Ländern ging der Anteil der leistungsschwächsten Schüler zwischen 2006 und 2009 zurück (Tabelle V.3.5). Unter den Ländern mit dem größten Anteil an Schülerinnen und Schülern, die Stufe 2, d.h. das Basisniveau naturwissenschaftlicher Kompetenz, nicht erreichten, ging dieser Anteil in Katar um 14 Prozentpunkte zurück,

Abbildung V.3.6

\section{Wie die Länder in Naturwissenschaften abschneiden und wie sich die Leistungen in Naturwissenschaften seit 2006 verändert haben}

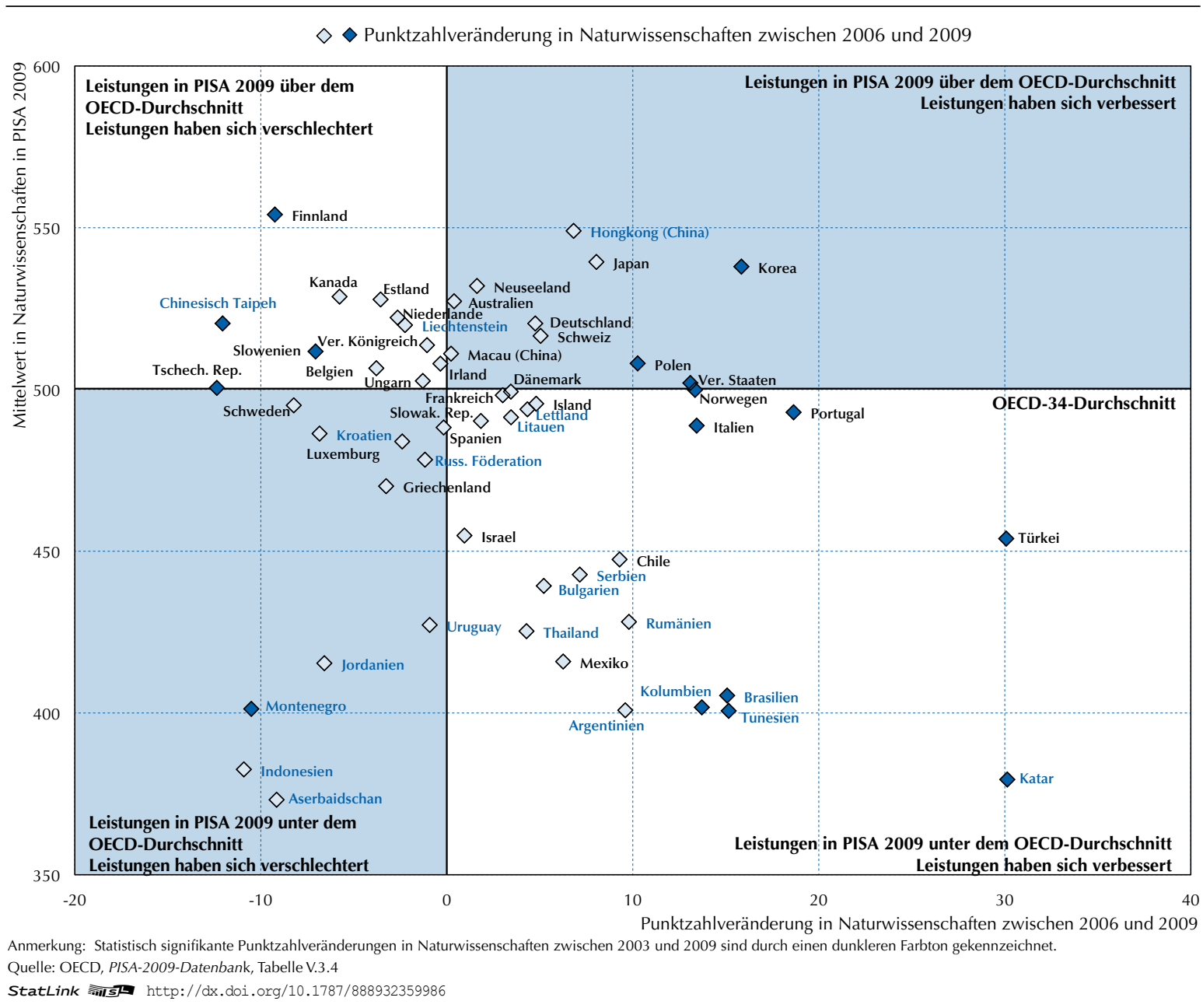


wenngleich nahezu zwei Drittel der Schülerinnen und Schüler in Katar nach wie vor keine ausreichenden Leistungen für Stufe 2 erbringen. Kirgisistan konnte 2009 einen Rückgang des Anteils der besonders leistungsschwachen Schüler um 4 Prozentpunkte verzeichnen, ist aber immer noch das Land, in dem dieser Anteil am höchsten ist. In Tunesien, Brasilien und Kolumbien sank der Prozentsatz der Schülerinnen und Schüler mit Leistungen unter Stufe 2 um 6-9 Prozentpunkte, obgleich er weiterhin etwa 54\% beträgt. In Mexiko ging der Anteil der Schülerinnen und Schüler mit Leistungen unter Stufe 2 um 4 Prozentpunkte zurück, verharrt aber ebenfalls auf einem recht hohen Niveau von $47 \%$, dem höchsten unter den OECD-Ländern.

In der Türkei verringerte sich der Prozentsatz der Schülerinnen und Schüler mit Leistungen unter Stufe 2 um 17 Prozentpunkte von $47 \%$ auf 30\%. Damit verzeichnete die Türkei unter allen Ländern den stärksten Rückgang. In Chile ist der Prozentsatz der leistungsschwächsten Schülerinnen und Schüler um 7 Prozentpunkte gesunken, womit der Anteil der Schülerinnen und Schüler, deren Leistungen im Bereich Naturwissenschaften den Anforderungen von Stufe 2 nicht entsprechen, dort nun bei 32\% liegt (vgl. Kasten V.F über Chile). In Italien beträgt der Anteil der Schülerinnen und Schüler unter Stufe 2 nun 21\%, was einem Rückgang um 5 Prozentpunkte gegenüber 2006 entspricht. In den Vereinigten Staaten und Island erbringen 18\% der Schülerinnen und Schüler keine ausreichenden Leistungen für Stufe 2, ihr Anteil ist in den Vereinigten Staaten um 6 Prozentpunkte und in Island um 3 Prozentpunkte gesunken. Im Partnerland Serbien ging dieser Prozentsatz um 4 Prozentpunkte auf 34\% zurück (Abb. V.3.7).

Unter den Ländern, in denen der Anteil der Schülerinnen und Schüler mit Leistungen unter Stufe 2 in Naturwissenschaften über dem Durchschnitt lag, mittlerweile aber unter dem Durchschnitt angesiedelt ist, ging er in Portugal um 8 Prozentpunkte auf $17 \%$ und in Norwegen um 5 Prozentpunkte auf 16\% zurück. Im Partnerland Litauen reduzierte sich dieser Schüleranteil um 3 Prozentpunkte auf $17 \%$.

Unter den Ländern, in denen der Anteil der Schülerinnen und Schüler mit Leistungen unter Stufe 2 in Naturwissenschaften bereits unter dem Durchschnitt lag, reduzierte er sich nur in Polen und Korea weiter um 4 bzw. 5 Prozentpunkte. In Polen ging der Prozentsatz der leistungsschwächsten Schüler von 17\% auf 13\% zurück, während er sich in Korea von $11 \%$ auf $6 \%$ verringerte, womit er sehr nahe beim niedrigsten Niveau unter den OECD-Ländern liegt.

Der Anteil der Schülerinnen und Schüler mit Leistungen unter Stufe 2 stieg in Schweden von 16\% auf 19\%. In Finnland erhöhte sich der Prozentsatz der Schülerinnen und Schüler mit Leistungen unter Stufe 2 von 4\% auf 6\%;

Abbildung V.3.7

Prozentsatz der Schüler unter Kompetenzstufe 2 in Naturwissenschaften, 2006 und 2009

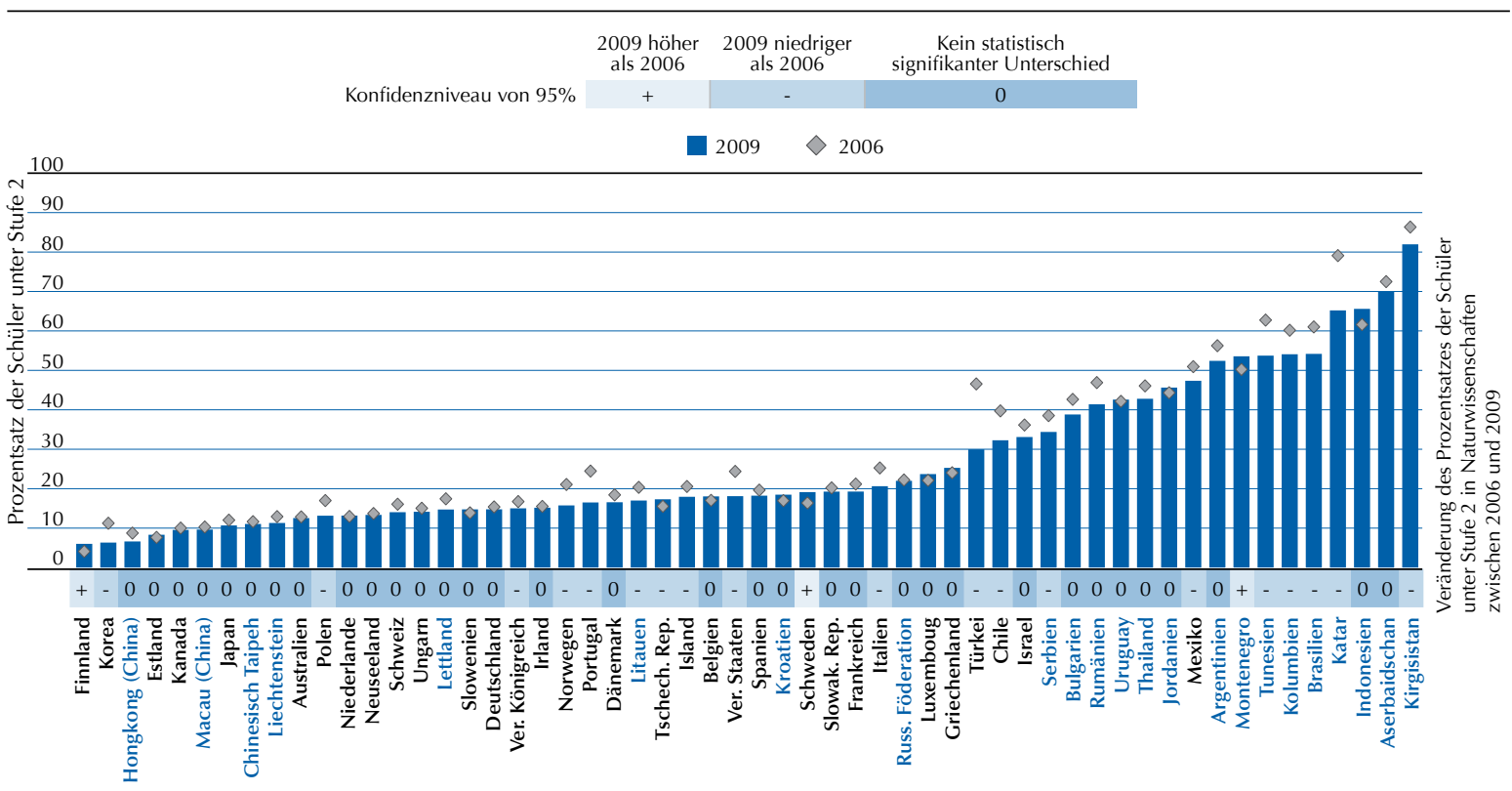

Die Länder sind in aufsteigender Reihenfolge des Prozentsatzes der Schüler unter Stufe 2 in Naturwissenschaften im Jahr 2009 angeordnet. Quelle: OECD, PISA-2009-Datenbank, Tabelle V.3.5

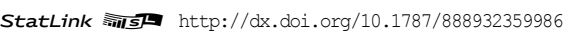




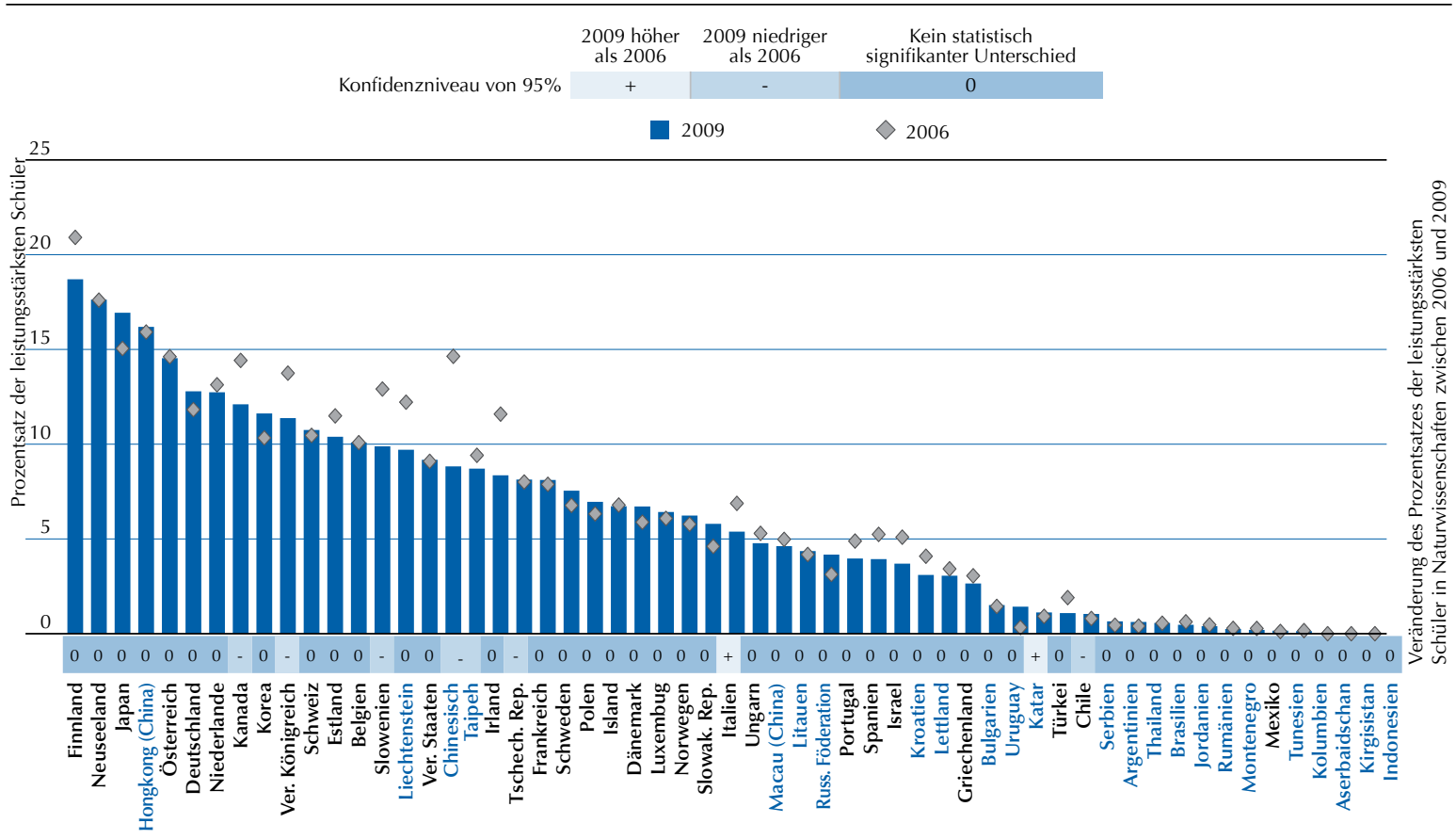

Die Länder sind in absteigender Reihenfolge des Prozentsatzes der leistungsstärksten Schüler in Naturwissenschaften im Jahr 2009 angeordnet. Quelle: OECD, PISA-2009-Datenbank, Tabelle V.3.5

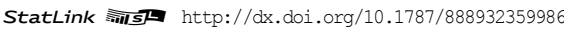

Finnland ist damit 2009, wie bereits 2006, aber immer noch das Land unter den PISA-Teilnehmer-ländern und -volkswirtschaften, in dem der Anteil dieser Schüler am niedrigsten ist. Im Partnerland Montenegro, wo der Anteil der Schülerinnen und Schüler mit Leistungen unter Stufe 22006 bei etwa 50\% lag, stieg er um 3 Prozentpunkte.

Der Prozentsatz der leistungsstärksten Schüler nahm in Naturwissenschaften nur in zwei Ländern zu (Tabelle V.3.5). In Italien erhöhte sich der Prozentsatz der Schülerinnen und Schüler auf den Stufen 5 und 6 von 5\% auf 6\%, während das Partnerland Katar 2006 kaum Schüler auf diesem Niveau zählte, inzwischen aber einen Anteil von mehr als $1 \%$ vorweisen kann.

Der Prozentsatz der Schülerinnen und Schüler auf Stufe 5 und darüber ging in Naturwissenschaften nur in Ländern zurück, die 2006 einen über dem Durchschnitt liegenden Prozentsatz dieser Schüler verzeichneten. In der Tschechischen Republik und Slowenien sank ihr Anteil um 3 Prozentpunkte, während er im Vereinigten Königreich und Kanada um 2 Prozentpunkte zurückging. Etwa 8\% der Schülerinnen und Schüler in der Tschechischen Republik erreichen nun mindestens Stufe 5, womit das Land nahe am OECD-Durchschnitt von 9\% liegt. In anderen Ländern verharrte der Prozentsatz der besonders leistungsstarken Schüler über dem Durchschnitt, so in Slowenien (rd. 10\%), im Vereinigten Königreich (11\%) und in Kanada (12\%). In Chile wurde ein leichter Rückgang gegenüber einem ohnehin bereits niedrigen Niveau von $2 \%$ auf $1 \%$ verzeichnet. Die Partnervolkswirtschaft Chinesisch Taipeh wies den größten Rückgang beim Prozentsatz der leistungsstärksten Schüler in Naturwissenschaften auf: Dieser sank um 6 Prozentpunkte von $15 \%$ auf $9 \%$. 


\section{Kasten V.D Portugal}

In PISA 2000 gehörte Portugal zu den OECD-Ländern mit den niedrigsten Ergebnissen im Bereich Lesekompetenz und wies einen der höchsten Anteile an Schülerinnen und Schülern auf, deren Leistungen den Anforderungen von Stufe 2, dem Basisniveau an Lesekompetenz, nicht entsprachen. Diese Ergebnisse lösten in der Öffentlichkeit intensive Debatten aus, bei denen sich die Erkenntnis herauskristallisierte, dass es zu vielen portugiesischen Schülerinnen und Schülern an den Kenntnissen und Fähigkeiten mangelt, die in einer modernen Gesellschaft und Wirtschaft notwendig sind. Außerdem setzte sich die Auffassung durch, dass hohe Wiederholungsraten bei Schülern aus sozial benachteiligten Milieus ein Erfolgshindernis sind.

Die Ergebnisse von PISA 2003 und PISA 2006 wurden im Kontext von Bildungsreformvorschlägen noch umfassender diskutiert. Der Bildungsminister hob die Bedeutung der Ergebnisse und Erkenntnisse hervor, die sich aus dem innovativen, auf die kreative Nutzung von Kenntnissen und Fähigkeiten ausgerichteten Ansatz der PISA-Studie gewinnen lassen. Seit 2005 hat Portugal ein breites Spektrum an Maßnahmen in Kraft gesetzt, die auf eine Verbesserung der Lernergebnisse abzielen.

Viele dieser Maßnahmen konzentrierten sich auf eine Verbesserung der Lebensbedingungen von Menschen aus sozial benachteiligten Milieus. In Portugal sind die Vermögensunterschiede relativ groß, und der Anteil der Hochschulabsolventen in der Erwerbsbevölkerung zählt mit zu den niedrigsten. Schätzungen zufolge wäre die Arbeitsproduktivität je Erwerbstätigenstunde um 14,4\% höher, wenn die Bevölkerung im erwerbsfähigen Alter in Portugal über dasselbe Bildungsniveau verfügen würde wie in den Vereinigten Staaten (OECD, 2010c). Die Reformen sollten diese Situation durch eine Verbesserung der Bildungsmöglichkeiten für Kinder und Erwachsene aus relativ benachteiligten Verhältnissen ändern.

Das portugiesische Schulsystem war zuvor sehr selektiv, zahlreiche Schülerinnen und Schüler mussten Klassen wiederholen, und viele von ihnen brachen die Schule letztlich ab. Häufige Klassenwiederholungen waren ein konstantes Merkmal des portugiesischen Schulsystems, und sie sind dies bis zu einem gewissen Grad auch noch heute; außerdem besteht zwischen schlechten schulischen Leistungen und einem niedrigen sozioökonomischen Status eine enge Korrelation.

Nach dem Konzept, das den seit 2005 umgesetzten Maßnahmen zu Grunde liegt, setzen Effizienz- und Qualitätssteigerungen im Bildungssystem eine Erhöhung der Chancengerechtigkeit voraus. Die Ergebnisse der PISA-Studie haben klar und deutlich gezeigt, dass mehr Chancengerechtigkeit nicht auf Kosten der Qualität gehen muss.

Obgleich das portugiesische Schulsystem nahezu vollständig öffentlich ist und der Unterricht bis zur Beendung der Pflichtschulzeit, d.h. bis zur 12. Klasse oder Vollendung des 18. Lebensjahrs kostenlos ist, hat die Regierung größere Mittel für die Unterstützung von Schülerinnen und Schülern aus einkommensschwachen Familien bereitgestellt. Ausgaben für Laptops, Mahlzeiten, Bücher, Breitband-Internetanschlüsse, Englischunterricht und sonstige außercurriculare Aktivitäten wurden von der Regierung bezuschusst; in wirtschaftlicher Hinsicht benachteiligten Schülerinnen und Schülern wurde zusätzliche Unterstützung zuteil. Anspruch auf diese Leistungen besteht vom ersten Grundschuljahr bis zum Ende der Sekundarschule. Zwischen 2005 und 2009 hat sich die Zahl der Leistungsempfänger aus dem Sozialen Aktionsprogramm für Schulen verdreifacht.

Zwischen 2004 und 2009 war ein drastischer Rückgang der Wiederholungsrate in der 9. Klasse von 21,5\% auf $12,8 \%$ zu verzeichnen. Dies ist allein schon ein positives Zeichen, da Klassenwiederholungen den PISAErgebnissen zufolge im Allgemeinen mit einem niedrigen Leistungsniveau und einem stärkeren Einfluss des sozioökonomischen Hintergrunds auf die Schülerleistungen assoziiert sind (vgl. Kapitel 2 in Band IV, Was macht eine Schule erfolgreich?). Dieser Rückgang war zugleich mit höheren Schulbesuchsquoten in Sekundarstufe II (Klasse 10-12) und einem Rückgang der Gesamtzahl der Schulabbrecher verbunden. 2007 beschloss das Bildungsministerium, dass alle portugiesischen Bürger mindestens bis zur 12. Klasse zur Schule gehen sollten. 2009 wurde ein Gesetz zur Verlängerung der Schulpflicht verabschiedet.

Parallel hierzu wurden Weiterbildungen für die Lehrkräfte angeboten, vornehmlich in Portugiesisch, Mathematik und Informationstechnologien. Außerdem wurde ein neues System der Lehrer- und Schulevaluierung eingeführt, um die Rechenschaftspflicht zu erhöhen. Obgleich sich die Umsetzung auf Grund des prinzipiellen Widerstands gegen an ein Beurteilungssystem gebundene Maßnahmen zur Stärkung der Rechenschaftspflicht verzögerte, 
hatte sich bereits eine Entwicklung hin zu einer stärker ergebnisorientierten Rechenschaftslegung vollzogen, durch die sich die Einstellung der Lehrkräfte und Schulen gegenüber externen Beurteilungen, darunter auch PISA, veränderte. Die Effizienz des Schulsystems wurde durch eine Reduzierung der Fehlzeiten der Lehrer und die Vertretung abwesender Lehrkräfte verbessert, was dazu beitrug, dass weniger Unterrichtsstunden ausfielen.

Der derzeitige Maßnahmenkatalog zielt auch darauf ab, die Schulverwaltung zu verändern. 2006 und 2009 hatte Portugal unter den OECD-Ländern einen der niedrigsten Mittelwerte auf dem Index der Schulautonomie bei der Ressourcenallokation und dem Index der Schulautonomie für Unterrichtsinhalte und Beurteilungen (vgl. Band IV, Was macht eine Schule erfolgreich?, sowie Tabelle IV.3.5 und IV.3.6). Die Maßnahmen, die derzeit umgesetzt werden, verleihen den Direktoren von Schulkomplexen mehr Autonomie. Ein "Schulkomplex" ist eine organisatorische Einheit, die sich aus mehreren Schulen vom Kindergarten bis zur 9. oder 12. Klasse zusammensetzt und vertikal auf einem einheitlichen Bildungsprojekt unter der Aufsicht des Direktors aufgebaut ist. Der Direktor wird von einem Rat gewählt, der sich aus Lehrkräften, Eltern, Schülern, Vertretern der Gemeinde und verschiedener Institutionen sowie wichtigen lokalen Akteuren zusammensetzt. Der überwiegenden Mehrzahl der Schulkomplexe stehen nun gewählte Direktoren vor, die bei der Verfolgung vorgeschlagener Bildungsprojekte über sehr viel mehr Autonomie verfügen. Der Maßnahmenkatalog umfasst zudem bedeutende Investitionen in die physische Infrastruktur, die im Jahr 2008 eingeleitet wurden.

Im Rahmen der Reformen werden alle Schülerinnen und Schüler der 4., 6. und 9. Klasse einer jährlichen nationalen Prüfung in portugiesischer Sprache und Mathematik unterzogen. Die Beurteilungen in der 4. und der 6. Klasse haben zwar keinen direkten Einfluss auf den weiteren schulischen Werdegang der einzelnen Schüler, sie finden nun aber in allen Schulen Anwendung und liefern für Schüler, Eltern und Lehrer wichtige Evaluierungen. Ferner bieten die Sekundarschulen den Schülerinnen und Schülern inzwischen als Alternative berufsbildende Programme an, die etwa von der Hälfte der Schüler in den Klassen 10, 11 und 12 besucht werden. Dies hatte zur Folge, dass die Schulbesuchszahlen im Sekundarbereich seit 2005 gestiegen sind, womit dem seit 1995 beobachteten beunruhigenden Rückgang der Schülerzahlen ein Ende gesetzt wurde.

Mathematik gilt für die Schülerinnen und Schüler in Portugal traditionell als das schwierigste Fach. Und in der Tat waren die PISA-Ergebnisse in Mathematik 2003 sogar schlechter als im Bereich Lesekompetenz, und nahezu ein Drittel der Schülerinnen und Schüler erreichte nicht die Kompetenzstufe 2 (vgl. Abb. V.3.3 und Tabelle V.3.2). Nach Bekanntgabe der PISA-Ergebnisse sowie der Resultate der 2005 in Mathematik in der 9. Klasse durchgeführten Prüfungen stieß das Bildungsministerium eine weitreichende Debatte zu diesem Thema an. Der Aktionsplan Mathematik, der 2005 ins Leben gerufen wurde und etwa 78000 Lehrkräfte und 400000 Schülerinnen und Schüler einbezieht, besteht aus sechs Komponenten: a) einem Mathematikprogramm für jede Schule, b) Fortbildungsmaßnahmen für Lehrkräfte im Sekundarbereich (Escola Básica und Escola Secundária), c) stärkere Gewichtung des Fachs Mathematik in der Lehrererstausbildung, d) Anpassung des Mathematiklehrplans im gesamten Pflichtschulsystem, e) Einrichtung einer Datenbank über die verfügbaren Ressourcen für den Mathematikunterricht sowie f) eine Evaluierung der Mathematiklehrbücher. Zugleich wurden mehr Mathematiklehrer ausgebildet und eingestellt.

Der Nationale Aktionsplan Leseverständnis wurde 2006 als gemeinsame Initiative des Ministeriums für Bildung, des Ministeriums für Kultur und des Ministeriums für Parlamentsangelegenheiten ins Leben gerufen. Dieser Plan soll die Lesekompetenz der Kinder verbessern und gute Lesegewohnheiten fördern. Über eine Million Kinder und Jugendliche in allen Schulkomplexen und Sekundarschulen nehmen an diesem Programm teil.

Die Ergebnisse der PISA-Studie 2009 zeigen, dass Portugal Fortschritte auf dem Weg zur Erreichung der von den Reformverantwortlichen aufgestellten Ziele macht. Unter den Ländern, deren Ergebnisse dem OECD-Durchschnitt entsprechen oder über diesem liegen, war Portugal das einzige Land, das seine Leistungen in allen drei PISAErhebungsbereichen gesteigert hat, wobei der Großteil der Verbesserungen zwischen 2006 und 2009 zu beobachten war (vgl. Abb. V.1.2 und Tabelle V.2.1, V.3.1, V.3.3 und V.3.4). Die Ergebnisse im Bereich Lesekompetenz haben sich seit dem Jahr 2000 um 19 Punkte verbessert; in Mathematik und Naturwissenschaften waren Leistungsfortschritte in ähnlicher Größenordnung festzustellen, die aber über einen kürzeren Zeitraum erzielt wurden.

In den Bereichen Lesekompetenz und Naturwissenschaften sind diese positiven Trends größtenteils auf Verbesserungen in der Gruppe der leistungsschwächsten Schülerinnen und Schüler zurückzuführen. Im Bereich 
Lesekompetenz konnte Portugal den Anteil der leistungsschwachen Schüler (mit Leistungen unter Stufe 2) um nahezu 9 Prozentpunkte reduzieren, während der Anteil der besonders leistungsstarken Schülerinnen und Schüler (Stufe 5 oder darüber) in etwa auf gleichem Niveau verharrte (vgl. Abb. V.2.4 und V.2.5 sowie Tabelle V.2.2). Ähnliche Ergebnisse sind im Bereich Naturwissenschaften festzustellen (vgl. Abb. V.3.7 und V.3.8 sowie Tabelle V.3.5). In Mathematik hingegen ist nicht nur der Prozentsatz der leistungsschwachen Schülerinnen und Schüler um 6 Prozentpunkte gesunken, sondern kam es auch zu einer Erhöhung des Anteils der besonders leistungsstarken Schülerinnen und Schüler um etwa 4 Prozentpunkte (vgl. Abb. V.3.3 und V.3.4 sowie Tabelle V.3.2).

Portugal zählt zu den sechs Ländern in PISA 2009, die sowohl ihr Gesamtergebnis im Bereich Lesekompetenz verbessert als auch die Leistungsvarianz verringert haben, was in erster Linie Leistungssteigerungen unter den schwächeren Schülern zuzuschreiben ist, während die leistungsstarken Schüler ihr Niveau hielten.

\section{Kasten V.E Türkei}

Die Türkei beteiligt sich seit 2003 an der PISA-Studie. Aus den Ergebnissen der Erhebung von 2003 geht hervor, dass die 15-jährigen Schülerinnen und Schüler in der Türkei, deren Leistungen im Durchschnitt bei rd. 425 Punkten liegen und von denen über die Hälfte die Stufe 2 nicht erreicht, weit hinter dem OECD-Durchschnitt zurückbleiben. 2006 war das Bild ähnlich, wenngleich zu jenem Zeitpunkt bereits einige große Reformen in Angriff genommen worden waren.

Die Türkei verbesserte ihre Mathematikleistungen zwischen 2003 und 2009 um mehr als 20 Punkte (vgl. Abb. V.3.1 und Tabelle V.3.1). Parallel zu diesem Anstieg ging der Prozentsatz der Schülerinnen und Schüler, deren Leistungen unter Stufe 2, dem Basisniveau mathematischer Kompetenz lagen, um 10 Prozentpunkte von $52 \%$ auf $42 \%$ zurück. Im Bereich Naturwissenschaften konnte die Türkei ihr Ergebnis seit 2006 um 30 Punkte verbessern, was nahezu einem vollen Schuljahr entspricht, wobei der Anteil der Schülerinnen und Schüler mit Leistungen unter Stufe 2 um 17 Prozentpunkte von 47\% auf 30\% gesunken ist. Hiermit verzeichnet die Türkei den stärksten Rückgang dieses Schüleranteils unter den 56 Ländern, deren Ergebnisse aus den PISA-Erhebungen 2006 und 2009 vergleichbar sind.

Unter den zahlreichen Programmen, die in der Türkei umgesetzt wurden, betraf das 1998 ins Leben gerufene Grundbildungsprogramm fast alle Schülerinnen und Schüler (OECD, 2007). Zu den Zielen dieses Programms, das sich an internationalen Bildungsstandards orientiert, zählen die Ausweitung der Primarschulbildung, die Verbesserung der Bildungsqualität und der Schülerleistungen insgesamt, die Beseitigung des Leistungsgefälles zwischen Jungen und Mädchen, die Erhöhung der Chancengerechtigkeit, die Anpassung an die Leistungsindikatoren der Europäischen Union, die Einrichtung von Schulbibliotheken, die Steigerung der Effizienz des Bildungssystems, die Sicherung eines ausreichenden Qualifikationsniveaus der Beschäftigten im Bildungsbereich, die Integration von Informations- und Kommunikationstechnologien in das Bildungssystem sowie die Schaffung von in den Schulen basierten lokalen Lernzentren, die allen offenstehen.

Eine der größten Veränderungen, die dieses Programm mit sich brachte, war die Einführung eines Schulpflichtgesetzes. Dieses Gesetz trat im Schuljahr 1997/1998 in Kraft, und im Jahr 2003 schlossen die ersten Schülerinnen und Schüler die mit ihm eingerichtete achtjährige Pflichtschulzeit ab. Seit der Lancierung dieses Programms stieg die Schulbesuchsquote im achtjährigen Grundbildungssystem von etwa $85 \%$ auf nahezu $100 \%$, während sich die Besuchsquote in Vorschulprogrammen von $10 \%$ auf $25 \%$ erhöhte. Zudem wurde das System erweitert, um 3,5 Millionen zusätzliche Schülerinnen und Schüler aufnehmen zu können, die durchschnittliche Klassengröße wurde auf ungefähr 30 Schüler reduziert, alle Schülerinnen und Schüler lernen zumindest eine Fremdsprache, in allen Grundschulen wurden Computerräume eingerichtet, und in allen 35000 Schulen in ländlichen Gebieten wurden die allgemeinen Arbeitsbedingungen deutlich verbessert. Für das Programm wurden umgerechnet über 11 Mrd. US-\$ zur Verfügung gestellt. Es hat keinen direkten Einfluss auf die Bildungsbeteiligung des Großteils der in PISA getesteten 15-Jährigen, die hauptsächlich Sekundarschulen besuchen, in denen die Schulbesuchsquoten bei nahezu $60 \%$ liegen. 
Im Einklang mit diesen Zielsetzungen wurden im Schuljahr 2006/2007 unter Berücksichtigung der Erfahrungen der Türkei mit internationalen Prüfungen, wie der PISA-Erhebung, neue Lehrpläne ab der 6. Klasse eingeführt. Die Sekundarschulcurricula in Mathematik und Sprachen wurden ebenfalls überarbeitet, und in der 9. Klasse kam ab dem Schuljahr 2008/2009 ein neues Lehrprogramm in Naturwissenschaften zum Einsatz. Die an PISA 2009 teilnehmenden Schüler wurden bereits ein Jahr lang nach dem neuen Lehrplan unterrichtet, hatten ihre Primarschulbildung aber immer noch im alten System erhalten. Die Standards des neuen Lehrplans sollten den PISAZielen gerecht werden: „Verstärkte Bedeutung wird mathematischen Aktivitäten der Schüler beigemessen, was konkret bedeutet Analyse mathematischer Gedankengänge, Problemlösung, Herstellung von Verknüpfungen zwischen mathematischen Ideen und deren Anwendung in realen Lebenssituationen" (Talim ve Terbiye Kurulu, 2008).

Mit der Lehrplanreform sollte nicht nur der Inhalt der Bildungsprogramme erneuert und die Einführung innovativer Unterrichtsmethoden vorangebracht werden, sondern vor allem eine Veränderung der Unterrichtsphilosophie und -kultur innerhalb der Schulen herbeigeführt werden. In den neuen Lehrplänen und Unterrichtsmaterialien wird das "schülerzentrierte Lernen“ hervorgehoben, das den Schülern eine aktivere Rolle zukommen lässt als zuvor, wo von den Schülern hauptsächlich verlangt wurde, dass sie Informationen auswendiglernen. Zudem drückt sich in ihnen der Gedanke aus, der auch der PISA-Studie zu Grunde liegt, nämlich dass die Schulen die Schülerinnen und Schüler mit den nötigen Fähigkeiten und Kompetenzen ausstatten sollen, um in der Schule und im späteren Leben insgesamt erfolgreich zu sein.

Mit mehreren Maßnahmen wurde versucht, die Schulkultur und die Schulverwaltung zu verändern. Die Schulen wurden zur Vorlage eines Arbeitsplans verpflichtet, der konkrete Zielsetzungen und strategische Umsetzungsvorgaben zu ihrer Erreichung enthalten muss. Vorgeschlagen wurden u.a. demokratischere Verwaltungsstrukturen, eine stärkere Einbeziehung der Eltern und mehr Teamarbeit. Im Jahr 2004 wurde in allen Grund- und Sekundarschulen ein Projekt ins Leben gerufen, das darauf abzielt, den Schülerinnen und Schülern demokratische Fähigkeiten zu vermitteln und in dessen Rahmen Schülervereinigungen viel Verantwortung übertragen wurde. Zugleich wurden neue, transparentere und stärker leistungsorientierte Inspektionsinstrumente entwickelt.

In der Türkei wurden auch private Investitionen eingesetzt, um die Kapazität des Schulsystems zu erhöhen. Im Zeitraum 2004-2005 wurde über Investitionen des privaten Sektors die Einrichtung von 14000 zusätzlichen Schulklassen finanziert. Privatunternehmen, die in Bildung investierten, erhielten Steuerermäßigungen. Das war in Provinzen mit starker inländischer Zuwanderung besonders hilfreich (OECD, 2006c).

Diese großen Veränderungen wurden von Maßnahmen flankiert, die direkt auf die Lehrkräfte ausgerichtet sind. Es wurden neue Bestimmungen eingeführt, um die Lehrerausbildung für die Sekundarstufe II im Rahmen eines fünfjährigen Magisterprogramms zu organisieren. Die Bestimmungen sehen ferner vor, dass Hochschulabsolventen in anderen Fachbereichen, wie Naturwissenschaften oder Literatur, die den Lehrerberuf ausüben möchten, ebenfalls an einem 18-monatigen Programm zur Erlangung der Lehrbefähigung teilnehmen müssen.

Mehrere der in der Türkei in den vergangenen zehn Jahren umgesetzten Projekte waren auf Fragen der Chancengerechtigkeit ausgerichtet. Eine 2003 ins Leben gerufene Kampagne zielte darauf ab, zu garantieren, dass 100\% der Mädchen die Grundschule besuchen (Altersgruppe 6-14 Jahre). Seit 2003 werden die Lehrbücher für alle Grundschüler vom Bildungsministerium kostenfrei zur Verfügung gestellt. Mit einem komplementären Ausbildungsprogramm, das 2008 gestartet wurde, wird seit kurzem versucht, zu gewährleisten, dass 10- bis 14-Jährige eine Grundausbildung erhalten, selbst wenn sie nie zur Schule gegangen sind oder die Schule abgebrochen haben.

Unabhängig vom Zusammenhang zwischen diesen Initiativen und den beobachteten Lernergebnissen ist die Türkei, die mit die größten Leistungsverbesserungen in Mathematik und Naturwissenschaften verzeichnet, auf dem besten Weg, die Bildungsstandards anderer OECD-Länder zu erreichen. 


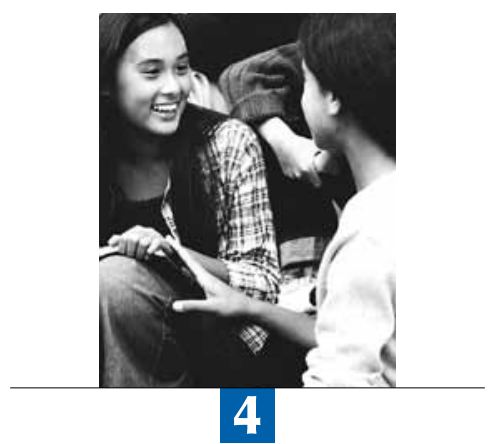

\section{Entwicklungen im Bereich der Chancengerechtigkeit}

In diesem Kapitel werden die Entwicklungen in Bezug auf die Chancengerechtigkeit bei Lernmöglichkeiten und Lernergebnissen untersucht. Der Schwerpunkt liegt auf der Frage, inwieweit sich im Zeitraum 2000-2009 die Varianz der Leistungen im Bereich Lesekompetenz sowie der Einfluss des sozioökonomischen Hintergrunds und des Migrationsstatus auf die Leistungen verändert haben. 


\section{ENTWICKLUNGEN BEI DER VARIANZ DER SCHÜLERLEISTUNGEN}

Die mittleren Punktzahlen geben zwar Aufschluss darüber, wie erfolgreich die Schülerinnen und Schüler in den einzelnen Ländern im Durchschnitt sind, für die politischen Entscheidungsträger ist es jedoch auch von Interesse zu wissen, wie stark die Leistungen der einzelnen Schüler von diesen Mittelwerten abweichen und wie hoch der Grad der Leistungsvarianz ist. Dank der Identifizierung von Ländern, in denen sich die Leistungsvarianz verringert hat, besonders derjenigen, wo dies mit einer Verbesserung der Schülerleistungen insgesamt einhergeht, können wichtige Erkenntnisse für die Politikgestaltung gewonnen werden.

Abbildung V.4.1 zeigt die Veränderungen der Varianz der Leistungen im Bereich Lesekompetenz, die im Zeitraum 2000-2009 zu beobachten waren (Tabelle V.4.1). Die Länder sind nach der Stärke der Varianz der Punktzahlen im Bereich Lesekompetenz, bezogen auf die statistische Varianz im Jahr 2009, angeordnet, und die Punkte stellen die entsprechenden Zahlen für das Jahr 2000 dar.

Im Durchschnitt der OECD-Länder verringerte sich die Varianz der Schülerleistungen im Bereich Lesekompetenz um 3\%; zwischen den Ländern bestanden hier jedoch erhebliche Unterschiede (Tabelle V.4.1). In der Gruppe der OECD-Länder, in denen die Varianz der Schülerleistungen bereits unter dem Durchschnitt lag, verringerte sie sich in Chile, Kanada und Ungarn weiter um 8-15\%. In der Gruppe der OECD-Länder, in denen sich die Varianz der Lesekompetenz in der Nähe des durchschnittlichen Niveaus bewegte, verzeichneten Dänemark, Polen und Portugal einen Rückgang der Varianz um über 20\%, während die Varianz in der Tschechischen Republik um $8 \%$ abnahm. Unter den OECD-Ländern mit überdurchschnittlich starker Varianz der Schülerleistungen im Jahr 2000 war in Deutschland ein Rückgang um 27\% und in Norwegen um 23\% zu beobachten, und auch in der Schweiz, den Vereinigten Staaten, in Neuseeland und Belgien kam es zu einer erheblichen Verringerung. Unter den Partnerländern beobachteten Lettland, Liechtenstein und Rumänien einen Rückgang der Leistungsvarianz um über 20\% auf ein unterdurchschnittlich niedriges Niveau. In den Partnerländern Indonesien und Thailand war eine Abnahme der Leistungsvarianz um 16\% bzw. 12\% festzustellen.

abbildung V.4.1

Vergleich der Varianz der Schülerleistungen im Bereich Lesekompetenz, 2000 und 2009

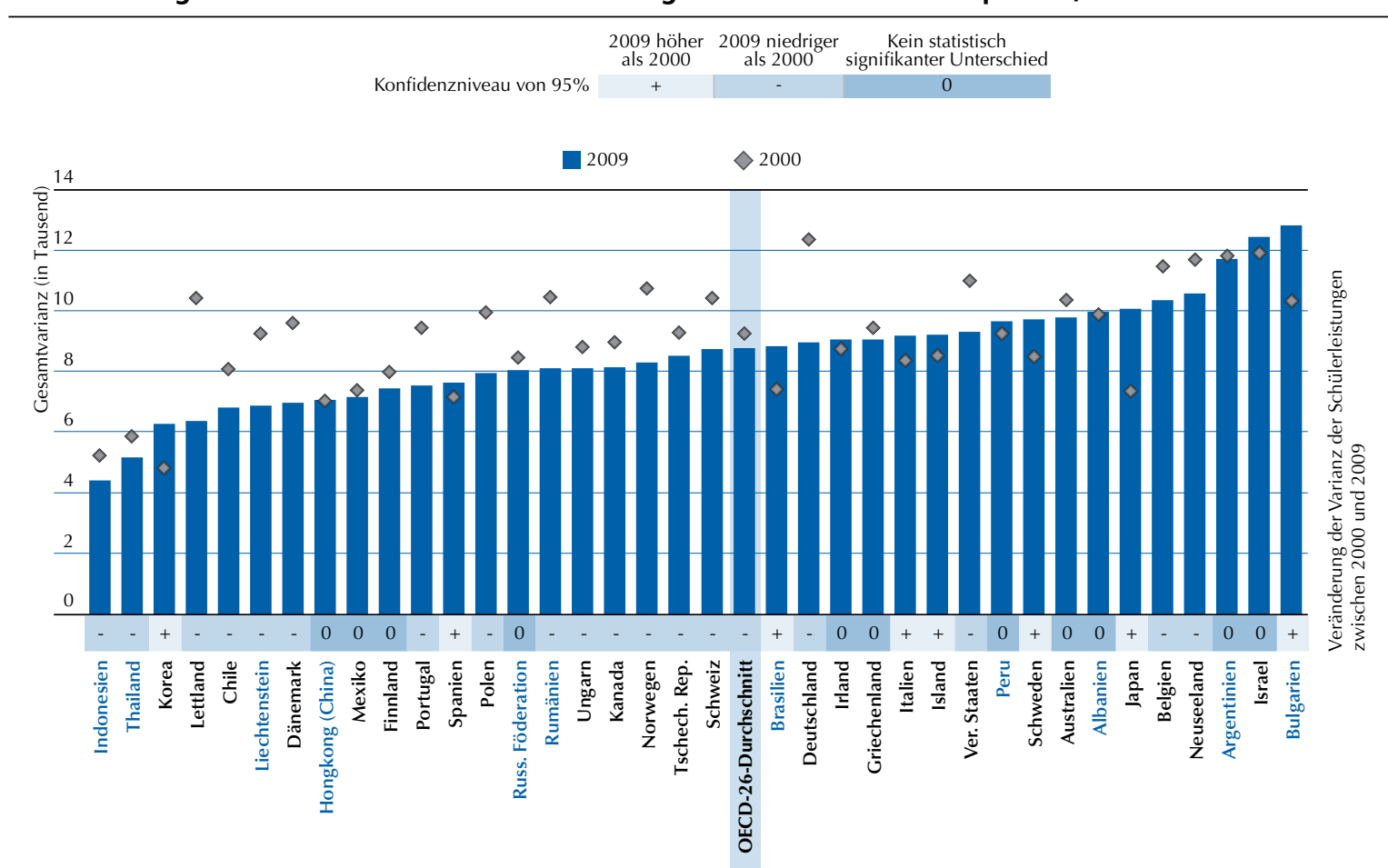

Die Länder sind in aufsteigender Reihenfolge nach der Höhe der Gesamtvarianz der Schülerleistungen im Jahr 2009 angeordnet.

Quelle: OECD, PISA-2009-Datenbank, Tabelle V.4.1.

StatLink 完开舟 http://dx.doi.org/10.1787/888932360005 
Mehrere Länder hatten eine Zunahme der Leistungsvarianz im Bereich Lesekompetenz zu verzeichnen. Während diese Zunahme in Schweden, Italien, Island und Spanien weniger als $15 \%$ betrug, belief sie sich in Japan und Korea auf 30\% oder mehr. In der Gruppe der Partnerländer hat sich die Leistungsvarianz in Bulgarien und Brasilien erheblich erhöht.

Diese Veränderungen lassen sich genauer untersuchen, wenn man die relative Erhöhung der Ergebnisse im Bereich Lesekompetenz der leistungsschwächsten und der leistungsstärksten Schüler betrachtet. Verbessern sich z.B. die Ergebnisse der leistungsschwachen Schüler, während die der leistungsstarken Schüler auf demselben Niveau verharren, dann verringert sich die Leistungsvarianz. Verbessern sich dagegen die Ergebnisse der leistungsstarken Schüler, die der leistungsschwachen jedoch nicht, dann erhöht sich die Gesamtvarianz. Die Ergebnisse in Abbildung V.4.1 können daher mit denen in Abbildung V.2.6 verglichen werden, in der die Veränderungen am 10. und 90. Perzentil der Lesekompetenz dargestellt sind.

In Ländern, in denen sich die Varianz der Schülerleistungen verringerte, haben sich häufig die Punktzahlen der leistungsschwächeren Schüler erhöht (Tabelle V.2.3). Dies war in Chile, Polen, Portugal, Deutschland, der Schweiz, Norwegen und Dänemark der Fall, da bei den leistungsschwächeren Schülern in Chile ein Anstieg um 51, in Polen um 39, in der Schweiz und Norwegen um 19 und in Dänemark um 16 Punkte festzustellen war (Beschreibung der in Chile und Polen für leistungsschwache Schüler konzipierten Maßnahmen in Kasten V.F und V.C). Das Partnerland Lettland zeigt den stärksten Rückgang der Leistungsvarianz und zugleich die bedeutendste Verbesserung der Ergebnisse der leistungsschwächeren Schüler - um 57 Punkte -, einen leicht positiven Trend bei Schülern mit durchschnittlichen Leistungen und keinerlei Veränderung, was die leistungsstärksten Schüler betrifft. Die Partnerländer Indonesien und Liechtenstein hatten ebenfalls eine leichte Verringerung der Leistungsvarianz zu verzeichnen, die durch eine Verbesserung der Ergebnisse der leistungsschwachen Schüler zustande kam. In all diesen Ländern haben sich die Ergebnisse der leistungsstarken Schüler nicht verschlechtert, und die Gesamtergebnisse haben sich verbessert (bzw. blieben zumindest unverändert). Interessant ist, dass die erhebliche Verringerung der Leistungsvarianz in zwei OECD-Ländern, Dänemark und Norwegen, nicht nur durch eine Verbesserung der Ergebnisse der leistungsschwachen Schüler, sondern auch durch eine Verschlechterung der Ergebnisse der leistungsstarken Schüler bzw. des Anteils der leistungsstärksten Schüler bedingt war, wobei der Gesamttrend bei der Lesekompetenz insgesamt nahe null lag.

In Schweden nahm die Leistungsvarianz auf Grund einer Verschlechterung der Ergebnisse der leistungsschwächeren Schüler zu, während bei den leistungsstarken Schülern keine Veränderung zu verzeichnen war (Tabelle V.2.3). Dagegen war die Zunahme der Varianz in Korea und im Partnerland Brasilien die Folge einer erheblichen Verbesserung bei den leistungsstarken Schülern, was in diesen Ländern zu einer Erhöhung der Gesamtergebnisse führte.

Es ist nützlich, zu wissen, inwieweit Varianzveränderungen mit den Entwicklungen bei den leistungsschwachen und den leistungsstarken Schülern in Verbindung stehen, wichtig ist es aber auch, den Zusammenhang zwischen den Veränderungen der Varianz und den Gesamtergebnissen zu kennen, um zu verstehen, wie sich die Leistungen im Lauf der aufeinanderfolgenden PISA-Erhebungen entwickelt haben. Dieser Zusammenhang ist in Abbildung V.4.2 dargestellt, wo die vertikale Achse die Veränderungen der Varianz und die horizontale Achse die Veränderungen der Durchschnittsleistungen anzeigt. Die Länder, die sich weiter rechts in der Abbildung befinden, hatten einen Anstieg der Durchschnittsleistungen, die Länder weiter links eine Verschlechterung der Durchschnittsleistungen zu verzeichnen. Die Leistungsvarianz hat sich im Verlauf der aufeinanderfolgenden Erhebungen in den Ländern im unteren Bereich der Abbildung verringert, in den Ländern im oberen Bereich dagegen erhöht. Länder, die statistisch signifikante Veränderungen in beiderlei Hinsicht aufweisen, sind dunkel markiert.

Interessanterweise geht eine Verringerung der Varianz oft mit einem Anstieg des Gesamtleistungsniveaus einher, d.h. die Länder mit der stärksten Verringerung der Varianz sind oft auch diejenigen, wo sich die mittleren Punktzahlen deutlich erhöht haben. In Deutschland, Polen, Portugal, Chile und Ungarn und in den Partnerländern Liechtenstein und Indonesien verringerte sich die Varianz und erhöhte sich das Leistungsniveau. Das Land mit der stärksten Verringerung der Leistungsvarianz, das Partnerland Lettland, kann auch einen Anstieg der Durchschnittsleistungen um über 20 Punkte vorweisen. In all diesen Ländern war die Gesamtverbesserung der Durchschnittsergebnisse und die Verringerung der Leistungsvarianz auf eine vergleichsweise stärkere Verbesserung bei den leistungsschwachen Schülern zurückzuführen. In der Tschechischen Republik ging eine Verringerung der Leistungsvarianz mit einem Rückgang des Gesamtleistungsniveaus einher. 
$\diamond \diamond$ Veränderung der Varianz und Punktzahlveränderung im Bereich Lesekompetenz

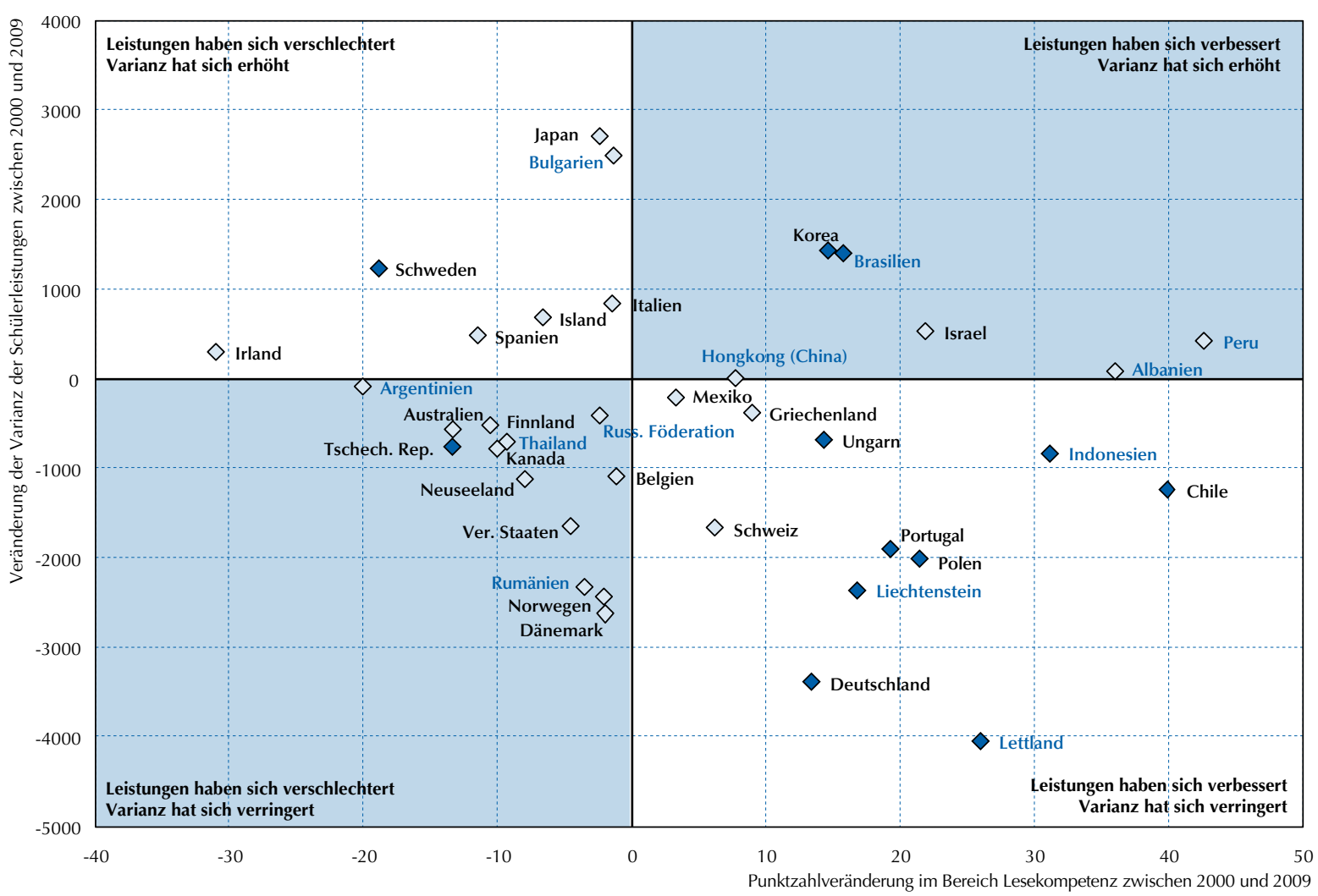

Anmerkung: Länder, in denen sowohl die Veränderung der Varianz als auch die Punktzahlveränderung statistisch signifikant sind, sind in einem dunkleren Farbton gekennzeichnet. Quelle: OECD, PISA-2009-Datenbank, Tabelle V.2.1 und V.4.1

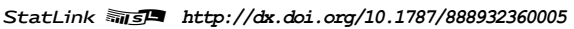

Bei diesem Muster gibt es Ausnahmen. Die Leistungsvarianz erhöhte sich in Korea, wo der Gesamtanstieg der Punktzahlen weitgehend auf Verbesserungen der Ergebnisse der leistungsstarken Schüler zurückzuführen war, während der Prozentsatz der leistungsschwachen Schüler unverändert blieb. Desgleichen war die Gesamtverbesserung der Leseleistungen im Partnerland Brasilien mit einem Anstieg der Leistungsvarianz verbunden, der weitgehend durch Verbesserungen bei den leistungsstarken Schülern bedingt war.

In Schweden erhöhte sich die Varianz, während die Gesamtleistung, großenteils auf Grund niedrigerer Punktwerte bei den leistungsschwachen Schülern, seit 2000 zurückgegangen ist.

Abbildung V.4.3 zeigt den Grad der Varianz der Leseleistungen 15-Jähriger zwischen den Schulen und innerhalb der Schulen und wie sich diese Varianz im Zeitverlauf entwickelt hat (Tabelle V.4.1) ${ }^{1}$. Die Länge des dunklen und des hellen Balkens zusammen genommen zeigt die Gesamtvarianz der Schülerleistungen auf der PISALesekompetenzskala. Für jedes Land unterscheidet die Abbildung zwischen der Varianz, die den Unterschieden zwischen den Durchschnittsergebnissen von Schülern verschiedener Schulen zuzuschreiben ist (Varianz zwischen den Schulen, dunkler Teil des Balkens), und dem Teil der Varianz, der sich aus Leistungsunterschieden zwischen den Schülern derselben Schulen erklärt (Varianz innerhalb der Schulen, heller Teil des Balkens)². Die Varianz zwischen den Schulen ist zudem für die Anordnung der Länder in der Abbildung maßgeblich. Längere dunkle Segmente zeigen eine stärkere Varianz der Durchschnittsergebnisse verschiedener Schulen, längere helle Segmente dagegen eine stärkere Varianz zwischen Schülern derselben Schulen. Die Rauten in der Abbildung stellen die Ergebnisse der Länder im Jahr 2000 dar. Im Falle signifikanter Veränderungen im Zeitraum 2000-2009 sind die entsprechenden Länder dunkel markiert, und zwar separat für die zwischenschulische und die innerschulische Varianz. 
Wie aus Abbildung V.4.3 ersichtlich, ist in allen Ländern eine erhebliche Varianz der Schülerleistungen innerhalb der Schulen zu beobachten, und in vielen Ländern ist auch eine starke Varianz zwischen den Schulen festzustellen. Der Prozentsatz der Leistungsvarianz zwischen den Schulen lässt sich als eine Messgröße für die schulische Inklusion interpretieren. Wo große Leistungsdifferenzen zwischen den Schulen bestehen, die Leistungsunterschiede zwischen den Schülern der einzelnen Schulen aber geringer sind, gilt in der Regel, dass die Schülerinnen und Schüler in Schulen zusammengefasst sind, in denen die meisten Schüler ein ähnliches Leistungsniveau aufweisen wie sie selbst. Der Grad der schulischen Inklusion eines Schulsystems kann auf bewusste Entscheidungen der Familien in Bezug auf die Schul- oder Wohnortwahl, die Aufnahmebestimmungen der Schulen oder die Verteilung der Schüler auf verschiedene Bildungsprogramme im Rahmen von Selektionsverfahren zurückzuführen sein. Wegen näherer Einzelheiten zur schulischen Inklusion, vgl. Band II, Potenziale nutzen und Chancengerechtigkeit sichern - Sozialer Hintergrund und Schülerleistungen.

Abbildung V.4.3

Varianz der Leistungen im Bereich Lesekompetenz innerhalb und zwischen den Schulen, 2000 und 2009

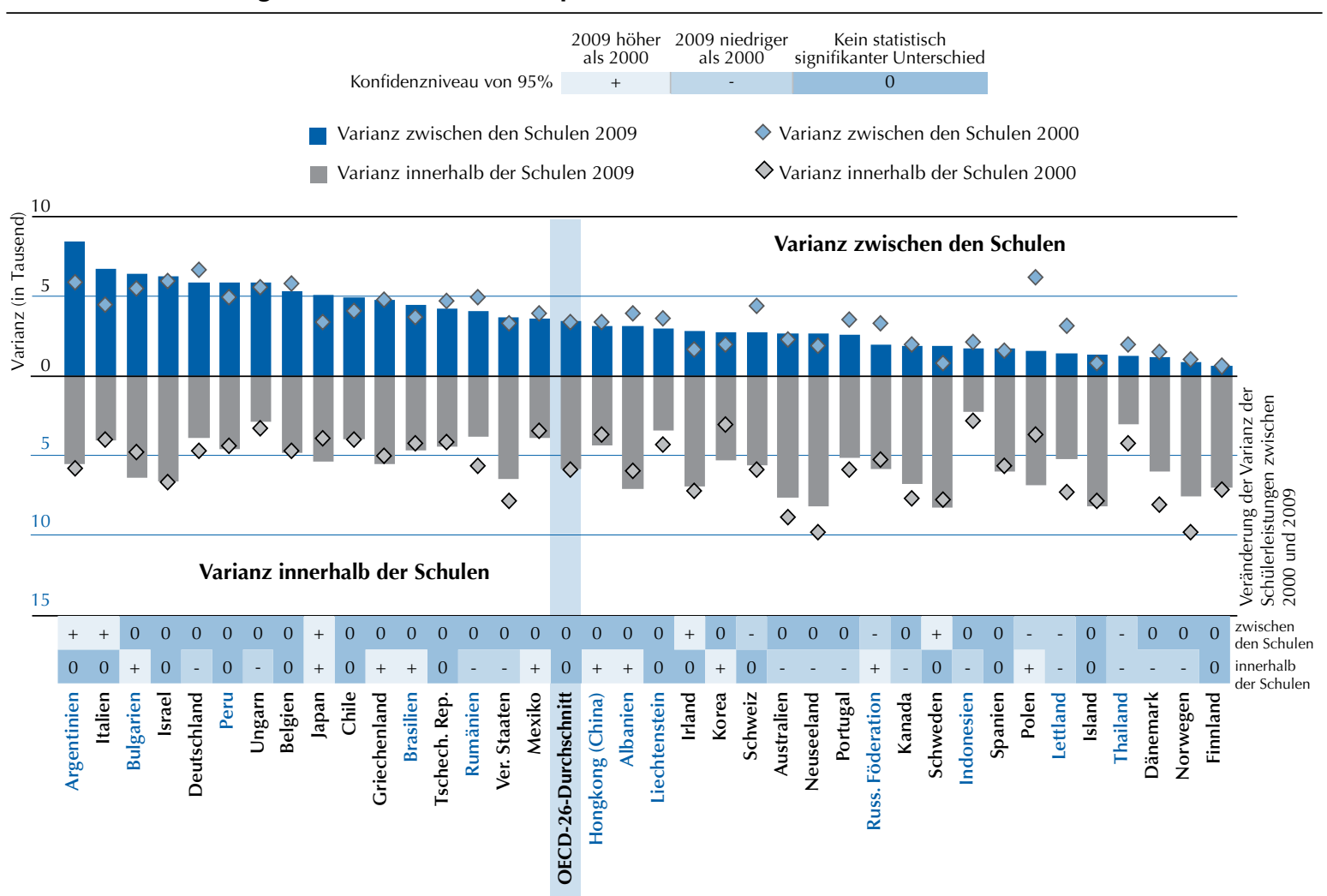

Die Länder sind in absteigender Reihenfolge nach der Höhe der Varianz zwischen den Schulen im Jahr 2009 angeordnet. Quelle: OECD, PISA-2009-Datenbank, Tabelle V.4.1.

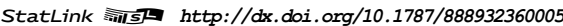

Im Gegensatz zur Gesamtvarianz blieb der relative Anteil der zwischenschulischen Varianz in den meisten Ländern im Zeitraum 2000-2009 unverändert. Zwei OECD-Länder, Polen und die Schweiz, und drei Partnerländer, Lettland, die Russische Föderation und Thailand, hatten eine Verringerung des Anteils der zwischenschulischen Leistungsvarianz zu verzeichnen. Der stärkste Rückgang war in Polen zu beobachten, wo die Varianz zwischen den Schulen von einem relativ hohen Niveau im Jahr 2000 ausgehend um drei Viertel und damit unter den Durchschnitt sank. Die Verringerung dieses Anteils war in der Schweiz zwar geringer, der Anteil der Varianz zwischen den Schulen lag dort im Jahr 2000 jedoch über dem Durchschnitt, während er jetzt unter dem Durchschnitt liegt. In Lettland und Thailand hat sich die Varianz zwischen den Schulen von einem niedrigen Niveau ausgehend sogar weiter verringert, während sie in der Russischen Föderation von einem unterdurchschnittlichen Niveau ausgehend um fast 40\% sank.

In Italien und im Partnerland Argentinien erhöhte sich die zwischenschulische Varianz gegenüber einem bereits hohen Ausgangsniveau. Diese Länder weisen 2009 die größten Unterschiede zwischen den Schulen auf. In den anderen Ländern mit hoher Varianz zwischen den Schulen kam es zu keinen Veränderungen. 


\section{ENTWICKLUNGEN BEI DEN HINTERGRUNDMERKMALEN DER SCHÜLER UND DEM ZUSAMMENHANG MIT DEN LESELEISTUNGEN}

\section{Sozioökonomischer Status}

Die Identifizierung der Merkmale der Schüler, Schulen und Bildungssysteme, die trotz eines ungünstigen sozioökonomischen Umfelds gute Ergebnisse erzielen, kann den politischen Entscheidungsträgern helfen, effektive Politikinstrumente zur Beseitigung von Chancenungleichheiten bei den Bildungsmöglichkeiten zu konzipieren.

Die Art und Weise, wie die Schülerinnen und Schüler auf verschiedene Schulen verteilt werden, kann zu erheblichen Leistungsunterschieden führen. Einige Länder haben integrative Schulsysteme, in denen zwischen den verschiedenen Einrichtungen nur begrenzte oder keine Unterschiede bestehen. Sie wollen allen Schülerinnen und Schülern gleichwertige Lernmöglichkeiten bieten und verlangen von sämtlichen Schulen und Lehrkräften, dass sie der gesamten Palette der Fähigkeiten, Interessen und Hintergrundmerkmale der Schüler Rechnung tragen. Andere Länder versuchen der Verschiedenheit der Schülerinnen und Schüler gerecht zu werden, indem sie die Schüler auf verschiedene Schulen bzw. innerhalb der Schulen auf verschiedene Klassen bzw. Bildungsgänge oder Leistungsgruppen verteilen, um sie so entsprechend ihrem jeweiligen schulischen Potenzial und/oder ihren Interessen an bestimmten Lehrprogrammen bestmöglich fördern zu können. In vielen Ländern werden diese beiden Ansätze auch miteinander kombiniert (wegen Einzelheiten dazu, wie die Schüler in den Schulsystemen ausgewählt und eingeteilt werden, vgl. Band IV, Was macht eine Schule erfolgreich?).

In allen Systemen können erhebliche Leistungsunterschiede zwischen den Schulen bestehen, bedingt durch die sozioökonomischen und kulturellen Merkmale des jeweiligen Einzugsgebiets oder durch geografische Unterschiede (zwischen einzelnen Regionen, Provinzen oder Bundesstaaten in föderativ gegliederten Ländern oder zwischen ländlichen und städtischen Gebieten). Zwischen einzelnen Schulen können auch Unterschiede bestehen, die sich schwerer quantifizieren lassen, wie z.B. Differenzen in der Qualität oder Effektivität des von ihnen angebotenen Unterrichts. Folglich kann das Leistungsniveau der Schülerinnen und Schüler verschiedener Schulen auch in integrativen Schulsystemen sehr unterschiedlich sein.

Unterschiede zwischen dem sozioökonomischen Hintergrund der Schüler können auch innerhalb der Schulen eine wichtige Rolle spielen. Die Schüler können innerhalb derselben Schule auf verschiedene Bildungsgänge verteilt werden. Die Schulen haben u.U. Probleme mit sozioökonomisch benachteiligten Schülern, und es kann auch sein, dass nur die Schüler aus günstigeren sozioökonomischen Verhältnissen zusätzliche Kursangebote in Anspruch nehmen, um ihr Bildungsniveau zu verbessern. All diese Faktoren können den Effekt des sozioökonomischen Hintergrunds auf die Leistungen der Schüler sogar innerhalb derselben Schulen verstärken.

Obwohl der sozioökonomische Hintergrund der Schüler im Laufe der PISA-Erhebungen weitgehend gleich blieb, führten in einigen Ländern wirtschaftliche und gesellschaftliche Umstellungen im Zeitraum 2000-2009 zu Veränderungen des sozioökonomischen Hintergrunds der Schüler ${ }^{3}$. Die stärkste Verschlechterung des sozioökonomischen Hintergrunds der Schüler war in den Partnerländern Albanien und Bulgarien zu verzeichnen (Tabelle V.4.2).

Band II, Potenziale nutzen und Chancengerechtigkeit sichern, analysiert den Zusammenhang zwischen dem familiären Hintergrund und den Leistungen der Schüler im Jahr 2009. In diesem Abschnitt werden Veränderungen dieses Zusammenhangs untersucht. Abbildung V.4.4 zeigt, wie sich der Zusammenhang zwischen dem sozioökonomischen Hintergrund und den Leistungen im Bereich Lesekompetenz, der hier als eine Messgröße der Gerechtigkeit bei der Verteilung der Bildungschancen verwendet wird, im Zeitraum 2000-2009 verändert hat (Tabelle V.4.3). Die dunklen Balken zeigen die Steigung der sozioökonomischen Gradienten im Jahr 2009, wobei die Länder nach der Höhe dieses Werts angeordnet sind ${ }^{4}$. Die Ergebnisse von 2000 sind in der Abbildung markiert, und neben den Ländernamen finden sich zusätzliche Angaben zur Signifikanz der Veränderung im Zeitraum 2000-2009.

Im OECD-Durchschnitt blieb der Effekt des sozioökonomischen Hintergrunds auf die Lernergebnisse im Zeitraum 2000-2009 unverändert. Einigen Ländern gelang es jedoch, die Chancengerechtigkeit bei der Verteilung der Bildungsmöglichkeiten zu verbessern. Unter den Ländern, in denen der Effekt des sozioökonomischen Hintergrunds auf die Lernergebnisse relativ stark war, gelang es der Tschechischen Republik, den Vereinigten Staaten und Deutschland, ihn zu verringern, so dass er dort jetzt näher am Durchschnitt liegt. In vier Ländern, die nahe am Durchschnitt lagen - Chile, Kanada und die Partnerländer Lettland und Albanien -, war der Effekt des sozioökonomischen Hintergrunds auf die Leistungen 2009 ebenfalls schwächer als 2000. In Mexiko und in der Partnervolkswirtschaft Hongkong (China) war der Zusammenhang zwischen dem sozioökonomischen Hintergrund und den Leistungen schon 2000 schwach, und er hat sich bis 2009 weiter verringert. 


\section{Zusammenhang zwischen dem sozioökonomischen Hintergrund der Schüler und ihren Leistungen im Bereich Lesekompetenz, 2000 und 2009}

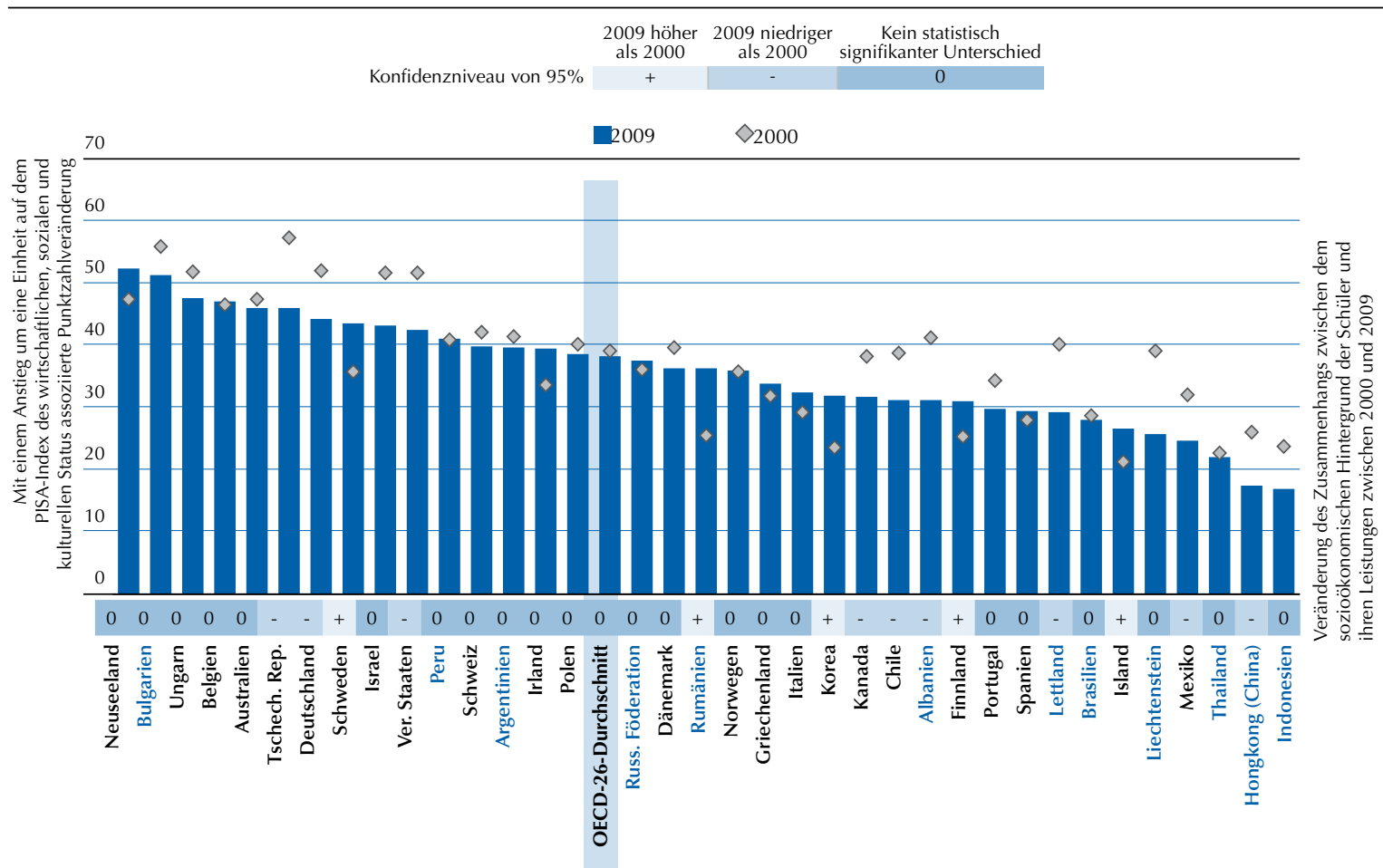

Die Länder sind in absteigender Reihenfolge nach dem Gesamtzusammenhang mit dem sozioökonomischen Hintergrund im Jahr 2009 angeordnet. Quelle: OECD, PISA-2009-Datenbank, Tabelle V.4.3.

StatLink 需西 http://dx.doi.org/10.1787/888932360005

Im Gegensatz dazu verstärkte sich die Steigung der sozioökonomischen Gradienten im Zeitraum 2000-2009 in fünf Ländern. Trotz dieser Zunahme liegt der Effekt des sozioökonomischen Hintergrunds auf die Schülerleistungen in Korea, Finnland und Island immer noch unter dem OECD-Durchschnitt, im Falle Schwedens liegt er aber darüber. Der stärkste Anstieg war im Partnerland Rumänien zu beobachten, wo sich die Steigung der sozioökonomischen Gradienten auf das Durchschnittsniveau erhöhte.

Einige Maßnahmen zur Überwindung sozioökonomischer Ungleichheiten zielen darauf ab, die Unterschiede in der sozioökonomischen Zusammensetzung der Schulen zu reduzieren. Beispiele hierfür sind Änderungen innerhalb der Schulsysteme mit dem Ziel, die Unterschiede zwischen den von den verschiedenen Schulen angegebenen Bildungsprogrammen zu verringern. Andere Maßnahmen sind darauf angelegt, den Effekt des sozioökonomischen Hintergrunds auf die Schülerleistungen innerhalb der Schulen zu begrenzen, z.B. durch zusätzliche Unterstützung für benachteiligte Schüler.

Abbildung V.4.5 zeigt, wie sich der Zusammenhang zwischen den Leistungen und dem sozioökonomischen Hintergrund der Schüler und Schulen innerhalb der Schulen und im Vergleich zwischen den Schulen entwickelt hat (Tabelle V.4.3). Die dunklen Balken im oberen Teil der Abbildung zeigen den Effekt des sozioökonomischen Hintergrunds im Vergleich zwischen den Schulen im Jahr 2009. Die Länder sind nach diesem Wert angeordnet, und die Rauten stellen die Ergebnisse von 2000 dar. Der zwischenschulische Effekt spiegelt die Stärke des Zusammenhangs zwischen dem durchschnittlichen sozioökonomischen Hintergrund der Schulen und den Durchschnittsergebnissen der Schulen wider. Die helleren Balken im unteren Teil der Abbildung stellen den Effekt innerhalb der Schulen dar, wobei die Rauten den Stand von 2000 zeigen. Der innerschulische Effekt spiegelt den durchschnittlichen Zusammenhang zwischen dem sozioökonomischen Hintergrund der Schüler und ihren Leistungen innerhalb der Schulen wider. Zusätzliche Informationen zur Erklärung statistisch signifikanter Veränderungen beider Aspekte sind neben den Ländernamen zu finden.

Im Durchschnitt der OECD-Länder ist der Zusammenhang zwischen dem sozioökonomischen Hintergrund und den Schülerleistungen im Vergleich zwischen den Schulen geringer geworden. Besonders stark war diese Verringerung in Polen und im Partnerland Lettland, wo der Zusammenhang 2009 gegenüber 2000 dreimal schwächer war. 
Abbildung V.4.5

\section{Zusammenhang zwischen dem sozioökonomischen Hintergrund der Schüler und ihren Leistungen im Bereich Lesekompetenz innerhalb und zwischen den Schulen, 2000 und 2009}

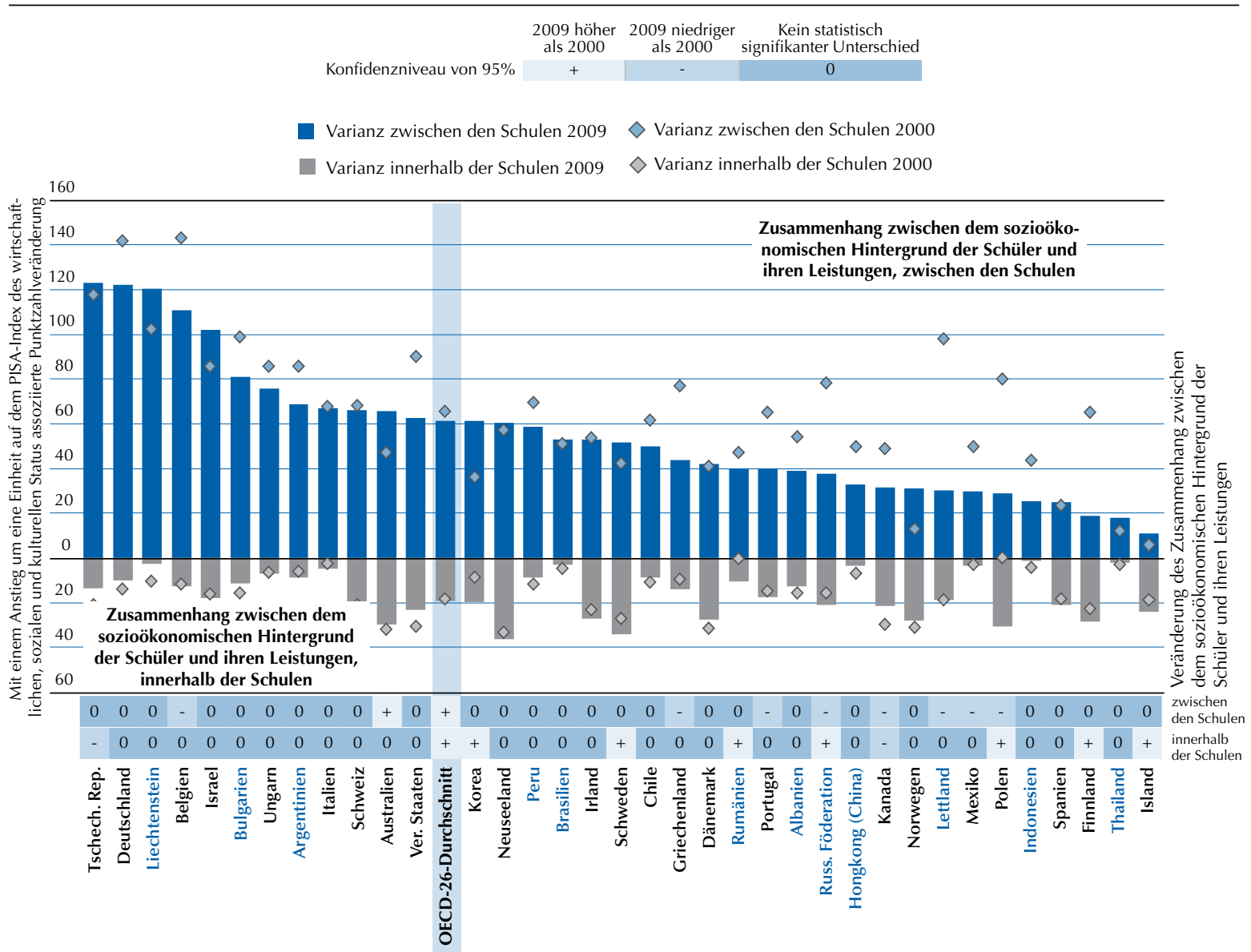

Die Länder sind in absteigender Reihenfolge nach dem Zusammenhang zwischen dem sozioökonomischen Hintergrund der Schüler und ihren Leistungen im Bereich Lesekompetenz im Jahr 2009 angeordnet.

Quelle: OECD, PISA-2009-Datenbank, Tabelle V.4.3.

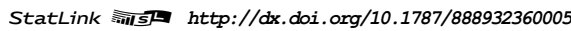

Doch während sich in Lettland auch der Gesamteffekt des sozioökonomischen Hintergrunds auf die Leistungen verringerte, erhöhte sich der innerschulische Effekt in Polen, so dass der Gesamteffekt unverändert dort blieb. Dies lässt vermuten, dass die Schulreform in Polen zwar den Effekt hatte, Schüler mit unterschiedlichem Hintergrund gleichmäßiger auf die verschiedenen Schulen zu verteilen, dass dies jedoch nichts daran änderte, wie die Schüler mit ungünstigerem Hintergrund insgesamt abschnitten (Kasten V.C über die Schulreform in Polen). In Griechenland, Belgien, Portugal, Mexiko, Kanada und im Partnerland Russische Föderation war zudem eine Abnahme der sozioökonomischen Disparitäten der Schulen zu beobachten, was allerdings nur in Mexiko und Kanada mit einer Abschwächung des Gesamteffekts des Hintergrunds der Schüler auf die Lernergebnisse einherging.

\section{Migrationsstatus und im Elternhaus gesprochene Sprache}

\section{Veränderung der Zahl der Schüler mit Migrationshintergrund und Zusammenhang mit den Leistungen}

In den OECD-Ländern mit vergleichbaren Daten ${ }^{5}$ hat sich der Prozentsatz der Schüler mit Migrationshintergrund im Zeitraum 2000-2009 durchschnittlich um 2 Prozentpunkte erhöht (Tabelle V.4.4). Der Prozentsatz der Schüler mit Migrationshintergrund ist jedoch in allen Ländern unterschiedlich.

In 13 Ländern erhöhte sich der Prozentsatz der Schüler mit Migrationshintergrund um über 2 Prozentpunkte, so dass diese Schüler jetzt über 5\% der Schülerpopulation ausmachen. In Spanien, Irland, den Vereinigten Staaten, Neuseeland und den Partnerländern Liechtenstein und Russische Föderation erhöhte sich der Prozentsatz der Schüler mit Migrationshintergrund um mindestens 5 Prozentpunkte, und diese Schüler stellen jetzt einen Anteil von 
8-30\% der Schülerpopulation dieser Länder. In Italien, Griechenland und Kanada stieg der Prozentsatz der Schüler mit Migrationshintergrund um 3-5 Prozentpunkte.

In Israel, dem Partnerland Lettland und der Partnervolkswirtschaft Hongkong (China) verringerte sich der Prozentsatz der Schüler mit Migrationshintergrund um 5, 18 bzw. 4 Prozentpunkte. Das hatte zur Folge, dass der Anteil der Schüler mit Migrationshintergrund in Lettland auf 4\% sank, in Israel und Hongkong (China) jedoch immer noch $20 \%$ bzw. 39\% der Schülerpopulation beträgt.

Im Durchschnitt der OECD-Länder blieb der Leistungsunterschied zwischen Schülern mit und ohne Migrationshintergrund in etwa gleich. Die Schüler ohne Migrationshintergrund erzielten 2000 ebenso wie 2009 im Schnitt über 40 Punkte mehr als die Schüler mit Migrationshintergrund (Tabelle V.4.4).

In den Ländern, die bei den Schülern mit Migrationshintergrund die stärkste relative Verbesserung verzeichnen konnten, hat sich der Leistungsabstand verringert. Gleichwohl schneiden Schüler ohne Migrationshintergrund in diesen Ländern immer noch besser ab als Schüler mit Migrationshintergrund. Zum Beispiel verringerte sich in Belgien und in der Schweiz der Leistungsabstand um nahezu 40 Punkte, doch erzielten die Schüler ohne Migrationshintergrund in Belgien immer noch durchschnittlich 68 Punkte und in der Schweiz 48 Punkte mehr als die Schüler mit Migrationshintergrund. In der Schweiz wurde diese Verringerung des Leistungsabstands erzielt, obwohl sich der Prozentsatz der Schüler mit Migrationshintergrund erhöht hatte. Deutschland, Neuseeland und das Partnerland Liechtenstein konnten ebenfalls eine Verringerung des Leistungsabstands zwischen Schülern mit und ohne Migrationshintergrund verzeichnen.

In Australien war eine Verringerung der Punktzahlen der Schüler ohne Migrationshintergrund zu beobachten, während das Leistungsniveau der Schüler mit Migrationshintergrund unverändert blieb, so dass die Schüler mit Migrationshintergrund dort jetzt besser abschneiden als die Schüler ohne Migrationshintergrund. Australien ist unter den Ländern, für die sich die entsprechenden Trends berechnen lassen, das einzige Land, in dem die Schüler ohne

Abbildung V.4.6

Prozentsatz der Schüler mit Migrationshintergrund, 2000 und 2009

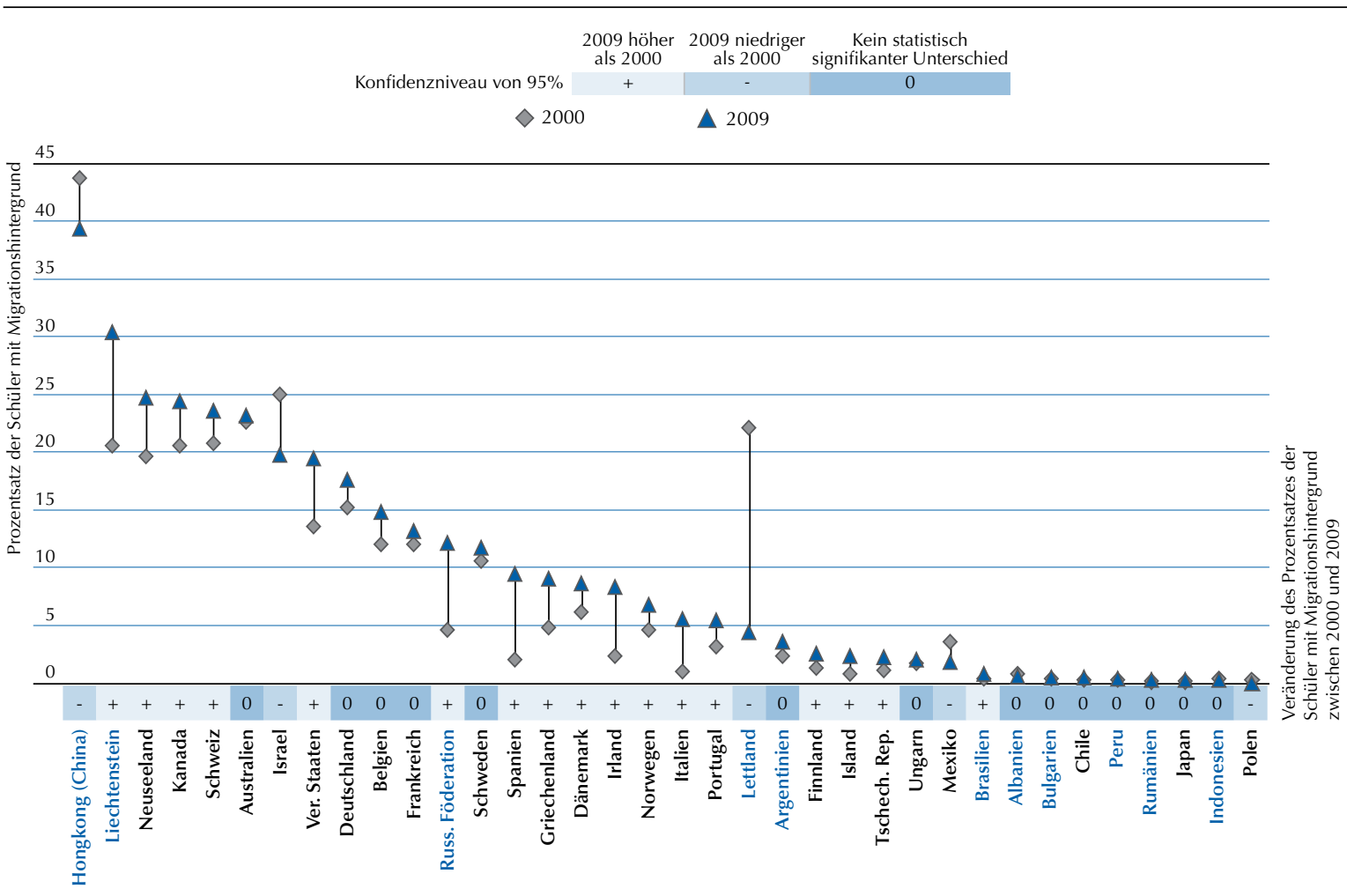

Die Länder sind in absteigender Reihenfolge nach dem Prozentsatz der Schüler mit Migrationshintergrund im Jahr 2009 angeordnet. Quelle: OECD, PISA-2009-Datenbank, Tabelle V.4.4.

StatLink त्राज़ http://dx.doi.org/10.1787/888932360005 


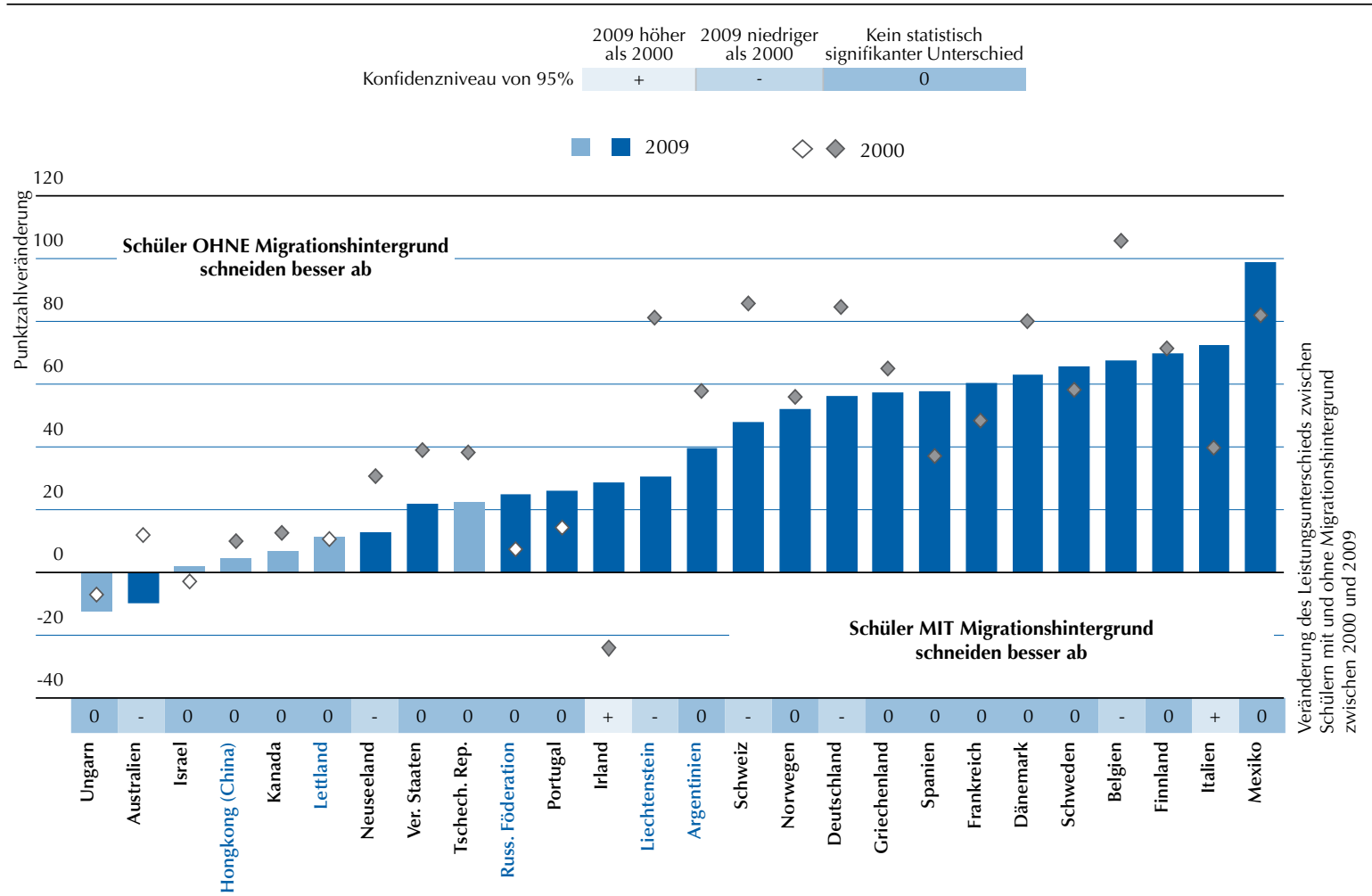

Anmerkung: Statistisch signifikante Punktzahlveränderungen sind durch einen dunkleren Farbton gekennzeichnet.

Die Länder sind in aufsteigender Reihenfolge nach der Leistungsdifferenz zwischen Schülern mit und ohne Migrationshintergrund im Jahr 2009 angeordnet.

Quelle: OECD, PISA-2009-Datenbank, Tabelle V.4.4.

StatLink 部促 http://dx.doi.org/10.1787/888932360005

Migrationshintergrund jetzt im Durchschnitt geringere Punktzahlen haben als die Schüler mit Migrationshintergrund. In Italien und Irland hat sich der Vorsprung der Schüler ohne Migrationshintergrund vergrößert. In beiden Ländern erhöhte sich der Prozentsatz der Schüler mit Migrationshintergrund im Zeitraum 2000-2009 um 5-6 Prozentpunkte. In Italien verharrten die Leistungen der Schüler ohne Migrationshintergrund auf demselben Niveau, während die Leistungen der Schüler mit Migrationshintergrund sanken. In Irland ging der Leistungsabfall der Schüler ohne Migrationshintergrund mit einem noch stärkeren Leistungsrückgang der Schüler mit Migrationshintergrund einher.

\section{Veränderung der Zahl der Schüler, die im Elternhaus eine andere Sprache sprechen, und Zusammenhang mit den Leistungen}

Im OECD-Durchschnitt hat sich der Prozentsatz der Schüler, die im Elternhaus eine andere Sprache als die Sprache sprechen, in der sie in PISA geprüft wurden, im Zeitraum 2000-2009 nicht verändert (Tabelle V.4.5) ${ }^{6}$. In den einzelnen Ländern verlief die Entwicklung jedoch unterschiedlich.

In neun Ländern erhöhte sich der Prozentsatz der betreffenden Schüler um mindestens 1 Prozentpunkt. Damit liegt der Prozentsatz der Schüler, die im Elternhaus eine andere Sprache sprechen, in Neuseeland, Kanada, Deutschland und im Partnerland Bulgarien jetzt bei mindestens 10\%. In anderen Ländern mit vergleichbarem Anstieg ist ihr prozentualer Anteil geringer.

Unter den OECD-Ländern mit einem überdurchschnittlich hohen Prozentsatz an Schülern, die im Elternhaus eine andere Sprache sprechen, ging dieser Anteil in Australien von 17\% auf 9\%, in Italien von 18\% auf $14 \%$ und in der Schweiz von $19 \%$ auf weniger als $16 \%$ zurück.

Unter den 32 Ländern, in denen ein Vergleich der Leistungen im Bereich Lesekompetenz der Schüler, die im Elternhaus die Testsprache sprechen, und derjenigen, die im Elternhaus eine andere Sprache sprechen, möglich 
Abbildung V.4.8

Prozentsatz der Schüler, die im Elternhaus eine andere Sprache als die Testsprache sprechen, 2000 und 2009

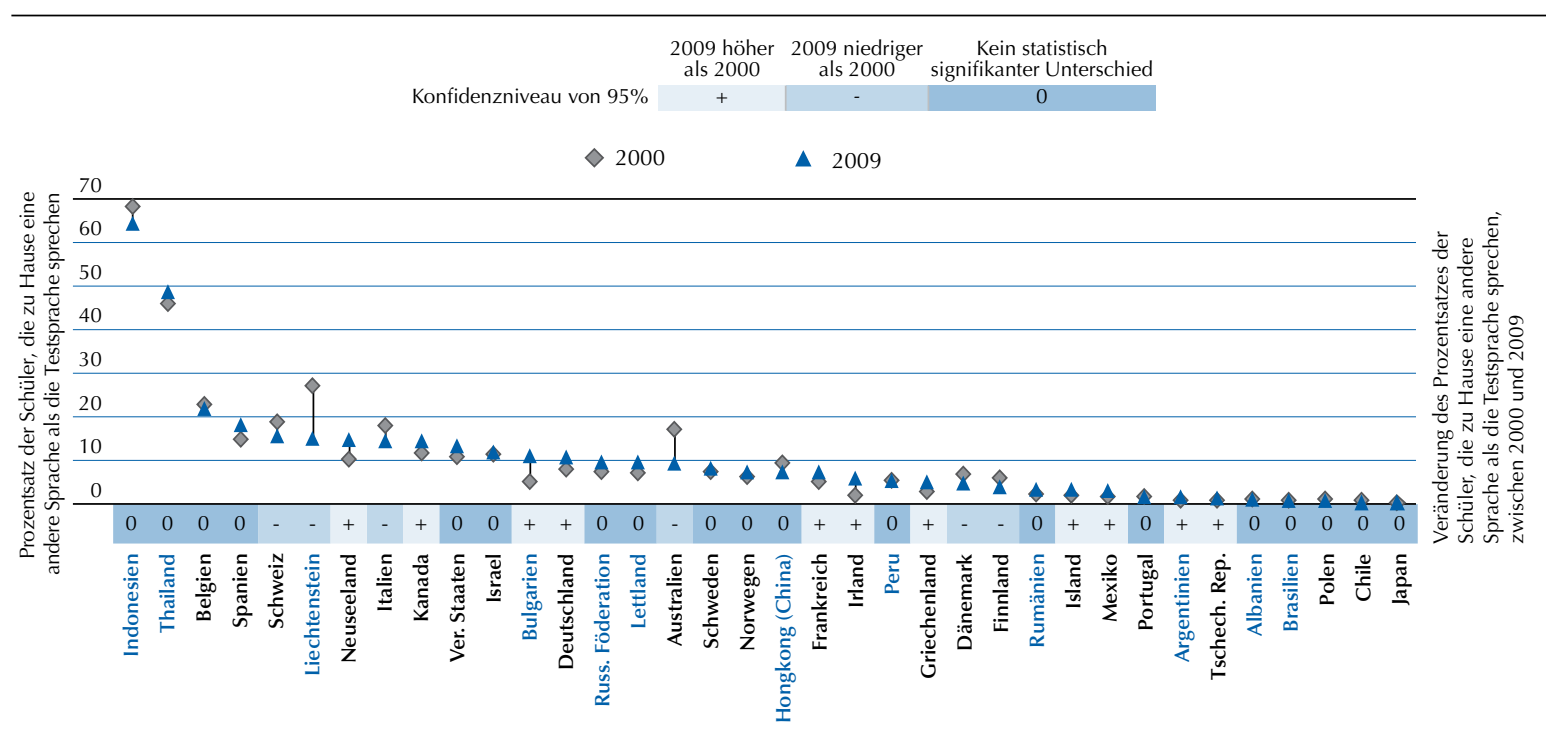

Die Länder sind in absteigender Reihenfolge nach dem Prozentsatz der Schüler angeordnet, die im Jahr 2009 im Elternhaus eine andere Sprache als die Testsprache sprachen. Quelle: OECD, PISA-2009-Datenbank, Tabelle V.4.5.

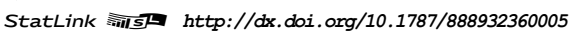

Abbildung V.4.9

Im Elternhaus gesprochene Sprache und Leistungen im Bereich Lesekompetenz, 2000 und 2009

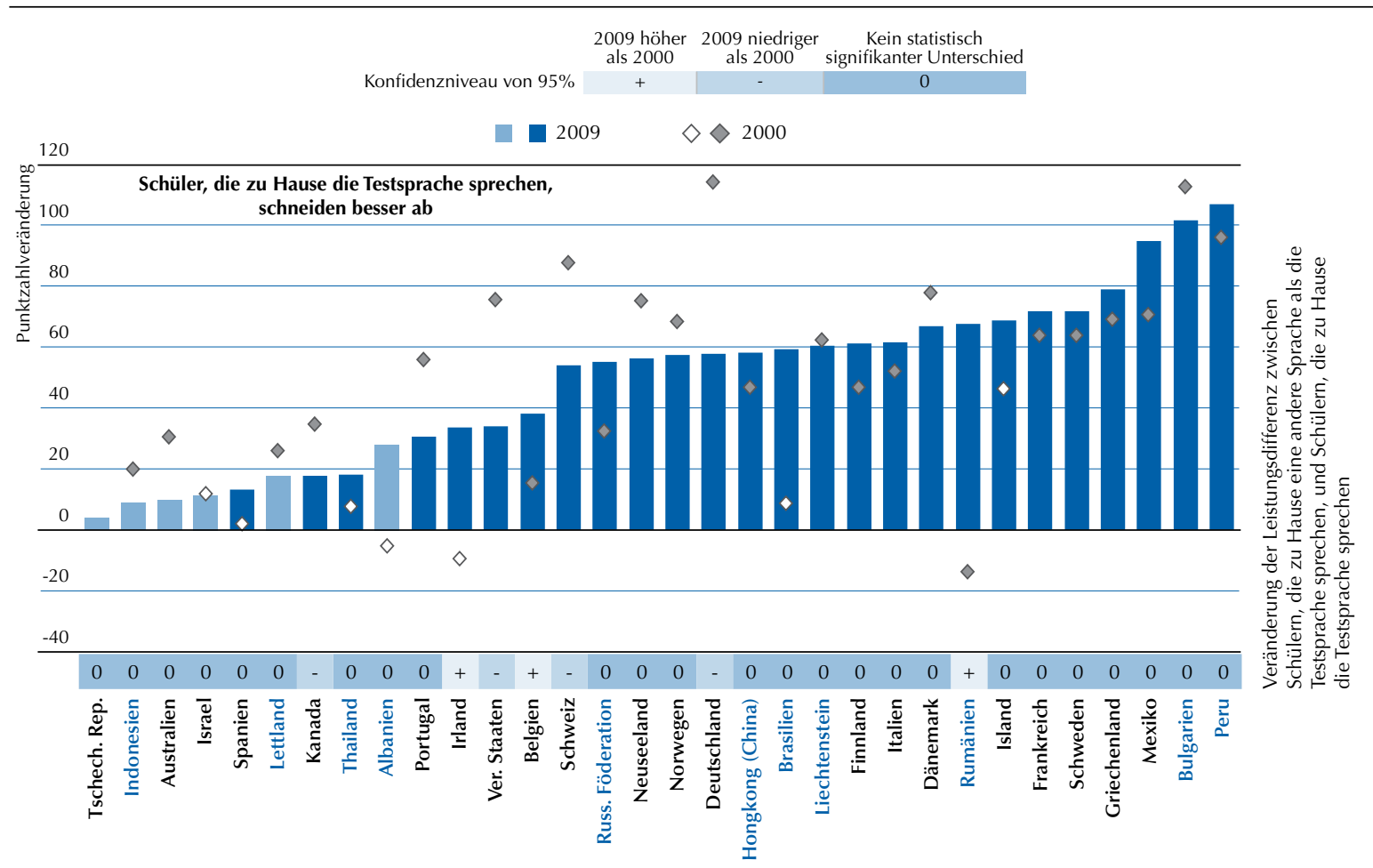

Anmerkung: Statistisch signifikante Punktzahlveränderungen sind durch einen dunkleren Farbton gekennzeichnet.

Die Länder sind in aufsteigender Reihenfolge nach der Leistungsdifferenz zwischen den Schülern angeordnet, die 2009 im Elternhaus eine andere Sprache als die Testsprache sprachen, und Schülern, die zu Hause die Testsprache sprachen.

Quelle: OECD, PISA-2009-Datenbank, Tabelle V.4.5.

StatLink 部西 http://dx.doi.org/10.1787/888932360005 
ist, war in vier Ländern ein Rückgang des Leistungsvorsprungs der Schüler zu verzeichnen, die im Elternhaus die Testsprache sprechen, während die Situation in drei Ländern umgekehrt ist (Tabelle V.4.5).

Deutschland, die Vereinigten Staaten, die Schweiz und Kanada hatten einen Rückgang des Leistungsvorsprungs der Schüler zu verzeichnen, die im Elternhaus die Testsprache sprechen. In Deutschland, den Vereinigten Staaten und der Schweiz war dieser Trend weitgehend auf die Verbesserung der Leistungen der Schüler zurückzuführen, die im Elternhaus eine andere Sprache sprechen. In Kanada war diese Veränderung die Folge eines Leistungsrückgangs der Schüler, die im Elternhaus die Testsprache sprechen, und einer Leistungsverbesserung der Schüler, die im Elternhaus eine andere Sprache sprechen. In all diesen Ländern verharrte der Leistungsvorsprung auf relativ hohem Niveau - über 30 Punkte -, außer in Kanada, wo er von 35 auf 18 Punkte sank.

Irland, Belgien und das Partnerland Rumänien sind die einzigen Länder, in denen der Vorsprung der Schüler, die zu Hause die Testsprache sprechen, zugenommen hat ${ }^{7}$. In Belgien vergrößerte sich der bereits 2000 ganz erhebliche Leistungsabstand auf Grund eines deutlichen Leistungsabfalls der Schüler, die zu Hause eine andere Sprache als die Testsprache sprechen, noch mehr. Während der Leistungsvorsprung der Schüler, die zu Hause die Testsprache sprechen, in Rumänien und Irland im Jahr 2000 nicht statistisch signifikant war, ist er jetzt relativ groß. In Irland erhöhte sich jedoch die Zahl der Schüler, die eine andere Sprache sprechen, und die Ergebnisse dieser Schüler unterschieden sich 2000 deutlich von denen von 2009. In Rumänien verschlechterten sich die Leistungen eines relativ geringen Prozentsatzes an Schülern, die zu Hause nicht die Testsprache sprechen, um 82 Punkte, während die Leistungen der Schüler, die zu Hause die Testsprache sprechen, nahezu unverändert blieben. 


\section{Anmerkungen}

1. Die bei PISA angewandte Mehrebenen-Modellierungsmethode wurde seit 2000 aktualisiert, so dass die in den Berichten 2000, 2003 und 2006 dargelegten Ergebnisse der Varianzzerlegung mit den hier angegebenen Werten nicht unmittelbar vergleichbar sind. Im vorliegenden Bericht werden sowohl auf Schüler- als auch auf Schulebene Gewichtungen vorgenommen, zuvor hingegen ausschließlich auf Schülerebene. Im PISA 2009 Technical Report wird die für die Daten von 2009 angewandte Gewichtungsmethode im Einzelnen beschrieben (OECD, erscheint demnächst).

2. Die Ergebnisse werden dadurch beeinflusst, wie die Schulen in den einzelnen Ländern definiert und organisiert sind und welche Einheiten für die Stichprobenziehung gewählt wurden. In einigen Ländern z.B. wurden die Schulen in der PISA-Stichprobe als Verwaltungseinheiten definiert (selbst wenn sie, wie in Italien, mehrere geografisch getrennte Einrichtungen umfassen), in anderen wiederum als die Teile größerer Bildungseinrichtungen, die von 15-Jährigen besucht werden; in manchen Ländern waren auch die einzelnen Schulgebäudeeinheiten maßgeblich, und in wieder anderen wurden die Schulen unter dem Aspekt der Verwaltung definiert (z.B. als Einheiten, die eine eigene Schulleitung haben). Anhang A2 und der PISA 2009 Technical Report (OECD, erscheint demnächst) liefern einen Überblick über die verschiedenen Methoden der Definition der Schulen. Zu beachten ist auch, dass die Varianz innerhalb der Schulen auf Grund der Art und Weise, wie die Schülerinnen und Schüler in der Stichprobe erfasst wurden, sowohl die Leistungsunterschiede zwischen den Klassen als auch zwischen den einzelnen Schülern umfasst.

3. Die verschiedenen Aspekte des sozioökonomischen Hintergrunds sind in der Regel stark miteinander verknüpft und werden in PISA zu einem einzigen Index, dem PISA-Index des wirtschaftlichen, sozialen und kulturellen Status (ESCS) der Schüler, zusammengefasst. Dieser Index wurde so konstruiert, dass rund zwei Drittel der OECD-Schülerpopulation zwischen den Werten -1 und 1 angesiedelt sind, wobei das Indexmittel bei 0 liegt (was heißt, dass der Mittelwert der gesamten Schülerpopulation der teilnehmenden OECD-Länder mit 0 und die Standardabweichung mit 1 angesetzt wurde). Einzelheiten dazu, wie der ESCS-Index bei den verschiedenen PISA-Erhebungen angeglichen wurde, damit sein Wert im Zeitraum 2000-2009 vergleichbar ist, finden sich in Anhang A1.

4. Die Stärke des Zusammenhangs wird gemessen durch einen Koeffizienten in einer linearen Regression der Lesekompetenz auf den PISA-Index des wirtschaftlichen, sozialen und kulturellen Status (ESCS) der Schüler.

5. Die Schülerleistungen werden nur für die Länder analysiert, in denen mindestens 30 Schüler in fünf verschiedenen Schulen mit anderen Schülern verglichen werden können. Dies bedeutet, dass die Schülerstichprobe eines Landes, um in der Analyse berücksichtigt zu werden, mindestens 30 Schüler mit Migrationshintergrund umfassen muss und dass diese Schüler auf fünf verschiedene Schulen verteilt sein müssen. Ebenso muss die Stichprobe, um im nächsten Abschnitt einen gültigen Leistungsvergleich zu ermöglichen, mindestens 30 Schüler umfassen, die im Elternhaus eine andere Sprache als die Testsprache sprechen und die aus fünf verschiedenen Schulen kommen. Diese Regel gilt für die Daten aus allen PISA-Erhebungen, so dass sie für den Vergleich der Lesekompetenz auf PISA 2000 und PISA 2009 anzuwenden ist.

6. Einige Länder verwendeten bei den PISA-Erhebungen verschiedene Sprachen.

7. In Irland zählten zu den Schülern, die zu Hause eine andere Sprache als die Testsprache sprechen, Schüler, die zu Hause weder Englisch noch Irisch sprechen (3,7\% aller Schüler), Schüler, die zu Hause Irisch sprechen, den Test aber auf Englisch ablegten $(0,5 \%)$, und Schüler, die zu Hause Englisch sprechen, an der Erhebung aber in Irisch teilnahmen (1,8\%). 


\section{Kasten V. F Chile}

Das durchschnittliche Leistungsniveau Chiles im Bereich Lesekompetenz ist zwischen 2000 und 2009 um 40 Punkte gestiegen, womit Chile im Hinblick auf den Grad der Ergebnisverbesserung in diesem Zeitraum unter den PISA-Teilnehmerländern an zweiter Stelle liegt (Abb. V.2.1 und Tabelle V.2.1). Das durchschnittliche Leistungsniveau Chiles ist zwar immer noch unter dem OECD-Durchschnitt angesiedelt, doch hat Chile dank dieser Leistungsverbesserung gegenüber dem Jahr 2000 Argentinien, Bulgarien, Mexiko, Rumänien und Thailand überholt, die 2000 ein ähnliches oder sogar höheres Leistungsniveau aufzuweisen hatten (Abb. V.2.3).

Die Verbesserungen sind zwar über das ganze Leistungsspektrum zu beobachten, bei den leistungsschwachen Schülern sind sie jedoch am deutlichsten. Das Leistungsniveau der Schüler mit den niedrigsten Ergebnissen ist seit 2000 um 51 Punkte gestiegen, und der Prozentsatz der Schüler, die im Bereich Lesekompetenz unter Stufe 2 liegen, hat sich um 17,6 Prozentpunkte verringert. 2009 lag der Anteil der 15-jährigen Schülerinnen und Schüler, die Stufe 2 nicht erreichten, bei 30,6\%, während es im Jahr 2000 fast der Hälfte der 15-Jährigen an den grundlegenden Fähigkeiten fehlte, die diesem Kompetenzniveau im Bereich Lesekompetenz entsprechen. Die Verbesserungen im obersten Bereich des Leistungsspektrums fielen dagegen geringer aus: Der Prozentsatz der am besten abschneidenden Schülerinnen und Schüler, gemessen am Anteil der Schüler, deren Leistungen über der Stufe 5 liegen, erhöhte sich nur um 0,8 Prozentpunkte (Abb. V.2.4 und V.2.5, und Tabelle V.2.2).

Infolge der stärkeren Ergebnisverbesserung der leistungsschwachen Schüler hat sich die Gesamtleistungsvarianz in Chile bedeutend verringert, wodurch der Abstand zwischen den leistungsstärksten und den leistungsschwächsten Schülerinnen und Schülern kleiner wurde. Und da leistungsschwache Schülerinnen und Schüler mit höherer Wahrscheinlichkeit aus sozioökonomisch benachteiligten Verhältnissen kommen, hat sich der Grad der Chancengerechtigkeit in Chile ebenfalls verbessert (Abb. V.4.4 und Tabelle V.4.3). Die mit einem Anstieg um eine Einheit auf dem PISA-Index des wirtschaftlichen, sozialen und kulturellen Status assoziierte Punktzahldifferenz sank um 8 Punkte.

In den 1980er Jahren war es Chile gelungen, allen Kindern Zugang zu Schulbildung zu bieten; in den Jahren danach bestand die Herausforderung darin, eine qualitative Verbesserung des Bildungsangebots für alle Schülerinnen und Schüler zu gewährleisten. Seit der Rückkehr zur Demokratie in den 1990er Jahren wurden mehrere Maßnahmen und Programme verabschiedet und umgesetzt, die darauf abzielten, die Qualität des Bildungsangebots und das Leistungsniveau zu erhöhen. Die meisten dieser Maßnahmen sind auf leistungsschwache und benachteiligte Schulen ausgerichtet, in denen, wie PISA 2000 zeigte, der Verbesserungsbedarf am größten ist (wegen einer Analyse der bildungspolitischen Maßnahmen in Chile, vgl. Cox, 2003).

Speziell auf leistungsschwache und benachteiligte Schülerinnen und Schüler ausgerichtete Programme sind u.a. das Anfang der 1990er Jahre eingerichtete P900 Escuelas und das einige Jahre später eingeführte Programa de Mejoramiento de la Educación con Calidad y Equidad (MECE). Das Programm P900 Escuelas war auf die leistungsschwächsten $10 \%$ der Grundschulen ausgerichtet, wobei die Lehrkräfte von Ausbildern aus denselben Kommunen technisch unterstützt wurden. Den betroffenen Schulen wurde auch in materieller Hinsicht geholfen, insbesondere indem ihnen Bildungsmittel zur Verfügung gestellt wurden. Das MECE-Programm verfolgte im Hinblick auf die Unterstützung der Schulen einen umfassenderen Ansatz: Es war darauf angelegt, Schulinfrastruktur und -ressourcen, Lehrerausbildung und Schulverwaltung zu verbessern und dafür zu sorgen, dass die Schulen über die notwendigen Kapazitäten verfügen, um ihre eigenen Bildungsprogramme gestalten zu können. Dieses Programm wurde schrittweise im ganzen Bildungssystem umgesetzt, angefangen bei den leistungsschwächsten Schulen bis hin zu den leistungsstärksten. Ebenfalls in den 1990er Jahren wurden die Klassenzimmer der unteren Grundschulklassen mit Büchern ausgestattet und in Klassenzimmerbibliotheken umgewandelt, und sowohl öffentliche als auch vom Staat abhängige private Schulen erhielten Lehrbücher, die an die einzelnen Schüler zu verteilen waren. Diese Bücher waren dann Eigentum der Schüler, so dass auch den sozial am stärksten benachteiligten Schülern zu Hause eine Erstausstattung an Büchern zur Verfügung stand, die die ganze Familie nutzen konnte.

Die chilenische Regierung nahm zudem ab den 1990er Jahren eine substanzielle Erhöhung ihrer Bildungsinvestitionen vor. Die Gesamtausgaben für die Grundschul- und Sekundarschulbildung haben 
sich von 1995 bis 2007 mehr als verdoppelt (Bildung auf einen Blick 2010, Tabelle B1.5, S. 234), und die Lehrergehälter stiegen im Zeitraum 2000-2006 real um über 7,7\% (Bildung auf einen Blick 2002, Tabelle D6.1, S. 504, und Bildung auf einen Blick 2008, Tabelle D3.1, S. 460).

Überdies wurde Ende der 1990er Jahre eine Lehrplanreform durchgeführt. Nachdem die Ergebnisse der PISAErhebung 2000 bekannt wurden, die auf ein niedriges Niveau der funktionellen Lesefähigkeiten chilenischer Schülerinnen und Schüler schließen ließen, wurden Veränderungen im Lehrplan für den Testsprachenunterricht vorgenommen, die eine Schwerpunktverlagerung von Literatur und Grammatik hin zu Leseverständnis und Kommunikation beinhaltete, d.h. Kompetenzen, die der im Rahmen von PISA gemessenen Lesekompetenz näher kommen (Gysling, 2003).

Zu den sonstigen Maßnahmen allgemeinerer Art, die durchgeführt wurden, um die Bildungsergebnisse zu verbessern, gehörte eine Erhöhung der Unterrichtsstundenzahl im Jahr 1997, die mit einer Umstellung von Vormittags- oder Nachmittagsunterricht auf Ganztagsunterricht für alle Schülerinnen und Schüler verbunden war. 1998 wurde das nationale System zur Messung der Bildungsergebnisse (SIMCE) erheblich verbessert. Seit 1995 werden die Ergebnisse dieser Bewertungen auf Schulebene öffentlich bekannt gegeben, und für die Schulen ist ein individuelles Feedback vorgesehen. In öffentlichen Schulen tätige Lehrkräfte werden einer detaillierten Beurteilung unterzogen, die sich u.a. auf Unterrichtsbeobachtungen und konkrete Belege zur Lehrtätigkeit der Betreffenden, stützt (Docente Más). Lehrkräfte, die diese Prüfung nicht bestehen, haben die Möglichkeit, eine kostenlose Lehrerweiterbildung zu absolvieren. Bei dreimaligem Nichtbestehen der Prüfung wird die Lehrkraft entlassen. Auf der Basis der SIMCE-Schülerleistungsdaten und anderer Informationen werden die Schulen im Sistema Nacional de Evaluación Docente (SNED) eingestuft, das den leistungsstärksten Schulen zusätzliche Mittel zuteilt, die diese direkt den Lehrkräften zukommen lassen können. Im Rahmen eines zweiten Lehrkräftebeurteilungsprogramms - Asignación de Excelencia Pedagógica -, das allen Lehrkräften auf freiwilliger Basis offensteht, werden Lehrkräfte, die ausgezeichnete pädagogische Fähigkeiten nachweisen können, durch Gehaltserhöhungen belohnt.

Die im Rahmen von PISA 2000 beurteilten Schüler waren nur zu Beginn ihrer Schulzeit von einigen dieser Programme betroffen. Die Schüler, die an PISA 2009 teilnahmen, wurden dagegen Ende der 1990er Jahre eingeschult, so dass ihnen diese Programme während ihrer ganzen Schulzeit zugute kamen. Viele der Programme zur Verbesserung der Bildungsergebnisse, insbesondere im Bereich Lesekompetenz, zielten schwerpunktmäßig darauf ab, leistungsschwachen und benachteiligten Schülern in den ersten Grundschuljahren zu helfen.

Die Einführung von Schulgutscheinen Anfang der 1980er Jahre führte in Chile zu einem Anstieg der Zahl der Privatschulen und trug dazu bei, dass mehr Kinder Zugang zu Schulbildung bekamen. Seit der Einführung der Gutscheine hat sich die Zahl der Schüler, die privat verwaltete, staatlich geförderte Schulen besuchen, erhöht, was zu Lasten des Besuchs öffentlicher Schulen ging (Carnoy, 1998). Infolgedessen ist der Anteil der Schüler der vom Staat abhängigen privaten Schulen an der Gesamtpopulation der 15-jährigen Schüler im Zeitraum 2000-2009 von 32,8\% (PISA-2000-Datenbank) auf 49,2\% gestiegen (Band IV, Was macht eine Schule erfolgreich?, und Tabelle IV.3.9), d.h. um 16,4 Prozentpunkte.

Während bei einigen Studien keinerlei Effekte der Gutscheine auf die Schülerleistungen nachgewiesen werden konnten (Hsieh und Urquiola, 2006), wurden im Rahmen anderer Arbeiten positive Auswirkungen festgestellt (Anand, Mizala und Repetto, 2006). Der entsprechende Effekt beträgt etwa 0,14 Standardabweichungen und ist sehr gering verglichen mit dem im Zeitraum 2000-2009 in Chile beobachteten Anstieg der Schülerleistungen um 40 Punkte (Abb. V.2.1, Tabelle V.2.1). Deutlicher zum Ausdruck kommt der Effekt der Gutscheine bei einem Blick auf die sozioökonomische Zusammensetzung der Schulen, denn die Gutschein-Programme führten zu einer stärkeren Segregation der Schulen nach schulischen Leistungen und sozioökonomischem Hintergrund (Hsieh und Urquiola, 2006; Belley, 2007, und Band IV, Was macht eine Schule erfolgreich?).

Trotz der bedeutenden Verbesserung der Ergebnisse Chiles im Bereich Lesekompetenz zeigt PISA 2009, dass das Land weiter unter dem OECD-Durchschnitt liegt und dass es drei von zehn Schülern nach wie vor an den grundlegenden Fähigkeiten im Bereich Lesekompetenz fehlt. Die Anhebung der Bildungsstandards ist in Chile 
eines der größten Anliegen sowohl der Öffentlichkeit als auch der Regierung. Es werden weiter Maßnahmen und Programme konzipiert und umgesetzt, um die Bildungsqualität zu verbessern, insbesondere was die Gruppe der benachteiligten und leistungsschwachen Schüler betrifft. Zum Beispiel wurden Änderungen im Gutschein-Programm vorgenommen, so dass die Gutscheine jetzt nach dem sozioökonomischen Status der Schüler gewichtet werden. Die Schulen können die Schüler nicht nach ihrem sozioökonomischen Status oder ihren Leistungen in der Grundschule auswählen, und die Schüler können nicht von der Schule verwiesen werden, ohne die Chance gehabt zu haben, eine Klassenstufe zu wiederholen. Es werden Anreize geboten, um Personen mit höherem Studienabschluss für den Lehrerberuf zu interessieren, und Studiengänge in Erziehungswissenschaften werden mit öffentlichen Mitteln gefördert, vorausgesetzt, dass die Personen, die diese Beihilfen in Anspruch nehmen, nach Abschluss ihres Studiums eine bestimmte Anzahl von Jahren in öffentlich finanzierten Schulen unterrichten. 


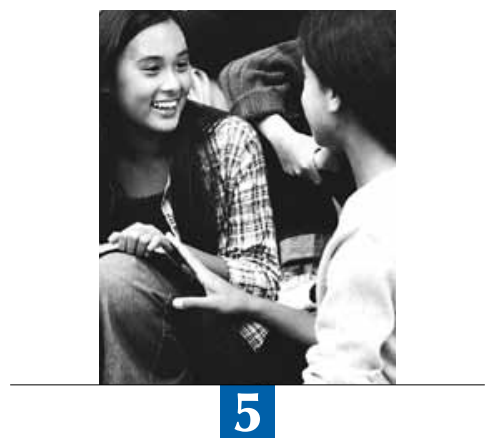

\section{Trends bei den Einstellungen der Schüler und den Beziehungen zwischen Schülern und Schulen}

Haben sich die Einstellungen der Schülerinnen und Schüler zum Lesen im Lauf der Jahre verändert? Dieses Kapitel beschreibt die zwischen 2000 und 2009 beobachteten Trends in Bezug darauf, ob und wenn ja was die Schüler zum Vergnügen lesen, und wie sich die Unterschiede zwischen Jungen und Mädchen bei den Lesevorlieben und Leseleistungen in diesem Zeitraum entwickelt haben. Das Kapitel untersucht auch die Trends bei den Lehrer-Schüler-Beziehungen und der Schuldisziplin. 


\section{TRENDS BEIM LESEENGAGEMENT}

\section{Veränderungen in Bezug auf das Lesen zum Vergnügen}

Schülerinnen und Schüler, die sich stark mit einer Vielzahl von Leseaktivitäten befassen und die bestimmte Lernstrategien verinnerlicht haben, werden mit größerer Wahrscheinlichkeit als andere Schüler effektive Lernende sein und in der Schule gute Ergebnisse erzielen. Forschungsergebnisse zeigen außerdem, dass unter Erwachsenen eine starke Verbindung zwischen dem Leseverhalten, der Lesemotivation und der Lesekompetenz besteht (OECD und Statistics Canada, 2000). Die in Band III, Learning to Learn (nur auf Englisch verfügbar), vorgestellten Ergebnisse deuten darauf hin, dass das Lesen zum Vergnügen mit der Lesekompetenz assoziiert ist. Die in Band III vorgestellten Daten belegen, dass der entscheidende Punkt, in dem sich Schüler mit guten Ergebnissen im PISA-Lesekompetenztest von solchen mit schlechten Ergebnissen unterscheiden, die Frage ist, ob sie täglich zum Vergnügen lesen, wohingegen der Umfang der mit Lesen verbrachten Zeit weniger ausschlaggebend ist.

Im Rahmen von PISA 2009 wurden die Schülerinnen und Schüler gefragt, wie viel Zeit sie in der Regel damit verbringen, zum Vergnügen zu lesen. Da ihnen in PISA 2000 die gleiche Frage gestellt wurde, können die Antworten der Schülerinnen und Schüler bei den beiden Erhebungen verglichen werden. Die Schüler wurden in zwei Kategorien eingeteilt: jene, die zum Vergnügen lesen, und die, die nicht zum Vergnügen lesen.

Im Jahr 2009 begeisterten sich die 15-jährigen Schülerinnen und Schüler tendenziell weniger für das Lesen als im Jahr 2000. Im Durchschnitt der OECD-Länder ist der Anteil der Schülerinnen und Schüler, die angaben, täglich zum Vergnügen zu lesen, um 5 Prozentpunkte gesunken (Tabelle V.5.1). Im Jahr 2000 gaben 69\% der Schülerinnen und Schüler an, täglich zum Vergnügen zu lesen, im Jahr 2009 waren es hingegen nur noch $64 \%$. In nicht weniger als 22 Ländern kam es zwischen 2000 und 2009 zu einem Rückgang des Anteils der Schülerinnen und Schüler, die zum Vergnügen lesen. In zehn anderen Ländern sind die Verhaltensmuster hinsichtlich des Lesens allerdings unverändert geblieben, und in Japan, Griechenland und Kanada sowie den Partnerländern und -volkswirtschaften Hongkong (China), Bulgarien und Thailand hat sich der Anteil der Schülerinnen und Schüler, die täglich zum Vergnügen lesen, gegenüber dem Jahr 2000 erhöht (Abb. V.5.1).

Abbildung V.5.1

Prozentsatz der Schüler, die zum Vergnügen lesen, 2000 und 2009

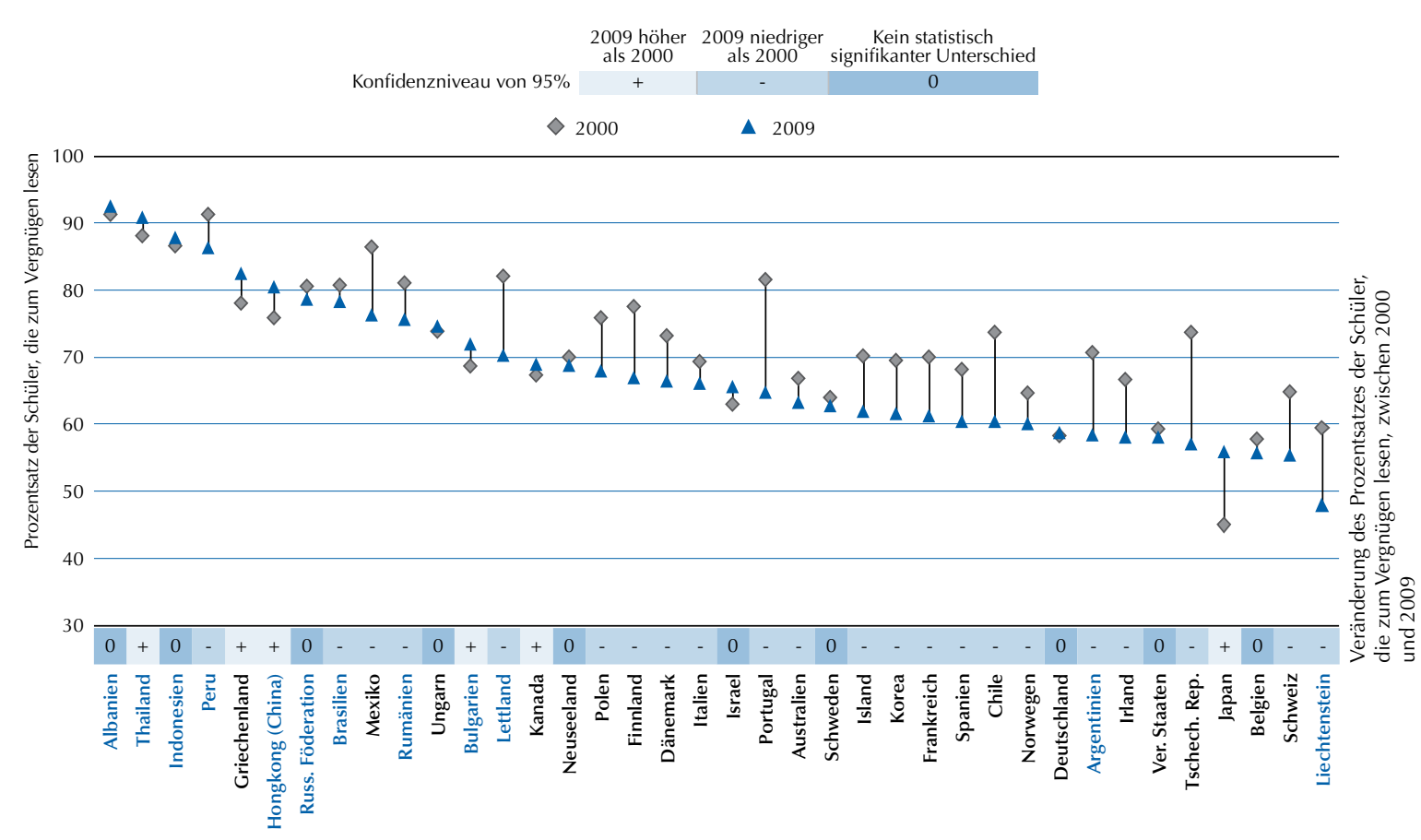

Die Länder sind in absteigender Reihenfolge nach dem Prozentsatz der Schüler, die zum Vergnügen lesen, im Jahr 2009 angeordnet. Quelle: OECD, PISA-2009-Datenbank, Tabelle V.5.1

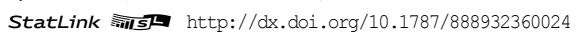


Manche der Länder, in denen der Anteil der Schüler, die zum Vergnügen lesen, zwischen 2000 und 2009 gesunken ist, sind Länder mit einem vergleichsweise hohen Anteil solcher Schüler. In Portugal, Finnland und Mexiko sowie dem Partnerland Lettland ist der Anteil der Schülerinnen und Schüler, die zum Vergnügen lesen, von einem relativ hohen Niveau ausgehend (über 75\% im Jahr 2000) um mehr als 10 Prozentpunkte gesunken.

Japan ist das einzige Land, in dem weniger als zwei Drittel der Schülerinnen und Schüler im Jahr 2009 täglich zum Vergnügen lasen, wobei dieser Anteil immerhin deutlich höher ist als im Jahr 2000. Japan war im Jahr 2000 das Land mit dem geringsten Anteil an Schülern, die zum Vergnügen lasen, ist so der Anteil dieser Schüler, selbst nachdem er sich um 11 Prozentpunkte erhöht hat, dort immer noch niedriger als in den meisten anderen Ländern.

Die Mädchen sind unter den Schülern, die zum Vergnügen lesen, wesentlich zahlreicher vertreten als die Jungen. Im OECD-Durchschnitt lesen 74\% der Mädchen täglich zum Vergnügen, jedoch nur 54\% der Jungen, was einer Differenz von 20 Prozentpunkten entspricht (Tabelle V.5.1). Der Abstand zwischen Jungen und Mädchen hat sich zwischen 2000 und 2009 im OECD-Durchschnitt um 3 Prozentpunkte ausgeweitet: Im Jahr 2000 lasen 60\% der Jungen und 77\% der Mädchen zum Vergnügen; 2009 nur noch 54\% bzw. 74\%. Interessanterweise war die Ausweitung des Abstands zwischen Jungen und Mädchen dadurch bedingt, dass zwar im Jahr 2009 im Durchschnitt ein geringerer Prozentsatz der Jungen und der Mädchen zum Vergnügen las als im Jahr 2000, der Rückgang jedoch bei den Jungen stärker ausfiel als bei den Mädchen. Mit anderen Worten war der Rückgang des Leseengagements bei den Jungen ausgeprägter als bei den Mädchen. Die Entwicklung der geschlechtsspezifischen Unterschiede beim Lesen zum Vergnügen zwischen 2000 und 2009 fällt in den einzelnen Ländern sehr unterschiedlich aus (vgl. Abb. V.5.2). Während der Anteil der Jungen, die zum Vergnügen lesen, in den meisten Ländern zwischen 2000 und 2009 gesunken ist, kann bei den Mädchen kein durchgehender Trend festgestellt werden.

Im Vergleich aller 38 Länder mit gültigen Ergebnissen aus dem Lesekompetenztest der Jahre 2000 und 2009 weisen nur zwei Länder einen Anstieg des Anteils der zum Vergnügen lesenden Jungen auf. In Japan ist der Anteil der zum Vergnügen lesenden Jungen um 9 Prozentpunkte gestiegen, in der Partnervolkswirtschaft Hongkong (China) um

Abbildung V.5.2

Veränderung des Prozentsatzes der Jungen und Mädchen, die zum Vergnügen lesen, zwischen 2000 und 2009

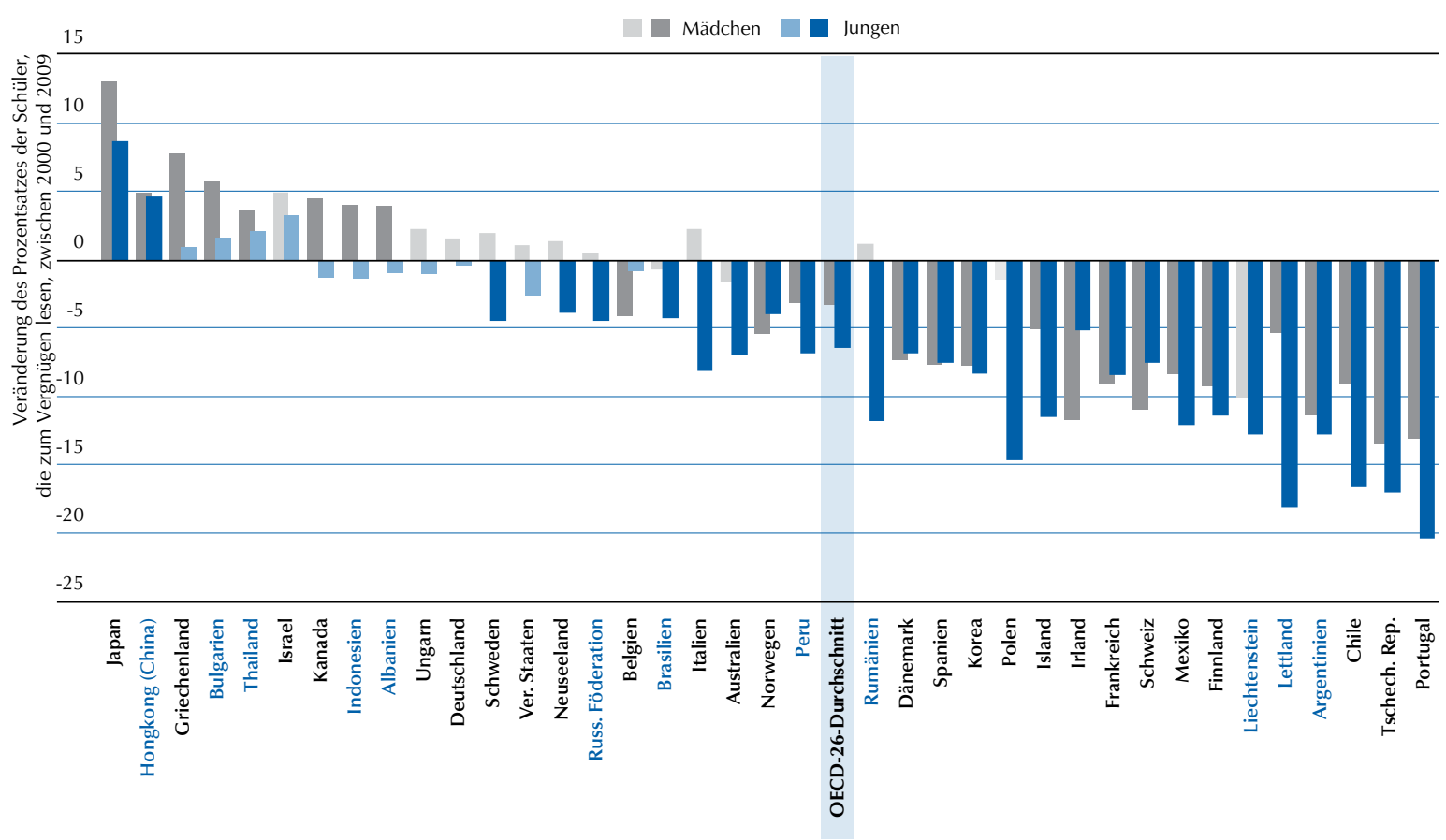

Anmerkung: Statistisch signifikante Punktzahlveränderungen sind durch einen dunkleren Farbton gekennzeichnet.

Die Länder sind in absteigender Reihenfolge nach der Veränderung des Prozentsatzes aller Schüler, die zum Vergnügen lesen, zwischen 2000 und 2009 angeordnet.

Quelle: OECD, PISA-2009-Datenbank, Tabelle V.5.1

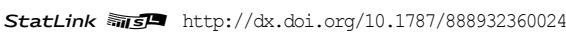


5 Prozentpunkte. In Japan war der Anstieg unter den Mädchen höher und ging deshalb mit einer Ausweitung des Abstands zwischen den Jungen und Mädchen einher. In Hongkong (China) stieg der Anteil der zum Vergnügen lesenden Jungen und Mädchen in gleichem Maße, so dass die Differenz bei etwa 8 Prozentpunkten stabil blieb. In 11 Ländern, darunter die OECD-Länder Belgien, Kanada, Deutschland, Griechenland, Ungarn, Israel und Vereinigte Staaten, hat sich der Anteil der Jungen, die zum Vergnügen lesen, nicht verändert. In 25 Ländern ist der Anteil der Jungen, die zum Vergnügen lesen, seit dem Jahr 2000 gesunken. Portugal, die Tschechische Republik, Chile und das Partnerland Lettland sind die Länder mit dem stärksten Rückgang. In diesen Ländern ist der Anteil der Jungen, die zum Vergnügen lesen, um mindestens 15 Prozentpunkte gesunken und liegt nun zwischen $44 \%$ und $55 \%$. Unter den anderen Ländern, in denen ein Rückgang des Anteils der Jungen zu verzeichnen war, die zum Vergnügen lesen, beträgt dieser Prozentsatz in der Schweiz und den Partnerländern Argentinien und Liechtenstein nun bis zu 50\% und in Island, Finnland, Frankreich, Italien, Spanien, Australien, Irland, Schweden und Norwegen bis zu 55\%.

Der Anteil der Mädchen, die zum Vergnügen lesen, ist in 17 Ländern zurückgegangen (vgl. Abb. V.5.2). In der Tschechischen Republik, Portugal, Irland, der Schweiz und dem Partnerland Argentinien ist ihr Anteil um 11-13 Prozentpunkte gesunken und beträgt nun fast 70\%, außer in Portugal, wo der Gesamtprozentsatz nahe bei $80 \%$ liegt - weit über dem OECD-Durchschnitt von 74\%. In zwölf weiteren Ländern ist der Prozentsatz der Mädchen, die zum Vergnügen lesen, um bis zu 10 Prozentpunkte zurückgegangen. In 13 OECD-Ländern ist der Anteil der zum Vergnügen lesenden Mädchen unverändert geblieben. In acht Ländern ist er gestiegen. In Griechenland und Kanada sowie den Partnerländern und -volkswirtschaften Bulgarien, Hongkong (China), Indonesien, Albanien und Thailand liegt er nun über $80 \%$, während er trotz des insgesamt größten Anstiegs in Japan noch immer unter $60 \%$ verharrt.

\section{Veränderungen in Bezug auf die Freude am Lesen}

Die Angaben der Schülerinnen und Schüler zu ihrer Einstellung zum Lesen geben Aufschluss darüber, inwieweit sie Freude am Lesen haben. Beispielsweise sollten die Schülerinnen und Schüler angeben, ob die Aussagen „Ich lese nur, wenn ich muss" oder "Ich spreche gerne mit anderen Leuten über Bücher" auf sie "überhaupt nicht", "eher nicht", "eher" oder "ganz genau“ zutreffen. Die Antworten der Schülerinnen und Schüler hierzu lassen sich in einem Index zusammenfassen, auf dem einem durchschnittlichen Schüler (d.h. einem Schüler mit durchschnittlicher Freude am Lesen) ein Indexwert von 0 zugeordnet wurde und etwa zwei Drittel der OECD-Schülerpopulation im Bereich zwischen -1 und 1 liegen (der Index hat also eine Standardabweichung von 1).

Im Durchschnitt der OECD-Länder ist der Anteil der Schülerinnen und Schüler, die angaben, dass sie nur lesen, wenn sie müssen, zwischen 2000 und 2009 um etwa 4 Prozentpunkte gestiegen: Im Jahr 2000 gaben 36\% der Schülerinnen und Schüler in den OECD-Ländern an, dass sie nur lesen, wenn sie müssen, während sich dieser Anteil im Jahr 2009 auf 40\% belief. Der Anteil der Schülerinnen und Schüler, die laut eigenen Angaben nur dann lesen, wenn sie müssen, stieg in 21 Ländern, und in Mexiko, Island, Korea und der Tschechischen Republik sowie in den Partnerländern Indonesien, Peru, Albanien und Liechtenstein war dieser Anstieg mit über 10 Prozentpunkten besonders deutlich. 2009 gab mehr als jeder zweite Schüler in Korea und Liechtenstein an, nur zu lesen, wenn dies notwendig ist. In Chile und den Partnerländern Thailand, Brasilien und Rumänien sank der Anteil der Schüler, die angaben, nur zu lesen, wenn sie müssen, zwischen 2000 und 2009 hingegen um über 10 Prozentpunkte (Tabelle V.5.3).

Der allgemeine Anstieg der Zahl der Schüler, die solche Angaben machten, deckt sich mit einem starken Rückgang der Zahl der Schüler, die aussagten, dass sie gerne in Buchhandlungen oder Büchereien/Bibliotheken gehen. Im Jahr 2000 gaben 49\% der Schülerinnen und Schüler an, gerne in Buchhandlungen oder Bibliotheken/Büchereien zu gehen, 2009 nur noch 43\% - ein Rückgang um 6 Prozentpunkte. Dieser Rückgang war besonders deutlich in Mexiko, der Tschechischen Republik, Portugal, Chile, Dänemark, Polen, Finnland, Spanien und Griechenland sowie den Partnerländern Peru und Rumänien. Der Anteil der Schülerinnen und Schüler, die laut eigenen Angaben gerne Buchhandlungen oder Büchereien/Bibliotheken besuchen, ist nur in Australien, Kanada und der Partnervolkswirtschaft Hongkong (China) gestiegen.

Abbildung V.5.4 beschreibt die Veränderungen auf dem Index der Freude am Lesen in den Teilnehmerländern von PISA 2000 und 2009 (vgl. auch Tabelle V.5.2). Anders als im vorhergehenden Abschnitt, in dem es darum ging, ob die Schülerinnen und Schüler zum Vergnügen lesen, fasst dieser Index zusammen, wie gerne sie lesen. Im Allgemeinen ist die Freude am Lesen in den 26 OECD-Ländern, für die vergleichbare Daten vorliegen, gesunken. In manchen Ländern begeisterten sich die Schülerinnen und Schüler im Jahr 2000 mehr für das Lesen als 2009, während in anderen das Gegenteil festgestellt wurde. Die Freude am Lesen hat in Deutschland, Kanada, Neuseeland, Japan 
Abbildung V.5.3

Prozentsatz der Schüler, die nur lesen, wenn sie müssen, und der Schüler, die gerne in Buchhandlungen oder Büchereien gehen, 2000 und 2009

Prozentsatz der Schüler, die für folgende Aussagen "stimmt eher" oder "stimmt ganz genau" angekreuzt haben

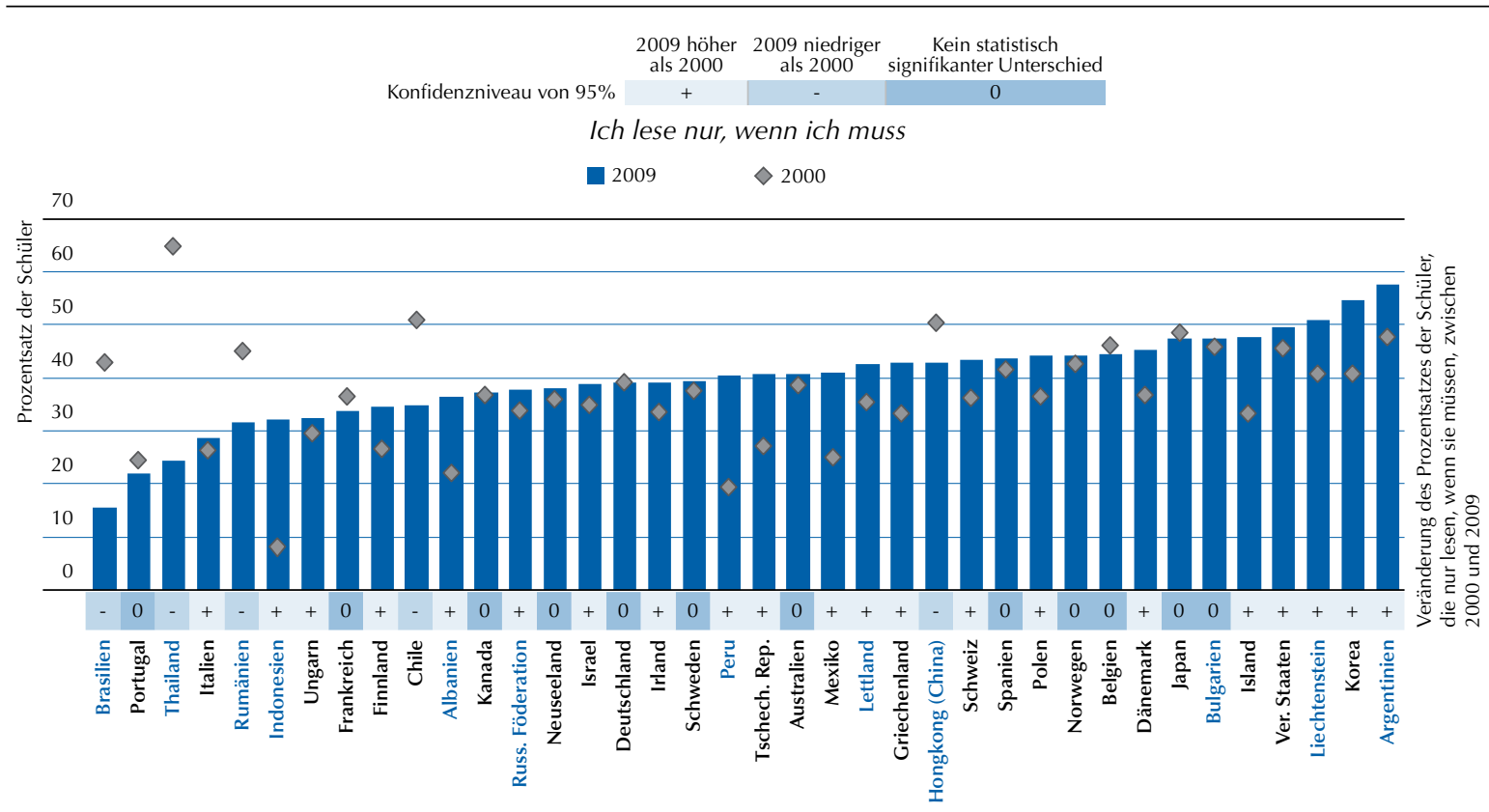

Ich gehe gerne in Buchhandlungen oder Büchereien/Bibliotheken

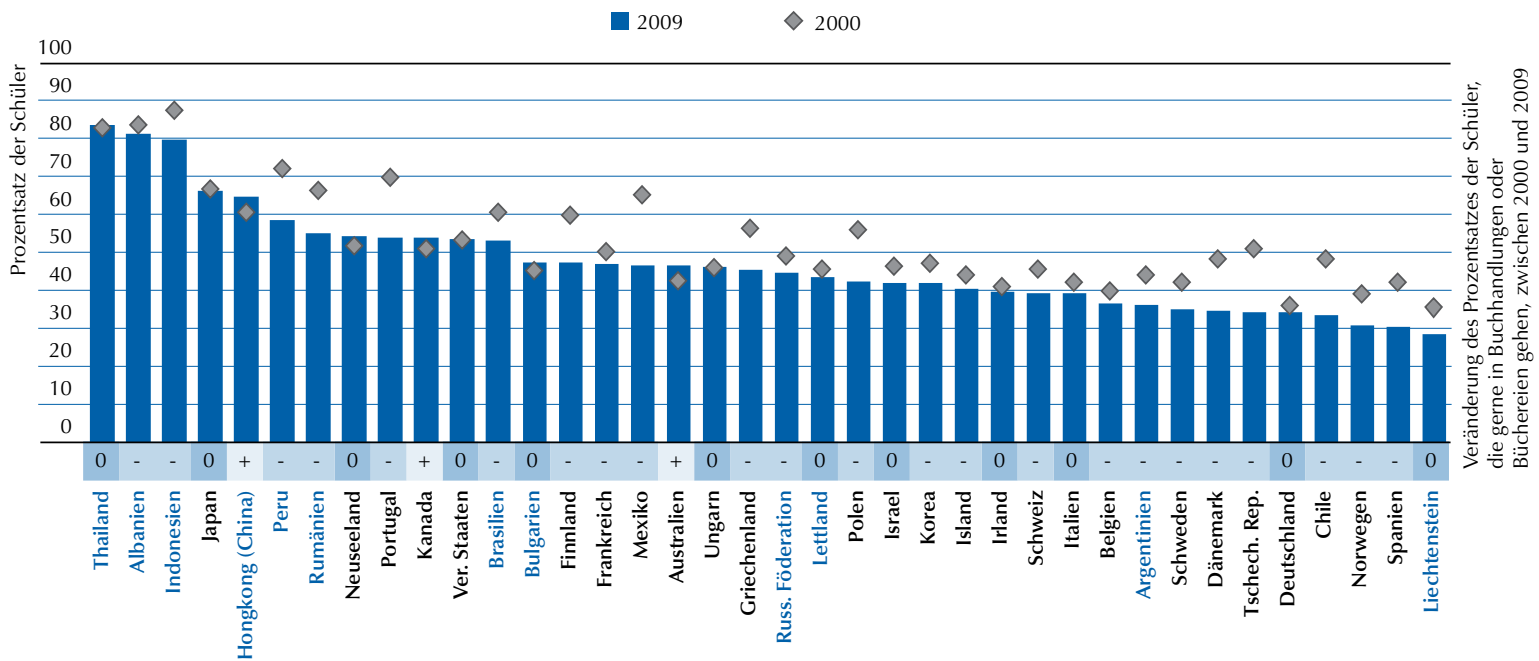

Die Länder sind in absteigender Reihenfolge nach dem Prozentsatz der Schüler für die jeweiligen Items im Jahr 2009 angeordnet. Quelle: OECD, PISA-2009-Datenbank, Tabelle V.5.3.

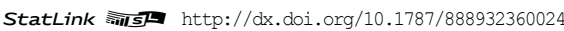

und Korea sowie in den Partnerländern und -volkswirtschaften Thailand, Hongkong (China), Albanien und Brasilien zugenommen. In 15 Ländern blieb die Freude am Lesen auf dem gleichen Niveau, während sie in 14 anderen Ländern abgenommen hat, wobei die Tschechische Republik, Mexiko und Finnland den stärksten Rückgang verzeichnet haben (um mindestens ein Fünftel der Standardabweichung dieses Indizes).

Die Unterschiede zwischen Jungen und Mädchen in Bezug auf die Zunahme bzw. Abnahme der Freude am Lesen zwischen 2000 und 2009 decken sich mehr oder minder mit den Entwicklungen, die bei Jungen und Mädchen im gleichen Zeitraum hinsichtlich des Lesens zum Vergnügen zu beobachten waren. Die Jungen bekundeten in PISA 2009 im Durchschnitt nicht nur weniger Freude am Lesen als die Mädchen, sondern der diesbezügliche Abstand 


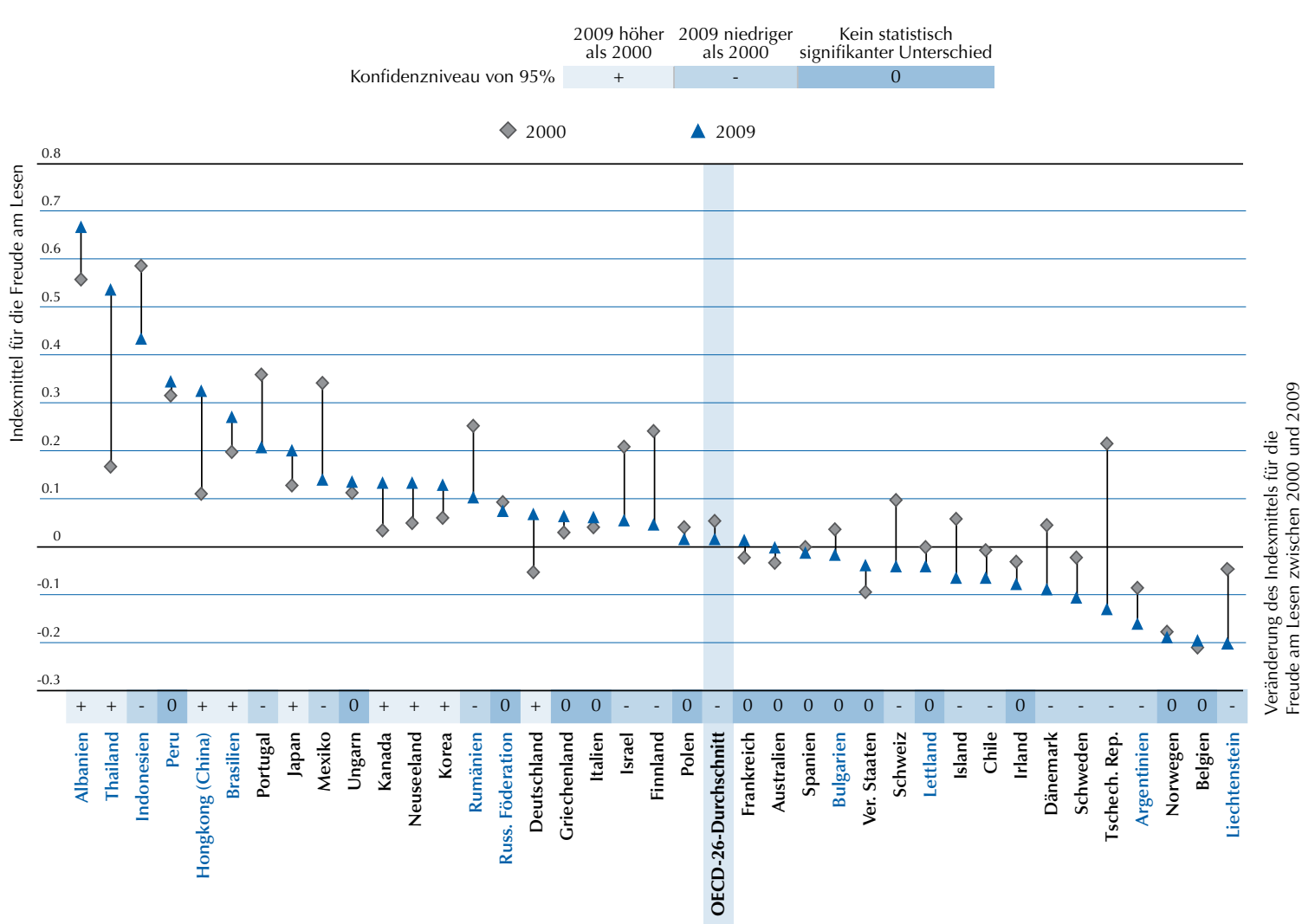

Länder sind in absteigender Reihenfolge nach dem Indexmittel für die Freude am Lesen im Jahr 2009 angeordnet. Quelle: OECD, PISA-2009-Datenbank, Tabelle V.5.2.

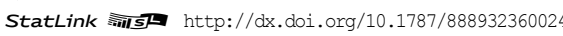

zwischen den Geschlechtern weitete sich auch aus: Die Jungen haben immer weniger Freude am Lesen, während der Rückgang bei den Mädchen weniger ausgeprägt ist. Und in manchen Ländern hat die Freude am Lesen bei den Mädchen 2009 im Vergleich zum Jahr 2000 zugenommen (Tabelle V.5.2). In den meisten Ländern ist die Freude am Lesen unter den Jungen zwar stärker zurückgegangen als unter den Mädchen, in Irland bekundeten die Jungen im Jahr 2009 jedoch genauso viel Freude am Lesen wie im Jahr 2000, während die Freude der Mädchen am Lesen im gleichen Zeitraum zurückgegangen ist. In der Tschechischen Republik, Finnland und Dänemark zeigten sich sowohl die Mädchen als auch die Jungen weniger am Lesen interessiert, der Rückgang war bei den Mädchen jedoch stärker als bei den Jungen (Abb. V.5.5). In diesen Ländern begeistern sich die Mädchen allerdings weiterhin mehr für das Lesen als die Jungen, obwohl die diesbezügliche Differenz zwischen den Geschlechtern zwischen 2000 und 2009 geschrumpft ist.

In zahlreichen Ländern war der Rückgang der Freude am Lesen unter den Jungen wesentlich deutlicher als unter den Mädchen, was zu einer Ausweitung der Unterschiede geführt hat. In Polen und dem Partnerland Albanien hat sich der Unterschied zwischen Jungen und Mädchen in Bezug auf die Freude am Lesen am stärksten vergrößert. In Albanien ist die Freude der Mädchen am Lesen zwischen 2000 und 2009 gestiegen, während die Jungen im Durchschnitt 2009 genauso viel Freude am Lesen bekundeten wie im Jahr 2000. In Polen nahm die Freude der Jungen am Lesen ab, während die der Mädchen zunahm.

Die Trends hinsichtlich der Zahl der Schüler, die laut eigenen Angaben zum Vergnügen lesen, und dem Niveau der Freude am Lesen stehen in engem Zusammenhang. In Ländern, in denen die Schülerinnen und Schüler öfter angeben, dass sie zum Vergnügen lesen, geben sie auch öfter an, Freude am Lesen zu haben. Da beide Aspekte in PISA 2009 auch auf der Ebene der einzelnen Schüler verbunden sind (vgl. Band III, Learning to Learn), zeigt dies, dass Schülerinnen und Schüler, die zum Vergnügen lesen, in der Regel Freude am Lesen haben, und dass ein Anstieg des Lesens zum Vergnügen auch mit einem allgemeinen Anstieg des Grads der Freude am Lesen einhergeht. 


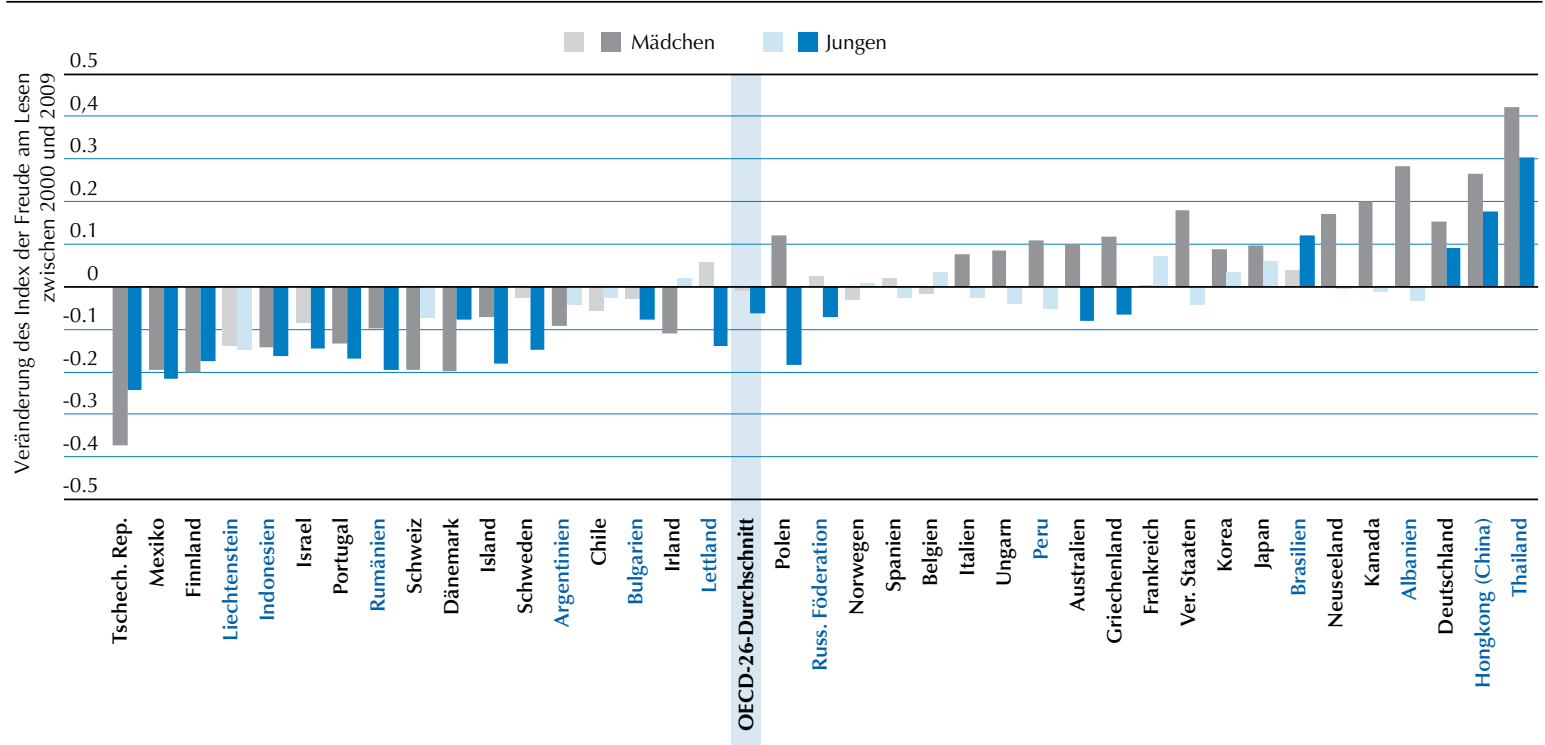

Anmerkung: Statistisch signifikante Punktzahlveränderungen sind durch einen dunkleren Farbton gekennzeichnet.

Die Länder sind in absteigender Reihenfolge nach der Höhe der Veränderung des Indexmittels für die Freude am Lesen für alle Schüler zwischen 2000 und 2009 angeordnet. Quelle: OECD, PISA-2009-Datenbank, Tabelle V.5.2.

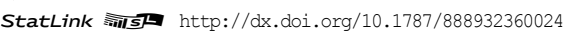

Abbildung V.5.6 zeigt den Zusammenhang zwischen den Trends bezüglich des Lesens zum Vergnügen und des Grads der Freude am Lesen. In den Ländern im rechten Teil der Abbildung gaben 2009 mehr Schülerinnen und Schüler an, zum Vergnügen zu lesen, als im Jahr 2000, während in den Ländern im oberen Teil der Mittelwert der Freude am Lesen zugenommen hat. Die hinsichtlich des Lesens zum Vergnügen und des Grads der Freude am Lesen beobachteten Trends sind eindeutig ähnlich. In Kanada, Japan und der Partnervolkswirtschaft Hongkong (China) sind sowohl der Prozentsatz der Schülerinnen und Schüler, die zum Vergnügen lesen, als auch der Grad der Lesefreude zwischen 2000 und 2009 im Durchschnitt gestiegen. In 12 Ländern ist der Prozentsatz der Schülerinnen und Schüler, die zum Vergnügen lesen, zurückgegangen, und in diesen Ländern war unter den Schülerinnen und Schülern auch ein Rückgang des Grads der Freude am Lesen zu beobachten. Lediglich in zwei Ländern, Korea und Brasilien, ging der Prozentsatz der Schülerinnen und Schüler, die zum Vergnügen lesen, zurück, während der Mittelwert der Freude am Lesen zunahm.

\section{Veränderungen bei den Inhalten, die Schülerinnen und Schüler zum Vergnügen lesen}

Band III, Learning to Learn, untersucht die Lesegewohnheiten der Schülerinnen und Schüler in den einzelnen Ländern sowie unterschiedlicher Schülergruppen innerhalb der einzelnen Länder. Im Rahmen von PISA wurden die Schülerinnen und Schüler gebeten, anzugeben, wie oft sie die folgenden Arten von Texten zum Vergnügen lesen: Zeitschriften, Comic-Hefte/Comics, Romane/Erzählungen/Geschichten, Sachbücher und Tageszeitungen. Die Häufigkeitskategorien reichten von "nie oder fast nie“ bis "mehrmals in der Woche”. Die in den Abbildungen V.5.7 und V.5.8 sowie in den Tabellen V.5.6, V.5.7, V.5.8 und V.5.9 vorgestellten Ergebnisse konzentrieren sich auf die Veränderungen der Zahl der Schüler, die die verschiedenen Arten von Texten regelmäßig lesen, also mehrmals im Monat oder in der Woche. Die in diesem Band veröffentlichten Ergebnisse zu den Veränderungen bei den von den Schülern zum Vergnügen gelesenen Arten von Texten zwischen 2000 und 2009 sollten im Licht einer etwaigen Zunahme der Zeit, die die Schüler mit Online-Leseaktivitäten zum Vergnügen verbringen, gesehen werden. Da die zur Messung der Online-Leseaktivitäten 2000 und 2009 eingesetzten Methoden nicht vergleichbar sind, können etwaige Substitutionseffekte zwischen dem Lesen gedruckter oder digitaler Texte hier nicht untersucht werden.

In Band III, Kapitel 1, wird ein besonders starker und positiver Zusammenhang zwischen den Leistungen im Bereich Lesekompetenz und dem regelmäßigen Lesen von Romanen, Erzählungen und Geschichten festgestellt, dem ein negativer Zusammenhang zwischen den Leseleistungen und dem regelmäßigen Lesen von Comic-Heften gegenübersteht. Im Durchschnitt der OECD-Länder ist der Anteil der Schülerinnen und Schüler, die angaben, 
Veränderung des Index der Freude am Lesen und des Prozentsatzes der Schüler, die zum Vergnügen lesen, zwischen 2000 und 2009

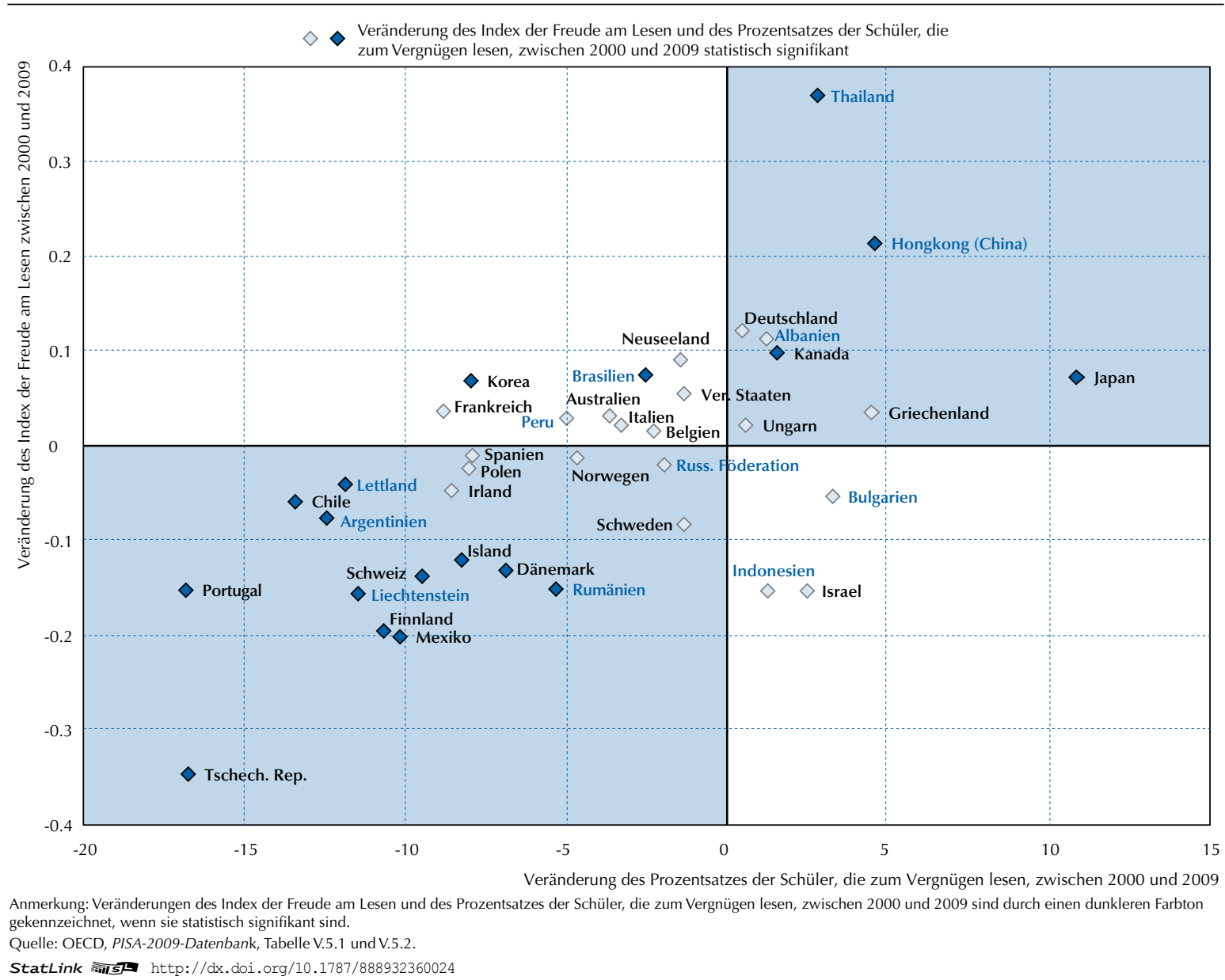

regelmäßig fiktionale Literatur zu lesen, zwischen 2000 und 2009 um 3 Prozentpunkte gestiegen. Dieser im OECDDurchschnitt beobachtete Anstieg spiegelt sich in vielen Ländern wider. Nur in drei Ländern ist das Lesen von Romanen, Erzählungen und Geschichten zurückgegangen, während es in 19 anderen Ländern stieg und in den übrigen 16 Ländern konstant blieb. Im Partnerland Indonesien war der Anstieg des Lesens fiktionaler Texte besonders ausgeprägt: Während dort im Jahr 2000 nur 37\% der Schülerinnen und Schüler angaben, regelmäßig solche Texte zu lesen, war dies im Jahr 2009 für fast 60\% der Schüler der Fall - ein Anstieg um 23\%. In Japan, Korea, Kanada und den Partnerländern und -volkswirtschaften Indonesien, Thailand, Peru und Hongkong (China) ist der Anteil der Schülerinnen und Schüler, die angaben, fiktionale Literatur zu lesen, zwischen 2000 und 2009 um über 10 Prozentpunkte gestiegen. Während in den meisten dieser Länder im Jahr 2000 nur etwa ein Drittel der Schülerinnen und Schüler angab, regelmäßig Romane, Erzählungen und Geschichten zu lesen, traf dies neun Jahre später auf über vier von zehn Schülerinnen und Schülern zu (vgl. Abb. V.5.7 und Tabelle V.5.6).

Band III identifiziert erhebliche geschlechterspezifische Unterschiede beim Anteil der Jungen und der Mädchen, die Romane, Erzählungen und Geschichten lesen: Im Jahr 2009 bestand bei den Mädchen im Durchschnitt der OECDLänder eine fast doppelt so hohe Wahrscheinlichkeit, dass sie angaben, solche Texte zu lesen. 2009 lasen mehr Jungen und Mädchen Romane, Erzählungen und Geschichten als im Jahr 2000. Im Durchschnitt der OECD-Länder ist der Prozentsatz der Jungen, die laut eigenen Angaben fiktionale Texte lesen, um fast 3 Prozentpunkte gestiegen, und unter den Mädchen war eine Zunahme um fast 4 Prozentpunkte festzustellen. In den meisten Ländern war der Anstieg bei den Mädchen höher als bei den Jungen. Eine bemerkenswerte Ausnahme bildet das Partnerland Russische Föderation, wo der Prozentsatz der Jungen, die Romane, Erzählungen und Geschichten lesen, um fast 8 Prozentpunkte gestiegen ist, während er bei den Mädchen nur um 3 Prozentpunkte zugenommen hat; eine 


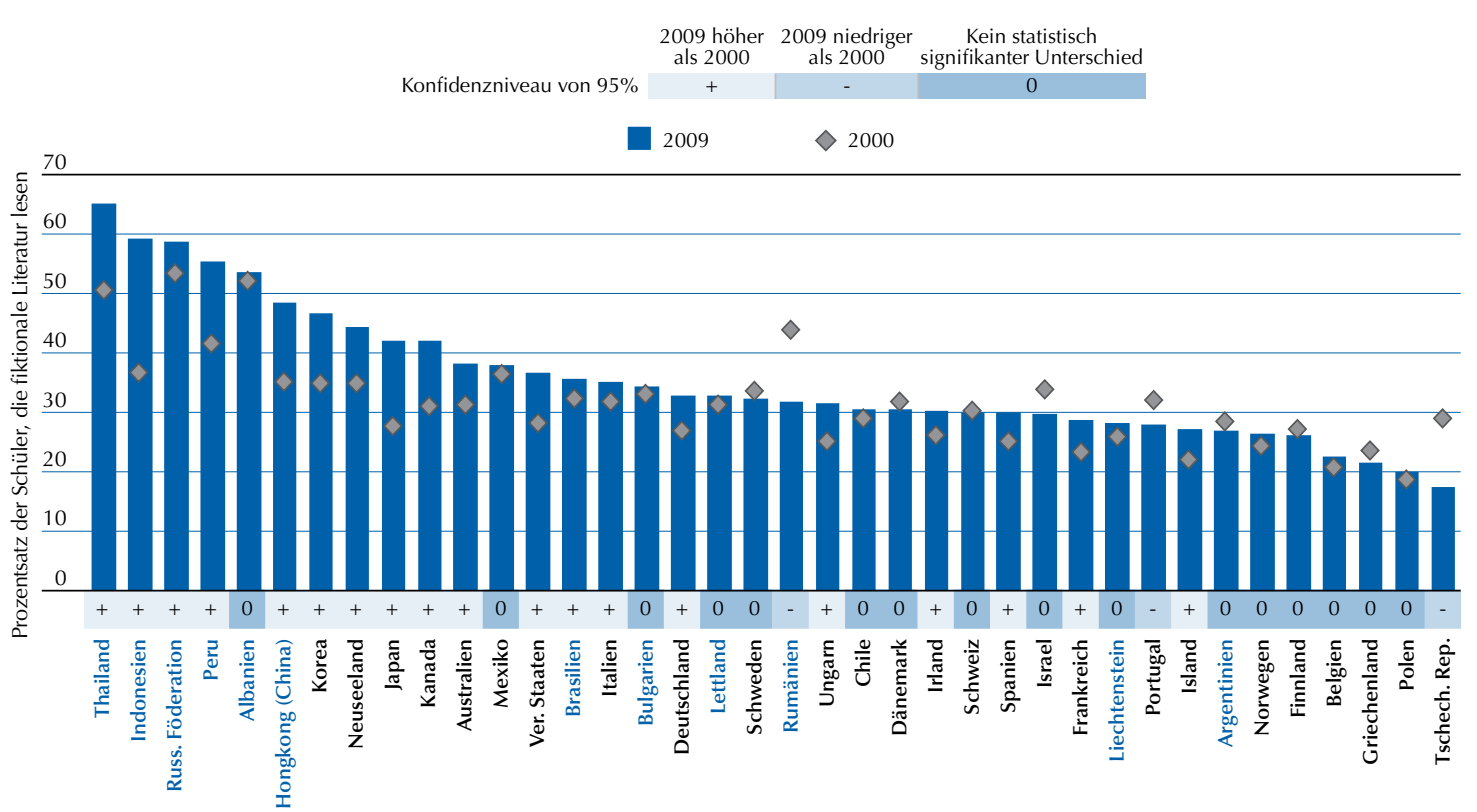

Die Länder sind in absteigender Reihenfolge nach dem Prozentsatz der Schüler, die fiktionale Literatur zum Vergnügen lesen, im Jahr 2009 angeordnet Quelle: OECD, PISA-2009-Datenbank, Tabelle V.5.6.

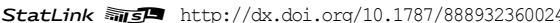

Ausnahme ist auch Norwegen, wo der Anteil der Jungen um 4 Prozentpunkte stieg, während der der Mädchen stabil blieb. In der Schweiz hat sich der erhebliche Abstand zwischen dem Prozentsatz der Jungen und der Mädchen, die fiktionale Texte lesen, zwischen 2000 und 2009 erheblich reduziert. Im Jahr 2009 gab fast einer von fünf Jungen an, regelmäßig fiktionale Literatur zu lesen, was einem Anstieg um über 4 Prozentpunkte im Vergleich zum Jahr 2000 entspricht, während dies 2009 für zwei von fünf Mädchen der Fall war, womit bei den Mädchen ein Rückgang um 4 Prozentpunkte gegenüber dem Jahr 2000 zu verzeichnen war. Die Tschechische Republik ist eines der Länder mit dem niedrigsten Anteil an Jungen und Mädchen, die angaben, regelmäßig Romane, Erzählungen und Geschichten zu lesen, und dort war der Rückgang in dieser Kategorie bei den Mädchen besonders deutlich. Im Jahr 2000 gaben fast 45\% der Mädchen an, regelmäßig fiktionale Texte zu lesen, im Jahr 2009 hingegen sank dieser Prozentsatz um 16 Prozentpunkte auf 29\% (Tabelle V.5.7).

Bei den Schülerinnen und Schülern war ein Rückgang des Interesses am regelmäßigen Lesen von Comic-Heften zu beobachten. Im Durchschnitt ist der Prozentsatz der Schülerinnen und Schüler, die angaben, regelmäßig ComicHefte zu lesen, in den OECD-Ländern zwischen 2000 und 2009 um fast 7 Prozentpunkte zurückgegangen. Der Anteil der Schüler, die laut eigenen Angaben regelmäßig Comic-Hefte lesen, sank in 19 Ländern, stieg in acht Ländern und blieb in den übrigen elf Ländern, die sowohl an PISA 2000 als auch an PISA 2009 teilnahmen, stabil. Im Allgemeinen gehören die Länder mit dem größten Anteil an Schülern, die regelmäßig Comic-Hefte lesen, zu den Ländern, in denen der stärkste Rückgang des Interesses der Schüler an Comic-Heften festzustellen war. Die einzige Ausnahme bildet Thailand, wo der Prozentsatz dieser Schülerinnen und Schüler um fast 12 Prozentpunkte gestiegen ist: Waren es im Jahr 2000 noch sechs von zehn Schülerinnen und Schülern, so waren es neun Jahre später sieben von zehn. In Dänemark ging die Zahl der Comic-Leser besonders stark zurück: Während dort im Jahr 2000 fast drei von fünf Schülern regelmäßig Comic-Hefte lasen, war dies neun Jahre später nur für einen von fünf der Fall - ein Rückgang um über 40 Prozentpunkte. Der Rückgang des Prozentsatzes der Schülerinnen und Schüler, die angaben, regelmäßig Comic-Hefte zu lesen, liegt auch in Korea, Island und Norwegen bei über 15 Prozentpunkten - alles Länder, in denen relativ viele Schülerinnen und Schüler im Jahr 2000 regelmäßig Comic-Hefte lasen (vgl. Abb. V.5.8 und Tabelle V.5.6).

In fast allen Ländern, die sowohl an PISA 2000 als auch an PISA 2009 teilgenommen haben, gab 2009 ein niedrigerer Prozentsatz an Schülerinnen und Schülern an, Tageszeitungen und Zeitschriften zu lesen, als im Jahr 2000 . Im 


\section{Prozentsatz der Schüler, die Comics lesen, 2000 und 2009}

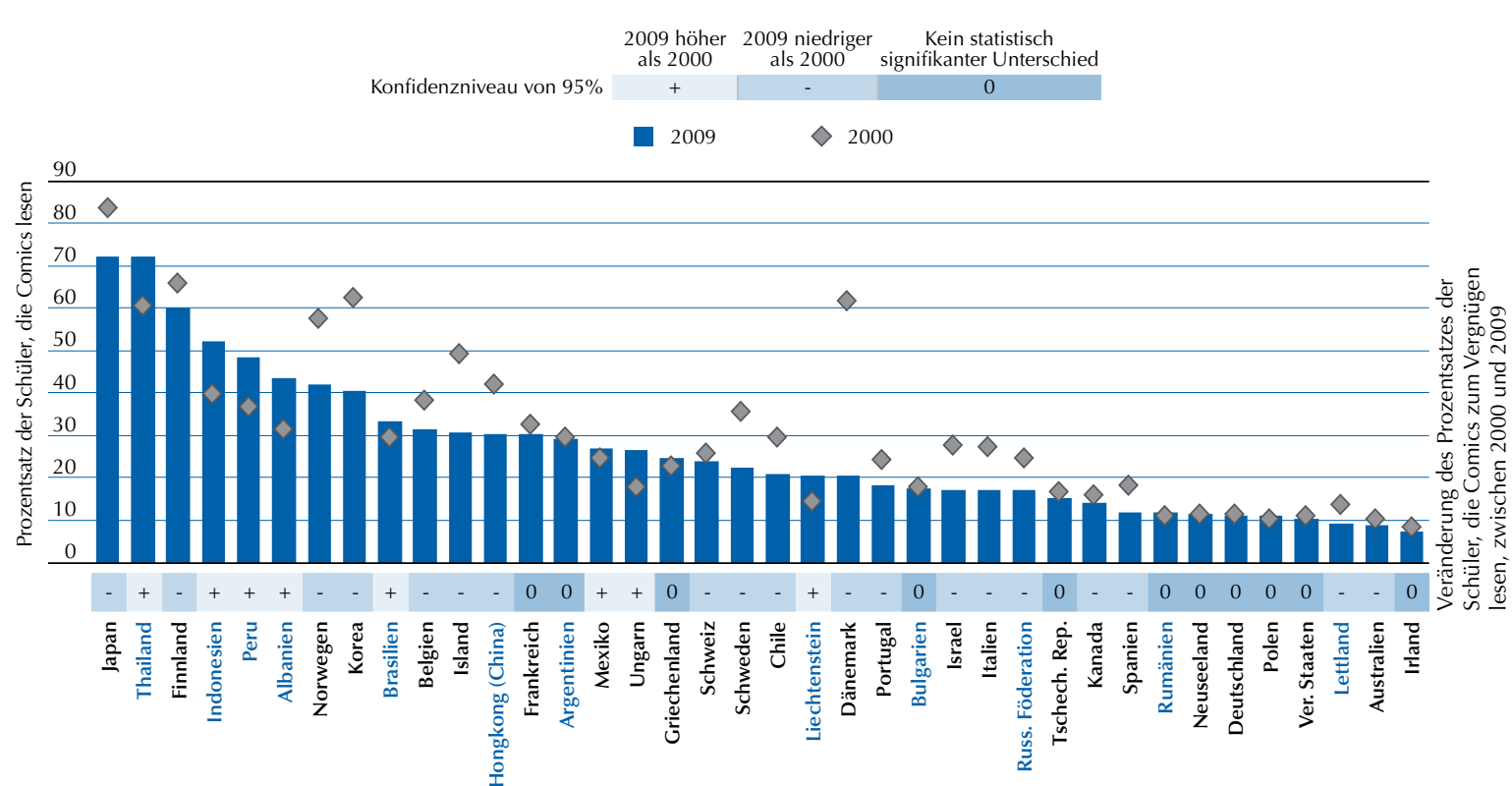

Die Länder sind in absteigender Reihenfolge nach dem Prozentsatz der Schüler, die Comics zum Vergnügen lesen, im Jahr 2009 angeordnet.

Quelle: OECD, PISA-2009-Datenbank, Tabelle V.5.6.

StatLink *inाs http://dx.doi.org/10.1787/888932360024

Durchschnitt der OECD-Länder ist die Zahl der Schülerinnen und Schüler, die angaben, Zeitschriften zu lesen, um 10 Prozentpunkte gesunken, und bis auf neun Länder verzeichneten alle einen Rückgang dieser Leseaktivität. Thailand, Peru und Indonesien sind die einzigen Länder mit einem Zuwachs des Prozentsatzes der Schülerinnen und Schüler, die angaben, regelmäßig Zeitschriften zu lesen, und alle drei gehören zu den Ländern, in denen weniger als einer von zwei Schülern im Jahr 2000 angegeben hatte, regelmäßig Zeitschriften zu lesen. Auch der Rückgang des Prozentsatzes der Schüler, die angaben, zum Vergnügen Zeitungen zu lesen, war im Durchschnitt der OECD-Länder mit 5 Prozentpunkten relativ ausgeprägt. Der Prozentsatz der Schülerinnen und Schüler, die angaben, regelmäßig Zeitungen zu lesen, ist in sieben Ländern gestiegen, in zehn Ländern stabil geblieben und in 21 Ländern zurückgegangen (Tabelle V.5.6). Der Prozentsatz der Jungen und der Mädchen, die angaben, Zeitungen zu lesen, ging im gleichen Umfang zurück, um fast 5 Prozentpunkte (Tabelle V.5.7).

Band III zeigt, dass vielseitige Leser - mit anderen Worten, Schülerinnen und Schüler, die sich nicht auf eine Lektüreart beschränken - kompetentere Leser sind als diejenigen, die nur bestimmte Arten von Texten lesen. Tabelle V.5.10 untersucht die Veränderungen bei der Vielfalt der gelesenen Texte zwischen 2000 und 2009. In sieben Ländern wurden die Schülerinnen und Schüler zu vergleichsweise vielseitigeren Lesern, in acht war keine Veränderung zu beobachten, und in 23 Ländern wurde die Lektüre der Jugendlichen weniger vielseitig. Die Vielseitigkeit der Lektüre stieg in Ungarn, der Schweiz, Polen und den Partnerländern Indonesien, Peru, Thailand und Albanien. Der Rückgang war in Dänemark am stärksten, und in Italien, Korea, Island, Schweden und den Partnerländern Russische Föderation und Bulgarien betrug er fast ein Drittel einer Standardabweichung auf dem entsprechenden Index.

In fast allen Ländern sind die Mädchen vielseitigere Leser als die Jungen, und diese Lücke hat sich vergrößert, da die Jungen im Zeitverlauf weniger vielseitige Leser geworden sind. Allerdings unterscheiden sich die Länder hinsichtlich der Entwicklung der Lesevielseitigkeit seit dem Jahr 2000. In Indonesien beispielsweise ist dieVielseitigkeit sowohl bei den Jungen als auch bei den Mädchen gleichermaßen gestiegen, und zwar fast um eine halbe Standardabweichung, während in anderen Ländern, in denen die Vielseitigkeit der Leseaktivitäten ebenfalls zugenommen hat, die Mädchen noch vielseitigere Leserinnen wurden. In keinem Land nahm die Lesevielseitigkeit bei den Jungen zu, während sie bei den Mädchen gleichblieb oder sank. In zahlreichen Ländern nahm die Lesevielseitigkeit bei den Jungen und den Mädchen gleichermaßen ab, während sie in Island, Schweden, Korea, Israel und Rumänien unter den Jungen stärker zurückging als unter den Mädchen, wodurch sich die Lücke weiter vergrößerte. 


\section{Veränderungen des Leseengagements von in sozioökonomischer Hinsicht benachteiligten Schülerinnen und Schülern}

Es wird oft behauptet, dass die Schüler immer weniger lesen, vor allem die Jungen. Daten aus PISA bestätigen, dass die Jungen nicht nur weniger lesen als die Mädchen, sondern dass der Prozentsatz der Schülerinnen und Schüler, die zum Vergnügen lesen, in vielen Ländern auch zurückgegangen ist, besonders unter den Jungen. Außerdem herrscht Besorgnis darüber, dass dieser negative Trend unter den in sozioökonomischer Hinsicht benachteiligten Schülerinnen und Schülern deutlicher ausgeprägt sein könnte. Daten aus PISA 2009 bestätigen dies, da sie darauf schließen lassen, dass Schülerinnen und Schüler mit ungünstigerem sozioökonomischem Hintergrund effektiv weniger lesen als andere (vgl. Band III). Dieser Abschnitt untersucht verschiedene Trends, um festzustellen, wie sich das Leseengagement von Jungen und Mädchen je nach sozioökonomischem Hintergrund verändert hat.

Tabelle V.5.4 und Abbildung V.5.10 zeigen die Veränderungen des Prozentsatzes der Jungen und der Mädchen mit unterschiedlichem sozioökonomischem Hintergrund, die zum Vergnügen lesen. Tabelle V.5.5 enthält ähnliche Daten bezüglich der Veränderungen der Freude am Lesen zwischen den Erhebungen der Jahre 2000 und 2009. Die Mehrzahl der Schülerinnen und Schüler, die zum Vergnügen lesen, ist sozioökonomisch besser gestellt (vgl. Abb. V.5.9)1. Diese Schülerinnen und Schüler lesen nicht nur mit höherer Wahrscheinlichkeit zum Vergnügen als die sozioökonomisch weniger gut gestellten Jugendlichen, sie haben auch mehr Freude daran. Dies gilt sowohl für 2000 als auch für 2009.

Sozioökonomisch benachteiligte Schülerinnen und Schüler gaben im Jahr 2009 öfter an, nicht gerne zu lesen, als dies im Jahr 2000 der Fall war (vgl. Abb. V.5.9 und Tabelle V.5.4). Im Durchschnitt der OECD-Länder sank der Anteil der sozioökonomisch benachteiligten Schüler, die zum Vergnügen lesen, um 9 Prozentpunkte, während der entsprechende Anteil unter den Schülerinnen und Schülern mit privilegierterem Hintergrund um 3 Prozentpunkte gesunken ist. Der Abstand zwischen den zwei Gruppen weitete sich von 10 Prozentpunkten im Jahr 2000 auf 16 Prozentpunkte im Jahr 2009 aus. Ebenso war der Grad der Freude am Lesen bei den sozioökonomisch benachteiligten Schülerinnen und Schülern laut eigenen Angaben im Durchschnitt niedriger als bei den besser gestellten Schülerinnen und Schülern. In PISA 2000 lag der Unterschied bei 0,33 einer Standardabweichung, in PISA 2009 stieg er auf 0,46 (Tabelle V.5.5).

Die relativen Veränderungen hinsichtlich der Angaben der Schülerinnen und Schüler dazu, ob sie zum Vergnügen lesen und wie viel Freude sie am Lesen haben, variieren je nach sozioökonomischem Hintergrund stark von Land zu Land (vgl. Tabelle V.5.4 und V.5.5). Im Partnerland Hongkong (China) ist der Prozentsatz der Schülerinnen und Schüler, die zum Vergnügen lesen, beispielsweise unter den benachteiligten Schülerinnen und Schülern gestiegen, während er bei den privilegierten Schülerinnen und Schülern unverändert blieb. In Kanada stieg dieser Prozentsatz nur unter den Schülerinnen und Schülern mit günstigem sozioökonomischem Hintergrund. In Irland hingegen blieb der

Abbildung V.5.9

Prozentsatz der Schüler, die zum Vergnügen lesen, 2000 und 2009, nach sozioökonomischem Hintergrund

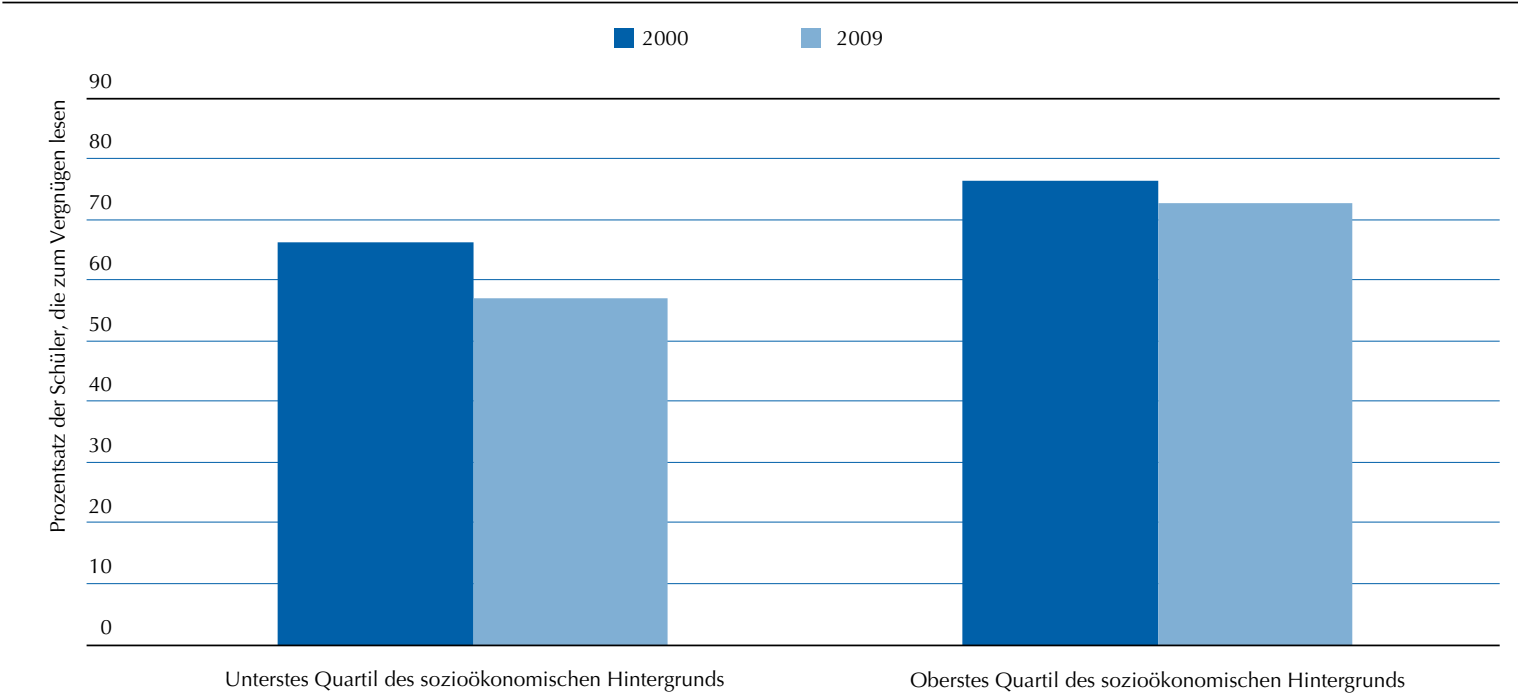

Quelle: OECD, PISA-2009-Datenbank, Tabelle V.5.4

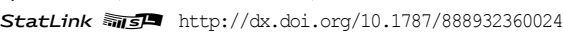


Veränderung des Prozentsatzes der Jungen und Mädchen, die zum Vergnügen lesen, zwischen 2000 und 2009, nach sozioökonomischem Hintergrund

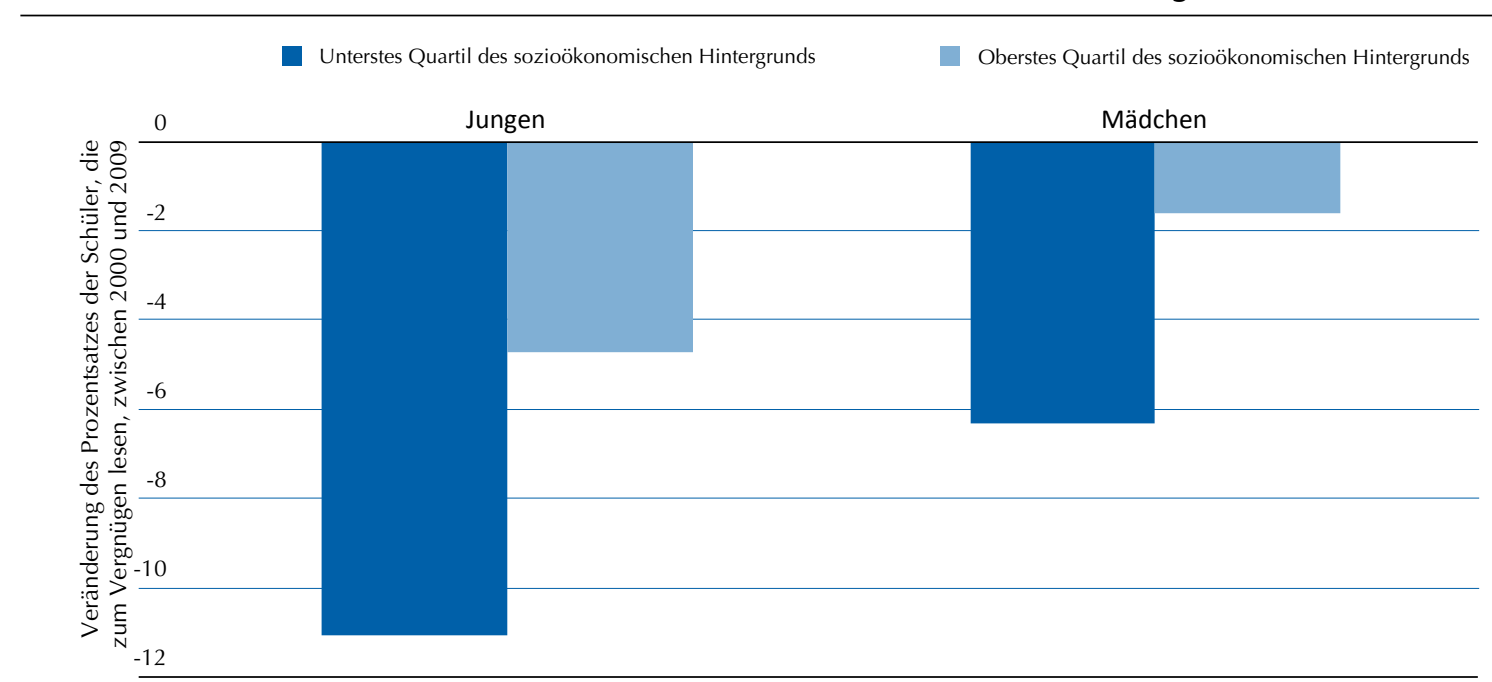

Quelle: OECD, PISA-2009-Datenbank, Tabelle V.5.4.

StatLink 部西 http://dx.doi.org/10.1787/888932360024

Prozentsatz der Schülerinnen und Schüler, die zum Vergnügen lesen, unter den Schülern mit privilegiertem Hintergrund unverändert, während er bei den sozioökonomisch benachteiligten Schülerinnen und Schülern um fast 18 Prozentpunkte zurückging. In Finnland sank die Zahl der Schülerinnen und Schüler mit sozioökonomisch ungünstigem Hintergrund, die angaben, zum Vergnügen zu lesen, zwischen 2000 und 2009 um 17 Prozentpunkte. Doch anders als in Irland nahm in Finnland auch der Prozentsatz der sozioökonomisch privilegierten Schülerinnen und Schüler ab, die angaben, zum Vergnügen zu lesen, und zwar um 6 Prozentpunkte.

Abbildung V.5.10 (vgl. auch Tabelle V.5.1 und V.5.4) stellt den Prozentsatz der Jungen und der Mädchen mit sozioökonomisch benachteiligtem und privilegiertem Hintergrund gegenüber, die in PISA 2000 und 2009 angaben, zum Vergnügen zu lesen. Die Abbildung zeigt, dass dieser Prozentsatz unter den Jungen unabhängig von ihrem sozioökonomischen Hintergrund stärker abgenommen hat (um 6 Prozentpunkte) als unter den Mädchen (um 3 Prozentpunkte). Der Prozentsatz der Mädchen mit benachteiligtem Hintergrund, die zum Vergnügen lesen, ist um 6 Prozentpunkte zurückgegangen, während der entsprechende Anteil unter den Jungen mit vergleichbarem Hintergrund um 11 Prozentpunkte gesunken ist. Unter den Jungen mit privilegiertem Hintergrund ging der Prozentsatz derjenigen, die zum Vergnügen lesen, um 5 Prozentpunkte zurück, während sich der Rückgang bei den Mädchen aus der gleichen Gruppe auf weniger als 2 Prozentpunkte belief. Sozioökonomisch benachteiligte Jungen sind außerdem die Schülergruppe, die am wenigsten zum Vergnügen liest und in der das Lesen zum Vergnügen zugleich am meisten zurückgegangen ist.

\section{Veränderungen der Leistungen im Bereich Lesekompetenz der Schülerinnen und Schüler, die Romane und Erzählungen lesen}

In Band III werden die erheblichen Kompetenzunterschiede aufgezeigt, die zwischen Schülerinnen und Schülern, die regelmäßig Romane, Erzählungen oder Geschichten lesen, und anderen Schülern festzustellen sind. In Tabelle V.5.8 sind die Veränderungen der Leistungen im Bereich Lesekompetenz von Schülerinnen und Schülern, die angaben, fiktionale Literatur zu lesen, zwischen 2000 und 2009 dargestellt. In 11 Ländern nahmen die Leistungen dieser Schülerinnen und Schüler im Bereich Lesekompetenz zu, in drei Ländern gingen sie zurück, und in 24 Ländern blieben sie unverändert. In 15 Ländern vergrößerte sich der mit dem Lesen von fiktionalen Texten verbundene Leistungsvorsprung, wobei die Veränderung in Frankreich, der Tschechischen Republik, Italien, Polen, Griechenland, Portugal und der Schweiz mindestens 20 Punkte betrug (Tabelle V.5.8).

Bei den Mädchen stieg der an das Lesen von fiktionaler Literatur geknüpfte Leistungsvorsprung zwischen 2000 und 2009 in 12 Ländern, blieb in 22 Ländern stabil und ging in vier Ländern zurück: in Korea, Mexiko und den Partnerländern Lettland und Argentinien (vgl. Tabelle V.5.9). Bei den Jungen erhöhte sich der mit dem Lesen von 
Romanen, Erzählungen und Geschichten verbundene Leistungsvorsprung in elf Ländern; in Mexiko nahm er ab, und in den anderen Ländern blieb er unverändert. Eine Zunahme des Leistungsvorsprungs um mindestens 25 Punkte war bei den Mädchen in der Tschechischen Republik und in Frankreich und bei den Jungen in Frankreich, Polen, Belgien, Italien und dem Partnerland Albanien festzustellen.

\section{TRENDS HINSICHTLICH DER SCHÜLEREINSTELLUNGEN ZU SCHULE UND LEHRERN}

\section{Veränderungen der Lehrer-Schüler-Beziehungen}

Positive Lehrer-Schüler-Beziehungen sind entscheidend für die Herstellung eines dem Lernen förderlichen Umfelds. Forschungsergebnisse zeigen, dass Schülerinnen und Schüler, insbesondere solche mit ungünstigem sozioökonomischem Hintergrund, mehr lernen und weniger Disziplinprobleme aufweisen, wenn sie den Eindruck gewinnen, dass ihre Lehrer sie ernst nehmen (Gamoran, 1993), und wenn sie eine starke Verbindung zu ihren Lehrern fühlen (Crosnoe et al., 2004). Eine Erklärung hierfür ist, dass positive Lehrer-Schüler-Beziehungen zur Weitergabe von sozialem Kapital beitragen, ein gemeinschaftliches Lernumfeld schaffen und die Einhaltung von lernförderlichen Normen unterstützen und stärken (Birch und Ladd, 1998).

Im Rahmen von PISA 2009 wurden die Schülerinnen und Schüler gebeten, ihre Meinung zu mehreren Aussagen hinsichtlich ihrer Beziehungen zu ihren Lehrern abzugeben. Zu diesen Aussagen gehörte, ob sie mit den meisten ihrer Lehrerinnen/Lehrer gut auskommen, ob es den meisten ihrer Lehrerinnen/Lehrer wichtig ist, dass sie sich wohlfühlen, ob die Lehrerinnen/Lehrer sich für das interessieren, was sie zu sagen haben, ob sie bei Bedarf zusätzliche Hilfe von den Lehrern bekommen und ob die Lehrerinnen/Lehrer sie fair behandeln. Die gleichen Fragen waren auch im Jahr 2000 gestellt worden, so dass die Lehrer-Schüler-Beziehungen im Zeitverlauf verglichen werden können.

Die Ergebnisse von PISA 2009 deuten darauf hin, dass die Schülerinnen und Schüler im OECD-Raum im Allgemeinen mit der Qualität der Lehrer-Schüler-Beziehungen zufrieden sind (vgl. Kapitel 2 in Band IV, Was macht eine Schule erfolgreich?). Der Unterschied zwischen den Antworten der Jahre 2000 und 2009 lässt darauf schließen, dass sich die Qualität der Lehrer-Schüler-Beziehungen in dieser Zeitspanne effektiv verbessert hat (Abb. V.5.11 und Tabelle V.5.11). Beispielsweise stimmten im Jahr 2000 in den 26 OECD-Ländern mit vergleichbaren Daten $74 \%$ der Schülerinnen und Schüler den Aussagen "wenn ich zusätzlich Hilfe brauche, erhalte ich sie von meinen Lehrerinnen/Lehrern" oder "die meisten meiner Lehrerinnen/Lehrer behandeln mich fair" eher oder ganz zu, während dies in PISA 2009 für 79\% der Schülerinnen und Schüler der Fall war, was einem Anstieg um 5 Prozentpunkte entspricht. Im Jahr 2000 stimmten 65\% der Schülerinnen und Schüler der Aussage "die meisten Lehrerinnen/Lehrer interessieren sich für das, was ich zu sagen habe“ eher oder ganz zu; bis zum Jahr 2009 hatte sich dieser Anteil auf fast 68\% erhöht, ein Zuwachs um 3 Prozentpunkte.

Der Anstieg des Anteils der Schülerinnen und Schüler, die angaben, dass ihre Lehrerinnen/Lehrer sich für das interessieren, was sie zu sagen haben, lag in Deutschland, Korea, Japan, Island und dem Partnerland Albanien bei über 10 Prozentpunkten. Der Anteil der Schüler, die bestätigten, dass sie bei Bedarf zusätzliche Hilfe von ihren Lehrerinnen/Lehrern erhalten, ist in vielen Ländern ebenfalls gestiegen. Dieser Anstieg war in Polen, Portugal, Deutschland und den Partnerländern Albanien und Lettland besonders deutlich, wo er sich auf über 10 Prozentpunkte belief. Die Länder, in denen die stärkste Zunahme des Anteils der Schülerinnen und Schüler verzeichnet wurde, die darauf vertrauen, dass sie bei Bedarf Hilfe von ihren Lehrerinnen/Lehrern erhalten, sind meist diejenigen, in denen der Anteil der Schülerinnen und Schüler, die großes Vertrauen in die Lehrerschaft geäußert hatten, im Jahr 2000 am geringsten war. Der Abstand zwischen den Ländern bezüglich des Prozentsatzes der Schülerinnen und Schüler, die sich von ihren Lehrerinnen und Lehrern "fair behandelt" fühlten, ist ebenfalls deutlich geschrumpft, da die Länder mit dem höchsten Zuwachs in der Regel diejenigen waren (z.B. Italien und Polen), in denen im Jahr 2000 ein vergleichsweise kleinerer Anteil der Schülerinnen und Schüler angegeben hatte, sich von den Lehrern fair behandelt zu fühlen. 


\section{Lehrer-Schüler-Beziehungen in PISA 2000 und PISA 2009}

Prozentsatz der Schüler, die angeben, dass Folgendes "nie oder fast nie“ bzw. "in einigen Stunden" vorkommt

\begin{tabular}{ccc}
$\begin{array}{c}2009 \text { höher } \\
\text { als } 2000\end{array}$ & $\begin{array}{c}2009 \text { niedriger } \\
\text { als } 2000\end{array}$ & $\begin{array}{c}\text { Kein statistisch } \\
\text { signifikanter Unterschied }\end{array}$ \\
\hline Konfidenzniveau von 95\% & + &
\end{tabular}

Die meisten meiner Lehrerinnen/Lehrer interessieren sich für das, was ich zu sagen habe

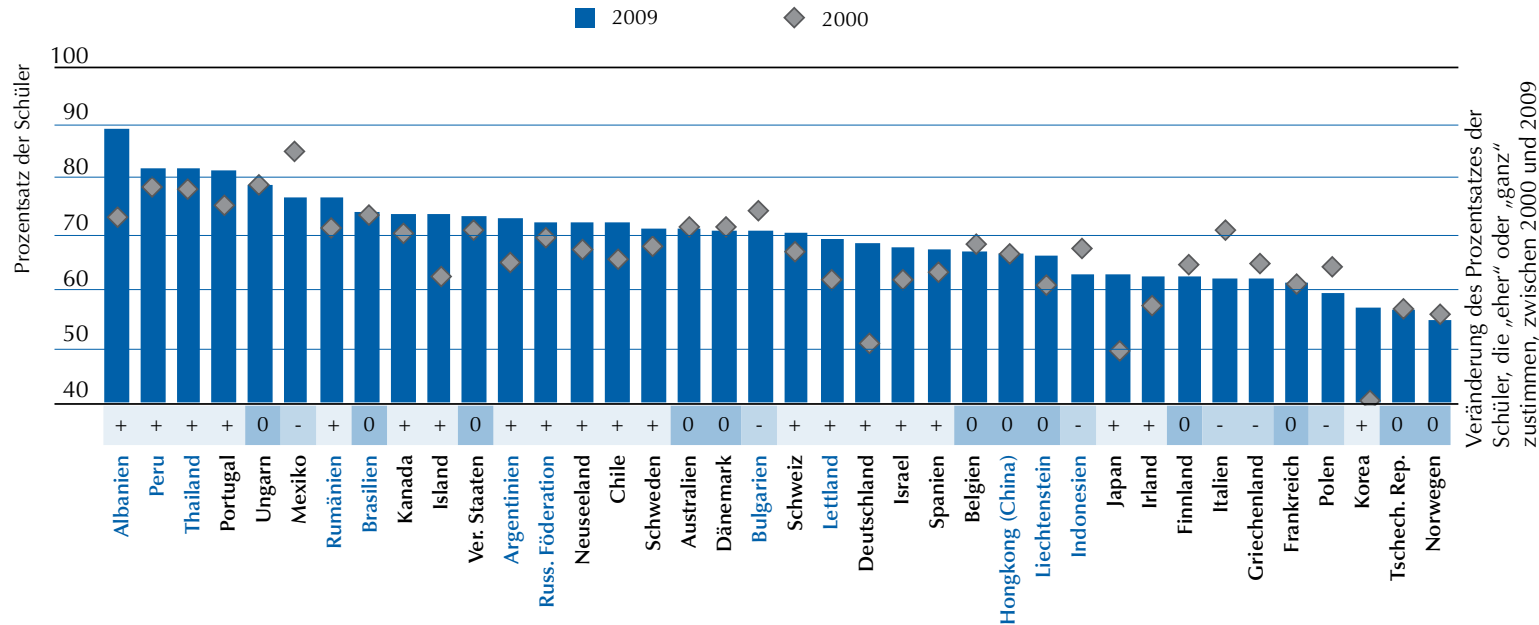

Wenn ich zusätzlich Hilfe brauche, bekomme ich sie von meinen Lehrerinnen/Lehrern

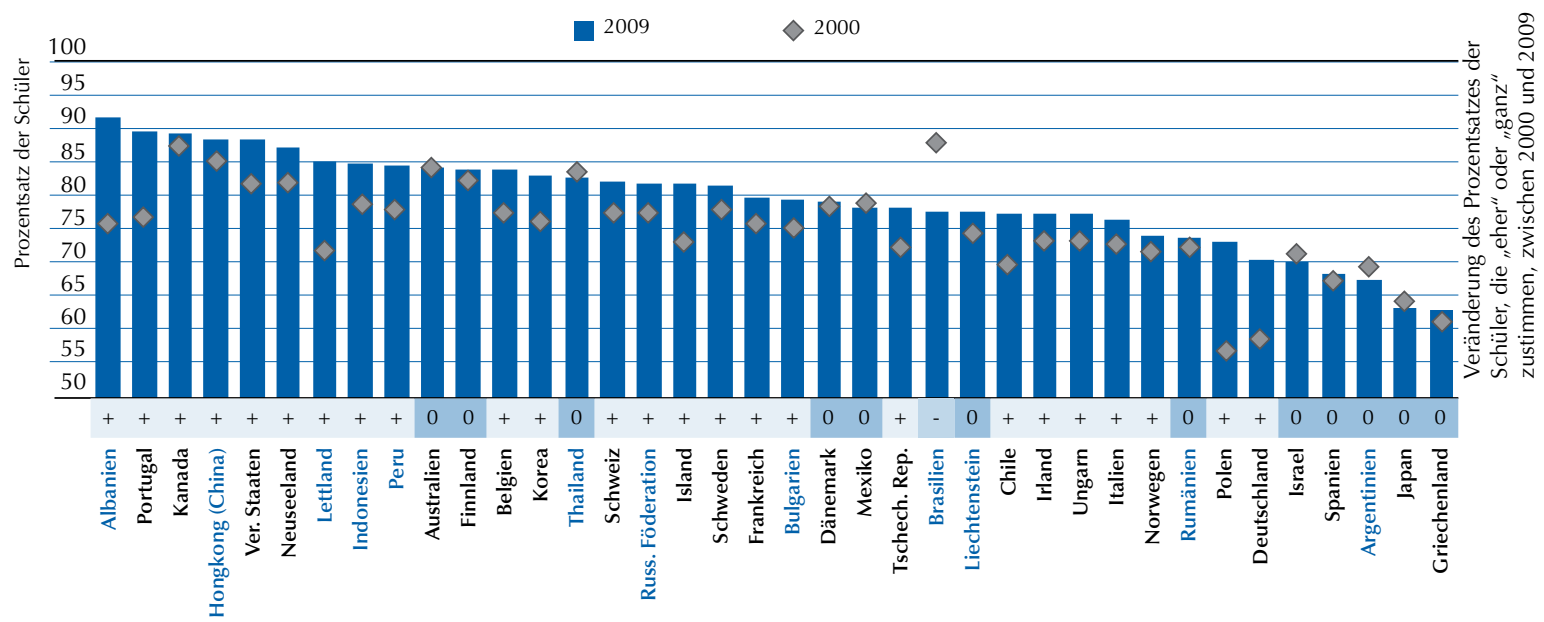

Die Länder sind in absteigender Reihenfolge nach dem Prozentsatz der Schüler für die jeweiligen Items im Jahr 2009 angeordnet. Quelle: OECD, PISA-2009-Datenbank, Tabelle V.5.11

StatLink 部吉 http://dx.doi.org/10.1787/888932360024 


\section{Veränderungen der Schuldisziplin}

Die im Unterricht und an der Schule herrschende Disziplin kann sich ebenfalls auf das Lernen auswirken. In Klassen und Schulen mit größeren Disziplinproblemen wird weniger gut gelernt, da die Lehrer mehr Zeit darauf verwenden müssen, Ruhe herzustellen, bevor der Unterricht beginnen kann (Gamoran und Nystrand in Newman et al., 1992). Störendes Verhalten im Unterricht beeinträchtigt die Konzentration der Schülerinnen und Schüler sowie ihre Fähigkeit, dem Unterricht zu folgen.

Die Schülerinnen und Schüler wurden gebeten, das Disziplinklima im Testsprachenunterricht zu beschreiben. Dazu wurden sie gefragt, wie häufig - nie, in einigen Stunden, in den meisten Stunden oder in allen Stunden - es vorkommt, dass die Schülerinnen und Schüler der Lehrerin/dem Lehrer nicht zuhören, es im Unterricht laut ist und alles durcheinandergeht, die Lehrerin/der Lehrer lange warten muss, bis Ruhe eintritt, die Schüler nicht ungestört arbeiten können oder erst lange nach Stundenbeginn zu arbeiten beginnen. In PISA 2000 wurden die gleichen Fragen gestellt, so dass sich die Antworten im Zeitverlauf vergleichen lassen.

Im Durchschnitt der OECD-Länder ist der Prozentsatz der Schülerinnen und Schüler, die angaben, dass ihre Lehrerinnen/Lehrer nie oder fast nie lange warten müssen, bis Ruhe einkehrt, bis zum Jahr 2009 um 6 Prozentpunkte gestiegen - auf 73\%, gegenüber 67\% im Jahr 2000 (TabelleV.5.12). Verbesserungen auf dem Index der Schuldisziplin waren in 25 Ländern festzustellen, während die Situation in den anderen 13 Ländern unverändert blieb. Besonders hoch - über 10 Prozentpunkte - war der Anstieg des Prozentsatzes der Schülerinnen und Schüler, die angaben, dass ihre Lehrerinnen/ihre Lehrer nie oder fast nie lang warten müssten, bis die Klasse ruhig ist, in Italien, Schweden, Deutschland, Spanien, Israel, dem Partnerland Indonesien und der Partnervolkswirtschaft Hongkong (China). Die größten Verbesserungen waren zumeist in den Ländern zu beobachten, in denen die Bedingungen anfangs ungünstiger waren, in Italien oder Indonesien gab beispielsweise im Jahr 2000 nur die Hälfte der Schülerinnen und Schüler an, ihre Lehrerinnen und Lehrer bräuchten nicht lang zu warten, bis in der Klasse Ruhe eintritt.

Abbildung V.5.12 und Tabelle V.5.12 zeigen, dass der Anteil der Schülerinnen und Schüler, die für die Aussage "Wir hören nicht auf das, was die Lehrerin/der Lehrer sagt" "nie" oder "in einigen Stunden" ankreuzten, im Durchschnitt der OECD-Länder im Jahr 20093 Prozentpunkte geringer war. Dieser Anteil bleibt jedoch hoch: 72\% im Jahr 2009, gegenüber 75\% im Jahr 2000. In 18 Ländern gaben weniger Schülerinnen und Schüler an, dass die Aussage "Wir hören nicht auf das, was die Lehrerin/der Lehrer sagt" für die meisten oder alle Stunden nicht zutrifft, was auf eine Verschlechterung der Schuldisziplin schließen lässt. In Griechenland, Polen, Irland, Australien, der Tschechischen Republik und dem Partnerland Liechtenstein war hier ein Rückgang um über 10 Prozentpunkte zu verzeichnen. In zehn anderen Ländern stieg allerdings der Anteil der Schülerinnen und Schüler, die dieser Aussage nicht zustimmten. In Korea und der Partnervolkswirtschaft Hongkong (China) war der stärkste Anstieg dieses Anteils festzustellen: über 10 Prozentpunkte. Eine Zunahme um 5-10 Prozentpunkte wurde in Japan, Deutschland, Israel und den Partnerländern Peru und Rumänien beobachtet.

Tabelle V.5.12 zeigt, dass der Anteil der Schülerinnen und Schüler, die angaben, dass im Unterricht Lärm und Unruhe herrschen, in den OECD-Ländern im Durchschnitt unverändert blieb, und dass der Anteil derer, die meinten, dass sie nie oder fast nie ungestört arbeiten können und dass sie erst lange nach dem Beginn der Stunde zu arbeiten anfangen können, um 2 Prozentpunkte gestiegen ist. In manchen Ländern, in denen fast jeder zweite Schüler angab, dass in einigen Stunden Lärm und Unruhe herrschten, verbesserte sich die Lage deutlich: Im Jahr 2000 hatten in Chile, Griechenland und Italien nur zwischen 51\% und 54\% Prozent der Schülerinnen und Schüler angegeben, dass nie oder fast nie Lärm und Unruhe zu beklagen seien. Im Jahr 2009 hatte sich dieser Anteil in Italien auf 68\%, in Griechenland auf 58\% und in Chile auf 63\% erhöht. Gleichzeitig verschlechterten sich die Bedingungen in vielen Ländern, in denen mehr als acht von zehn Schülerinnen und Schülern angegeben hatten, bei ihnen herrschten weder Lärm noch Unruhe im Unterricht: In der Schweiz und in Polen sowie den Partnerländern Liechtenstein, Lettland und Albanien sank dieser Prozentsatz um 4-9 Prozentpunkte. 
Abbildung V.5.12

Schuldisziplin in PISA 2000 und PISA 2009

Prozentsatz der Schüler, die angeben, dass Folgendes "nie oder fast nie" bzw. "in einigen Stunden" vorkommt

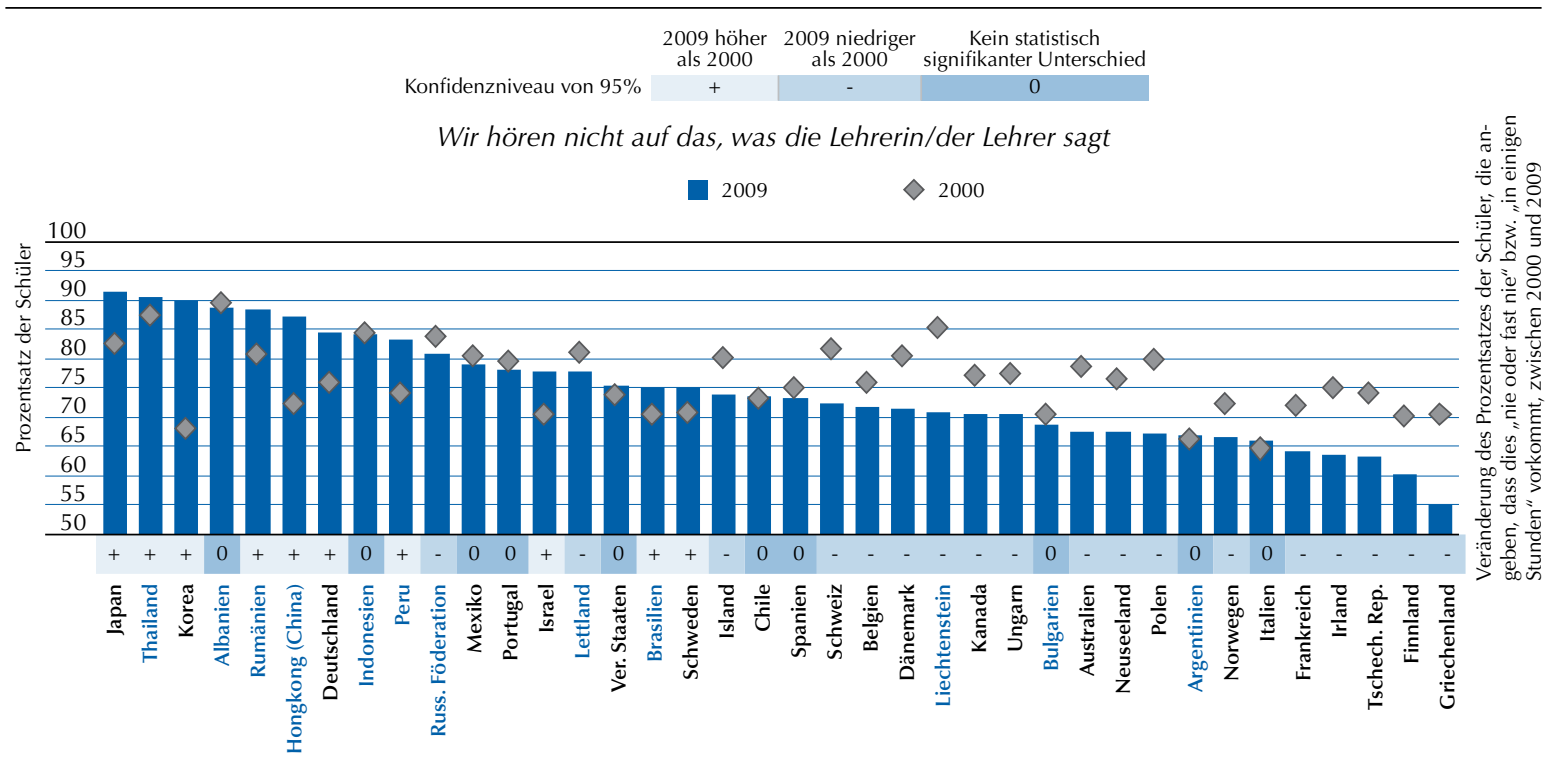

Der Lehrer muss lange warten, bis Ruhe eintritt

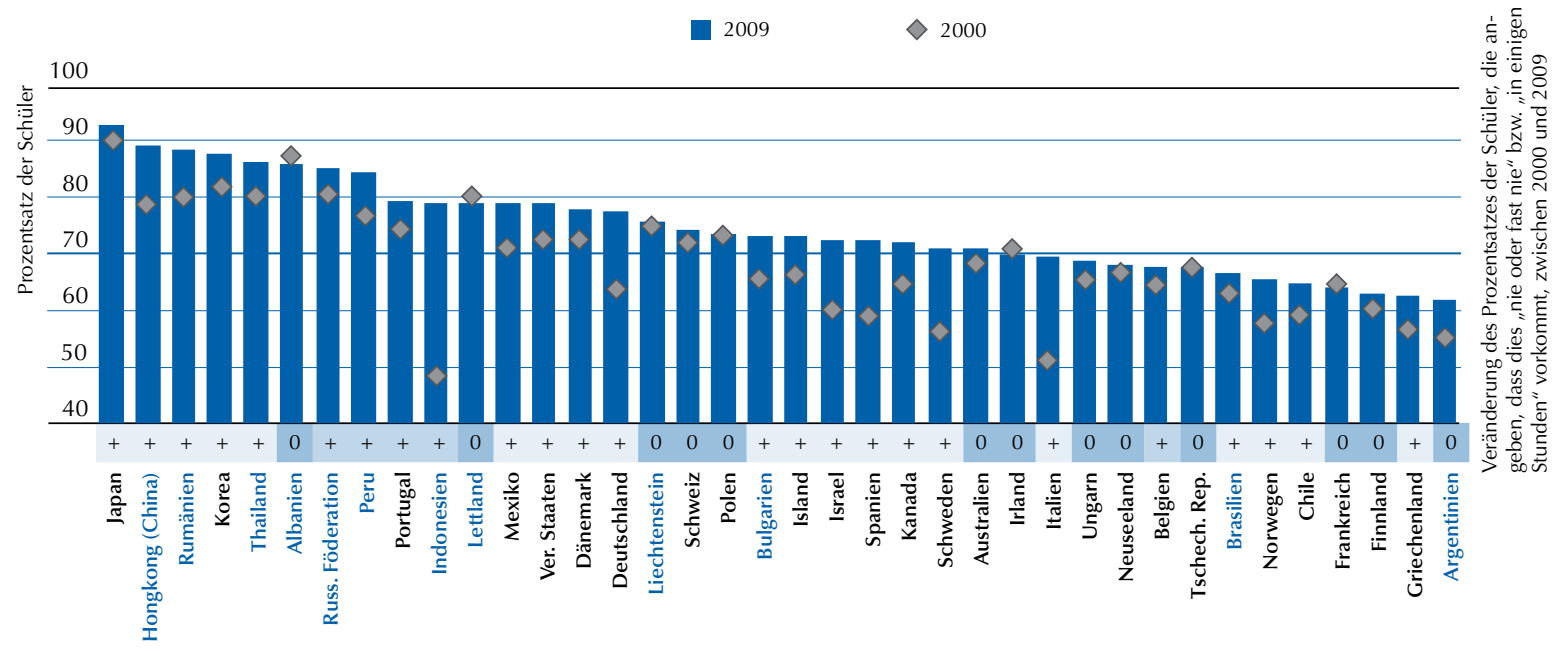

Die Länder sind in absteigender Reihenfolge nach dem Prozentsatz der Schüler für die jeweiligen Items im Jahr 2009 angeordnet. Quelle: OECD, PISA-2009-Datenbank, Tabelle V.5.12

StatLink त्ञात http://dx.doi.org/10.1787/888932360024

\section{Anmerkungen}

1. In diesem Kontext werden Schülerinnen und Schüler, die auf dem PISA-Index des wirtschaftlichen, sozialen und kulturellen Status ihres Landes im unteren Quartil liegen, als benachteiligt betrachtet, während diejenigen im obersten Quartil als sozioökonomisch besser gestellt angesehen werden. 


\section{Kasten V.G Brasilien}

Brasilien ist ein gutes Beispiel dafür, dass Länder mit schwachen Ergebnissen internationale Vergleiche nutzen können, um ihr Bildungssystem zu verbessern.

In den 1950er Jahren lebten 64\% der brasilianischen Bevölkerung noch in ländlichen Gebieten, und von ihnen waren über 50\% Analphabeten. Die stufenweise Verbesserung der Bildung wurde zu einer Priorität der politischen Führung des Landes, die jedoch vor der Herausforderung stand, die Eltern von der Bedeutung einer längeren und besseren Schulbildung ihrer Kinder zu überzeugen, da viele dieser Eltern ihre Kinder zum Arbeiten schicken mussten, um zum Lebensunterhalt der Familie beizutragen.

Die Verbesserung des Bildungszugangs wurde zudem durch die brasilianische Geografie erschwert: Die 193 Millionen Einwohner des Landes sind über gut 8,5 Millionen Quadratkilometer verteilt - etwas weniger als die Gesamtfläche der Vereinigten Staaten. Angesichts von etwa 83000 über das ganze Land verstreuten Schulen in ländlichen Gebieten, viele mit nur ein oder zwei Lehrkräften, war sowohl die Qualität der Lehrkräfte als auch des von ihnen angebotenen Unterrichts begrenzt. Außerdem führte der ausgiebige Rückgriff auf Klassenwiederholungen in diesem Schulsystem dazu, dass der Altersunterschied der Schülerinnen und Schüler in jeder Klasse zwischen zwei und sechs Jahren liegen konnte, was das Unterrichten erschwerte.

Im Jahr 1995 waren 90\% der Kinder eingeschult, jedoch schloss nur die Hälfte von ihnen die 8. Klasse ab. Und diejenigen, die soweit kamen, benötigten dafür im Durchschnitt 12 Jahre, was durch die schlechte Qualität des Unterrichts und ihre schwachen Leistungen bedingt war, die zu Klassenwiederholungen führten. Im Jahr 2000 galten 13,6\% der brasilianischen Erwachsenenbevölkerung als Analphabeten, und $75 \%$ waren funktionale Analphabeten, was bedeutet, dass sie keine langen Texte lesen, Untertitel verfolgen, zwei Texte vergleichen, Schlüsse ziehen und Synthesen erstellen, mathematische Probleme lösen oder mit Karten und Grafiken arbeiten konnten. In diesem Jahr verzeichnete Brasilien die niedrigsten Ergebnisse bei der PISA-Erhebung: Über 50\% der Schülerinnen und Schüler erreichten weniger als Stufe 1 bei der Lesekompetenz, während weniger als 1\% die höchste Stufe erreichten.

Über die letzten zehn Jahre hat Brasilien jedoch allem Anschein nach messbare Verbesserungen der Schülerleistungen in den verschiedenen untersuchten Bereichen erzielt (vgl. Abb. V.1.2 sowie Tabellen V.2.1, V.3.1 und V.3.4). Das Land hat erheblich mehr Ressourcen in Bildung investiert, die Ausgaben für Bildungseinrichtungen sind von 4\% des BIP im Jahr 2000 auf 5,2\% des BIP im Jahr 2009 gestiegen, und ein größerer Anteil dieser Ressourcen kam der Anhebung der Lehrergehälter zugute. Die Gelder werden heute auch wesentlich gerechter verteilt als in der Vergangenheit. Bundesmittel fließen nun in die ärmsten der 26 Bundesstaaten, so dass die Schulen in diesen Bundesstaaten über vergleichbare Ressourcen wie die der vermögenderen Staaten verfügen.

Außerdem nennen Pädagogen in Brasilien den im Jahr 2005 geschaffenen Entwicklungsindex für den Grundschulunterricht (Índice de Desenvolvimento da Educação Básica - IDEB) als einen Schlüssel zur landesweiten Verbesserung der Schulergebnisse. Der Index basiert sowohl auf den durchschnittlichen Ergebnissen der landesweiten Prüfungen in portugiesischer Sprache und Mathematik, die in der 4., 8. und 11. Klasse durchgeführt werden, als auch auf der Quote der versetzten Schüler. Die Berechnung ergibt einen Notenspiegel von 1 bis 10, wobei die Stufen an die internationale PISA-Skala angepasst sind. Die Verwendung dieser beiden Faktoren garantiert, dass Schulen keine Anreize haben, Schüler in den geprüften Stufen zu halten bzw. sie dazu zu bewegen, die Schule zu verlassen. Das explizite Ziel der brasilianischen Regierung ist es, im Jahr 2021, also im Vorjahr der Zweihundertjahrfeier der Unabhängigkeit Brasiliens, das Durchschnittsniveau von PISA zu erreichen.

Der Index wird für jede Schule des Landes einzeln errechnet, so dass ein Entwicklungspfad entsteht, der mit dem Niveau der Schule im Jahr 2005 beginnt und dort endet, wo die Schule den PISA-Durchschnitt des Jahres 2021 erreicht. Die Pädagogen haben das System akzeptiert, weil sie glauben, dass es fairer ist, die derzeitige Leistung einer Schule mit den Leistungen der Vergangenheit zu vergleichen, als ein willkürliches Ziel zu setzen, das alle Schulen erreichen sollten. Anders als viele Länder schließt Brasilien sowohl die Privatschulen als auch die öffentlichen Schulen in die Beurteilung und die Zielsetzung ein. Seit Einführung des Index sind die Leistungen der Grundschulen (1. bis 4. Klasse) landesweit von 3,8 im Jahr 2005 auf 4,6 im Jahr 2009 gestiegen 
und übertrafen somit das Ziel von 4,2. In den mittleren Klassenstufen (5. bis 8. Klasse) ist der Index von 3,5 im Jahr 2005 auf 4,0 im Jahr 2009 gestiegen und übertraf ebenfalls die Zielmarke von 3,7. In der Sekundarstufe (9. bis 11. Klasse) sind die Ergebnisse leicht gestiegen, von 3,4 auf 3,6 im gleichen Zeitraum.

Die Ergebnisse im Bereich Lesekompetenz in PISA haben sich zwischen 2000 und 2009 ebenfalls verbessert. Brasilien hat sich in der Lesekompetenz um 16 Punkte verbessert (vgl. Abb. V.2.1 und Tabelle V.2.1), wodurch sich der Anteil der Schülerinnen und Schüler, die unter Stufe 2 liegen, reduziert hat, und der Prozentsatz der Schüler, die auf Stufe 5 oder darüber angesiedelt sind, leicht gestiegen ist (vgl. Abb. V.2.4 und V.2.5 sowie Tabelle V.2.2).

Die allgemeine Verbesserung der PISA-Lesekompetenz wurde von einem Anstieg der Leistungsvarianz begleitet. Dies war weitgehend dadurch bedingt, dass Brasilien, relativ gesehen, die Lesekompetenz seiner besten Schülerinnen und Schüler steigern konnte, und das Kompetenzniveau der schwächsten Schülerinnen und Schüler gleichzeitig stabil blieb. Außerdem war der positive Trend hinsichtlich der Lesekompetenz hauptsächlich durch größere Verbesserungen bei den Mädchen gegenüber den Jungen bedingt. Ein allgemeiner Rückgang des Anteils der Schülerinnen und Schüler mit schwachen Ergebnissen war hauptsächlich auf die besseren Ergebnisse der Mädchen zurückzuführen, da von ihnen 9 Prozentpunkte weniger unter Stufe 2 lagen - bei den Jungen waren es 3 Prozentpunkte weniger (vgl. Abb. V.2.8 und Tabellen V.2.5 und V.2.6).

In Mathematik haben die Schülerinnen und Schüler Brasiliens ihre PISA-Leistungen seit dem Jahr 2003 um 30 Punkte verbessert, und der Prozentsatz der Schülerinnen und Schüler unter Stufe 2 wurde um 4 Prozentpunkte reduziert (vgl. Abb. V.3.1 und V.3.3 sowie Tabelle V.3.1 und V.3.2). In den Naturwissenschaften hat Brasilien seit 2006 seine Leistung um etwa 15 Punkte gesteigert und den Anteil der Schüler, die unter Stufe 2 liegen, um 7 Prozentpunkte gesenkt (vgl. Abb. V.3.5 und V.3.7 sowie Tabelle V.3.4 und V.3.5).

Trotz dieser Fortschritte bleiben die Mittelwerte Brasiliens mit 412 Punkten in der Lesekompetenz, 386 Punkten in Mathematik und 405 Punkten in den Naturwissenschaften in der Erhebungsrunde 2009 weit unter dem OECD-Durchschnitt.

Veränderungen des Evaluierungssystems gingen mit Umgestaltungen der Lehrerausbildung einher. Als Gegenleistung für zusätzliche Ressourcen arbeiten die Bundesuniversitäten mit leistungsschwachen Schulen in ihren Gemeinden zusammen, um die Bedürfnisse dieser Schulen zu identifizieren sowie Lehrer auszubilden und zu begleiten. Das nationale Bildungsministerium stellt außerdem Ausstattung und Materialien, Transportdienste und Technologie zur Verfügung, um durch die Fernuniversität die Lehrerausbildung in ländlichen Schulen zu unterstützen. Diese Schulen unterrichten $13 \%$ der Schülerinnen und Schüler des Landes, die an einer Grundbildung teilnehmen. Jeder Bundesstaat Brasiliens ist im Grunde ein „Innovationslabor" für Bildungspolitik und -praxis.

So zum Beispiel der Bundesstaat Acre. Es handelt sich um einen der kleinsten und am wenigsten entwickelten Staaten Brasiliens, im fernen Nordwesten und im Regenwald des Amazonas gelegen. Mehr als die Hälfte der 690000 Einwohner leben in zwei Städten. Die anderen sind auf Kleinstädte und entlegene Gebiete verteilt, mit Flüssen als einzigem Transportweg. 1999 rangierte Acre bei den Bildungsergebnissen an letzter Stelle des Landes, es gab keine Pläne zur Verbesserung der Schulen, die Schulgebäude waren in schlechtem Zustand, nur 14 der 22 Gemeinden boten eine Sekundarstufe an, und nur 27\% der Lehrkräfte konnten eine Tertiärbildung vorweisen.

Die Verbesserungen fingen mit den Lehrkräften an. Die Einstiegsgehälter der Lehrkräfte wurden auf 26\% über dem nationalen Mindestanfangsgehalt angehoben, und in Zusammenarbeit mit der Bundesuniversität in Acre wurde ein Lehrerausbildungsprogramm erarbeitet, so dass die Attraktivität des Lehrerberufs in Acre stieg. Auch wurden Gebäudenormen entwickelt, und allen Schülern wurde die Möglichkeit einer Sekundarschulbildung garantiert. Der Staat dezentralisierte die Ausstattungshaushalte auf Pro-Schüler-Basis und verlangte Ausgabenpläne von den Schulen. Acre erstellte in Zusammenarbeit mit der Roberto-Marinho-Stiftung zwei Sonderprogramme, um Schülerinnen und Schülern der 5. bis 8. Klasse und der Sekundarstufe zu helfen, die mehrere Klassen wiederholt hatten. Dank dieser Reformen steigerte Acre seinen IDEB-Index von 3,3 im Jahr 2005 auf 4,5 im Jahr 2009. 
São Paulo, Brasiliens am dichtesten besiedelter und reichster Bundesstaat, hat eine vergleichbare Wandlung seines Bildungssystems vollzogen. Wie in der Mehrzahl der Bundesstaaten besuchen die meisten Schülerinnen und Schüler der oberen Mittelschicht Privatschulen, so dass die Priorität darin bestand, allen Kindern den Schulbesuch zu ermöglichen. Dann erstellte der Staat sein eigenes Beurteilungssystem und eigene Indikatoren, die ein zweijähriges, individuell für jede Schule auf der Basis von PISA und internationalen Standards festgelegtes Ziel setzten. Während das nationale Ziel darin besteht, bis 2021 den PISA-Durchschnitt zu erreichen, sind die Ziele in São Paulo differenzierter: Die Leistung der Schule wird je nach Anteil der Schülerinnen und Schüler in jeder Kategorie betrachtet: Unter dem Grundniveau, Grundniveau, angemessen und fortgeschritten. Dadurch werden Eltern und Öffentlichkeit besser über die Qualität der schulischen Leistungen informiert und die Bezirke sowie der Bundesstaat können besser einschätzen, wo die Schule sich noch steigern muss.

Der Bildungsminister des Bundesstaats arbeitete mit Lehrern und Universitätsprofessoren zusammen, um einen klar definierten gemeinsamen Lehrplan für jede Stufe und jedes Fach zu entwickeln und die Lehrer auszubilden. Es wurden etwa 12000 pädagogische Assistenten eingestellt, damit jede Schule über einen Coach verfügt, der mit den Lehrern an der Verbesserung ihrer Praxis arbeiten kann. Ein besonders wichtiger Teil des Programms bestand aus Schulungen in Datenanalyse. Es wurde für alle Schulen ein Anreizsystem geschaffen, um alle zu belohnen, die ihre Verbesserungsziele erreicht hatten. Je besser die Leistung der Schule desto mehr Autonomie wurde gewährt. Schulen, die ihre Ziele nicht erreichten, erhielten zusätzliche technische Hilfe, die auf die Lehrerentwicklung und einen Ausbau der Lernressourcen ausgerichtet war. Im Jahr 2007 wurden die 1000 leistungsschwächsten Schulen ermittelt, die dann technische Unterstützung zu Gunsten der beruflichen Entwicklung der Lehrkräfte sowie zusätzliche Lernressourcen erhielten. Ein Jahr später hatten 95\% ihre Ziele erreicht. Inzwischen haben die öffentlichen Schulen im Bundesstaat São Paulo ihren IDEB-Index in der 4. Klasse von 4,5 im Jahr 2005 auf 5,4 im Jahr 2009 gesteigert, und für die 8. Klasse von 3,8 auf 4,3 im gleichen Zeitraum. 



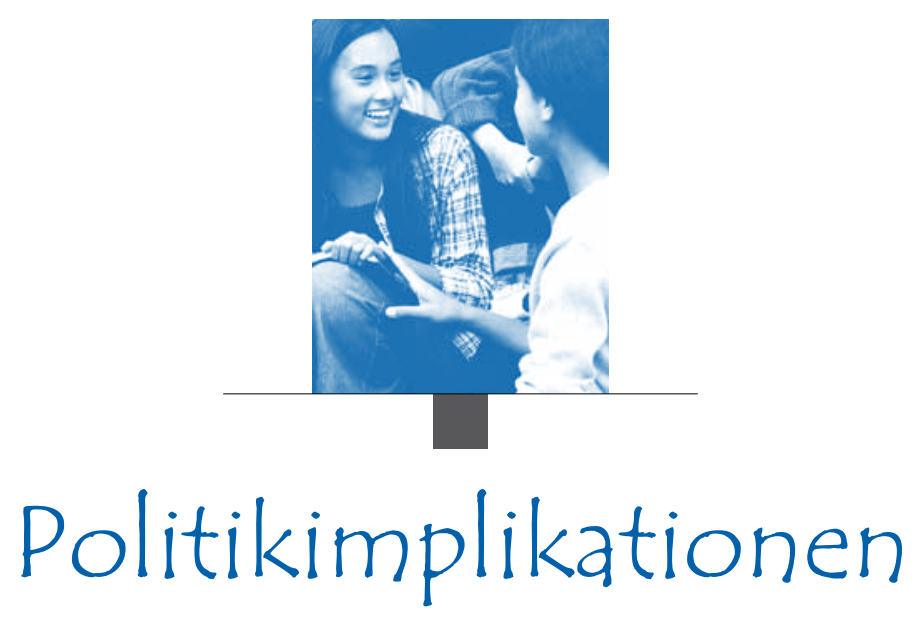

Der Aufbau der PISA-Studie ermöglicht nicht nur einen Vergleich des relativen Stands der einzelnen Länder hinsichtlich ihrer Lernergebnisse. Er erlaubt es auch jedem Land, Veränderungen dieser Ergebnisse im Zeitverlauf zu verfolgen. Diese Veränderungen zeigen, wie erfolgreich die Bildungssysteme bisher bei der Entwicklung der Kenntnisse und Kompetenzen der 15-Jährigen waren. Tatsächlich haben manche Länder in den letzten zehn Jahren beeindruckende Fortschritte erzielt, die in manchen Fällen für die gesamte Schülerpopulation eine Verbesserung um mehr als ein durchschnittliches Schuljahr darstellten. Manche dieser Länder haben ausgehend von relativ schwachen Leistungen aufgeholt, während andere auf der Basis bereits hoher Leistungen weitere Fortschritte erzielten. Alle Länder, die ihre Leistungen steigern möchten, können Inspiration und Vorbilder bei denen finden, die es in einer relativ kurzen Zeitspanne bereits geschafft haben.

Allerdings können Veränderungen der Schülerleistungen auch darauf hindeuten, dass sich die demografischen Bedingungen und die sozioökonomische Zusammensetzung der Schülerpopulationen geändert haben. So könnte z.B. in manchen Ländern das Wachstum benachteiligter Populationen in dem Maße einen allgemeinen Rückgang der Lernergebnisse verursachen, wie der sozioökonomische Hintergrund und die Lernergebnisse verbunden sind. Diese Länder werden sich darauf konzentrieren müssen, das Lernen unter den sozioökonomisch benachteiligten Schülerinnen und Schülern zu verbessern, um eine langfristige Schwächung ihrer Leistung zu verhindern.

PISA zeigt auch bedeutsame Veränderungen des Anteils der leistungsstarken und der leistungsschwachen Schülerinnen und Schüler, der Varianz der Schülerleistungen, der Leistungsunterschiede zwischen den Geschlechtern, des Zusammenhangs zwischen Leistung und sozioökonomischem Hintergrund sowie Veränderungen der Bedingungen, die sich auf das Lernen auswirken, wie die Einstellungen der Schüler und die Schuldisziplin.

\section{VERÄNDERTE LERNBEDINGUNGEN}

Die Medien berichten häufig, dass die Lern- und Lehrbedingungen schwieriger werden, da die Schulen mit zahlreichen verschiedenen Faktoren konkurrieren müssen, die das Leben junger Menschen beeinflussen und wovon einige sie vom Lernen abhalten könnten. Die PISA-Ergebnisse zeichnen ein gemischtes Bild. Einerseits ist die Freude am Lesen tendenziell zurückgegangen, insbesondere unter den Jungen, was für die Schulen die Herausforderung birgt, die Schüler in Leseaktivitäten einzubinden, die 15-Jährige relevant und interessant finden. Andererseits waren die Veränderungen der Lehrer-Schüler-Beziehungen und der Klassendisziplin generell positiv oder verschlechterten sich zumindest nicht, wie es viele erwartet hätten. Im Allgemeinen vertrauen die Schülerinnen und Schüler heute mehr darauf, dass sie von ihren Lehrern Hilfe erhalten können. Insgesamt haben sich auch einige Aspekte der Klassendisziplin verbessert, wenngleich sich in manchen Ländern, wo die Schüler Berichten zufolge mit der geringsten Wahrscheinlichkeit den Lehrkräften zuhören, diese Situation noch verschlimmert hat. Es gibt jedoch keine Belege für die Behauptung, dass die Schülerinnen und Schüler immer mehr das Interesse an der Schule verlieren. 


\section{FORTSCHRITTE IM HINBLICK AUF DIE HEBUNG DES LEISTUNGSNIVEAUS UND DIE HERSTELLUNG GLEICHER RAHMENBEDINGUNGEN}

Die Trends hinsichtlich der Schülerleistungen unterscheiden sich von Land zu Land, die Veränderungen zeigen jedoch, dass das Profil der Lesekompetenzleistung nicht in Stein gemeißelt ist. Die Bildungsergebnisse können sich sowohl absolut als auch relativ verbessern, und sie dürfen auch weder als Teil unveränderlicher "kultureller" Unterschiede zwischen den Ländern, noch als unvermeidlich mit dem Stand der wirtschaftlichen Entwicklung des Landes verbunden betrachtet werden. Insgesamt lässt sich zwischen 2000 und 2009 Folgendes feststellen:

- Die durchschnittlichen Ergebnisse im Bereich Lesekompetenz sind in 13 Ländern mit vergleichbaren Daten deutlich gestiegen und in 4 dieser Länder gesunken.

- In vielen Ländern wurden die besseren Ergebnisse insbesondere von Fortschritten am unteren Ende der Leistungsverteilung induziert, was auf Fortschritte in der Verbesserung der Chancengerechtigkeit hindeutet. Unter den OECD-Ländern ist die Varianz der Schülerleistungen um 3\% gesunken. Andererseits haben sich die Genderdifferenzen ausgeweitet oder sind stabil geblieben, statt zurückzugehen. In neun Ländern hat der Vorsprung der Mädchen weiter zugenommen. Während der Anteil der Jungen mit niedrigem Leistungsniveau in sieben Ländern rückläufig war, ist er in acht Ländern gestiegen.

- Im OECD-Vergleich ist der Prozentsatz der Schülerinnen und Schüler mit Migrationshintergrund zwischen 2000 und 2009 im Durchschnitt um 2 Prozentpunkte gestiegen. Allerdings hat sich die Lage der Schülerinnen und Schüler mit Migrationshintergrund in vielen Ländern verbessert. Der Abstand in der Lesekompetenz zwischen den Schülerinnen und Schülern mit und ohne Migrationshintergrund hat sich in sechs Ländern verringert und lediglich in zwei Ländern ausgeweitet. Auch hat sich der Abstand zwischen den Schülern, die daheim die Testsprache sprechen, und denen, die eine andere Sprache sprechen, in vier Ländern verringert und nur in drei Ländern vergrößert. Trotz dieser positiven Entwicklungen liegen die Schülerinnen und Schüler mit Migrationshintergrund - selbst die im Inland Geborenen mit im Ausland geborenen Eltern - in den meisten Ländern noch hinter den Mitschülern ohne Migrationshintergrund zurück.

Um zu verstehen, was bisher erreicht wurde, ist es hilfreich, die Länder genauer zu betrachten, die entweder hinsichtlich der Qualität oder der Chancengerechtigkeit Fortschritte erzielt haben.

Korea war in der Lage, sein bereits hohes Leistungsniveau im Bereich Lesekompetenz sogar noch weiter zu steigern, indem sich der Anteil der Schülerinnen und Schüler, die mindestens Stufe 5 erreichen, dort mehr als verdoppelte. Polen und das Partnerland Liechtenstein gehören zu den Ländern, die im Jahr 2000 unter dem OECD-Durchschnitt lagen, jedoch im Jahr 2009 ein überdurchschnittliches Leistungsniveau erreichten. Portugal, Ungarn und Deutschland sind von einem unterdurchschnittlichen auf ein durchschnittliches Niveau aufgestiegen. Wenngleich die Leistungen Israels und des Partnerlands Lettland noch immer unter dem OECD-Durchschnitt liegen, haben sie ihre Punktwerte deutlich gesteigert, so dass ihre Leistung derjenigen der meisten OECD-Länder näherkommt. Chile, das sich in den vergangenen neun Jahren erheblich verbessert hat, liegt nun ebenfalls näher am OECD-Durchschnitt. Peru, Albanien, Indonesien und Brasilien steigerten ihre Leistungen in Lesekompetenz ebenfalls, wenngleich von einem niedrigen Ausgangsniveau aus.

Die Länder mit den raschesten Fortschritten - Chile und die Partnerländer Peru, Albanien und Indonesien - haben mehrere Schlüsselfaktoren gemeinsam. Alle erzielten in der 2000er-Testrunde eine stark unterdurchschnittliche mittlere Punktzahl. Jedes dieser Länder hat seine mittlere Punktzahl bis 2009 um zwischen 31 und 43 Punkten verbessert - etwa die Hälfte einer Kompetenzstufe bzw. etwa das Äquivalent eines Schuljahrs -, was in jeder Hinsicht über einen Zeitraum von neun Jahren eine deutliche Verbesserung darstellt. Dies erreichten sie teilweise durch eine Verringerung des Anteils der Schüler auf den niedrigsten Kompetenzstufen. Beispielsweise ist in Chile der Anteil an Schülerinnen und Schülern mit Leistungen unter der Stufe 2 von nahezu der Hälfte (48\%) auf unter ein Drittel (31\%) gesunken. In Albanien, Indonesien und Peru ging der Anteil der Schülerinnen und Schüler, die in PISA mindestens Stufe 2 erreichen, um 14-15 Prozentpunkte zurück. In allen vier Ländern stieg außerdem die Leistung der relativ starken Schülerinnen und Schüler, wenngleich Chile das einzige Land war, in dem der Anteil der Schüler, die Stufe 5 oder 6 erreichten, zunahm. Außerdem ist die Beziehung zwischen dem sozioökonomischen Hintergrund und den Lernergebnissen in Albanien, Chile und Indonesien inzwischen weniger ausgeprägt. Dies zeigt, dass eine Verbesserung der Ergebnisse unter den Leistungsschwachen nicht unbedingt auf Kosten der Leistungsträger geschehen muss und mit größerer Chancengerechtigkeit einhergehen kann. In Peru führten die allgemeinen Verbesserungen hingegen nicht zu einer größeren Chancengerechtigkeit im Bildungswesen. 
Polen und das Partnerland Liechtenstein, die von unterdurchschnittlichen zu überdurchschnittlichen Ergebnissen vorgerückt sind, sowie Deutschland, Ungarn und Portugal, die sich von einem unterdurchschnittlichen zu einem durchschnittlichen Leistungsniveau entwickelten, und Lettland, das sich verbessert hat und nun knapp unter dem OECD-Durchschnitt liegt, weisen eine wichtige Gemeinsamkeit auf: In allen sechs Ländern ist der Anteil der Schülerinnen und Schüler mit Leistungen unter der Stufe 2 gesunken und derjenige der leistungsstarken Schüler, die mindestens Stufe 5 erreichten, unverändert geblieben. Dies spiegelt sich in einem all-gemeinen Rückgang der Leistungsvarianz wider. In Portugal ist der Anteil der Schülerinnen und Schüler mit Leistungen unter der Stufe 2 von $26 \%$ auf $18 \%$ und in Lettland von $30 \%$ auf $18 \%$ zurückgegangen. Dies bedeutet, dass in beiden Ländern eine deutliche Verbesserung bei den Leistungsschwachen stattgefunden hat. Zudem verbesserte sich in Lettland und Polen die Chancengerechtigkeit unter den Schulen, was mit Reformen zusammenhängen kann, durch die die Aufteilung der Schülerinnen und Schüler in schulische und berufsbildende Programme auf einen späteren Zeitpunkt verschoben wurde. Der Zusammenhang zwischen sozioökonomischem Hintergrund und Leistungsniveau der Schülerinnen und Schüler hat sich auch in Deutschland abgeschwächt. Infolge der durch den PISA-Test 2000 ans Licht gebrachten erheblichen Ungleichheiten haben die deutschen Schulen und Bundesländer stark in sozioökonomisch benachteiligte Schülerinnen und Schüler investiert, einschließlich derjenigen mit Migrationshintergrund.

In Korea und Brasilien, und bis zu einem gewissen Grad in Israel, war die beobachtete Leistungssteigerung hauptsächlich durch die besseren Noten der leistungsstärksten Schüler bedingt. In allen drei Ländern hat sich der Anteil der Schülerinnen und Schüler, die die Anforderungen von Stufe 5 oder darüber erfüllen, verdoppelt. Korea hat bereits im Jahr 2000 ein hohes Leistungsniveau erreicht und zählte nur sehr wenige leistungsschwache Schülerinnen und Schüler, doch erzielte nur ein kleiner Anteil der Schüler hervorragende Leistungen in der Lesekompetenz. Indem es den Anteil der leistungsstarken Schülerinnen und Schüler fast verdoppeln konnte, auf 13\%, hat Korea zu den stärksten Ländern aufgeschlossen. In Israel stieg der Anteil der leistungsstarken Schülerinnen und Schüler deutlich, dennoch bleibt der Anteil unter dem OECD-Durchschnitt. Die Verbesserungen in Brasilien wurden durch die leistungsstarken Schülerinnen und Schüler angeführt, wenngleich der Anteil dieser Jugendlichen in Brasilien im Vergleich zu dem der OECD-Länder gering ist.

Diese allgemeinen Veränderungen kaschieren deutliche Unterschiede bei den Fortschritten der Jungen und Mädchen. Seit dem Jahr 2000 hat sich die Lesekompetenzleistung der Jungen nur in fünf Ländern verbessert, während die der Mädchen in 13 Ländern gestiegen ist. Wie bereits festgestellt, schneiden Mädchen im Bereich Lesekompetenz wie schon im Jahr 2000 weiterhin in allen Teilnehmerländern besser ab als Jungen, doch war der Abstand im Jahr 2009 noch größer. In acht Ländern ist der Prozentsatz der Jungen, die die Stufe 2, d.h. das Basisniveau an Lesekompetenz, nicht erreichten, gestiegen, während er in sieben Ländern zurückging. Ferner besteht bei den Jungen, insbesondere solche mit sozioökonomisch benachteiligtem Hintergrund, eine größere Tendenz, nicht zum Vergnügen zu lesen oder negative Einstellungen zum Lesen anzugeben. Obgleich die Notwendigkeit einer Anhebung des Leseengagements der Jungen, insbesondere derjenigen aus einem sozial benachteiligten Milieu, im Jahr 2000 erkannt worden war, deutet die jüngste PISA-Erhebung darauf hin, dass in dieser Hinsicht wenig Fortschritte erzielt wurden.

PISA ermöglicht auch einen Vergleich der Schülerleistungen in Mathematik und Naturwissenschaften im Zeitverlauf, wenngleich diese über kürzere Zeiträume beobachtet werden. In Mathematik konnten die Schüler in Mexiko, der Türkei, Griechenland, Portugal, Italien, Deutschland und den Partnerländern Brasilien und Tunesien ihre Punktwerte seit dem Jahr 2003 erheblich steigern. Mexiko reduzierte den Anteil der Schülerinnen und Schüler, deren Leistungen in Mathematik unter dem Basisniveau der PISA-Kompetenzstufe 2 liegen, seit 2003 um 15 Prozentpunkte von 66\% auf $51 \%$, und die Türkei im selben Zeitraum von $52 \%$ auf $42 \%$. Griechenland, Italien, Portugal und die Partnerländer Brasilien und Tunesien senkten ebenfalls den Anteil der Schülerinnen und Schüler, die in Mathematik unter Stufe 2 abschnitten. In Portugal ist der Prozentsatz der Schülerinnen und Schüler, die in Mathematik mindestens Stufe 5 erreichen, um 4 Prozentpunkte gestiegen, während der Anteil dieser leistungsstärksten Schüler in Italien und Griechenland um fast 2 Prozentpunkte gestiegen ist.

In den Naturwissenschaften konnten 11 der 56 Länder, deren Ergebnisse zwischen 2006 und 2009 vergleichbar sind, Steigerungen des Leistungsniveaus der Schülerinnen und Schüler verzeichnen. In der Türkei kam es beispielsweise in nur drei Jahren zu einem Anstieg von 30 Punkten, was einer halben Kompetenzstufe entspricht. Die Ergebnisse im Bereich Naturwissenschaften verbesserten sich ebenfalls in den OECD-Ländern Portugal, Korea, Italien, Norwegen, den Vereinigten Staaten und Polen sowie in den Partnerländern Katar, Tunesien, Brasilien und Kolumbien. In der Türkei, in Portugal, Chile, den Vereinigten Staaten, Norwegen, Korea und Italien sank der Anteil der leistungsschwächsten Schüler in den Naturwissenschaften wie auch in den Partnerländern Katar, Tunesien, Brasilien und Kolumbien um 
mindestens etwa 5 Prozentpunkte. Die Türkei reduzierte den Anteil dieser Schülerinnen und Schüler von 47\% auf $30 \%$. Im Partnerland Katar ist der Anteil der Schülerinnen und Schüler, die die Anforderungen von Stufe 2 nicht erfüllten, um 14 Prozentpunkte gesunken, wenngleich fast zwei Drittel der Schülerinnen und Schüler in Katar in den Naturwissenschaften noch immer nicht die Stufe 2 erreichen.

Einige Länder konnten sich in verschiedenen Erhebungsbereichen verbessern. Korea erreichte sowohl in der Lesekompetenz als auch in den Naturwissenschaften ein sehr hohes Niveau. Auch Polen verbesserte sich in diesen beiden Bereichen. Deutschland steigerte sich in Lesekompetenz und Mathematik. Portugal verbesserte sich in allen Erhebungsbereichen. Mehrere Länder, die in der Lesekompetenz noch immer unter dem OECD-Durchschnitt liegen, verzeichneten Verbesserungen in anderen Bereichen.

Insgesamt zeigt sich, dass Länder mit unterschiedlichster Ausgangsbasis Potenzial zur Leistungssteigerung unter Beweis gestellt haben, indem sie sowohl die Durchschnittsniveaus der Lesekompetenz angehoben als auch die Ungleichheiten bei den Ergebnissen reduziert haben, ohne dass sich die Leistungen unter den fähigsten Schülerinnen und Schülern verschlechtert hätten. In manchen, jedoch nicht allen, Fällen wirkten sich diese Verbesserungen auch positiv auf die soziale Gerechtigkeit aus. Allerdings bleibt die Geschlechterlücke im Bereich Lesekompetenz groß und stellt für die meisten Länder, einschließlich derjenigen mit sehr hohem Leistungsniveau, eine wachsende Herausforderung dar. 


\section{Literaturverzeichnis}

Anand, P., A. Mizala und A. Repetto (2006), "Using School Scholarships to Estimate the Effect of Government Subsidized Private Education on Academic Achievement in Chile", Documentos de Trabajo, No. 220, Universidad de Chile, Santiago.

Belley, C. (2007), "Expansión de la educación privada y mejoramiento de la educación en Chile. Evaluación a partir de la evidencia" (Expansion of Private Education and Improvement of Education in Chile. Evidence-based evaluation), Revista Pensamiento Educativo, Vol. 40, No. 1, S. 1-21.

Birch, S.H. und G.W. Ladd (1998), "Children's interpersonal behaviors and teacher-child relationships", Developmental Psychology, Vol. 34, S. 934-946.

Carnoy, M. (1998), "National Voucher Plans in Chile and Sweden: Did Privatization Make for Better Education?", Comparative Education Review, Vol. 42, No. 3, S. 309-337.

Crosnoe, R., G.H. Elder und M. Johnson (2004), "Intergenerational Bonding in School: The Behavioral and Contextual Correlates of Student-Teacher Relationships", Sociology of Education, Vol. 77, No. 1, S. 60-81.

Gamoran, A. (1993), "Alternative uses of ability grouping in secondary schools: Can we bring high-quality instruction to low-ability classes?", American Journal of Education, Vol. 102, No. 1, S. 1-22.

Gamoran, A. und M. Nystrand (1992), "Taking students seriously" in F.M. Newman (Hrsg.), Student engagement and achievement in American secondary schools, Teachers College Press, New York.

Ganzeboom, H.B.G., P.M. De Graaf und D.J. Treiman (1992), "A Standard International Socio-economic Index of Occupational Status", Social Science Research 21.1, S. 1-56.

Gebhardt, E. und R.J. Adams (2007), "The influence of equating methodology on reported trends in PISA", Journal of Applied Measurement, Vol. 8, No. 3, S. 305-322.

GUS (2009), Kształcenie dorosłych, Warschau.

Gysling, J. (2003), Reforma Curricular: Itinerario de una transformación cultural (Curricular Reform: Itinerary of a Cultural Transformation), in C. Cox (Hrsg.), Políticas Educacionales en el cambio de siglo. La reforma del sistema escolar de Chile, Editorial Universitaria, Santiago.

Hsieh, C. und M. Urquiola (2006), "The Effects of Generalized School Choice on Achievement and Stratification: Evidence from Chile's Voucher Program", Journal of Public Economics, Vol. 90, S. 1477-1503.

ILO (Internationale Arbeitsorganisation) (1990), Internationale Standardklassifikation der Berufe (ISCO-88), Genf.

Jakubowski, M., H. Patrinos, E. Porta und J. Wisniewski (2010), "The Impact of the 1999 Education Reform in Poland", OECD Education Working Papers, No. 49.

MEHRD (Ministry of Education and Human Resources Development) (2007), 3-1 Mathematics textbook (in Koreanisch), Seoul.

Neuwirth, E. (2006), "PISA 2000: Sample Weight Problems in Austria", OECD Education Working Papers, No. 5.

Matthews, P., E. Klaver, J. Lannert, G. Ó Conluain und A. Ventura (2008), Policy measures implemented in the first cycle of compulsory education in Portugal (International evaluation), OECD Publishing.

Pinto Ferreira, C. und A. Serrão (2008), "Literacy competences of the Portuguese students: cross-national comparison among some Mediterranean countries", Präsentation für eine Tagung, PISA 2006: The performance of educational systems in countries and regions, Trient, Italien.

OECD (1999), Classifying Educational Programmes: Manual for ISCED-97 Implementation in OECD Countries, OECD Publishing.

OECD (2001), Lernen für das Leben: Erste Ergebnisse von PISA 2000, OECD Publishing.

OECD (2002a), PISA 2000 Technical Report, OECD Publishing.

OECD (2002b), Bildung auf einen Blick 2002: OECD-Indikatoren, W. Bertelsmann Verlag, Bielefeld.

PISA 2009 ERGEBNISSE: LERNFORTSCHRITTE IM GLOBALEN WETTBEWERB - BANDV 
OECD (2005), PISA 2003 Technical Report, OECD Publishing.

OECD (2006a), Economic Survey of Poland: 2006, OECD Publishing.

OECD (2006b), Assessing Scientific, Reading and Mathematical Literacy: A Framework for PISA 2006, OECD Publishing.

OECD (2006c), Economic Survey of Turkey: 2006, OECD Publishing.

OECD (2007), Reviews of National Policies for Education: Basic Education in Turkey, OECD Publishing.

OECD (2008), PISA 2006 Technical Report, OECD Publishing.

OECD (2010a), Pathways to Success: How knowledge and skills at age 15 shape future lives in Canada, OECD Publishing.

OECD (2010b), Bildung auf einen Blick 2010: OECD-Indikatoren, W. Bertelsmann Verlag, Bielefeld.

OECD (2010c), Economic Survey of Portugal: 2010, OECD Publishing.

OECD (2010d), Quality time for students. Learning in and out of school, OECD Publishing.

OECD (erscheint demnächst), PISA 2009 Technical Report, OECD Publishing.

OECD und Statistics Canada (2000), Literacy in the Information Age: Final Report of the International Adult Literacy Survey, OECD Publishing.

Royston, P. (2004), "Multiple imputation of missing values", Stata Journal, Vol. 4, No. 3, S. 227-241.

Rubin, D.B. (1987), "Multiple imputation for non-response in surveys", John Wiley \& Sons, New York.

Santiago, P., D. Roseveare, G. van Amelsvoort, J. Manzi und P. Matthews (2009), OECD Review of Teacher Evaluation in Portugal, OECD Publishing.

Talim ve Terbiye Kurulu (TTKB) (2008), İlkögretim Matematik Dersi 6-8 Sınıflar Öğretim Programı ve Kılavuzu (Teaching Syllabus and Curriculum Guidebook for Elementary school mathematics course: Grades 6 to 8), Ankara, Milli Eğitim Bakanlığı.

Warm, T.A. (1985), "Weighted Maximum Likelihood Estimation of Ability Item Response Theory with Tests of Finite Length", Technical Report CGI-TR-85-08, U.S. Coast Guard Institute, Oklahoma City. 


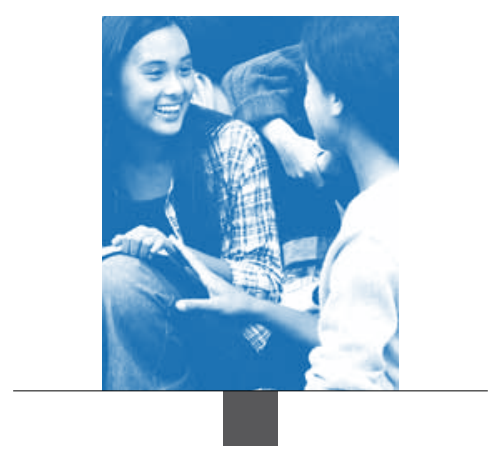

\section{Anhang A}

\section{TECHNISCHE HINWEISE}

Alle Tabellen in Anhang A sind online verfügbar

Anhang A1: Konstruktion der auf den Kontextfragebogen für Schüler, Schulen und Eltern beruhenden Lesekompetenzskalen und -indizes

Anhang A2: PISA-Zielpopulation, PISA-Stichproben und Definition der Schulen

Anhang A3: Standardfehler, Signifikanztests und Vergleiche zwischen Untergruppen

Anhang A4: Qualitätssicherung

Anhang A5: Teilnahme der Länder an den PISA-Erhebungen

Anhang A6: Lineare und bereinigte Trends 


\section{ANHANG A1 \\ KONSTRUKTION DER AUF DEN KONTEXTFRAGEBOGEN FÜR SCHÜLER, SCHULEN UND ELTERN BERUHENDEN LESEKOMPETENZSKALEN UND -INDIZES}

\section{Gestaltung, Analyse und Skalierung der Lesekompetenztests von Pisa 2009}

Die in PISA 2009 verwendeten Leseaufgaben wurden von einem von der OECD beauftragten internationalen Konsortium von Bildungsforschungseinrichtungen unter der Leitung einer Gruppe von Lesekompetenzexperten aus den Teilnehmerländern entwickelt. Die Teilnehmerländer schlugen Stimulusmaterial und Aufgaben vor, die in den drei Jahren bis zur Testdurchführung im Jahr 2009 in einem iterativen Prozess geprüft, getestet und verfeinert wurden. Der Entwicklungsprozess umfasste mehrere Runden, in denen die Teilnehmerländer Kommentare abgeben konnten, sowie einen kleineren Pilotversuch und einen formellen Feldtest mit Stichproben der Population der 15-Jährigen aus allen Teilnehmerländern. Die Expertengruppe Lesen machte eine Empfehlung für die endgültige Auswahl der Aufgaben, in der von 21 Teilnehmerländern eingereichtes Material berücksichtigt wurde. Die Auswahl wurde sowohl im Hinblick auf die technische Qualität der Aufgaben - die anhand der Ergebnisse des Feldtests bewertet wurde - als auch auf ihre kulturelle Angemessenheit und den Grad des Interesses getroffen, den sie bei 15-Jährigen hervorrufen dürften, was von den Teilnehmerländern beurteilt wurde. Ein weiteres wesentliches Kriterium für die Auswahl des Materials insgesamt war, wie gut es in den in Band I, Was Schülerinnen und Schüler wissen und können, beschriebenen Rahmen passte, was die Wahrung eines ausgewogenen Verhältnisses zwischen den verschiedenen Text-, Aspekt- und Situationskategorien anbelangte. Darüber hinaus wurde darauf geachtet, dass der Aufgabenkatalog ein breites Spektrum von Schwierigkeitsgraden abdeckte, um eine genaue Messung und Beschreibung der Lesekompetenz aller 15-jährigen Schülerinnen und Schüler, von den leistungsschwächsten bis zu den leistungsstärksten, zu ermöglichen.

Über 130 Aufgaben zum Lesen gedruckter Texte kamen in PISA 2009 zum Einsatz, die Schülerinnen und Schüler in der Stichprobe sahen jedoch jeweils nur einen Teil der Aufgaben, da ihnen unterschiedliche Testhefte ausgehändigt wurden. Die für PISA 2009 ausgewählten Aufgaben im Bereich Lesekompetenz waren zu halbstündigen Aufgabenblöcken zusammengelegt. Diese wurden mit Aufgabenblöcken aus den Bereichen Mathematik und Naturwissenschaften zu Testheften zusammengefasst, die jeweils vier Aufgabenblöcke enthielten. Die teilnehmenden Schülerinnen und Schüler wurden daraufhin zwei Stunden lang geprüft. Da bei der PISA-Erhebung 2009 die Lesekompetenz im Mittelpunkt stand, enthielt jedes Testheft mindestens einen Aufgabenblock mit Leseaufgaben. Die Blöcke wurden unterschiedlich angeordnet, so dass jeder Aufgabenblock in den Testheften in jeder der vier möglichen Positionen erschien und jedes Paar von Aufgabenblöcken in mindestens einem der 13 Testhefte enthalten war.

Dank dieses Testaufbaus, der sich mit der Gestaltung der vergangenen PISA-Erhebungen deckte, war es möglich, eine einzige Skala für die Lesekompetenz zu konstruieren, auf der jede Aufgabe einem bestimmten Punkt zugeordnet werden kann, der ihren Schwierigkeitsgrad anzeigt, und die Leistung jedes Schülers mit einem bestimmten Punkt verbunden werden kann, der seiner geschätzten Lesekompetenz entspricht. Eine Beschreibung des Modellierungsverfahrens, das zur Konstruktion dieser Skala verwendet wurde, findet sich im PISA 2009 Technical Report (OECD, erscheint demnächst).

Der relative Schwierigkeitsgrad der Testaufgaben lässt sich anhand des Anteils der Testteilnehmer schätzen, die alle Aufgaben richtig beantworten. Die relative Leistungsfähigkeit der Schülerinnen und Schüler, die an einem bestimmten Test teilnehmen, lässt sich anhand des Anteils der Testaufgaben schätzen, die sie richtig beantworten. Der Zusammenhang zwischen der Schwierigkeit der Testaufgaben und der Leistung der Schülerinnen und Schüler kann auf einer einzigen kontinuierlichen Skala aufgezeigt werden. Durch die Konstruktion einer Skala, die den Schwierigkeitsgrad jeder Aufgabe anzeigt, ist es möglich, die Lesekompetenzstufe zu ermitteln, die einer bestimmten Aufgabe entspricht. Durch die Übertragung der Leistung der Schülerinnen und Schüler auf diese Skala ist es dann möglich, den Grad der Lesekompetenz zu beschreiben, über den diese verfügen.

Die Position des Leistungsniveaus der Schülerinnen und Schüler auf dieser Skala wird mit der im Test konkret verwendeten Aufgabengruppe in Beziehung gesetzt. Die einzelnen in der Erhebung verwendeten Aufgaben wurden allerdings so gestaltet, dass sie repräsentativ für die PISA-Definition der Lesekompetenz sind, ebenso wie die Stichprobe der 2009 an PISA teilnehmenden Schülerinnen und Schüler so ausgewählt wurde, dass sie repräsentativ für alle 15-Jährigen in den Teilnehmerländern ist. Das geschätzte Leistungsniveau der Schülerinnen und Schüler spiegelt die Art von Aufgaben wider, von denen anzunehmen ist, dass sie sie erfolgreich lösen können. Dies bedeutet, dass die Schülerinnen und Schüler in der Lage sein dürften, Aufgaben bis zu dem Schwierigkeitsgrad zu lösen, der ihrer Position auf der Skala entspricht (auch wenn dies vielleicht nicht immer der Fall ist). Umgekehrt werden sie Aufgaben über dem Schwierigkeitsgrad, der ihrer Position auf der Skala entspricht, wahrscheinlich nicht lösen können (auch wenn dies bei manchen Aufgaben doch der Fall sein kann).

Je weiter das Kompetenzniveau eines Schülers über dem Schwierigkeitsgrad einer gegebenen Aufgabe liegt, desto höher ist die Wahrscheinlichkeit, dass er die Aufgabe (und andere Aufgaben desselben Schwierigkeitsgrads) erfolgreich lösen kann; je weiter das Kompetenzniveau eines Schülers unter dem Schwierigkeitsgrad einer gegebenen Aufgabe liegt, desto geringer ist die Wahrscheinlichkeit, dass er die Aufgabe - und andere ähnlich schwierige Aufgaben - lösen kann. 


\section{Anpassung der Gesamtskala Lesekompetenz von PISA 2009 an die Lesekompetenzskalen der PISA-Erhebungen 2000, 2003 und 2006}

In den PISA-Erhebungen 2000, 2003, 2006 und 2009 wurde jeweils dieselbe Gesamtskala Lesekompetenz verwendet, so dass die Punkte auf dieser Skala im Zeitverlauf unmittelbar miteinander vergleichbar sind. Möglich ist dies durch die Verwendung von Link-Items, die in allen Erhebungen zum Einsatz kamen und sich für das Equating-Verfahren zur Anpassung der Leistungsskalen nutzen lassen.

Die Abbildung der Ergebnisse im Bereich Lesekompetenz aus PISA 2009 auf der Gesamtskala Lesekompetenz von PISA 2000 erfolgte anhand eines zweistufigen Equating-Verfahrens. Im ersten Schritt wurde eine Korrektur zur Anpassung der Items berechnet. Auch wenn 28 der 101 Aufgaben Link-Items waren, die in allen früheren PISA-Erhebungen verwendet worden waren, wurden letztlich lediglich 26 Link-Items berücksichtigt, da die Ergebnisse bei zwei der Aufgaben als für Linking-Zwecke ungeeignet erachtet wurden. Der durchschnittliche Schwierigkeitsgrad der 26 Link-Items wurde für die Erhebungen 2009 und 2006 berechnet, und anhand der sich daraus ergebenden Differenz wurden die Ergebnisse der Erhebung 2009 so verschoben, dass sie sich an die Skala 2006 anpassten. Die Gesamtskala Lesekompetenz 2006 war bereits an die Skala 2003 angepasst worden, und die Skala aus der Erhebung 2003 an die Skala 2000, so dass die Leistungsskala 2009 somit an die erstmals im Jahr 2000 konstruierte Skala angepasst war.

Im zweiten Schritt wurde die nötige Korrektur zur Anpassung der aus den Link-Items bestehenden Skala an die aus den Link-Items sowie aus neuen Items bestehende Skala mittels folgender Methode berechnet: Die Daten aus PISA 2009 wurden zweimal skaliert, einmal unter Verwendung aller Items und einmal unter Verwendung lediglich der Link-Items. Aus diesen zwei Skalierungen wurde die Differenz zwischen den OECD-Mittelwerten berechnet und die sich daraus ergebende Verschiebung dann zur Anpassung der lediglich die Link-Items umfassenden Skala an die alle Items umfassende Skala verwendet. Nach dieser Korrektur wurden die aus den Item-Response-Theorie-(IRT)-Modellen abgeleiteten Punktzahlen nach dem Geschlecht unterteilt auf die PISA-Skala übertragen.

Da durch das Equating-Verfahren ein Zufallsfehler im Zusammenhang mit den Leistungsveränderungen bei den Link-Items entsteht, wurden die Standardfehler der Schätzwerte für die Leistungstrends angepasst. Diese konservativeren Standardfehler spiegeln nicht nur die Messpräzision und die Stichprobenvarianz wie für die herkömmlichen PISA-Ergebnisse, sondern auch den Linking-Fehler wider.

Anzumerken ist, dass zusätzlich zu den 26 Link-Items, die in den drei aufeinanderfolgenden PISA-Erhebungsrunden 2000, 2003 und 2006 berücksichtigt wurden, zusätzlich 11 Items aus PISA 2000 in die PISA-Erhebung 2009 Eingang fanden. Die 39 Items, die in beiden Erhebungsrunden verwendet wurden, wurden zur Schätzung des Linking-Fehlers zwischen der Gesamtskala Lesekompetenz von PISA 2000 und der Gesamtskala Lesekompetenz von PISA 2009 genutzt, während 28 Items zur Berechnung des Linking-Fehlers der anderen Erhebungsrunden verwendet wurden. Die Linking-Fehler wurden bei den Vergleichen der Ergebnisse aus den einzelnen Erhebungen zu den jeweiligen Ergebnissen hinzugerechnet. Diese Linking-Fehler sind in Tabelle A1.1 aufgeführt.

Tabelle A1.1 Schätzungen der Linking-Fehler

\begin{tabular}{ll} 
& Linking-Fehler auf der PISA-Skala \\
\hline PISA-Gesamtskala Lesekompetenz 2000-2003 & 5.307 \\
\hline PISA-Gesamtskala Lesekompetenz 2000-2006 & 4.976 \\
\hline PISA-Gesamtskala Lesekompetenz 2000-2009 & 4.936 \\
\hline PISA-Gesamtskala Lesekompetenz 2003-2006 & 4.474 \\
\hline PISA-Gesamtskala Lesekompetenz 2003-2009 & 4.088 \\
\hline PISA-Gesamtskala Lesekompetenz 2006-2009 & 4.069 \\
\hline PISA-Gesamtskala Mathematik 2003-2009 & 1.990 \\
\hline PISA-Gesamtskala Mathematik 2006-2009 & 1.333 \\
\hline PISA-Gesamtskala Mathematik 2003-2006 & 1.382 \\
\hline PISA-Gesamtskala Naturwissenschaften 2006-2009 & 2.566 \\
\hline
\end{tabular}

\section{Wie die Lesekompetenzstufen in PISA 2009 definiert sind}

In PISA 2009 wurde mit einer Gesamtskala Lesekompetenz, die sich auf alle Aufgaben des Lesekompetenztests stützt, sowie drei aspektbezogenen Skalen und zwei Textformatskalen gearbeitet. Das Maßsystem für die Gesamtskala Lesekompetenz basiert auf einem Mittelwert für die OECD-Länder, der in PISA 2000 auf 500 gesetzt wurde, mit einer Standardabweichung von 100. Um leichter interpretieren zu können, was die Punktzahlen der Schülerinnen und Schüler konkret bedeuten, wurde die Skala nach einer Reihe statistischer Prinzipien in Stufen unterteilt. Danach wurde, ausgehend von den Aufgaben, die den verschiedenen Stufen zugeordnet sind, beschrieben, welche Art von Kenntnissen und Fertigkeiten zur Lösung dieser Aufgaben jeweils nötig sind.

In PISA 2009 erlaubt das Schwierigkeitsspektrum der Aufgaben die Beschreibung von sieben Lesekompetenzstufen: Stufe 1b ist die niedrigste Stufe, dann folgt Stufe 1a, Stufe 2, Stufe 3 usw. bis hin zu Stufe 6. 
Schülerinnen und Schüler, deren Leistung im Bereich von Stufe 1b liegt, können Aufgaben der Kompetenzstufe 1b (und andere ähnliche Aufgaben) wahrscheinlich erfolgreich lösen, dürften jedoch nicht imstande sein, Aufgaben auf höheren Stufen zu lösen. Stufe 6 entspricht den Aufgaben, die die größten Anforderungen an die Lesekompetenz und die Kenntnisse der Schüler stellen. Schülerinnen und Schüler mit Punktzahlen in diesem Bereich dürften in der Lage sein, Leseaufgaben dieser Stufe ebenso wie alle anderen PISA-Leseaufgaben erfolgreich zu lösen.

Die Konstruktion der Kompetenzskalen erfolgt bei PISA nach einer Standardmethode. Auf der Grundlage der Ergebnisse, die die Schülerinnen und Schüler bei den Testaufgaben erzielen, wird ihre Punktzahl ermittelt und in einem bestimmten Teil der Skala positioniert, wodurch diese Punktzahl einem festgelegten Kompetenzniveau zugeordnet werden kann. Die Stufe, in der die Punktzahl eines Schülers liegt, ist die höchste Stufe, in der zu erwarten ist, dass er den Großteil einer nach dem Zufallsprinzip erfolgten Auswahl von Testaufgaben richtig beantwortet. So wäre z.B. bei einem Test, der aus Aufgaben besteht, die sich gleichmäßig auf Stufe 3 verteilen, von allen Schülerinnen und Schülern, deren Punktzahl Stufe 3 zuzuordnen ist, anzunehmen, dass sie mindestens 50\% der Aufgaben richtig lösen. Da eine Stufe einer ganzen Bandbreite verschiedener Schwierigkeitsgrade und Leistungsniveaus entspricht, variiert die Erfolgsquote innerhalb dieses Spektrums. Schülerinnen und Schüler am unteren Ende der Stufe werden wahrscheinlich knapp über 50\% der gleichmäßig über die Stufe verteilten Aufgaben erfolgreich lösen können, wohingegen Schülerinnen und Schüler am oberen Ende der Stufe deutlich über 70\% dieser Aufgaben richtig beantworten dürften.

Abbildung I.2.12 in Band I enthält nähere Angaben zur Art der Lesefähigkeiten, Kenntnisse und Verständniskapazitäten, die auf den einzelnen Stufen der Lesekompetenzskala erforderlich sind.

\section{Erläuterung der Indizes}

Dieser Abschnitt erklärt die auf den Kontextfragebogen für Schülerinnen und Schüler, Schulen und Eltern beruhenden Indizes, die in PISA 2009 verwendet werden. Die auf den Elternfragebogen beruhenden Indizes sind lediglich für die 14 Länder verfügbar, die sich für den Einsatz dieses optionalen Elternfragebogens entschieden haben.

Bei mehreren PISA-Messgrößen handelt es sich um Indizes, die Antworten von Schülern, ihren Eltern oder Schulvertretern (in der Regel Schulleiterinnen und Schulleiter) auf eine Reihe miteinander verknüpfter Fragen zusammenfassen. Die Fragen wurden auf der Basis theoretischer Überlegungen und vorangegangener Forschungen aus einem größeren Fragenkatalog ausgewählt. Zur Bestätigung des theoretisch erwarteten Verhaltens der Indizes und zur Validierung ihrer Vergleichbarkeit zwischen den Ländern wurden Strukturgleichungsmodelle verwendet. Zu diesem Zweck wurden Modelle für jedes Land separat und für alle OECDLänder insgesamt geschätzt.

Eine Beschreibung anderer PISA-Indizes und Einzelheiten zu den gewählten Methoden sind im PISA 2000 Technical Report (OECD, 2002a), im PISA 2003 Technical Report (OECD, 2005), im PISA 2006 Technical Report (OECD, 2008) und im PISA 2009 Technical Report (OECD, erscheint demnächst) enthalten.

Es gibt zwei Arten von Indizes: einfache Indizes und Skalenindizes.

Einfache Indizes sind Variablen, die durch arithmetische Transformation oder Umkodierung eines oder mehrerer Items in den einzelnen Erhebungen jeweils auf genau dieselbe Art und Weise konstruiert werden. Hier werden die Antworten je Item (item response) zur Berechnung aussagekräftiger Variablen verwendet, etwa bei der Umkodierung der vierstelligen ISCO-88-Codes zur Erstellung des Index der höchsten beruflichen Stellung der Eltern (HISEI) oder bei der Berechnung der Schüler/Lehrer-Quote auf der Grundlage der Daten aus dem Schulleiterfragebogen.

Skalenindizes sind Variablen, die durch Skalierung mehrerer Items konstruiert werden. Sofern nicht anders vermerkt, wurden die fraglichen Indizes mit Hilfe einer gewichteten Maximum-Likelihood-Schätzung (Warm, 1985) auf der Grundlage eines einparametrigen Item-Response-Modells skaliert (im Fall von Items mit mehr als zwei Antwortkategorien wurde ein Modell abgestufter Punktwerte - partial credit model - eingesetzt). Analog zu den Lesekompetenzskalen müssen die auf den Schülerfragebogen beruhenden Indizes gleichgesetzt werden. Dies erfolgte durch Schätzung der Itemparameter anhand der Antwortdaten aus allen Erhebungsrunden, woraus ein Trendindex zu Tage tritt. Dies wird als konkurrente Schätzung der Itemparameter bezeichnet. Alle Items, die in einer bestimmten Erhebung fehlten, wurden im Schätzverfahren als strukturell fehlende Daten behandelt.

Die Skalierung erfolgte in drei Stufen:

- Die Itemparameter wurden auf der Basis gleich großer Teilstichproben von Schülerinnen und Schülern aus jedem OECD-Land und aus allen PISA-Erhebungen geschätzt.

- Die Schätzungen wurden für alle Schüler und alle Schulen durch die im vorangegangenen Schritt ermittelten Itemparameter geankert.

- Die Indizes wurden dann für die Erhebungsdaten des Jahres 2009 standardisiert, so dass der mittlere Indexwert für die OECDSchülerpopulation 2009 gleich null und die Standardabweichung gleich eins gesetzt wurde (wobei den Ländern bei dem Standardisierungsprozess dieselbe Gewichtung gegeben wurde).

Den verschiedenen Antwortkategorien der Fragen wurden in der Reihenfolge, in der letztere in den Schüler-, Schulleiter- und Elternfragebogen erschienen, sequenzielle Codes zugewiesen. Diese Codes wurden zum Zweck der Konstruktion der Indizes bzw. Skalen z.T. umgepolt, worauf in diesem Abschnitt jeweils hingewiesen wird. Es ist zu beachten, dass negative Werte bei einem Index 
[Teil 1/1]

Tabelle A1.2 Bildungsabschluss der Eltern, umgerechnet in Bildungsjahre

\begin{tabular}{|c|c|c|c|c|c|c|c|c|}
\hline & & $\begin{array}{c}\text { Kein } \\
\text { Schul- } \\
\text { besuch }\end{array}$ & $\begin{array}{l}\text { Abschluss } \\
\text { ISCED 1 } \\
\text { (Primar- } \\
\text { bereich) } \\
\end{array}$ & $\begin{array}{l}\text { Abschluss } \\
\text { ISCED 2 } \\
\text { (Sekundar- } \\
\text { bereich I) } \\
\end{array}$ & $\begin{array}{c}\text { Abschluss ISCED 3B oder } \\
\text { 3C (Sekundarbereich II, } \\
\text { zur Berufsreife oder zu } \\
\text { ISCED-5B-Programmen } \\
\text { führend) }\end{array}$ & $\begin{array}{c}\text { Abschluss ISCED 3A } \\
\text { (Sekundarbereich II, } \\
\text { Zugang zu ISCED-5A- } \\
\text { und 5B-Programmen) } \\
\text { oder ISCED 4 } \\
\text { (postsekundärer } \\
\text { nichttertiärer Bereich) }\end{array}$ & $\begin{array}{c}\text { Abschluss ISCED } \\
\text { 5A (universitärer } \\
\text { Tertiärbereich) } \\
\text { oder ISCED 6 } \\
\text { (weiterführende } \\
\text { Forschungs- } \\
\text { programme) } \\
\end{array}$ & $\begin{array}{c}\text { Abschluss ISCED 5B } \\
\text { (nichtuniversitärer } \\
\text { Tertiärbereich) }\end{array}$ \\
\hline \multirow{35}{*}{ 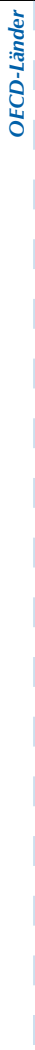 } & Australien & 0.0 & 6.0 & 10.0 & 11.0 & 12.0 & 15.0 & 14.0 \\
\hline & Österreich & 0.0 & 4.0 & 9.0 & 12.0 & 12.5 & 17.0 & 15.0 \\
\hline & Belgien & 0.0 & 6.0 & 9.0 & 12.0 & 12.0 & 17.0 & 14.5 \\
\hline & Kanada & 0.0 & 6.0 & 9.0 & 12.0 & 12.0 & 17.0 & 15.0 \\
\hline & Chile & 0.0 & 6.0 & 8.0 & 12.0 & 12.0 & 17.0 & 16.0 \\
\hline & Tschech. Rep. & 0.0 & 5.0 & 9.0 & 11.0 & 13.0 & 16.0 & 16.0 \\
\hline & Dänemark & 0.0 & 6.0 & 9.0 & 12.0 & 12.0 & 17.0 & 15.0 \\
\hline & Estland & 0.0 & 4.0 & 9.0 & 12.0 & 12.0 & 16.0 & 15.0 \\
\hline & Finnland & 0.0 & 6.0 & 9.0 & 12.0 & 12.0 & 16.5 & 14.5 \\
\hline & Frankreich & 0.0 & 5.0 & 9.0 & 12.0 & 12.0 & 15.0 & 14.0 \\
\hline & Deutschland & 0.0 & 4.0 & 10.0 & 13.0 & 13.0 & 18.0 & 15.0 \\
\hline & Griechenland & 0.0 & 6.0 & 9.0 & 11.5 & 12.0 & 17.0 & 15.0 \\
\hline & Ungarn & 0.0 & 4.0 & 8.0 & 10.5 & 12.0 & 16.5 & 13.5 \\
\hline & Island & 0.0 & 7.0 & 10.0 & 13.0 & 14.0 & 18.0 & 16.0 \\
\hline & Irland & 0.0 & 6.0 & 9.0 & 12.0 & 12.0 & 16.0 & 14.0 \\
\hline & Israel & 0.0 & 6.0 & 9.0 & 12.0 & 12.0 & 15.0 & 15.0 \\
\hline & Italien & 0.0 & 5.0 & 8.0 & 12.0 & 13.0 & 17.0 & 16.0 \\
\hline & Japan & 0.0 & 6.0 & 9.0 & 12.0 & 12.0 & 16.0 & 14.0 \\
\hline & Korea & 0.0 & 6.0 & 9.0 & 12.0 & 12.0 & 16.0 & 14.0 \\
\hline & Luxemburg & 0.0 & 6.0 & 9.0 & 12.0 & 13.0 & 17.0 & 16.0 \\
\hline & Mexiko & 0.0 & 6.0 & 9.0 & 12.0 & 12.0 & 16.0 & 14.0 \\
\hline & Niederlande & 0.0 & 6.0 & 10.0 & a & 12.0 & 16.0 & a \\
\hline & Neuseeland & 0.0 & 5.5 & 10.0 & 11.0 & 12.0 & 15.0 & 14.0 \\
\hline & Norwegen & 0.0 & 6.0 & 9.0 & 12.0 & 12.0 & 16.0 & 14.0 \\
\hline & Polen & 0.0 & a & 8.0 & 11.0 & 12.0 & 16.0 & 15.0 \\
\hline & Portugal & 0.0 & 6.0 & 9.0 & 12.0 & 12.0 & 17.0 & 15.0 \\
\hline & Schottland & 0.0 & 7.0 & 11.0 & 13.0 & 13.0 & 16.0 & 16.0 \\
\hline & Slowak. Rep. & 0.0 & 4.5 & 8.5 & 12.0 & 12.0 & 17.5 & 13.5 \\
\hline & Slowenien & 0.0 & 4.0 & 8.0 & 11.0 & 12.0 & 16.0 & 15.0 \\
\hline & Spanien & 0.0 & 5.0 & 8.0 & 10.0 & 12.0 & 16.5 & 13.0 \\
\hline & Schweden & 0.0 & 6.0 & 9.0 & 11.5 & 12.0 & 15.5 & 14.0 \\
\hline & Schweiz & 0.0 & 6.0 & 9.0 & 12.5 & 12.5 & 17.5 & 14.5 \\
\hline & Türkei & 0.0 & 5.0 & 8.0 & 11.0 & 11.0 & 15.0 & 13.0 \\
\hline & Ver. Königreich & 0.0 & 6.0 & 9.0 & 12.0 & 13.0 & 16.0 & 15.0 \\
\hline & Ver. Staaten & 0.0 & 6.0 & 9.0 & a & 12.0 & 16.0 & 14.0 \\
\hline \multirow{31}{*}{ 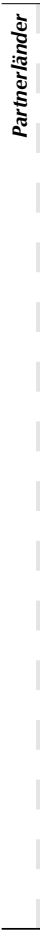 } & Albanien & 0.0 & 6.0 & 9.0 & 12.0 & 12.0 & 16.0 & 16.0 \\
\hline & Argentinien & 0.0 & 6.0 & 10.0 & 12.0 & 12.0 & 17.0 & 14.5 \\
\hline & Aserbaidschan & 0.0 & 4.0 & 9.0 & 11.0 & 11.0 & 17.0 & 14.0 \\
\hline & Brasilien & 0.0 & 4.0 & 8.0 & 11.0 & 11.0 & 16.0 & 14.5 \\
\hline & Bulgarien & 0.0 & 4.0 & 8.0 & 12.0 & 12.0 & 17.5 & 15.0 \\
\hline & Kolumbien & 0.0 & 5.0 & 9.0 & 11.0 & 11.0 & 15.5 & 14.0 \\
\hline & Kroatien & 0.0 & 4.0 & 8.0 & 11.0 & 12.0 & 17.0 & 15.0 \\
\hline & Dubai (VAE) & 0.0 & 5.0 & 9.0 & 12.0 & 12.0 & 16.0 & 15.0 \\
\hline & Hongkong (China) & 0.0 & 6.0 & 9.0 & 11.0 & 13.0 & 16.0 & 14.0 \\
\hline & Indonesien & 0.0 & 6.0 & 9.0 & 12.0 & 12.0 & 15.0 & 14.0 \\
\hline & Jordanien & 0.0 & 6.0 & 10.0 & 12.0 & 12.0 & 16.0 & 14.5 \\
\hline & Kasachstan & 0.0 & 4.0 & 9.0 & 11.5 & 12.5 & 15.0 & 14.0 \\
\hline & Kirgisistan & 0.0 & 4.0 & 8.0 & 11.0 & 10.0 & 15.0 & 13.0 \\
\hline & Lettland & 0.0 & 3.0 & 8.0 & 11.0 & 11.0 & 16.0 & 16.0 \\
\hline & Liechtenstein & 0.0 & 5.0 & 9.0 & 11.0 & 13.0 & 17.0 & 14.0 \\
\hline & Litauen & 0.0 & 3.0 & 8.0 & 11.0 & 11.0 & 16.0 & 15.0 \\
\hline & Macau (China) & 0.0 & 6.0 & 9.0 & 11.0 & 12.0 & 16.0 & 15.0 \\
\hline & Montenegro & 0.0 & 4.0 & 8.0 & 11.0 & 12.0 & 16.0 & 15.0 \\
\hline & Panama & 0.0 & 6.0 & 9.0 & 12.0 & 12.0 & 16.0 & a \\
\hline & Peru & 0.0 & 6.0 & 9.0 & 11.0 & 11.0 & 17.0 & 14.0 \\
\hline & Katar & 0.0 & 6.0 & 9.0 & 12.0 & 12.0 & 16.0 & 15.0 \\
\hline & Rumänien & 0.0 & 4.0 & 8.0 & 11.5 & 12.5 & 16.0 & 14.0 \\
\hline & Russ. Föderation & 0.0 & 4.0 & 9.0 & 11.5 & 12.0 & 15.0 & a \\
\hline & Serbien & 0.0 & 4.0 & 8.0 & 11.0 & 12.0 & 17.0 & 14.5 \\
\hline & Shanghai (China) & 0.0 & 6.0 & 9.0 & 12.0 & 12.0 & 16.0 & 15.0 \\
\hline & Singapur & 0.0 & 6.0 & 8.0 & 10.5 & 10.5 & 12.5 & 12.5 \\
\hline & Chinesisch Taipeh & 0.0 & 6.0 & 9.0 & 12.0 & 12.0 & 16.0 & 14.0 \\
\hline & Thailand & 0.0 & 6.0 & 9.0 & 12.0 & 12.0 & 16.0 & 14.0 \\
\hline & Trinidad und Tobago & 0.0 & 5.0 & 9.0 & 12.0 & 12.0 & 16.0 & 15.0 \\
\hline & Tunesien & 0.0 & 6.0 & 9.0 & 12.0 & 13.0 & 17.0 & 16.0 \\
\hline & Uruguay & 0.0 & 6.0 & 9.0 & 12.0 & 12.0 & 17.0 & 15.0 \\
\hline
\end{tabular}

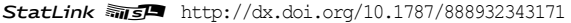


[Teil 1/1]

Mehr-Ebenen-Modell zur Schätzung von Klasseneffekten im Bereich Lesekompetenz nach Tabelle A1.3 Berücksichtigung mehrerer Hintergrundvariablen

\begin{tabular}{|c|c|c|c|c|c|c|c|c|c|c|c|c|c|c|c|c|c|}
\hline & \multicolumn{2}{|c|}{ Klassenstufe } & \multicolumn{2}{|c|}{$\begin{array}{l}\text { PISA-Index des } \\
\text { wirtschaftl., } \\
\text { sozialen und } \\
\text { kulturellen } \\
\text { Status }\end{array}$} & \multicolumn{2}{|c|}{$\begin{array}{c}\text { PISA- } \\
\text { Index des } \\
\text { wirtschaftl., } \\
\text { sozialen und } \\
\text { kulturellen } \\
\text { Status, } \\
\text { quadriert }\end{array}$} & \multicolumn{2}{|c|}{\begin{tabular}{|c|} 
Durchschnittl. \\
PISA-Index des \\
wirtschaftl., \\
sozialen und \\
kulturellen \\
Status der \\
Schule
\end{tabular}} & \multicolumn{2}{|c|}{$\begin{array}{l}\text { Schüler der } \\
\text { 1. Generation }\end{array}$} & \multicolumn{2}{|c|}{$\begin{array}{c}\text { Anteil } \\
\text { Schüler der } \\
\text { 1. Generation } \\
\text { in der Schule }\end{array}$} & $\begin{array}{r}\text { Gesc } \\
\text { we }\end{array}$ & $\begin{array}{l}\text { lecht - } \\
\text { blich }\end{array}$ & Auffang & variable \\
\hline & & Koeff. & S.E. & Koeff. & S.E. & Koeff. & S.E. & Koeff. & S.E. & Koeff. & S.E. & Koeff. & S.E. & Koeff. & S.E. & Koeff. & S.E. \\
\hline ‡ & Australien & 33.2 & (1.95) & 30.0 & (1.36) & -3.8 & (1.05) & 66.4 & $(1.87)$ & -7.4 & (2.82) & 0.1 & $(0.07)$ & 32.9 & (1.91) & 466.0 & (1.39) \\
\hline : & Österreich & 35.3 & (2.18) & 11.4 & (1.66) & -0.5 & $(1.00)$ & 89.7 & (3.86) & -33.1 & (6.11) & 1.4 & $(0.13)$ & 19.9 & $(2.67)$ & 467.9 & $(2.45)$ \\
\hline 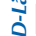 & Belgien & 48.9 & (1.98) & 10.0 & (1.12) & -0.1 & $(0.63)$ & 79.9 & $(1.73)$ & -3.2 & (5.18) & 0.3 & $(0.11)$ & 11.3 & $(1.81)$ & 507.0 & (1.70) \\
\hline & Kanada & 45.0 & (2.14) & 19.4 & $(1.52)$ & 1.5 & $(0.91)$ & 33.9 & $(2.28)$ & -13.7 & (3.18) & 0.3 & $(0.04)$ & 30.4 & $(1.60)$ & 483.4 & (1.76) \\
\hline & Chile & 35.5 & (1.55) & 8.6 & (1.52) & 0.3 & $(0.63)$ & 37.4 & $(1.61)$ & c & c & c & c & 13.8 & (2.33) & 478.6 & $(1.60)$ \\
\hline & Tschech. Rep. & 44.6 & (3.39) & 13.4 & (1.89) & -2.3 & (1.47) & 111.5 & $(3.12)$ & -8.9 & (12.29) & 0.4 & $(0.33)$ & 32.3 & $(2.84)$ & 460.7 & (2.39) \\
\hline & Dänemark & 36.1 & (3.02) & 27.9 & (1.51) & -2.8 & (1.10) & 35.1 & $(2.91)$ & -37.5 & $(5.97)$ & 0.0 & $(0.14)$ & 25.5 & (2.59) & 474.0 & (1.95) \\
\hline & Estland & 44.4 & $(2.74)$ & 14.1 & $(1.80)$ & 1.6 & (1.43) & 52.1 & $(4.52)$ & -18.7 & (14.08) & -3.3 & $(0.44)$ & 36.7 & (2.45) & 485.8 & $(2.02)$ \\
\hline & Finnland & 37.3 & $(3.60)$ & 27.7 & (1.66) & -2.5 & (1.30) & 10.4 & (3.28) & -56.0 & (13.09) & -0.1 & $(0.29)$ & 51.5 & $(2.26)$ & 500.6 & $(2.02)$ \\
\hline & Frankreich & 47.1 & (5.14) & 12.5 & $(1.70)$ & -1.9 & (1.12) & 81.6 & $(4.04)$ & -11.6 & (9.24) & 0.2 & $(0.15)$ & 25.9 & $(2.67)$ & 516.5 & (2.35) \\
\hline & Deutschland & 34.4 & (1.74) & 9.2 & (1.23) & -1.6 & $(0.74)$ & 109.1 & (2.16) & -13.2 & $(4.80)$ & 0.2 & $(0.12)$ & 27.2 & (1.92) & 458.0 & (1.46) \\
\hline & Griechenland & 22.6 & (10.86) & 15.9 & (1.46) & 1.5 & (1.07) & 41.2 & (2.84) & -15.0 & (7.82) & 0.0 & $(0.18)$ & 36.2 & (2.55) & 469.0 & (2.04) \\
\hline & Ungarn & 25.6 & (2.19) & 8.3 & (1.39) & 0.9 & $(0.87)$ & 74.8 & (2.09) & 2.8 & $(7.92)$ & 0.0 & $(0.27)$ & 21.4 & $(2.22)$ & 494.1 & (1.65) \\
\hline & Island & c & c & 29.8 & (2.56) & -5.1 & (1.56) & -3.8 & $(5.12)$ & -52.2 & (11.45) & -1.3 & $(0.40)$ & 44.9 & (2.59) & 469.1 & (4.23) \\
\hline & Irland & 18.2 & (1.99) & 29.7 & (1.78) & -3.5 & (1.44) & 43.6 & (2.68) & -32.8 & (6.52) & -0.1 & $(0.20)$ & 33.9 & (3.62) & 474.8 & (2.77) \\
\hline & Israel & 36.6 & (3.85) & 19.9 & (1.90) & 3.4 & (1.04) & 104.7 & $(2.10)$ & -11.0 & (6.13) & 1.5 & $(0.08)$ & 29.4 & $(2.81)$ & 460.1 & (2.13) \\
\hline & Italien & 36.1 & (1.67) & 4.5 & $(0.69)$ & -1.4 & $(0.42)$ & 76.4 & (1.07) & -29.7 & (3.36) & 0.2 & $(0.08)$ & 24.0 & (1.29) & 491.4 & $(0.85)$ \\
\hline & Japan & $\mathrm{a}$ & $\mathrm{a}$ & 4.1 & (1.51) & 0.1 & (1.47) & 144.2 & $(2.40)$ & C & c & C & C & 27.9 & (2.43) & 508.6 & (1.58) \\
\hline & Korea & 31.2 & $(9.77)$ & 12.9 & (1.42) & 1.9 & (1.18) & 64.9 & $(2.24)$ & a & $\mathrm{a}$ & $\mathrm{a}$ & $\mathrm{a}$ & 30.6 & (3.21) & 537.7 & (2.08) \\
\hline & Luxemburg & 45.3 & (1.95) & 16.6 & (1.31) & -2.6 & (1.08) & 62.0 & (2.89) & -10.4 & (5.11) & -0.2 & $(0.10)$ & 33.0 & $(2.22)$ & 435.7 & (2.40) \\
\hline & Mexiko & 32.6 & (1.59) & 7.5 & $(0.92)$ & 0.8 & $(0.34)$ & 27.8 & $(0.80)$ & -41.9 & (6.36) & -1.8 & $(0.15)$ & 17.9 & (1.03) & 473.7 & (1.02) \\
\hline & Niederlande & 26.6 & (2.04) & 6.0 & (1.52) & -1.2 & (1.02) & 106.7 & $(2.32)$ & -11.6 & $(5.72)$ & 1.7 & $(0.14)$ & 15.3 & (1.85) & 484.5 & (2.33) \\
\hline & Neuseeland & 44.2 & (4.15) & 38.9 & $(1.82)$ & -1.7 & (1.44) & 56.3 & (3.35) & -12.2 & (3.84) & 0.0 & $(0.10)$ & 44.8 & $(2.62)$ & 496.5 & (2.44) \\
\hline & Norwegen & 37.6 & (18.19) & 34.2 & $(2.00)$ & -3.4 & $(1.62)$ & 31.1 & $(4.32)$ & -33.4 & $(7.52)$ & 0.4 & $(0.25)$ & 48.3 & (2.56) & 453.2 & $(2.87)$ \\
\hline & Polen & 73.8 & $(4.44)$ & 29.4 & (1.59) & -1.8 & (1.21) & 19.4 & (2.99) & c & c & c & c & 44.2 & (2.41) & 498.9 & (1.89) \\
\hline & Portugal & 48.9 & $(1.71)$ & 12.0 & $(0.94)$ & 1.0 & $(0.64)$ & 21.3 & (1.33) & -5.3 & (5.75) & 0.0 & $(0.23)$ & 22.9 & (1.84) & 518.6 & $(1.92)$ \\
\hline & Slowak. Rep. & 34.2 & (3.85) & 14.7 & (1.44) & -3.2 & $(0.98)$ & 64.3 & $(6.30)$ & c & c & c & c & 39.1 & (2.58) & 483.2 & $(2.33)$ \\
\hline & Slowenien & 22.8 & (3.41) & 4.8 & (1.28) & 0.0 & (1.25) & 100.2 & $(2.74)$ & -23.4 & (7.48) & -0.2 & $(0.24)$ & 27.7 & (2.16) & 452.4 & (1.63) \\
\hline & Spanien & 61.7 & (1.22) & 9.8 & $(0.83)$ & 0.4 & $(0.64)$ & 22.7 & (1.25) & -29.7 & (2.86) & 0.4 & $(0.04)$ & 18.0 & (1.42) & 511.3 & (1.07) \\
\hline & Schweden & 63.8 & $(6.69)$ & 31.4 & $(1.82)$ & -1.3 & (1.04) & 49.0 & $(6.55)$ & -38.8 & (8.53) & 0.3 & $(0.34)$ & 43.2 & $(2.41)$ & 454.4 & (3.62) \\
\hline & Schweiz & 45.5 & (2.75) & 18.2 & $(1.27)$ & -1.0 & (1.23) & 59.5 & (2.95) & -25.1 & (3.99) & -0.7 & $(0.11)$ & 27.0 & $(2.00)$ & 488.8 & (1.50) \\
\hline & Türkei & 33.7 & (1.96) & 7.7 & (1.50) & 0.3 & $(0.61)$ & 46.3 & $(1.70)$ & C & c & c & c & 27.9 & (1.74) & 524.0 & (1.59) \\
\hline & Ver. Königreich & 35.9 & $(6.21)$ & 27.7 & (2.01) & -0.3 & (1.51) & 65.7 & (2.49) & -13.6 & (8.49) & -0.3 & $(0.13)$ & 23.1 & (2.48) & 468.7 & (1.73) \\
\hline & Ver. Staaten & 36.3 & $(2.17)$ & 23.5 & $(1.70)$ & 4.4 & (1.15) & 50.4 & $(2.56)$ & -5.6 & $(5.57)$ & 0.8 & $(0.14)$ & 25.4 & $(2.36)$ & 463.5 & $(2.01)$ \\
\hline 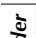 & Albanien & 11.9 & $(5.07)$ & 20.8 & (3.04) & 3.2 & (1.35) & 43.0 & $(2.47)$ & c & c & c & c & 56.5 & $(3.40)$ & 421.5 & (3.44) \\
\hline & Argentinien & 33.6 & $(2.50)$ & 11.2 & (1.96) & 0.9 & $(0.87)$ & 52.6 & $(2.03)$ & -27.0 & (10.55) & 0.5 & $(0.20)$ & 24.0 & (2.38) & 439.7 & $(2.32)$ \\
\hline & Aserbaidschan & 13.2 & (1.78) & 10.5 & $(1.67)$ & 1.3 & $(0.90)$ & 36.4 & $(2.00)$ & -9.8 & (12.34) & -0.3 & $(0.49)$ & 22.6 & (2.16) & 390.9 & $(2.12)$ \\
\hline & Brasilien & 36.1 & $(1.23)$ & 7.7 & (1.54) & 1.3 & $(0.57)$ & 38.3 & (1.25) & -71.7 & (17.16) & -0.9 & $(0.47)$ & 20.2 & (1.63) & 445.5 & (1.33) \\
\hline & Bulgarien & 27.8 & (5.08) & 15.7 & (1.93) & 0.2 & (1.29) & 75.7 & (3.99) & C & c & c & C & 42.1 & (3.51) & 423.7 & $(2.61)$ \\
\hline & Kolumbien & 33.2 & $(1.12)$ & 6.9 & $(2.01)$ & 0.9 & $(0.72)$ & 39.4 & $(1.53)$ & C & c & c & C & 3.2 & $(2.17)$ & 477.7 & $(1.83)$ \\
\hline & Kroatien & 31.8 & (2.33) & 10.3 & (1.36) & -4.0 & $(0.99)$ & 75.3 & $(2.01)$ & -13.0 & $(5.71)$ & -0.1 & $(0.22)$ & 31.4 & $(2.56)$ & 472.8 & (1.69) \\
\hline & Dubai (VAE) & 34.6 & (1.56) & 15.2 & (1.52) & 3.2 & (1.03) & 25.9 & (3.13) & 21.5 & (3.25) & 1.1 & $(0.05)$ & 28.2 & (3.94) & 362.4 & (2.92) \\
\hline & Hongkong (China) & 33.6 & (2.03) & -0.9 & $(1.70)$ & -1.0 & $(0.76)$ & 41.9 & (1.64) & 23.4 & $(3.70)$ & -0.4 & $(0.06)$ & 21.9 & $(2.42)$ & 575.8 & $(1.83)$ \\
\hline & Indonesien & 14.4 & $(2.00)$ & 4.7 & (2.44) & 0.9 & $(0.62)$ & 29.1 & $(1.83)$ & C & c & c & c & 28.0 & (1.48) & 430.8 & $(2.46)$ \\
\hline & Jordanien & 47.6 & (6.38) & 17.7 & $(1.52)$ & 0.7 & $(0.81)$ & 26.9 & $(1.55)$ & -11.5 & $(7.50)$ & -0.2 & $(0.20)$ & 48.1 & $(2.73)$ & 415.5 & (2.04) \\
\hline & Kasachstan & 22.2 & $(2.42)$ & 16.2 & $(2.12)$ & -1.7 & (1.31) & 55.7 & $(2.70)$ & -12.2 & (6.78) & 0.0 & $(0.10)$ & 38.1 & $(2.23)$ & 411.1 & $(1.57)$ \\
\hline & Kirgisistan & 20.8 & (2.92) & 18.3 & $(2.23)$ & 1.7 & (1.10) & 75.2 & $(2.03)$ & -23.4 & (21.78) & 3.3 & $(0.50)$ & 46.0 & $(2.45)$ & 345.7 & $(1.83)$ \\
\hline & Lettland & 43.8 & $(3.07)$ & 16.2 & (1.89) & -0.8 & $(1.35)$ & 37.0 & $(2.77)$ & c & c & c & c & 38.9 & (2.36) & 479.6 & (1.77) \\
\hline & Liechtenstein & 23.8 & (7.40) & 2.1 & (4.18) & -5.3 & (3.07) & 112.5 & (12.17) & -12.6 & $(10.22)$ & -0.7 & $(0.44)$ & 20.3 & (6.86) & 499.8 & $(8.42)$ \\
\hline & Litauen & 27.4 & $(2.87)$ & 18.1 & (1.56) & 0.2 & (1.04) & 44.0 & $(2.45)$ & c & c & C & C & 51.1 & $(2.34)$ & 447.6 & (1.87) \\
\hline & Macau (China) & 36.7 & (1.01) & 1.8 & (1.61) & -1.1 & $(0.78)$ & 1.0 & $(4.75)$ & 16.7 & (2.17) & -0.1 & $(0.23)$ & 14.1 & (1.51) & 511.0 & (3.47) \\
\hline & Montenegro & 22.9 & (3.44) & 12.1 & (1.38) & -0.3 & (1.05) & 64.2 & (6.54) & -1.8 & (6.69) & -1.2 & $(0.32)$ & 39.3 & $(2.63)$ & 409.5 & (2.58) \\
\hline & Panama & 32.6 & $(3.41)$ & 7.9 & (2.42) & 1.2 & $(0.79)$ & 45.8 & $(2.60)$ & -3.4 & (10.77) & -1.4 & $(0.16)$ & 15.8 & $(4.48)$ & 431.3 & (3.22) \\
\hline & Peru & 27.5 & $(1.23)$ & 10.5 & $(2.05)$ & 0.9 & $(0.64)$ & 47.2 & $(1.46)$ & C & C & C & C & 8.3 & $(2.17)$ & 445.6 & (1.59) \\
\hline & Katar & 30.7 & (1.70) & 5.3 & $(0.98)$ & 0.4 & $(0.85)$ & 12.7 & (2.91) & 31.5 & (2.98) & 1.7 & $(0.07)$ & 31.4 & (3.71) & 302.5 & (2.94) \\
\hline & Rumänien & 19.6 & $(4.19)$ & 10.7 & $(1.63)$ & -0.3 & $(0.79)$ & 63.9 & (2.34) & C & c & c & c & 13.7 & $(2.56)$ & 446.4 & (1.70) \\
\hline & Russ. Föderation & 31.0 & (2.01) & 18.2 & (1.93) & -1.6 & $(1.40)$ & 38.8 & (3.32) & -9.1 & (5.88) & -0.4 & $(0.22)$ & 38.7 & $(2.28)$ & 452.9 & (1.89) \\
\hline & Serbien & 21.3 & $(4.48)$ & 9.2 & $(1.25)$ & -0.8 & $(0.74)$ & 55.1 & $(3.42)$ & 1.2 & (5.65) & 0.3 & $(0.13)$ & 27.1 & $(2.22)$ & 425.1 & (1.60) \\
\hline & Shanghai (China) & 21.8 & (3.34) & 4.6 & (1.41) & 0.1 & $(0.85)$ & 57.3 & $(1.48)$ & C & c & c & c & 29.3 & (1.98) & 583.5 & (2.04) \\
\hline & Singapur & 28.9 & (2.09) & 22.2 & (2.19) & -2.8 & $(1.14)$ & 104.7 & $(2.86)$ & 0.4 & $(4.21)$ & -1.0 & $(0.13)$ & 24.6 & $(2.57)$ & 590.2 & $(2.76)$ \\
\hline & Chinesisch Taipeh & 15.4 & $(4.12)$ & 15.5 & $(1.50)$ & -1.2 & (1.05) & 82.8 & (3.06) & c & c & c & c & 36.8 & $(2.25)$ & 515.6 & (2.03) \\
\hline & Thailand & 22.1 & (2.05) & 10.4 & (1.54) & 2.4 & $(0.66)$ & 28.8 & (1.31) & $\mathrm{a}$ & $\mathrm{a}$ & $\mathrm{a}$ & $\mathrm{a}$ & 31.3 & (1.78) & 454.6 & $(1.67)$ \\
\hline & Trinidad und Tobago & 35.3 & $(1.60)$ & -0.6 & $(2.00)$ & -0.2 & $(0.91)$ & 123.2 & $(3.42)$ & -9.2 & (13.59) & -0.7 & $(0.28)$ & 40.4 & $(2.90)$ & 484.9 & $(2.77)$ \\
\hline & Tunesien & 49.7 & $(1.57)$ & 3.7 & (1.76) & 0.7 & $(0.56)$ & 17.8 & $(1.25)$ & c & c & C & c & 14.4 & (1.84) & 449.6 & (1.63) \\
\hline & Uruguay & 41.4 & (1.49) & 12.4 & $(1.58)$ & 0.5 & $(0.75)$ & 29.7 & $(1.58)$ & c & c & c & c & 30.1 & $(2.48)$ & 464.2 & $(2.29)$ \\
\hline
\end{tabular}

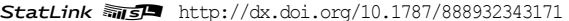


nicht zwangsläufig auf negative Antworten der Schülerinnen und Schüler auf die gestellten Fragen schließen lassen. Ein negativer Wert weist lediglich darauf hin, dass die jeweiligen Befragten weniger positiv antworteten, als das für den Durchschnitt aller Befragten in den OECD-Ländern der Fall war. Dementsprechend bedeutet ein positiver Wert bei einem Index, dass die jeweiligen Befragten positivere Antworten gaben, als dies für den Durchschnitt der Befragten in den OECD-Ländern der Fall war.

Wie oben erwähnt, wurde der Durchschnitt des Indexwerts der Schülerpopulation der OECD-Länder 2009 für die neu geschätzten Indizes gleich null und die Standardabweichung gleich eins gesetzt. Die Mittelwerte und Standardabweichungen früherer Erhebungsrunden können jedoch hiervon abweichen. Die für die Trendanalyse neu geschätzten Indizes müssen daher nicht mit den in früheren Berichten angegebenen Werten übereinstimmen. Die Länderdurchschnitte und sonstigen Statistiken dürften zwar nahe bei den zuvor angegebenen Werten liegen, sie könnten jedoch oftmals leicht abweichen, da die Indizes in den früheren Studien anhand der Daten aus der früheren Erhebung standardisiert wurden und nicht anhand der Ergebnisse aus PISA 2009.

Neben den in diesem Anhang beschriebenen einfachen Indizes und Skalenindizes gibt es eine Reihe von Variablen aus den Fragebogen, die Einzelitems entsprechen, die nicht zur Konstruktion der Indizes verwendet wurden. Diese nicht umkodierten Variablen weisen das Präfix „ST" für die Items im Schülerfragebogen, „SC" für die Items im Schulleiterfragebogen sowie "PA“ für die Items im Elternfragebogen auf. Alle Kontextfragebogen sowie die internationale PISA-Datenbank mit sämtlichen Variablen sind auf www.pisa.oecd.org verfügbar.

\section{Einfache Indizes auf Schülerebene}

\section{Alter}

Die Variable ALTER wird für Trends anders berechnet als für die Ergebnisse aus PISA 2009, da die zur Konstruktion des Altersindex verwendeten Daten zum Monat, in dem die Tests tatsächlich durchgeführt wurden, für das Jahr 2000 nicht verfügbar sind. Für die Trendanalyse wurden somit stattdessen die Daten zum mittleren Monat des Testzeitraums verwendet. Die Ergebnisse aus allen Erhebungen wurden als Differenz zwischen dem mittleren Monat und dem Jahr, in dem die Schülerinnen und Schüler getestet wurden, und ihrem Geburtsmonat und -jahr, ausgedrückt in Jahren und Monaten, neu berechnet.

\section{Berufliche Stellung der Eltern}

Sowohl über die Väter als auch über die Mütter der Schülerinnen und Schüler wurden mit Hilfe von offenen Fragen im Schülerfragebogen berufsspezifische Daten erhoben (ST9a, ST9b, ST12, ST13a, ST13b und ST16). Die Antworten wurden in vierstellige ISCO-Codes kodiert (ILO, 1990) und dann im sozioökonomischen Index (SEI) von Ganzeboom et al. abgebildet (1992). Je höher die SEI-Werte, desto höher die berufliche Stellung. Auf diese Weise wurden die folgenden drei Indizes erstellt:

- Der Index der beruflichen Stellung der Mutter (BMMJ).

- Der Index der beruflichen Stellung des Vaters (BFMJ).

- Der Index der höchsten beruflichen Stellung der Eltern (HISEI) entspricht dem SEI-Wert des Elternteils mit der jeweils höheren beruflichen Stellung bzw. des Elternteils, für den ein entsprechender Wert vorhanden ist.

\section{Bildungsabschluss der Eltern}

Der Bildungsabschluss der Eltern wird auf der Grundlage der Antworten der Schülerinnen und Schüler im Schülerfragebogen (ST10, ST11, ST14 und ST15) nach ISCED klassifiziert (OECD, 1999). Es sei darauf hingewiesen, dass sich das Frageformat für die Schulbildung in PISA 2009 von dem in den PISA-Erhebungsrunden 2000, 2003 und 2006 verwendeten zwar unterscheidet, die Methode für die Berechnung des Bildungsabschlusses der Eltern jedoch nach wie vor dieselbe ist.

Wie in PISA 2000, PISA 2003 und PISA 2006 wurden die Indizes konstruiert, indem für jeden Elternteil der höchste Abschluss ausgewählt und einer der folgenden Kategorien zugeordnet wurde: (0) Kein Abschluss, (1) ISCED 1 (Primarbereich), (2) ISCED 2 (Sekundarbereich I), (3) ISCED 3B oder 3C (berufsbildender/vorberuflicher Sekundarbereich II), (4) ISCED 3A (Sekundarbereich II) und/oder ISCED 4 (nichttertiärer postsekundärer Bereich), (5) ISCED 5B (berufsbildender Tertiärbereich) und (6) ISCED 5A, 6 (theoretisch orientierter Tertiär- und Postgraduiertenbereich). Anhand dieser Kategorien wurden die folgenden drei Indizes konzipiert:

- Index des Bildungsabschlusses der Mutter (MISCED).

- Index des Bildungsabschlusses des Vaters (FISCED).

- Index des höchsten Bildungsabschlusses der Eltern (HISCED), entspricht der ISCED-Stufe des Elternteils mit dem jeweils höheren Bildungsabschluss.

Der höchste Bildungsabschluss der Eltern wurde ferner in die Anzahl der Ausbildungsjahre (PARED) umgerechnet. Wegen der Umrechnung des Bildungsstands in Ausbildungsjahre vgl. Tabelle A1.2.

\section{Relative Klassenstufe}

Die Daten zur Klassenstufe der Schülerinnen und Schüler können sowohl dem Schülerfragebogen (ST01) als auch den Unterlagen über den bisherigen Bildungsweg der Schülerinnen und Schüler entnommen werden. Wie bei allen Variablen, die sowohl in den 
Unterlagen über den bisherigen Bildungsweg als auch in dem Fragebogen zu finden sind, werden Unstimmigkeiten zwischen den beiden Quellen geprüft und während der Datenbereinigung gelöst. Zur Berücksichtigung der Varianz zwischen den Ländern gibt der relative Klassenstufenindex (GRADE) an, ob sich die Schülerinnen und Schüler in Bezug auf ein gegebenes Land in der Regelklassenstufe (Wert $=0$ ) oder über bzw. unter der Regelklassenstufe befinden (+x-Klassenstufen, -x-Klassenstufen).

Der Zusammenhang zwischen Klassenstufe und Schülerleistungen wurde mit Hilfe eines Mehrebenen-Modells geschätzt, in dem folgende Hintergrundvariablen berücksichtigt wurden: a) der PISA-Index des wirtschaftlichen, sozialen und kulturellen Status, b) der PISA-Index des wirtschaftlichen, sozialen und kulturellen Status zum Quadrat, c) der Mittelwert der Schule auf dem PISA-Index des wirtschaftlichen, sozialen und kulturellen Status, d) ein Indikator für im Ausland geborene Schülerinnen und Schüler der ersten Generation, e) der Prozentsatz der Schülerinnen und Schüler der ersten Generation in der Schule sowie f) das Geschlecht der Schülerinnen und Schüler.

In Tabelle A1.3 sind die Ergebnisse des Mehrebenen-Modells dargestellt. Spalte 1 in Tabelle A1.3 enthält eine Schätzung der mit einer Klassenstufe (oder einem Schuljahr) assoziierten Punktzahldifferenz. Schätzen lässt sich diese Differenz für die 32 OECDLänder, in denen eine beträchtliche Zahl 15-Jähriger in den PISA-Stichproben mindestens zwei unterschiedliche Klassenstufen besuchte. Da nicht davon ausgegangen werden kann, dass die 15-Jährigen nach dem Zufallsprinzip auf die Klassenstufen verteilt sind, mussten für die oben genannten Umfeldfaktoren, die einen Einfluss auf die Zuordnung der Schülerinnen und Schüler zu bestimmten Klassenstufen haben können, Anpassungen vorgenommen werden. Diese Anpassungen sind in den Spalten 2-7 der Tabelle dargestellt. Es ist zwar möglich, den typischen Leistungsunterschied zwischen Schülerinnen und Schülern aus zwei aufeinanderfolgenden Klassenstufen, korrigiert um Auswahl- und Kontexteffekte, zu schätzen, dieser Unterschied kann jedoch nicht automatisch mit den Lernfortschritten gleichgesetzt werden, die die Schülerinnen und Schüler im vorausgegangenen Jahr gemacht haben, sondern sollte vielmehr als Untergrenze der erzielten Fortschritte interpretiert werden. Dies ist nicht nur darauf zurückzuführen, dass unterschiedliche Schülerinnen und Schüler getestet wurden, sondern auch auf die Tatsache, dass der Inhalt der PISA-Tests nicht ausdrücklich auf den Lehrstoff abstellte, den die Schülerinnen und Schüler im vorausgegangenen Jahr durchgenommen hatten, sondern ganz allgemein auf die Bewertung der kumulativen Lernstände von Schülerinnen und Schülern bis zum Alter von 15 Jahren. Enthält beispielsweise das Curriculum der von 15-Jährigen besuchten Klassenstufen hauptsächlich Unterrichtsstoff, der in PISA nicht beurteilt wird (aber in früheren Schuljahren auf dem Lehrplan stand), wird der erzielte Lernfortschritt durch den beobachteten Leistungsunterschied unterzeichnet.

\section{Migrationsstatus und sprachlicher Hintergrund}

Die Daten über das Geburtsland der Schülerinnen und Schüler sowie ihrer Eltern (ST17) wurden anhand von länderspezifischen ISOCodes auf ähnliche Weise erhoben wie in PISA 2000, PISA 2003 und PISA 2006. Die ISO-Codes der Geburtsländer der Schülerinnen und Schüler sowie ihrer Eltern sind in der internationalen PISA-Datenbank verfügbar (COBN_S, COBN_M und COBN_F).

Für die Trendanalysen wurde ein dichotomer Index konstruiert, der die folgenden Kategorien umfasst: (1) einheimische Schülerinnen und Schüler bzw. Schüler ohne Migrationshintergrund (im Erhebungsland geborene Schülerinnen und Schüler mit mindestens einem im Erhebungsland geborenen Elternteil; Schülerinnen und Schüler, die in einem anderen Land geboren wurden, aber wenigstens einen Elternteil haben, der im Erhebungsland geboren wurde, werden ebenfalls als „einheimisch" eingestuft), (2) Schülerinnen und Schüler der zweiten und der ersten Generation (im Erhebungsland geborene Schülerinnen und Schüler mit in einem anderen Land geborenen Eltern sowie nicht im Erhebungsland geborene Schülerinnen und Schüler mit ebenfalls in einem anderen Land geborenen Eltern). Schülerinnen und Schüler, die entweder zu ihrem eigenen Geburtsland, dem ihrer Mutter oder dem ihres Vaters oder zu allen dreien keine Angaben machten, wurden bei dieser Variablen mit Fehlwerten erfasst.

Die Schülerinnen und Schüler gaben die Sprache an, die sie in der Regel zu Hause sprechen. Die Daten werden in länderspezifischen Sprachencodes erfasst, die mit folgenden beiden Werten in die Variable ST19Q01 umkodiert wurden: (1) Die zu Hause gesprochene Sprache ist mit der Testsprache identisch und (2) die zu Hause gesprochene Sprache ist nicht mit der Testsprache identisch. Eine ähnliche Umkodierung erfolgte für 2003 und 2006. Im Jahr 2000 gaben die Schülerinnen und Schüler unmittelbar an, ob sie die Testsprache oder eine andere Sprache zu Hause sprechen. Diese Antworten wurden dichotomisiert und mit den umkodierten Werten für 2003, 2006 und 2009 verglichen.

\section{Skalenindizes auf Schülerebene}

Vier Indizes wurden auf der Grundlage der Ausstattung des Elternhauses erstellt, nämlich WEALTH, CULTPOSS, HEDRES und HOMEPOS. Diese sind weiter unten ausführlicher beschrieben. Diese Indizes wurden in zwei Schritten geschätzt. Da internationale Itemparameter als nicht geeignet erachtet wurden, um Skalen für die Ausstattung des Elternhauses zu schätzen, wurde ein zweistufiges Verfahren verwendet. In Schritt 1 wurde eine konkurrente Schätzung vorgenommen, um diese Indizes anhand der nationalen Itemparameter zu berechnen (d.h. die Itemparameter wurden auf der Ebene der einzelnen Länder geschätzt). Auf diese Weise war es möglich, länderspezifische Trends bei den Ausstattungsindizes zu betrachten. Um jedoch Vergleiche dieser Skalen zwischen den Ländern zu ermöglichen, wurden die relativen Positionen der Länder anhand einer gemeinsamen Skala geschätzt. Die resultierenden Differenzen bei den Mittelwerten der Ausstattungsindizes wurden anhand einer linearen Transformation auf die gewichteten Maximum-Likelihood-Schätzwerte (aus Schritt 1) angewendet. 


\section{Wohlstand der Familie}

Der Index des Wohlstands der Familie (WEALTH) beruht auf den Angaben der Schülerinnen und Schüler über das Vorhandensein der folgenden Dinge in ihrem Elternhaus: ein eigenes Zimmer für sie, ein Internetanschluss, eine Geschirrspülmaschine (als länderspezifischer Gegenstand), ein DVD-Player sowie drei weitere länderspezifische Gegenstände (einige der in ST20 aufgelisteten Items); ebenfalls berücksichtigt wurden ihre Angaben zur Zahl der Handys, Fernseher, Computer, Autos sowie Zimmer mit Badewanne oder Dusche in ihrem Elternhaus (ST21).

\section{Bildungsressourcen im Elternhaus}

Der Index der Bildungsressourcen im Elternhaus (HEDRES) beruht auf Items, die zur Messung der im Elternhaus vorhandenen Bildungsressourcen dienen, darunter ein Schreibtisch und ein ruhiger Platz zum Lernen, ein Computer, den die Schülerinnen und Schüler für Schularbeiten verwenden können, Lernsoftware, Bücher, die den Schülerinnen und Schülern bei den Schularbeiten helfen, technische Nachschlagewerke sowie ein Wörterbuch (einige der in ST20 aufgelisteten Items).

\section{Besitz von Kulturgütern}

Der Kulturgüterindex (CULTPOSS) beruht auf den Angaben der Schülerinnen und Schüler über das Vorhandensein der folgenden Dinge in ihrem Elternhaus: klassische Literatur, Gedichtbände und Kunstwerke (einige der in ST20 aufgelisteten Items).

\section{Wirtschaftlicher, sozialer und kultureller Status}

Der PISA-Index des wirtschaftlichen, sozialen und kulturellen Status (ESCS) wurde aus den folgenden drei Indizes abgeleitet: dem Index der höchsten beruflichen Stellung der Eltern (HISEI), dem Index des höchsten Bildungsabschlusses der Eltern in Ausbildungsjahren gemäß ISCED (PARED) und dem Index der Ausstattung des Elternhauses (HOMEPOS). Der Index der Ausstattung des Elternhauses (HOMEPOS) umfasst alle Items der Indizes WEALTH, CULTPOSS und HEDRES ebenso wie die Zahl der Bücher im Elternhaus, die in eine vierstufige Kategorienvariable umkodiert wurde (0-10 Bücher, 11-25 bzw. 26-100 Bücher, 101-200 bzw. 201-500 Bücher, über 500 Bücher). Um eine Trendstudie zu ermöglichen, wurden für jede Erhebungsrunde ähnliche Abbildungen der ISCEDStufen im Index PARED verwendet, und der Index der Ausstattung des Elternhauses (HOMEPOS) wurde ebenfalls konkurrent für die einzelnen Erhebungsrunden geschätzt.

Der PISA-Index des wirtschaftlichen, sozialen und kulturellen Status (ESCS) wurde aus einer Hauptkomponentenanalyse standardisierter Variablen (in der jede Variable einen OECD-Mittelwert von null und eine Standardabweichung von eins hat) abgeleitet, wobei die Faktorwerte für die erste Hauptkomponente als Messgrößen des ESCS-Index dienten.

Die Hauptkomponentenanalyse wurde ebenfalls für jedes Teilnehmerland durchgeführt, um festzustellen, inwieweit die Indexkomponenten in den Ländern auf dieselbe Art und Weise operieren. Aus der Analyse ging hervor, dass die Strukturen für die Gewichtung der einzelnen Faktoren in den Ländern sehr ähnlich waren und dass alle drei Komponenten in gleichem Ausmaß im Index berücksichtigt wurden. Für die berufliche Komponente betrug die Faktorgewichtung im Länderdurchschnitt 0,80, wobei die Bandbreite von 0,66 bis 0,87 reichte. Für die Bildungskomponente lag die Faktorgewichtung im Länderdurchschnitt bei 0,79, bei einer Bandbreite von 0,69 bis 0,87. Für die Elternhausausstattungskomponente betrug die Faktorgewichtung im Länderdurchschnitt 0,73 , bei einer Bandbreite von 0,60 bis 0,84. Die Reliabilität des Index reichte von 0,41 bis 0,81. Diese Ergebnisse untermauern die länderübergreifende Validität des PISA-Index des wirtschaftlichen, sozialen und kulturellen Status.

Für Schülerinnen und Schüler, für die Daten zu einer der Komponenten fehlten, erfolgte die Berechnung der Komponenten auf der Grundlage einer Regression der anderen beiden Variablen unter Hinzufügung einer Zufallsfehlerkomponente. Die Endwerte auf dem PISA-Index des wirtschaftlichen, sozialen und kulturellen Status (ESCS) haben einen Mittelwert von null und eine Standardabweichung von eins.

\section{Freude am Lesen}

Der Index der Freude am Lesen (ENJOY) beruht darauf, inwieweit die Schülerinnen und Schüler folgenden Aussagen (ST24) zustimmten: a) Ich lese nur, wenn ich muss, b) Lesen ist eines meiner liebsten Hobbys, $c$ ) ich spreche gerne mit anderen Leuten über Bücher, d) es fällt mir schwer, Bücher zu Ende zu lesen, e) ich freue mich, wenn ich ein Buch geschenkt bekomme, $f$ ) für mich ist Lesen Zeitverschwendung, $g$ ) ich gehe gerne in Buchhandlungen oder Büchereien/Bibliotheken, $h$ ) ich lese nur, um Informationen zu bekommen, die ich brauche, i) ich kann nicht länger als ein paar Minuten stillsitzen und lesen, $j$ ) ich sage gerne meine Meinung über Bücher, die ich gelesen habe, $k$ ) ich tausche gerne Bücher mit meinen Freundinnen und Freunden.

Da alle Items, die negativ formuliert sind (Items $a, d, f, h$ und $i$ ), für die IRT-Skalierung umgepolt wurden, weisen höhere Werte auf diesem Index auf eine größere Freude am Lesen hin.

\section{Vielfalt des Lesestoffs}

Der Index der Vielfalt des Lesestoffs (DIVREAD) beruht darauf, wie häufig die Schülerinnen und Schüler aus eigenem Antrieb folgende Arten von Lesestoff lesen (ST25 im Jahr 2009 und ST36 im Jahr 2000): Zeitschriften, Comics, Romane/Erzählungen/ Geschichten, Sachbücher und Zeitungen. Höhere Werte auf diesem Index weisen auf eine größere Vielfalt des Lesestoffs hin. 


\section{ANHANG A2}

\section{PISA-ZIELPOPULATION, PISA-STICHPROBEN UND DEFINITION DER SCHULEN}

\section{Definition der PISA-Zielpopulation}

PISA 2009 erfasst die kumulativen Bildungserträge und Lernergebnisse zu einem Zeitpunkt, an dem sich die meisten Jugendlichen noch in der Phase der Erstausbildung befinden.

Eine große Herausforderung bei einer internationalen Erhebung besteht darin, sicherzustellen, dass die internationale Vergleichbarkeit der Zielpopulationen in den verschiedenen Ländern gewährleistet ist.

Auf Grund länderspezifischer Unterschiede bei Art und Verbreitung von Einrichtungen des Elementarbereichs, dem regulären Einschulungsalter und der institutionellen Struktur der Bildungssysteme ist die Definition über bestimmte Klassenstufen für internationale Vergleiche ungeeignet. Daher werden bei internationalen Vergleichen von Schulleistungen die Populationen in der Regel in Bezug auf ein Zielalter definiert. Einige frühere internationale Erhebungen definierten ihre Zielpopulationen auf der Basis der Klassenstufe, die eine maximale Erfassung einer bestimmten Alterskohorte gewährleistet. Dieser Ansatz weist den Nachteil auf, dass leichte Abweichungen bei der altersmäßigen Verteilung der Schülerinnen und Schüler auf die einzelnen Klassenstufen sowie auch Unterschiede zwischen den Bildungssystemen innerhalb der einzelnen Länder häufig dazu führen, dass in den verschiedenen Ländern unterschiedliche Zielklassen ausgewählt werden, was die Vergleichbarkeit der Ergebnisse zwischen den einzelnen Ländern und zuweilen auch innerhalb der Länder ernsthaft in Frage stellt. Darüber hinaus kann es, da gewöhnlich nicht alle Schülerinnen und Schüler einer gewünschten Altersgruppe bei klassenspezifischen Stichproben repräsentiert sind, zu potenziell gravierenderen Verzerrungen der Ergebnisse kommen, wenn sich die unterrepräsentierten Schülerinnen und Schüler in einigen Ländern typischerweise in der nächsthöheren Klasse, in anderen Ländern wiederum in der gerade darunter liegenden Klasse befinden. Dadurch blieben Schülerinnen und Schüler mit einem potenziell höheren Leistungsniveau in den erstgenannten Ländern und Schülerinnen und Schüler mit einem potenziell niedrigeren Leistungsniveau in den an zweiter Stelle genannten Ländern unberücksichtigt.

Um dieses Problem zu vermeiden, geht PISA von einer altersspezifischen Definition der Zielpopulation aus, d.h. einer Definition, die von der institutionellen Struktur der Bildungssysteme der jeweiligen Länder unabhängig ist. PISA erfasst Schülerinnen und Schüler, die zu Beginn der Testperiode im Alter von 15 Jahren und 3 (vollen) Monaten bis zu 16 Jahren und 2 (vollen) Monaten waren, wobei eine Abweichung von plus/minus einem Monat akzeptiert wird, gleichgültig welche Klasse oder Art von Bildungseinrichtung sie besuchten und unabhängig davon, ob es sich um eine Ganztags- oder Halbtagsschule handelte, solange sie sich in Klassenstufe 7 oder höher befanden. Bildungseinrichtungen werden in dieser Veröffentlichung generell mit dem Begriff Schulen bezeichnet, obwohl einige davon (insbesondere manche Formen von berufsbildenden Einrichtungen) im landesüblichen Sprachgebrauch vielleicht nicht als Schulen bezeichnet werden. Wie auf Grund dieser Definition zu erwarten, betrug das Durchschnittsalter der Schülerinnen und Schüler OECD-weit 15 Jahre und 9 Monate. Die Varianz des Durchschnittsalters in den verschiedenen Ländern belief sich auf 2 Monate und 5 Tage (0,18 Jahre), wobei das niedrigste Durchschnittsalter 15 Jahre und 8 Monate und das höchste 15 Jahre und 10 Monate betrug.

Auf Grund dieser Populationsdefinition gelangt PISA zu Feststellungen über die Kenntnisse und Fähigkeiten einer Gruppe von Schülern, die in einer vergleichbaren Referenzperiode geboren sind, aber über unterschiedliche inner- wie außerschulische Bildungserfahrungen verfügen können. Im Rahmen von PISA werden diese Kenntnisse und Fähigkeiten als Erträge der Bildung in einem für die verschiedenen Länder gleichen Alter bezeichnet. Je nach der von den einzelnen Ländern verfolgten Politik in Bezug auf Einschulung, Auswahl und Versetzung können sich diese Schülerinnen und Schüler auf ein engeres oder ein breiteres Spektrum von Klassenstufen, und auf verschiedene Bildungssysteme, Bildungsgänge oder -zweige verteilen. Diese Unterschiede sollten beim Vergleich der PISA-Ergebnisse in den verschiedenen Ländern berücksichtigt werden, da die zu beobachtenden Unterschiede zwischen Schülern im Alter von 15 Jahren später auf Grund der konvergierenden Bildungserfahrungen möglicherweise nicht mehr zu erkennen sind.

Wenn ein Land auf den Skalen für Lesekompetenz, mathematische und naturwissenschaftliche Grundbildung eine wesentlich höhere Punktzahl erreicht als ein anderes, ist daraus nicht automatisch zu folgern, dass die Schulen oder bestimmte Teile des Bildungssystems in diesem Land effektiver sind als in dem anderen. Gleichwohl lässt sich aber durchaus die Schlussfolgerung ableiten, dass der kumulative Effekt der Lernerfahrungen in dem Land mit der höheren Punktzahl von der frühen Kindheit bis zum Alter von 15 Jahren, und zwar sowohl im schulischen als auch im außerschulischen Umfeld, zu positiveren Ergebnissen in den von PISA getesteten Grundbildungsbereichen geführt hat.

In der PISA-Zielpopulation nicht enthalten sind Schülerinnen und Schüler des jeweiligen Landes, die eine Schule im Ausland besuchen. Erfasst sind hingegen ausländische Schülerinnen und Schüler, die eine Schule im Erhebungsland besuchen.

Um den Ländern entgegenzukommen, die zum Zweck nationaler Analysen nach Klassenstufen aufgeschlüsselte Ergebnisse wünschten, stand bei PISA 2009 eine Stichprobenoption zur Ergänzung der altersbezogenen Stichprobe durch eine klassenstufenbezogene Stichprobe offen. 


\section{Erfassungsbereich der PISA-Schülerpopulation}

Alle Länder waren um eine möglichst breite Erfassung der 15-jährigen Schülerinnen und Schüler in ihren nationalen Stichproben bemüht, darunter auch solche in Sondereinrichtungen. Daher erreichte PISA 2009 einen für internationale Erhebungen dieser Art beispiellosen Standard der Populationserfassung.

Die PISA-Stichprobenstandards erlaubten es den Ländern, bis zu insgesamt 5\% der relevanten Population auszuschließen, sei es durch Ausschluss von Schulen oder durch Ausschluss von Schülern innerhalb der Schulen. Mit Ausnahme von fünf Ländern, nämlich Dänemark (8,17\%), Luxemburg (8,15\%), Kanada (6,00\%), Norwegen (5,93\%) und den Vereinigten Staaten $(5,16 \%)$, erreichten alle Länder diesen Standard, und in 36 Ländern und Volkswirtschaften lag die Gesamtausschlussrate bei weniger als $2 \%$. Wenn sprachlich bedingte Ausschlüsse berücksichtigt, d.h. aus der Gesamtausschlussrate herausgerechnet werden, liegen die Ausschlussraten in den Vereinigten Staaten nicht mehr über 5\%. Wegen näherer Einzelheiten vgl. www.pisa.oecd.org.

Zu den Ausschlüssen innerhalb der oben genannten Grenzen zählen:

- Auf Schulebene: a) Schulen, die geografisch unzugänglich waren oder wo die Durchführung der PISA-Erhebung als nicht praktikabel angesehen wurde, sowie b) Schulen, deren Unterrichtsangebot sich auf die Kategorien beschränkt, die unter der Rubrik „Ausschlüsse innerhalb der Schulen“ definiert sind, wie z.B. Blindenschulen. Der Prozentsatz der 15-Jährigen, die solche Schulen besuchten, musste weniger als 2,5\% der angestrebten Grundgesamtheit auf Länderebene betragen [maximal 0,5\% für a) und maximal 2\% für b)]. Der PISA 2009 Technical Report (OECD, erscheint demnächst) enthält Angaben über die Größenordnung, Art und Begründung der Ausschlüsse auf Schulebene.

- Auf Schülerebene: a) Schülerinnen und Schüler mit kognitiver Behinderung, b) Schülerinnen und Schüler mit funktionaler Behinderung, c) Schülerinnen und Schüler mit unzureichender Kenntnis der Testsprache, d) sonstige Schülerinnen und Schüler, eine Kategorie, die von den nationalen Zentren definiert und vom Internationalen Konsortium genehmigt werden muss, und e) Schülerinnen und Schüler, die in dem Haupttestbereich in einer Unterrichtssprache unterrichtet werden, für die keine Unterlagen verfügbar waren. Es war nicht möglich, Schülerinnen und Schüler allein auf Grund eines niedrigen Leistungsniveaus oder normaler Disziplinprobleme auszuschließen. Der Prozentsatz der innerhalb der Schulen ausgeschlossenen 15-Jährigen musste weniger als 2,5\% der auf Länderebene angestrebten Grundgesamtheit betragen.

Tabelle A2.1 beschreibt die Zielpopulation der an PISA 2009 teilnehmenden Länder. Nähere Einzelheiten über die Zielpopulation und die Anwendung der PISA-Stichprobenstandards enthält PISA 2009 Technical Report (OECD, erscheint demnächst).

• Spalte 1 zeigt die Gesamtzahl der 15-Jährigen gemäß den aktuellsten vorliegenden Informationen, d.h. für die meisten Länder aus dem Jahr 2008, das der Erhebung vorausging.

- Spalte 2 zeigt die Zahl der 15-Jährigen in Klassenstufe 7 oder darüber (gemäß obiger Definition), also die in Betracht kommende Population.

- Spalte 3 zeigt die angestrebte Grundgesamtheit auf Länderebene. Die Länder konnten a priori bis zu 0,5\% der Schülerinnen und Schüler von der in Betracht kommenden Population ausschließen, im Wesentlichen aus praktischen Gründen. In folgenden Fällen wurde diese Höchstgrenze überschritten, was aber im Einvernehmen mit dem PISA-Konsortium geschah: Kanada hat 1,1\% seiner Population ausgeschlossen, da es sich um Schüler in Indigenengebieten (Territories and Aboriginal Reserves) handelt; in Frankreich blieben 1,7\% der in Betracht kommenden Schülerinnen und Schüler unberücksichtigt, weil sie in Überseeterritorien (Territoires d'Outre-Mer) leben oder besondere Institutionen besuchen; im Fall Indonesiens wurden 4,7\% der Schülerinnen und Schüler aus vier Provinzen aus Sicherheitsgründen ausgeschlossen; in Kirgisistan wurden 2,3\% der Zielpopulation nicht einbezogen, weil sie entlegene, schwer erreichbare Schulen besuchen, und in Serbien wurden 2\% der Schüler ausgeschlossen, die im Kosovo in serbischer Sprache unterrichtet werden.

- Spalte 4 zeigt die Zahl der Schülerinnen und Schüler in Schulen, die von der angestrebten Grundgesamtheit auf Länderebene bei der Stichprobenziehung oder zu einem späteren Zeitpunkt während der Datensammlung ausgeschlossen wurden.

- Spalte 5 zeigt die Größe der angestrebten Grundgesamtheit auf Länderebene nach Abzug der Schülerinnen und Schüler in den ausgeschlossenen Schulen. Sie ergibt sich durch Subtraktion der Spalte 4 von Spalte 3.

- Spalte 6 zeigt den Prozentsatz der Schülerinnen und Schüler in den ausgeschlossenen Schulen. Er wird durch Division der Spalte 4 durch Spalte 3 und durch Multiplikation mit 100 ermittelt.

- Spalte 7 zeigt die Zahl der an PISA 2009 teilnehmenden Schülerinnen und Schüler. Dabei ist zu beachten, dass in manchen Fällen die im Rahmen nationaler Optionen zusätzlich einbezogenen Schülerinnen und Schüler in diesem Wert nicht berücksichtigt sind.

- Spalte 8 zeigt die gewichtete Zahl der teilnehmenden Schülerinnen und Schüler, d.h. die Zahl der Schülerinnen und Schüler in der definierten Grundgesamtheit auf Länderebene, die die PISA-Stichprobe repräsentiert.

- Jedes Land war bestrebt, den Erfassungsbereich der PISA-Zielpopulation bei den in die Stichprobe einbezogenen Schulen zu maximieren. Für jede einbezogene Schule wurden alle in Betracht kommenden Schülerinnen und Schüler, d.h. die 15-Jährigen, unabhängig von ihrer Klasse, zuerst aufgelistet. Auch die von der Stichprobe auszuschließenden Schülerinnen und Schüler mussten in der Stichprobendokumentation aufgeführt sein, und auf einer Liste waren die Gründe für ihren Ausschluss darzulegen. 
[Teil 1/2]

Tabelle A2.1 PISA-Zielpopulationen und -Stichproben

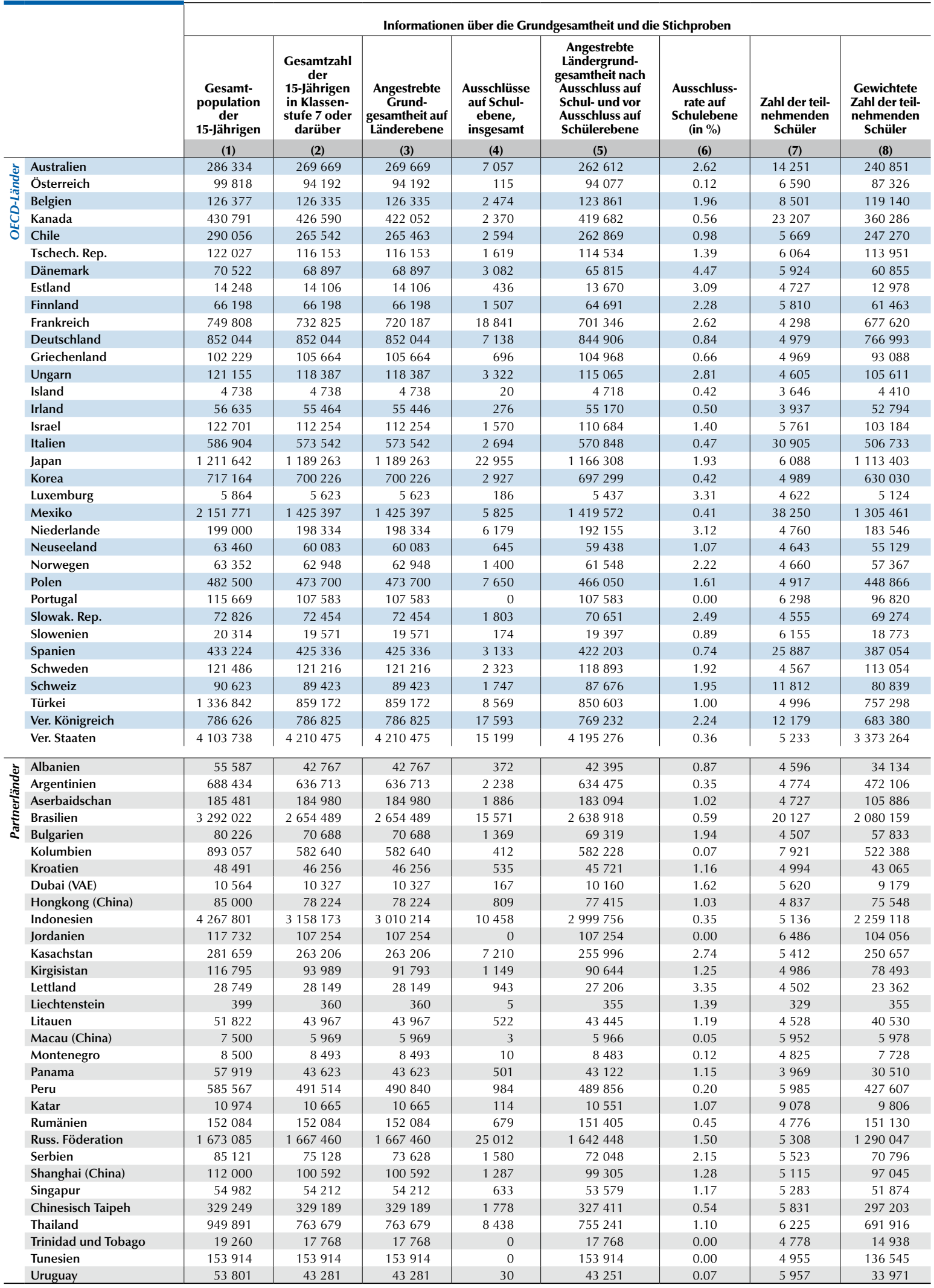

Anmerkung: Wegen einer vollständigen Erklärung der Einzelheiten dieser Tabelle vgl. PISA 2009 Technical Report (OECD, erscheint demnächst). Die in Spalte 1 angegebene Zahl der nationalen Gesamtpopulation der eingeschulten 15-Jährigen kann auf Grund unterschiedlicher Datenquellen in einzelnen Fällen größer sein als die Gesamtpopulation der 15-Jährigen in Spalte 2. In Griechenland sind die Zuwanderer in Spalte 1 nicht berücksichtigt, in Spalte 2 hingegen sind sie enthalten. StatLink *iाls http://dx.doi.org/10.1787/888932343190 
[Teil 2/2]

Tabelle A2.1 PISA-Zielpopulationen und -Stichproben

\begin{tabular}{|c|c|c|c|c|c|c|c|c|}
\hline & & \multicolumn{4}{|c|}{ Informationen über die Grundgesamtheit und die Stichproben } & \multicolumn{3}{|c|}{ Erfassungsindizes } \\
\hline & & $\begin{array}{l}\text { Zahl der } \\
\text { ausgeschloss. } \\
\text { Schüler }\end{array}$ & $\begin{array}{c}\text { Gewichtete Zahl } \\
\text { der ausgeschloss. } \\
\text { Schüler }\end{array}$ & $\begin{array}{l}\text { Ausschlussrate } \\
\text { innerhalb der } \\
\text { Schulen (in \%) }\end{array}$ & $\begin{array}{l}\text { Ausschlussrate } \\
\text { insg. (in \%) }\end{array}$ & $\begin{array}{l}\text { Erfassungs- } \\
\text { index 1: } \\
\text { Erfassung der } \\
\text { angestrebten } \\
\text { Ländergrund- } \\
\text { gesamtheit }\end{array}$ & $\begin{array}{l}\text { Erfassungs- } \\
\text { index 2: } \\
\text { Erfassung der } \\
\text { nationalen } \\
\text { Schüler- } \\
\text { population }\end{array}$ & $\begin{array}{c}\text { Erfassungs- } \\
\text { index 3: } \\
\text { Erfassung der } \\
\text { Population der } \\
\text { 15-Jährigen }\end{array}$ \\
\hline & & (9) & (10) & (11) & (12) & (13) & (14) & (15) \\
\hline \multirow{34}{*}{ 氞 } & Australien & 313 & 4389 & 1.79 & 4.36 & 0.956 & 0.956 & 0.841 \\
\hline & Österreich & 45 & 607 & 0.69 & 0.81 & 0.992 & 0.992 & 0.875 \\
\hline & Belgien & 30 & 292 & 0.24 & 2.20 & 0.978 & 0.978 & 0.943 \\
\hline & Kanada & 1607 & 20837 & 5.47 & 6.00 & 0.940 & 0.930 & 0.836 \\
\hline & Chile & 15 & 620 & 0.25 & 1.22 & 0.988 & 0.987 & 0.852 \\
\hline & Tschech. Rep. & 24 & 423 & 0.37 & 1.76 & 0.982 & 0.982 & 0.934 \\
\hline & Dänemark & 296 & 2448 & 3.87 & 8.17 & 0.918 & 0.918 & 0.863 \\
\hline & Estland & 32 & 97 & 0.74 & 3.81 & 0.962 & 0.962 & 0.911 \\
\hline & Finnland & 77 & 717 & 1.15 & 3.40 & 0.966 & 0.966 & 0.928 \\
\hline & Frankreich & 1 & 304 & 0.04 & 2.66 & 0.973 & 0.957 & 0.904 \\
\hline & Deutschland & 28 & 3591 & 0.47 & 1.30 & 0.987 & 0.987 & 0.900 \\
\hline & Griechenland & 142 & 2977 & 3.10 & 3.74 & 0.963 & 0.963 & 0.911 \\
\hline & Ungarn & 10 & 361 & 0.34 & 3.14 & 0.969 & 0.969 & 0.872 \\
\hline & Island & 187 & 189 & 4.10 & 4.50 & 0.955 & 0.955 & 0.931 \\
\hline & Irland & 136 & 1492 & 2.75 & 3.23 & 0.968 & 0.967 & 0.932 \\
\hline & Israel & 86 & 1359 & 1.30 & 2.68 & 0.973 & 0.973 & 0.841 \\
\hline & Italien & 561 & 10663 & 2.06 & 2.52 & 0.975 & 0.975 & 0.863 \\
\hline & Japan & 0 & 0 & 0.00 & 1.93 & 0.981 & 0.981 & 0.919 \\
\hline & Korea & 16 & 1748 & 0.28 & 0.69 & 0.993 & 0.993 & 0.879 \\
\hline & Luxemburg & 196 & 270 & 5.01 & 8.15 & 0.919 & 0.919 & 0.874 \\
\hline & Mexiko & 52 & 1951 & 0.15 & 0.56 & 0.994 & 0.994 & 0.607 \\
\hline & Niederlande & 19 & 648 & 0.35 & 3.46 & 0.965 & 0.965 & 0.922 \\
\hline & Neuseeland & 184 & 1793 & 3.15 & 4.19 & 0.958 & 0.958 & 0.869 \\
\hline & Norwegen & 207 & 2260 & 3.79 & 5.93 & 0.941 & 0.941 & 0.906 \\
\hline & Polen & 15 & 1230 & 0.27 & 1.88 & 0.981 & 0.981 & 0.930 \\
\hline & Portugal & 115 & 1544 & 1.57 & 1.57 & 0.984 & 0.984 & 0.837 \\
\hline & Slowak. Rep. & 106 & 1516 & 2.14 & 4.58 & 0.954 & 0.954 & 0.951 \\
\hline & Slowenien & 43 & 138 & 0.73 & 1.61 & 0.984 & 0.984 & 0.924 \\
\hline & Spanien & 775 & 12673 & 3.17 & 3.88 & 0.961 & 0.961 & 0.893 \\
\hline & Schweden & 146 & 3360 & 2.89 & 4.75 & 0.953 & 0.953 & 0.931 \\
\hline & Schweiz & 209 & 940 & 1.15 & 3.08 & 0.969 & 0.969 & 0.892 \\
\hline & Türkei & 11 & 1497 & 0.20 & 1.19 & 0.988 & 0.988 & 0.566 \\
\hline & Ver. Königreich & 318 & 17094 & 2.44 & 4.62 & 0.954 & 0.954 & 0.869 \\
\hline & Ver. Staaten & 315 & 170542 & 4.81 & 5.16 & 0.948 & 0.948 & 0.822 \\
\hline \multirow{31}{*}{ 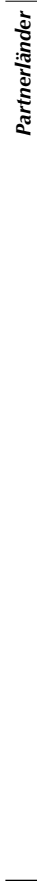 } & Albanien & 0 & 0 & 0.00 & 0.87 & 0.991 & 0.991 & 0.614 \\
\hline & Argentinien & 14 & 1225 & 0.26 & 0.61 & 0.994 & 0.994 & 0.686 \\
\hline & Aserbaidschan & 0 & 0 & 0.00 & 1.02 & 0.990 & 0.990 & 0.571 \\
\hline & Brasilien & 24 & 2692 & 0.13 & 0.72 & 0.993 & 0.993 & 0.632 \\
\hline & Bulgarien & 0 & 0 & 0.00 & 1.94 & 0.981 & 0.981 & 0.721 \\
\hline & Kolumbien & 11 & 490 & 0.09 & 0.16 & 0.998 & 0.998 & 0.585 \\
\hline & Kroatien & 34 & 273 & 0.63 & 1.78 & 0.982 & 0.982 & 0.888 \\
\hline & Dubai (VAE) & 5 & 7 & 0.07 & 1.69 & 0.983 & 0.983 & 0.869 \\
\hline & Hongkong (China) & 9 & 119 & 0.16 & 1.19 & 0.988 & 0.988 & 0.889 \\
\hline & Indonesien & 0 & 0 & 0.00 & 0.35 & 0.997 & 0.950 & 0.529 \\
\hline & Jordanien & 24 & 443 & 0.42 & 0.42 & 0.996 & 0.996 & 0.884 \\
\hline & Kasachstan & 82 & 3844 & 1.51 & 4.21 & 0.958 & 0.958 & 0.890 \\
\hline & Kirgisistan & 86 & 1384 & 1.73 & 2.96 & 0.970 & 0.948 & 0.672 \\
\hline & Lettland & 19 & 102 & 0.43 & 3.77 & 0.962 & 0.962 & 0.813 \\
\hline & Liechtenstein & 0 & 0 & 0.00 & 1.39 & 0.986 & 0.986 & 0.890 \\
\hline & Litauen & 74 & 632 & 1.53 & 2.70 & 0.973 & 0.973 & 0.782 \\
\hline & Macau (China) & 0 & 0 & 0.00 & 0.05 & 0.999 & 0.999 & 0.797 \\
\hline & Montenegro & 0 & 0 & 0.00 & 0.12 & 0.999 & 0.999 & 0.909 \\
\hline & Panama & 0 & 0 & 0.00 & 1.15 & 0.989 & 0.989 & 0.527 \\
\hline & Peru & 9 & 558 & 0.13 & 0.33 & 0.997 & 0.995 & 0.730 \\
\hline & Katar & 28 & 28 & 0.28 & 1.35 & 0.986 & 0.986 & 0.894 \\
\hline & Rumänien & 0 & 0 & 0.00 & 0.45 & 0.996 & 0.996 & 0.994 \\
\hline & Russ. Föderation & 59 & 15247 & 1.17 & 2.65 & 0.973 & 0.973 & 0.771 \\
\hline & Serbien & 10 & 133 & 0.19 & 2.33 & 0.977 & 0.957 & 0.832 \\
\hline & Shanghai (China) & 7 & 130 & 0.13 & 1.41 & 0.986 & 0.986 & 0.866 \\
\hline & Singapur & 48 & 417 & 0.80 & 1.96 & 0.980 & 0.980 & 0.943 \\
\hline & Chinesisch Taipeh & 32 & 1662 & 0.56 & 1.09 & 0.989 & 0.989 & 0.903 \\
\hline & Thailand & 6 & 458 & 0.07 & 1.17 & 0.988 & 0.988 & 0.728 \\
\hline & Trinidad und Tobago & 11 & 36 & 0.24 & 0.24 & 0.998 & 0.998 & 0.776 \\
\hline & Tunesien & 7 & 184 & 0.13 & 0.13 & 0.999 & 0.999 & 0.887 \\
\hline & Uruguay & 14 & 67 & 0.20 & 0.26 & 0.997 & 0.997 & 0.631 \\
\hline
\end{tabular}


[Teil 1/1]

Tabelle A2.2 Ausschlüsse

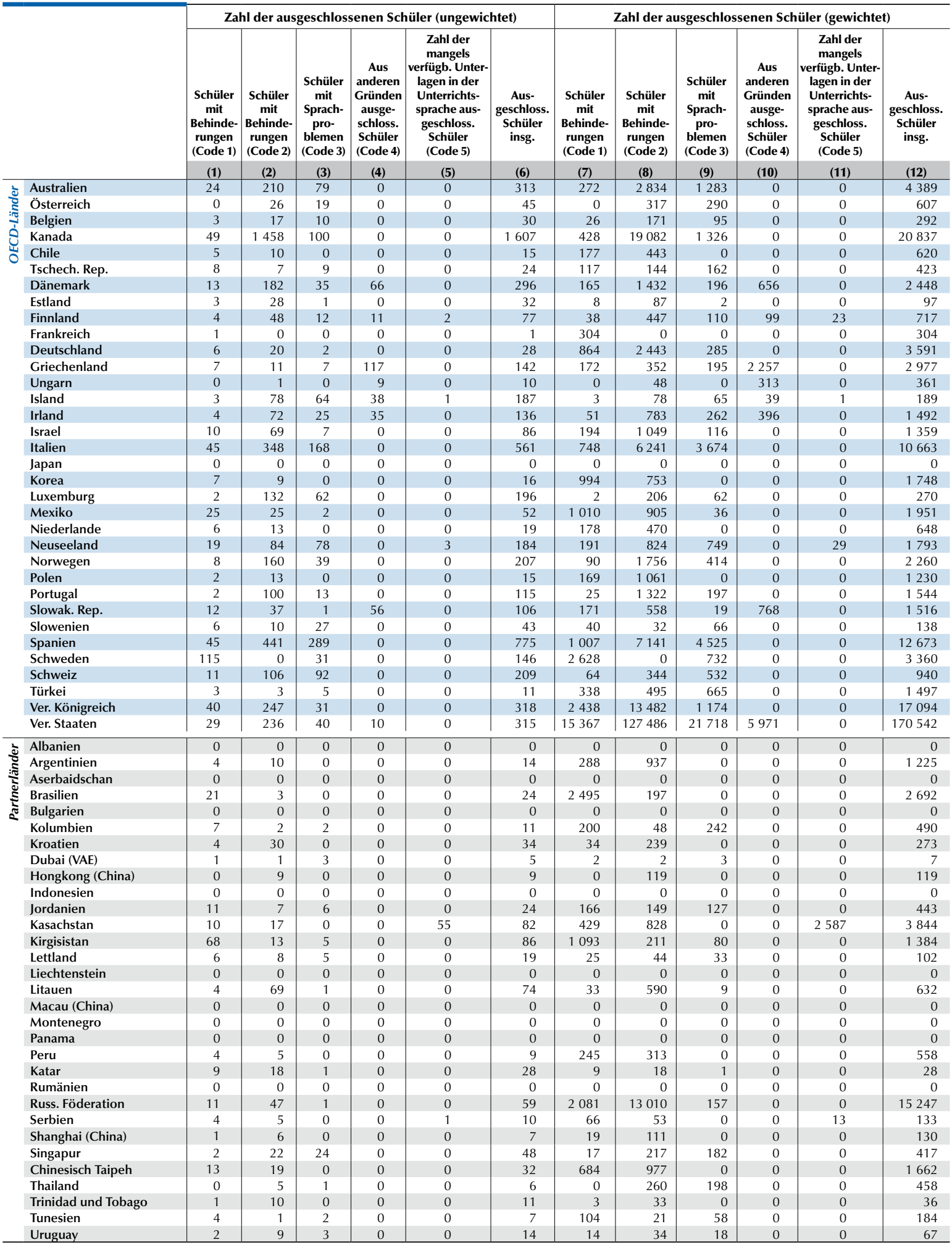

\section{Ausschlusscodes:}

Code 1 Funktionale Behinderung - die Schülerin/der Schüler hat eine mittelschwere bis schwere dauerhafte körperliche Behinderung.

Code 2 Kognitive Behinderung - die Schülerin/der Schüler hat eine mentale oder emotionale Behinderung und wurde nach entsprechenden Tests bzw. nach der professionellen Meinung qualifizierter Kräfte als kognitiv retardiert eingestuft.

Code 3 Unzureichende Kenntnis der Testsprache - keine der Testsprachen des jeweiligen Landes ist die Muttersprache der Schülerin/des Schülers und der Aufenthalt in dem betreffenden Land beträgt weniger als ein Jahr.

Constige - von den nationalen Zentren definierte und dem internationalen Konsortium genehmigte Ausschlussgründe.

Code 5 Keine Unterlagen in der Unterrichtssprache verfügbar.

Anmerkung: Wegen einer ausführlicheren Erklärung der in dieser Tabelle enthaltenen Einzelheiten vgl. PISA 2009 Technical Report (OECD, erscheint demnächst).

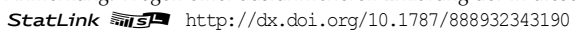


Spalte 9 gibt die Gesamtzahl der ausgeschlossenen Schülerinnen und Schüler an, die in Tabelle A2.2 näher beschrieben und in spezifische Kategorien unterteilt wird. Der in Spalte 10 angegebene Wert steht für die gewichtete Zahl der ausgeschlossenen Schülerinnen und Schüler, d.h. die Gesamtzahl der Schüler in der definierten Grundgesamtheit auf Länderebene, die die Zahl der von der Stichprobe ausgeschlossenen Schülerinnen und Schüler repräsentiert, die ebenfalls in Tabelle A2.2 näher beschrieben und in verschiedene Kategorien unterteilt wurde. Der Ausschluss der Schülerinnen und Schüler erfolgt auf der Basis von fünf Kategorien: a) Schülerinnen und Schüler mit kognitiver Behinderung, d.h. die eine mentale oder emotionale Behinderung aufweisen und kognitiv retardiert sind, so dass sie nicht in der Lage sind, an einer Erhebung unter den PISATestbedingungen teilzunehmen; b) Schülerinnen und Schüler mit funktionaler Behinderung, d.h. die an einer mittelschweren bis schweren dauerhaften körperlichen Behinderung leiden, so dass sie nicht in der Lage sind, an einer Erhebung unter den PISA-Testbedingungen teilzunehmen; c) Schülerinnen und Schüler mit unzureichender Kenntnis der Testsprache, die nicht in der Lage sind, in einer der Testsprachen des Landes zu lesen oder zu sprechen und die die Sprachbarriere in der Testsituation nicht hätten überwinden können (in der Regel können Schülerinnen und Schüler, die weniger als ein Jahr Unterricht in der Testsprache hatten, von der Erhebung ausgeschlossen werden); d) sonstige Schülerinnen und Schüler, eine Kategorie, die von den nationalen Zentren definiert und vom Internationalen Konsortium genehmigt werden muss, und e) Schülerinnen und Schüler, die in dem Haupttestbereich in einer Unterrichtssprache unterrichtet werden, für die keine Unterlagen verfügbar waren.

- Spalte 11 zeigt den Prozentsatz der Schülerinnen und Schüler, die innerhalb der Schulen ausgeschlossen wurden. Er ist berechnet als die gewichtete Zahl der ausgeschlossenen Schülerinnen und Schüler (Spalte 10), dividiert durch die gewichtete Zahl der ausgeschlossenen und der teilnehmenden Schülerinnen und Schüler (Spalte 8 plus Spalte 10), multipliziert mit 100.

- Spalte 12 zeigt die Ausschlussrate insgesamt, die dem gewichteten Prozentsatz der angestrebten Grundgesamtheit auf Länderebene entspricht, der von PISA entweder auf Schulebene oder auf innerschulischer Ebene von der Erhebung ausgeschlossen wurde. Sie wird wie folgt berechnet: Ausschlussrate auf Schulebene (Spalte 6 dividiert durch 100), zuzüglich Ausschlussrate innerhalb der Schulen (Spalte 11 dividiert durch 100), multipliziert mit 1, abzüglich der Ausschlussrate auf Schulebene (Spalte 6 dividiert durch 100). Dieses Ergebnis wird dann mit 100 multipliziert. In fünf Ländern, nämlich in Dänemark, Luxemburg, Kanada, Norwegen und den Vereinigten Staaten war die Ausschlussrate größer als $5 \%$. Wenn sprachlich bedingte Ausschlüsse berücksichtigt, d.h. aus der Gesamtausschlussrate herausgerechnet werden, liegen die Ausschlussraten in den Vereinigten Staaten nicht mehr über $5 \%$.

- Spalte 13 zeigt einen Index für den Erfassungsgrad der angestrebten Grundgesamtheit auf Länderebene durch die PISAStichprobe. Dänemark, Luxemburg, Kanada, Norwegen und die Vereinigten Staaten waren die einzigen Länder, in denen weniger als 95\% der Population von der PISA-Erhebung erfasst wurden.

- Spalte 14 zeigt einen Index für den Erfassungsgrad der 15-jährigen Schülerinnen und Schüler durch die PISA-Stichprobe. Der Index misst den Gesamtanteil der nationalen Schülerpopulation, der durch die nach den Ausschlüssen verbleibende Schülerstichprobe erfasst wird. Bei dem Index werden Ausschlüsse sowohl auf Schulebene als auch auf Schülerebene berücksichtigt. Nahe bei 100 liegende Werte weisen darauf hin, dass die PISA-Stichprobe das gesamte Schulsystem nach Definition für PISA 2009 repräsentiert. Der Index ergibt sich aus der gewichteten Zahl der teilnehmenden Schülerinnen und Schüler (Spalte 8), dividiert durch die gewichtete Zahl der teilnehmenden und der ausgeschlossenen Schüler (Spalte 8 plus Spalte 10), multipliziert mit der definierten Grundgesamtheit auf Länderebene (Spalte 5), dividiert durch die in Betracht kommende Population (Spalte 2) (mal 100).

- Spalte 15 zeigt einen Index des prozentualen Anteils der erfassten Schülerpopulation. Dieser Index ergibt sich aus der gewichteten Gesamtzahl der teilnehmenden Schülerinnen und Schüler (Spalte 8), dividiert durch die Gesamtpopulation der 15-jährigen Schülerinnen und Schüler (Spalte 1).

Dieser hohe Erfassungsgrad trägt mit zur Vergleichbarkeit der Erhebungsergebnisse bei. Selbst wenn man z.B. annimmt, dass die ausgeschlossenen Schülerinnen und Schüler durchgehend schlechtere Ergebnisse erzielt hätten als die teilnehmenden und dass diese Korrelation mittelstark ausgeprägt ist, würde eine Ausschlussrate in einer Größenordnung von 5\% wahrscheinlich nur zu einer Überzeichnung der nationalen Durchschnittsergebnisse um weniger als 5 Punkte führen (auf einer Skala mit einem internationalen Mittelwert von 500 Punkten und einer Standardabweichung von 100 Punkten). Diese Bewertung basiert auf den folgenden Berechnungen: Bei einer Korrelation zwischen der Ausschlussneigung und den Schülerleistungen von 0,3 wären die resultierenden durchschnittlichen Ergebnisse bei einer Ausschlussrate von 1\% um 1 Punkt, bei einer Ausschlussrate von 5\% um 3 Punkte und bei einer Ausschlussrate von 10\% um 6 Punkte überzeichnet. Betrüge die Korrelation zwischen der Ausschlussneigung und den Schülerleistungen 0,5, wären die resultierenden Durchschnittsergebnisse bei einer Ausschlussrate von 1\% um 1 Punkt, von $5 \%$ um 5 Punkte und von $10 \%$ um 10 Punkte überzeichnet. Für diese Berechnung wurde ein Modell verwendet, in dem von einer bivariaten Normalverteilung der Teilnahmeneigung und der Leistungen ausgegangen wurde. Nähere Einzelheiten hierzu enthält PISA 2009 Technical Report (OECD, erscheint demnächst).

\section{Stichprobenziehung und Beteiligungsquoten}

Die Genauigkeit jeglicher Untersuchungsergebnisse hängt von der Qualität der Informationen ab, auf denen die nationalen Stichproben basieren, sowie von der Stichprobenziehung. Für PISA wurden Qualitätsstandards, Verfahren, Instrumente und Verifikationsmechanismen entwickelt, die gewährleisteten, dass die nationalen Stichproben vergleichbare Daten ergaben und die Ergebnisse ohne Vorbehalte miteinander verglichen werden können. 
[Teil 1/2]

Tabelle A2.3 Beteiligungsquoten

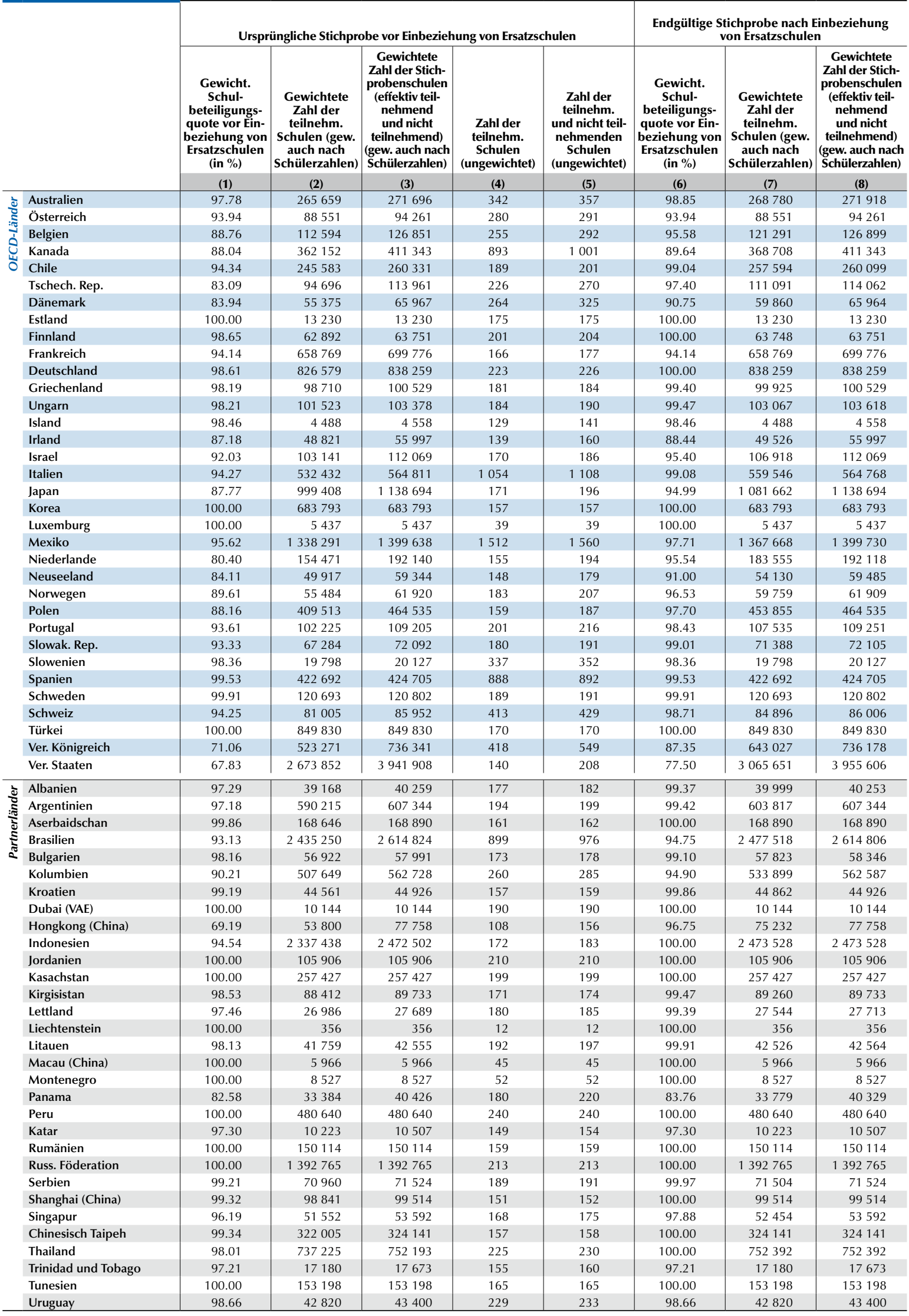

StatLink त्ता Sמ http://dx.doi.org/10.1787/888932343190 
[Teil 2/2]

Tabelle A2.3 Beteiligungsquoten

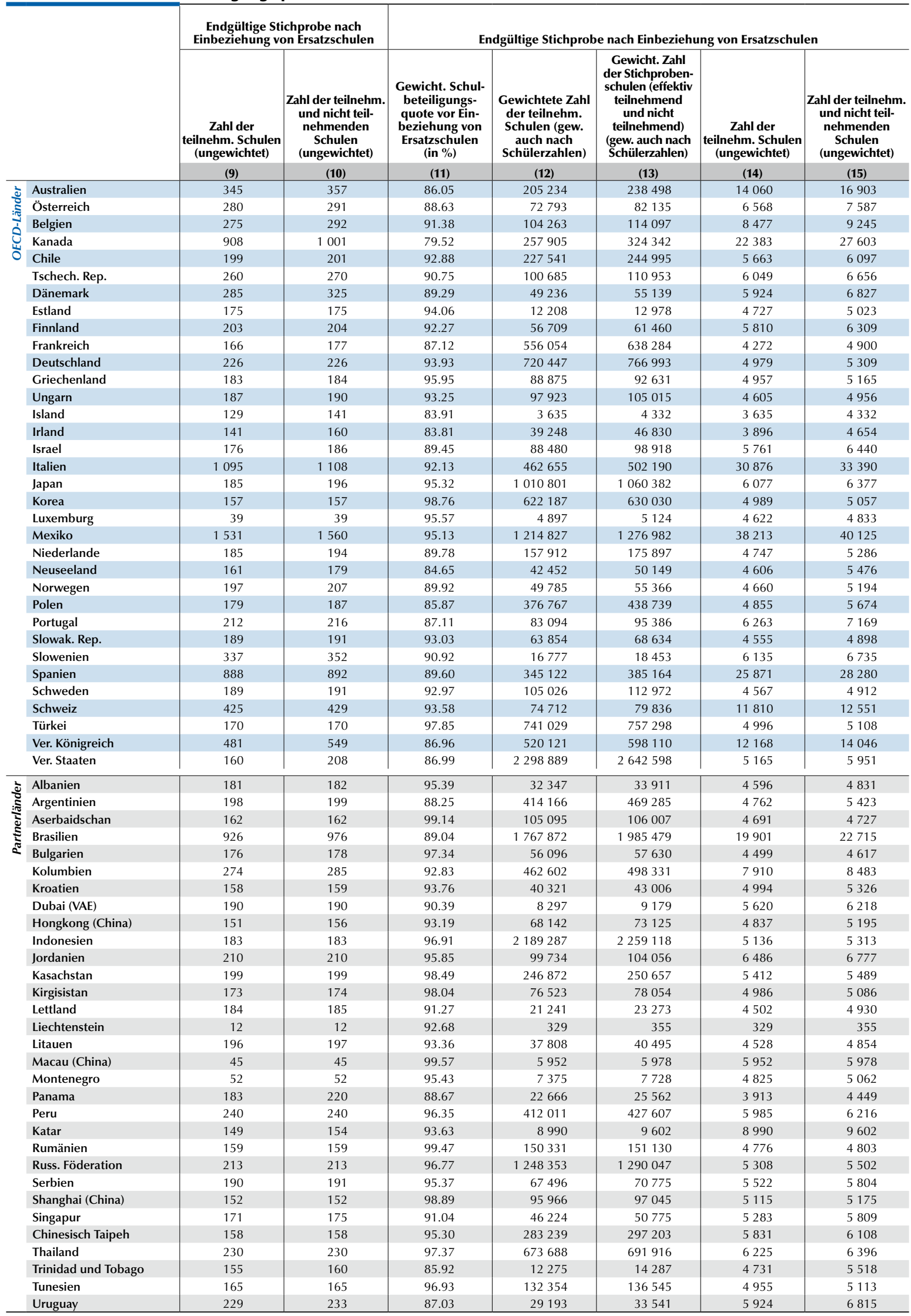

StatLink त्ताI 
Die meisten PISA-Stichproben wurden nach dem Konzept der zweistufigen geschichteten Stichproben konzipiert (soweit die Länder unterschiedliche Stichprobenkonzepte anwandten, sind diese dokumentiert im PISA 2009 Technical Report, OECD, erscheint demnächst). Die erste Stufe bestand darin, eine Stichprobe einzelner Schulen zu ziehen, die von 15-jährigen Schülerinnen und Schülern besucht wurden. Die Schulstichprobe wurde systematisch gezogen, mit Wahrscheinlichkeiten proportional zur Größe, die wiederum in Abhängigkeit zur geschätzten Zahl der in Betracht kommenden (15-jährigen) Schülerinnen und Schüler stand. In jedem Land wurden mindestens 150 Schulen (sofern vorhanden) ausgewählt, wobei die Anforderungen für die nationalen Analysen allerdings häufig eine etwas größere Stichprobe bedingten. Gleichzeitig mit der Stichprobenziehung wurden Ersatzschulen ermittelt für den Fall, dass eine der ausgewählten Schulen nicht an der PISA-2009-Erhebung teilnehmen wollte.

In Island, Katar, Liechtenstein, Luxemburg und Macau (China) wurden alle Schulen und alle innerhalb der Schulen in Betracht kommenden Schülerinnen und Schüler in die Stichprobe aufgenommen.

Experten des PISA-Konsortiums führten den Auswahlprozess der Stichproben in den meisten Teilnehmerländern durch und überwachten diesen Prozess sorgfältig in jenen Ländern, die ihre eigenen Stichproben auswählten. In der zweiten Phase des Auswahlprozesses wurden Schülerstichproben innerhalb der für die Stichprobe ermittelten Schulen gezogen. Nach der Auswahl der Schulen wurde eine Liste aller 15-jährigen Schülerinnen und Schüler an den für die Stichprobe gezogenen Schulen erstellt. Aus dieser Liste wurden 35 Schülerinnen und Schüler mit gleicher Wahrscheinlichkeit ausgewählt (wenn es weniger als 35 gab, wurden alle 15-jährigen Schülerinnen und Schüler ausgewählt). Die Zahl der für die Stichproben ausgewählten Schülerinnen und Schüler je Schule konnte von 35 abweichen, durfte jedoch 20 nicht unterschreiten.

Die Qualitätsstandards der PISA-Daten setzten eine Mindestteilnahmequote sowohl für die Schulen als auch für die Schülerinnen und Schüler voraus. Diese Standards wurden aufgestellt, um mögliche beteiligungsbedingte Verzerrungen zu minimieren. Bei den Ländern, die diese Standards erfüllten, dürften etwaige Verzerrungen infolge von Nichtbeteiligung unerheblich, d.h. in der Regel geringer als der Stichprobenfehler sein.

Für die ursprünglich ausgewählten Schulen galt eine Mindestbeteiligungsquote von $85 \%$ als erforderlich. Wenn die ursprüngliche Beteiligungsquote auf Schulebene zwischen 65\% und 85\% lag, konnte eine akzeptable Quote jedoch immer noch durch die Einbeziehung von Ersatzschulen erzielt werden. Dieses Verfahren war mit dem Risiko eines erhöhten Beteiligungsbias verbunden. Daher wurden die Teilnehmerländer ersucht, so viele der ursprünglich für die Stichprobe gezogenen Schulen wie möglich zur Teilnahme zu veranlassen. Wenn die Beteiligungsquote der Schülerinnen und Schüler zwischen 25\% und 50\% lag, galt die betreffende Schule nicht als teilnehmende Schule, aber die diesbezüglichen Daten wurden in die Datenbank aufgenommen und bei den verschiedenen Schätzungen berücksichtigt. Daten von Schulen mit einer Schülerbeteiligungsquote von weniger als $25 \%$ wurden nicht in die Datenbank aufgenommen.

Für PISA 2009 wurde auch eine minimale Schülerbeteiligungsquote von 80\% innerhalb der teilnehmenden Schulen festgesetzt. Diese Mindestquote musste auf Landesebene und nicht zwangsläufig von jeder teilnehmenden Schule erreicht werden. Folgesitzungen waren in Schulen erforderlich, in denen zu wenig Schülerinnen und Schüler an der ursprünglichen Testsitzung teilgenommen hatten. Die Schülerbeteiligungsquoten wurden für alle ursprünglichen Schulen sowie für alle Schulen, d.h. die ursprünglich ausgewählten und die Ersatzschulen, auf der Basis der Teilnahme der Schülerinnen und Schüler an der ursprünglichen Testsitzung wie auch an etwaigen Folgesitzungen errechnet. Schüler, die bei der ersten Testsitzung oder einer Folgesitzung anwesend waren, galten als Teilnehmer. Solche, die sich nur an der Beantwortung der Fragebogen beteiligten, wurden in die internationale Datenbank aufgenommen und bei den Statistiken in dieser Veröffentlichung berücksichtigt, wenn sie zumindest Angaben über die berufliche Tätigkeit des Vaters oder der Mutter gemacht hatten.

Tabelle A2.3 gibt Auskunft über die Beteiligungsquoten auf Schüler- und auf Schulebene, vor und nach Einbeziehung von Ersatzschulen.

- Spalte 1 zeigt die gewichtete Beteiligungsquote der Schulen vor Einbeziehung von Ersatzschulen. Sie wird durch Division von Spalte 2 durch Spalte 3 ermittelt.

- Spalte 2 zeigt die gewichtete Zahl der teilnehmenden Schulen vor Einbeziehung von Ersatzschulen (gewichtet nach der Schülerzahl).

- Spalte 3 zeigt die gewichtete Zahl der für die Stichprobe gezogenen Schulen vor Einbeziehung von Ersatzschulen (sowohl effektiv teilnehmende als auch nicht teilnehmende Schulen, gewichtet nach der Schülerzahl).

- Spalte 4 zeigt die ungewichtete Zahl der teilnehmenden Schulen vor Einbeziehung von Ersatzschulen.

- Spalte 5 zeigt die ungewichtete Zahl der teilnehmenden und nicht teilnehmenden Schulen vor Einbeziehung von Ersatzschulen.

- Spalte 6 zeigt die gewichtete Schulbeteiligungsquote nach Einbeziehung von Ersatzschulen. Sie ergibt sich durch Division von Spalte 7 durch Spalte 8.

- Spalte 7 zeigt die gewichtete Zahl der teilnehmenden Schulen nach Einbeziehung von Ersatzschulen (gewichtet nach der Schülerzahl).

- Spalte 8 zeigt die gewichtete Zahl der für die Stichprobe gezogenen Schulen nach Einbeziehung von Ersatzschulen (sowohl effektiv teilnehmende als auch nicht teilnehmende Schulen, gewichtet nach der Schülerzahl).

- Spalte 9 zeigt die ungewichtete Zahl der teilnehmenden Schulen nach Einbeziehung von Ersatzschulen. 
[Teil 1/1]

Tabelle A2.4a Prozentsatz der Schüler in den einzelnen Klassenstufen

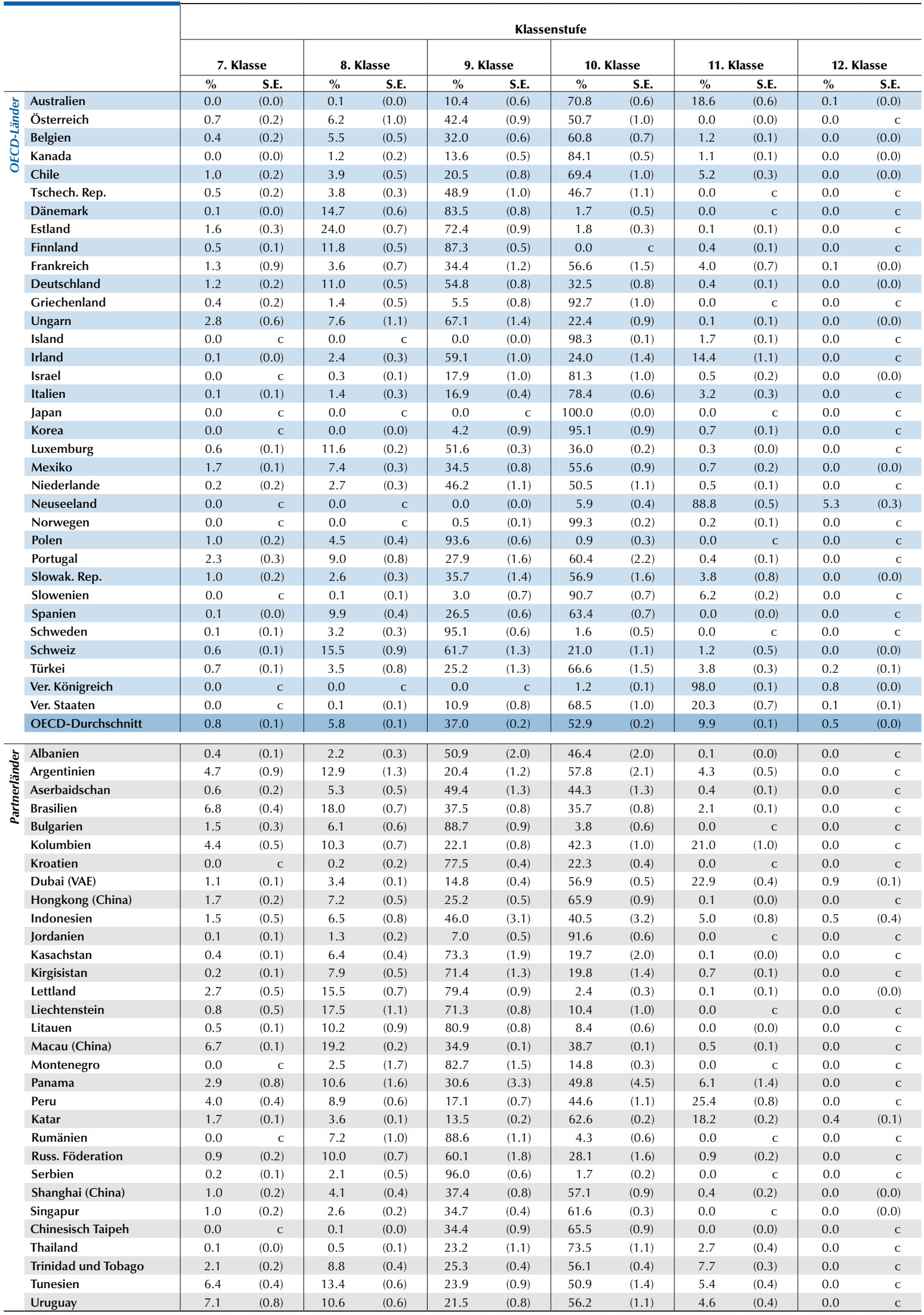

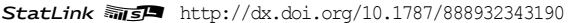


[Teil 1/2]

Tabelle A2.4b Prozentsatz der Schüler in den einzelnen Klassenstufen, nach Geschlecht

\begin{tabular}{|c|c|c|c|c|c|c|c|c|c|c|c|c|c|}
\hline & & \multicolumn{12}{|c|}{ Jungen - Klassenstufe } \\
\hline & & \multicolumn{2}{|c|}{ 7. Klasse } & \multicolumn{2}{|c|}{ 8. Klasse } & \multicolumn{2}{|c|}{ 9. Klasse } & \multicolumn{2}{|c|}{ 10. Klasse } & \multicolumn{2}{|c|}{ 11. Klasse } & \multicolumn{2}{|c|}{ 12. Klasse } \\
\hline & & $\%$ & S.E. & $\%$ & S.E. & $\%$ & S.E. & $\%$ & S.E. & $\%$ & S.E. & $\%$ & S.E. \\
\hline \multirow{35}{*}{ 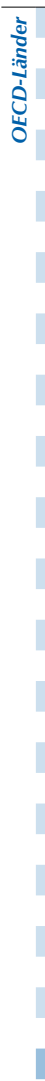 } & Australien & 0.0 & $\mathrm{C}$ & 0.1 & $(0.0)$ & 13.1 & (0.9) & 69.6 & (1.1) & 17.1 & (0.8) & 0.1 & $(0.0)$ \\
\hline & Österreich & 0.7 & $(0.2)$ & 7.4 & $(1.2)$ & 42.6 & (1.3) & 49.3 & (1.3) & 0.0 & $(0.0)$ & 0.0 & C \\
\hline & Belgien & 0.6 & $(0.2)$ & 6.4 & $(0.7)$ & 34.6 & (0.9) & 57.3 & (1.0) & 1.1 & $(0.2)$ & 0.0 & $(0.0)$ \\
\hline & Kanada & 0.0 & $(0.0)$ & 1.4 & $(0.3)$ & 14.6 & $(0.6)$ & 82.9 & (0.6) & 1.1 & $(0.1)$ & 0.0 & $(0.0)$ \\
\hline & Chile & 1.3 & $(0.3)$ & 4.9 & (0.6) & 23.2 & $(1.0)$ & 65.9 & (1.3) & 4.7 & $(0.3)$ & 0.0 & c \\
\hline & Tschech. Rep. & 0.7 & $(0.2)$ & 4.5 & (0.5) & 52.5 & $(2.2)$ & 42.3 & (2.4) & 0.0 & c & 0.0 & C \\
\hline & Dänemark & 0.1 & $(0.0)$ & 19.5 & (0.9) & 79.5 & $(1.0)$ & 0.8 & $(0.3)$ & 0.0 & c & 0.0 & c \\
\hline & Estland & 2.4 & $(0.5)$ & 27.0 & (1.0) & 69.6 & (1.1) & 1.0 & $(0.3)$ & 0.0 & c & 0.0 & C \\
\hline & Finnland & 0.6 & $(0.2)$ & 14.0 & $(0.8)$ & 85.2 & $(0.8)$ & 0.0 & c & 0.2 & $(0.1)$ & 0.0 & c \\
\hline & Frankreich & 1.3 & (0.9) & 4.0 & (0.6) & 39.6 & (1.5) & 51.4 & (1.9) & 3.6 & (0.8) & 0.0 & $(0.0)$ \\
\hline & Deutschland & 1.4 & $(0.3)$ & 13.1 & $(0.7)$ & 56.1 & (1.0) & 28.8 & (0.9) & 0.6 & $(0.1)$ & 0.0 & C \\
\hline & Griechenland & 0.5 & $(0.2)$ & 1.9 & (0.5) & 6.2 & $(1.2)$ & 91.4 & (1.5) & 0.0 & c & 0.0 & C \\
\hline & Ungarn & 3.2 & (0.8) & 9.3 & (1.3) & 68.8 & (1.6) & 18.7 & (0.9) & 0.0 & $(0.0)$ & 0.0 & $(0.0)$ \\
\hline & Island & 0.0 & c & 0.0 & c & 0.0 & c & 98.7 & $(0.2)$ & 1.3 & $(0.2)$ & 0.0 & C \\
\hline & Irland & 0.1 & $(0.0)$ & 2.8 & $(0.5)$ & 60.9 & (1.3) & 22.4 & (1.5) & 13.8 & (1.4) & 0.0 & c \\
\hline & Israel & 0.0 & C & 0.5 & $(0.2)$ & 19.9 & (1.1) & 78.7 & $(1.2)$ & 1.0 & (0.4) & 0.0 & c \\
\hline & Italien & 0.1 & $(0.1)$ & 1.7 & $(0.4)$ & 20.1 & (0.6) & 75.7 & $(0.7)$ & 2.5 & (0.3) & 0.0 & c \\
\hline & Japan & 0.0 & C & 0.0 & C & 0.0 & C & 100.0 & $(0.0)$ & 0.0 & C & 0.0 & c \\
\hline & Korea & 0.0 & C & 0.1 & $(0.1)$ & 4.7 & (1.3) & 94.5 & (1.4) & 0.7 & $(0.2)$ & 0.0 & c \\
\hline & Luxemburg & 0.8 & $(0.2)$ & 12.5 & (0.4) & 52.4 & (0.5) & 34.0 & (0.4) & 0.3 & $(0.1)$ & 0.0 & c \\
\hline & Mexiko & 2.0 & $(0.2)$ & 8.8 & $(0.5)$ & 37.6 & (0.9) & 51.0 & (0.9) & 0.5 & $(0.2)$ & 0.0 & c \\
\hline & Niederlande & 0.4 & $(0.3)$ & 3.0 & (0.4) & 48.9 & (1.3) & 47.3 & (1.3) & 0.3 & $(0.1)$ & 0.0 & C \\
\hline & Neuseeland & 0.0 & c & 0.0 & c & 0.0 & c & 6.9 & $(0.5)$ & 87.9 & (0.6) & 5.2 & (0.5) \\
\hline & Norwegen & 0.0 & C & 0.0 & C & 0.5 & $(0.1)$ & 99.2 & $(0.2)$ & 0.3 & $(0.2)$ & 0.0 & c \\
\hline & Polen & 1.5 & $(0.3)$ & 6.5 & (0.6) & 91.6 & $(0.7)$ & 0.5 & $(0.2)$ & 0.0 & C & 0.0 & c \\
\hline & Portugal & 3.4 & $(0.5)$ & 10.5 & (0.9) & 30.9 & $(2.0)$ & 54.9 & (2.6) & 0.4 & $(0.1)$ & 0.0 & c \\
\hline & Slowak. Rep. & 1.4 & (0.3) & 3.7 & $(0.5)$ & 40.1 & (1.9) & 51.6 & $(2.1)$ & 3.3 & $(0.7)$ & 0.0 & c \\
\hline & Slowenien & 0.0 & c & 0.1 & $(0.1)$ & 4.0 & (1.2) & 91.1 & $(1.2)$ & 4.7 & (0.4) & 0.0 & c \\
\hline & Spanien & 0.1 & $(0.0)$ & 12.2 & (0.6) & 28.7 & $(0.8)$ & 58.9 & (0.9) & 0.0 & $(0.0)$ & 0.0 & c \\
\hline & Schweden & 0.0 & $(0.0)$ & 4.1 & $(0.4)$ & 94.7 & (0.6) & 1.1 & $(0.3)$ & 0.0 & c & 0.0 & C \\
\hline & Schweiz & 0.8 & $(0.2)$ & 18.0 & $(1.2)$ & 60.7 & (1.8) & 19.4 & (1.8) & 1.0 & $(0.4)$ & 0.1 & $(0.1)$ \\
\hline & Türkei & 1.0 & $(0.2)$ & 4.0 & (0.9) & 30.2 & (1.4) & 61.3 & (1.7) & 3.2 & $(0.3)$ & 0.2 & $(0.1)$ \\
\hline & Ver. Königreich & 0.0 & c & 0.0 & C & 0.0 & $\mathrm{c}$ & 1.3 & $(0.2)$ & 98.0 & $(0.2)$ & 0.7 & $(0.1)$ \\
\hline & Ver. Staaten & 0.0 & c & 0.1 & $(0.0)$ & 13.2 & $(1.0)$ & 68.6 & (1.4) & 17.9 & (0.9) & 0.1 & $(0.1)$ \\
\hline & OECD-Durchschnitt & 1.0 & $(0.1)$ & 7.0 & $(0.1)$ & 40.8 & $(0.2)$ & 50.8 & $(0.2)$ & 9.8 & $(0.1)$ & 0.7 & $(0.0)$ \\
\hline む & Albanien & 0.5 & $(0.2)$ & 2.6 & $(0.4)$ & 54.0 & (2.0) & 42.9 & (2.1) & 0.0 & $(0.0)$ & 0.0 & c \\
\hline 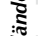 & Argentinien & 5.9 & (1.1) & 15.4 & (1.4) & 22.7 & (1.5) & 52.5 & (2.4) & 3.5 & $(0.5)$ & 0.0 & C \\
\hline$\frac{2 \pi}{2}$ & Aserbaidschan & 0.6 & $(0.2)$ & 4.7 & (0.5) & 47.8 & (1.4) & 46.5 & (1.5) & 0.3 & $(0.1)$ & 0.0 & c \\
\hline อั้ & Brasilien & 8.4 & $(0.6)$ & 21.0 & (0.9) & 37.8 & (0.8) & 31.1 & (0.9) & 1.7 & $(0.2)$ & 0.0 & c \\
\hline & Bulgarien & 2.0 & $(0.4)$ & 7.4 & (0.9) & 86.9 & $(1.2)$ & 3.7 & $(0.6)$ & 0.0 & c & 0.0 & c \\
\hline & Kolumbien & 5.5 & (0.9) & 11.5 & (0.9) & 21.9 & (1.1) & 42.4 & (1.4) & 18.7 & $(1.2)$ & 0.0 & c \\
\hline & Kroatien & 0.0 & c & 0.1 & $(0.1)$ & 79.1 & $(0.6)$ & 20.7 & $(0.6)$ & 0.0 & $\mathrm{C}$ & 0.0 & $\mathrm{c}$ \\
\hline & Dubai (VAE) & 1.6 & $(0.2)$ & 4.5 & $(0.3)$ & 16.0 & (0.6) & 53.6 & $(0.7)$ & 23.1 & (0.6) & 1.1 & $(0.2)$ \\
\hline & Hongkong (China) & 1.9 & $(0.3)$ & 7.3 & (0.6) & 26.6 & $(0.7)$ & 64.1 & (1.0) & 0.1 & $(0.1)$ & 0.0 & C \\
\hline & Indonesien & 1.8 & $(0.7)$ & 8.2 & (1.0) & 49.3 & (3.4) & 36.2 & (3.6) & 4.0 & $(0.9)$ & 0.5 & $(0.3)$ \\
\hline & Jordanien & 0.1 & $(0.1)$ & 1.2 & $(0.4)$ & 7.5 & $(0.8)$ & 91.2 & (0.9) & 0.0 & c & 0.0 & c \\
\hline & Kasachstan & 0.5 & $(0.1)$ & 7.1 & (0.6) & 75.2 & $(2.2)$ & 17.2 & (2.3) & 0.1 & $(0.0)$ & 0.0 & c \\
\hline & Kirgisistan & 0.2 & $(0.1)$ & 8.9 & $(0.7)$ & 72.9 & (1.6) & 17.4 & (1.6) & 0.5 & $(0.2)$ & 0.0 & C \\
\hline & Lettland & 3.6 & (0.9) & 19.9 & (1.1) & 74.7 & (1.4) & 1.6 & (0.4) & 0.1 & $(0.1)$ & 0.0 & $(0.0)$ \\
\hline & Liechtenstein & 1.1 & $(0.7)$ & 19.7 & (1.6) & 68.9 & $(1.2)$ & 10.3 & $(1.2)$ & 0.0 & c & 0.0 & c \\
\hline & Litauen & 0.6 & $(0.2)$ & 12.3 & (1.2) & 80.0 & $(1.2)$ & 7.2 & $(0.7)$ & 0.0 & C & 0.0 & C \\
\hline & Macau (China) & 8.9 & $(0.2)$ & 22.0 & $(0.2)$ & 34.9 & $(0.2)$ & 33.6 & $(0.2)$ & 0.5 & $(0.1)$ & 0.0 & c \\
\hline & Montenegro & 0.0 & c & 3.0 & (2.0) & 85.0 & (1.8) & 12.0 & (0.4) & 0.0 & c & 0.0 & C \\
\hline & Panama & 3.4 & (1.1) & 13.6 & (2.5) & 32.6 & (4.4) & 45.7 & (5.5) & 4.7 & (1.8) & 0.0 & c \\
\hline & Peru & 4.9 & $(0.5)$ & 11.2 & $(0.8)$ & 18.8 & (1.0) & 42.3 & (1.4) & 22.9 & $(0.9)$ & 0.0 & c \\
\hline & Katar & 1.9 & $(0.1)$ & 4.3 & $(0.2)$ & 14.8 & $(0.3)$ & 60.4 & $(0.3)$ & 18.2 & $(0.2)$ & 0.4 & $(0.1)$ \\
\hline & Rumänien & 0.0 & c & 6.3 & (1.1) & 89.9 & (1.3) & 3.9 & $(0.7)$ & 0.0 & c & 0.0 & c \\
\hline & Russ. Föderation & 1.4 & $(0.3)$ & 10.4 & (0.9) & 61.2 & (1.9) & 26.3 & (1.9) & 0.8 & $(0.2)$ & 0.0 & c \\
\hline & Serbien & 0.3 & $(0.1)$ & 2.7 & $(0.7)$ & 95.6 & $(0.8)$ & 1.4 & $(0.2)$ & 0.0 & c & 0.0 & c \\
\hline & Shanghai (China) & 1.2 & (0.3) & 5.1 & (0.6) & 38.8 & (1.2) & 54.7 & (1.4) & 0.2 & $(0.1)$ & 0.0 & c \\
\hline & Singapur & 0.8 & $(0.2)$ & 2.9 & $(0.3)$ & 35.7 & (0.6) & 60.6 & $(0.5)$ & 0.0 & c & 0.0 & c \\
\hline & Chinesisch Taipeh & 0.0 & c & 0.2 & $(0.1)$ & 35.2 & (1.5) & 64.7 & (1.5) & 0.0 & c & 0.0 & c \\
\hline & Thailand & 0.2 & $(0.1)$ & 0.8 & $(0.2)$ & 26.3 & (1.4) & 70.5 & (1.4) & 2.2 & $(0.5)$ & 0.0 & C \\
\hline & Trinidad und Tobago & 2.7 & $(0.3)$ & 10.7 & $(0.5)$ & 28.4 & $(0.6)$ & 51.0 & $(0.5)$ & 7.1 & $(0.4)$ & 0.0 & c \\
\hline & Tunesien & 8.9 & (0.6) & 16.8 & (0.9) & 24.4 & (1.1) & 45.3 & (1.5) & 4.7 & $(0.5)$ & 0.0 & c \\
\hline & Uruguay & 9.1 & (1.0) & 12.0 & $(0.8)$ & 24.9 & $(0.8)$ & 50.4 & (1.3) & 3.6 & $(0.4)$ & 0.0 & c \\
\hline
\end{tabular}

StatLink 需西 http://dx.doi.org/10.1787/888932343190 
[Teil 2/2]

Tabelle A2.4b Prozentsatz der Schüler in den einzelnen Klassenstufen, nach Geschlecht

\begin{tabular}{|c|c|c|c|c|c|c|c|c|c|c|c|c|c|}
\hline & & \multicolumn{12}{|c|}{ Mädchen - Klassenstufe } \\
\hline & & \multicolumn{2}{|c|}{ 7. Klasse } & \multicolumn{2}{|c|}{ 8. Klasse } & \multicolumn{2}{|c|}{ 9. Klasse } & \multicolumn{2}{|c|}{ 10. Klasse } & \multicolumn{2}{|c|}{ 11. Klasse } & \multicolumn{2}{|c|}{ 12. Klasse } \\
\hline & & $\%$ & S.E. & $\%$ & S.E. & $\%$ & S.E. & $\%$ & S.E. & $\%$ & S.E. & $\%$ & S.E. \\
\hline \multirow{35}{*}{ 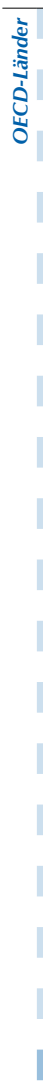 } & Australien & 0.0 & $(0.0)$ & 0.1 & $(0.0)$ & 7.9 & $(0.5)$ & 72.0 & (0.8) & 20.0 & (0.8) & 0.1 & $(0.0)$ \\
\hline & Österreich & 0.6 & $(0.4)$ & 5.0 & $(1.2)$ & 42.2 & (1.4) & 52.1 & (1.5) & 0.0 & $(0.0)$ & 0.0 & C \\
\hline & Belgien & 0.3 & $(0.1)$ & 4.5 & $(0.5)$ & 29.3 & (1.1) & 64.5 & (1.1) & 1.3 & $(0.2)$ & 0.0 & $(0.0)$ \\
\hline & Kanada & 0.0 & $(0.0)$ & 1.0 & $(0.2)$ & 12.5 & $(0.5)$ & 85.3 & $(0.5)$ & 1.1 & $(0.1)$ & 0.0 & $(0.0)$ \\
\hline & Chile & 0.7 & $(0.1)$ & 2.9 & $(0.5)$ & 17.7 & $(0.9)$ & 73.0 & (1.1) & 5.6 & $(0.4)$ & 0.0 & $(0.0)$ \\
\hline & Tschech. Rep. & 0.3 & $(0.2)$ & 3.1 & (0.4) & 44.8 & (1.9) & 51.8 & (1.9) & 0.0 & C & 0.0 & C \\
\hline & Dänemark & 0.1 & $(0.0)$ & 10.0 & $(0.7)$ & 87.3 & (0.9) & 2.5 & $(0.8)$ & 0.0 & c & 0.0 & c \\
\hline & Estland & 0.9 & $(0.3)$ & 20.8 & (0.9) & 75.4 & (1.1) & 2.7 & $(0.5)$ & 0.2 & $(0.2)$ & 0.0 & C \\
\hline & Finnland & 0.4 & $(0.1)$ & 9.6 & (0.6) & 89.4 & (0.6) & 0.0 & c & 0.6 & $(0.2)$ & 0.0 & c \\
\hline & Frankreich & 1.3 & (0.9) & 3.2 & (0.9) & 29.4 & (1.5) & 61.6 & (1.7) & 4.4 & (0.8) & 0.1 & $(0.1)$ \\
\hline & Deutschland & 1.1 & $(0.2)$ & 8.8 & $(0.6)$ & 53.4 & (1.1) & 36.4 & (1.1) & 0.3 & $(0.1)$ & 0.0 & $(0.0)$ \\
\hline & Griechenland & 0.2 & $(0.2)$ & 0.9 & (0.5) & 4.9 & $(0.7)$ & 94.0 & (0.9) & 0.0 & c & 0.0 & C \\
\hline & Ungarn & 2.3 & $(0.7)$ & 5.9 & (1.1) & 65.4 & (1.6) & 26.2 & $(1.2)$ & 0.2 & $(0.1)$ & 0.0 & c \\
\hline & Island & 0.0 & c & 0.0 & C & 0.0 & $(0.1)$ & 97.9 & $(0.2)$ & 2.1 & $(0.2)$ & 0.0 & c \\
\hline & Irland & 0.1 & $(0.1)$ & 2.0 & $(0.4)$ & 57.3 & (1.5) & 25.7 & (2.0) & 15.1 & (1.5) & 0.0 & C \\
\hline & Israel & 0.0 & C & 0.1 & $(0.1)$ & 15.9 & (1.0) & 83.8 & (1.1) & 0.2 & $(0.1)$ & 0.0 & $(0.0)$ \\
\hline & Italien & 0.2 & $(0.1)$ & 1.0 & $(0.2)$ & 13.5 & (0.6) & 81.4 & $(0.7)$ & 3.9 & $(0.3)$ & 0.0 & c \\
\hline & Japan & 0.0 & C & 0.0 & C & 0.0 & C & 100.0 & $(0.0)$ & 0.0 & C & 0.0 & C \\
\hline & Korea & 0.0 & c & 0.0 & c & 3.6 & (1.0) & 95.6 & $(1.0)$ & 0.8 & $(0.1)$ & 0.0 & c \\
\hline & Luxemburg & 0.4 & $(0.1)$ & 10.6 & $(0.3)$ & 50.8 & $(0.4)$ & 38.0 & $(0.3)$ & 0.2 & $(0.1)$ & 0.0 & c \\
\hline & Mexiko & 1.5 & $(0.2)$ & 6.1 & $(0.4)$ & 31.5 & (0.9) & 60.1 & (1.0) & 0.8 & $(0.3)$ & 0.0 & $(0.0)$ \\
\hline & Niederlande & 0.1 & $(0.1)$ & 2.3 & $(0.4)$ & 43.4 & (1.4) & 53.5 & (1.3) & 0.7 & $(0.2)$ & 0.0 & c \\
\hline & Neuseeland & 0.0 & c & 0.0 & C & 0.1 & (0.1) & 4.8 & $(0.5)$ & 89.8 & (0.6) & 5.4 & (0.5) \\
\hline & Norwegen & 0.0 & C & 0.0 & C & 0.4 & $(0.1)$ & 99.4 & $(0.2)$ & 0.1 & $(0.1)$ & 0.0 & c \\
\hline & Polen & 0.6 & $(0.2)$ & 2.5 & $(0.3)$ & 95.6 & $(0.7)$ & 1.3 & $(0.6)$ & 0.0 & c & 0.0 & c \\
\hline & Portugal & 1.4 & $(0.2)$ & 7.7 & $(0.8)$ & 25.1 & (1.4) & 65.4 & (1.9) & 0.4 & $(0.1)$ & 0.0 & C \\
\hline & Slowak. Rep. & 0.7 & $(0.2)$ & 1.5 & $(0.3)$ & 31.4 & (1.8) & 62.1 & $(2.1)$ & 4.3 & (0.9) & 0.0 & (0.0) \\
\hline & Slowenien & 0.0 & C & 0.0 & C & 1.9 & $(0.7)$ & 90.3 & $(0.8)$ & 7.8 & (0.5) & 0.0 & c \\
\hline & Spanien & 0.1 & $(0.1)$ & 7.6 & $(0.4)$ & 24.2 & $(0.7)$ & 68.0 & $(0.8)$ & 0.0 & $(0.0)$ & 0.0 & C \\
\hline & Schweden & 0.1 & $(0.1)$ & 2.3 & $(0.3)$ & 95.4 & $(0.7)$ & 2.2 & $(0.7)$ & 0.0 & C & 0.0 & C \\
\hline & Schweiz & 0.4 & $(0.1)$ & 12.9 & (0.9) & 62.6 & (1.8) & 22.7 & (2.0) & 1.4 & (0.6) & 0.0 & c \\
\hline & Türkei & 0.4 & $(0.2)$ & 2.9 & (0.8) & 19.8 & (1.3) & 72.3 & (1.6) & 4.4 & $(0.4)$ & 0.2 & $(0.1)$ \\
\hline & Ver. Königreich & 0.0 & c & 0.0 & c & 0.0 & c & 1.0 & $(0.1)$ & 98.1 & $(0.1)$ & 0.9 & $(0.1)$ \\
\hline & Ver. Staaten & 0.0 & c & 0.2 & $(0.2)$ & 8.5 & $(0.7)$ & 68.4 & (1.1) & 22.8 & (1.0) & 0.1 & $(0.1)$ \\
\hline & OECD-Durchschnitt & 0.6 & $(0.1)$ & 5.0 & $(0.1)$ & 35.6 & $(0.2)$ & 55.0 & $(0.2)$ & 10.2 & $(0.1)$ & 0.5 & $(0.0)$ \\
\hline ¿ & Albanien & 0.2 & $(0.1)$ & 1.8 & (0.4) & 47.6 & (2.3) & 50.2 & (2.3) & 0.2 & $(0.1)$ & 0.0 & c \\
\hline : & Argentinien & 3.6 & (0.9) & 10.7 & (1.5) & 18.4 & $(1.2)$ & 62.3 & $(2.2)$ & 4.9 & (0.6) & 0.0 & c \\
\hline छั & Aserbaidschan & 0.6 & $(0.3)$ & 5.8 & $(0.6)$ & 51.0 & (1.5) & 42.1 & (1.4) & 0.4 & $(0.1)$ & 0.0 & c \\
\hline 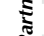 & Brasilien & 5.4 & (0.4) & 15.3 & $(0.6)$ & 37.1 & (0.9) & 39.7 & (0.9) & 2.5 & $(0.2)$ & 0.0 & c \\
\hline 2 & Bulgarien & 0.9 & $(0.3)$ & 4.6 & $(0.7)$ & 90.6 & (1.0) & 3.9 & $(0.7)$ & 0.0 & c & 0.0 & c \\
\hline & Kolumbien & 3.3 & (0.4) & 9.1 & (0.8) & 22.4 & (1.0) & 42.2 & (1.1) & 23.0 & (1.1) & 0.0 & c \\
\hline & Kroatien & 0.0 & c & 0.2 & $(0.2)$ & 75.8 & (0.6) & 24.1 & $(0.5)$ & 0.0 & c & 0.0 & c \\
\hline & Dubai (VAE) & 0.6 & $(0.1)$ & 2.2 & $(0.2)$ & 13.5 & $(0.5)$ & 60.4 & (0.6) & 22.7 & $(0.7)$ & 0.6 & $(0.1)$ \\
\hline & Hongkong (China) & 1.5 & $(0.2)$ & 7.1 & $(0.6)$ & 23.5 & (0.6) & 67.9 & (1.0) & 0.0 & c & 0.0 & c \\
\hline & Indonesien & 1.2 & $(0.3)$ & 4.9 & (0.8) & 42.7 & (3.7) & 44.6 & (3.8) & 6.0 & (1.1) & 0.6 & $(0.5)$ \\
\hline & Jordanien & 0.1 & $(0.0)$ & 1.3 & $(0.3)$ & 6.5 & $(0.7)$ & 92.1 & $(0.9)$ & 0.0 & $\mathrm{c}$ & 0.0 & c \\
\hline & Kasachstan & 0.4 & $(0.1)$ & 5.7 & (0.5) & 71.5 & $(2.0)$ & 22.3 & $(2.1)$ & 0.2 & $(0.1)$ & 0.0 & c \\
\hline & Kirgisistan & 0.1 & $(0.1)$ & 7.1 & $(0.6)$ & 69.9 & (1.5) & 22.0 & (1.6) & 0.9 & $(0.2)$ & 0.0 & c \\
\hline & Lettland & 1.7 & $(0.4)$ & 11.2 & $(0.6)$ & 83.9 & $(0.8)$ & 3.1 & (0.4) & 0.1 & $(0.1)$ & 0.0 & C \\
\hline & Liechtenstein & 0.6 & (0.6) & 15.0 & (1.5) & 74.0 & $(1.2)$ & 10.4 & (1.6) & 0.0 & c & 0.0 & c \\
\hline & Litauen & 0.3 & $(0.1)$ & 8.1 & $(0.8)$ & 81.9 & (0.9) & 9.6 & $(0.7)$ & 0.0 & $(0.0)$ & 0.0 & C \\
\hline & Macau (China) & 4.4 & $(0.1)$ & 16.3 & $(0.2)$ & 34.9 & $(0.2)$ & 43.9 & $(0.2)$ & 0.5 & $(0.1)$ & 0.0 & c \\
\hline & Montenegro & 0.0 & c & 2.0 & (1.4) & 80.3 & (1.3) & 17.8 & (0.4) & 0.0 & C & 0.0 & c \\
\hline & Panama & 2.4 & (0.6) & 7.7 & (1.1) & 28.7 & (3.0) & 53.8 & $(4.0)$ & 7.5 & (1.6) & 0.0 & c \\
\hline & Peru & 3.2 & (0.4) & 6.5 & $(0.6)$ & 15.4 & (0.8) & 47.0 & $(1.2)$ & 27.9 & (1.2) & 0.0 & C \\
\hline & Katar & 1.4 & $(0.1)$ & 3.0 & $(0.1)$ & 12.1 & $(0.2)$ & 64.9 & $(0.2)$ & 18.1 & $(0.2)$ & 0.5 & (0.1) \\
\hline & Rumänien & 0.0 & c & 8.1 & (1.5) & 87.3 & (1.5) & 4.7 & (0.6) & 0.0 & c & 0.0 & c \\
\hline & Russ. Föderation & 0.5 & $(0.1)$ & 9.7 & $(0.8)$ & 59.0 & (2.0) & 29.8 & (1.8) & 1.0 & $(0.2)$ & 0.0 & c \\
\hline & Serbien & 0.1 & $(0.1)$ & 1.4 & $(0.5)$ & 96.4 & $(0.6)$ & 2.0 & $(0.2)$ & 0.0 & c & 0.0 & c \\
\hline & Shanghai (China) & 0.8 & $(0.2)$ & 3.0 & $(0.4)$ & 36.1 & (1.0) & 59.5 & (1.0) & 0.6 & $(0.2)$ & 0.0 & $(0.0)$ \\
\hline & Singapur & 1.2 & $(0.2)$ & 2.3 & $(0.3)$ & 33.7 & $(0.5)$ & 62.7 & (0.4) & 0.0 & c & 0.0 & $(0.0)$ \\
\hline & Chinesisch Taipeh & 0.0 & c & 0.0 & $(0.0)$ & 33.7 & (1.5) & 66.3 & (1.5) & 0.0 & $(0.0)$ & 0.0 & C \\
\hline & Thailand & 0.0 & C & 0.3 & $(0.1)$ & 20.9 & (1.4) & 75.8 & (1.4) & 3.0 & $(0.4)$ & 0.0 & C \\
\hline & Trinidad und Tobago & 1.5 & $(0.3)$ & 6.9 & $(0.5)$ & 22.3 & $(0.6)$ & 61.0 & $(0.6)$ & 8.3 & $(0.4)$ & 0.0 & C \\
\hline & Tunesien & 4.2 & (0.4) & 10.3 & (0.5) & 23.4 & (1.0) & 56.1 & (1.4) & 6.0 & (0.5) & 0.0 & c \\
\hline & Uruguay & 5.4 & (0.6) & 9.4 & (0.5) & 18.5 & (0.9) & 61.4 & (1.2) & 5.4 & (0.6) & 0.0 & C \\
\hline
\end{tabular}

StatLink त्ताज़ http://dx.doi.org/10.1787/888932343190 
- Spalte 10 zeigt die ungewichtete Zahl der teilnehmenden und nicht teilnehmenden Schulen nach Einbeziehung von Ersatzschulen.

- Spalte 11 zeigt die gewichtete Schülerbeteiligungsquote nach Einbeziehung von Ersatzschulen. Sie ergibt sich durch Division der Spalte 12 durch Spalte 13.

- Spalte 12 zeigt die gewichtete Zahl der getesteten Schülerinnen und Schüler.

- Spalte 13 zeigt die gewichtete Zahl der Stichprobenschüler (an der Testsitzung teilnehmende und nicht teilnehmende Schülerinnen und Schüler).

- Spalte 14 zeigt die ungewichtete Zahl der getesteten Schülerinnen und Schüler. Zu beachten ist, dass Schüler von Schulen mit einer Schülerbeteiligungsquote von weniger als 50\% nicht in die (gewichteten und ungewichteten) Quoten einbezogen wurden.

- Spalte 15 zeigt die ungewichtete Zahl der Stichprobenschüler (an der Testsitzung teilnehmende und nicht teilnehmende Schülerinnen und Schüler). Zu beachten ist, dass Schülerinnen und Schüler von Schulen, in denen weniger als die Hälfte der in Betracht kommenden Schüler an der Erhebung teilgenommen hat, nicht in den (gewichteten und ungewichteten) Quoten berücksichtigt wurden.

\section{Definition der Schulen}

In einigen Ländern wurden anstelle von Schulen Untereinheiten von Schulen für die Stichprobe herangezogen, und das kann die Schätzung der Anteile der Varianz zwischen Schulen beeinflussen. In Deutschland, Japan, Österreich, Rumänien, Slowenien, der Tschechischen Republik und Ungarn wurden Schulen mit mehr als einem Bildungsgang in die den jeweiligen Bildungsgängen entsprechenden Einheiten unterteilt. In den Niederlanden wurden Schulen, die sowohl die Sekundarstufe I als auch II umfassen, in die jeweiligen Stufen unterteilt. Was Belgien betrifft, so wurden in der flämischen Gemeinschaft bei Multi-Campus-Schulen die verschiedenen Einrichtungen in die Stichprobe einbezogen, während in der französischen Gemeinschaft im Fall von MultiCampus-Schulen die jeweils größere Verwaltungseinheit berücksichtigt wurde. In Australien wurden im Fall von Schulen mit mehr als einem Campus die einzelnen Campuseinheiten erfasst. In Argentinien, Dubai (VAE) und Kroatien wurden im Fall solcher Schulen die einzelnen Standorte berücksichtigt. In Spanien wurden die Schulen im Baskenland, die Mehrsprachenmodelle anbieten, für die Stichprobenziehung in die einzelnen Programme unterteilt.

\section{Klassenstufen}

Die in PISA 2009 getesteten Schülerinnen und Schüler besuchen unterschiedliche Klassenstufen. Der Anteil der Schülerinnen und Schüler in jeder Klassenstufe wird in Tabelle A2.4a nach Ländern geordnet und in Tabelle A2.4b innerhalb jedes Landes geschlechtsspezifisch dargestellt.

\section{Schülerinnen und Schüler innerhalb und außerhalb des regulären Bildungssystems in Argentinien}

Die niedrige Leistung der 15-jährigen Schülerinnen und Schüler in Argentinien ist zu einem gewissen Grad dadurch bedingt, dass ein recht hoher Anteil der 15-Jährigen in Bildungsgängen außerhalb des regulären Bildungssystems eingeschrieben ist. Tabelle A2.5 zeigt jeweils den Anteil der Schülerinnen und Schüler innerhalb und außerhalb des regulären Bildungssystems sowie ihre Leistungen in PISA 2009.

Prozentsatz der Schüler und Mittelwerte in Lesekompetenz, Mathematik und Naturwissenschaften in Tabelle A2.5 Argentinien nach Teilnahme oder Nichtteilnahme am regulären Bildungssystem

\begin{tabular}{|c|c|c|c|c|c|c|c|c|}
\hline & & & \multicolumn{6}{|c|}{ Mittelwerte } \\
\hline & \multicolumn{2}{|c|}{ Prozentsatz der Schüler } & \multicolumn{2}{|c|}{ Lesekompetenz } & \multicolumn{2}{|c|}{ Mathematik } & \multicolumn{2}{|c|}{ Naturwissenschaften } \\
\hline & $\%$ & S.E. & Punktzahl & S.E. & Punktzahl & S.E. & Punktzahl & S.E. \\
\hline Schüler im regulären Bildungssystem ${ }^{1}$ & 60.9 & 2.2 & 439 & 5.1 & 421 & 4.8 & 439 & 4.9 \\
\hline Schüler außerhalb des regulären Bildungssystems ${ }^{2}$ & 39.1 & 2.2 & 335 & 8.0 & 337 & 6.7 & 341 & 8.3 \\
\hline
\end{tabular}

1. Schüler, die nicht die Klassenstufen 10 oder 11 besuchen und nicht in Programm 3, 4, 5, 6, 7 oder 8 eingeschrieben sind.

2. Schüler, die die Klassenstufen 10 oder 11 besuchen und in Programm 3, 4, 5, 6, 7 oder 8 eingeschrieben sind.

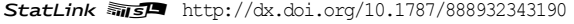




\section{ANHANG A3 \\ STANDARDFEHLER, SIGNIFIKANZTESTS UND VERGLEICHE ZWISCHEN UNTERGRUPPEN}

Die in diesem Bericht enthaltenen Statistiken stellen Schätzwerte der nationalen Leistung auf der Basis der Schülerstichproben dar und nicht etwa Werte, die ausgehend von Antworten sämtlicher Schülerinnen und Schüler eines Landes auf sämtliche Fragen errechnet worden wären. Daher ist es wichtig, die mögliche Höhe des Messfehlers dieser Schätzungen zu ermitteln. In PISA wird bei jeder Schätzung ein Messfehler angegeben, der durch den Standardfehler (S.E.) ausgedrückt ist. Die Verwendung von Konfidenzintervallen ermöglicht es, Schlüsse in Bezug auf die Populationsdurchschnittswerte und -prozentsätze zu ziehen und dabei den an die Stichprobenschätzungen geknüpften Messfehler zu berücksichtigen. Unter Annahme einer Normalverteilung kann davon ausgegangen werden, dass das tatsächlich beobachtete statistische Ergebnis einer gegebenen Population in 95 von 100 Wiederholungsmessungen mit unterschiedlichen Stichproben derselben Population innerhalb des Konfidenzintervalls liegen würde.

Die Leser sind häufig in erster Linie daran interessiert, ob sich ein bestimmter Wert für ein gegebenes Land von einem zweiten Wert für dasselbe Land oder für ein anderes Land unterscheidet, z.B. ob in einem bestimmten Land Mädchen bessere Leistungen erzielen als Jungen. In den Tabellen und Abbildungen dieses Berichts werden Unterschiede als statistisch signifikant bezeichnet, wenn die betreffenden kleineren oder größeren Differenzen in weniger als 5\% der Fälle beobachtet würden, obwohl die entsprechenden Populationswerte in Wirklichkeit nicht voneinander abweichen. Entsprechend wird das Risiko, dass eine Korrelation als signifikant eingestuft wird, wenn effektiv keine Korrelation zwischen zwei Messgrößen besteht, auf 5\% begrenzt.

Für sämtliche Teile des Berichts wurden Signifikanztests durchgeführt, um die statistische Signifikanz der vorgenommenen Vergleiche zu prüfen.

\section{Geschlechtsspezifische Unterschiede}

Die geschlechtsspezifischen Unterschiede bei den Schülerleistungen oder anderen Indizes wurden auf ihre statistische Signifikanz hin getestet. Positive Unterschiede weisen auf höhere Punktzahlen für Jungen hin, negative Unterschiede auf höhere Punktzahlen für Mädchen. Die in den Tabellen dieses Bands fettgedruckten Unterschiede sind im Allgemeinen bei einem Konfidenzniveau von 95\% statistisch signifikant.

\section{Leistungsdifferenzen zwischen dem obersten und dem untersten Quartil der PISA-Indizes und -Skalen}

Unterschiede bei den Durchschnittsleistungen zwischen dem obersten und dem untersten Quartil auf den PISA-Indizes und -Skalen wurden auf ihre statistische Signifikanz hin getestet. Angaben in Fettdruck weisen darauf hin, dass bei einem Konfidenzniveau von 95\% ein statistisch signifikanter Unterschied zwischen der Leistung des obersten und des untersten Quartils der Schüler auf dem jeweiligen Index besteht.

\section{Veränderung der Leistung je Indexeinheit}

Für viele Tabellen wurde die Veränderung der Leistung je Indexeinheit berechnet. Angaben in Fettdruck weisen auf Veränderungen hin, die bei einem Konfidenzniveau von 95\% statistisch signifikant von null abweichen.

\section{Unterschiede bei der Lesekompetenz zwischen Schülern mit und ohne Migrationshintergrund}

Leistungsunterschiede zwischen Schülern ohne Migrationshintergrund und Schülern mit Migrationshintergrund wurden auf ihre statistische Signifikanz hin getestet. Zu diesem Zweck wurden Schüler der ersten Generation und der zweiten Generation in der Kategorie „Schüler mit Migrationshintergrund“ zusammengefasst. Positive Differenzen deuten auf höhere Punktzahlen für Schüler ohne Migrationshintergrund hin, negative Differenzen auf höhere Punktzahlen für Schüler der ersten und der zweiten Generation. Angaben in Fettdruck weisen in den Datentabellen dieses Bands auf statistisch signifikante Punktzahlveränderungen bei einem Konfidenzniveau von $95 \%$ hin. 


\section{ANHANG A4 \\ QUALITÄTSSICHERUNG}

Wie bereits in allen früheren PISA-Erhebungen wurden in sämtlichen Teilen von PISA 2009 Qualitätssicherungsverfahren durchgeführt.

Die einheitliche Qualität und sprachliche Äquivalenz der in PISA 2009 verwendeten Erhebungsinstrumente wurden dadurch erleichtert, dass den Ländern äquivalente Originalfassungen der Erhebungsinstrumente in Englisch und Französisch vorgelegt und die Länder (in denen die Schülerleistungen nicht in diesen zwei Sprachen erhoben wurden) aufgefordert wurden, ausgehend von den beiden Originalversionen zwei unabhängige Übersetzungen anzufertigen und dann zusammenzufassen. Es wurden genaue Übersetzungs- und Bearbeitungsrichtlinien vorgegeben und Anweisungen hinsichtlich der Auswahl und Ausbildung der Übersetzer erteilt. Für jedes Land wurden die Übersetzung und das Format der Erhebungsinstrumente (einschließlich des Testmaterials, der Kodieranweisungen, der Fragebogen und der Handbücher) durch vom PISA-Konsortium eingesetzte erfahrene Übersetzer überprüft, bevor sie im Feldtest und in der Haupterhebung von PISA 2009 eingesetzt wurden. Die Muttersprache dieser Übersetzer war die Unterrichtssprache in dem betreffenden Land und sie verfügten über ausreichende Kenntnisse bezüglich der Bildungssysteme. Nähere Informationen über die PISA-Übersetzungsverfahren enthält PISA 2009 Technical Report (OECD, erscheint demnächst).

Die Erhebung wurde mit Hilfe standardisierter Verfahren durchgeführt. Das PISA-Konsortium stellte umfassende Handbücher zur Verfügung, die den Ablauf der Erhebung erklärten und u.a. präzise Anweisungen für die Arbeit der Schulkoordinatoren sowie Vorlagen für die Testleiter zum Gebrauch bei den Testsitzungen enthielten. Vorgeschlagene Anpassungen der Erhebungsmethoden oder vorgeschlagene Änderungen der Testsitzungen wurden vor der Überprüfung dem PISA-Konsortium zur Genehmigung vorgelegt. Das PISA-Konsortium überprüfte dann die nationalen Übersetzungen und die Anpassung der Handbücher.

Um die Glaubwürdigkeit von PISA im Hinblick auf Validität und Unvoreingenommenheit zu gewährleisten und einen einheitlichen Ablauf der Testsitzungen zu fördern, wurden die Testleiterinnen und Testleiter in den Teilnehmerländern nach folgenden Kriterien ausgewählt: Vorgeschrieben wurde, dass die Leiterinnen und Leiter der vorgesehenen PISA-Testsitzungen nicht zugleich die Fachlehrer der teilnehmenden Schülerinnen und Schüler in den Bereichen Lesen, Mathematik und Naturwissenschaften sein durften; empfohlen wurde, dass die Testleiterinnen und Testleiter nicht Mitglied des Kollegiums einer Schule sein sollten, an der sie die Leitung der PISA-Tests übernahmen; als wünschenswert wurde erachtet, dass die Testleiterinnen und Testleiter nicht zum Kollegium einer für die PISA-Stichprobe gezogenen Schule gehören sollten. Die Teilnehmerländer organisierten für die Testleiterinnen und Testleiter eine individuelle Schulung.

Es war Aufgabe der Teilnehmerländer sicherzustellen, dass die Testleiterinnen und Testleiter mit den Schulkoordinatoren bei der Vorbereitung der Testsitzung zusammenarbeiteten, u.a. bei der Aktualisierung der Unterlagen über den bisherigen Bildungsweg der Schülerinnen und Schüler sowie der Identifizierung ausgeschlossener Schülerinnen und Schüler, dass für die kognitiven Items keine zusätzliche Zeit eingeräumt wurde (während es erlaubt war, für die Beantwortung des Schülerfragebogens mehr Zeit zuzugestehen), dass kein Instrument vor Beginn der zwei einstündigen Teile der kognitiven Sitzung bekannt gegeben wurde, dass die Testleiterinnen und Testleiter den Stand der Schülerbeteiligung auf den Unterlagen über die Bildungswege der Schülerinnen und Schüler festhielten und ein Formular für den Sitzungsbericht ausfüllten, dass kein kognitives Instrument fotokopiert werden durfte, dass kein kognitives Instrument von dem Schulpersonal vor der Testsitzung gesehen werden durfte und die Testleiterinnen und Testleiter das Material unmittelbar nach Abschluss der Testsitzungen an das nationale Zentrum zurückgaben.

Die nationalen Projektmanager wurden dazu angehalten, eine Folgesitzung zu organisieren, wenn mehr als 15\% der PISA-Stichprobe nicht an der ursprünglichen Testsitzung teilnehmen konnten.

Für das Qualitätsmonitoring zuständige nationale Vertreter des PISA-Konsortiums besuchten alle nationalen Zentren, um die Verfahren der Datenerhebung zu überprüfen. Schließlich besuchten „School Quality Monitors“ aus dem PISA-Konsortium eine Stichprobe von 15 Schulen während der Erhebung. Nähere Informationen über die Feldoperationen enthält PISA 2009 Technical Report (OECD, erscheint demnächst).

Um eine konsistente und genaue Anwendung der in den PISA-Handbüchern dargelegten Kodieranweisungen zu gewährleisten, wurden entsprechende Verfahren entwickelt. Die nationalen Projektleiter wurden gebeten, dem Konsortium Änderungsvorschläge zur Genehmigung vorzulegen. Es wurden Reliabilitätsuntersuchungen durchgeführt, um die Konsistenz des Kodierungsprozesses zu analysieren, worauf weiter unten noch näher eingegangen wird.

Eine speziell für PISA konzipierte Software erleichterte die Dateneingabe, deckte weitverbreitete Fehler während der Dateneingabe auf und vereinfachte den Prozess der Datenbereinigung. Durch Schulungen wurden die nationalen Projektleiter mit diesen Verfahren vertraut gemacht.

Eine Beschreibung der für PISA angewandten Verfahren zur Qualitätssicherung und deren Ergebnisse enthält PISA 2009 Technical Report (OECD, erscheint demnächst).

Die Ergebnisse der Datenbeurteilung zeigen, dass den technischen Standards von PISA in allen PISA-2009-Teilnehmerländern und -volkswirtschaften gänzlich entsprochen wurde, wenngleich für ein Land ernsthafte Zweifel erhoben wurden. Die Analyse der 
Daten für Aserbaidschan deutet aus folgenden vier Gründen darauf hin, dass die technischen Standards von PISA möglicherweise nicht genau eingehalten wurden: a) Die Reihenfolge der Schwierigkeitsgrade der Aufgabenblöcke entspricht nicht den Erfahrungen und die Reihenfolge ist je nach Testheft unterschiedlich, b) der Anteil der richtigen Antworten ist bei manchen Items höher als in den Ländern mit den höchsten Ergebnissen, c) der Schwierigkeitsgrad der Aufgabenblöcke schwankt je nach Testheft stark, und d) die Kodierung der Items in Aserbaidschan stimmt von einem unabhängigen Kodierer zum anderen extrem überein und wurde bei manchen Items als zu nachsichtig erachtet. Allerdings ergaben sich bei näherer Untersuchung der Erhebungsinstrumente, der Testabläufe und der Kodierung der Schülerantworten auf nationaler Ebene keine ausreichenden Hinweise auf systematische Fehler oder Verstöße gegen die technischen Standards von PISA. Deshalb wurden die Daten für Aserbaidschan in die internationale Datenreihe von PISA 2009 eingefügt.

In Österreich kam es aus Anlass der PISA-Erhebung 2009 zu einem Streit zwischen den Lehrergewerkschaften und der Bildungsministerin, der zur Ankündigung eines PISA-Boykotts führte, welcher dann aber nach der ersten Testwoche zurückgenommen wurde. Auf Grund des Boykotts musste die OECD identifizierbare Fälle aus der Datenreihe entfernen. Wenngleich die österreichischen Daten nach der Entfernung dieser Fälle die technischen Standards von PISA 2009 erfüllten, hat die negative Stimmung gegenüber Bildungserhebungen die Testbedingungen belastet und könnte die Motivation der Schüler zur Beantwortung der PISA-Aufgaben ungünstig beeinflusst haben. Die Vergleichbarkeit der Erhebungsdaten des Jahres 2009 mit denen früherer PISA-Erhebungen kann dementsprechend nicht gewährleistet werden, weshalb die österreichischen Daten aus den Trendvergleichen ausgeschlossen wurden. 


\section{ANHANG A5}

\section{TEILNAHME DER LÄNDER AN DEN PISA-ERHEBUNGEN}

Nicht alle OECD-Mitgliedsländer haben an allen PISA-Erhebungen teilgenommen, und die Liste der Teilnehmerländer und -volkswirtschaften ist seit dem Jahr 2000 erheblich gewachsen. Wie in Kapitel 1 erläutert, werden die Leistungstrends im Bereich Lesekompetenz für alle Länder mit vergleichbaren Ergebnissen in den Erhebungen von 2000 und 2009 aufgeführt, da die Lesekompetenz Schwerpunktbereich von PISA 2000 war, wo eine Leistungsskala erstellt wurde, die mit allen künftigen Erhebungen vergleichbar ist. Da in PISA 2003 der Schwerpunkt auf Mathematik lag und eine Leistungsskala erstellt wurde, die als Maßstab für nachfolgende Beurteilungen der Mathematikleistungen dient, sind die Trends in Mathematik nur für Länder mit vergleichbaren Daten aus den Erhebungen 2006 und 2009 aufgeführt. Im Bereich Naturwissenschaften liefern nur die Erhebungen von 2006 und 2009 vergleichbare Ergebnisse, da Naturwissenschaften Schwerpunkt von PISA 2006 waren, wo eine Referenzskala für Naturwissenschaften erstellt wurde.

Folglich ist die Zusammensetzung der Länder, für die Leistungstrends erfasst werden, in den Erhebungsbereichen unterschiedlich (Tabelle A5.1). Auch die Gruppe der OECD-Länder, für die sich der OECD-Durchschnitt im Zeitverlauf vergleichen lässt, weicht in den Erhebungsbereichen ab.

Wie in Kapitel 1 erläutert, bleiben einige Länder aus methodischen Gründen in den Vergleichen zwischen PISA 2000, 2003, 2006 und 2009 unberücksichtigt. Die niederländische Stichprobe entsprach im Jahr 2000 wegen der zu geringen Beteiligungsquote nicht den PISA-Standards. Deshalb wurden für die Niederlande im Jahr 2000 keine Mittelwerte angegeben. In Luxemburg wurden die Testverfahren 2003 im Vergleich zu 2000 stark geändert. Daher sind nur die Ergebnisse von PISA 2003, 2006 und 2009 vergleichbar. Die Stichproben für das Vereinigte Königreich wurden 2000 und 2003 den in Bezug auf die Beteiligungsquote geltenden Regeln von PISA nicht gerecht, weshalb die Daten für das Vereinigte Königreich nicht mit denen der anderen Länder vergleichbar sind. Aus den Vereinigten Staaten liegen für 2006 keine Ergebnisse zur Lesekompetenz vor. In Österreich wurden die Stichprobengewichtungen für die PISA-Erhebung 2000 angepasst, um Vergleiche mit nachfolgenden PISA-Erhebungen zu ermöglichen. Auf Grund eines PISABoykotts in einigen österreichischen Schulen konnte die Vergleichbarkeit der Daten von 2009 mit Daten früherer Erhebungen nicht gewährleistet werden. Daher wurden die österreichischen Daten aus den Trendvergleichen ausgeschlossen. Nähere Einzelheiten hierzu finden sich im Haupttext und in den Fußnoten zu Kapitel 1.

Beim Vergleich der Trends im Bereich Lesekompetenz sind in diesem Band 38 Länder mit vergleichbaren Ergebnissen aus den PISAErhebungen 2000 und 2009 berücksichtigt. Hierzu zählen auch 26 OECD-Länder. Von den 34 aktuellen OECD-Mitgliedsländern nahmen Estland, die Slowakische Republik, Slowenien und die Türkei an PISA 2000 nicht teil, während die PISA-2000-Daten für Luxemburg, die Niederlande und das Vereinigte Königreich sowie die PISA-2009-Daten für Österreich als nicht hinreichend vergleichbar mit denen anderer PISA-Erhebungen gelten und von der Analyse ausgeklammert wurden. Der Durchschnitt der 26 OECD-Länder wird für die meisten Vergleiche in diesem Band zu Grunde gelegt, wann immer die Ergebnisse aus PISA 2009 mit denen aus PISA 2000 verglichen werden. Jedoch gibt es drei weitere OECD-Länder, für die keine validen Ergebnisse aus den Erhebungen der Jahre 2003 bzw. 2006 vorliegen. Chile und Israel nahmen an der Erhebung 2003 nicht teil, während 2006 für die Vereinigten Staaten keine Daten zur Lesekompetenz zur Verfügung standen. So kann für die vier Erhebungen im Bereich Lesekompetenz nur der OECD-23Durchschnitt berechnet werden. Der OECD-23-Durchschnitt ist in den Tabellen aufgeführt, in denen die Daten aus PISA 2000 und PISA 2009 zusammen mit den Ergebnissen aus PISA 2003 und PISA 2006 angegeben sind.

Beim Vergleich der Trends in Mathematik werden die Resultate der 39 Länder mit vergleichbaren Ergebnissen aus PISA 2003 und PISA 2009 erörtert, wozu auch 28 OECD-Länder zählen. Chile, Estland, Israel und Slowenien nahmen an PISA 2003 nicht teil, während die Daten für das Vereinigte Königreich aus PISA 2003 und die Daten für Österreich aus PISA 2009 als nicht vergleichbar erachtet wurden.

Beim Vergleich der Trends in Naturwissenschaften werden die Leistungen von 56 Teilnehmerländern an den Erhebungen 2006 und 2009 verglichen, darunter 33 OECD-Länder. Die PISA-2009-Daten für Österreich wurden als nicht vergleichbar erachtet.

Daher werden in diesem Band unterschiedliche OECD-Durchschnitte angegeben. Der Durchschnitt der 26 OECD-Länder wird bei allen Leistungsvergleichen zwischen PISA 2000 und PISA 2009 angegeben. Bei Leistungsvergleichen der Lesekompetenz über alle vier PISA-Erhebungen ist der Durchschnitt der 23 OECD-Länder in den Tabellen V.2.1, V.2.3 und V2.7 aufgeführt. In Tabelle V.2.9 wird für Vergleiche zwischen PISA 2003 und PISA 2009 der Durchschnitt der 28 OECD-Länder angegeben und bei Vergleichen zwischen PISA 2006 und PISA 2009 der Durchschnitt der 32 OECD-Länder. Leistungsvergleiche im Bereich Mathematik beziehen sich auf den Durchschnitt der 28 OECD-Länder. Im Bereich Naturwissenschaften wird der Durchschnitt der 33 OECD-Länder angegeben.

Infolgedessen weichen die in anderen Bänden dieses Berichts bei Querschnittsvergleichen enthaltenen OECD-Durchschnitte von den Durchschnitten ab, auf die in Band V bei Vergleichen der Schülerleistungen und sonstigen Messgrößen im Zeitverlauf Bezug genommen wird.

Der OECD-Durchschnitt wird für jede Erhebung getrennt berechnet und umfasst alle OECD-Länder mit validen Ergebnissen aus der jeweiligen Erhebung. In einigen Fällen werden die Ergebnisse für ein oder zwei OECD-Länder auf Grund eines zu geringen Stichprobenumfangs (durch ein "," gekennzeichnet), fehlender Daten (, $\left.\mathrm{m}^{\prime \prime}\right)$ oder weil Daten zurückgezogen oder nicht erhoben wurden (,, $\left.\mathrm{w}^{\prime \prime}\right)$ nicht aufgeführt. In derartigen Fällen lässt sich der OECD-Durchschnitt in einer Erhebung für eine kleinere Zahl von Ländern berechnen als in der anderen. Die Veränderung des OECD-Durchschnitts betrifft nur Länder mit validen Ergebnissen aus beiden Erhebungen. Daher stimmt in einigen seltenen Fällen der Unterschied zwischen zwei OECD-Durchschnitten, die für zwei Erhebungen getrennt berechnet wurden, nicht mit der Veränderung des OECD-Durchschnitts überein. Da beispielsweise in PISA 2000 für Japan keine sozioökonomischen Daten erhoben wurden, ist Japan in dem in Tabelle V.4.3 wiedergegebenen OECD-Durchschnitt nicht berücksichtigt. Entsprechend wird dieVeränderung beim OECD-Durchschnitt ohne Japan berechnet. Jedoch ist Japan im Durchschnitt von 2009 berücksichtigt. Analog hierzu werden die Durchschnittswerte in den Tabellen V.4.4 und V.4.5 für Länder berechnet, die über eine hinreichend große Zahl an Beobachtungen verfügen, um Leistungslücken zwischen verschiedenen Schülergruppen aufzuzeigen. Bei den OECD-Durchschnitten in den Tabellen V.4.1 und V.4.3 ist Frankreich berücksichtigt; die Daten für Frankreich wurden aber aus den Tabellen zurückgezogen. 
[Teil 1/1]

Tabelle A5.1 Teilnahme der Länder an unterschiedlichen PISA-Erhebungen

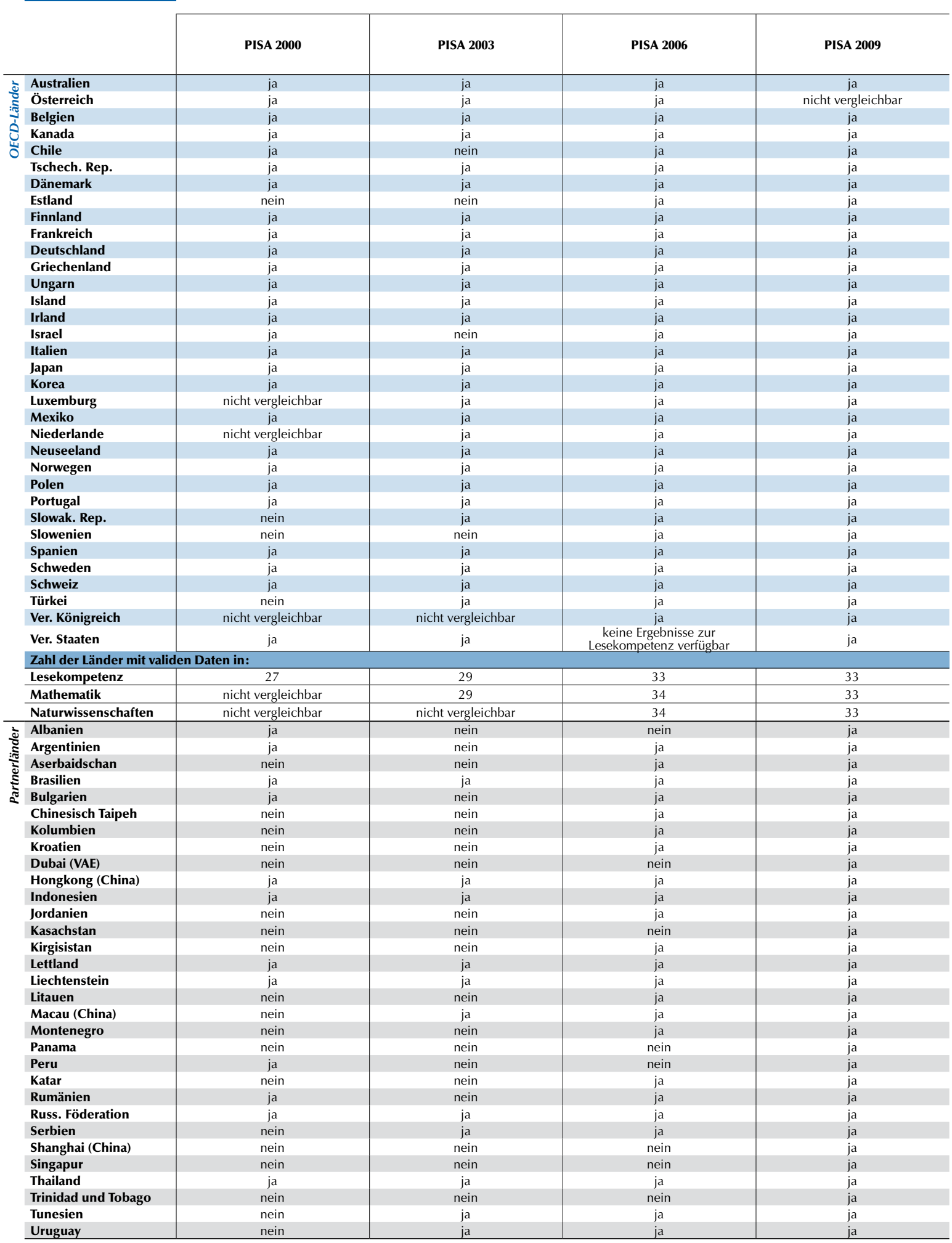




\section{ANHANG A6}

\section{LINEARE UND BEREINIGTE TRENDS}

Untersuchungsergebnisse können auf Grund von Stichproben- und Messfehlern zwischen den Erhebungsrunden variieren, selbst wenn sich das eigentliche Leistungsniveau der Schülerinnen und Schüler nicht verändert. Die Genauigkeit der Ergebnisse lässt sich durch die Berücksichtigung von Informationen aus allen Erhebungsrunden erhöhen. Hierdurch wird die Gelegenheit geboten, Trends genauer zu verfolgen, als es der Rückgriff auf nur zwei Beobachtungen zulassen würde. In diesem Anhang wird beschrieben, wie ein lineares Regressionsmodell zur Schätzung linearer Trends auf die Ergebnisse der vier PISA-Erhebungen zugeschnitten wurde.

Ferner ist es bei der Untersuchung und Interpretation der Veränderungen der PISA-Ergebnisse auf Länderebene wichtig, dem potenziellen Einfluss vonVeränderungen bei Faktoren, wie der demografischen Entwicklung und der Stichprobenermittlungsmethode auf die Ergebnisse Rechnung zu tragen. Dieser Aspekt wurde von Gebhardt und Adams (2007) hervorgehoben, die veranschaulichten, wie Veränderungen im Zeitverlauf bei Faktoren wie der geschätzten Verteilung des sozioökonomischen Hintergrunds und des geschätzten Prozentsatzes an Schülerinnen und Schülern einen wesentlichen Einfluss auf die Trendergebnisse haben können.

Gebhardt und Adams (2007) bezeichneten Trends, die um diese Veränderungen nicht bereinigt waren, als marginale Trends und bereinigte Trends als bedingte Trends. Sie kamen zu dem Schluss, dass ein umfassenderes Verständnis der Trendergebnisse eines Landes erreicht werden könnte, wenn sowohl die marginalen als auch die bedingten Trends untersucht würden. In diesem Bericht werden diese beiden Ergebniskategorien als unbereinigte bzw. bereinigte Trends bezeichnet.

Wie die in Kapitel 2 und in diesem Anhang dargelegten Ergebnisse veranschaulichen, verändern diese Bereinigungen die Hauptschlussfolgerungen hinsichtlich der in den verschiedenen Ländern beobachteten Trends nicht. Kapitel 2 des vorliegenden Bands erörtert jene Fälle, in denen diese Bereinigungen doch zu unterschiedlichen Schlussfolgerungen führen.

\section{Lineare Trends}

Trends können nicht vollständig beurteilt werden, wenn nur der Leistungsunterschied zwischen zwei Zeitpunkten betrachtet wird. In einigen Ländern variieren die Durchschnittsleistungen zwischen den Erhebungsrunden, wobei die Veränderungen gegenüber dem Vorjahr in unterschiedliche Richtungen gehen. Um herauszufinden, ob die Leistungen in einem bestimmten Land um ein ähnliches Niveau schwanken oder sich im Zeitverlauf durchgehend steigern oder abschwächen, wurde die nachstehende Methode der Kombination von Informationen aus sukzessiven PISA-Erhebungen verwendet.

Kapitel 2 fasst die Leistungen im Bereich Lesekompetenz aus allen Erhebungen in einem einzigen Indikator zusammen. Dieser Indikator ist das Ergebnis einer linearen Regression, die auf Länderebene mit den Ergebnissen aller verfügbaren PISA-Erhebungen vorgenommen wurde. Obgleich bei Ländern mit Ergebnissen aus zwei, drei oder vier Erhebungen dieselbe Methode zum Einsatz kommt, ist der lineare Trendindikator bei Ländern mit validen Ergebnissen aus allen vier PISA-Erhebungen im Bereich Lesekompetenz präziser. In Fällen, in denen Länder nur über Daten aus zwei Erhebungen verfügen, sind die linearen Trends und die annualisierte Differenz zwischen diesen beiden Erhebungen identisch.

In allen Fällen werden lineare Trends als Leistungsunterschiede in einem Jahr ausgedrückt, so dass die Ergebnisse selbst dann zwischen Ländern verglichen werden können, wenn diese an unterschiedlichen Erhebungen teilgenommen haben, die verschiedene Zeiträume abdecken. Daher werden lineare Trends auf einer ähnlichen Skala dargestellt wie annualisierte Trends, die in Kapitel 2 ebenfalls erörtert werden. Jedoch tragen lineare Trends Daten aus mehreren Erhebungen Rechnung, sofern diese verfügbar sind, während der annualisierte Trend einen Wert wiedergibt, der dem Unterschied zwischen den Daten aus zwei Erhebungen, dividiert durch die Zahl der zwischen den Erhebungen liegenden Jahre, entspricht.

Einige Länder führten die PISA-2000-Erhebung ein oder zwei Jahre später durch (vgl. Anmerkung 6 in Kapitel 1). Dies wird bei der Schätzung der linearen Trends berücksichtigt.

Wie bei allen in diesem Bericht enthaltenen Statistiken muss die Präzision der Trendschätzungen geschätzt werden. Bei linearen Trends müssen die Standardfehler zwei Quellen von Zufallsvariationen Rechnung tragen: a) Variationen, die durch Stichprobenunterschiede entstehen und $b$ ) Variationen, die durch den Linking-Fehler beim Ergebnisvergleich zwischen sukzessiven Erhebungen bedingt sind (vgl. Anhang A1 wegen näherer Einzelheiten zum Linking-Fehler).

Der Linking-Fehler spiegelt die Genauigkeit wider, mit der die Schülerpunktwerte in den verschiedenen Erhebungen aufeinander abgestimmt sind. Bei Leistungsveränderungen zwischen zwei Erhebungen wurden die Linking-Fehler geschätzt und in die dargelegten Ergebnisse einbezogen (vgl. Anhang A1 wegen näherer Einzelheiten). Bei linearen Trends kam die Monte-Carlo-Methode zum Einsatz, um die Standardfehler der Regressionsparameter zu schätzen (d.h. den linearen Trend). Im Rahmen des Monte-CarloVerfahrens wurde für jedes Land mit 500 möglichen Mittelwerten gearbeitet. Bei der Aufstellung dieser Mittelwerte wurde davon ausgegangen, dass sich die mit jedem nationalen Durchschnitt einhergehende Ungewissheit im weiteren Verlauf unabhängig entwickelt und normalerweise um den geschätzten Mittelwert mit einer Varianz verteilt wurde, die anhand einer Kombination der Stichproben- und Linking-Fehler geschätzt wurde. Lineare Regressionen wurden für jeden der 500 Werte durchgeführt, und die Standardfehler wurden anhand der Standardabweichungen der 500 geschätzten Regressionskoeffizienten geschätzt. 


\section{Bereinigte Trends}

PISA hält im Zeitverlauf an seinen technischen Standards fest. Obwohl das theoretisch bedeutet, dass Trends über vergleichbare Populationen berechnet werden können, wurden in einigen Ländern bei den Stichprobenauswahlmethoden leichte methodische Abweichungen beobachtet. Außerdem können sich die demografischen Merkmale und der sozioökonomische Hintergrund verschiedener Bevölkerungsgruppen 15-Jähriger auch ändern. Um auf der Grundlage von Trendergebnissen verlässliche Schlussfolgerungen ziehen zu können, ist es wichtig, zu prüfen, ob die unbereinigt aufgeführten Trendergebnisse nach der Bereinigung um demografische Faktoren und den sozioökonomischen Hintergrund der Schülerinnen und Schüler Bestand hatten.

Lineare Regressionen können zur Bereinigung von Leistungsergebnissen um Unterschiede beim sozioökonomischen Hintergrund der Schülerinnen und Schüler verwendet werden. Das in diesem Bericht verwendete Regressionsmodell enthält die berücksichtigten Hintergrundmerkmale, so dass der Zusammenhang zwischen diesen Merkmalen und den Schülerleistungen in den einzelnen Erhebungen variieren kann. Auf diese Weise wurden für jedes Land drei Arten von Ergebnissen getrennt berechnet: a) die bereinigten Leistungsergebnisse jeder Erhebung, b) der bereinigte Unterschied zwischen zwei Erhebungen und c) der bereinigte lineare Trend über mehrere Erhebungen.

Die in Kapitel 2 dargelegten bereinigten Ergebnisse im Bereich Lesekompetenz verwenden die PISA-2009-Stichprobe als Bezugsgröße. Daher wurden die Ergebnisse früherer Erhebungen angepasst, um sie mit den PISA-2009-Ergebnissen vergleichen zu können. Erreicht wurde dies über eine Zentrierung der Hintergrundmerkmale auf die Durchschnittswerte von PISA 2009 für jedes Land und die anschließende Durchführung einer Regression mit den zentrierten Hintergrundmerkmalen zur Ermittlung bereinigter Trends. Die Ergebnisse aus PISA 2000, 2003 und 2006 wurden also angepasst, um mit den Daten von 2009 kompatibel zu sein.

Tabelle A6.1 enthält Durchschnittswerte für Hintergrundvariablen, wobei folgende Messgrößen für die Anpassung zu Grunde gelegt wurden: Geschlecht und Alter der Schülerinnen und Schüler ebenso wie Indikatoren, die Aufschluss über die Schüler geben, deren zu Hause gesprochene Sprache von der Testsprache abweicht, sowie darüber, ob der Schüler in einem anderen Land geboren wurde, ob die Mutter des Schülers in einem anderen Land geboren wurde und ob der Vater des Schülers in einem anderen Land geboren wurde. In den letzten Spalten sind die Unterschiede bei diesen Merkmalen aufgeführt. Die Ergebnisse wurden auch um Veränderungen beim sozioökonomischen Hintergrund bereinigt, die anhand des PISA-Index des wirtschaftlichen, sozialen und kulturellen Status (Variable ESCS) gemessen wurden. Wie in Anhang A1 erläutert wird, wurde der ESCS-Index für die Erhebungen 2000, 2003 und 2006 neu geschätzt, um mit den Ergebnissen von 2009 kompatibel zu sein. Mittelwerte, die Standardabweichung und Veränderungen in diesen Statistiken für den neu geschätzten ESCS-Index sind in Tabelle V.4.3 ausgewiesen. Diese Statistiken könnten von den Statistiken in den Berichten 2000, 2003 und 2006 abweichen, da die neu geschätzten Werte des ESCS-Index, die mit den Ergebnissen von PISA 2009 vergleichbar sind, geringfügig von den in früheren Erhebungen ausgewiesenen Werten abweichen können. In beiden Tabellen weisen Veränderungen in Fettdruck darauf hin, dass sich die Mittelwerte für die jeweilige Messgröße zwischen den Erhebungen verändert haben. In diesem Fall spiegelt der Unterschied zwischen unbereinigten und bereinigten Trends diese Veränderung wider, die in den bereinigten Trends zum Ausdruck kommt.

Für unbereinigte Leistungsergebnisse wird der Durchschnitt über alle an den PISA-Erhebungen teilnehmenden Schülerinnen und Schülern abgebildet. Daher sollten die bereinigten Ergebnisse auch für alle Teilnehmer in jedem Land berechnet werden. Das ist nicht immer möglich, da in einigen Fällen Informationen über Hintergrundmerkmale der Schülerinnen und Schüler auf Grund von fehlenden oder unzulänglichen Antworten nicht verfügbar sind. Eine Imputation fehlender Werte war vor den Anpassungen erforderlich, um Stichprobenumfänge und Vergleiche mit unbereinigten Ergebnissen aufrechtzuerhalten. Dies erfolgte unter Verwendung multipler Imputationsmodelle, durch die der Zusammenhang zwischen Leistung und Hintergrundmerkmalen bei der Imputation fehlender Informationen gewahrtwurde (Rubin, 1987; Royston, 2004). Das Imputationsmodell wurde auf jeden plausiblen Wert einmal angewendet und umfasste die Gesamtheit der im vorangegangenen Absatz dargelegten Hintergrundmerkmale der Schülerinnen und Schüler. Nach der Imputation wurden alle Berechnungen jeweils fünfmal durchgeführt, für jeden imputierten Datensatz, der eine der fünf plausiblen Werte für die Leistungsmessgrößen enthält, einmal. Die Endergebnisse wurden aus dem Durchschnitt der Regressionsergebnisse jedes einzelnen imputierten Datensatzes ermittelt, wobei dem Imputationsfehler durch Verwendung der sogenannten Kombinationsregeln von Rubin Rechnung getragen wurde (Rubin, 1987). Die Ergebnisse nach der Imputation weichen geringfügig von den ohne die Imputation erzielten Ergebnissen ab, da die Zahl der fehlenden Beobachtungen in den meisten Ländern und Erhebungen relativ niedrig war.

\section{Bereinigung der Trends um Veränderungen bei Alter und Geschlecht der Schülerinnen und Schüler}

Bei der in PISA untersuchten Bevölkerung handelt es sich um 15-jährige Schülerinnen und Schüler. In einigen Ländern kann sich das Testfenster zwischen den PISA-Erhebungen aber leicht verschoben haben, was die Trends beeinflussen kann. Wenn beispielsweise während einer Erhebung die Schülerinnen und Schüler 2 Monate jünger waren als der in PISA getestete Durchschnittsschüler, hätte dies keinen Einfluss auf Vergleiche mit anderen Ländern, da ein Altersunterschied von zwei Monaten unerheblich ist. Wenn aber die Schülerinnen und Schüler in einer anderen Erhebung zwei Monate älter sind, könnte das Durchschnittsalter in den beiden Stichproben um vier Monate abweichen, was stärker ins Gewicht fällt. Sind diese Unterschiede dann auch noch mit anderen Diskrepanzen zwischen Schülerstichproben im Zeitverlauf verbunden, kann darunter die Vergleichbarkeit der Trends leiden, so dass sich für Länder, in denen der durchschnittliche Altersunterschied zwischen zwei Erhebungen größer ist, höhere Werte 
ergeben. Faktisch sind die Altersunterschiede zwischen PISA-Erhebungen minimal und betragen unter den Ländern höchstens einen Monat.

Ein weiteres gemeinsames Kriterium bei der Stichprobenauswahl der Schülerpopulation ist die Geschlechterrepräsentation. Da die Lesekompetenz der Mädchen gewöhnlich höher ist als die der Jungen, können Ungleichgewichte bei der geschlechtlichen Zusammensetzung der Stichproben aus unterschiedlichen PISA-Erhebungen die Verlässlichkeit der Trendschätzungen beeinträchtigen. Wenn beispielsweise Mädchen in PISA 2000 besser abschnitten und zahlreicher waren als in PISA 2009, ergibt sich hieraus eine Leistungsverschlechterung, da die Zusammensetzung der PISA-2000-Stichprobe günstiger war. Ungleichgewichte in der Geschlechterzusammensetzung sind in den PISA-Erhebungen sehr selten, doch wurde in der PISA-Studie untersucht, ob kleine Veränderungen im prozentualen Anteil der Jungen und Mädchen die Trends beeinflusst haben.

Ergebnisse für Trends, die um Alters- und Geschlechtsunterschiede in der Stichprobenauswahl bereinigt sind, werden in Abbildung A6.1 mit unbereinigten Trendschätzungen verglichen (vgl. auch Tabelle A6.2, die bereinigte Trends und bereinigte Ergebnisse für die Jahre 2000, 2003 und 2006 enthält). Die Trends sind sehr ähnlich. Das zeigt, dass die Stichprobenauswahlverfahren in PISA kohärent sind und die Vergleichbarkeit der Ergebnisse zwischen und in den Ländern langfristig gewährleisten. In Kapitel 2 werden Ergebnisse unter dem Gesichtspunkt weiterer Anpassungen bei Berücksichtigung von Veränderungen im demografischen und sozioökonomischen Kontext der Schülerinnen und Schüler erörtert.

Abbildung A.6.1

Beobachtete Punktzahlveränderungen und um Stichprobenunterschiede zwischen 2000 und 2009 bereinigte Punktzahlveränderungen

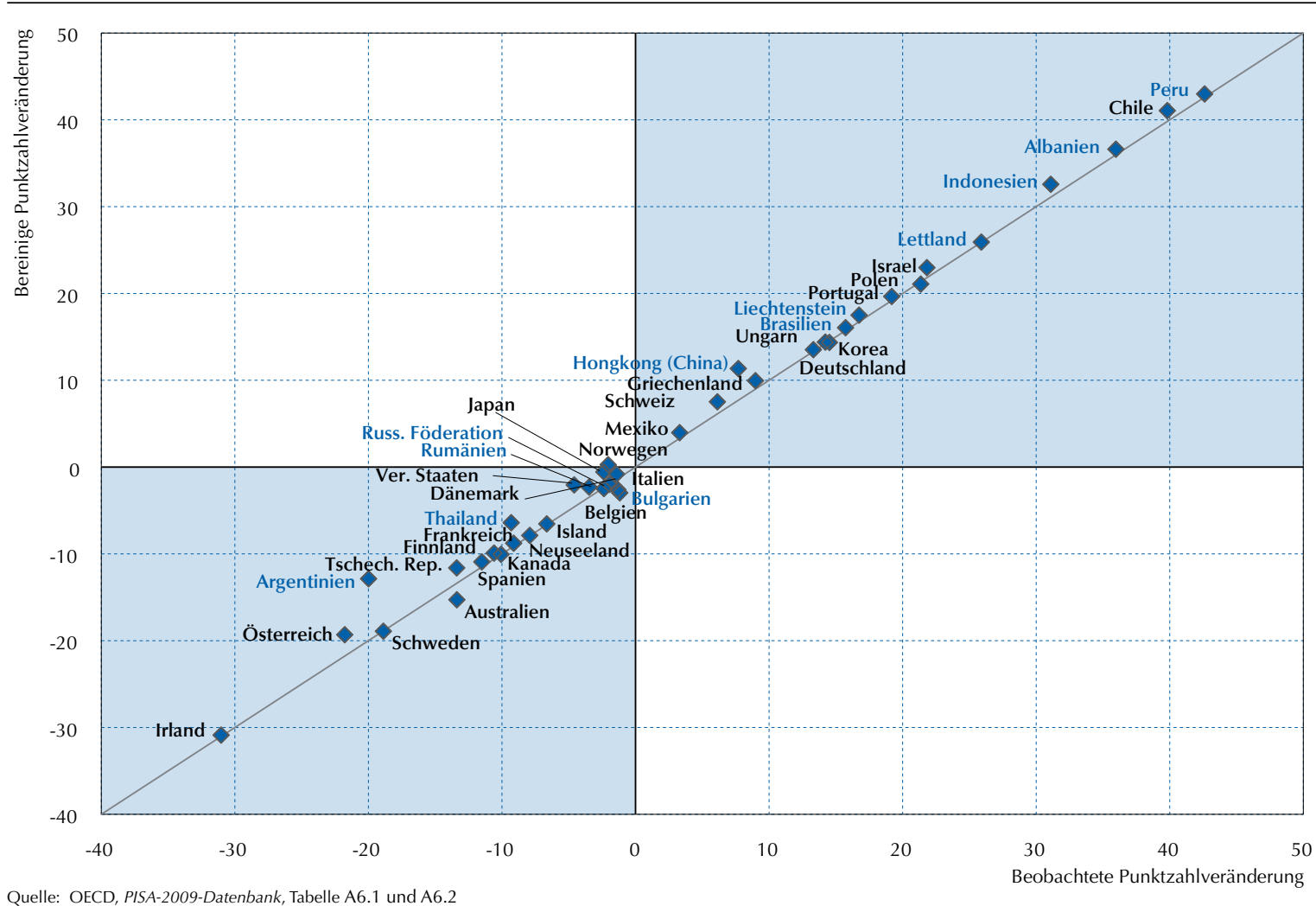

Quelle: OECD, PISA-2009-Datenbank, Tabelle A6.1 und A6.2 
Tabelle A6.1 Hintergrundmerkmale der Schülerinnen und Schüler in PISA 2000 und PISA 2009

\begin{tabular}{|c|c|c|c|c|c|c|c|c|c|c|}
\hline & \multicolumn{10}{|c|}{ PISA 2000} \\
\hline & \multicolumn{2}{|c|}{$\begin{array}{c}\text { Prozentsatz } \\
\text { der Mädchen }\end{array}$} & \multicolumn{2}{|c|}{ Alter } & \multicolumn{2}{|c|}{$\begin{array}{l}\text { Im Ausland } \\
\text { geborener Schüler }\end{array}$} & \multicolumn{2}{|c|}{$\begin{array}{l}\text { Mutter des Schülers } \\
\text { im Ausland geboren }\end{array}$} & \multicolumn{2}{|c|}{$\begin{array}{l}\text { Vater des Schülers } \\
\text { im Ausland geboren }\end{array}$} \\
\hline & $\%$ & S.E. & Mittelwert & S.E. & $\%$ & S.E. & $\%$ & S.E. & $\%$ & S.E. \\
\hline Australien & 47.5 & (2.2) & 15.7 & $(0.0)$ & 13.0 & (1.2) & 31.5 & (1.6) & 33.0 & $(1.7)$ \\
\hline : స్ Österreich & 48.8 & (2.3) & 15.8 & $(0.0)$ & 8.1 & $(0.7)$ & 14.1 & (1.0) & 13.7 & (0.9) \\
\hline Belgien & 47.9 & (1.7) & 15.7 & $(0.0)$ & 5.8 & $(0.5)$ & 16.9 & (1.1) & 18.6 & (1.2) \\
\hline Kanada & 50.1 & $(0.5)$ & 15.8 & $(0.0)$ & 10.7 & (0.6) & 24.9 & (1.0) & 26.8 & (1.1) \\
\hline Chile & 53.0 & (1.8) & 15.8 & $(0.0)$ & 1.5 & $(0.2)$ & 0.9 & $(0.2)$ & 1.1 & $(0.2)$ \\
\hline Tschech. Rep. & 51.7 & (1.8) & 15.8 & $(0.0)$ & 1.0 & $(0.1)$ & 4.5 & $(0.3)$ & 4.8 & $(0.3)$ \\
\hline Dänemark & 49.7 & $(0.9)$ & 15.7 & $(0.0)$ & 6.3 & $(0.5)$ & 9.5 & (0.6) & 10.0 & $(0.7)$ \\
\hline Finnland & 51.4 & $(0.8)$ & 15.7 & $(0.0)$ & 2.5 & $(0.3)$ & 2.1 & $(0.3)$ & 2.6 & $(0.3)$ \\
\hline Frankreich & 51.3 & (1.3) & 15.9 & $(0.0)$ & 3.5 & $(0.3)$ & 17.2 & (1.0) & 19.6 & (1.0) \\
\hline Deutschland & 49.7 & (1.5) & 15.8 & $(0.0)$ & 11.3 & (0.6) & 18.0 & $(0.9)$ & 19.0 & (0.9) \\
\hline Griechenland & 49.8 & (1.3) & 15.8 & $(0.0)$ & 6.6 & (1.0) & 8.6 & (1.0) & 6.8 & (1.0) \\
\hline Ungarn & 49.6 & $(2.1)$ & 15.7 & $(0.0)$ & 2.2 & $(0.2)$ & 2.7 & $(0.3)$ & 2.8 & $(0.3)$ \\
\hline Island & 50.4 & $(0.8)$ & 15.7 & $(0.0)$ & 5.9 & $(0.4)$ & 3.2 & (0.4) & 3.8 & $(0.4)$ \\
\hline Irland & 50.4 & (1.8) & 15.7 & $(0.0)$ & 4.2 & (0.4) & 7.9 & $(0.5)$ & 6.0 & $(0.5)$ \\
\hline Israel & 58.2 & $(2.7)$ & 15.7 & $(0.0)$ & 10.9 & (1.2) & 34.2 & (1.9) & 36.3 & (2.1) \\
\hline Italien & 49.3 & $(2.7)$ & 15.7 & $(0.0)$ & 2.2 & $(0.3)$ & 3.7 & $(0.3)$ & 2.2 & $(0.3)$ \\
\hline Japan & 50.5 & (2.4) & 15.8 & $(0.0)$ & 0.2 & $(0.1)$ & 0.4 & $(0.1)$ & 0.3 & $(0.1)$ \\
\hline Korea & 44.1 & (3.5) & 15.7 & $(0.0)$ & $\mathrm{m}$ & $\mathrm{m}$ & $\mathrm{m}$ & $\mathrm{m}$ & $\mathrm{m}$ & $\mathrm{m}$ \\
\hline Luxemburg & $\mathrm{m}$ & $\mathrm{m}$ & $\mathrm{m}$ & $\mathrm{m}$ & $\mathrm{m}$ & $\mathrm{m}$ & $\mathrm{m}$ & $\mathrm{m}$ & $\mathrm{m}$ & $\mathrm{m}$ \\
\hline Mexiko & 50.0 & (1.2) & 15.8 & $(0.0)$ & 3.2 & $(0.4)$ & 4.5 & $(0.4)$ & 4.7 & $(0.4)$ \\
\hline Niederlande & $\mathrm{m}$ & $\mathrm{m}$ & $\mathrm{m}$ & $\mathrm{m}$ & $\mathrm{m}$ & $\mathrm{m}$ & $\mathrm{m}$ & $\mathrm{m}$ & $\mathrm{m}$ & $\mathrm{m}$ \\
\hline Neuseeland & 49.7 & (2.4) & 15.7 & $(0.0)$ & 16.7 & $(0.9)$ & 27.3 & (1.1) & 29.4 & $(1.2)$ \\
\hline Norwegen & 49.0 & $(0.9)$ & 15.8 & $(0.0)$ & 5.5 & (0.4) & 7.6 & (0.5) & 7.9 & $(0.5)$ \\
\hline Polen & 49.1 & (2.6) & 15.7 & $(0.0)$ & 1.0 & $(0.2)$ & 0.7 & $(0.2)$ & 1.5 & $(0.3)$ \\
\hline Portugal & 52.0 & $(0.9)$ & 15.7 & $(0.0)$ & 6.0 & $(0.5)$ & 6.9 & $(0.4)$ & 6.1 & $(0.4)$ \\
\hline Spanien & 50.8 & (1.3) & 15.8 & $(0.0)$ & 2.5 & $(0.4)$ & 4.3 & $(0.4)$ & 3.6 & $(0.4)$ \\
\hline Schweden & 49.2 & $(0.9)$ & 15.7 & $(0.0)$ & 8.3 & $(0.6)$ & 15.7 & $(0.9)$ & 16.1 & (1.1) \\
\hline Schweiz & 49.8 & (1.0) & 15.9 & $(0.0)$ & 14.1 & $(0.7)$ & 28.3 & $(0.9)$ & 28.9 & (1.0) \\
\hline Ver. Königreich & $\mathrm{m}$ & $\mathrm{m}$ & $\mathrm{m}$ & $\mathrm{m}$ & $\mathrm{m}$ & $\mathrm{m}$ & $\mathrm{m}$ & $\mathrm{m}$ & $\mathrm{m}$ & $\mathrm{m}$ \\
\hline Ver. Staaten & 51.6 & $(1.0)$ & 15.8 & $(0.0)$ & 7.3 & (1.0) & 15.8 & (2.3) & 17.2 & (2.5) \\
\hline OECD-23-Durchschnitt & 49.7 & $(0.4)$ & 15.8 & $(0.0)$ & 6.0 & $(0.1)$ & 11.2 & $(0.2)$ & 11.6 & $(0.2)$ \\
\hline OECD-26-Durchschnitt & 50.2 & $(0.3)$ & 15.8 & $(0.0)$ & 6.1 & $(0.1)$ & 11.9 & $(0.2)$ & 12.4 & $(0.2)$ \\
\hline ¿ Albanien & 51.0 & $(1.2)$ & 15.7 & $(0.0)$ & 0.5 & $(0.1)$ & 1.2 & $(0.2)$ & 1.3 & $(0.2)$ \\
\hline Argentinien & 56.4 & (2.5) & 15.9 & $(0.0)$ & 0.8 & $(0.2)$ & 5.1 & $(0.8)$ & 5.5 & $(0.4)$ \\
\hline Brasilien & 54.0 & $(1.2)$ & 15.8 & $(0.0)$ & 0.2 & $(0.1)$ & 0.8 & $(0.2)$ & 1.1 & $(0.2)$ \\
\hline Bulgarien & 48.5 & (1.9) & 15.7 & $(0.0)$ & 1.1 & $(0.2)$ & 2.3 & $(0.3)$ & 1.6 & $(0.3)$ \\
\hline Hongkong (China) & 49.8 & $(2.1)$ & 15.8 & $(0.0)$ & 20.7 & $(0.9)$ & 52.3 & $(1.0)$ & 54.2 & $(1.2)$ \\
\hline Indonesien & 51.1 & (1.8) & 15.7 & $(0.0)$ & 0.3 & $(0.1)$ & 0.5 & $(0.1)$ & 0.6 & $(0.1)$ \\
\hline Lettland & 51.3 & (1.6) & 15.7 & $(0.0)$ & 30.5 & (3.4) & 30.8 & (2.6) & 30.9 & (2.5) \\
\hline Liechtenstein & 49.7 & (2.9) & 15.7 & $(0.0)$ & 12.9 & (1.8) & 35.4 & (2.6) & 30.4 & (2.6) \\
\hline Peru & 49.9 & $(2.2)$ & 15.9 & $(0.0)$ & 0.6 & $(0.1)$ & 0.7 & $(0.2)$ & 1.1 & $(0.2)$ \\
\hline Rumänien & 52.7 & (1.1) & 15.8 & $(0.0)$ & 0.2 & $(0.1)$ & 0.5 & $(0.2)$ & 0.6 & $(0.2)$ \\
\hline Russ. Föderation & 50.1 & $(0.9)$ & 15.8 & $(0.0)$ & 5.4 & (0.5) & 8.1 & $(0.7)$ & 9.6 & $(0.8)$ \\
\hline Thailand & 58.8 & (2.0) & 15.8 & (0.0) & 0.1 & $(0.0)$ & 0.9 & (0.5) & 1.1 & $(0.5)$ \\
\hline
\end{tabular}

Anmerkung: Statistisch signifikante Werte sind durch Fettdruck gekennzeichnet (siehe Anhang A3).

StatLink הत्ताज http://dx.doi.org/10.1787/888932360100 
[Teil 2/3]

Tabelle A6.1 Hintergrundmerkmale der Schülerinnen und Schüler in PISA 2000 und PISA 2009

\begin{tabular}{|c|c|c|c|c|c|c|c|c|c|c|}
\hline & \multicolumn{10}{|c|}{ PISA 2009} \\
\hline & \multicolumn{2}{|c|}{$\begin{array}{c}\text { Prozentsatz } \\
\text { der Mädchen }\end{array}$} & \multicolumn{2}{|c|}{ Alter } & \multicolumn{2}{|c|}{$\begin{array}{l}\text { Im Ausland } \\
\text { geborener Schüler }\end{array}$} & \multicolumn{2}{|c|}{$\begin{array}{l}\text { Mutter des Schülers } \\
\text { im Ausland geboren }\end{array}$} & \multicolumn{2}{|c|}{$\begin{array}{l}\text { Vater des Schülers } \\
\text { im Ausland geboren }\end{array}$} \\
\hline & $\%$ & S.E. & Mittelwert & S.E. & $\%$ & S.E. & $\%$ & S.E. & $\%$ & S.E. \\
\hline Australien & 51.1 & (1.3) & 15.7 & $(0.0)$ & 12.8 & (0.6) & 32.6 & (1.1) & 33.1 & (1.1) \\
\hline Österreich & $\mathrm{m}$ & $\mathrm{m}$ & $\mathrm{m}$ & $\mathrm{m}$ & $\mathrm{m}$ & $\mathrm{m}$ & $\mathrm{m}$ & $\mathrm{m}$ & $\mathrm{m}$ & $\mathrm{m}$ \\
\hline Delgien & 48.9 & $(1.2)$ & 15.8 & $(0.0)$ & 9.2 & $(0.7)$ & 20.5 & $(1.2)$ & 21.7 & (1.1) \\
\hline Kanada & 49.7 & $(0.5)$ & 15.8 & $(0.0)$ & 12.3 & $(0.7)$ & 29.4 & (1.3) & 30.3 & (1.3) \\
\hline Chile & 49.0 & (1.1) & 15.8 & $(0.0)$ & 1.1 & $(0.1)$ & 1.1 & $(0.2)$ & 1.4 & $(0.2)$ \\
\hline Tschech. Rep. & 46.8 & (1.8) & 15.8 & $(0.0)$ & 1.4 & $(0.2)$ & 4.8 & $(0.3)$ & 6.2 & $(0.4)$ \\
\hline Dänemark & 50.5 & $(0.7)$ & 15.7 & $(0.0)$ & 4.7 & $(0.3)$ & 12.2 & $(0.5)$ & 13.1 & $(0.5)$ \\
\hline Finnland & 49.9 & $(0.5)$ & 15.7 & $(0.0)$ & 2.7 & $(0.3)$ & 4.7 & $(0.4)$ & 5.0 & $(0.5)$ \\
\hline Frankreich & 51.3 & $(1.2)$ & 15.9 & $(0.0)$ & 5.0 & $(0.6)$ & 18.5 & (1.4) & 20.3 & (1.6) \\
\hline Deutschland & 48.9 & $(1.0)$ & 15.8 & $(0.0)$ & 7.2 & $(0.4)$ & 21.3 & (1.1) & 22.0 & $(1.0)$ \\
\hline Griechenland & 50.9 & (1.1) & 15.7 & $(0.0)$ & 9.0 & $(0.8)$ & 15.6 & $(0.9)$ & 11.2 & $(0.9)$ \\
\hline Ungarn & 49.6 & (1.5) & 15.7 & $(0.0)$ & 2.0 & $(0.3)$ & 3.8 & $(0.3)$ & 3.3 & $(0.3)$ \\
\hline Island & 50.3 & $(0.3)$ & 15.7 & $(0.0)$ & 6.3 & $(0.4)$ & 6.6 & $(0.4)$ & 5.5 & $(0.4)$ \\
\hline Irland & 49.4 & (1.1) & 15.7 & $(0.0)$ & 14.9 & $(0.7)$ & 17.0 & $(0.8)$ & 16.4 & $(0.7)$ \\
\hline Israel & 50.9 & $(0.9)$ & 15.7 & $(0.0)$ & 9.2 & $(0.8)$ & 26.8 & (1.1) & 27.3 & (1.1) \\
\hline Italien & 48.6 & $(0.9)$ & 15.7 & $(0.0)$ & 5.8 & $(0.2)$ & 9.7 & $(0.3)$ & 7.6 & $(0.3)$ \\
\hline Japan & 48.4 & (1.8) & 15.7 & $(0.0)$ & 0.4 & $(0.1)$ & 0.9 & $(0.1)$ & 0.5 & $(0.1)$ \\
\hline Korea & 47.3 & (1.8) & 15.7 & $(0.0)$ & 0.4 & $(0.1)$ & 0.3 & $(0.1)$ & $\mathrm{m}$ & $\mathrm{m}$ \\
\hline Luxemburg & 49.3 & $(0.2)$ & 15.8 & $(0.0)$ & 19.3 & $(0.5)$ & 48.0 & $(0.6)$ & 49.2 & $(0.7)$ \\
\hline Mexiko & 50.6 & $(0.4)$ & 15.7 & $(0.0)$ & 2.4 & $(0.1)$ & 2.9 & $(0.2)$ & 3.0 & $(0.2)$ \\
\hline Niederlande & 50.3 & $(0.7)$ & 15.7 & $(0.0)$ & 4.8 & $(0.5)$ & 16.0 & (1.6) & 16.7 & (1.6) \\
\hline Neuseeland & 49.0 & $(1.2)$ & 15.7 & $(0.0)$ & 20.8 & $(0.7)$ & 32.5 & $(1.2)$ & 32.8 & $(1.2)$ \\
\hline Norwegen & 48.9 & $(0.5)$ & 15.7 & $(0.0)$ & 5.4 & $(0.4)$ & 11.0 & (0.6) & 10.9 & $(0.7)$ \\
\hline Polen & 50.0 & $(0.5)$ & 15.7 & $(0.0)$ & 0.4 & $(0.1)$ & 0.1 & $(0.1)$ & 0.5 & $(0.1)$ \\
\hline Portugal & 51.1 & $(0.6)$ & 15.7 & $(0.0)$ & 7.4 & $(0.5)$ & 13.0 & $(0.6)$ & 11.2 & $(0.6)$ \\
\hline Spanien & 49.2 & $(0.6)$ & 15.8 & $(0.0)$ & 10.0 & $(0.5)$ & 13.1 & $(0.5)$ & 11.8 & $(0.5)$ \\
\hline Schweden & 49.2 & $(0.5)$ & 15.7 & $(0.0)$ & 5.8 & $(0.5)$ & 16.6 & $(1.3)$ & 18.0 & $(1.3)$ \\
\hline Schweiz & 49.2 & $(1.1)$ & 15.8 & $(0.0)$ & 10.9 & $(0.6)$ & 33.7 & $(0.9)$ & 32.2 & $(1.0)$ \\
\hline Ver. Königreich & 50.9 & (1.6) & 15.7 & $(0.0)$ & 6.8 & $(0.5)$ & 14.2 & $(1.0)$ & 15.8 & $(1.2)$ \\
\hline Ver. Staaten & 48.7 & $(0.8)$ & 15.7 & $(0.0)$ & 7.4 & $(0.5)$ & 23.3 & (1.4) & 23.6 & $(1.5)$ \\
\hline OECD-23-Durchschnitt & 49.5 & $(0.2)$ & 15.7 & $(0.0)$ & 6.8 & $(0.1)$ & 13.9 & $(0.2)$ & 14.4 & $(0.2)$ \\
\hline OECD-26-Durchschnitt & 49.5 & $(0.2)$ & 15.7 & $(0.0)$ & 6.7 & $(0.1)$ & 14.3 & $(0.2)$ & 14.8 & $(0.2)$ \\
\hline ¿ Albanien & 48.7 & $(0.9)$ & 15.8 & $(0.0)$ & 1.5 & $(0.2)$ & 0.8 & $(0.2)$ & 0.8 & $(0.2)$ \\
\hline Argentinien & 53.7 & $(1.1)$ & 15.7 & $(0.0)$ & 1.9 & $(0.3)$ & 6.3 & $(0.7)$ & 6.3 & $(0.7)$ \\
\hline Brasilien & 53.1 & $(0.4)$ & 15.9 & $(0.0)$ & 0.5 & $(0.1)$ & 1.1 & $(0.2)$ & 1.7 & $(0.2)$ \\
\hline Bulgarien & 48.1 & $(2.2)$ & 15.8 & $(0.0)$ & 1.3 & $(0.2)$ & 1.9 & $(0.3)$ & 1.3 & $(0.2)$ \\
\hline Hongkong (China) & 47.1 & (1.8) & 15.7 & $(0.0)$ & 22.8 & $(1.0)$ & 53.0 & (1.4) & 45.8 & (1.4) \\
\hline Indonesien & 50.5 & (1.9) & 15.7 & $(0.0)$ & 0.6 & $(0.1)$ & 0.3 & $(0.1)$ & 0.5 & $(0.2)$ \\
\hline Lettland & 50.7 & $(0.9)$ & 15.7 & $(0.0)$ & 1.6 & $(0.3)$ & 11.3 & $(0.9)$ & 12.7 & (1.0) \\
\hline Liechtenstein & 47.0 & $(1.2)$ & 15.7 & $(0.0)$ & 26.1 & $(2.3)$ & 54.2 & $(2.7)$ & 48.1 & (2.8) \\
\hline Peru & 49.5 & $(1.2)$ & 15.8 & $(0.0)$ & 0.7 & $(0.1)$ & 0.9 & $(0.1)$ & 0.9 & $(0.1)$ \\
\hline Rumänien & 50.9 & (1.4) & 15.7 & $(0.0)$ & 0.6 & $(0.1)$ & 0.5 & $(0.1)$ & 0.6 & $(0.1)$ \\
\hline Russ. Föderation & 50.4 & $(0.7)$ & 15.8 & $(0.0)$ & 6.9 & $(0.5)$ & 16.7 & $(0.8)$ & 17.2 & $(0.8)$ \\
\hline Thailand & 56.7 & (1.5) & 15.7 & $(0.0)$ & 0.1 & $(0.0)$ & 0.0 & $(0.0)$ & 0.1 & $(0.1)$ \\
\hline
\end{tabular}

Anmerkung: Statistisch signifikante Werte sind durch Fettdruck gekennzeichnet (siehe Anhang A3).

StatLink त्ताs http://dx.doi.org/10.1787/888932360100 
[Teil 3/3]

Tabelle A6.1 Hintergrundmerkmale der Schülerinnen und Schüler in PISA 2000 und PISA 2009

\begin{tabular}{|c|c|c|c|c|c|c|c|c|c|c|}
\hline & \multicolumn{10}{|c|}{ Veränderung zwischen PISA 2000 und PISA 2009 (PISA 2009 - PISA 2000) } \\
\hline & \multicolumn{2}{|c|}{$\begin{array}{c}\text { Prozentsatz } \\
\text { der Mädchen }\end{array}$} & \multicolumn{2}{|c|}{ Alter } & \multicolumn{2}{|c|}{$\begin{array}{c}\text { Im Ausland } \\
\text { geborener Schüler }\end{array}$} & \multicolumn{2}{|c|}{$\begin{array}{l}\text { Mutter des Schülers } \\
\text { im Ausland geboren }\end{array}$} & \multicolumn{2}{|c|}{$\begin{array}{l}\text { Vater des Schülers } \\
\text { im Ausland geboren }\end{array}$} \\
\hline & Diff. \% & S.E. & Diff. & S.E. & Diff. \% & S.E. & Diff. \% & S.E. & Diff. \% & S.E. \\
\hline ¿ Australien & 3.6 & (2.5) & 0.02 & $(0.01)$ & -0.1 & $(1.4)$ & 1.1 & $(2.0)$ & 0.1 & $(2.0)$ \\
\hline : Österreich & $\mathrm{m}$ & $\mathrm{m}$ & $\mathrm{m}$ & $\mathrm{m}$ & $\mathrm{m}$ & $\mathrm{m}$ & $\mathrm{m}$ & $\mathrm{m}$ & $\mathrm{m}$ & $\mathrm{m}$ \\
\hline Belgien & 1.1 & $(2.1)$ & 0.09 & $(0.00)$ & 3.3 & $(0.9)$ & 3.6 & $(1.7)$ & 3.1 & $(1.7)$ \\
\hline Kanada & -0.4 & $(0.7)$ & 0.01 & $(0.00)$ & 1.6 & $(1.0)$ & 4.5 & (1.6) & 3.5 & $(1.7)$ \\
\hline Chile & -4.0 & $(2.1)$ & -0.02 & $(0.01)$ & -0.4 & $(0.3)$ & 0.3 & $(0.2)$ & 0.2 & $(0.2)$ \\
\hline Tschech. Rep. & -4.9 & $(2.5)$ & -0.00 & $(0.01)$ & 0.4 & $(0.2)$ & 0.3 & $(0.4)$ & 1.4 & $(0.5)$ \\
\hline Dänemark & 0.8 & $(1.2)$ & -0.01 & $(0.01)$ & -1.5 & $(0.6)$ & 2.8 & $(0.8)$ & 3.1 & $(0.9)$ \\
\hline Finnland & -1.5 & $(0.9)$ & 0.01 & $(0.01)$ & 0.1 & $(0.4)$ & 2.6 & $(0.5)$ & 2.4 & $(0.5)$ \\
\hline Frankreich & -0.1 & (1.8) & -0.01 & $(0.01)$ & 1.5 & $(0.6)$ & 1.2 & (1.7) & 0.6 & (1.9) \\
\hline Deutschland & -0.7 & (1.8) & 0.00 & $(0.01)$ & -4.1 & $(0.7)$ & 3.3 & (1.4) & 3.0 & (1.3) \\
\hline Griechenland & 1.1 & $(1.7)$ & -0.08 & $(0.01)$ & 2.3 & (1.3) & 7.0 & (1.3) & 4.5 & (1.3) \\
\hline Ungarn & -0.0 & $(2.6)$ & -0.01 & $(0.01)$ & -0.2 & $(0.4)$ & 1.1 & $(0.4)$ & 0.5 & $(0.4)$ \\
\hline Island & -0.2 & $(0.9)$ & 0.00 & $(0.01)$ & 0.4 & $(0.5)$ & 3.4 & $(0.6)$ & 1.7 & $(0.5)$ \\
\hline Irland & -1.1 & $(2.1)$ & 0.01 & $(0.01)$ & 10.7 & $(0.8)$ & 9.1 & $(1.0)$ & 10.4 & $(0.9)$ \\
\hline Israel & -7.3 & (2.8) & 0.02 & $(0.01)$ & -1.7 & (1.4) & -7.4 & $(2.2)$ & -9.0 & (2.3) \\
\hline Italien & -0.7 & $(2.9)$ & -0.02 & $(0.00)$ & 3.6 & $(0.4)$ & 6.0 & $(0.4)$ & 5.4 & $(0.4)$ \\
\hline Japan & -2.1 & $(2.9)$ & -0.09 & $(0.01)$ & 0.2 & $(0.1)$ & 0.6 & $(0.1)$ & 0.2 & $(0.1)$ \\
\hline Korea & 3.1 & $(4.0)$ & -0.04 & $(0.01)$ & $\mathrm{m}$ & $\mathrm{m}$ & $\mathrm{m}$ & $\mathrm{m}$ & $\mathrm{m}$ & $\mathrm{m}$ \\
\hline Luxemburg & $\mathrm{m}$ & $\mathrm{m}$ & $\mathrm{m}$ & $\mathrm{m}$ & $\mathrm{m}$ & $\mathrm{m}$ & $\mathrm{m}$ & $\mathrm{m}$ & $\mathrm{m}$ & $\mathrm{m}$ \\
\hline Mexiko & 0.6 & $(1.3)$ & -0.07 & $(0.01)$ & -0.7 & $(0.4)$ & -1.6 & $(0.5)$ & -1.7 & $(0.4)$ \\
\hline Niederlande & $\mathrm{m}$ & $\mathrm{m}$ & $\mathrm{m}$ & $\mathrm{m}$ & $\mathrm{m}$ & $\mathrm{m}$ & $\mathrm{m}$ & $\mathrm{m}$ & $\mathrm{m}$ & $\mathrm{m}$ \\
\hline Neuseeland & -0.8 & $(2.7)$ & 0.01 & $(0.01)$ & 4.0 & $(1.2)$ & 5.2 & (1.6) & 3.4 & (1.6) \\
\hline Norwegen & -0.1 & $(1.0)$ & -0.09 & $(0.01)$ & -0.1 & $(0.6)$ & 3.4 & $(0.8)$ & 3.1 & $(0.8)$ \\
\hline Polen & 0.8 & $(2.7)$ & -0.00 & $(0.01)$ & -0.6 & $(0.3)$ & -0.6 & $(0.2)$ & -1.1 & $(0.3)$ \\
\hline Portugal & -0.8 & $(1.1)$ & -0.01 & $(0.01)$ & 1.3 & $(0.7)$ & 6.1 & $(0.7)$ & 5.1 & $(0.7)$ \\
\hline Spanien & -1.6 & (1.5) & -0.01 & $(0.00)$ & 7.6 & $(0.7)$ & 8.8 & $(0.7)$ & 8.2 & $(0.7)$ \\
\hline Schweden & -0.0 & $(1.0)$ & 0.00 & $(0.01)$ & -2.5 & $(0.8)$ & 0.9 & (1.6) & 1.9 & $(1.7)$ \\
\hline Schweiz & -0.6 & (1.5) & -0.07 & $(0.01)$ & -3.2 & $(0.9)$ & 5.3 & (1.3) & 3.4 & (1.4) \\
\hline Ver. Königreich & $\mathrm{m}$ & $\mathrm{m}$ & $\mathrm{m}$ & $\mathrm{m}$ & $\mathrm{m}$ & $\mathrm{m}$ & $\mathrm{m}$ & $\mathrm{m}$ & $\mathrm{m}$ & $\mathrm{m}$ \\
\hline Ver. Staaten & -2.9 & $(1.2)$ & -0.06 & $(0.01)$ & 0.1 & $(1.1)$ & 7.5 & $(2.7)$ & 6.4 & $(3.0)$ \\
\hline OECD-23-Durchschnitt & -0.2 & $(0.4)$ & -0.0 & $(0.0)$ & 1.1 & $(0.2)$ & 3.2 & $(0.2)$ & 2.8 & $(0.2)$ \\
\hline OECD-26-Durchschnitt & -0.7 & $(0.4)$ & -0.0 & $(0.0)$ & 0.9 & $(0.2)$ & 2.9 & $(0.2)$ & 2.4 & $(0.3)$ \\
\hline đalbanien & -2.3 & (1.5) & 0.03 & $(0.01)$ & 1.0 & $(0.3)$ & -0.3 & $(0.3)$ & -0.5 & $(0.3)$ \\
\hline Argentinien & -2.7 & $(2.7)$ & -0.16 & $(0.01)$ & 1.1 & $(0.3)$ & 1.2 & (1.1) & 0.8 & $(0.8)$ \\
\hline Brasilien & -0.9 & $(1.2)$ & 0.07 & $(0.01)$ & 0.3 & $(0.2)$ & 0.3 & $(0.2)$ & 0.6 & $(0.3)$ \\
\hline Bulgarien & -0.4 & $(2.9)$ & 0.09 & $(0.01)$ & 0.1 & $(0.3)$ & -0.4 & $(0.4)$ & -0.3 & $(0.3)$ \\
\hline Hongkong (China) & -2.8 & $(2.7)$ & -0.11 & $(0.01)$ & 2.1 & (1.4) & 0.7 & (1.8) & -8.4 & (1.8) \\
\hline Indonesien & -0.7 & $(2.7)$ & -0.07 & $(0.01)$ & 0.3 & $(0.2)$ & -0.2 & $(0.2)$ & -0.1 & $(0.2)$ \\
\hline Lettland & -0.7 & (1.8) & 0.02 & $(0.01)$ & -28.9 & (3.4) & -19.5 & $(2.7)$ & -18.2 & $(2.7)$ \\
\hline Liechtenstein & -2.7 & $(3.1)$ & 0.01 & $(0.02)$ & 13.2 & $(3.0)$ & 18.8 & $(3.7)$ & 17.7 & (3.8) \\
\hline Peru & -0.4 & $(2.5)$ & -0.07 & $(0.01)$ & 0.2 & $(0.2)$ & 0.2 & $(0.2)$ & -0.2 & $(0.2)$ \\
\hline Rumänien & -1.8 & $(1.8)$ & -0.04 & $(0.01)$ & 0.4 & $(0.1)$ & 0.0 & $(0.2)$ & 0.0 & $(0.2)$ \\
\hline Russ. Föderation & 0.4 & $(1.1)$ & -0.01 & $(0.01)$ & 1.5 & $(0.7)$ & 8.6 & $(1.1)$ & 7.6 & $(1.1)$ \\
\hline Thailand & -2.1 & (2.6) & -0.14 & $(0.01)$ & -0.0 & $(0.1)$ & -0.9 & $(0.5)$ & -0.9 & $(0.5)$ \\
\hline
\end{tabular}

Anmerkung: Statistisch signifikante Werte sind durch Fettdruck gekennzeichnet (siehe Anhang A3).

StatLink 需s http://dx.doi.org/10.1787/888932360100 
[Teil 1/1]

Tabelle A6.2 Um Stichprobenunterschiede bereinigte Trends

\begin{tabular}{|c|c|c|c|c|c|c|c|c|c|c|}
\hline & \multicolumn{2}{|c|}{$\begin{array}{c}\text { Bereinigte Ergebnisse } \\
\text { von PISA } 2000\end{array}$} & \multicolumn{2}{|c|}{$\begin{array}{c}\text { Bereinigte Ergebnisse } \\
\text { von PISA } 2003\end{array}$} & \multicolumn{2}{|c|}{$\begin{array}{c}\text { Bereinigte Ergebnisse } \\
\text { von PISA } 2006\end{array}$} & \multicolumn{2}{|c|}{$\begin{array}{c}\text { Bereinigte Ergebnisse } \\
\text { von PISA } 2009\end{array}$} & \multicolumn{2}{|c|}{$\begin{array}{l}\text { Veränderung } \\
\text { zwischen 2000 und 2009 } \\
\text { (PISA 2009 - PISA 2000) }\end{array}$} \\
\hline & Mittelwert & S.E. & Mittelwert & S.E. & Mittelwert & S.E. & Mittelwert & S.E. & Punktdiff. & S.E. \\
\hline Australien & 530 & (3.4) & 526 & (2.0) & 514 & (1.9) & 515 & (2.3) & -15.3 & (6.5) \\
\hline Österreich & 490 & (2.5) & 490 & (3.5) & 491 & (4.3) & $\mathrm{m}$ & $\mathrm{m}$ & $\mathrm{m}$ & $\mathrm{m}$ \\
\hline í Belgien & 509 & (3.6) & 507 & (2.5) & 502 & (3.0) & 506 & (2.3) & -3.0 & (6.5) \\
\hline Kanada & 534 & (1.6) & 528 & (1.8) & 527 & (2.4) & 524 & (1.5) & -10.0 & (5.4) \\
\hline Chile & 408 & (3.5) & $\mathrm{m}$ & $\mathrm{m}$ & 443 & (4.9) & 449 & (3.1) & 41.1 & (6.8) \\
\hline Tschech. Rep. & 490 & (2.5) & 488 & (3.5) & 484 & $(4.2)$ & 478 & (2.9) & -11.6 & (6.3) \\
\hline Dänemark & 497 & (2.4) & 492 & (2.8) & 495 & (3.2) & 495 & (2.1) & -2.0 & (5.9) \\
\hline Estland & $\mathrm{m}$ & $\mathrm{m}$ & $\mathrm{m}$ & $\mathrm{m}$ & 501 & (2.9) & 501 & (2.6) & $\mathrm{m}$ & $\mathrm{m}$ \\
\hline Finnland & 546 & (2.6) & 543 & (1.6) & 548 & (2.1) & 536 & (2.3) & -9.9 & (6.0) \\
\hline Frankreich & 504 & (2.6) & 496 & $(2.7)$ & 490 & (4.1) & 496 & (3.4) & -8.8 & (6.6) \\
\hline Deutschland & 484 & (2.4) & 493 & (3.5) & 495 & (4.4) & 497 & $(2.7)$ & 13.5 & (6.1) \\
\hline Griechenland & 473 & (4.9) & 472 & $(4.1)$ & 460 & (3.8) & 483 & $(4.3)$ & 10.0 & $(8.2)$ \\
\hline Ungarn & 480 & (3.9) & 483 & (2.5) & 483 & (3.2) & 494 & (3.2) & 14.4 & (7.1) \\
\hline Island & 507 & (1.4) & 493 & (1.5) & 485 & (1.9) & 500 & (1.4) & -6.5 & (5.3) \\
\hline Irland & 527 & (3.2) & 515 & (2.6) & 517 & (3.4) & 496 & (3.0) & -30.9 & (6.6) \\
\hline Israel & 451 & (8.6) & $\mathrm{m}$ & $\mathrm{m}$ & 438 & (4.5) & 474 & (3.6) & 23.0 & (10.6) \\
\hline Italien & 487 & (2.8) & 474 & (3.1) & 468 & (2.4) & 486 & (1.6) & -0.8 & (5.9) \\
\hline Japan & 520 & $(5.2)$ & 495 & $(4.1)$ & 497 & (3.7) & 520 & (3.5) & -0.6 & (8.0) \\
\hline Korea & 525 & (2.2) & 535 & (2.8) & 554 & (3.5) & 539 & (3.5) & 14.4 & (6.4) \\
\hline Luxemburg & $\mathrm{m}$ & $\mathrm{m}$ & 481 & (1.5) & 479 & (1.3) & 472 & (1.3) & $\mathrm{m}$ & $\mathrm{m}$ \\
\hline Mexiko & 421 & (3.3) & 399 & $(4.1)$ & 410 & (3.0) & 425 & (2.0) & 4.0 & $(6.2)$ \\
\hline Niederlande & $\mathrm{m}$ & $\mathrm{m}$ & 513 & (2.8) & 507 & (2.9) & 508 & (5.1) & $\mathrm{m}$ & $\mathrm{m}$ \\
\hline Neuseeland & 529 & $(2.5)$ & 521 & (2.3) & 520 & $(2.7)$ & 521 & (2.4) & -7.9 & $(6.0)$ \\
\hline Norwegen & 503 & $(2.7)$ & 499 & (2.9) & 485 & (3.2) & 503 & (2.6) & 0.3 & $(6.2)$ \\
\hline Polen & 479 & (4.6) & 497 & (2.8) & 508 & $(2.7)$ & 500 & (2.6) & 21.1 & $(7.2)$ \\
\hline Portugal & 470 & $(4.4)$ & 475 & (3.9) & 472 & (3.5) & 489 & (3.1) & 19.7 & (7.3) \\
\hline Slowak. Rep. & $\mathrm{m}$ & $\mathrm{m}$ & 470 & (3.0) & 467 & (3.0) & 477 & (2.5) & $\mathrm{m}$ & $\mathrm{m}$ \\
\hline Slowenien & $\mathrm{m}$ & $\mathrm{m}$ & $\mathrm{m}$ & $\mathrm{m}$ & 494 & $(1.0)$ & 483 & (1.0) & $\mathrm{m}$ & $\mathrm{m}$ \\
\hline Spanien & 492 & $(2.7)$ & 479 & $(2.7)$ & 461 & $(2.2)$ & 481 & $(2.0)$ & -10.9 & (6.0) \\
\hline Schweden & 516 & $(2.2)$ & 514 & (2.4) & 507 & (3.4) & 497 & (2.9) & -18.9 & (6.1) \\
\hline Schweiz & 493 & $(4.1)$ & 500 & (3.0) & 500 & (3.0) & 501 & (2.4) & 7.5 & (6.9) \\
\hline Türkei & $\mathrm{m}$ & $\mathrm{m}$ & 441 & $(5.7)$ & 447 & $(4.2)$ & 464 & (3.5) & $\mathrm{m}$ & $\mathrm{m}$ \\
\hline Ver. Königreich & $\mathrm{m}$ & $\mathrm{m}$ & $\mathrm{m}$ & $\mathrm{m}$ & 495 & $(2.2)$ & 494 & $(2.3)$ & $\mathrm{m}$ & $\mathrm{m}$ \\
\hline Ver. Staaten & 502 & $(7.2)$ & 494 & (3.2) & $\mathrm{m}$ & $\mathrm{m}$ & 500 & $(3.7)$ & -2.1 & (9.5) \\
\hline OECD-23-Durchschnitt & 501 & $(0.7)$ & 497 & $(0.6)$ & 495 & $(0.7)$ & 499 & $(0.6)$ & -1 & (1.4) \\
\hline OECD-26-Durchschnitt & 495 & $(0.8)$ & 497 & $(0.6)$ & 490 & $(0.7)$ & 496 & $(0.5)$ & 1 & (1.3) \\
\hline
\end{tabular}

\begin{tabular}{|c|c|c|c|c|c|c|c|c|c|c|}
\hline A Albanien & 348 & (2.9) & $\mathrm{m}$ & $\mathrm{m}$ & $\mathrm{m}$ & $\mathrm{m}$ & 385 & (4.0) & 36.6 & (7.0) \\
\hline Argentinien & 411 & (8.6) & $\mathrm{m}$ & $\mathrm{m}$ & 374 & (6.9) & 398 & (4.6) & -12.9 & (10.9) \\
\hline § Aserbaidschan & $\mathrm{m}$ & $\mathrm{m}$ & $\mathrm{m}$ & $\mathrm{m}$ & 353 & (3.4) & 362 & (3.3) & $\mathrm{m}$ & $\mathrm{m}$ \\
\hline ¿ Brasilien & 396 & (3.0) & 405 & (4.6) & 393 & (4.0) & 412 & (2.7) & 16.1 & (6.4) \\
\hline Bulgarien & 432 & (4.8) & $\mathrm{m}$ & $\mathrm{m}$ & 404 & (6.5) & 429 & (6.7) & -2.6 & (9.6) \\
\hline Kolumbien & $\mathrm{m}$ & $\mathrm{m}$ & $\mathrm{m}$ & $\mathrm{m}$ & 385 & (4.9) & 413 & (3.7) & $\mathrm{m}$ & $\mathrm{m}$ \\
\hline Kroatien & $\mathrm{m}$ & $\mathrm{m}$ & $\mathrm{m}$ & $\mathrm{m}$ & 476 & $(2.7)$ & 476 & (2.9) & $\mathrm{m}$ & $\mathrm{m}$ \\
\hline Hongkong (China) & 522 & (3.1) & 509 & (3.6) & 533 & (2.4) & 533 & (2.1) & 11.4 & (6.2) \\
\hline Indonesien & 369 & (3.9) & 381 & (3.3) & 391 & (5.8) & 402 & (3.7) & 32.6 & (7.3) \\
\hline Jordanien & $\mathrm{m}$ & $\mathrm{m}$ & $\mathrm{m}$ & $\mathrm{m}$ & 400 & (3.3) & 405 & (3.3) & $\mathrm{m}$ & $\mathrm{m}$ \\
\hline Kirgisistan & $\mathrm{m}$ & $\mathrm{m}$ & $\mathrm{m}$ & $\mathrm{m}$ & 284 & (3.4) & 314 & (3.2) & $\mathrm{m}$ & $\mathrm{m}$ \\
\hline Lettland & 458 & (4.9) & 489 & (3.5) & 479 & (3.6) & 484 & (3.0) & 25.9 & (7.5) \\
\hline Liechtenstein & 482 & $(4.0)$ & 525 & (3.7) & 507 & $(4.0)$ & 499 & (2.8) & 17.5 & (7.0) \\
\hline Litauen & $\mathrm{m}$ & $\mathrm{m}$ & $\mathrm{m}$ & $\mathrm{m}$ & 470 & (3.0) & 468 & (2.4) & $\mathrm{m}$ & $\mathrm{m}$ \\
\hline Macau (China) & $\mathrm{m}$ & $\mathrm{m}$ & 497 & $(2.2)$ & 492 & (1.1) & 487 & $(0.9)$ & $\mathrm{m}$ & $\mathrm{m}$ \\
\hline Montenegro & $\mathrm{m}$ & $\mathrm{m}$ & $\mathrm{m}$ & $\mathrm{m}$ & 392 & (1.2) & 408 & (1.7) & $\mathrm{m}$ & $\mathrm{m}$ \\
\hline Peru & 327 & (4.6) & $\mathrm{m}$ & $\mathrm{m}$ & $\mathrm{m}$ & $\mathrm{m}$ & 370 & (4.0) & 43.0 & (7.8) \\
\hline Katar & $\mathrm{m}$ & $\mathrm{m}$ & $\mathrm{m}$ & $\mathrm{m}$ & 312 & (1.2) & 372 & $(0.8)$ & $\mathrm{m}$ & $\mathrm{m}$ \\
\hline Rumänien & 427 & (3.5) & $\mathrm{m}$ & $\mathrm{m}$ & 396 & (4.5) & 424 & $(4.1)$ & -2.3 & (7.3) \\
\hline Russ. Föderation & 462 & (4.1) & 442 & (3.8) & 439 & $(4.2)$ & 459 & (3.3) & -2.5 & $(7.2)$ \\
\hline Serbien & $\mathrm{m}$ & $\mathrm{m}$ & 411 & (3.2) & 401 & (3.2) & 442 & (2.4) & $\mathrm{m}$ & $\mathrm{m}$ \\
\hline Chinesisch Taipeh & $\mathrm{m}$ & $\mathrm{m}$ & $\mathrm{m}$ & $\mathrm{m}$ & 507 & (4.1) & 495 & (2.6) & $\mathrm{m}$ & $\mathrm{m}$ \\
\hline Thailand & 428 & (3.0) & 421 & $(2.7)$ & 418 & (2.5) & 421 & (2.6) & -6.4 & (6.3) \\
\hline Tunesien & $\mathrm{m}$ & $\mathrm{m}$ & 375 & (2.8) & 380 & (3.9) & 404 & (2.9) & $\mathrm{m}$ & $\mathrm{m}$ \\
\hline Uruguay & $\mathrm{m}$ & $\mathrm{m}$ & 436 & (3.4) & 413 & (3.3) & 426 & (2.6) & $\mathrm{m}$ & $\mathrm{m}$ \\
\hline
\end{tabular}

Anmerkung: Statistisch signifikante Werte sind durch Fettdruck gekennzeichnet (siehe Anhang A3).

StatLink *ills http://dx.doi.org/10.1787/888932360100 


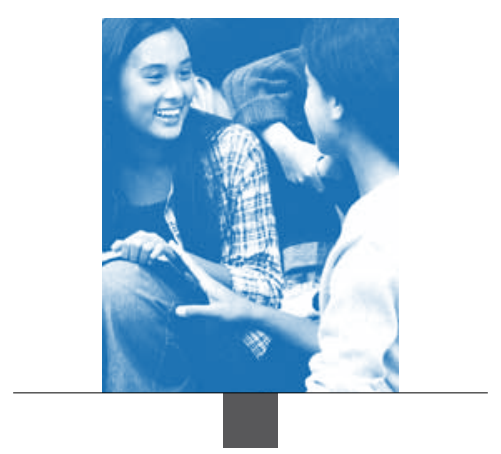

\section{Anhang B}

\section{ERGEBNISTABELLEN}

Alle Tabellen in Anhang B sind online verfügbar

Anhang B1: Ergebnisse für Länder und Volkswirtschaften

Anhang B2: Ergebnisse für einzelne Regionen innerhalb der Länder (nur auf Englisch verfügbar)

\section{Regionen, deren Stichproben international überprüft} wurden

Daten, bei denen die Einhaltung der PISA-

Stichprobenstandards und die internationale Vergleichbarkeit international überprüft wurden.

Regionen, deren Stichproben national festgelegt und überprüft wurden

Daten, bei denen die Einhaltung der PISA-

Stichprobenstandards auf den subnationalen Ebenen von den betreffenden Ländern bewertet wurde.

In diesen Ländern wurden die Einhaltung der PISA-Stichprobenstandards und die internationale

Vergleichbarkeit nur für die Gesamtheit aller subnationalen Einheiten international überprüft.

Anmerkung: Sofern nicht anders spezifiziert, sind alle in den nachstehenden Tabellen enthaltenen Daten der PISA-Datenbank der OECD entnommen. 


\section{ANHANG B1}

\section{ERGEBNISSE FÜR LÄNDER UND VOLKSWIRTSCHAFTEN}

[Teil 1/1]

Mittlere Punktzahlen im Bereich Lesekompetenz aus den PISA-Erhebungen 2000, 2003, 2006 und Tabelle V.2.1 2009

\begin{tabular}{|c|c|c|c|c|c|c|c|c|c|c|c|c|}
\hline & \multicolumn{2}{|c|}{ PISA 2000} & \multicolumn{2}{|c|}{ PISA 2003} & \multicolumn{2}{|c|}{ PISA 2006} & \multicolumn{2}{|c|}{ PISA 2009} & \multicolumn{3}{|c|}{$\begin{array}{c}\text { Veränderung zwischen } \\
2000 \text { und } 2009 \\
\text { (PISA } 2009 \text { - PISA 2000) }\end{array}$} \\
\hline & & Mittelwert & S.E. & Mittelwert & S.E. & Mittelwert & S.E. & Mittelwert & S.E. & Punktdiff. & S.E. & p-Wert \\
\hline \multirow{36}{*}{ } & Australien & 528 & (3.5) & 525 & (2.1) & 513 & (2.1) & 515 & (2.3) & -13 & (6.5) & 0.04 \\
\hline & Österreich & 492 & $(2.7)$ & 491 & (3.8) & 490 & (4.1) & $\mathrm{m}$ & $\mathrm{m}$ & $\mathrm{m}$ & $\mathrm{m}$ & $\mathrm{m}$ \\
\hline & Belgien & 507 & (3.6) & 507 & (2.6) & 501 & (3.0) & 506 & (2.3) & -1 & (6.5) & 0.86 \\
\hline & Kanada & 534 & (1.6) & 528 & $(1.7)$ & 527 & (2.4) & 524 & (1.5) & -10 & (5.4) & 0.06 \\
\hline & Chile & 410 & (3.6) & $\mathrm{m}$ & $\mathrm{m}$ & 442 & (5.0) & 449 & (3.1) & 40 & (6.9) & 0.00 \\
\hline & Tschech. Rep. & 492 & $(2.4)$ & 489 & (3.5) & 483 & $(4.2)$ & 478 & (2.9) & -13 & $(6.2)$ & 0.03 \\
\hline & Dänemark & 497 & (2.4) & 492 & (2.8) & 494 & (3.2) & 495 & $(2.1)$ & -2 & (5.8) & 0.74 \\
\hline & Estland & $\mathrm{m}$ & $\mathrm{m}$ & $\mathrm{m}$ & $\mathrm{m}$ & 501 & (2.9) & 501 & (2.6) & $\mathrm{m}$ & $\mathrm{m}$ & $\mathrm{m}$ \\
\hline & Finnland & 546 & (2.6) & 543 & (1.6) & 547 & (2.1) & 536 & (2.3) & -11 & (6.0) & 0.08 \\
\hline & Frankreich & 505 & (2.7) & 496 & $(2.7)$ & 488 & (4.1) & 496 & (3.4) & -9 & (6.6) & 0.17 \\
\hline & Deutschland & 484 & (2.5) & 491 & (3.4) & 495 & (4.4) & 497 & $(2.7)$ & 13 & (6.1) & 0.03 \\
\hline & Griechenland & 474 & (5.0) & 472 & $(4.1)$ & 460 & $(4.0)$ & 483 & (4.3) & 9 & $(8.2)$ & 0.28 \\
\hline & Ungarn & 480 & $(4.0)$ & 482 & (2.5) & 482 & (3.3) & 494 & (3.2) & 14 & $(7.1)$ & 0.04 \\
\hline & Island & 507 & (1.5) & 492 & (1.6) & 484 & (1.9) & 500 & (1.4) & -7 & (5.3) & 0.21 \\
\hline & Irland & 527 & (3.2) & 515 & (2.6) & 517 & (3.5) & 496 & (3.0) & -31 & (6.6) & 0.00 \\
\hline & Israel & 452 & $(8.5)$ & $\mathrm{m}$ & $\mathrm{m}$ & 439 & (4.6) & 474 & (3.6) & 22 & (10.5) & 0.04 \\
\hline & Italien & 487 & (2.9) & 476 & (3.0) & 469 & (2.4) & 486 & (1.6) & -1 & (5.9) & 0.81 \\
\hline & Japan & 522 & $(5.2)$ & 498 & (3.9) & 498 & (3.6) & 520 & (3.5) & -2 & (8.0) & 0.77 \\
\hline & Korea & 525 & (2.4) & 534 & (3.1) & 556 & (3.8) & 539 & (3.5) & 15 & (6.5) & 0.03 \\
\hline & Luxemburg & $\mathrm{m}$ & $\mathrm{m}$ & 479 & (1.5) & 479 & (1.3) & 472 & (1.3) & $\mathrm{m}$ & $\mathrm{m}$ & $\mathrm{m}$ \\
\hline & Mexiko & 422 & (3.3) & 400 & $(4.1)$ & 410 & (3.1) & 425 & (2.0) & 3 & (6.3) & 0.60 \\
\hline & Niederlande & $\mathrm{m}$ & $\mathrm{m}$ & 513 & (2.9) & 507 & (2.9) & 508 & (5.1) & $\mathrm{m}$ & $\mathrm{m}$ & $\mathrm{m}$ \\
\hline & Neuseeland & 529 & (2.8) & 522 & (2.5) & 521 & (3.0) & 521 & (2.4) & -8 & (6.1) & 0.20 \\
\hline & Norwegen & 505 & $(2.8)$ & 500 & $(2.8)$ & 484 & (3.2) & 503 & (2.6) & -2 & $(6.2)$ & 0.74 \\
\hline & Polen & 479 & $(4.5)$ & 497 & (2.9) & 508 & (2.8) & 500 & (2.6) & 21 & $(7.1)$ & 0.00 \\
\hline & Portugal & 470 & $(4.5)$ & 478 & (3.7) & 472 & (3.6) & 489 & (3.1) & 19 & $(7.4)$ & 0.01 \\
\hline & Slowak. Rep. & $\mathrm{m}$ & $\mathrm{m}$ & 469 & (3.1) & 466 & (3.1) & 477 & (2.5) & $\mathrm{m}$ & $\mathrm{m}$ & $\mathrm{m}$ \\
\hline & Slowenien & $\mathrm{m}$ & $\mathrm{m}$ & $\mathrm{m}$ & $\mathrm{m}$ & 494 & (1.0) & 483 & (1.0) & $\mathrm{m}$ & $\mathrm{m}$ & $\mathrm{m}$ \\
\hline & Spanien & 493 & $(2.7)$ & 481 & (2.6) & 461 & $(2.2)$ & 481 & $(2.0)$ & -12 & (6.0) & 0.05 \\
\hline & Schweden & 516 & $(2.2)$ & 514 & (2.4) & 507 & (3.4) & 497 & (2.9) & -19 & (6.1) & 0.00 \\
\hline & Schweiz & 494 & $(4.2)$ & 499 & (3.3) & 499 & (3.1) & 501 & (2.4) & 6 & $(7.0)$ & 0.38 \\
\hline & Türkei & $\mathrm{m}$ & $\mathrm{m}$ & 441 & (5.8) & 447 & $(4.2)$ & 464 & (3.5) & $\mathrm{m}$ & $\mathrm{m}$ & $\mathrm{m}$ \\
\hline & Ver. Königreich & $\mathrm{m}$ & $\mathrm{m}$ & $\mathrm{m}$ & $\mathrm{m}$ & 495 & (2.3) & 494 & (2.3) & $\mathrm{m}$ & $\mathrm{m}$ & $\mathrm{m}$ \\
\hline & Ver. Staaten & 504 & $(7.0)$ & 495 & $(3.2)$ & $\mathrm{m}$ & $\mathrm{m}$ & 500 & (3.7) & -5 & (9.3) & 0.62 \\
\hline & OECD-23-Durchschnitt & 501 & $(0.7)$ & 497 & $(0.6)$ & 495 & $(0.7)$ & 499 & $(0.6)$ & -2 & $(5.0)$ & 0.73 \\
\hline & OECD-26-Durchschnitt & 496 & $(0.8)$ & $\mathrm{m}$ & $\mathrm{m}$ & $\mathrm{m}$ & $\mathrm{m}$ & 496 & $(0.5)$ & 1 & $(5.0)$ & 0.90 \\
\hline \multirow{25}{*}{ 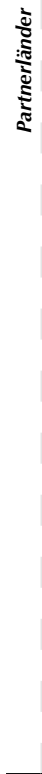 } & Albanien & 349 & (3.3) & $\mathrm{m}$ & $\mathrm{m}$ & $\mathrm{m}$ & $\mathrm{m}$ & 385 & $(4.0)$ & 36 & $(7.2)$ & 0.00 \\
\hline & Argentinien & 418 & (9.9) & $\mathrm{m}$ & $\mathrm{m}$ & 374 & $(7.2)$ & 398 & $(4.6)$ & -20 & $(12.0)$ & 0.09 \\
\hline & Aserbaidschan & $\mathrm{m}$ & $\mathrm{m}$ & $\mathrm{m}$ & $\mathrm{m}$ & 353 & (3.1) & 362 & (3.3) & $\mathrm{m}$ & $\mathrm{m}$ & $\mathrm{m}$ \\
\hline & Brasilien & 396 & (3.1) & 403 & (4.6) & 393 & (3.7) & 412 & $(2.7)$ & 16 & (6.4) & 0.01 \\
\hline & Bulgarien & 430 & $(4.9)$ & $\mathrm{m}$ & $\mathrm{m}$ & 402 & (6.9) & 429 & $(6.7)$ & -1 & (9.6) & 0.89 \\
\hline & Kolumbien & $\mathrm{m}$ & $\mathrm{m}$ & $\mathrm{m}$ & $\mathrm{m}$ & 385 & (5.1) & 413 & (3.7) & $\mathrm{m}$ & $\mathrm{m}$ & $\mathrm{m}$ \\
\hline & Kroatien & $\mathrm{m}$ & $\mathrm{m}$ & $\mathrm{m}$ & $\mathrm{m}$ & 477 & (2.8) & 476 & (2.9) & $\mathrm{m}$ & $\mathrm{m}$ & $\mathrm{m}$ \\
\hline & Hongkong (China) & 525 & (2.9) & 510 & (3.7) & 536 & (2.4) & 533 & $(2.1)$ & 8 & $(6.1)$ & 0.21 \\
\hline & Indonesien & 371 & $(4.0)$ & 382 & (3.4) & 393 & (5.9) & 402 & (3.7) & 31 & (7.4) & 0.00 \\
\hline & Jordanien & $\mathrm{m}$ & $\mathrm{m}$ & $\mathrm{m}$ & $\mathrm{m}$ & 401 & (3.3) & 405 & (3.3) & $\mathrm{m}$ & $\mathrm{m}$ & $\mathrm{m}$ \\
\hline & Kirgisistan & $\mathrm{m}$ & $\mathrm{m}$ & $\mathrm{m}$ & $\mathrm{m}$ & 285 & (3.5) & 314 & $(3.2)$ & $\mathrm{m}$ & $\mathrm{m}$ & $\mathrm{m}$ \\
\hline & Lettland & 458 & (5.3) & 491 & (3.7) & 479 & (3.7) & 484 & (3.0) & 26 & (7.8) & 0.00 \\
\hline & Liechtenstein & 483 & $(4.1)$ & 525 & (3.6) & 510 & (3.9) & 499 & (2.8) & 17 & $(7.0)$ & 0.02 \\
\hline & Litauen & $\mathrm{m}$ & $\mathrm{m}$ & $\mathrm{m}$ & $\mathrm{m}$ & 470 & (3.0) & 468 & (2.4) & $\mathrm{m}$ & $\mathrm{m}$ & $\mathrm{m}$ \\
\hline & Macau (China) & $\mathrm{m}$ & $\mathrm{m}$ & 498 & $(2.2)$ & 492 & (1.1) & 487 & $(0.9)$ & $\mathrm{m}$ & $\mathrm{m}$ & $\mathrm{m}$ \\
\hline & Montenegro & $\mathrm{m}$ & $\mathrm{m}$ & $\mathrm{m}$ & $\mathrm{m}$ & 392 & $(1.2)$ & 408 & (1.7) & $\mathrm{m}$ & $\mathrm{m}$ & $\mathrm{m}$ \\
\hline & Peru & 327 & $(4.4)$ & $\mathrm{m}$ & $\mathrm{m}$ & $\mathrm{m}$ & $\mathrm{m}$ & 370 & $(4.0)$ & 43 & $(7.7)$ & 0.00 \\
\hline & Katar & $\mathrm{m}$ & $\mathrm{m}$ & $\mathrm{m}$ & $\mathrm{m}$ & 312 & $(1.2)$ & 372 & $(0.8)$ & $\mathrm{m}$ & $\mathrm{m}$ & $\mathrm{m}$ \\
\hline & Rumänien & 428 & (3.5) & $\mathrm{m}$ & $\mathrm{m}$ & 396 & (4.7) & 424 & $(4.1)$ & -3 & (7.3) & 0.63 \\
\hline & Russ. Föderation & 462 & $(4.2)$ & 442 & (3.9) & 440 & (4.3) & 459 & (3.3) & -2 & (7.3) & 0.74 \\
\hline & Serbien & $\mathrm{m}$ & $\mathrm{m}$ & 412 & (3.6) & 401 & (3.5) & 442 & (2.4) & $\mathrm{m}$ & $\mathrm{m}$ & $\mathrm{m}$ \\
\hline & Chinesisch Taipeh & $\mathrm{m}$ & $\mathrm{m}$ & $\mathrm{m}$ & $\mathrm{m}$ & 496 & (3.4) & 495 & (2.6) & $\mathrm{m}$ & $\mathrm{m}$ & $\mathrm{m}$ \\
\hline & Thailand & 431 & $(3.2)$ & 420 & (2.8) & 417 & (2.6) & 421 & (2.6) & -9 & (6.5) & 0.15 \\
\hline & Tunesien & $\mathrm{m}$ & $\mathrm{m}$ & 375 & (2.8) & 380 & (4.0) & 404 & (2.9) & $\mathrm{m}$ & $\mathrm{m}$ & $\mathrm{m}$ \\
\hline & Uruguay & $\mathrm{m}$ & $\mathrm{m}$ & 434 & (3.4) & 413 & (3.4) & 426 & (2.6) & $\mathrm{m}$ & $\mathrm{m}$ & $\mathrm{m}$ \\
\hline
\end{tabular}

Anmerkung: Statistisch signifikante Werte sind durch Fettdruck gekennzeichnet (siehe Anhang A3). StatLink ints http://dx.doi.org/10.1787/888932343285 
[Teil 1/1]

Prozentsatz der Schüler unter Stufe 2 sowie auf Stufe 5 oder höher auf der Gesamtskala Tabelle V.2.2 Lesekompetenz in PISA 2000 und PISA 2009

\begin{tabular}{|c|c|c|c|c|c|c|c|c|c|c|c|c|c|}
\hline & \multicolumn{4}{|c|}{ Kompetenzstufen in PISA 2000} & \multicolumn{4}{|c|}{ Kompetenzstufen in PISA 2009} & \multicolumn{4}{|c|}{$\begin{array}{c}\text { Veränderung zwischen } 2000 \text { und } 2009 \\
\text { (PISA 2009 - PISA 2000) }\end{array}$} \\
\hline & & \multicolumn{2}{|c|}{$\begin{array}{c}\text { Unter Stufe } 2 \\
\text { (unter 407 Punkte) }\end{array}$} & \multicolumn{2}{|c|}{$\begin{array}{l}\text { Stufe } 5 \text { oder höher } \\
\text { (über } 626 \text { Punkte) }\end{array}$} & \multicolumn{2}{|c|}{$\begin{array}{c}\text { Unter Stufe } 2 \\
\text { (unter 407 Punkte) }\end{array}$} & \multicolumn{2}{|c|}{$\begin{array}{l}\text { Stufe } 5 \text { oder höher } \\
\text { (über } 626 \text { Punkte) }\end{array}$} & \multicolumn{2}{|c|}{$\begin{array}{c}\text { Unter Stufe } 2 \\
\text { (unter 407 Punkte) }\end{array}$} & \multicolumn{2}{|c|}{$\begin{array}{l}\text { Stufe } 5 \text { oder höher } \\
\text { (über } 626 \text { Punkte) }\end{array}$} \\
\hline & & $\%$ & S.E. & $\%$ & S.E. & $\%$ & S.E. & $\%$ & S.E. & Diff. \% & S.E. & Diff. \% & S.E. \\
\hline \multirow{31}{*}{ 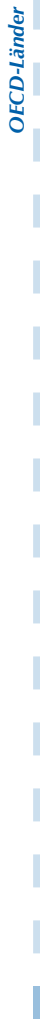 } & Australien & 12.5 & (0.9) & 17.6 & $(1.2)$ & 14.2 & $(0.6)$ & 12.8 & $(0.8)$ & 1.8 & $(1.0)$ & -4.9 & (1.4) \\
\hline & Österreich & 19.3 & $(0.9)$ & 7.5 & $(0.7)$ & $\mathrm{m}$ & $\mathrm{m}$ & $\mathrm{m}$ & $\mathrm{m}$ & $\mathrm{m}$ & $\mathrm{m}$ & $\mathrm{m}$ & $\mathrm{m}$ \\
\hline & Belgien & 19.0 & (1.3) & 12.0 & $(0.7)$ & 17.7 & $(0.9)$ & 11.2 & $(0.6)$ & -1.2 & (1.6) & -0.8 & $(0.9)$ \\
\hline & Kanada & 9.6 & $(0.4)$ & 16.8 & $(0.5)$ & 10.3 & $(0.5)$ & 12.8 & $(0.5)$ & 0.7 & $(0.6)$ & -4.0 & $(0.7)$ \\
\hline & Chile & 48.2 & (1.9) & 0.5 & $(0.1)$ & 30.6 & (1.5) & 1.3 & $(0.3)$ & -17.6 & (2.4) & 0.8 & $(0.3)$ \\
\hline & Tschech. Rep. & 17.5 & $(0.8)$ & 7.0 & (0.6) & 23.1 & (1.3) & 5.1 & $(0.5)$ & 5.6 & (1.5) & -1.9 & $(0.7)$ \\
\hline & Dänemark & 17.9 & $(0.9)$ & 8.1 & $(0.5)$ & 15.2 & $(0.9)$ & 4.7 & $(0.5)$ & -2.7 & (1.3) & -3.4 & $(0.7)$ \\
\hline & Finnland & 7.0 & $(0.7)$ & 18.5 & (0.9) & 8.1 & $(0.5)$ & 14.5 & (0.8) & 1.2 & $(0.8)$ & -4.0 & $(1.2)$ \\
\hline & Frankreich & 15.2 & (1.1) & 8.5 & $(0.5)$ & 19.8 & $(1.2)$ & 9.6 & (1.0) & 4.6 & (1.6) & 1.1 & (1.1) \\
\hline & Deutschland & 22.6 & (1.0) & 8.8 & $(0.5)$ & 18.5 & (1.1) & 7.6 & $(0.6)$ & -4.2 & (1.4) & -1.2 & (0.8) \\
\hline & Griechenland & 24.4 & (2.1) & 5.0 & $(0.7)$ & 21.3 & (1.8) & 5.6 & $(0.5)$ & -3.1 & (2.8) & 0.6 & $(0.8)$ \\
\hline & Ungarn & 22.7 & (1.5) & 5.1 & $(0.8)$ & 17.6 & (1.4) & 6.1 & $(0.7)$ & -5.1 & (2.1) & 1.0 & (1.0) \\
\hline & Island & 14.5 & $(0.7)$ & 9.1 & $(0.7)$ & 16.8 & (0.6) & 8.5 & (0.6) & 2.3 & $(0.9)$ & -0.5 & $(0.9)$ \\
\hline & Irland & 11.0 & (1.0) & 14.2 & $(0.8)$ & 17.2 & (1.0) & 7.0 & (0.5) & 6.2 & (1.4) & -7.3 & (1.0) \\
\hline & Israel & 33.2 & (3.2) & 4.2 & (0.8) & 26.5 & $(1.2)$ & 7.4 & (0.6) & -6.7 & (3.4) & 3.3 & (1.0) \\
\hline & Italien & 18.9 & (1.1) & 5.3 & $(0.5)$ & 21.0 & (0.6) & 5.8 & $(0.3)$ & 2.1 & (1.3) & 0.5 & $(0.6)$ \\
\hline & Japan & 10.1 & (1.5) & 9.9 & (1.1) & 13.6 & (1.1) & 13.4 & $(0.9)$ & 3.5 & (1.9) & 3.6 & (1.4) \\
\hline & Korea & 5.8 & $(0.7)$ & 5.7 & (0.6) & 5.8 & $(0.8)$ & 12.9 & (1.1) & 0.0 & (1.1) & 7.2 & $(1.2)$ \\
\hline & Luxemburg & $\mathrm{m}$ & $\mathrm{m}$ & $\mathrm{m}$ & $\mathrm{m}$ & 26.0 & (0.6) & 5.7 & $(0.5)$ & $\mathrm{m}$ & $\mathrm{m}$ & $\mathrm{m}$ & $\mathrm{m}$ \\
\hline & Mexiko & 44.1 & (1.7) & 0.9 & $(0.2)$ & 40.1 & (1.0) & 0.4 & $(0.1)$ & -4.0 & (2.0) & -0.5 & $(0.2)$ \\
\hline & Niederlande & $\mathrm{m}$ & $\mathrm{m}$ & $\mathrm{m}$ & $\mathrm{m}$ & 14.3 & (1.5) & 9.8 & (1.1) & $\mathrm{m}$ & $\mathrm{m}$ & $\mathrm{m}$ & $\mathrm{m}$ \\
\hline & Neuseeland & 13.7 & $(0.8)$ & 18.7 & (1.0) & 14.3 & $(0.7)$ & 15.7 & $(0.8)$ & 0.6 & (1.1) & -3.0 & (1.3) \\
\hline & Norwegen & 17.5 & (1.1) & 11.2 & $(0.7)$ & 15.0 & $(0.8)$ & 8.4 & $(0.9)$ & -2.5 & (1.3) & -2.8 & (1.1) \\
\hline & Polen & 23.2 & (1.4) & 5.9 & (0.9) & 15.0 & $(0.8)$ & 7.2 & (0.6) & -8.2 & (1.7) & 1.3 & (1.1) \\
\hline & Portugal & 26.3 & (1.9) & 4.2 & $(0.5)$ & 17.6 & (1.2) & 4.8 & $(0.5)$ & -8.6 & $(2.2)$ & 0.6 & $(0.8)$ \\
\hline & Spanien & 16.3 & (1.1) & 4.2 & $(0.5)$ & 19.6 & $(0.9)$ & 3.3 & $(0.3)$ & 3.3 & (1.4) & -0.9 & $(0.6)$ \\
\hline & Schweden & 12.6 & $(0.7)$ & 11.2 & $(0.7)$ & 17.4 & $(0.9)$ & 9.0 & $(0.7)$ & 4.9 & (1.2) & -2.2 & (1.0) \\
\hline & Schweiz & 20.4 & (1.3) & 9.2 & (1.0) & 16.8 & $(0.9)$ & 8.1 & $(0.7)$ & -3.6 & (1.6) & -1.1 & $(1.3)$ \\
\hline & Ver. Königreich & $\mathrm{m}$ & $\mathrm{m}$ & $\mathrm{m}$ & $\mathrm{m}$ & 18.4 & (0.8) & 8.0 & (0.5) & $\mathrm{m}$ & $\mathrm{m}$ & $\mathrm{m}$ & $\mathrm{m}$ \\
\hline & Ver. Staaten & 17.9 & $(2.2)$ & 12.2 & (1.4) & 17.6 & (1.1) & 9.9 & (0.9) & -0.3 & (2.4) & -2.4 & (1.6) \\
\hline & OECD-26-Durchschnitt & 19.3 & $(0.3)$ & 9.0 & $(0.2)$ & 18.1 & $(0.2)$ & 8.2 & $(0.1)$ & -1.2 & $(0.3)$ & -0.8 & $(0.2)$ \\
\hline \multirow{12}{*}{ 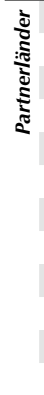 } & Albanien & 70.4 & (1.1) & 0.1 & $(0.1)$ & 56.7 & (1.9) & 0.2 & $(0.1)$ & -13.7 & $(2.2)$ & 0.1 & $(0.1)$ \\
\hline & Argentinien & 43.9 & (4.5) & 1.7 & $(0.5)$ & 51.6 & (1.9) & 1.0 & $(0.2)$ & 7.7 & (4.9) & -0.7 & $(0.5)$ \\
\hline & Brasilien & 55.8 & (1.7) & 0.6 & $(0.2)$ & 49.6 & (1.3) & 1.3 & $(0.2)$ & -6.2 & $(2.1)$ & 0.8 & $(0.3)$ \\
\hline & Bulgarien & 40.3 & $(2.1)$ & 2.2 & (0.6) & 41.0 & (2.6) & 2.8 & $(0.5)$ & 0.7 & (3.3) & 0.6 & $(0.8)$ \\
\hline & Hongkong (China) & 9.1 & (1.0) & 9.5 & $(0.8)$ & 8.3 & $(0.7)$ & 12.4 & (0.8) & -0.8 & $(1.2)$ & 2.9 & (1.1) \\
\hline & Indonesien & 68.7 & (2.5) & 0.0 & c & 53.4 & (2.3) & 0.0 & C & -15.2 & (3.4) & C & c \\
\hline & Lettland & 30.1 & (2.0) & 4.2 & (0.6) & 17.6 & (1.2) & 2.9 & $(0.4)$ & -12.5 & (2.4) & -1.2 & $(0.8)$ \\
\hline & Liechtenstein & 22.1 & $(2.1)$ & 5.1 & (1.6) & 15.7 & (1.8) & 4.6 & (1.4) & -6.4 & $(2.7)$ & -0.4 & $(2.1)$ \\
\hline & Peru & 79.5 & (1.5) & 0.1 & $(0.1)$ & 64.8 & (1.7) & 0.5 & $(0.2)$ & -14.8 & $(2.2)$ & 0.4 & $(0.2)$ \\
\hline & Rumänien & 41.3 & $(1.5)$ & 2.2 & $(0.3)$ & 40.4 & (2.0) & 0.7 & $(0.2)$ & -0.9 & (2.5) & -1.5 & $(0.3)$ \\
\hline & Russ. Föderation & 27.4 & $(1.7)$ & 3.2 & $(0.5)$ & 27.4 & (1.3) & 3.2 & $(0.5)$ & -0.1 & (2.2) & 0.0 & $(0.7)$ \\
\hline & Thailand & 37.1 & (1.7) & 0.5 & $(0.2)$ & 42.9 & (1.5) & 0.3 & $(0.2)$ & 5.8 & (2.3) & -0.2 & $(0.2)$ \\
\hline
\end{tabular}

Anmerkung: Statistisch signifikante Werte sind durch Fettdruck gekennzeichnet (siehe Anhang A3).

StatLink ज्ञात $\mathrm{http}: / / \mathrm{dx}$.doi.org/10.1787/888932343285 
[Teil 1/2]

Tabelle V.2.3 Perzentile auf der Gesamtskala Lesekompetenz in PISA 2000 und PISA 2009

\begin{tabular}{|c|c|c|c|c|c|c|c|c|c|c|c|c|c|c|c|c|c|c|c|c|c|c|c|}
\hline & \multicolumn{7}{|c|}{ PISA 2000} & \multicolumn{8}{|c|}{ PISA 2003} & \multicolumn{8}{|c|}{ PISA 2006} \\
\hline & $\begin{array}{c}10 . \\
\text { Perzentil }\end{array}$ & \multicolumn{2}{|c|}{$\begin{array}{c}25 . \\
\text { Perzentil }\end{array}$} & \multicolumn{2}{|c|}{$\begin{array}{c}75 . \\
\text { Perzentil }\end{array}$} & \multicolumn{2}{|c|}{$\begin{array}{c}90 . \\
\text { Perzentil }\end{array}$} & \multicolumn{2}{|c|}{$\begin{array}{c}10 . \\
\text { Perzentil }\end{array}$} & \multicolumn{2}{|c|}{$\begin{array}{c}25 . \\
\text { Perzentil }\end{array}$} & \multicolumn{2}{|c|}{$\begin{array}{c}75 . \\
\text { Perzentil }\end{array}$} & \multicolumn{2}{|c|}{$\begin{array}{c}90 . \\
\text { Perzentil }\end{array}$} & \multicolumn{2}{|c|}{$\begin{array}{c}10 . \\
\text { Perzentil }\end{array}$} & \multicolumn{2}{|c|}{$\begin{array}{c}25 . \\
\text { Perzentil }\end{array}$} & \multicolumn{2}{|c|}{$\begin{array}{c}75 . \\
\text { Perzentil }\end{array}$} & \multicolumn{2}{|c|}{$\begin{array}{c}90 . \\
\text { Perzentil }\end{array}$} \\
\hline & $\begin{array}{l}\text { Punkt- } \\
\text { zahl } \quad \text { S.E. }\end{array}$ & $\begin{array}{l}\text { Punkt- } \\
\text { zahl }\end{array}$ & S.E. & $\begin{array}{l}\text { Punkt- } \\
\text { zahl }\end{array}$ & S.E. & $\begin{array}{c}\text { Punkt- } \\
\text { zahl }\end{array}$ & S.E. & \begin{tabular}{|c} 
Punkt- \\
zahl
\end{tabular} & S.E. & $\begin{array}{l}\text { Punkt- } \\
\text { zahl }\end{array}$ & S.E. & $\begin{array}{l}\text { Punkt- } \\
\text { zahl }\end{array}$ & S.E. & $\begin{array}{l}\text { Punkt- } \\
\text { zahl }\end{array}$ & S.E. & $\begin{array}{l}\text { Punkt- } \\
\text { zahl }\end{array}$ & S.E. & $\begin{array}{l}\text { Punkt- } \\
\text { zahl }\end{array}$ & S.E. & $\begin{array}{l}\text { Punkt- } \\
\text { zahl }\end{array}$ & S.E. & $\begin{array}{l}\text { Punkt- } \\
\text { zahl }\end{array}$ & S.E. \\
\hline Australien & $394 \quad(4.4)$ & 458 & (4.4) & 602 & (4.6) & 656 & (4.2) & 395 & (3.6) & 464 & (3.0) & 594 & (2.5) & 644 & $(2.7)$ & 388 & (3.4) & 453 & (2.4) & 579 & (2.3) & 628 & (2.9) \\
\hline Österreich & $359 \quad(5.8)$ & 428 & (3.4) & 563 & (3.4) & 614 & (3.8) & 354 & (6.3) & 423 & (4.9) & 565 & (4.2) & 617 & (3.7) & 348 & (9.4) & 421 & (5.5) & 568 & (3.7) & 621 & (3.1) \\
\hline Belgien & $354 \quad(8.9)$ & 437 & (6.6) & 587 & (2.3) & 634 & (2.5) & 355 & (6.6) & 440 & (4.2) & 587 & (2.1) & 635 & (2.1) & 347 & (8.3) & 433 & (4.7) & 581 & (2.3) & 631 & (2.2) \\
\hline Kanada & $410 \quad(2.4)$ & 472 & (2.0) & 600 & (1.5) & 652 & (1.9) & 410 & (3.1) & 472 & (2.3) & 590 & (2.1) & 636 & (2.1) & 402 & (3.9) & 468 & (3.0) & 593 & (2.6) & 644 & (2.7) \\
\hline Chile & (5.3) & 350 & (4.4) & 472 & (3.9) & 524 & (3.8) & $\mathrm{m}$ & $\mathrm{m}$ & $\mathrm{m}$ & $\mathrm{m}$ & $\mathrm{m}$ & $\mathrm{m}$ & $\mathrm{m}$ & $\mathrm{m}$ & 310 & (5.8) & 373 & (5.4) & 513 & (6.4) & 575 & (6.7) \\
\hline Tschech. & $368 \quad(4.9)$ & 433 & (2.7) & 557 & (2.8) & 610 & (3.2) & 362 & (6.9) & 428 & (4.7) & 555 & (4.0) & 607 & (3.8) & 335 & (7.0) & 408 & (6.2) & 564 & (3.8) & 621 & (4.2) \\
\hline Dänen & $367 \quad(5.0)$ & 434 & (3.3) & 566 & (2.7) & 617 & (2.9) & 376 & (4.6) & 438 & (4.0) & 553 & (3.0) & 600 & (2.7) & 378 & (5.0) & 437 & (3.9) & 557 & (2.9) & 604 & (3.7) \\
\hline Estland & $\mathrm{m}$ & $\mathrm{m}$ & $\mathrm{m}$ & $\mathrm{m}$ & $\mathrm{m}$ & $\mathrm{m}$ & $\mathrm{m}$ & $\mathrm{m}$ & $\mathrm{m}$ & $\mathrm{m}$ & $\mathrm{m}$ & $\mathrm{m}$ & $\mathrm{m}$ & $\mathrm{m}$ & $\mathrm{m}$ & 389 & (5.4) & 448 & (3.8) & 560 & (2.8) & 606 & (3.2) \\
\hline Finnland & (5.1) & 492 & (2.9) & 608 & (2.6) & 654 & (2.8) & 437 & (3.1) & 494 & (2.4) & 599 & (1.7) & 641 & (2.2) & 441 & (3.8) & 494 & (2.9) & 603 & (2.2) & 649 & (2.5) \\
\hline Frankre & $381 \quad(5.2)$ & 444 & (4.5) & 570 & (2.4) & 619 & (2.9) & 367 & (7.0) & 436 & (4.0) & 565 & (2.8) & 614 & (2.7) & 346 & (7.5) & 421 & (6.1) & 564 & (3.8) & 614 & (4.0) \\
\hline Deut & $335 \quad(6.3)$ & 417 & (4.6) & 563 & (3.1) & 619 & (2.8) & 341 & (6.8) & 419 & (5.6) & 572 & (3.4) & 624 & (3.2) & 350 & (8.0) & 429 & (5.9) & 573 & (3.4) & 625 & (3.7) \\
\hline Grie & (8.4) & 409 & (7.4) & 543 & (4.5) & 595 & (5.1) & 333 & (6.2) & 406 & (5.2) & 546 & (4.4) & 599 & (4.4) & 321 & (8.5) & 398 & (5.2) & 531 & (3.8) & 583 & (4.2) \\
\hline Unga & 354 & 414 & (5.3) & 549 & (4.5) & 598 & (4.4) & 361 & (4.2) & 422 & (3.3) & 546 & (3.3) & 597 & (3.4) & 359 & (5.0) & 422 & (4.8) & 549 & (3.6) & 595 & (4.4) \\
\hline Island & $383 \quad$ (3.6) & 447 & (3.1) & 573 & (2.1) & 621 & (3.5) & 362 & (4.8) & 431 & (2.3) & 560 & (2.2) & 612 & (2.8) & 356 & (4.1) & 423 & (3.0) & 552 & (2.8) & 603 & (3.2) \\
\hline Irland & $401 \quad(6.4)$ & 468 & (4.3) & 593 & (3.6) & 641 & (4.0) & 401 & (4.6) & 460 & (3.8) & 577 & (2.8) & 622 & (3.0) & 395 & (5.5) & 457 & (4.7) & 582 & (3.9) & 633 & (3.5) \\
\hline Israel & $305(13.0)$ & 379 & (11.1) & 532 & (8.1) & 587 & (7.1) & $\mathrm{m}$ & $\mathrm{m}$ & $\mathrm{m}$ & $\mathrm{m}$ & $\mathrm{m}$ & $\mathrm{m}$ & $\mathrm{m}$ & $\mathrm{m}$ & 280 & (8.0) & 356 & (6.2) & 526 & (4.8) & 588 & (4.9) \\
\hline Italier & $368 \quad(5.8)$ & 429 & (4.1) & 552 & (3.2) & 601 & (2.7) & 341 & (6.8) & 411 & (4.4) & 547 & (2.5) & 598 & (2.1) & 325 & (4.8) & 402 & (3.6) & 546 & (2.3) & 599 & (2.9) \\
\hline Japan & (9.8) & 471 & (7.0) & 582 & (4.4) & 625 & (4.5) & 355 & (6.5) & 431 & (5.4) & 574 & (3.7) & 624 & (4.8) & 361 & (6.6) & 433 & (6.1) & 569 & (3.4) & 623 & (3.5) \\
\hline Korea & $433 \quad(4.4)$ & 481 & (2.9) & 574 & (2.6) & 608 & (2.9) & 428 & (5.2) & 484 & (4.1) & 590 & (2.8) & 634 & (4.1) & 440 & (7.9) & 503 & (4.8) & 617 & (3.4) & 663 & (4.3) \\
\hline Luxembu & $\mathrm{m}$ & $\mathrm{m}$ & $\mathrm{m}$ & $\mathrm{m}$ & $\mathrm{m}$ & $\mathrm{m}$ & $\mathrm{m}$ & 344 & (2.9) & 416 & (2.8) & 551 & (1.9) & 601 & (2.1) & 344 & (3.3) & 415 & (2.3) & 552 & (1.8) & 602 & (2.5) \\
\hline Mexiko & $311 \quad(3.4)$ & 360 & (3.6) & 482 & (4.8) & 535 & (5.5) & 274 & (5.5) & 335 & (4.9) & 467 & (4.3) & 521 & (6.1) & 285 & (6.2) & 348 & (4.2) & 478 & (2.8) & 530 & (3.1) \\
\hline & $\mathrm{m}$ & $\mathrm{m}$ & $\mathrm{m}$ & $\mathrm{m}$ & $\mathrm{m}$ & $\mathrm{m}$ & $\mathrm{m}$ & 400 & (5.2) & 454 & (4.5) & 576 & (3.2) & 621 & (2.9) & 379 & (6.4) & 446 & (4.3) & 578 & (2.5) & 622 & (2.4) \\
\hline & $82 \quad(5.2)$ & 59 & (4.0) & 06 & (3.0) & 51 & (4.4) & 381 & (4.4) & 53 & (3.5) & 596 & (2.8) & 652 & (2.9) & 381 & (4.6) & 453 & (4.5) & 595 & (2.9) & 651 & (2.8) \\
\hline Norwegen & $364 \quad(5.5)$ & 440 & (4.5) & 579 & (2.7) & 631 & (3.1) & 364 & (4.7) & 434 & (3.8) & 571 & (3.6) & 625 & (3.9) & 346 & (5.5) & 416 & (4.6) & 558 & (3.0) & 613 & (4.1) \\
\hline Polen & $343 \quad(6.8)$ & 414 & (5.8) & 551 & (6.0) & 603 & (6.6) & 374 & (5.0) & 436 & (3.6) & 563 & (3.1) & 616 & (3.4) & 374 & (4.6) & 441 & (3.5) & 579 & (3.2) & 633 & (3.4) \\
\hline Ports & $337 \quad(6.2)$ & 403 & (6.4) & 541 & (4.5) & 592 & (4.2) & 351 & (7.1) & 418 & (5.2) & 544 & (3.5) & 592 & (3.5) & 339 & (6.3) & 408 & (5.3) & 543 & (3.6) & 594 & (3.7) \\
\hline Slow & $\mathrm{m}$ & $\mathrm{m}$ & $\mathrm{m}$ & $\mathrm{m}$ & $\mathrm{m}$ & $\mathrm{m}$ & $\mathrm{m}$ & 348 & (5.8) & 408 & (4.6) & 535 & (3.2) & 587 & (3.0) & 326 & (6.6) & 398 & (4.3) & 542 & (3.4) & 597 & (3.8) \\
\hline Slowe & $\mathrm{m}$ & $\mathrm{m}$ & $\mathrm{m}$ & $\mathrm{m}$ & $\mathrm{m}$ & $\mathrm{m}$ & $\mathrm{m}$ & $\mathrm{m}$ & $\mathrm{m}$ & $\mathrm{m}$ & $\mathrm{m}$ & $\mathrm{m}$ & $\mathrm{m}$ & $\mathrm{m}$ & $\mathrm{m}$ & 377 & (2.6) & 437 & (1.8) & 558 & (2.2) & 603 & (2.1) \\
\hline Spanien & $379 \quad(5.0)$ & 436 & (4.6) & 553 & (2.6) & 597 & (2.6) & 354 & (4.9) & 421 & (3.4) & 548 & (2.8) & 597 & (2.8) & 343 & (4.1) & 405 & (2.9) & 523 & (2.3) & 569 & (2.7) \\
\hline Schweden & $392 \quad(4.0)$ & 456 & (3.1) & 581 & (3.1) & 630 & (2.9) & 390 & (4.3) & 453 & (3.4) & 582 & (2.9) & 631 & (2.9) & 378 & (5.6) & 445 & (3.8) & 575 & (3.3) & 629 & (4.0) \\
\hline Schweiz & $355 \quad(5.8)$ & 426 & (5.5) & 567 & (4.6) & 621 & (5.5) & 373 & (5.6) & 439 & (4.5) & 565 & (3.7) & 615 & (3.9) & 373 & (5.1) & 440 & (3.5) & 566 & (3.1) & 615 & (3.6) \\
\hline Türkei & $\mathrm{m}$ & $\mathrm{m}$ & $\mathrm{m}$ & $\mathrm{m}$ & $\mathrm{m}$ & $\mathrm{m}$ & $\mathrm{m}$ & 324 & (5.3) & 377 & (5.7) & 500 & (6.6) & 562 & (11.4) & 330 & (6.4) & 388 & (4.4) & 510 & (5.2) & 564 & (6.5) \\
\hline Ver. Königreich & $\mathrm{m}$ & $\mathrm{m}$ & $\mathrm{m}$ & $\mathrm{m}$ & $\mathrm{m}$ & $\mathrm{m}$ & $\mathrm{m}$ & $\mathrm{m}$ & $\mathrm{m}$ & $\mathrm{m}$ & $\mathrm{m}$ & $\mathrm{m}$ & $\mathrm{m}$ & $\mathrm{m}$ & $\mathrm{m}$ & 359 & (4.0) & 431 & (2.8) & 566 & (2.5) & 621 & (3.1) \\
\hline Ver. Staaten & 363 (11.4) & 436 & (8.8) & 577 & (6.8) & 636 & (6.5) & 361 & (5.2) & 429 & (4.1) & 568 & (3.6) & 622 & (3.5) & $\mathrm{m}$ & $\mathrm{m}$ & $\mathrm{m}$ & $\mathrm{m}$ & $\mathrm{m}$ & $\mathrm{m}$ & $\mathrm{m}$ & $\mathrm{m}$ \\
\hline OECD-23-Durchschnitt & $373 \quad(1.2)$ & 439 & (1.0) & 569 & $(0.7)$ & 618 & (0.8) & 369 & (1.1) & 436 & (0.8) & 565 & (0.6) & 615 & $(0.7)$ & 364 & (1.2) & 432 & (0.9) & 564 & $(0.7)$ & 615 & $(0.7)$ \\
\hline OECD-26-Durchschnitt| & $367 \quad(1.3)$ & 433 & (1.0) & 564 & $(0.8)$ & 614 & $(0.8)$ & $\mathrm{m}$ & $\mathrm{m}$ & $\mathrm{m}$ & $\mathrm{m}$ & $\mathrm{m}$ & $\mathrm{m}$ & $\mathrm{m}$ & $\mathrm{m}$ & $\mathrm{m}$ & $\mathrm{m}$ & $\mathrm{m}$ & $\mathrm{m}$ & $\mathrm{m}$ & & $\mathrm{m}$ & \\
\hline
\end{tabular}

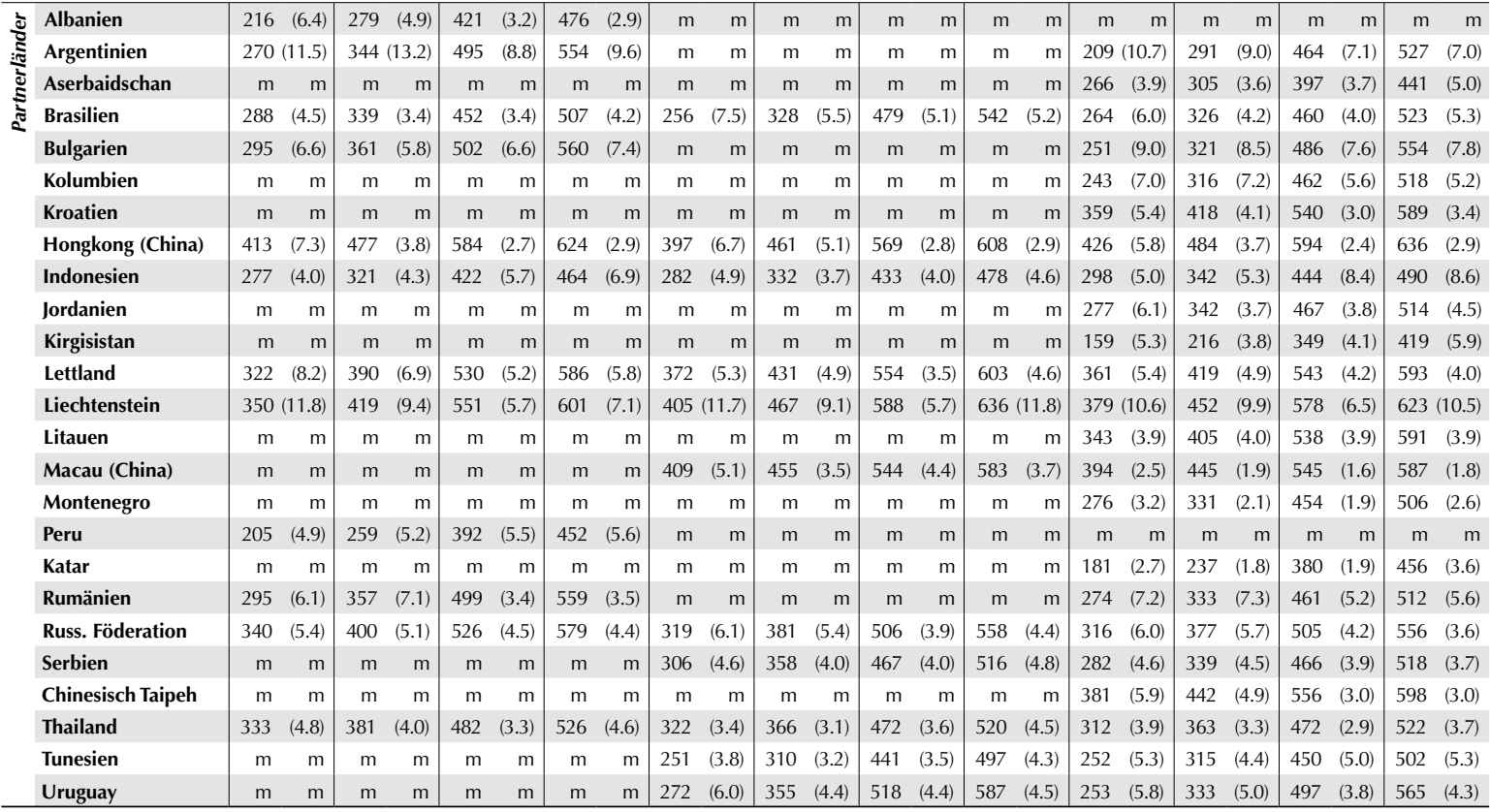

Anmerkung: Statistisch signifikante Werte sind durch Fettdruck gekennzeichnet (siehe Anhang A3)

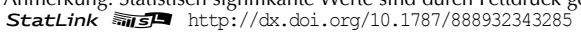


[Teil 2/2]

Tabelle V.2.3 Perzentile auf der Gesamtskala Lesekompetenz in PISA 2000 und PISA 2009

\begin{tabular}{|c|c|c|c|c|c|c|c|c|c|c|c|c|c|c|c|c|c|}
\hline & \multicolumn{8}{|c|}{ PISA 2009} & \multicolumn{8}{|c|}{ Veränderung bei den Perzentilen zwischen 2009 und 2000} \\
\hline & & \multicolumn{2}{|c|}{ 10. Perzentil } & \multicolumn{2}{|c|}{ 25. Perzentil } & \multicolumn{2}{|c|}{ 75. Perzentil } & \multicolumn{2}{|c|}{ 90. Perzentil } & \multicolumn{2}{|c|}{ 10. Perzentil } & \multicolumn{2}{|c|}{ 25. Perzentil } & 75. Pe & rzentil & 90. Per & rzentil \\
\hline & & $\begin{array}{c}\text { Punkt- } \\
\text { zahl }\end{array}$ & S.E. & $\begin{array}{c}\text { Punkt- } \\
\text { zahl }\end{array}$ & S.E. & $\begin{array}{c}\text { Punkt- } \\
\text { zahl }\end{array}$ & S.E. & $\begin{array}{c}\text { Punkt- } \\
\text { zahl }\end{array}$ & S.E. & $\begin{array}{l}\begin{array}{c}\text { Punkt- } \\
\text { diff. }\end{array} \\
\text { int }\end{array}$ & S.E. & $\begin{array}{c}\text { Punkt- } \\
\text { diff. }\end{array}$ & S.E. & $\begin{array}{c}\text { Punkt- } \\
\text { diff. }\end{array}$ & S.E. & $\begin{array}{c}\text { Punkt- } \\
\text { diff. }\end{array}$ & S.E. \\
\hline & Australien & 384 & $(3.1)$ & 450 & (2.9) & 584 & $(2.7)$ & 638 & (3.2) & -10 & (7.3) & -8 & (7.2) & -18 & (7.3) & -18 & $(7.2)$ \\
\hline & Österreich & $\mathrm{m}$ & $\mathrm{m}$ & $\mathrm{m}$ & $\mathrm{m}$ & $\mathrm{m}$ & $\mathrm{m}$ & $\mathrm{m}$ & $\mathrm{m}$ & $\mathrm{m}$ & $\mathrm{m}$ & $\mathrm{m}$ & $\mathrm{m}$ & $\mathrm{m}$ & $\mathrm{m}$ & $\mathrm{m}$ & $\mathrm{m}$ \\
\hline & Belgien & 368 & (4.3) & 436 & (3.8) & 583 & (2.2) & 631 & $(2.7)$ & 14 & (11.0) & -1 & (9.1) & -4 & (5.9) & -3 & (6.2) \\
\hline & Kanada & 406 & $(2.7)$ & 464 & (1.9) & 588 & (1.7) & 637 & (1.9) & -4 & $(6.1)$ & -8 & (5.7) & -12 & (5.4) & -15 & (5.6) \\
\hline & Chile & 342 & (5.0) & 393 & (4.1) & 506 & (3.3) & 556 & (3.6) & 51 & (8.8) & 44 & (7.8) & 35 & (7.1) & 32 & $(7.2)$ \\
\hline & Tschech. Rep. & 357 & (4.9) & 413 & $(4.2)$ & 545 & (3.3) & 598 & (3.2) & -11 & (8.5) & -20 & (7.0) & -13 & (6.6) & -12 & (6.7) \\
\hline & Dänemark & 383 & (3.7) & 440 & (2.9) & 554 & (2.8) & 599 & (3.0) & 16 & (8.0) & 6 & (6.6) & -12 & (6.3) & -18 & (6.4) \\
\hline & Estland & 392 & (4.4) & 446 & (3.3) & 559 & (2.8) & 605 & (3.6) & $\mathrm{m}$ & $\mathrm{m}$ & $\mathrm{m}$ & $\mathrm{m}$ & $\mathrm{m}$ & $\mathrm{m}$ & $\mathrm{m}$ & $\mathrm{m}$ \\
\hline & Finnland & 419 & (3.6) & 481 & (2.7) & 597 & (2.2) & 642 & (2.6) & -10 & (8.0) & -11 & (6.3) & -11 & (6.0) & -11 & (6.2) \\
\hline & Frankreich & 352 & (7.0) & 429 & (4.7) & 572 & $(4.0)$ & 624 & (3.9) & -29 & (10.0) & -15 & (8.2) & 2 & (6.8) & 6 & (6.9) \\
\hline & Deutschland & 367 & (5.1) & 432 & (4.5) & 567 & (2.8) & 615 & (3.2) & 31 & (9.5) & 15 & $(8.1)$ & 5 & (6.4) & -5 & (6.5) \\
\hline & Griechenland & 355 & (8.0) & 420 & (6.3) & 550 & (3.1) & 601 & (3.7) & 13 & (12.6) & 11 & (10.9) & 7 & (7.4) & 7 & (8.0) \\
\hline & Ungarn & 371 & (6.9) & 435 & (4.3) & 559 & (3.6) & 607 & (3.5) & 17 & (10.1) & 20 & $(8.4)$ & 11 & (7.6) & 9 & (7.5) \\
\hline & Island & 371 & $(4.1)$ & 439 & (2.9) & 567 & (2.0) & 619 & (2.6) & -12 & (7.4) & -8 & (6.5) & -5 & (5.7) & -2 & (6.6) \\
\hline & Irland & 373 & (4.7) & 435 & (3.9) & 562 & (2.8) & 611 & (2.8) & -28 & $(9.3)$ & -32 & (7.6) & -31 & (6.7) & -31 & (7.0) \\
\hline & Israel & 322 & (7.8) & 401 & (4.4) & 554 & (3.4) & 611 & $(4.0)$ & 17 & (15.9) & 22 & (12.9) & 22 & (10.1) & 25 & (9.5) \\
\hline & Italien & 358 & (2.6) & 422 & (2.3) & 556 & (1.7) & 604 & $(1.7)$ & -10 & $(8.0)$ & -7 & (6.8) & 4 & $(6.1)$ & 3 & (5.9) \\
\hline & Japan & 386 & (7.1) & 459 & (4.8) & 590 & (3.0) & 639 & (3.6) & -22 & (13.0) & -12 & (9.8) & 9 & (7.2) & 14 & (7.6) \\
\hline & Korea & 435 & (5.9) & 490 & (4.1) & 595 & (3.4) & 635 & (3.0) & 2 & $(8.9)$ & 9 & (7.0) & 22 & (6.6) & 27 & (6.5) \\
\hline & Luxemburg & 332 & (3.6) & 403 & (2.4) & 547 & (1.7) & 600 & (2.0) & $\mathrm{m}$ & $\mathrm{m}$ & $\mathrm{m}$ & $\mathrm{m}$ & $\mathrm{m}$ & $\mathrm{m}$ & $\mathrm{m}$ & $\mathrm{m}$ \\
\hline & Mexiko & 314 & (2.9) & 370 & (2.4) & 485 & (1.9) & 531 & (2.2) & 3 & (6.6) & 9 & (6.6) & 3 & (7.1) & -4 & (7.7) \\
\hline & Niederlande & 390 & (5.0) & 442 & (6.1) & 575 & (5.4) & 625 & (4.6) & $\mathrm{m}$ & $\mathrm{m}$ & $\mathrm{m}$ & $\mathrm{m}$ & $\mathrm{m}$ & $\mathrm{m}$ & $\mathrm{m}$ & $\mathrm{m}$ \\
\hline & Neuseeland & 383 & (4.5) & 452 & (3.1) & 595 & (2.8) & 649 & (2.7) & 1 & (8.5) & -8 & (7.1) & -11 & (6.4) & -12 & $(7.2)$ \\
\hline & Norwegen & 382 & (4.0) & 443 & (3.6) & 568 & (2.9) & 619 & (3.9) & 19 & (8.4) & 3 & (7.6) & -11 & (6.3) & -13 & (7.0) \\
\hline & Polen & 382 & $(4.2)$ & 441 & (3.4) & 565 & (3.2) & 613 & (3.3) & 39 & (9.4) & 27 & (8.3) & 14 & (8.4) & 9 & (8.8) \\
\hline & Portugal & 373 & (4.9) & 432 & (4.4) & 551 & (3.4) & 599 & (3.5) & 35 & (9.3) & 29 & $(9.2)$ & 10 & (7.4) & 7 & (7.3) \\
\hline & Slowak. Rep. & 358 & (5.2) & 416 & (4.1) & 543 & (2.7) & 594 & (3.2) & $\mathrm{m}$ & $\mathrm{m}$ & $\mathrm{m}$ & $\mathrm{m}$ & $\mathrm{m}$ & $\mathrm{m}$ & $\mathrm{m}$ & $\mathrm{m}$ \\
\hline & Slowenien & 359 & $(2.1)$ & 421 & (1.9) & 550 & (1.7) & 598 & (2.9) & $\mathrm{m}$ & $\mathrm{m}$ & $\mathrm{m}$ & $\mathrm{m}$ & $\mathrm{m}$ & $\mathrm{m}$ & $\mathrm{m}$ & $\mathrm{m}$ \\
\hline & Spanien & 364 & (3.5) & 426 & (3.3) & 543 & (2.0) & 588 & (2.0) & -15 & (7.8) & -11 & (7.5) & -11 & (5.9) & -9 & (5.9) \\
\hline & Schweden & 368 & (5.5) & 437 & (3.3) & 565 & (3.2) & 620 & (3.7) & -23 & (8.4) & -20 & (6.7) & -16 & (6.6) & -10 & (6.8) \\
\hline & Schweiz & 374 & (4.0) & 437 & (3.6) & 569 & (3.0) & 617 & (3.3) & 19 & (8.6) & 11 & (8.2) & 2 & (7.4) & -4 & (8.1) \\
\hline & Türkei & 356 & (4.3) & 409 & (3.8) & 522 & (4.5) & 569 & (5.2) & $\mathrm{m}$ & $\mathrm{m}$ & $\mathrm{m}$ & $\mathrm{m}$ & $\mathrm{m}$ & $\mathrm{m}$ & $\mathrm{m}$ & $\mathrm{m}$ \\
\hline & Ver. Königreich & 370 & (3.1) & 430 & (2.8) & 561 & (3.2) & 616 & (2.6) & $\mathrm{m}$ & $\mathrm{m}$ & $\mathrm{m}$ & $\mathrm{m}$ & $\mathrm{m}$ & $\mathrm{m}$ & $\mathrm{m}$ & $\mathrm{m}$ \\
\hline & Ver. Staaten & 372 & (3.9) & 433 & (4.0) & 569 & (4.6) & 625 & (5.0) & 9 & (13.1) & -3 & (10.8) & -8 & (9.6) & -11 & (9.6) \\
\hline & OECD-23-Durchschnitt & 375 & (1.0) & 438 & $(0.8)$ & 566 & (0.6) & 615 & $(0.6)$ & 1 & $(5.2)$ & -1 & $(5.1)$ & -3 & (5.0) & -4 & (5.0) \\
\hline & OECD-26-Durchschnitt & 372 & (1.0) & 435 & $(0.7)$ & 563 & (0.6) & 613 & (0.6) & 4 & (5.2) & 2 & (5.1) & -1 & (5.0) & -1 & (5.0) \\
\hline ま & Albanien & 254 & (5.4) & 319 & $(4.9)$ & 458 & $(4.8)$ & 509 & $(4.9)$ & 38 & $(9.7)$ & 39 & $(8.5)$ & 36 & (7.6) & 33 & (7.5) \\
\hline & Argentinien & 257 & (8.3) & 329 & (5.8) & 473 & (6.3) & 535 & (7.1) & -14 & (15.0) & -15 & (15.2) & -22 & (11.9) & -19 & (12.9) \\
\hline & Aserbaidschan & 263 & (4.8) & 311 & (4.3) & 413 & (4.0) & 458 & (4.4) & $\mathrm{m}$ & $\mathrm{m}$ & $\mathrm{m}$ & $\mathrm{m}$ & $\mathrm{m}$ & $\mathrm{m}$ & $\mathrm{m}$ & $\mathrm{m}$ \\
\hline \pm & Brasilien & 293 & (3.2) & 348 & (2.7) & 474 & (3.9) & 537 & $(4.2)$ & 5 & $(7.4)$ & 9 & (6.6) & 22 & $(7.2)$ & 30 & (7.7) \\
\hline & Bulgarien & 276 & (7.8) & 351 & (8.6) & 512 & (6.5) & 572 & (7.3) & -19 & (11.4) & -9 & (11.4) & 10 & (10.4) & 12 & (11.5) \\
\hline & Kolumbien & 302 & (5.2) & 355 & (4.4) & 473 & (3.9) & 524 & $(4.1)$ & $\mathrm{m}$ & $\mathrm{m}$ & $\mathrm{m}$ & $\mathrm{m}$ & $\mathrm{m}$ & $\mathrm{m}$ & $\mathrm{m}$ & $\mathrm{m}$ \\
\hline & Kroatien & 359 & (3.6) & 416 & (4.5) & 539 & (3.1) & 586 & (3.5) & $\mathrm{m}$ & $\mathrm{m}$ & $\mathrm{m}$ & $\mathrm{m}$ & $\mathrm{m}$ & $\mathrm{m}$ & $\mathrm{m}$ & $\mathrm{m}$ \\
\hline & Hongkong (China) & 418 & (4.5) & 482 & (3.0) & 592 & (2.5) & 634 & (2.9) & 5 & (9.9) & 6 & (6.9) & 8 & (6.2) & 11 & (6.4) \\
\hline & Indonesien & 315 & (5.0) & 357 & (4.1) & 447 & (4.6) & 487 & (5.0) & 38 & (8.1) & 36 & (7.7) & 25 & (8.8) & 24 & (9.8) \\
\hline & Jordanien & 284 & (5.0) & 350 & $(4.1)$ & 468 & (3.5) & 515 & (3.9) & $\mathrm{m}$ & $\mathrm{m}$ & $\mathrm{m}$ & $\mathrm{m}$ & $\mathrm{m}$ & $\mathrm{m}$ & $\mathrm{m}$ & $\mathrm{m}$ \\
\hline & Kirgisistan & 190 & $(4.7)$ & 249 & $(4.1)$ & 377 & $(4.2)$ & 441 & (6.4) & $\mathrm{m}$ & $\mathrm{m}$ & $\mathrm{m}$ & $\mathrm{m}$ & $\mathrm{m}$ & $\mathrm{m}$ & $\mathrm{m}$ & $\mathrm{m}$ \\
\hline & Lettland & 379 & $(4.2)$ & 429 & (3.8) & 541 & (3.3) & 584 & (3.2) & 57 & (10.4) & 39 & (9.3) & 11 & (7.9) & -2 & (8.3) \\
\hline & Liechtenstein & 385 & (10.6) & 442 & (6.5) & 56 & $(4.7)$ & 599 & (7.9) & 36 & (16.6) & 24 & (12.4) & 9 & (8.9) & -2 & (11.7) \\
\hline & Litauen & 353 & $(4.2)$ & 40 & (3.3) & 53 & (3.1) & 580 & (3.4) & $\mathrm{m}$ & $\mathrm{m}$ & $\mathrm{m}$ & $\mathrm{m}$ & $\mathrm{m}$ & $\mathrm{m}$ & $\mathrm{m}$ & $\mathrm{m}$ \\
\hline & Macau (China) & 388 & (1.9) & 437 & (1.4) & 540 & (1.4) & 582 & (1.8) & $\mathrm{m}$ & $\mathrm{m}$ & $\mathrm{m}$ & $\mathrm{m}$ & $\mathrm{m}$ & $\mathrm{m}$ & $\mathrm{m}$ & $\mathrm{m}$ \\
\hline & Montenegro & 288 & (3.8) & 345 & (2.6) & 473 & (2.4) & 526 & (2.7) & $\mathrm{m}$ & $\mathrm{m}$ & $\mathrm{m}$ & $\mathrm{m}$ & $\mathrm{m}$ & $\mathrm{m}$ & $\mathrm{m}$ & $\mathrm{m}$ \\
\hline & Peru & 241 & (3.9) & 302 & (4.3) & 437 & (5.2) & 496 & (6.4) & 36 & (8.0) & 43 & (8.4) & 45 & (9.0) & 43 & (9.8) \\
\hline & Katar & 228 & (2.2) & 288 & (1.4) & 450 & (1.4) & 529 & $(2.1)$ & $\mathrm{m}$ & $\mathrm{m}$ & $\mathrm{m}$ & $\mathrm{m}$ & $\mathrm{m}$ & $\mathrm{m}$ & $\mathrm{m}$ & $\mathrm{m}$ \\
\hline & Rumänien & 304 & $(5.7)$ & 365 & (6.0) & 488 & $(4.7)$ & 537 & $(4.0)$ & 9 & (9.7) & 8 & (10.6) & -11 & (7.6) & -22 & (7.3) \\
\hline & Russ. Föderation & 344 & (5.5) & 401 & (3.6) & 519 & (3.2) & 572 & $(4.5)$ & 4 & (9.2) & 1 & (7.9) & -7 & (7.4) & -7 & (8.0) \\
\hline & Serbien & 331 & (3.8) & 388 & (3.2) & 501 & (2.5) & 547 & $(2.7)$ & $\mathrm{m}$ & $\mathrm{m}$ & $\mathrm{m}$ & $\mathrm{m}$ & $\mathrm{m}$ & $\mathrm{m}$ & $\mathrm{m}$ & $\mathrm{m}$ \\
\hline & Chinesisch Taipeh & 380 & (3.9) & 439 & (3.2) & 555 & (2.9) & 600 & (4.6) & $\mathrm{m}$ & $\mathrm{m}$ & $\mathrm{m}$ & $\mathrm{m}$ & $\mathrm{m}$ & $\mathrm{m}$ & $\mathrm{m}$ & $\mathrm{m}$ \\
\hline & Thailand & 331 & (3.8) & 373 & (3.2) & 469 & (2.6) & 514 & $(4.0)$ & -2 & (7.9) & -8 & (7.1) & -13 & (6.5) & -13 & (7.9) \\
\hline & Tunesien & 293 & (3.8) & 348 & (3.4) & 462 & (3.4) & 510 & (4.8) & $\mathrm{m}$ & $\mathrm{m}$ & $\mathrm{m}$ & $\mathrm{m}$ & $\mathrm{m}$ & $\mathrm{m}$ & $\mathrm{m}$ & $\mathrm{m}$ \\
\hline & Uruguay & 297 & $(4.2)$ & 359 & (3.5) & 495 & (3.1) & 552 & (3.3) & $\mathrm{m}$ & $\mathrm{m}$ & $\mathrm{m}$ & $\mathrm{m}$ & $\mathrm{m}$ & $\mathrm{m}$ & $\mathrm{m}$ & $\mathrm{m}$ \\
\hline
\end{tabular}

Anmerkung: Statistisch signifikante Werte sind durch Fettdruck gekennzeichnet (siehe Anhang A3).

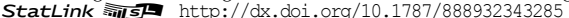


[Teil 1/1]

Leistungsabstand zwischen Jungen und Mädchen im Bereich Lesekompetenz in PISA 2000

Tabelle V.2.4 und PISA 2009

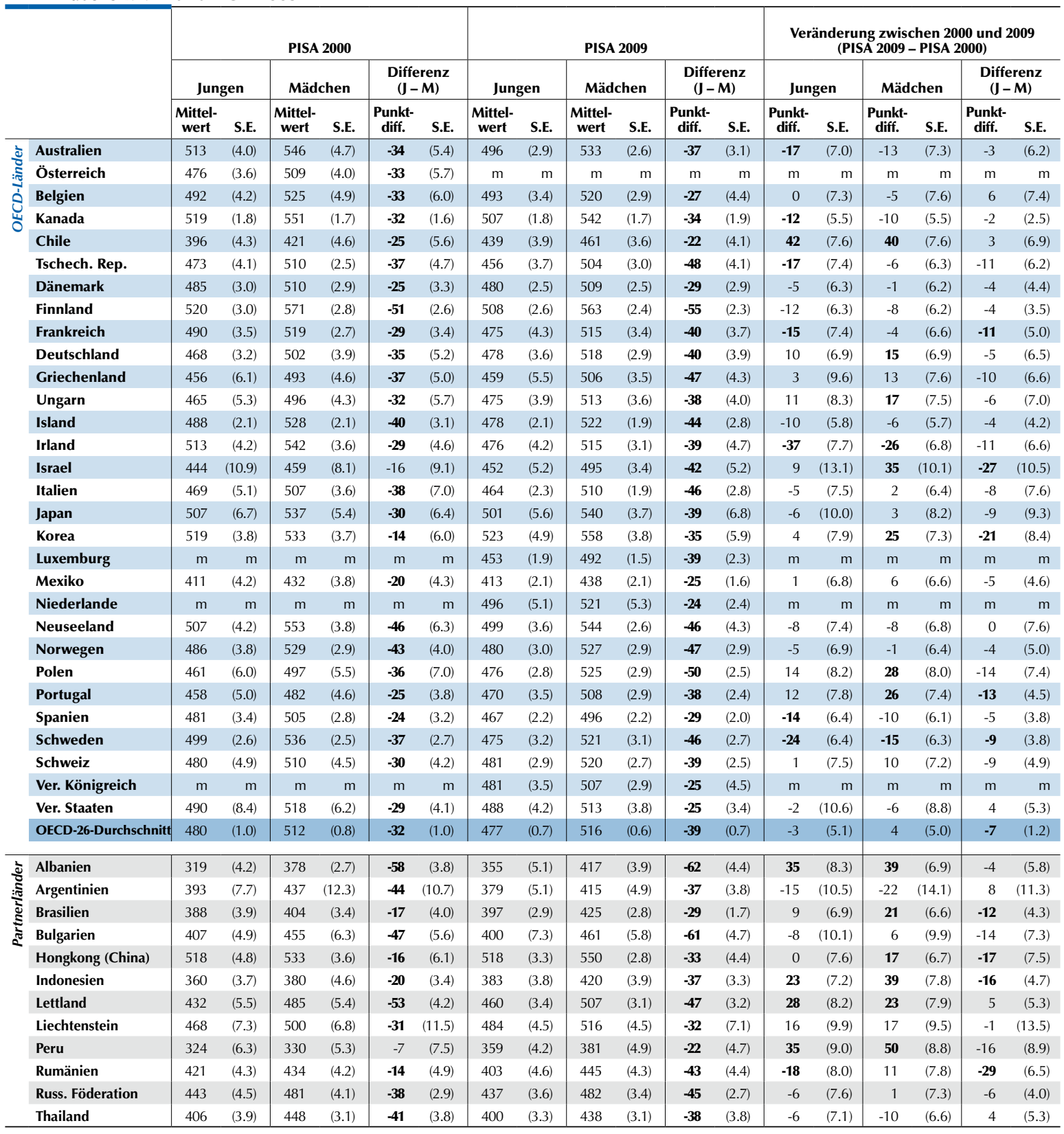

Anmerkung: Statistisch signifikante Werte sind durch Fettdruck gekennzeichnet (siehe Anhang A3).

StatLink त्राज़ http://dx.doi.org/10.1787/888932343285 
[Teil 1/1]

Prozentsatz der Jungen unter Stufe 2 und auf Stufe 5 oder höher auf der Gesamtskala Tabelle V.2.5 Lesekompetenz in PISA 2000 und PISA 2009

\begin{tabular}{|c|c|c|c|c|c|c|c|c|c|c|c|c|c|}
\hline & \multicolumn{4}{|c|}{ Jungen - Kompetenzstufen in PISA 2000} & \multicolumn{4}{|c|}{ Jungen - Kompetenzstufen in PISA 2009} & \multicolumn{4}{|c|}{$\begin{array}{l}\text { Veränderung zwischen } 2000 \text { und } 2009 \\
\text { (PISA 2009 - PISA 2000) }\end{array}$} \\
\hline & & \multicolumn{2}{|c|}{$\begin{array}{c}\text { Unter Stufe } 2 \\
\text { (unter 407 Punkte) }\end{array}$} & \multicolumn{2}{|c|}{$\begin{array}{l}\text { Stufe } 5 \text { oder höher } \\
\text { (über } 626 \text { Punkte) }\end{array}$} & \multicolumn{2}{|c|}{$\begin{array}{c}\text { Unter Stufe } 2 \\
\text { (unter 407 Punkte) }\end{array}$} & \multicolumn{2}{|c|}{$\begin{array}{l}\text { Stufe } 5 \text { oder höher } \\
\text { (über } 626 \text { Punkte) }\end{array}$} & \multicolumn{2}{|c|}{$\begin{array}{c}\text { Unter Stufe } 2 \\
\text { (unter 407 Punkte) }\end{array}$} & \multicolumn{2}{|c|}{$\begin{array}{l}\text { Stufe } 5 \text { oder höher } \\
\text { (über } 626 \text { Punkte) }\end{array}$} \\
\hline & & $\%$ & S.E. & $\%$ & S.E. & $\%$ & S.E. & $\%$ & S.E. & Diff. \% & S.E. & Diff. \% & S.E. \\
\hline \multirow{31}{*}{ 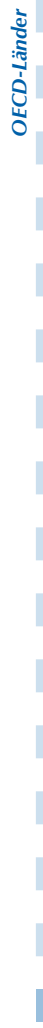 } & Australien & 16.0 & (1.3) & 14.2 & (1.1) & 19.7 & $(0.8)$ & 9.8 & $(0.8)$ & 3.7 & (1.6) & -4.4 & (1.3) \\
\hline & Österreich & 23.8 & (1.5) & 5.1 & $(0.7)$ & $\mathrm{m}$ & $\mathrm{m}$ & $\mathrm{m}$ & $\mathrm{m}$ & $\mathrm{m}$ & $\mathrm{m}$ & $\mathrm{m}$ & $\mathrm{m}$ \\
\hline & Belgien & 22.8 & (1.4) & 9.9 & $(0.9)$ & 21.5 & $(1.3)$ & 9.4 & $(0.8)$ & -1.3 & (1.9) & -0.5 & $(1.2)$ \\
\hline & Kanada & 12.7 & $(0.6)$ & 12.8 & $(0.6)$ & 14.5 & $(0.7)$ & 9.4 & $(0.5)$ & 1.8 & $(0.9)$ & -3.5 & $(0.8)$ \\
\hline & Chile & 53.6 & (2.2) & 0.4 & $(0.2)$ & 36.1 & (2.0) & 1.0 & $(0.4)$ & -17.5 & (3.0) & 0.7 & $(0.4)$ \\
\hline & Tschech. Rep. & 23.6 & (1.6) & 5.3 & $(0.7)$ & 30.8 & (1.9) & 2.8 & $(0.4)$ & 7.2 & (2.5) & -2.6 & $(0.8)$ \\
\hline & Dänemark & 21.8 & (1.3) & 6.8 & $(0.7)$ & 19.0 & (1.3) & 3.2 & $(0.5)$ & -2.7 & (1.8) & -3.6 & $(0.9)$ \\
\hline & Finnland & 11.0 & (0.9) & 11.0 & $(0.9)$ & 13.0 & $(0.9)$ & 8.1 & $(0.8)$ & 2.0 & (1.3) & -2.9 & $(1.2)$ \\
\hline & Frankreich & 19.9 & (1.5) & 6.4 & $(0.7)$ & 25.7 & (1.7) & 6.9 & $(0.8)$ & 5.7 & (2.3) & 0.5 & (1.1) \\
\hline & Deutschland & 26.6 & $(1.2)$ & 6.7 & $(0.8)$ & 24.0 & (1.5) & 4.4 & $(0.5)$ & -2.6 & (1.9) & -2.3 & $(0.9)$ \\
\hline & Griechenland & 30.9 & $(2.7)$ & 3.6 & $(0.7)$ & 29.7 & (2.4) & 3.4 & $(0.6)$ & -1.1 & (3.6) & -0.2 & (1.0) \\
\hline & Ungarn & 27.2 & $(2.2)$ & 3.5 & $(0.8)$ & 23.6 & $(1.8)$ & 3.9 & $(0.7)$ & -3.6 & (2.9) & 0.3 & (1.0) \\
\hline & Island & 20.1 & (1.1) & 6.4 & (0.9) & 23.8 & (1.0) & 5.6 & $(0.6)$ & 3.8 & (1.5) & -0.8 & (1.1) \\
\hline & Irland & 13.5 & (1.3) & 11.2 & $(1.1)$ & 23.1 & $(1.7)$ & 4.5 & $(0.6)$ & 9.6 & $(2.1)$ & -6.7 & (1.3) \\
\hline & Israel & 36.5 & $(4.0)$ & 4.2 & (1.1) & 34.1 & (1.6) & 6.3 & $(0.9)$ & -2.4 & (4.4) & 2.2 & (1.4) \\
\hline & Italien & 24.6 & $(2.1)$ & 3.7 & (0.6) & 28.9 & $(0.9)$ & 3.9 & $(0.3)$ & 4.3 & (2.3) & 0.1 & $(0.7)$ \\
\hline & Japan & 14.2 & (2.3) & 7.5 & (1.3) & 18.9 & (1.8) & 10.1 & (1.1) & 4.6 & (3.0) & 2.6 & (1.6) \\
\hline & Korea & 7.3 & (1.1) & 4.4 & $(0.6)$ & 8.8 & (1.4) & 9.3 & $(1.2)$ & 1.5 & (1.7) & 4.9 & (1.4) \\
\hline & Luxemburg & $\mathrm{m}$ & $\mathrm{m}$ & $\mathrm{m}$ & $\mathrm{m}$ & 32.8 & $(1.1)$ & 3.7 & $(0.5)$ & $\mathrm{m}$ & $\mathrm{m}$ & $\mathrm{m}$ & $\mathrm{m}$ \\
\hline & Mexiko & 49.8 & (2.0) & 0.8 & $(0.3)$ & 46.2 & $(1.1)$ & 0.3 & $(0.1)$ & -3.6 & $(2.3)$ & -0.5 & $(0.3)$ \\
\hline & Niederlande & $\mathrm{m}$ & $\mathrm{m}$ & $\mathrm{m}$ & $\mathrm{m}$ & 17.9 & (1.9) & 7.8 & (1.0) & $\mathrm{m}$ & $\mathrm{m}$ & $\mathrm{m}$ & $\mathrm{m}$ \\
\hline & Neuseeland & 18.5 & (1.4) & 13.7 & $(1.2)$ & 20.6 & $(1.2)$ & 11.9 & $(1.1)$ & 2.2 & $(1.8)$ & -1.8 & (1.6) \\
\hline & Norwegen & 23.2 & (1.6) & 8.1 & $(0.8)$ & 21.4 & $(1.2)$ & 5.0 & $(0.8)$ & -1.8 & (2.0) & -3.1 & $(1.2)$ \\
\hline & Polen & 30.3 & (2.5) & 4.1 & $(0.8)$ & 22.6 & $(1.2)$ & 4.3 & $(0.6)$ & -7.7 & (2.8) & 0.2 & (1.0) \\
\hline & Portugal & 31.3 & $(2.2)$ & 3.8 & $(0.6)$ & 24.7 & (1.6) & 3.3 & $(0.5)$ & -6.6 & $(2.7)$ & -0.5 & $(0.7)$ \\
\hline & Spanien & 20.4 & (1.4) & 3.6 & $(0.7)$ & 24.4 & $(1.0)$ & 2.4 & $(0.3)$ & 4.0 & (1.7) & -1.2 & $(0.8)$ \\
\hline & Schweden & 16.8 & $(1.0)$ & 7.4 & $(0.8)$ & 24.2 & (1.3) & 6.0 & $(0.6)$ & 7.3 & (1.7) & -1.4 & (1.1) \\
\hline & Schweiz & 24.6 & (1.8) & 7.3 & $(0.9)$ & 22.0 & $(1.2)$ & 5.1 & $(0.6)$ & -2.6 & $(2.1)$ & -2.1 & (1.1) \\
\hline & Ver. Königreich & $\mathrm{m}$ & $\mathrm{m}$ & $\mathrm{m}$ & $\mathrm{m}$ & 23.1 & $(1.2)$ & 6.9 & $(0.7)$ & $\mathrm{m}$ & $\mathrm{m}$ & $\mathrm{m}$ & $\mathrm{m}$ \\
\hline & Ver. Staaten & 23.0 & (3.0) & 11.0 & (1.6) & 21.4 & (1.4) & 8.2 & $(1.0)$ & -1.6 & (3.3) & -2.8 & (1.9) \\
\hline & OECD-26-Durchschnitt & 23.8 & $(0.4)$ & 6.8 & $(0.2)$ & 24.0 & $(0.3)$ & 5.7 & $(0.1)$ & 0.1 & $(0.5)$ & -1.1 & $(0.2)$ \\
\hline \multirow{12}{*}{ 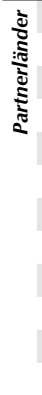 } & Albanien & 80.6 & $(1.2)$ & 0.1 & $(0.1)$ & 69.0 & (2.4) & 0.0 & c & -11.6 & $(2.7)$ & c & $\mathrm{c}$ \\
\hline & Argentinien & 53.3 & (3.4) & 0.9 & $(0.4)$ & 58.8 & $(2.1)$ & 0.7 & $(0.2)$ & 5.6 & $(4.0)$ & -0.2 & $(0.4)$ \\
\hline & Brasilien & 59.5 & (1.9) & 0.4 & $(0.2)$ & 56.5 & (1.4) & 1.0 & $(0.2)$ & -3.0 & (2.4) & 0.5 & $(0.3)$ \\
\hline & Bulgarien & 50.3 & $(2.4)$ & 1.2 & $(0.4)$ & 52.0 & $(3.0)$ & 1.5 & $(0.5)$ & 1.7 & (3.8) & 0.2 & $(0.6)$ \\
\hline & Hongkong (China) & 11.9 & (1.5) & 9.0 & $(1.1)$ & 11.3 & $(1.2)$ & 8.9 & (1.0) & -0.6 & (1.9) & -0.1 & (1.5) \\
\hline & Indonesien & 74.5 & $(2.4)$ & 0.0 & $\mathrm{C}$ & 65.5 & (2.3) & 0.0 & c & -9.0 & (3.3) & c & $\mathrm{c}$ \\
\hline & Lettland & 40.3 & (2.5) & 2.5 & $(0.5)$ & 26.6 & (1.8) & 1.6 & $(0.4)$ & -13.7 & (3.1) & -0.9 & $(0.7)$ \\
\hline & Liechtenstein & 27.1 & (3.9) & 3.9 & (1.9) & 21.2 & (3.3) & 3.1 & (1.5) & -5.9 & (5.1) & -0.8 & (2.4) \\
\hline & Peru & 80.7 & (2.3) & 0.1 & $(0.2)$ & 69.7 & $(1.8)$ & 0.6 & $(0.3)$ & -11.0 & (2.9) & 0.4 & $(0.3)$ \\
\hline & Rumänien & 44.2 & $(2.2)$ & 2.0 & $(0.4)$ & 50.7 & $(2.5)$ & 0.3 & $(0.2)$ & 6.5 & (3.3) & -1.7 & $(0.4)$ \\
\hline & Russ. Föderation & 35.1 & $(1.9)$ & 2.3 & $(0.5)$ & 36.3 & (1.8) & 1.7 & $(0.4)$ & 1.2 & $(2.7)$ & -0.6 & $(0.6)$ \\
\hline & Thailand & 51.1 & $(2.1)$ & 0.3 & $(0.2)$ & 55.5 & $(1.9)$ & 0.1 & $(0.1)$ & 4.4 & $(2.9)$ & -0.2 & $(0.2)$ \\
\hline
\end{tabular}

Anmerkung: Statistisch signifikante Werte sind durch Fettdruck gekennzeichnet (siehe Anhang A3).

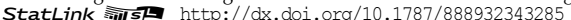


[Teil 1/1]

Prozentsatz der Mädchen unter Stufe 2 und auf Stufe 5 oder höher auf der Gesamtskala Tabelle V.2.6 Lesekompetenz in PISA 2000 und PISA 2009

\begin{tabular}{|c|c|c|c|c|c|c|c|c|c|c|c|c|c|}
\hline & \multicolumn{4}{|c|}{ Mädchen - Kompetenzstufen in PISA 2000} & \multicolumn{4}{|c|}{ Mädchen - Kompetenzstufen in PISA 2009} & \multicolumn{4}{|c|}{$\begin{array}{l}\text { Veränderung zwischen } 2000 \text { und } 2009 \\
\text { (PISA 2009 - PISA 2000) }\end{array}$} \\
\hline & & \multicolumn{2}{|c|}{$\begin{array}{c}\text { Unter Stufe } 2 \\
\text { (unter 407 Punkte) }\end{array}$} & \multicolumn{2}{|c|}{$\begin{array}{l}\text { Stufe } 5 \text { oder höher } \\
\text { (über } 626 \text { Punkte) }\end{array}$} & \multicolumn{2}{|c|}{$\begin{array}{c}\text { Unter Stufe } 2 \\
\text { (unter 407 Punkte) }\end{array}$} & \multicolumn{2}{|c|}{$\begin{array}{l}\text { Stufe } 5 \text { oder höher } \\
\text { (über } 626 \text { Punkte) }\end{array}$} & \multicolumn{2}{|c|}{$\begin{array}{c}\text { Unter Stufe } 2 \\
\text { (unter 407 Punkte) }\end{array}$} & \multicolumn{2}{|c|}{$\begin{array}{l}\text { Stufe } 5 \text { oder höher } \\
\text { (über } 626 \text { Punkte) }\end{array}$} \\
\hline & & $\%$ & S.E. & $\%$ & S.E. & $\%$ & S.E. & $\%$ & S.E. & Diff. \% & S.E. & Diff. \% & S.E. \\
\hline \multirow{31}{*}{ 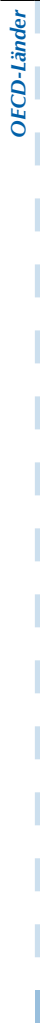 } & Australien & 8.4 & $(0.9)$ & 21.6 & $(2.0)$ & 9.1 & (0.6) & 15.6 & $(0.9)$ & 0.7 & $(1.1)$ & -6.0 & $(2.2)$ \\
\hline & Österreich & 14.6 & $(1.0)$ & 10.0 & $(1.1)$ & $\mathrm{m}$ & $\mathrm{m}$ & $\mathrm{m}$ & $\mathrm{m}$ & $\mathrm{m}$ & $\mathrm{m}$ & $\mathrm{m}$ & $\mathrm{m}$ \\
\hline & Belgien & 14.1 & $(1.7)$ & 14.5 & (1.0) & 13.8 & $(1.0)$ & 13.0 & $(0.8)$ & -0.3 & (2.0) & -1.5 & (1.3) \\
\hline & Kanada & 6.0 & $(0.4)$ & 21.0 & $(0.7)$ & 6.0 & $(0.4)$ & 16.2 & $(0.7)$ & 0.0 & (0.6) & -4.8 & (1.0) \\
\hline & Chile & 43.4 & (2.3) & 0.6 & $(0.2)$ & 24.8 & (1.5) & 1.6 & $(0.4)$ & -18.6 & $(2.7)$ & 0.9 & $(0.4)$ \\
\hline & Tschech. Rep. & 11.5 & $(0.8)$ & 8.6 & $(0.7)$ & 14.3 & $(1.2)$ & 7.8 & $(0.8)$ & 2.8 & (1.4) & -0.8 & (1.1) \\
\hline & Dänemark & 13.3 & (1.0) & 9.6 & $(0.9)$ & 11.5 & $(0.9)$ & 6.2 & $(0.6)$ & -1.9 & (1.4) & -3.4 & (1.1) \\
\hline & Finnland & 3.2 & $(0.7)$ & 25.5 & (1.4) & 3.2 & $(0.5)$ & 20.9 & (1.1) & 0.0 & $(0.8)$ & -4.6 & (1.7) \\
\hline & Frankreich & 10.5 & (1.1) & 10.5 & $(0.8)$ & 14.1 & (1.0) & 12.1 & (1.3) & 3.6 & (1.5) & 1.6 & (1.5) \\
\hline & Deutschland & 18.2 & (1.4) & 11.1 & $(0.8)$ & 12.7 & $(1.1)$ & 11.0 & (1.0) & -5.5 & (1.8) & -0.1 & (1.3) \\
\hline & Griechenland & 17.7 & (2.0) & 6.4 & $(0.9)$ & 13.2 & (1.4) & 7.7 & $(0.9)$ & -4.5 & (2.5) & 1.3 & $(1.2)$ \\
\hline & Ungarn & 17.9 & (1.7) & 6.7 & (1.0) & 11.4 & (1.5) & 8.3 & $(1.0)$ & -6.6 & (2.3) & 1.6 & (1.4) \\
\hline & Island & 8.0 & $(0.8)$ & 11.9 & $(0.9)$ & 9.9 & $(0.8)$ & 11.4 & $(0.9)$ & 1.9 & (1.1) & -0.5 & (1.3) \\
\hline & Irland & 8.3 & (1.1) & 17.4 & $(1.2)$ & 11.2 & $(1.0)$ & 9.5 & $(0.9)$ & 3.0 & (1.5) & -7.9 & (1.5) \\
\hline & Israel & 30.6 & (3.1) & 4.2 & (1.0) & 19.3 & (1.3) & 8.5 & $(0.8)$ & -11.3 & (3.4) & 4.3 & (1.3) \\
\hline & Italien & 12.6 & (1.4) & 7.0 & $(0.7)$ & 12.7 & $(0.7)$ & 7.9 & $(0.5)$ & 0.1 & (1.5) & 0.8 & $(0.8)$ \\
\hline & Japan & 6.0 & (1.2) & 12.1 & (1.4) & 8.0 & (1.0) & 16.9 & (1.4) & 2.0 & (1.5) & 4.8 & (2.0) \\
\hline & Korea & 3.7 & $(0.7)$ & 7.4 & (1.0) & 2.4 & $(0.5)$ & 16.9 & (1.6) & -1.3 & (0.9) & 9.5 & (1.9) \\
\hline & Luxemburg & $\mathrm{m}$ & $\mathrm{m}$ & $\mathrm{m}$ & $\mathrm{m}$ & 19.1 & $(0.9)$ & 7.7 & $(0.7)$ & $\mathrm{m}$ & $\mathrm{m}$ & $\mathrm{m}$ & $\mathrm{m}$ \\
\hline & Mexiko & 38.9 & (2.1) & 0.9 & $(0.3)$ & 34.1 & (1.1) & 0.5 & $(0.1)$ & -4.8 & (2.3) & -0.4 & $(0.3)$ \\
\hline & Niederlande & $\mathrm{m}$ & $\mathrm{m}$ & $\mathrm{m}$ & $\mathrm{m}$ & 10.8 & (1.4) & 11.8 & (1.3) & $\mathrm{m}$ & $\mathrm{m}$ & $\mathrm{m}$ & $\mathrm{m}$ \\
\hline & Neuseeland & 8.3 & $(0.7)$ & 24.0 & (1.5) & 7.8 & $(0.7)$ & 19.7 & (1.1) & -0.5 & (1.0) & -4.3 & (1.9) \\
\hline & Norwegen & 10.4 & (1.0) & 14.7 & (1.0) & 8.3 & $(0.8)$ & 12.0 & (1.3) & -2.1 & (1.3) & -2.8 & (1.6) \\
\hline & Polen & 15.9 & (1.7) & 7.7 & (1.3) & 7.4 & $(0.8)$ & 10.1 & $(0.9)$ & -8.4 & (1.9) & 2.4 & (1.6) \\
\hline & Portugal & 21.2 & (1.9) & 4.6 & $(0.7)$ & 10.8 & (1.1) & 6.2 & $(0.8)$ & -10.4 & $(2.2)$ & 1.6 & (1.1) \\
\hline & Spanien & 11.5 & (1.1) & 4.9 & $(0.5)$ & 14.6 & $(0.9)$ & 4.3 & $(0.3)$ & 3.1 & (1.5) & -0.6 & $(0.6)$ \\
\hline & Schweden & 7.8 & (0.8) & 15.1 & (1.1) & 10.5 & (1.0) & 12.2 & (1.0) & 2.7 & $(1.2)$ & -2.9 & (1.5) \\
\hline & Schweiz & 15.7 & $(1.3)$ & 11.3 & (1.4) & 11.4 & $(0.8)$ & 11.2 & (1.1) & -4.3 & $(1.5)$ & -0.1 & (1.8) \\
\hline & Ver. Königreich & $\mathrm{m}$ & $\mathrm{m}$ & $\mathrm{m}$ & $\mathrm{m}$ & 14.0 & $(0.9)$ & 9.1 & $(0.8)$ & $\mathrm{m}$ & $\mathrm{m}$ & $\mathrm{m}$ & $\mathrm{m}$ \\
\hline & Ver. Staaten & 13.1 & (1.7) & 13.4 & (1.6) & 13.6 & (1.1) & 11.6 & $(1.2)$ & 0.5 & (2.0) & -1.8 & (2.0) \\
\hline & OECD-26-Durchschnitt & 14.5 & $(0.3)$ & 11.3 & $(0.2)$ & 12.2 & $(0.2)$ & 10.7 & $(0.2)$ & -2.3 & $(0.3)$ & -0.5 & $(0.3)$ \\
\hline \multirow{12}{*}{ 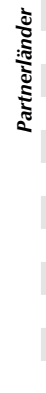 } & Albanien & 60.4 & (1.4) & 0.1 & $(0.1)$ & 43.6 & $(2.2)$ & 0.3 & $(0.2)$ & -16.8 & (2.6) & 0.3 & $(0.2)$ \\
\hline & Argentinien & 36.7 & (5.6) & 2.3 & $(0.8)$ & 45.3 & $(2.1)$ & 1.2 & $(0.4)$ & 8.6 & $(6.0)$ & -1.1 & $(0.9)$ \\
\hline & Brasilien & 52.1 & (2.0) & 0.7 & $(0.3)$ & 43.4 & (1.3) & 1.6 & $(0.3)$ & -8.7 & (2.4) & 0.9 & $(0.4)$ \\
\hline & Bulgarien & 29.8 & (2.3) & 3.3 & (1.0) & 29.2 & $(2.2)$ & 4.2 & $(0.7)$ & -0.6 & $(3.2)$ & 0.9 & $(1.2)$ \\
\hline & Hongkong (China) & 6.3 & (1.0) & 10.1 & $(1.2)$ & 4.9 & $(0.7)$ & 16.4 & (1.0) & -1.4 & $(1.2)$ & 6.3 & (1.6) \\
\hline & Indonesien & 63.1 & (2.9) & 0.0 & C & 41.6 & (2.6) & 0.0 & $\mathrm{C}$ & -21.5 & (3.9) & c & $\mathrm{c}$ \\
\hline & Lettland & 19.7 & (1.8) & 5.8 & (1.0) & 8.8 & $(1.2)$ & 4.3 & $(0.6)$ & -10.9 & $(2.1)$ & -1.6 & $(1.2)$ \\
\hline & Liechtenstein & 15.8 & (3.2) & 6.4 & (2.6) & 9.4 & (2.0) & 6.4 & (2.3) & -6.4 & (3.8) & 0.0 & (3.4) \\
\hline & Peru & 78.3 & (2.0) & 0.1 & $(0.1)$ & 59.8 & $(2.2)$ & 0.4 & $(0.2)$ & -18.5 & (3.0) & 0.3 & $(0.2)$ \\
\hline & Rumänien & 38.6 & (1.9) & 2.4 & $(0.4)$ & 30.4 & $(2.2)$ & 1.1 & $(0.3)$ & -8.2 & (2.8) & -1.3 & $(0.5)$ \\
\hline & Russ. Föderation & 19.6 & (1.6) & 4.1 & $(0.6)$ & 18.6 & (1.3) & 4.6 & $(0.8)$ & -1.0 & $(2.1)$ & 0.5 & $(1.0)$ \\
\hline & Thailand & 27.3 & $(1.6)$ & 0.6 & $(0.2)$ & 33.3 & (1.9) & 0.4 & $(0.2)$ & 6.0 & $(2.5)$ & -0.2 & $(0.3)$ \\
\hline
\end{tabular}

Anmerkung: Statistisch signifikante Werte sind durch Fettdruck gekennzeichnet (siehe Anhang A3).

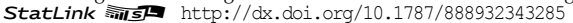


[Teil 1/1]

Leistungstrends im Bereich Lesekompetenz, nach Berücksichtigung demografischer Tabelle V.2.7 Veränderungen

\begin{tabular}{|c|c|c|c|c|c|c|c|c|c|c|c|c|}
\hline & & \multicolumn{2}{|c|}{$\begin{array}{l}\text { Bereinigte Ergebnisse } \\
\text { von PISA } 2000\end{array}$} & \multicolumn{2}{|c|}{$\begin{array}{c}\text { Bereinigte Ergebnisse } \\
\text { von PISA } 2003\end{array}$} & \multicolumn{2}{|c|}{$\begin{array}{l}\text { Bereinigte Ergebnisse } \\
\text { von PISA } 2006\end{array}$} & \multicolumn{2}{|c|}{$\begin{array}{c}\text { Ursprüngl. Ergebnisse } \\
\text { von PISA } 2009\end{array}$} & \multicolumn{3}{|c|}{$\begin{array}{l}\text { Veränderung zwischen } 2000 \\
\text { und } 2009 \text { (PISA 2009 - PISA 2000) }\end{array}$} \\
\hline & & Mittelwert & S.E. & Mittelwert & S.E. & Mittelwert & S.E. & Mittelwert & S.E. & Punktdiff. & S.E. & p-Wert \\
\hline \multirow{36}{*}{ 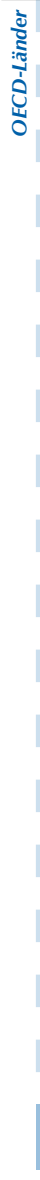 } & Australien & 535 & (2.7) & 526 & (1.6) & 511 & (1.6) & 515 & (2.3) & -20.2 & (6.1) & 0.00 \\
\hline & Österreich & 491 & (2.4) & 488 & (2.9) & 486 & (3.9) & $\mathrm{m}$ & $\mathrm{m}$ & $\mathrm{m}$ & $\mathrm{m}$ & $\mathrm{m}$ \\
\hline & Belgien & 508 & (3.4) & 506 & $(2.0)$ & 501 & (2.7) & 506 & (2.3) & -1.8 & (6.4) & 0.78 \\
\hline & Kanada & 529 & (1.4) & 530 & (1.5) & 527 & $(2.1)$ & 524 & (1.5) & -4.6 & (5.3) & 0.39 \\
\hline & Chile & 410 & (2.6) & $\mathrm{m}$ & $\mathrm{m}$ & 447 & (3.5) & 449 & (3.1) & 39.4 & (6.4) & 0.00 \\
\hline & Tschech. Rep. & 489 & (2.0) & 482 & (3.1) & 483 & (3.7) & 478 & (2.9) & -10.6 & $(6.0)$ & 0.08 \\
\hline & Dänemark & 498 & (1.9) & 496 & $(2.2)$ & 494 & (2.8) & 495 & $(2.1)$ & -3.4 & (5.7) & 0.55 \\
\hline & Estland & $\mathrm{m}$ & $\mathrm{m}$ & $\mathrm{m}$ & $\mathrm{m}$ & 504 & (2.8) & 501 & (2.6) & $\mathrm{m}$ & $\mathrm{m}$ & $\mathrm{m}$ \\
\hline & Finnland & 555 & (2.2) & 546 & (1.5) & 550 & (2.0) & 536 & (2.3) & -18.9 & (5.8) & 0.00 \\
\hline & Frankreich & 504 & (2.1) & 491 & $(2.2)$ & 488 & (3.7) & 496 & (3.4) & -8.7 & (6.4) & 0.17 \\
\hline & Deutschland & 480 & (2.4) & 491 & (2.8) & 489 & (3.5) & 497 & $(2.7)$ & 17.6 & $(6.1)$ & 0.00 \\
\hline & Griechenland & 473 & (4.4) & 475 & (3.2) & 462 & (3.2) & 483 & (4.3) & 9.8 & (7.9) & 0.22 \\
\hline & Ungarn & 484 & (2.6) & 481 & (1.9) & 481 & $(2.7)$ & 494 & $(3.2)$ & 10.6 & (6.4) & 0.10 \\
\hline & Island & 510 & (1.4) & 495 & (1.5) & 486 & (2.0) & 500 & (1.4) & -10.0 & (5.3) & 0.06 \\
\hline & Irland & 530 & (2.9) & 516 & $(2.0)$ & 516 & (2.9) & 496 & (3.0) & -34.6 & (6.5) & 0.00 \\
\hline & Israel & 445 & (8.1) & $\mathrm{m}$ & $\mathrm{m}$ & 430 & (4.4) & 474 & (3.6) & 29.3 & $(10.2)$ & 0.00 \\
\hline & Italien & 483 & $(2.7)$ & 474 & (3.0) & 467 & $(2.2)$ & 486 & (1.6) & 2.7 & $(5.8)$ & 0.65 \\
\hline & Japan & 521 & (5.1) & 493 & (3.6) & 495 & (3.5) & 520 & (3.5) & -0.7 & (7.9) & 0.93 \\
\hline & Korea & 530 & (2.0) & 538 & (2.6) & 553 & $(3.2)$ & 539 & (3.5) & 9.8 & (6.3) & 0.12 \\
\hline & Luxemburg & $\mathrm{m}$ & $\mathrm{m}$ & 479 & (1.6) & 479 & (1.4) & 472 & (1.3) & $\mathrm{m}$ & $\mathrm{m}$ & $\mathrm{m}$ \\
\hline & Mexiko & 422 & (2.3) & 399 & (2.5) & 410 & $(2.1)$ & 425 & (2.0) & 3.6 & (5.8) & 0.53 \\
\hline & Niederlande & $\mathrm{m}$ & $\mathrm{m}$ & 515 & (2.4) & 505 & (2.5) & 508 & (5.1) & $\mathrm{m}$ & $\mathrm{m}$ & $\mathrm{m}$ \\
\hline & Neuseeland & 525 & $(2.1)$ & 518 & (2.0) & 515 & (2.5) & 521 & (2.4) & -4.6 & (5.9) & 0.43 \\
\hline & Norwegen & 506 & (2.4) & 499 & (2.6) & 486 & (2.9) & 503 & (2.6) & -3.2 & $(6.1)$ & 0.60 \\
\hline & Polen & 478 & $(4.0)$ & 494 & (2.4) & 509 & (2.4) & 500 & (2.6) & 22.6 & (6.8) & 0.00 \\
\hline & Portugal & 471 & (3.3) & 479 & (3.0) & 480 & $(2.6)$ & 489 & $(3.1)$ & 18.6 & $(6.7)$ & 0.01 \\
\hline & Slowak. Rep. & $\mathrm{m}$ & $\mathrm{m}$ & 466 & (2.0) & 469 & $(2.7)$ & 477 & (2.5) & $\mathrm{m}$ & $\mathrm{m}$ & $\mathrm{m}$ \\
\hline & Slowenien & $\mathrm{m}$ & $\mathrm{m}$ & $\mathrm{m}$ & $\mathrm{m}$ & 494 & $(1.0)$ & 483 & (1.0) & $\mathrm{m}$ & $\mathrm{m}$ & $\mathrm{m}$ \\
\hline & Spanien & 495 & (2.0) & 479 & $(2.2)$ & 462 & (1.7) & 481 & (2.0) & -14.4 & (5.7) & 0.01 \\
\hline & Schweden & 516 & $(1.7)$ & 517 & $(1.8)$ & 507 & $(3.2)$ & 497 & $(2.9)$ & -18.4 & $(6.0)$ & 0.00 \\
\hline & Schweiz & 495 & $(3.2)$ & 502 & (2.3) & 501 & (2.6) & 501 & (2.4) & 5.3 & (6.4) & 0.40 \\
\hline & Türkei & $\mathrm{m}$ & $\mathrm{m}$ & 433 & (3.6) & 449 & (3.5) & 464 & (3.5) & $\mathrm{m}$ & $\mathrm{m}$ & $\mathrm{m}$ \\
\hline & Ver. Königreich & $\mathrm{m}$ & $\mathrm{m}$ & $\mathrm{m}$ & $\mathrm{m}$ & 492 & $(2.0)$ & 494 & $(2.3)$ & $\mathrm{m}$ & $\mathrm{m}$ & $\mathrm{m}$ \\
\hline & Ver. Staaten & 497 & $(4.5)$ & 486 & $(2.6)$ & $\mathrm{m}$ & $\mathrm{m}$ & 500 & (3.7) & 2.9 & (7.6) & 0.71 \\
\hline & OECD-23-Durchschnitt & 502 & $(0.6)$ & 497 & $(0.5)$ & 494 & $(0.6)$ & 499 & $(0.6)$ & -2.3 & $(5.0)$ & 0.64 \\
\hline & OECD-26-Durchschnitt & 496 & $(0.6)$ & $\mathrm{m}$ & $\mathrm{m}$ & $\mathrm{m}$ & $\mathrm{m}$ & 496 & $(0.5)$ & 0.7 & $(5.0)$ & 0.89 \\
\hline \multirow{25}{*}{ 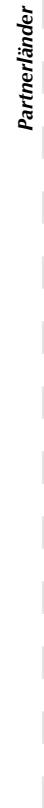 } & Albanien & 336 & (2.8) & $\mathrm{m}$ & $\mathrm{m}$ & $\mathrm{m}$ & $\mathrm{m}$ & 385 & $(4.0)$ & 48.3 & $(7.0)$ & 0.00 \\
\hline & Argentinien & 411 & $(7.1)$ & $\mathrm{m}$ & $\mathrm{m}$ & 374 & $(5.1)$ & 398 & (4.6) & -12.6 & (9.8) & 0.20 \\
\hline & Aserbaidschan & $\mathrm{m}$ & $\mathrm{m}$ & $\mathrm{m}$ & $\mathrm{m}$ & 352 & (3.4) & 362 & (3.3) & m & $\mathrm{m}$ & $\mathrm{m}$ \\
\hline & Brasilien & 393 & (2.6) & 399 & (3.9) & 395 & (3.5) & 412 & $(2.7)$ & 18.3 & $(6.2)$ & 0.00 \\
\hline & Bulgarien & 416 & $(3.2)$ & $\mathrm{m}$ & $\mathrm{m}$ & 407 & $(4.7)$ & 429 & $(6.7)$ & 12.6 & (8.9) & 0.16 \\
\hline & Kolumbien & $\mathrm{m}$ & $\mathrm{m}$ & $\mathrm{m}$ & $\mathrm{m}$ & 384 & $(4.1)$ & 413 & (3.7) & $\mathrm{m}$ & $\mathrm{m}$ & $\mathrm{m}$ \\
\hline & Kroatien & $\mathrm{m}$ & $\mathrm{m}$ & $\mathrm{m}$ & $\mathrm{m}$ & 476 & (2.6) & 476 & (2.9) & $\mathrm{m}$ & $\mathrm{m}$ & $\mathrm{m}$ \\
\hline & Hongkong (China) & 523 & (2.9) & 510 & $(3.2)$ & 534 & (2.3) & 533 & $(2.1)$ & 10.6 & $(6.1)$ & 0.08 \\
\hline & Indonesien & 369 & $(3.2)$ & 377 & (2.8) & 392 & $(5.1)$ & 402 & $(3.7)$ & 32.7 & $(7.0)$ & 0.00 \\
\hline & Jordanien & $\mathrm{m}$ & $\mathrm{m}$ & $\mathrm{m}$ & $\mathrm{m}$ & 398 & (2.8) & 405 & (3.3) & $\mathrm{m}$ & $\mathrm{m}$ & $\mathrm{m}$ \\
\hline & Kirgisistan & $\mathrm{m}$ & $\mathrm{m}$ & $\mathrm{m}$ & $\mathrm{m}$ & 289 & $(3.1)$ & 314 & $(3.2)$ & $\mathrm{m}$ & $\mathrm{m}$ & $\mathrm{m}$ \\
\hline & Lettland & 452 & (5.4) & 487 & (3.3) & 479 & $(3.2)$ & 484 & (3.0) & 31.5 & (7.9) & 0.00 \\
\hline & Liechtenstein & 477 & $(7.2)$ & 522 & (6.9) & 509 & $(4.3)$ & 499 & (2.8) & 22.4 & $(9.2)$ & 0.01 \\
\hline & Litauen & $\mathrm{m}$ & $\mathrm{m}$ & $\mathrm{m}$ & $\mathrm{m}$ & 469 & (2.6) & 468 & (2.4) & $\mathrm{m}$ & $\mathrm{m}$ & $\mathrm{m}$ \\
\hline & Macau (China) & $\mathrm{m}$ & $\mathrm{m}$ & 499 & $(2.1)$ & 482 & $(7.8)$ & 487 & $(0.9)$ & $\mathrm{m}$ & $\mathrm{m}$ & $\mathrm{m}$ \\
\hline & Montenegro & $\mathrm{m}$ & $\mathrm{m}$ & $\mathrm{m}$ & $\mathrm{m}$ & 395 & (1.9) & 408 & (1.7) & $\mathrm{m}$ & m & $\mathrm{m}$ \\
\hline & Peru & 323 & $(3.2)$ & $\mathrm{m}$ & $\mathrm{m}$ & $\mathrm{m}$ & $\mathrm{m}$ & 370 & $(4.0)$ & 47.0 & $(7.1)$ & 0.00 \\
\hline & Katar & $\mathrm{m}$ & $\mathrm{m}$ & $\mathrm{m}$ & $\mathrm{m}$ & 319 & $(1.2)$ & 372 & $(0.8)$ & $\mathrm{m}$ & $\mathrm{m}$ & $\mathrm{m}$ \\
\hline & Rumänien & 434 & $(4.0)$ & $\mathrm{m}$ & $\mathrm{m}$ & 397 & (3.8) & 424 & $(4.1)$ & -9.7 & (7.5) & 0.20 \\
\hline & Russ. Föderation & 472 & (3.1) & 441 & (3.3) & 440 & $(3.2)$ & 459 & (3.3) & -12.1 & $(6.7)$ & 0.07 \\
\hline & Serbien & $\mathrm{m}$ & $\mathrm{m}$ & 414 & (2.6) & 402 & $(2.7)$ & 442 & (2.4) & m & $\mathrm{m}$ & $\mathrm{m}$ \\
\hline & Chinesisch Taipeh & $\mathrm{m}$ & $\mathrm{m}$ & $\mathrm{m}$ & $\mathrm{m}$ & 505 & (3.5) & 495 & (2.6) & $\mathrm{m}$ & $\mathrm{m}$ & $\mathrm{m}$ \\
\hline & Thailand & 435 & (2.9) & 421 & (2.4) & 420 & $(2.2)$ & 421 & (2.6) & -13.7 & (6.3) & 0.03 \\
\hline & Tunesien & $\mathrm{m}$ & $\mathrm{m}$ & 357 & $(9.0)$ & 377 & $(3.1)$ & 404 & (2.9) & $\mathrm{m}$ & $\mathrm{m}$ & $\mathrm{m}$ \\
\hline & Uruguay & $\mathrm{m}$ & $\mathrm{m}$ & 418 & (3.2) & 406 & $(3.2)$ & 426 & (2.6) & $\mathrm{m}$ & $\mathrm{m}$ & $\mathrm{m}$ \\
\hline
\end{tabular}

Anmerkung: Statistisch signifikante Werte sind durch Fettdruck gekennzeichnet (siehe Anhang A3).

StatLink : 
[Teil 1/1]

Lineare Trends und jährliche Veränderungen bei den Leseleistungen auf der Grundlage Tabelle V.2.8 aller PISA-Erhebungen

\begin{tabular}{|c|c|c|c|c|c|c|c|c|}
\hline & \multirow{3}{*}{$\begin{array}{c}\text { Anzahl der Jahre, für } \\
\text { die PISA-Ergebnisse } \\
\text { vorliegen }\end{array}$} & \multicolumn{6}{|c|}{ Jährliche Punktzahlveränderung1 } \\
\hline & & & \multicolumn{2}{|c|}{ Beobachteter linearer Trend } & \multicolumn{2}{|c|}{$\begin{array}{c}\text { Jahresrate der beobachteten } \\
\text { Veränderung zwischen } 2000 \\
\text { und } 2009,2003 \text { und } 2009 \text { bzw. } \\
2006 \text { und } 2009\end{array}$} & \multicolumn{2}{|c|}{$\begin{array}{l}\text { Jahresrate der beobachteten } \\
\text { Veränderung zwischen } 2000 \text { und } 2009 \text {, } \\
2003 \text { und } 2009 \text { bzw. } 2006 \text { und } 2009 \text { nach } \\
\text { Berücksicht. demograf. Veränderungen }\end{array}$} \\
\hline & & & Punktdiff. & S.E. & Punktdiff. & S.E. & Punktdiff. & S.E. \\
\hline \multirow{36}{*}{ 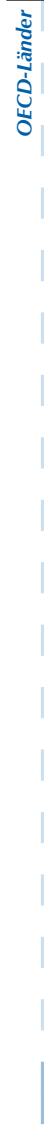 } & Australien & 9 & -1.8 & $(0.7)$ & -1.5 & $(0.7)$ & -2.2 & $(0.7)$ \\
\hline & Österreich & 6 & $\mathrm{~m}$ & $\mathrm{~m}$ & $\mathrm{~m}$ & $\mathrm{~m}$ & $\mathrm{~m}$ & $\mathrm{~m}$ \\
\hline & Belgien & 9 & -0.3 & $(0.7)$ & -0.1 & $(0.7)$ & -0.2 & $(0.7)$ \\
\hline & Kanada & 9 & -1.0 & $(0.6)$ & -1.1 & $(0.6)$ & -0.5 & $(0.6)$ \\
\hline & Chile & 8 & 5.1 & $(0.8)$ & 5.0 & $(0.9)$ & 4.9 & $(0.8)$ \\
\hline & Tschech. Rep. & 9 & -1.5 & $(0.7)$ & -1.5 & $(0.7)$ & -1.2 & $(0.7)$ \\
\hline & Dänemark & 9 & -0.1 & $(0.6)$ & -0.2 & (0.6) & -0.4 & $(0.6)$ \\
\hline & Estland & 3 & 0.1 & $(2.7)$ & 0.1 & (1.9) & -0.9 & (1.9) \\
\hline & Finnland & 9 & -0.9 & $(0.6)$ & -1.2 & $(0.7)$ & -2.1 & $(0.6)$ \\
\hline & Frankreich & 9 & -1.2 & $(0.7)$ & -1.0 & $(0.7)$ & -1.0 & $(0.7)$ \\
\hline & Deutschland & 9 & 1.5 & $(0.7)$ & 1.5 & $(0.7)$ & 2.0 & $(0.7)$ \\
\hline & Griechenland & 9 & 0.5 & (0.9) & 1.0 & (0.9) & 1.1 & $(0.9)$ \\
\hline & Ungarn & 9 & 1.4 & $(0.7)$ & 1.6 & $(0.8)$ & 1.2 & $(0.7)$ \\
\hline & Island & 9 & -0.9 & $(0.6)$ & -0.7 & $(0.6)$ & -1.1 & $(0.6)$ \\
\hline & Irland & 9 & -3.0 & $(0.7)$ & -3.4 & $(0.7)$ & -3.8 & $(0.7)$ \\
\hline & Israel & 8 & 2.2 & (1.4) & 2.7 & (1.3) & 3.7 & (1.3) \\
\hline & Italien & 9 & -0.4 & $(0.7)$ & -0.2 & $(0.7)$ & 0.3 & $(0.6)$ \\
\hline & Japan & 9 & -0.2 & $(0.9)$ & -0.3 & (0.9) & -0.1 & (0.9) \\
\hline & Korea & 9 & 2.2 & $(0.7)$ & 1.6 & $(0.7)$ & 1.1 & $(0.7)$ \\
\hline & Luxemburg & 6 & -1.2 & $(1.2)$ & -1.2 & $(0.8)$ & -1.1 & $(0.8)$ \\
\hline & Mexiko & 9 & 0.7 & $(0.8)$ & 0.4 & $(0.7)$ & 0.4 & (0.6) \\
\hline & Niederlande & 6 & -0.8 & (1.6) & -0.8 & $(1.2)$ & -1.0 & $(1.2)$ \\
\hline & Neuseeland & 9 & -0.8 & $(0.7)$ & -0.9 & $(0.7)$ & -0.5 & $(0.7)$ \\
\hline & Norwegen & 9 & -0.7 & $(0.5)$ & -0.2 & $(0.7)$ & -0.4 & $(0.7)$ \\
\hline & Polen & 9 & 2.5 & $(0.7)$ & 2.4 & $(0.8)$ & 2.5 & (0.8) \\
\hline & Portugal & 9 & 1.7 & $(0.8)$ & 2.1 & (0.8) & 2.1 & $(0.7)$ \\
\hline & Slowak. Rep. & 6 & 1.4 & (1.4) & 1.4 & (1.0) & 1.9 & $(0.9)$ \\
\hline & Slowenien & 3 & -3.8 & (2.4) & -3.8 & (1.4) & -3.8 & (1.4) \\
\hline & Spanien & 9 & -1.8 & $(0.7)$ & -1.3 & $(0.7)$ & -1.6 & $(0.6)$ \\
\hline & Schweden & 9 & -2.1 & $(0.7)$ & -2.1 & $(0.7)$ & -2.0 & $(0.7)$ \\
\hline & Schweiz & 9 & 0.6 & $(0.8)$ & 0.7 & $(0.8)$ & 0.6 & $(0.7)$ \\
\hline & Türkei & 6 & 3.9 & (1.7) & 3.9 & (1.3) & 5.3 & (1.1) \\
\hline & Ver. Königreich & 3 & -0.3 & (2.5) & -0.3 & (1.7) & 0.8 & (1.7) \\
\hline & Ver. Staaten & 9 & -0.3 & $(1.0)$ & -0.5 & (1.0) & 0.3 & $(0.8)$ \\
\hline & OECD-26-Durchschnitt & 9 & 0.0 & $(0.6)$ & 0.1 & $(0.6)$ & 0.1 & $(0.6)$ \\
\hline & OECD-33-Durchschnitt & 9 & 0.0 & $(0.6)$ & 0.1 & $(0.6)$ & 0.1 & $(0.6)$ \\
\hline \multirow{25}{*}{ 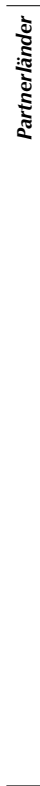 } & Albanien & 8 & 4.5 & $(0.9)$ & 4.5 & (0.9) & 6.0 & (0.9) \\
\hline & Argentinien & 8 & -3.2 & (1.5) & -2.5 & (1.5) & -1.6 & $(1.2)$ \\
\hline & Aserbaidschan & 3 & 2.9 & (2.8) & 2.9 & (2.0) & 3.0 & $(2.1)$ \\
\hline & Brasilien & 9 & 1.2 & $(0.7)$ & 1.7 & $(0.7)$ & 2.0 & $(0.7)$ \\
\hline & Bulgarien & 8 & -0.7 & (1.1) & -0.2 & $(1.2)$ & 1.6 & (1.1) \\
\hline & Kolumbien & 3 & 9.3 & (3.1) & 9.3 & (2.5) & 9.6 & (2.3) \\
\hline & Kroatien & 3 & -0.5 & $(2.7)$ & -0.5 & (1.9) & 0.0 & (1.9) \\
\hline & Hongkong (China) & 8 & 2.0 & $(0.8)$ & 1.0 & (0.8) & 1.3 & $(0.8)$ \\
\hline & Indonesien & 8 & 3.8 & $(0.9)$ & 3.9 & $(0.9)$ & 4.1 & $(0.9)$ \\
\hline & Jordanien & 3 & 1.5 & (2.8) & 1.5 & $(2.1)$ & 2.2 & $(2.0)$ \\
\hline & Kirgisistan & 3 & 9.8 & (2.9) & 9.8 & $(2.1)$ & 8.3 & $(2.0)$ \\
\hline & Lettland & 9 & 2.2 & $(0.9)$ & 2.9 & (0.9) & 3.5 & $(0.9)$ \\
\hline & Liechtenstein & 9 & 1.2 & $(0.8)$ & 1.9 & (0.8) & 2.5 & (1.0) \\
\hline & Litauen & 3 & -0.5 & $(2.7)$ & -0.5 & (1.9) & -0.1 & (1.8) \\
\hline & Macau (China) & 6 & -1.8 & (1.3) & -1.8 & $(0.8)$ & -2.0 & $(0.8)$ \\
\hline & Montenegro & 3 & 5.2 & (2.4) & 5.2 & (1.5) & 4.1 & (1.6) \\
\hline & Peru & 8 & 5.3 & (1.0) & 5.3 & (1.0) & 5.9 & $(0.9)$ \\
\hline & Katar & 3 & 19.8 & (2.4) & 19.8 & (1.4) & 17.6 & (1.4) \\
\hline & Rumänien & 7 & -0.9 & (1.0) & -0.5 & (1.0) & -1.4 & (1.1) \\
\hline & Russ. Föderation & 9 & -0.3 & $(0.8)$ & -0.3 & $(0.8)$ & -1.3 & $(0.7)$ \\
\hline & Serbien & 6 & 5.0 & (1.4) & 5.0 & (1.0) & 4.6 & $(0.9)$ \\
\hline & Chinesisch Taipeh & 3 & -0.3 & (2.4) & -0.3 & (2.0) & -3.3 & (2.0) \\
\hline & Thailand & 8 & -1.0 & $(0.8)$ & -1.2 & $(0.8)$ & -1.7 & $(0.8)$ \\
\hline & Tunesien & 6 & 4.8 & (1.4) & 4.8 & (1.0) & 7.8 & (1.7) \\
\hline & Uruguay & 6 & -1.4 & (1.4) & -1.4 & (1.0) & 1.3 & $(1.0)$ \\
\hline
\end{tabular}

Anmerkung: Statistisch signifikante Werte sind durch Fettdruck gekennzeichnet (siehe Anhang A3).

1. Die linearen Trends werden mittels einer linearen Regression geschätzt, die auf Daten aus allen PISA-Erhebungsrunden angewendet wird. Die Jahresrate der Veränderung wird durch Division des Leistungsabstands durch die Zahl der zwischen den beiden Erhebungen liegenden Jahre berechnet. Das Ergebnis spiegelt die einem Jahr entsprechende durchschnittliche Punktzahlveränderung wider.

StatLink त्ताs http://dx.doi.org/10.1787/888932343285 
[Teil 1/1]

Veränderung der durchschnittlichen Punktzahl in Lesekompetenz zwischen 2003 und 2009 Tabelle V.2.9 sowie zwischen 2006 und 2009

\begin{tabular}{|c|c|c|c|c|c|}
\hline & & \multicolumn{2}{|c|}{ Veränderung zwischen 2003 und 2009} & \multicolumn{2}{|c|}{ Veränderung zwischen 2006 und 2009} \\
\hline & & Punktzahlveränderung & S.E. & Punktzahlveränderung & S.E. \\
\hline \multirow{36}{*}{ 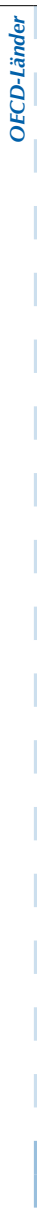 } & Australien & -11 & $(5.2)$ & 2 & $(5.1)$ \\
\hline & Österreich & $\mathrm{m}$ & $\mathrm{m}$ & $\mathrm{m}$ & $\mathrm{m}$ \\
\hline & Belgien & -1 & $(5.4)$ & 5 & $(5.6)$ \\
\hline & Kanada & -4 & $(4.7)$ & -3 & (5.0) \\
\hline & Chile & $\mathrm{m}$ & $\mathrm{m}$ & 7 & $(7.2)$ \\
\hline & Tschech. Rep. & -10 & (6.1) & -5 & (6.5) \\
\hline & Dänemark & 3 & $(5.4)$ & 0 & (5.6) \\
\hline & Estland & $\mathrm{m}$ & $\mathrm{m}$ & 0 & $(5.7)$ \\
\hline & Finnland & -8 & $(5.0)$ & -11 & $(5.1)$ \\
\hline & Frankreich & -1 & $(6.0)$ & 8 & (6.7) \\
\hline & Deutschland & 6 & $(5.9)$ & 2 & (6.6) \\
\hline & Griechenland & 11 & $(7.2)$ & 23 & (7.2) \\
\hline & Ungarn & 12 & $(5.7)$ & 12 & (6.1) \\
\hline & Island & 9 & (4.6) & 16 & (4.7) \\
\hline & Irland & -20 & $(5.7)$ & -22 & (6.2) \\
\hline & Israel & $\mathrm{m}$ & $\mathrm{m}$ & 35 & (7.1) \\
\hline & Italien & 10 & (5.3) & 18 & (5.0) \\
\hline & Japan & 22 & (6.6) & 22 & (6.5) \\
\hline & Korea & 5 & $(6.2)$ & -17 & (6.6) \\
\hline & Luxemburg & -7 & (4.5) & -7 & (4.4) \\
\hline & Mexiko & 26 & $(6.1)$ & 15 & (5.5) \\
\hline & Niederlande & -5 & $(7.2)$ & 2 & $(7.2)$ \\
\hline & Neuseeland & -1 & (5.3) & 0 & (5.6) \\
\hline & Norwegen & 3 & (5.6) & 19 & (5.8) \\
\hline & Polen & 4 & (5.6) & -7 & (5.6) \\
\hline & Portugal & 12 & (6.3) & 17 & (6.2) \\
\hline & Slowak. Rep. & 8 & (5.7) & 11 & (5.7) \\
\hline & Slowenien & $\mathrm{m}$ & $\mathrm{m}$ & -11 & (4.3) \\
\hline & Spanien & 1 & (5.3) & 20 & (5.1) \\
\hline & Schweden & -17 & (5.6) & -10 & (6.1) \\
\hline & Schweiz & 1 & (5.8) & 1 & (5.6) \\
\hline & Türkei & 23 & $(7.9)$ & 17 & (6.8) \\
\hline & Ver. Königreich & $\mathrm{m}$ & $\mathrm{m}$ & -1 & (5.2) \\
\hline & Ver. Staaten & 5 & (6.4) & $\mathrm{m}$ & $\mathrm{m}$ \\
\hline & OECD-28-Durchschnitt & 3 & $(4.2)$ & $\mathrm{m}$ & $\mathrm{m}$ \\
\hline & OECD-32-Durchschnitt & $\mathrm{m}$ & $\mathrm{m}$ & 5 & $(4.1)$ \\
\hline \multirow{23}{*}{ 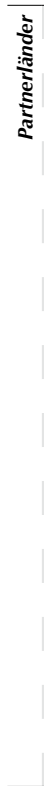 } & Argentinien & $\mathrm{m}$ & $\mathrm{m}$ & 25 & (9.5) \\
\hline & Aserbaidschan & $\mathrm{m}$ & $\mathrm{m}$ & 9 & (6.1) \\
\hline & Brasilien & 9 & (6.7) & 19 & $(6.2)$ \\
\hline & Bulgarien & $\mathrm{m}$ & $\mathrm{m}$ & 27 & (10.4) \\
\hline & Kolumbien & $\mathrm{m}$ & $\mathrm{m}$ & 28 & $(7.5)$ \\
\hline & Kroatien & $\mathrm{m}$ & $\mathrm{m}$ & -2 & (5.7) \\
\hline & Hongkong (China) & 24 & (5.9) & -3 & $(5.2)$ \\
\hline & Indonesien & 20 & (6.5) & 9 & (8.1) \\
\hline & Jordanien & $\mathrm{m}$ & $\mathrm{m}$ & 4 & (6.2) \\
\hline & Kirgisistan & $\mathrm{m}$ & $\mathrm{m}$ & 29 & (6.2) \\
\hline & Lettland & -7 & $(6.2)$ & 4 & (6.3) \\
\hline & Liechtenstein & -26 & (6.1) & -11 & (6.3) \\
\hline & Litauen & $\mathrm{m}$ & $\mathrm{m}$ & -2 & (5.6) \\
\hline & Macau (China) & -11 & $(4.7)$ & -6 & $(4.3)$ \\
\hline & Montenegro & $\mathrm{m}$ & $\mathrm{m}$ & 16 & (4.6) \\
\hline & Katar & $\mathrm{m}$ & $\mathrm{m}$ & 60 & (4.3) \\
\hline & Rumänien & $\mathrm{m}$ & $\mathrm{m}$ & 29 & $(7.4)$ \\
\hline & Russ. Föderation & 17 & (6.6) & 20 & (6.8) \\
\hline & Serbien & 30 & (5.9) & 41 & (5.9) \\
\hline & Chinesisch Taipeh & $\mathrm{m}$ & $\mathrm{m}$ & -1 & (5.9) \\
\hline & Thailand & 1 & (5.6) & 5 & (5.5) \\
\hline & Tunesien & 29 & $(5.7)$ & 23 & (6.4) \\
\hline & Uruguay & -8 & (5.9) & 13 & (5.9) \\
\hline
\end{tabular}

Anmerkung: Statistisch signifikante Werte sind durch Fettdruck gekennzeichnet (siehe Anhang A3).

StatLink ints http://dx.doi.org/10.1787/888932343285 
[Teil 1/1]

Tabelle V.3.1 Mittlere Punktzahlen in Mathematik in PISA 2003, PISA 2006 und PISA 2009

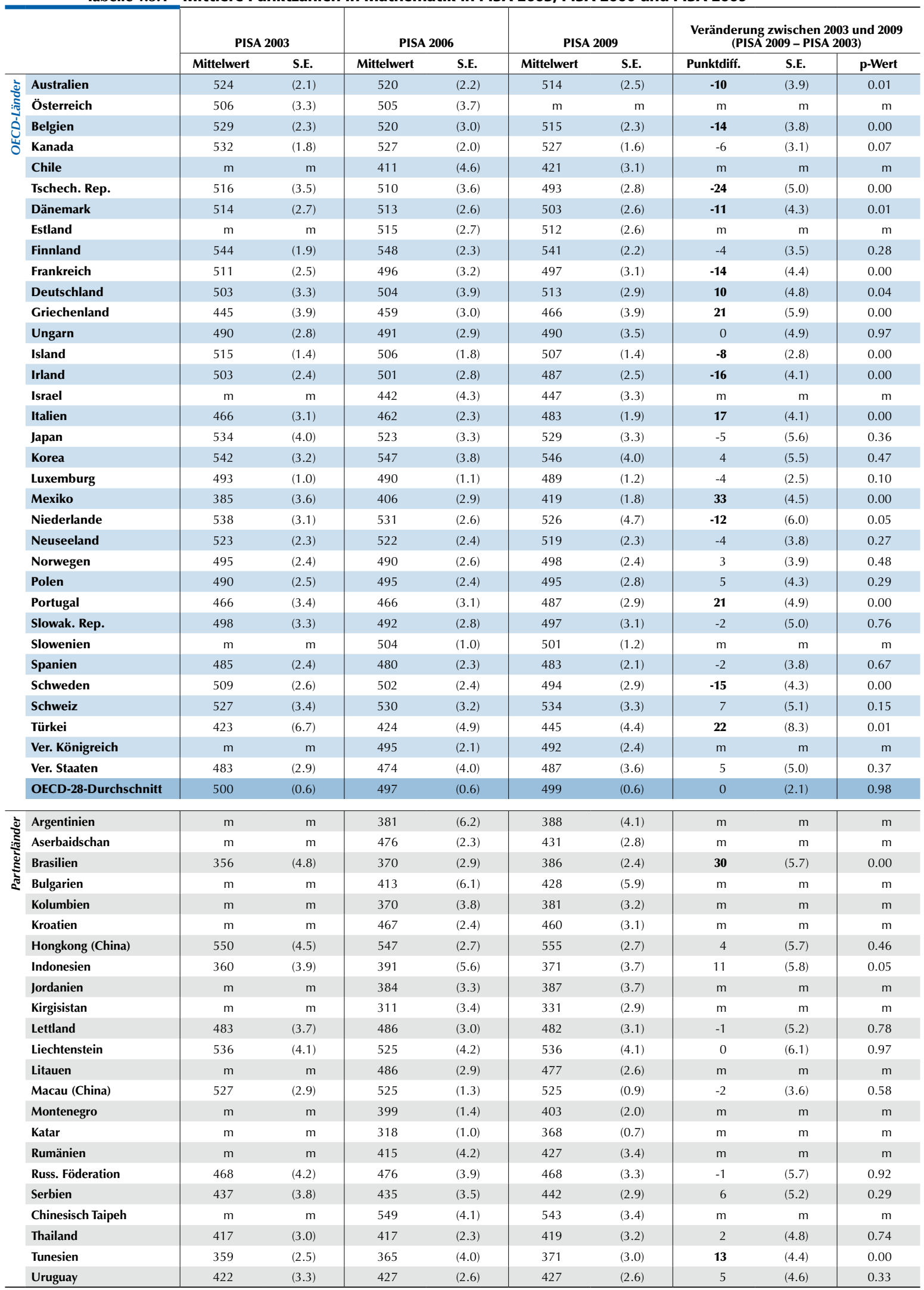

Anmerkung: Statistisch signifikante Werte sind durch Fettdruck gekennzeichnet (siehe Anhang A3).

StatLink o्राst http://dx.doi.org/10.1787/888932343285 
[Teil 1/1]

Prozentsatz der Schüler unter Stufe 2 und auf Stufe 5 oder höher auf der Gesamtskala Tabelle V.3.2 Mathematik in PISA 2003 und PISA 2009

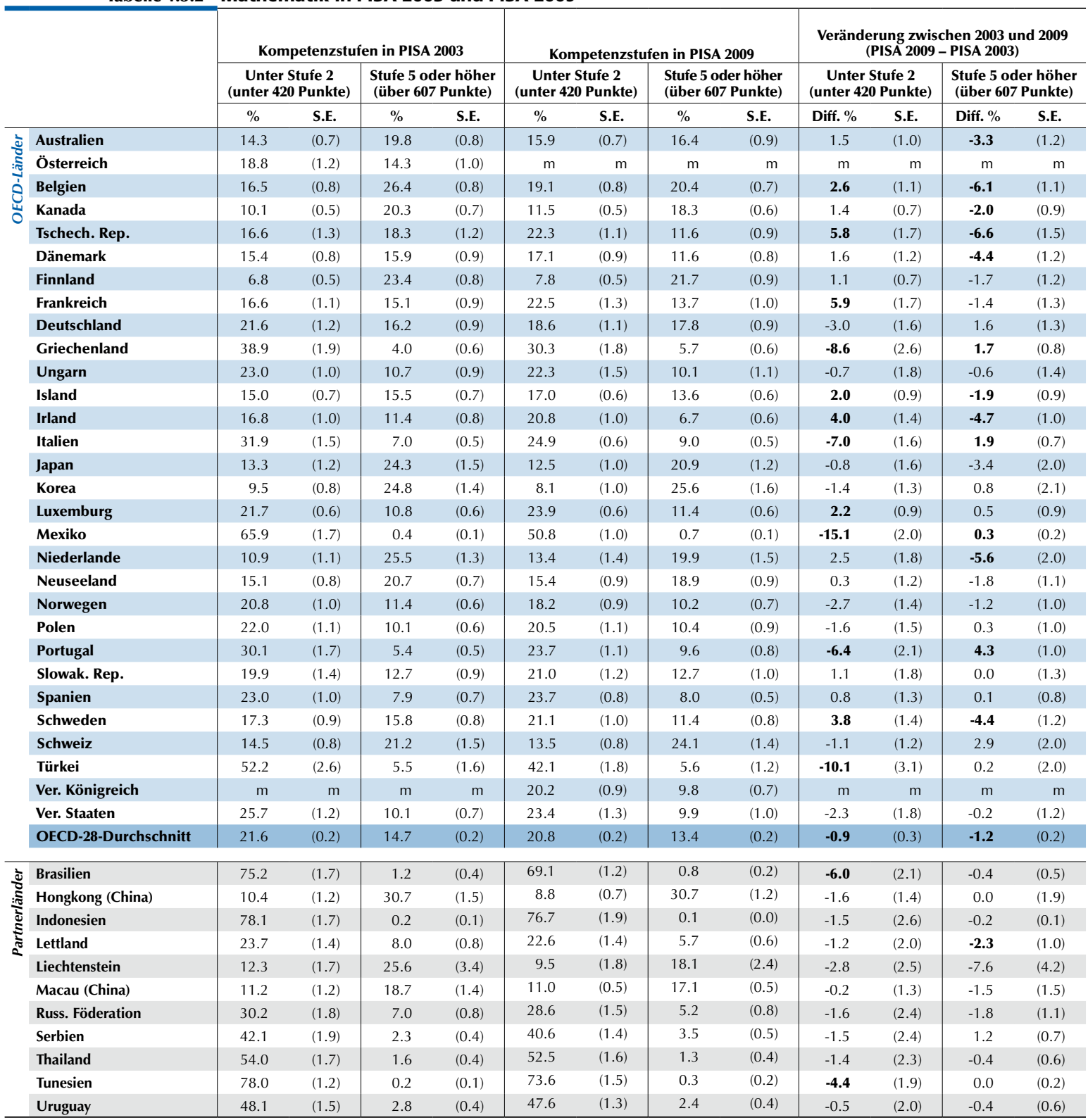

Anmerkung: Statistisch signifikante Werte sind durch Fettdruck gekennzeichnet (siehe Anhang A3).

StatLink *iाst http://dx.doi.org/10.1787/888932343285 
[Teil 1/1]

Tabelle V.3.3 Annualisierte Veränderung der Schülerleistungen in Mathematik seit 2003

\begin{tabular}{|c|c|c|c|c|c|c|c|}
\hline & & \multicolumn{2}{|c|}{$\begin{array}{l}\text { Jahresrate der Veränderung } \\
\text { zwischen } 2003 \text { und 20091 } \\
\text { (PISA 2009 - PISA 2003) }\end{array}$} & \multicolumn{2}{|c|}{$\begin{array}{l}\text { Veränderung zwischen } 2006 \text { und } 2009 \\
\text { (PISA 2009 - PISA 2006) }\end{array}$} & \multicolumn{2}{|c|}{$\begin{array}{l}\text { Jahresrate der Veränderung } \\
\text { zwischen } 2006 \text { und 20091 } \\
\text { (PISA 2009 - PISA 2006) }\end{array}$} \\
\hline & & Punktdifferenz & S.E. & Punktdifferenz & S.E. & Punktdifferenz & S.E. \\
\hline \multirow{35}{*}{ 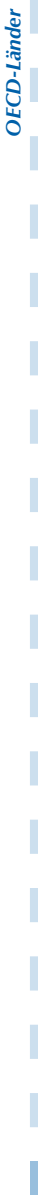 } & Australien & -1.7 & (0.6) & -6 & (3.6) & -1.9 & (1.2) \\
\hline & Österreich & $\mathrm{m}$ & $\mathrm{m}$ & $\mathrm{m}$ & $\mathrm{m}$ & $\mathrm{m}$ & $\mathrm{m}$ \\
\hline & Belgien & -2.3 & (0.6) & -5 & (3.9) & -1.7 & (1.3) \\
\hline & Kanada & -0.9 & (0.5) & 0 & (2.9) & -0.1 & (1.0) \\
\hline & Chile & $\mathrm{m}$ & $\mathrm{m}$ & 10 & (5.7) & 3.2 & (1.9) \\
\hline & Tschech. Rep. & -3.9 & $(0.8)$ & -17 & (4.7) & -5.7 & (1.6) \\
\hline & Dänemark & -1.8 & $(0.7)$ & -10 & (3.9) & -3.2 & (1.3) \\
\hline & Estland & $\mathrm{m}$ & $\mathrm{m}$ & -2 & (4.0) & -0.8 & (1.3) \\
\hline & Finnland & -0.6 & $(0.6)$ & -8 & (3.4) & -2.6 & (1.1) \\
\hline & Frankreich & -2.3 & $(0.7)$ & 1 & (4.6) & 0.4 & (1.5) \\
\hline & Deutschland & 1.6 & $(0.8)$ & 9 & (5.0) & 3.0 & (1.7) \\
\hline & Griechenland & 3.5 & (1.0) & 7 & (5.1) & 2.3 & (1.7) \\
\hline & Ungarn & 0.0 & $(0.8)$ & -1 & $(4.7)$ & -0.3 & (1.6) \\
\hline & Island & -1.4 & $(0.5)$ & 1 & (2.6) & 0.4 & $(0.9)$ \\
\hline & Irland & -2.6 & $(0.7)$ & -14 & $(4.0)$ & -4.8 & (1.3) \\
\hline & Israel & $\mathrm{m}$ & $\mathrm{m}$ & 5 & (5.6) & 1.7 & (1.9) \\
\hline & Italien & 2.9 & $(0.7)$ & 21 & (3.2) & 7.1 & (1.1) \\
\hline & Japan & -0.9 & $(0.9)$ & 6 & (4.9) & 2.0 & (1.6) \\
\hline & Korea & 0.7 & $(0.9)$ & -1 & (5.7) & -0.4 & (1.9) \\
\hline & Luxemburg & -0.7 & $(0.4)$ & -1 & (2.1) & -0.3 & $(0.7)$ \\
\hline & Mexiko & 5.5 & $(0.8)$ & 13 & (3.7) & 4.3 & $(1.2)$ \\
\hline & Niederlande & -2.0 & (1.0) & -5 & (5.6) & -1.6 & (1.9) \\
\hline & Neuseeland & -0.7 & (0.6) & -3 & (3.6) & -0.9 & (1.2) \\
\hline & Norwegen & 0.5 & $(0.7)$ & 8 & (3.8) & 2.7 & (1.3) \\
\hline & Polen & 0.8 & $(0.7)$ & -1 & $(4.0)$ & -0.2 & (1.3) \\
\hline & Portugal & 3.5 & $(0.8)$ & 21 & (4.4) & 6.9 & (1.5) \\
\hline & Slowak. Rep. & -0.3 & $(0.8)$ & 5 & (4.4) & 1.5 & (1.5) \\
\hline & Slowenien & m & $\mathrm{m}$ & -3 & (2.1) & -1.0 & $(0.7)$ \\
\hline & Spanien & -0.3 & $(0.6)$ & 4 & (3.4) & 1.2 & (1.1) \\
\hline & Schweden & -2.5 & $(0.7)$ & -8 & (4.0) & -2.7 & (1.3) \\
\hline & Schweiz & 1.2 & $(0.9)$ & 4 & (4.8) & 1.4 & (1.6) \\
\hline & Türkei & 3.7 & (1.4) & 22 & (6.7) & 7.2 & $(2.2)$ \\
\hline & Ver. Königreich & $\mathrm{m}$ & $\mathrm{m}$ & -3 & (3.5) & -1.0 & $(1.2)$ \\
\hline & Ver. Staaten & 0.8 & $(0.8)$ & 13 & (5.5) & 4.3 & (1.8) \\
\hline & OECD-28-Durchschnitt & 0.0 & $(0.4)$ & 2 & (1.5) & 0.7 & $(0.5)$ \\
\hline \multirow{23}{*}{ 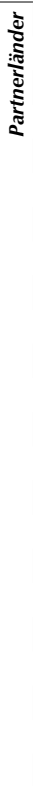 } & Argentinien & $\mathrm{m}$ & $\mathrm{m}$ & 7 & (7.6) & 2.3 & (2.5) \\
\hline & Aserbaidschan & $\mathrm{m}$ & $\mathrm{m}$ & -45 & (3.8) & -15.0 & (1.3) \\
\hline & Brasilien & 5.0 & (1.0) & 16 & $(4.0)$ & 5.4 & (1.3) \\
\hline & Bulgarien & $\mathrm{m}$ & $\mathrm{m}$ & 15 & (8.6) & 4.9 & (2.9) \\
\hline & Kolumbien & $\mathrm{m}$ & $\mathrm{m}$ & 11 & (5.2) & 3.6 & (1.7) \\
\hline & Kroatien & $\mathrm{m}$ & $\mathrm{m}$ & -7 & (4.1) & -2.4 & (1.4) \\
\hline & Hongkong (China) & 0.7 & $(0.9)$ & 7 & (4.0) & 2.4 & (1.3) \\
\hline & Indonesien & 1.9 & (1.0) & -20 & (6.9) & -6.6 & (2.3) \\
\hline & Jordanien & $\mathrm{m}$ & $\mathrm{m}$ & 3 & (5.1) & 0.9 & (1.7) \\
\hline & Kirgisistan & $\mathrm{m}$ & $\mathrm{m}$ & 21 & $(4.7)$ & 6.9 & (1.6) \\
\hline & Lettland & -0.2 & $(0.9)$ & -4 & (4.5) & -1.4 & (1.5) \\
\hline & Liechtenstein & 0.0 & (1.0) & 11 & (6.0) & 3.7 & (2.0) \\
\hline & Litauen & $\mathrm{m}$ & $\mathrm{m}$ & -10 & $(4.1)$ & -3.3 & (1.4) \\
\hline & Macau (China) & -0.3 & (0.6) & 0 & (2.1) & 0.1 & $(0.7)$ \\
\hline & Montenegro & $\mathrm{m}$ & $\mathrm{m}$ & 3 & (2.8) & 1.1 & $(0.9)$ \\
\hline & Katar & $\mathrm{m}$ & $\mathrm{m}$ & 50 & (1.8) & 16.7 & (0.6) \\
\hline & Rumänien & $\mathrm{m}$ & $\mathrm{m}$ & 12 & (5.6) & 4.1 & (1.9) \\
\hline & Russ. Föderation & -0.1 & $(0.9)$ & -8 & (5.3) & -2.6 & (1.8) \\
\hline & Serbien & 0.9 & $(0.9)$ & 7 & (4.8) & 2.3 & (1.6) \\
\hline & Chinesisch Taipeh & $\mathrm{m}$ & $\mathrm{m}$ & -6 & (5.5) & -2.1 & (1.8) \\
\hline & Thailand & 0.3 & (0.8) & 2 & $(4.2)$ & 0.5 & (1.4) \\
\hline & Tunesien & 2.1 & $(0.7)$ & 6 & (5.1) & 2.0 & (1.7) \\
\hline & Uruguay & 0.8 & $(0.8)$ & 0 & (3.9) & 0.0 & (1.3) \\
\hline
\end{tabular}

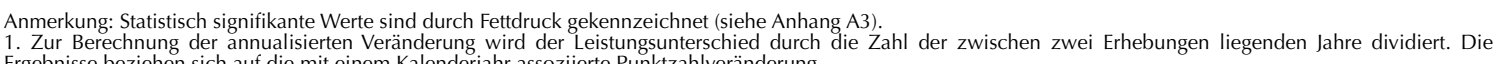

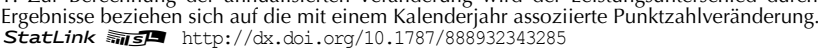


[Teil 1/1]

Tabelle V.3.4 Mittlere Punktzahlen in Naturwissenschaften in PISA 2006 und PISA 2009

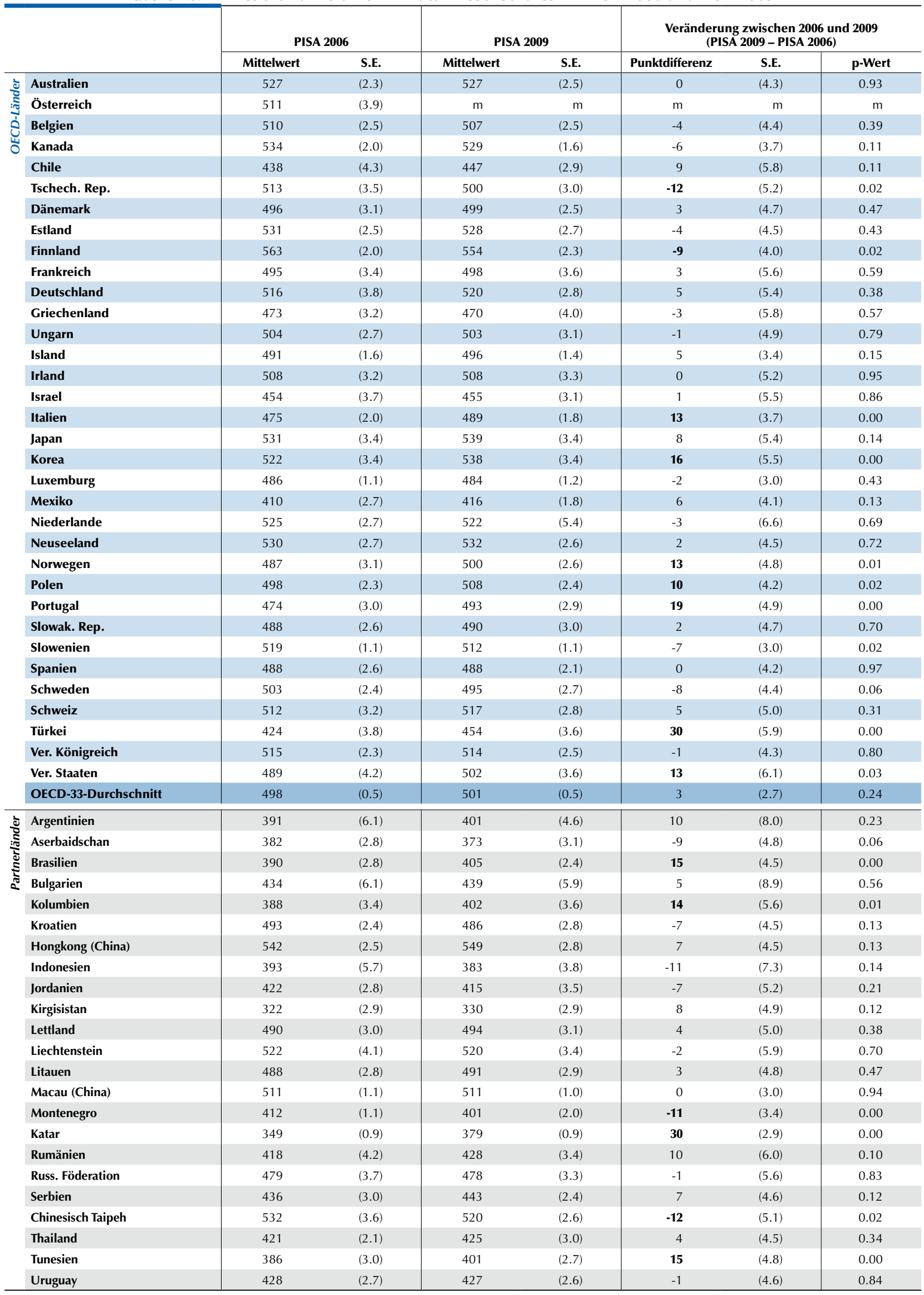

Anmerkung: Statistisch signifikante Werte sind durch Fettdruck gekennzeichnet (siehe Anhang A3).

StatLink הत्ताज http://dx.doi.org/10.1787/888932343285 
[Teil 1/1]

Prozentsatz der Schüler unter Stufe 2 und auf Stufe 5 oder höher auf der Gesamtskala Tabelle V.3.5 Naturwissenschaften in PISA 2006 und PISA 2009

\begin{tabular}{|c|c|c|c|c|c|c|c|c|c|c|c|c|c|}
\hline & \multicolumn{4}{|c|}{ Kompetenzstufen in PISA 2006} & \multicolumn{4}{|c|}{ Kompetenzstufen in PISA 2009} & \multicolumn{4}{|c|}{$\begin{array}{c}\text { Veränderung zwischen } 2006 \text { und } 2009 \\
\text { (PISA 2009 - PISA 2006) }\end{array}$} \\
\hline & & \multicolumn{2}{|c|}{$\begin{array}{c}\begin{array}{c}\text { Unter Stufe } 2 \\
\text { (unter } 410 \text { Punkte) }\end{array} \\
\end{array}$} & \multicolumn{2}{|c|}{$\begin{array}{l}\text { Stufe } 5 \text { oder höher } \\
\text { (über } 633 \text { Punkte) }\end{array}$} & \multicolumn{2}{|c|}{$\begin{array}{c}\text { Unter Stufe } 2 \\
\text { (unter } 410 \text { Punkte) }\end{array}$} & \multicolumn{2}{|c|}{$\begin{array}{c}\text { Stufe } 5 \text { oder höher } \\
\text { (über } 633 \text { Punkte) }\end{array}$} & \multicolumn{2}{|c|}{$\begin{array}{c}\text { Unter Stufe } 2 \\
\text { (unter } 410 \text { Punkte) }\end{array}$} & \multicolumn{2}{|c|}{$\begin{array}{c}\text { Stufe } 5 \text { oder höher } \\
\text { (über } 633 \text { Punkte) }\end{array}$} \\
\hline & & $\%$ & S.E. & $\%$ & S.E. & $\%$ & S.E. & $\%$ & S.E. & Diff. \% & S.E. & Diff. $\%$ & S.E. \\
\hline \multirow{35}{*}{ 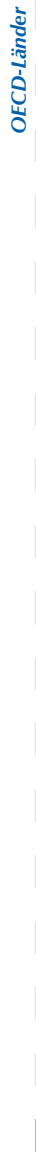 } & Australien & 12.9 & $(0.6)$ & 14.6 & $(0.7)$ & 12.6 & $(0.6)$ & 14.5 & $(0.8)$ & -0.3 & $(0.9)$ & -0.1 & $(1.1)$ \\
\hline & Österreich & 16.3 & (1.4) & 10.0 & $(0.8)$ & $\mathrm{m}$ & $\mathrm{m}$ & $\mathrm{m}$ & $\mathrm{m}$ & $\mathrm{m}$ & $\mathrm{m}$ & $\mathrm{m}$ & $\mathrm{m}$ \\
\hline & Belgien & 17.0 & (1.0) & 10.1 & $(0.5)$ & 18.0 & $(0.8)$ & 10.1 & $(0.7)$ & 1.0 & $(1.3)$ & 0.0 & $(0.9)$ \\
\hline & Kanada & 10.0 & $(0.6)$ & 14.4 & $(0.5)$ & 9.6 & $(0.5)$ & 12.1 & $(0.5)$ & -0.5 & $(0.7)$ & -2.3 & $(0.7)$ \\
\hline & Chile & 39.7 & (2.1) & 1.9 & $(0.3)$ & 32.3 & (1.4) & 1.1 & $(0.2)$ & -7.4 & (2.5) & -0.8 & $(0.4)$ \\
\hline & Tschech. Rep. & 15.5 & $(1.2)$ & 11.6 & $(0.9)$ & 17.3 & $(1.2)$ & 8.4 & $(0.7)$ & 1.8 & (1.6) & -3.2 & $(1.2)$ \\
\hline & Dänemark & 18.4 & (1.1) & 6.8 & $(0.7)$ & 16.6 & $(0.8)$ & 6.7 & $(0.6)$ & -1.9 & (1.4) & -0.1 & $(0.9)$ \\
\hline & Estland & 7.7 & $(0.6)$ & 11.5 & $(0.8)$ & 8.3 & $(0.8)$ & 10.4 & $(0.8)$ & 0.7 & $(1.0)$ & -1.1 & (1.1) \\
\hline & Finnland & 4.1 & $(0.5)$ & 20.9 & (0.8) & 6.0 & (0.5) & 18.7 & (0.9) & 1.9 & $(0.7)$ & -2.2 & $(1.2)$ \\
\hline & Frankreich & 21.2 & (1.4) & 8.0 & $(0.7)$ & 19.3 & (1.3) & 8.1 & $(0.8)$ & -1.9 & (1.9) & 0.1 & (1.0) \\
\hline & Deutschland & 15.4 & (1.3) & 11.8 & $(0.7)$ & 14.8 & $(1.0)$ & 12.8 & $(0.8)$ & -0.6 & $(1.7)$ & 1.0 & $(1.0)$ \\
\hline & Griechenland & 24.0 & (1.3) & 3.4 & (0.4) & 25.3 & (1.6) & 3.1 & $(0.4)$ & 1.2 & $(2.1)$ & -0.4 & $(0.5)$ \\
\hline & Ungarn & 15.0 & (1.0) & 6.9 & $(0.6)$ & 14.1 & (1.4) & 5.4 & $(0.6)$ & -0.9 & $(1.7)$ & -1.5 & $(0.9)$ \\
\hline & Island & 20.6 & $(0.8)$ & 6.3 & $(0.5)$ & 17.9 & $(0.7)$ & 7.0 & $(0.4)$ & -2.6 & $(1.1)$ & 0.6 & $(0.6)$ \\
\hline & Irland & 15.5 & (1.1) & 9.4 & $(0.7)$ & 15.2 & (1.1) & 8.7 & $(0.8)$ & -0.3 & (1.5) & -0.7 & (1.0) \\
\hline & Israel & 36.1 & (1.4) & 5.2 & (0.6) & 33.1 & $(1.2)$ & 3.9 & $(0.4)$ & -3.0 & (1.9) & -1.3 & $(0.7)$ \\
\hline & Italien & 25.3 & $(0.9)$ & 4.6 & $(0.3)$ & 20.6 & $(0.6)$ & 5.8 & $(0.3)$ & -4.6 & (1.1) & 1.2 & $(0.5)$ \\
\hline & Japan & 12.0 & $(1.0)$ & 15.1 & $(0.8)$ & 10.7 & (1.0) & 16.9 & $(0.9)$ & -1.4 & (1.5) & 1.9 & $(1.2)$ \\
\hline & Korea & 11.2 & $(1.1)$ & 10.3 & (1.1) & 6.3 & $(0.8)$ & 11.6 & (1.1) & -4.9 & (1.4) & 1.3 & (1.5) \\
\hline & Luxemburg & 22.1 & $(0.5)$ & 5.9 & (0.4) & 23.7 & $(0.8)$ & 6.7 & $(0.5)$ & 1.6 & (1.0) & 0.8 & $(0.6)$ \\
\hline & Mexiko & 50.9 & (1.4) & 0.3 & $(0.1)$ & 47.4 & $(1.0)$ & 0.2 & $(0.0)$ & -3.6 & $(1.7)$ & -0.1 & $(0.1)$ \\
\hline & Niederlande & 13.0 & $(1.0)$ & 13.1 & $(0.9)$ & 13.2 & (1.6) & 12.7 & $(1.2)$ & 0.2 & (1.9) & -0.4 & (1.5) \\
\hline & Neuseeland & 13.7 & $(0.7)$ & 17.6 & $(0.8)$ & 13.4 & $(0.7)$ & 17.6 & $(0.8)$ & -0.3 & (1.0) & 0.0 & (1.1) \\
\hline & Norwegen & 21.1 & $(1.3)$ & 6.1 & $(0.5)$ & 15.8 & $(0.9)$ & 6.4 & $(0.6)$ & -5.3 & (1.6) & 0.3 & $(0.8)$ \\
\hline & Polen & 17.0 & $(0.8)$ & 6.8 & $(0.5)$ & 13.1 & $(0.8)$ & 7.5 & $(0.5)$ & -3.8 & $(1.2)$ & 0.8 & $(0.7)$ \\
\hline & Portugal & 24.5 & (1.4) & 3.1 & $(0.4)$ & 16.5 & (1.1) & 4.2 & (0.5) & -8.0 & (1.7) & 1.0 & $(0.6)$ \\
\hline & Slowak. Rep. & 20.2 & (1.0) & 5.8 & $(0.5)$ & 19.3 & $(1.2)$ & 6.2 & $(0.6)$ & -0.9 & (1.5) & 0.5 & $(0.8)$ \\
\hline & Slowenien & 13.9 & $(0.6)$ & 12.9 & (0.6) & 14.8 & $(0.5)$ & 9.9 & $(0.6)$ & 0.9 & $(0.8)$ & -3.0 & $(0.9)$ \\
\hline & Spanien & 19.6 & $(0.9)$ & 4.9 & $(0.4)$ & 18.2 & $(0.9)$ & 4.0 & $(0.3)$ & -1.4 & (1.3) & -0.9 & $(0.5)$ \\
\hline & Schweden & 16.4 & $(0.8)$ & 7.9 & $(0.5)$ & 19.1 & $(1.0)$ & 8.1 & (0.6) & 2.8 & $(1.3)$ & 0.2 & $(0.8)$ \\
\hline & Schweiz & 16.1 & $(0.9)$ & 10.5 & $(0.8)$ & 14.0 & $(0.8)$ & 10.7 & $(0.9)$ & -2.0 & $(1.2)$ & 0.3 & $(1.2)$ \\
\hline & Türkei & 46.6 & (1.6) & 0.9 & $(0.3)$ & 30.0 & (1.5) & 1.1 & $(0.3)$ & -16.6 & $(2.2)$ & 0.2 & $(0.5)$ \\
\hline & Ver. Königreich & 16.7 & $(0.8)$ & 13.7 & $(0.6)$ & 15.0 & $(0.8)$ & 11.4 & $(0.7)$ & -1.7 & $(1.1)$ & -2.4 & $(0.9)$ \\
\hline & Ver. Staaten & 24.4 & (1.6) & 9.1 & $(0.7)$ & 18.1 & $(1.1)$ & 9.2 & $(1.0)$ & -6.3 & (1.9) & 0.1 & $(1.2)$ \\
\hline & OECD-33-Durchschnitt & 19.9 & $(0.2)$ & 8.8 & $(0.1)$ & 17.9 & $(0.2)$ & 8.5 & $(0.1)$ & -2.1 & $(0.3)$ & -0.3 & $(0.2)$ \\
\hline \multirow{23}{*}{ 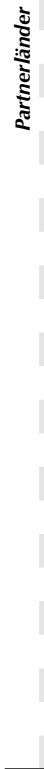 } & Argentinien & 56.3 & (2.5) & 0.4 & $(0.1)$ & 52.4 & (1.9) & 0.7 & $(0.2)$ & -3.8 & (3.2) & 0.2 & $(0.2)$ \\
\hline & Aserbaidschan & 72.5 & (1.9) & 0.0 & c & 70.0 & (1.5) & 0.0 & $(0.0)$ & -2.5 & (2.4) & C & c \\
\hline & Brasilien & 61.0 & (1.4) & 0.6 & $(0.2)$ & 54.2 & $(1.3)$ & 0.6 & $(0.1)$ & -6.8 & (1.9) & 0.0 & $(0.2)$ \\
\hline & Bulgarien & 42.6 & (2.4) & 3.1 & $(0.6)$ & 38.8 & (2.5) & 2.6 & $(0.5)$ & -3.8 & (3.5) & -0.4 & $(0.8)$ \\
\hline & Kolumbien & 60.2 & (1.8) & 0.2 & $(0.1)$ & 54.1 & (1.9) & 0.1 & $(0.1)$ & -6.1 & (2.6) & 0.0 & $(0.1)$ \\
\hline & Kroatien & 17.0 & (0.9) & 5.1 & $(0.5)$ & 18.5 & (1.1) & 3.7 & $(0.6)$ & 1.5 & (1.4) & -1.4 & $(0.8)$ \\
\hline & Hongkong (China) & 8.7 & $(0.8)$ & 15.9 & $(0.9)$ & 6.6 & $(0.7)$ & 16.2 & (1.0) & -2.1 & (1.1) & 0.3 & (1.4) \\
\hline & Indonesien & 61.6 & (3.4) & 0.0 & $\mathrm{C}$ & 65.6 & (2.3) & 0.0 & $\mathrm{c}$ & 4.0 & $(4.1)$ & c & C \\
\hline & Jordanien & 44.3 & $(1.2)$ & 0.6 & $(0.2)$ & 45.6 & (1.7) & 0.5 & $(0.2)$ & 1.3 & $(2.1)$ & -0.2 & $(0.3)$ \\
\hline & Kirgisistan & 86.3 & $(1.0)$ & 0.0 & $\mathrm{C}$ & 82.0 & (1.1) & 0.0 & $(0.0)$ & -4.4 & (1.5) & c & c \\
\hline & Lettland & 17.4 & $(1.2)$ & 4.1 & $(0.4)$ & 14.7 & $(1.2)$ & 3.1 & $(0.5)$ & -2.7 & $(1.7)$ & -1.0 & (0.6) \\
\hline & Liechtenstein & 12.9 & $(2.2)$ & 12.2 & (1.7) & 11.3 & (1.9) & 9.7 & (1.8) & -1.6 & (2.9) & -2.5 & (2.5) \\
\hline & Litauen & 20.3 & (1.0) & 5.0 & $(0.7)$ & 17.0 & (1.1) & 4.6 & $(0.5)$ & -3.3 & (1.5) & -0.4 & $(0.8)$ \\
\hline & Macau (China) & 10.3 & $(0.5)$ & 5.3 & $(0.4)$ & 9.6 & $(0.4)$ & 4.8 & $(0.5)$ & -0.7 & $(0.7)$ & -0.5 & (0.6) \\
\hline & Montenegro & 50.2 & $(0.9)$ & 0.3 & $(0.1)$ & 53.6 & (1.0) & 0.2 & $(0.1)$ & 3.3 & (1.4) & 0.0 & $(0.2)$ \\
\hline & Katar & 79.1 & $(0.4)$ & 0.3 & $(0.1)$ & 65.2 & $(0.6)$ & 1.4 & $(0.1)$ & -13.9 & $(0.7)$ & 1.1 & $(0.2)$ \\
\hline & Rumänien & 46.9 & (2.4) & 0.5 & $(0.1)$ & 41.4 & $(2.1)$ & 0.4 & $(0.1)$ & -5.5 & $(3.2)$ & -0.1 & $(0.2)$ \\
\hline & Russ. Föderation & 22.2 & (1.4) & 4.2 & $(0.5)$ & 22.0 & (1.4) & 4.4 & $(0.5)$ & -0.2 & (2.0) & 0.2 & $(0.7)$ \\
\hline & Serbien & 38.5 & (1.6) & 0.8 & $(0.2)$ & 34.4 & (1.3) & 1.0 & $(0.2)$ & -4.1 & $(2.0)$ & 0.2 & $(0.3)$ \\
\hline & Chinesisch Taipeh & 11.6 & $(1.0)$ & 14.6 & $(0.9)$ & 11.1 & $(0.7)$ & 8.8 & $(0.9)$ & -0.6 & $(1.2)$ & -5.8 & $(1.2)$ \\
\hline & Thailand & 46.1 & $(1.2)$ & 0.4 & $(0.1)$ & 42.8 & (1.6) & 0.6 & $(0.3)$ & -3.3 & $(2.0)$ & 0.2 & $(0.3)$ \\
\hline & Tunesien & 62.8 & (1.4) & 0.1 & $(0.1)$ & 53.7 & (1.4) & 0.2 & $(0.1)$ & -9.0 & (2.0) & 0.0 & $(0.1)$ \\
\hline & Uruguay & 42.1 & (1.4) & 1.4 & $(0.2)$ & 42.6 & (1.1) & 1.5 & $(0.2)$ & 0.4 & (1.8) & 0.1 & (0.3) \\
\hline
\end{tabular}

Anmerkung: Statistisch signifikante Werte sind durch Fettdruck gekennzeichnet (siehe Anhang A3).

StatLink הत्नाज http://dx.doi.org/10.1787/888932343285 
[Teil 1/1]

Varianz der Schülerleistungen im Bereich Lesekompetenz innerhalb und zwischen den Schulen, Tabelle V.4.1 PISA 2000 und PISA 2009

\begin{tabular}{|c|c|c|c|c|c|c|c|c|c|c|c|c|c|c|c|}
\hline & \multicolumn{3}{|c|}{ PISA 2000} & \multicolumn{3}{|c|}{ PISA 2009} & \multicolumn{5}{|c|}{$\begin{array}{l}\text { Veränderung zwischen } 2000 \text { und } 2009 \\
\text { (PISA 2009 - PISA 2000) }\end{array}$} & \multicolumn{3}{|c|}{$\begin{array}{c}\text { Veränderung zwischen } 2000 \\
\text { und } 2009 \text { in \% der Varianz } \\
\text { von } 2000 \text { (PISA } 2009 \text { - PISA } \\
2000 \text { )/PISA } 2000\end{array}$} \\
\hline & & \multirow{2}{*}{$\begin{array}{l}\text { Gesamt- } \\
\text { varianz }\end{array}$} & \multirow{2}{*}{$\begin{array}{c}\text { Varianz } \\
\text { zwischen } \\
\text { den } \\
\text { Schulen }\end{array}$} & \multirow{2}{*}{$\begin{array}{l}\text { Varianz } \\
\text { inner- } \\
\text { halb der } \\
\text { Schulen }\end{array}$} & \multirow[b]{2}{*}{$\begin{array}{l}\text { Gesamt- } \\
\text { varianz }\end{array}$} & \multirow{2}{*}{$\begin{array}{c}\text { Varianz } \\
\text { zwischen } \\
\text { den } \\
\text { Schulen }\end{array}$} & \multirow{2}{*}{$\begin{array}{l}\text { Varianz } \\
\text { inner- } \\
\text { halb der } \\
\text { Schulen }\end{array}$} & $\begin{array}{l}\text { Gesamt- } \\
\text { varianz }\end{array}$ & \multicolumn{2}{|c|}{$\begin{array}{l}\text { Varianz } \\
\text { zwischen den } \\
\text { Schulen }\end{array}$} & \multicolumn{2}{|c|}{$\begin{array}{c}\text { Varianz } \\
\text { innerhalb der } \\
\text { Schulen }\end{array}$} & \multirow{2}{*}{\begin{tabular}{|c|}
$\begin{array}{c}\text { Gesamt- } \\
\text { varianz }\end{array}$ \\
$\begin{array}{c}\text { Veränd. } \\
\text { in \% }\end{array}$ \\
\end{tabular}} & \multirow{2}{*}{\begin{tabular}{|c|}
$\begin{array}{c}\text { Varianz } \\
\text { zwischen } \\
\text { den } \\
\text { Schulen }\end{array}$ \\
$\begin{array}{c}\text { Veränd. } \\
\text { in \% }\end{array}$ \\
\end{tabular}} & \multirow{2}{*}{\begin{tabular}{|c|}
$\begin{array}{c}\text { Varianz } \\
\text { inner- } \\
\text { halb der } \\
\text { Schulen }\end{array}$ \\
$\begin{array}{c}\text { Veränd. } \\
\text { in \% }\end{array}$ \\
\end{tabular}} \\
\hline & & & & & & & & Diff. S.E. & Diff. & S.E. & Diff. & S.E. & & & \\
\hline \multirow{31}{*}{ 离 } & Australien & 10357 & 2221 & 8850 & 9783 & 2692 & 7631 & $-574(297)$ & 471 & (490) & -1219 & (288) & -5.5 & 21.2 & -13.8 \\
\hline & Österreich & 9703 & 6046 & 4408 & m & $\mathrm{m}$ & m & $\mathrm{m} \quad \mathrm{m}$ & $\mathrm{m}$ & $\mathrm{m}$ & $\mathrm{m}$ & $\mathrm{m}$ & $\mathrm{m}$ & $\mathrm{m}$ & $\mathrm{m}$ \\
\hline & Belgien & 11454 & 5797 & 4702 & 10360 & 5343 & 4833 & -1 094 (305) & -453 & (604) & 132 & (222) & -9.6 & -7.8 & 2.8 \\
\hline & Kanada & 8954 & 1934 & 7632 & 8163 & 1877 & 6780 & -791 (163) & -57 & (242) & -853 & (158) & -8.8 & -2.9 & -11.2 \\
\hline & Chile & 8074 & 4081 & 3981 & 6833 & 4893 & 4005 & -1241 & 813 & (729) & 24 & (154) & -15.4 & 19.9 & 0.6 \\
\hline & Tschech. Rep. & 9277 & 4651 & 4152 & 8516 & 4249 & 4428 & \begin{tabular}{|l|}
-761 \\
\end{tabular} & -402 & (725) & 276 & (204) & -8.2 & -8.6 & 6.7 \\
\hline & Dänemark & 9615 & 1472 & 8068 & 6987 & 1134 & 6012 & -2628 & -338 & (430) & -2056 & (274) & -27.3 & -22.9 & -25.5 \\
\hline & Finnland & 7994 & 591 & 7117 & 7467 & 665 & 6993 & $-526(327)$ & 74 & (198) & -124 & (279) & -6.6 & 12.5 & -1.7 \\
\hline & Frankreich & w & w & w & w & w & w & w w & w & w & w & w & w & w & w \\
\hline & Deutschland & 12367 & 6667 & 4717 & 8978 & 5890 & 3890 & -3 389 (485) & -777 & (950) & -827 & (197) & -27.4 & -11.7 & -17.5 \\
\hline & Griechenland & 9436 & 4762 & 4984 & 9054 & 4745 & 5558 & $-383(355)$ & -17 & (924) & 574 & (239) & -4.1 & -0.4 & 11.5 \\
\hline & Ungarn & 8810 & 5571 & 3275 & 8133 & 5846 & 2923 & $\mathbf{- 6 7 8}(344)$ & 275 & (749) & -352 & (129) & -7.7 & 4.9 & -10.8 \\
\hline & Island & 8529 & 732 & 7805 & 9211 & 1348 & 8186 & $682(340)$ & 616 & (429) & 381 & (365) & 8.0 & 84.1 & 4.9 \\
\hline & Irland & 8756 & 1593 & 7181 & 9053 & 2805 & 6966 & $297 \quad(340)$ & 1211 & $(587)$ & -215 & (324) & 3.4 & 76.0 & -3.0 \\
\hline & Israel & 11909 & 5923 & 6634 & 12438 & 6250 & 6615 & 529 (467) & 327 & (1 463) & -19 & (388) & 4.4 & 5.5 & -0.3 \\
\hline & Italien & 8355 & 4453 & 4001 & 9193 & 6695 & 4085 & 838 (282) & 2242 & (1 011) & 84 & (145) & 10.0 & 50.3 & 2.1 \\
\hline & Japan & 7359 & 3378 & 3907 & 10072 & 5087 & 5386 & 2713 & 1709 & (833) & 1480 & (240) & 36.9 & 50.6 & 37.9 \\
\hline & Korea & 4834 & 1937 & 3087 & 6271 & 2741 & 5283 & 1437 (209) & 804 & (724) & 2196 & (530) & 29.7 & 41.5 & 71.1 \\
\hline & Luxemburg & $\mathrm{m}$ & $\mathrm{m}$ & $\mathrm{m}$ & 10759 & 5335 & 6906 & $\mathrm{~m}$ & $\mathrm{~m}$ & $\mathrm{~m}$ & $\mathrm{~m}$ & $\mathrm{~m}$ & $\mathrm{~m}$ & $\mathrm{~m}$ & $\mathrm{~m}$ \\
\hline & Mexiko & 7371 & 3907 & 3484 & 7158 & 3583 & 3869 & $-213(235)$ & -324 & (504) & 385 & (142) & -2.9 & -8.3 & 11.1 \\
\hline & Niederlande & $\mathrm{m}$ & m & $\mathrm{m}$ & 7857 & 5107 & 2795 & $\mathrm{~m} \quad \mathrm{~m}$ & $\mathrm{~m}$ & $\mathrm{~m}$ & $\mathrm{~m}$ & $\mathrm{~m}$ & $\mathrm{~m}$ & $\mathrm{~m}$ & $\mathrm{~m}$ \\
\hline & Neuseeland & 11700 & 1867 & 9765 & 10575 & 2622 & 8228 & -1124 (428) & 755 & (576) & -1537 & (441) & -9.6 & 40.4 & -15.7 \\
\hline & Norwegen & 10743 & 1040 & 9753 & 8310 & 874 & 7598 & -2433 & -167 & (276) & -2155 & (373) & -22.6 & -16.0 & -22.1 \\
\hline & Polen & 9958 & 6125 & 3712 & 7950 & 1585 & 6869 & -2 008 & -4540 & (710) & 3157 & (261) & -20.2 & -74.1 & 85.1 \\
\hline & Portugal & 9436 & 3536 & 5855 & 7534 & 2565 & 5191 & -1902 (278) & -971 & $(527)$ & -664 & $(270)$ & -20.2 & -27.4 & -11.3 \\
\hline & Spanien & 7180 & 1533 & 5662 & 7658 & 1690 & 6048 & 478 (231) & 158 & (212) & 385 & (209) & 6.7 & 10.3 & 6.8 \\
\hline & Schweden & 8495 & 786 & 7729 & 9729 & 1877 & 8290 & 1234 & 1090 & (398) & 561 & (309) & 14.5 & 138.6 & 7.3 \\
\hline & Schweiz & 10409 & 4340 & 5867 & 8735 & 2740 & 5652 & -1674 (286) & -1600 & (521) & -215 & (264) & -16.1 & -36.9 & -3.7 \\
\hline & Ver. Königreich & $\mathrm{m}$ & $\mathrm{m}$ & $\mathrm{m}$ & 9096 & 2775 & 6684 & $\mathrm{~m}$ & $\mathrm{~m}$ & $\mathrm{~m}$ & $\mathrm{~m}$ & $\mathrm{~m}$ & $\mathrm{~m}$ & $\mathrm{~m}$ & $\mathrm{~m}$ \\
\hline & Ver. Staaten & 10979 & 3306 & 7846 & 9330 & 3638 & 6476 & -1649 (386) & 332 & (1 130) & -1370 & (345) & -15.0 & 10.0 & -17.5 \\
\hline & OECD-26-Durchschnitt & 9260 & 3324 & 5922 & 8793 & 3420 & 5875 & $-467 \quad(64)$ & 96 & $(140)$ & -47 & (56) & -3.1 & 14.6 & 4.3 \\
\hline \multirow{12}{*}{ 离 } & Albanien & 9882 & 3915 & 5946 & 9969 & 3127 & 7105 & $87(370)$ & -788 & $(654)$ & 1159 & (336) & 0.9 & -20.1 & 19.5 \\
\hline & Argentinien & 11800 & 5885 & 5763 & 11714 & 8456 & 5523 & $-85(558)$ & 2572 & (1 258) & -240 & (300) & -0.7 & 43.7 & -4.2 \\
\hline & Brasilien & 7427 & 3651 & 4206 & 8838 & 4417 & 4702 & 1410 & 766 & $(520)$ & 496 & (162) & 19.0 & 21.0 & 11.8 \\
\hline & Bulgarien & 10332 & 5435 & 4795 & 12823 & 6418 & 6439 & 2491 (407) & 983 & $(945)$ & 1644 & (273) & 24.1 & 18.1 & 34.3 \\
\hline & Hongkong (China) & 7050 & 3357 & 3646 & 7058 & 3143 & 4360 & $8(275)$ & -214 & (556) & 714 & (224) & 0.1 & -6.4 & 19.6 \\
\hline & Indonesien & 5246 & 2117 & 2785 & 4418 & 1749 & 2298 & $\mathbf{- 8 2 8}(172)$ & -368 & (300) & -487 & (97) & -15.8 & -17.4 & -17.5 \\
\hline & Lettland & 10434 & 3121 & 7297 & 6394 & 1391 & 5200 & -4041 & -1730 & (498) & -2096 & (306) & -38.7 & -55.4 & -28.7 \\
\hline & Liechtenstein & 9254 & 3581 & 4293 & 6896 & 2944 & 3453 & -2357 (981) & -637 & (1 625) & -840 & (631) & -25.5 & -17.8 & -19.6 \\
\hline & Peru & 9243 & 4906 & 4368 & 9670 & 5886 & 4623 & 426 (328) & 980 & (1 067$)$ & 254 & (192) & 4.6 & 20.0 & 5.8 \\
\hline & Rumänien & 10438 & 4922 & 5624 & 8105 & 4057 & 3832 & -2 333 (484) & -865 & (851) & -1792 & (239) & -22.4 & -17.6 & -31.9 \\
\hline & Russ. Föderation & 8465 & 3238 & 5221 & 8050 & 1965 & 5826 & $-416(277)$ & -1273 & $(472)$ & 605 & (241) & -4.9 & -39.3 & 11.6 \\
\hline & Thailand & 5871 & 1918 & 4212 & 5164 & 1231 & 3052 & $\mathbf{- 7 0 7}(193)$ & -687 & (328) & -1161 & (176) & -12.0 & -35.8 & -27.6 \\
\hline
\end{tabular}

Anmerkung: Statistisch signifikante Werte sind durch Fettdruck gekennzeichnet (siehe Anhang A3).

StatLink הiाls http://dx.doi.org/10.1787/888932343285 
[Teil 1/1]

Sozioökonomischer Hintergrund der Schüler, PISA 2000 und PISA 2009

Tabelle V.4.2 Auf Grundlage der Schülerangaben



Anmerkung: Statistisch signifikante Werte sind durch Fettdruck gekennzeichnet (siehe Anhang A3).

StatLink *ills http://dx.doi.org/10.1787/888932343285 
[Teil 1/1]

Zusammenhang zwischen Schülerleistungen im Bereich Lesekompetenz und PISA-Index des wirtschaftlichen, sozialen und kulturellen Status (ESCS), PISA 2000 und PISA 2009

Tabelle V.4.3 Auf Grundlage der Schülerangaben

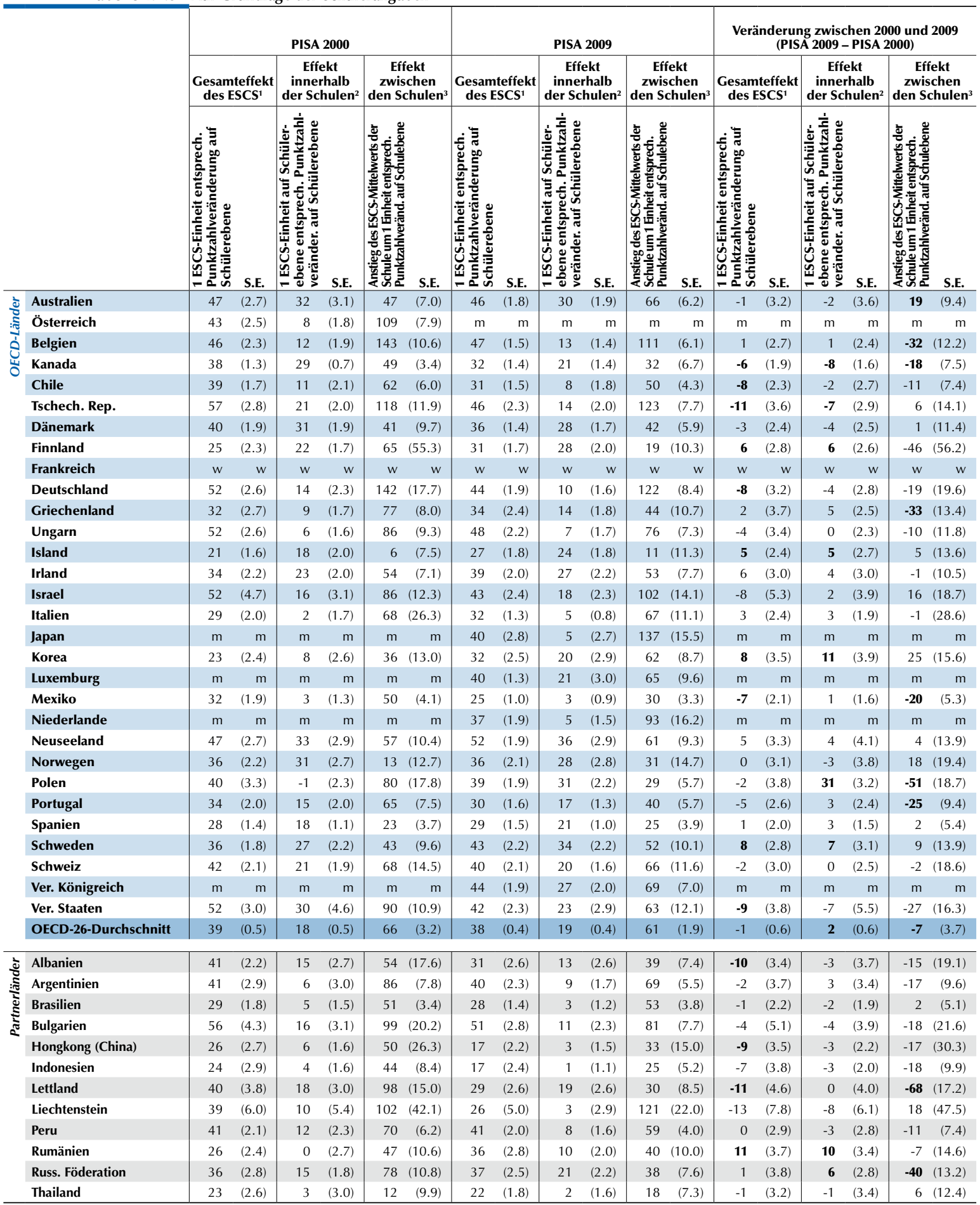

Anmerkung: Statistisch signifikante Werte sind durch Fettdruck gekennzeichnet (siehe Anhang A3).

1. Einstufige bivariate Regression der Leistungen im Bereich Lesekompetenz auf den Index des wirtschaftlichen, sozialen und kulturellen Status (ESCS), wobei die Steigung der sozioökonomischen Gradienten dem ESCS-Regressionskoeffizienten entspricht.

2. Zweistufige Regression der Leistungen im Bereich Lesekompetenz auf den ESCS der Schüler und den ESCS-Mittelwert der Schulen: innerschulische Steigung des ESCS auf Schülerebene.

3. Zweistufige Regression der Leistungen im Bereich Lesekompetenz auf den ESCS der Schüler und den ESCS-Mittelwert der Schulen: zwischenschulische Steigung des ESCS auf Schulebenel.

StatLink *iाls http://dx.doi.org/10.1787/888932343285 
[Teil 1/1]

Schülerleistungen im Bereich Lesekompetenz nach Migrationsstatus, PISA 2000 und PISA 2009 Tabelle V.4.4 Auf Grundlage der Schülerangaben

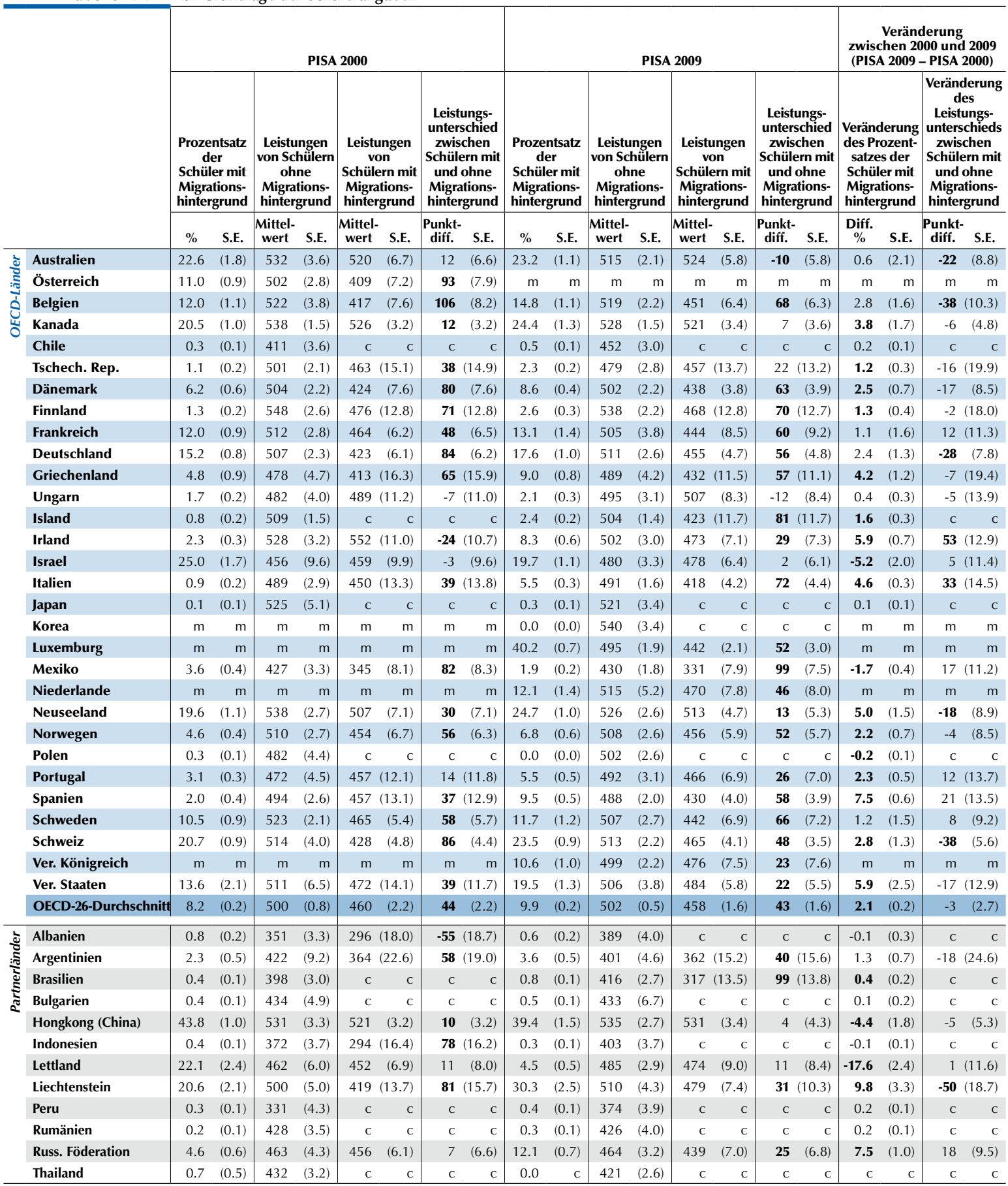

Anmerkung: Statistisch signifikante Werte sind durch Fettdruck gekennzeichnet (siehe Anhang A3).

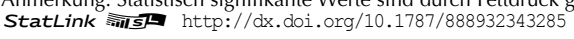


[Teil 1/1]

Schülerleistungen im Bereich Lesekompetenz, nach im Elternhaus gesprochener Sprache,

PISA 2000 und PISA 2009

Tabelle V.4.5 Auf Grundlage der Schülerangaben

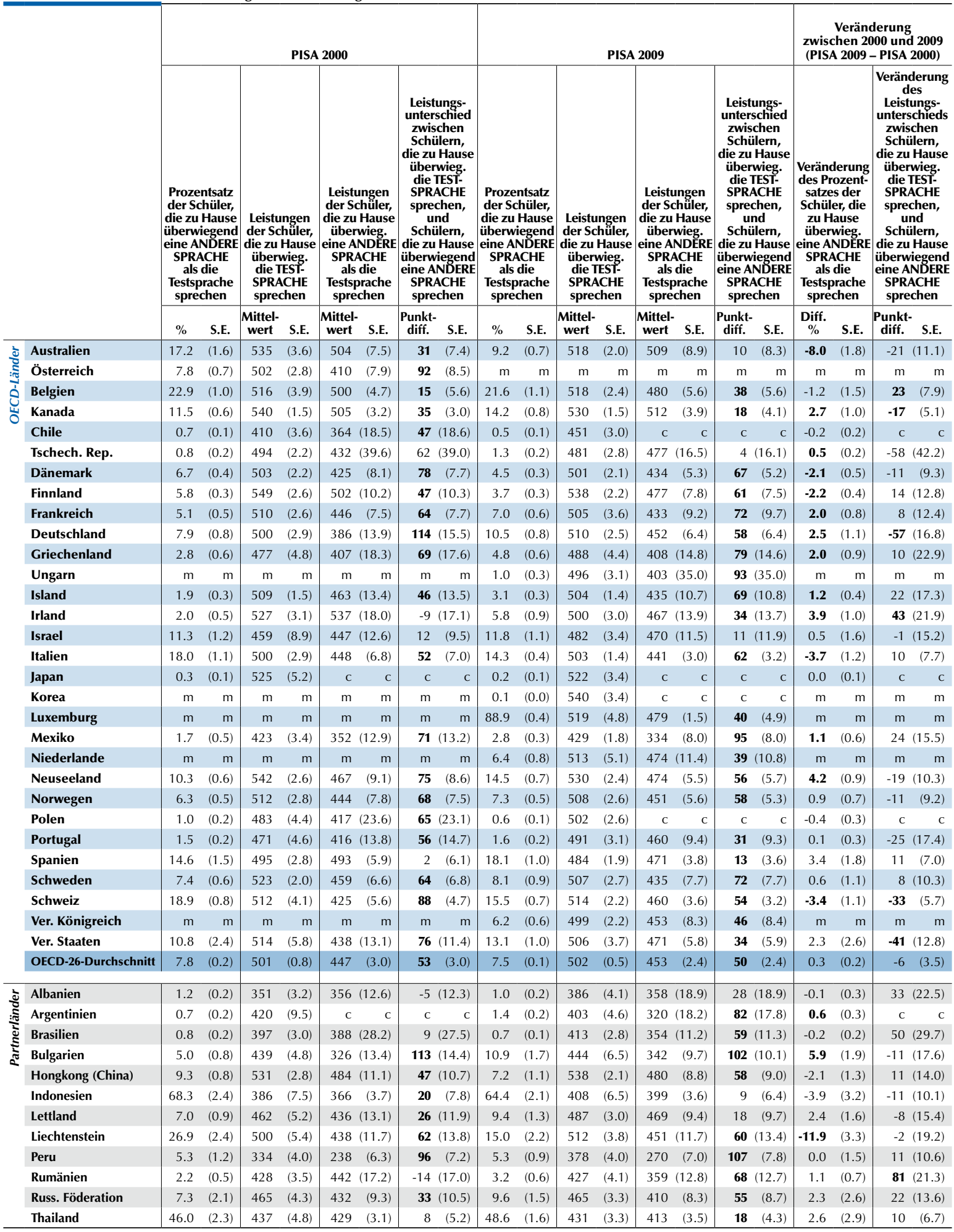

Anmerkung: Statistisch signifikante Werte sind durch Fettdruck gekennzeichnet (siehe Anhang A3).

StatLink ज्ञात http://dx.doi.org/10.1787/888932343285 
[Teil 1/1]

Prozentsatz der Schüler in PISA 2000 und PISA 2009, die zum Vergnügen lesen, nach Geschlecht Tabelle V.5.1 Auf Grundlage der Schülerangaben

\begin{tabular}{|c|c|c|c|c|c|c|c|c|c|c|c|c|c|c|c|c|c|c|c|}
\hline & \multicolumn{6}{|c|}{ PISA 2000} & \multicolumn{6}{|c|}{ PISA 2009} & \multicolumn{6}{|c|}{$\begin{array}{c}\text { Veränderung zwischen } 2000 \text { und } 2009 \\
\text { (PISA 2009 - PISA 2000) }\end{array}$} \\
\hline & & \multicolumn{2}{|c|}{ Alle Schüler } & \multicolumn{2}{|c|}{ Mädchen } & \multicolumn{2}{|c|}{ Jungen } & \multicolumn{2}{|c|}{ Alle Schüler } & \multicolumn{2}{|c|}{ Mädchen } & \multicolumn{2}{|c|}{ Jungen } & \multicolumn{2}{|c|}{ Alle Schüler } & \multicolumn{2}{|c|}{ Mädchen } & \multicolumn{2}{|c|}{ Jungen } \\
\hline & & $\%$ & S.E. & $\%$ & S.E. & $\%$ & S.E. & $\%$ & S.E. & $\%$ & S.E. & $\%$ & S.E. & Diff. \% & S.E. & Diff. \% & S.E. & Diff. \% & S.E. \\
\hline \multirow{31}{*}{ 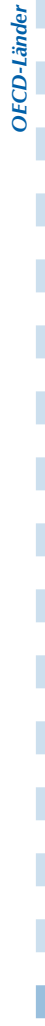 } & Australien & 66.9 & $(1.2)$ & 74.6 & (1.5) & 59.9 & (1.7) & 63.3 & $(0.6)$ & 73.1 & $(0.8)$ & 53.0 & $(0.8)$ & -3.6 & (1.3) & -1.5 & (1.6) & -6.9 & (1.9) \\
\hline & Österreich & 56.5 & $(1.2)$ & 68.6 & (1.1) & 44.9 & (1.3) & $\mathrm{m}$ & $\mathrm{m}$ & $\mathrm{m}$ & $\mathrm{m}$ & $\mathrm{m}$ & $\mathrm{m}$ & $\mathrm{m}$ & $\mathrm{m}$ & $\mathrm{m}$ & $\mathrm{m}$ & $\mathrm{m}$ & $\mathrm{m}$ \\
\hline & Belgien & 57.8 & $(0.9)$ & 69.4 & $(0.7)$ & 46.9 & (1.4) & 55.6 & $(0.8)$ & 65.4 & (1.0) & 46.2 & (1.0) & -2.2 & (1.2) & -4.1 & $(1.2)$ & -0.7 & (1.7) \\
\hline & Kanada & 67.3 & $(0.4)$ & 77.0 & $(0.5)$ & 57.4 & $(0.7)$ & 68.9 & $(0.5)$ & 81.6 & $(0.5)$ & 56.2 & $(0.8)$ & 1.6 & $(0.7)$ & 4.5 & $(0.7)$ & -1.3 & (1.0) \\
\hline & Chile & 73.7 & $(0.8)$ & 79.3 & (1.0) & 67.3 & (1.1) & 60.3 & $(0.8)$ & 70.3 & $(0.9)$ & 50.7 & (1.0) & -13.4 & (1.1) & -9.0 & (1.3) & -16.6 & (1.5) \\
\hline & Tschech. Rep. & 73.8 & $(0.8)$ & 84.9 & $(0.7)$ & 61.3 & (1.4) & 57.0 & $(0.8)$ & 71.5 & $(1.2)$ & 44.3 & $(1.0)$ & -16.7 & $(1.2)$ & -13.4 & (1.4) & -17.0 & (1.7) \\
\hline & Dänemark & 73.3 & $(0.8)$ & 82.6 & (1.0) & 64.2 & (1.3) & 66.4 & $(0.9)$ & 75.3 & (1.1) & 57.3 & (1.1) & -6.9 & (1.2) & -7.3 & (1.5) & -6.8 & (1.7) \\
\hline & Finnland & 77.6 & $(0.7)$ & 89.7 & (0.6) & 64.7 & $(1.1)$ & 67.0 & $(0.8)$ & 80.6 & (1.0) & 53.3 & $(1.1)$ & -10.7 & (1.0) & -9.2 & $(1.2)$ & -11.4 & (1.6) \\
\hline & Frankreich & 70.0 & $(0.8)$ & 78.8 & $(0.9)$ & 60.5 & (1.1) & 61.2 & (1.0) & 69.8 & (1.3) & 52.1 & (1.3) & -8.8 & (1.3) & -9.0 & (1.6) & -8.4 & (1.7) \\
\hline & Deutschland & 58.2 & $(0.9)$ & 70.9 & $(0.9)$ & 45.5 & $(1.2)$ & 58.7 & $(0.9)$ & 72.5 & (1.1) & 45.1 & $(1.1)$ & 0.5 & (1.2) & 1.6 & (1.4) & -0.4 & (1.6) \\
\hline & Griechenland & 78.0 & $(0.8)$ & 80.6 & $(0.9)$ & 75.4 & (1.4) & 82.5 & $(0.8)$ & 88.4 & (0.9) & 76.4 & (1.1) & 4.5 & (1.1) & 7.8 & (1.3) & 1.0 & (1.8) \\
\hline & Ungarn & 73.9 & $(0.9)$ & 81.2 & $(1.0)$ & 66.7 & $(1.2)$ & 74.5 & $(0.8)$ & 83.5 & (0.9) & 65.7 & $(1.2)$ & 0.6 & (1.2) & 2.3 & (1.4) & -1.0 & (1.7) \\
\hline & Island & 70.2 & $(0.7)$ & 77.3 & (1.0) & 63.0 & (1.0) & 62.0 & $(0.8)$ & 72.3 & (1.0) & 51.5 & (1.3) & -8.2 & (1.0) & -5.0 & (1.5) & -11.5 & (1.7) \\
\hline & Irland & 66.6 & $(0.9)$ & 75.5 & (1.0) & 57.6 & (1.4) & 58.1 & (1.0) & 63.8 & (1.3) & 52.5 & (1.4) & -8.5 & (1.3) & -11.7 & (1.6) & -5.1 & (1.9) \\
\hline & Israel & 63.0 & (2.4) & 70.1 & (2.9) & 51.9 & (2.0) & 65.5 & (0.9) & 75.1 & (1.0) & 55.2 & (1.5) & 2.5 & (2.6) & 5.0 & (3.0) & 3.3 & (2.5) \\
\hline & Italien & 69.4 & (1.1) & 76.7 & (1.1) & 62.0 & (1.3) & 66.1 & (0.6) & 79.0 & (0.6) & 53.9 & $(0.8)$ & -3.3 & (1.2) & 2.3 & (1.3) & -8.1 & (1.5) \\
\hline & Japan & 45.0 & (1.2) & 45.1 & (1.5) & 44.8 & (1.6) & 55.8 & $(0.9)$ & 58.2 & (1.3) & 53.6 & (1.1) & 10.9 & (1.6) & 13.1 & (2.0) & 8.8 & (1.9) \\
\hline & Korea & 69.4 & $(0.8)$ & 70.3 & (1.4) & 68.8 & $(1.2)$ & 61.5 & (0.8) & 62.6 & (1.4) & 60.5 & $(1.0)$ & -8.0 & (1.2) & -7.7 & (2.0) & -8.3 & (1.5) \\
\hline & Luxemburg & $\mathrm{m}$ & m & $\mathrm{m}$ & $\mathrm{m}$ & $\mathrm{m}$ & $\mathrm{m}$ & 51.8 & $(0.8)$ & 64.2 & (1.0) & 39.6 & (1.1) & $\mathrm{m}$ & $\mathrm{m}$ & $\mathrm{m}$ & $\mathrm{m}$ & $\mathrm{m}$ & $\mathrm{m}$ \\
\hline & Mexiko & 86.4 & $(0.7)$ & 91.1 & $(0.8)$ & 81.6 & (1.1) & 76.2 & $(0.4)$ & 82.8 & $(0.4)$ & 69.5 & $(0.7)$ & -10.2 & $(0.8)$ & -8.3 & $(0.9)$ & -12.1 & (1.3) \\
\hline & Niederlande & $\mathrm{m}$ & $\mathrm{m}$ & $\mathrm{m}$ & $\mathrm{m}$ & $\mathrm{m}$ & $\mathrm{m}$ & 51.4 & (1.3) & 66.8 & (1.4) & 35.8 & (1.5) & $\mathrm{m}$ & $\mathrm{m}$ & $\mathrm{m}$ & $\mathrm{m}$ & $\mathrm{m}$ & $\mathrm{m}$ \\
\hline & Neuseeland & 70.1 & $(0.9)$ & 76.9 & (1.0) & 63.2 & (1.3) & 68.7 & (0.8) & 78.3 & $(1.0)$ & 59.4 & (1.1) & -1.4 & (1.2) & 1.4 & (1.4) & -3.8 & (1.7) \\
\hline & Norwegen & 64.6 & $(0.8)$ & 75.3 & (1.1) & 54.4 & (1.3) & 60.0 & $(0.9)$ & 70.0 & (1.1) & 50.4 & (1.1) & -4.6 & $(1.2)$ & -5.3 & (1.6) & -4.0 & (1.7) \\
\hline & Polen & 75.8 & (1.1) & 83.9 & (1.0) & 67.8 & (1.8) & 67.8 & $(0.8)$ & 82.5 & $(0.9)$ & 53.1 & (1.3) & -8.0 & (1.4) & -1.3 & (1.3) & -14.6 & (2.2) \\
\hline & Portugal & 81.6 & $(0.8)$ & 91.7 & (0.6) & 70.6 & (1.3) & 64.8 & $(0.7)$ & 78.7 & $(0.8)$ & 50.2 & (1.0) & -16.8 & (1.1) & -13.0 & (1.0) & -20.4 & (1.7) \\
\hline & Spanien & 68.2 & $(0.9)$ & 77.6 & (1.1) & 58.5 & $(1.2)$ & 60.4 & $(0.7)$ & 70.0 & $(0.8)$ & 51.0 & $(0.9)$ & -7.9 & (1.1) & -7.6 & (1.4) & -7.5 & (1.5) \\
\hline & Schweden & 64.0 & $(1.0)$ & 73.0 & (1.3) & 55.1 & $(1.2)$ & 62.7 & $(0.9)$ & 75.0 & (1.0) & 50.7 & (1.1) & -1.3 & (1.3) & 2.0 & $(1.7)$ & -4.5 & (1.6) \\
\hline & Schweiz & 64.9 & (1.1) & 78.5 & (1.1) & 51.1 & (1.6) & 55.4 & $(0.9)$ & 67.6 & $(1.0)$ & 43.6 & $(1.1)$ & -9.5 & (1.4) & -10.9 & (1.5) & -7.6 & (1.9) \\
\hline & Ver. Königreich & $\mathrm{m}$ & $\mathrm{m}$ & $\mathrm{m}$ & $\mathrm{m}$ & $\mathrm{m}$ & $\mathrm{m}$ & 60.4 & $(0.9)$ & 69.7 & $(1.1)$ & 50.7 & (1.0) & $\mathrm{m}$ & $\mathrm{m}$ & $\mathrm{m}$ & $\mathrm{m}$ & $\mathrm{m}$ & $\mathrm{m}$ \\
\hline & Ver. Staaten & 59.3 & $(1.3)$ & 68.0 & (1.5) & 49.9 & (1.8) & 58.0 & $(1.0)$ & 69.2 & $(1.3)$ & 47.4 & $(1.2)$ & -1.3 & (1.7) & 1.2 & $(2.0)$ & -2.5 & $(2.2)$ \\
\hline & OECD-26-Durchschnitt & 68.8 & $(0.2)$ & 76.9 & $(0.2)$ & 60.4 & $(0.3)$ & 63.8 & $(0.2)$ & 73.7 & $(0.2)$ & 54.0 & $(0.2)$ & -5.0 & $(0.3)$ & -3.2 & $(0.3)$ & -6.4 & $(0.3)$ \\
\hline \multirow{12}{*}{ 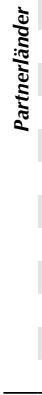 } & Albanien & 91.4 & $(0.6)$ & 93.4 & $(0.6)$ & 88.9 & (1.1) & 92.6 & $(0.5)$ & 97.4 & $(0.4)$ & 88.0 & $(0.8)$ & 1.2 & $(0.8)$ & 4.0 & $(0.7)$ & -0.9 & (1.4) \\
\hline & Argentinien & 70.7 & $(0.9)$ & 77.1 & $(1.2)$ & 62.2 & (1.3) & 58.3 & (1.0) & 65.8 & (1.3) & 49.4 & $(1.2)$ & -12.4 & (1.3) & -11.3 & (1.8) & -12.8 & (1.8) \\
\hline & Brasilien & 80.7 & $(1.0)$ & 87.2 & $(0.9)$ & 72.9 & (1.4) & 78.2 & $(0.6)$ & 86.6 & $(0.5)$ & 68.7 & (1.0) & -2.5 & (1.1) & -0.6 & (1.1) & -4.2 & (1.7) \\
\hline & Bulgarien & 68.7 & $(1.0)$ & 76.9 & $(1.3)$ & 60.2 & $(1.2)$ & 72.0 & (1.3) & 82.7 & $(1.1)$ & 61.9 & (1.6) & 3.3 & $(1.7)$ & 5.8 & $(1.7)$ & 1.7 & (2.0) \\
\hline & Hongkong (China) & 75.9 & $(0.9)$ & 80.0 & $(0.9)$ & 71.8 & (1.3) & 80.5 & $(0.6)$ & 84.9 & $(0.9)$ & 76.5 & $(0.8)$ & 4.6 & (1.1) & 5.0 & (1.3) & 4.7 & (1.5) \\
\hline & Indonesien & 86.5 & (1.1) & 88.1 & (1.5) & 84.7 & (1.1) & 87.9 & (0.6) & 92.2 & $(0.6)$ & 83.4 & $(0.9)$ & 1.3 & $(1.2)$ & 4.1 & (1.6) & -1.3 & (1.4) \\
\hline & Lettland & 82.1 & (1.1) & 90.5 & $(0.9)$ & 73.2 & (1.6) & 70.3 & $(0.9)$ & 85.2 & $(0.9)$ & 55.1 & (1.5) & -11.8 & (1.4) & -5.3 & (1.3) & -18.2 & (2.2) \\
\hline & Liechtenstein & 59.5 & $(2.7)$ & 68.5 & (3.9) & 51.5 & (3.9) & 48.0 & (2.4) & 58.4 & (3.7) & 38.8 & (3.4) & -11.5 & (3.7) & -10.1 & (5.4) & -12.7 & (5.2) \\
\hline & Peru & 91.3 & $(0.6)$ & 92.2 & $(0.7)$ & 90.4 & $(0.9)$ & 86.3 & $(0.5)$ & 89.1 & $(0.6)$ & 83.5 & $(0.8)$ & -5.0 & $(0.8)$ & -3.1 & $(0.9)$ & -6.8 & (1.2) \\
\hline & Rumänien & 81.0 & $(0.8)$ & 83.4 & (1.0) & 78.2 & (1.3) & 75.7 & $(0.9)$ & 84.6 & $(1.0)$ & 66.4 & (1.3) & -5.3 & $(1.2)$ & 1.2 & (1.4) & -11.8 & (1.8) \\
\hline & Russ. Föderation & 80.6 & $(0.7)$ & 86.1 & $(0.7)$ & 75.0 & (0.9) & 78.6 & $(0.8)$ & 86.6 & $(0.9)$ & 70.6 & $(1.2)$ & -1.9 & (1.1) & 0.5 & $(1.1)$ & -4.4 & (1.5) \\
\hline & Thailand & 88.1 & $(0.9)$ & 91.4 & $(0.8)$ & 83.0 & (1.7) & 90.8 & $(0.5)$ & 95.1 & $(0.4)$ & 85.1 & $(0.9)$ & 2.8 & $(1.0)$ & 3.7 & $(0.9)$ & 2.1 & (2.0) \\
\hline
\end{tabular}

Anmerkung: Statistisch signifikante Werte sind durch Fettdruck gekennzeichnet (siehe Anhang A3).

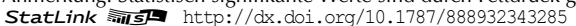


[Teil 1/1]

Index der Freude am Lesen in PISA 2000 und PISA 2009, nach Geschlecht Tabelle V.5.2 Auf Grundlage der Schülerangaben

\begin{tabular}{|c|c|c|c|c|c|c|c|c|c|c|c|c|c|c|c|c|c|c|c|}
\hline & \multicolumn{6}{|c|}{ PISA 2000} & \multicolumn{6}{|c|}{ PISA 2009} & \multicolumn{6}{|c|}{$\begin{array}{l}\text { Veränderung zwischen } 2000 \text { und } 2009 \\
\text { (PISA 2009 - PISA 2000) }\end{array}$} \\
\hline & & \multicolumn{2}{|c|}{ Alle Schüler } & \multicolumn{2}{|c|}{ Mädchen } & \multicolumn{2}{|c|}{ Jungen } & \multicolumn{2}{|c|}{ Alle Schüler } & \multicolumn{2}{|c|}{ Mädchen } & \multicolumn{2}{|c|}{ Jungen } & \multicolumn{2}{|c|}{ Alle Schüler } & \multicolumn{2}{|c|}{ Mädchen } & \multicolumn{2}{|c|}{ Jungen } \\
\hline & & $\begin{array}{l}\text { Index- } \\
\text { mittel }\end{array}$ & S.E. & $\begin{array}{l}\text { Index- } \\
\text { mittel }\end{array}$ & S.E. & $\begin{array}{l}\text { Index- } \\
\text { mittel }\end{array}$ & S.E. & $\begin{array}{l}\text { Index- } \\
\text { mittel }\end{array}$ & S.E. & $\begin{array}{l}\text { Index- } \\
\text { mittel }\end{array}$ & S.E. & $\begin{array}{l}\text { Index- } \\
\text { mittel }\end{array}$ & S.E. & Diff. & S.E. & Diff. & S.E. & Diff. & S.E. \\
\hline \multirow{31}{*}{ 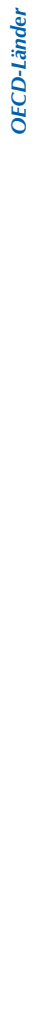 } & Australien & -0.03 & $(0.03)$ & 0.21 & $(0.03)$ & -0.25 & $(0.03)$ & 0.00 & $(0.02)$ & 0.31 & $(0.02)$ & -0.33 & $(0.02)$ & 0.03 & $(0.03)$ & 0.10 & $(0.04)$ & -0.08 & $(0.03)$ \\
\hline & Österreich & -0.09 & $(0.03)$ & 0.34 & (0.03) & -0.51 & $(0.03)$ & $\mathrm{m}$ & $\mathrm{m}$ & $\mathrm{m}$ & $\mathrm{m}$ & $\mathrm{m}$ & $\mathrm{m}$ & $\mathrm{m}$ & $\mathrm{m}$ & $\mathrm{m}$ & $\mathrm{m}$ & $\mathrm{m}$ & $\mathrm{m}$ \\
\hline & Belgien & -0.21 & $(0.02)$ & 0.09 & $(0.02)$ & -0.49 & $(0.02)$ & -0.20 & $(0.02)$ & 0.07 & $(0.02)$ & -0.45 & $(0.02)$ & 0.01 & $(0.02)$ & -0.01 & $(0.03)$ & 0.03 & $(0.03)$ \\
\hline & Kanada & 0.04 & $(0.01)$ & 0.34 & $(0.02)$ & -0.27 & $(0.01)$ & 0.13 & $(0.01)$ & 0.55 & $(0.02)$ & -0.28 & $(0.02)$ & 0.10 & $(0.02)$ & 0.20 & $(0.02)$ & -0.01 & $(0.02)$ \\
\hline & Chile & -0.01 & $(0.02)$ & 0.22 & $(0.02)$ & -0.26 & $(0.02)$ & -0.06 & $(0.01)$ & 0.16 & $(0.02)$ & -0.28 & $(0.02)$ & -0.06 & $(0.02)$ & -0.06 & $(0.03)$ & -0.02 & $(0.03)$ \\
\hline & Tschech. Rep. & 0.22 & $(0.02)$ & 0.59 & $(0.02)$ & -0.20 & $(0.03)$ & -0.13 & $(0.02)$ & 0.22 & $(0.02)$ & -0.44 & $(0.02)$ & -0.35 & $(0.03)$ & -0.37 & $(0.04)$ & -0.24 & $(0.03)$ \\
\hline & Dänemark & 0.04 & $(0.02)$ & 0.37 & $(0.03)$ & -0.27 & $(0.02)$ & -0.09 & $(0.02)$ & 0.17 & $(0.02)$ & -0.35 & $(0.02)$ & -0.13 & $(0.02)$ & -0.20 & $(0.03)$ & -0.08 & $(0.03)$ \\
\hline & Finnland & 0.24 & $(0.02)$ & 0.69 & $(0.02)$ & -0.24 & $(0.02)$ & 0.05 & $(0.02)$ & 0.50 & $(0.02)$ & -0.41 & $(0.02)$ & -0.20 & $(0.03)$ & -0.20 & $(0.03)$ & -0.17 & $(0.03)$ \\
\hline & Frankreich & -0.02 & $(0.02)$ & 0.24 & $(0.02)$ & -0.30 & $(0.02)$ & 0.01 & $(0.03)$ & 0.24 & $(0.03)$ & -0.23 & $(0.03)$ & 0.04 & $(0.03)$ & 0.00 & $(0.04)$ & 0.07 & $(0.04)$ \\
\hline & Deutschland & -0.05 & $(0.03)$ & 0.36 & $(0.03)$ & -0.47 & $(0.03)$ & 0.07 & $(0.02)$ & 0.52 & $(0.03)$ & -0.38 & $(0.02)$ & 0.12 & $(0.03)$ & 0.16 & $(0.04)$ & 0.09 & $(0.04)$ \\
\hline & Griechenland & 0.03 & $(0.02)$ & 0.24 & $(0.02)$ & -0.18 & $(0.02)$ & 0.07 & $(0.02)$ & 0.36 & $(0.02)$ & -0.24 & $(0.02)$ & 0.03 & $(0.03)$ & 0.12 & $(0.03)$ & -0.06 & $(0.03)$ \\
\hline & Ungarn & 0.11 & $(0.02)$ & 0.34 & $(0.03)$ & -0.11 & $(0.03)$ & 0.14 & $(0.02)$ & 0.43 & $(0.02)$ & -0.15 & $(0.03)$ & 0.02 & $(0.03)$ & 0.09 & $(0.04)$ & -0.04 & $(0.04)$ \\
\hline & Island & 0.06 & $(0.02)$ & 0.32 & $(0.02)$ & -0.20 & $(0.02)$ & -0.06 & $(0.02)$ & 0.25 & $(0.02)$ & -0.38 & $(0.02)$ & -0.12 & $(0.02)$ & -0.07 & $(0.03)$ & -0.18 & (0.03) \\
\hline & Irland & -0.03 & $(0.02)$ & 0.25 & (0.03) & -0.32 & $(0.03)$ & -0.08 & $(0.02)$ & 0.15 & $(0.03)$ & -0.30 & $(0.03)$ & -0.05 & $(0.03)$ & -0.11 & $(0.04)$ & 0.02 & (0.04) \\
\hline & Israel & 0.21 & $(0.06)$ & 0.43 & (0.08) & -0.11 & $(0.04)$ & 0.06 & $(0.02)$ & 0.35 & $(0.03)$ & -0.26 & $(0.03)$ & -0.15 & $(0.07)$ & -0.08 & $(0.08)$ & -0.14 & (0.06) \\
\hline & Italien & 0.04 & $(0.03)$ & 0.33 & (0.03) & -0.24 & $(0.02)$ & 0.06 & $(0.01)$ & 0.41 & $(0.01)$ & -0.27 & $(0.01)$ & 0.02 & (0.03) & 0.08 & $(0.03)$ & -0.03 & (0.03) \\
\hline & Japan & 0.13 & $(0.03)$ & 0.28 & $(0.04)$ & -0.03 & $(0.03)$ & 0.20 & $(0.02)$ & 0.38 & $(0.02)$ & 0.02 & $(0.03)$ & 0.07 & $(0.03)$ & 0.10 & $(0.04)$ & 0.06 & (0.04) \\
\hline & Korea & 0.06 & $(0.02)$ & 0.18 & $(0.04)$ & -0.04 & $(0.02)$ & 0.13 & $(0.02)$ & 0.27 & $(0.02)$ & 0.00 & $(0.02)$ & 0.07 & $(0.03)$ & 0.09 & $(0.04)$ & 0.04 & $(0.03)$ \\
\hline & Luxemburg & $\mathrm{m}$ & $\mathrm{m}$ & $\mathrm{m}$ & $\mathrm{m}$ & $\mathrm{m}$ & $\mathrm{m}$ & -0.16 & $(0.02)$ & 0.20 & $(0.03)$ & -0.51 & $(0.02)$ & $\mathrm{m}$ & & $\mathrm{m}$ & $\mathrm{m}$ & $\mathrm{m}$ & $\mathrm{m}$ \\
\hline & Mexiko & 0.34 & $(0.02)$ & 0.51 & $(0.02)$ & 0.18 & $(0.02)$ & 0.14 & $(0.01)$ & 0.32 & $(0.01)$ & -0.04 & $(0.01)$ & -0.20 & $(0.02)$ & -0.19 & $(0.02)$ & -0.21 & $(0.02)$ \\
\hline & Niederlande & $\mathrm{m}$ & $\mathrm{m}$ & $\mathrm{m}$ & $\mathrm{m}$ & $\mathrm{m}$ & $\mathrm{m}$ & -0.32 & $(0.03)$ & 0.02 & $(0.03)$ & -0.66 & $(0.03)$ & $\mathrm{m}$ & $\mathrm{m}$ & $\mathrm{m}$ & $\mathrm{m}$ & $\mathrm{m}$ & $\mathrm{m}$ \\
\hline & Neuseeland & 0.05 & $(0.02)$ & 0.27 & $(0.02)$ & -0.17 & $(0.02)$ & 0.13 & $(0.02)$ & 0.44 & $(0.02)$ & -0.17 & $(0.02)$ & 0.08 & $(0.03)$ & 0.17 & $(0.03)$ & 0.00 & $(0.03)$ \\
\hline & Norwegen & -0.18 & $(0.02)$ & 0.16 & (0.03) & -0.50 & $(0.02)$ & -0.19 & $(0.02)$ & 0.13 & $(0.03)$ & -0.50 & $(0.02)$ & -0.01 & $(0.03)$ & -0.03 & $(0.04)$ & 0.01 & (0.03) \\
\hline & Polen & 0.04 & $(0.03)$ & 0.27 & (0.03) & -0.18 & $(0.03)$ & 0.02 & $(0.02)$ & 0.39 & $(0.03)$ & -0.36 & $(0.02)$ & -0.02 & $(0.03)$ & 0.12 & $(0.04)$ & -0.18 & $(0.04)$ \\
\hline & Portugal & 0.36 & $(0.02)$ & 0.68 & $(0.02)$ & 0.02 & $(0.02)$ & 0.21 & $(0.02)$ & 0.54 & $(0.02)$ & -0.15 & $(0.02)$ & -0.15 & $(0.03)$ & -0.13 & $(0.03)$ & -0.17 & $(0.03)$ \\
\hline & Spanien & 0.00 & $(0.02)$ & 0.24 & $(0.03)$ & -0.26 & $(0.02)$ & -0.01 & $(0.01)$ & 0.26 & $(0.01)$ & -0.28 & $(0.02)$ & -0.01 & $(0.02)$ & 0.02 & $(0.03)$ & -0.02 & $(0.03)$ \\
\hline & Schweden & -0.02 & $(0.02)$ & 0.28 & $(0.03)$ & -0.32 & $(0.03)$ & -0.11 & $(0.02)$ & 0.26 & $(0.03)$ & -0.47 & $(0.02)$ & -0.08 & $(0.03)$ & -0.02 & $(0.04)$ & -0.15 & $(0.03)$ \\
\hline & Schweiz & 0.10 & $(0.03)$ & 0.56 & (0.03) & -0.37 & $(0.03)$ & -0.04 & $(0.02)$ & 0.37 & $(0.03)$ & -0.44 & $(0.02)$ & -0.14 & $(0.04)$ & -0.19 & $(0.04)$ & -0.07 & $(0.04)$ \\
\hline & Ver. Königreich & $\mathrm{m}$ & $\mathrm{m}$ & $\mathrm{m}$ & $\mathrm{m}$ & $\mathrm{m}$ & $\mathrm{m}$ & -0.12 & $(0.02)$ & 0.13 & $(0.02)$ & -0.37 & $(0.02)$ & $\mathrm{m}$ & $\mathrm{m}$ & $\mathrm{m}$ & $\mathrm{m}$ & $\mathrm{m}$ & $\mathrm{m}$ \\
\hline & Ver. Staaten & -0.09 & $(0.03)$ & 0.10 & $(0.04)$ & -0.30 & $(0.04)$ & -0.04 & $(0.03)$ & 0.28 & $(0.03)$ & -0.35 & $(0.03)$ & 0.05 & $(0.04)$ & 0.18 & $(0.05)$ & -0.04 & $(0.05)$ \\
\hline & OECD-26-Durchschnitt & 0.05 & $(0.00)$ & 0.33 & $(0.01)$ & -0.23 & $(0.01)$ & 0.02 & $(0.00)$ & 0.32 & $(0.00)$ & -0.29 & $(0.00)$ & -0.04 & $(0.01)$ & -0.01 & $(0.01)$ & -0.06 & $(0.01)$ \\
\hline \multirow{12}{*}{ 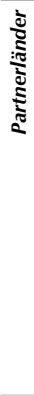 } & Albanien & 0.56 & $(0.01)$ & 0.70 & $(0.02)$ & 0.39 & $(0.02)$ & 0.67 & $(0.02)$ & 0.99 & $(0.02)$ & 0.36 & $(0.02)$ & 0.11 & $(0.02)$ & 0.28 & (0.03) & -0.03 & $(0.03)$ \\
\hline & Argentinien & -0.08 & $(0.03)$ & 0.09 & (0.04) & -0.30 & $(0.04)$ & -0.16 & $(0.02)$ & -0.01 & $(0.02)$ & -0.34 & $(0.02)$ & -0.08 & $(0.03)$ & -0.09 & $(0.04)$ & -0.04 & $(0.04)$ \\
\hline & Brasilien & 0.20 & $(0.02)$ & 0.43 & $(0.02)$ & -0.08 & $(0.02)$ & 0.27 & $(0.01)$ & 0.47 & $(0.01)$ & 0.05 & $(0.01)$ & 0.07 & $(0.02)$ & 0.04 & $(0.03)$ & 0.12 & $(0.02)$ \\
\hline & Bulgarien & 0.04 & $(0.03)$ & 0.26 & $(0.03)$ & -0.18 & $(0.02)$ & -0.02 & $(0.03)$ & 0.23 & $(0.03)$ & -0.25 & $(0.03)$ & -0.05 & $(0.04)$ & -0.03 & $(0.05)$ & -0.08 & $(0.03)$ \\
\hline & Hongkong (China) & 0.11 & $(0.01)$ & 0.24 & $(0.02)$ & -0.02 & $(0.02)$ & 0.32 & $(0.01)$ & 0.51 & $(0.02)$ & 0.16 & $(0.02)$ & 0.21 & $(0.02)$ & 0.27 & $(0.03)$ & 0.18 & $(0.03)$ \\
\hline & Indonesien & 0.59 & $(0.01)$ & 0.69 & $(0.02)$ & 0.48 & $(0.02)$ & 0.43 & $(0.01)$ & 0.55 & $(0.01)$ & 0.32 & $(0.01)$ & -0.15 & $(0.02)$ & -0.14 & $(0.02)$ & -0.16 & $(0.02)$ \\
\hline & Lettland & 0.00 & $(0.02)$ & 0.24 & $(0.03)$ & -0.25 & $(0.03)$ & -0.04 & $(0.02)$ & 0.30 & $(0.02)$ & -0.39 & $(0.02)$ & -0.04 & $(0.03)$ & 0.06 & $(0.03)$ & -0.14 & $(0.03)$ \\
\hline & Liechtenstein & -0.04 & $(0.06)$ & 0.35 & (0.09) & -0.43 & $(0.07)$ & -0.20 & $(0.05)$ & 0.21 & $(0.08)$ & -0.57 & $(0.07)$ & -0.16 & $(0.08)$ & -0.14 & $(0.12)$ & -0.15 & $(0.10)$ \\
\hline & Peru & 0.32 & $(0.02)$ & 0.37 & $(0.02)$ & 0.26 & $(0.02)$ & 0.35 & $(0.01)$ & 0.48 & $(0.02)$ & 0.21 & $(0.02)$ & 0.03 & $(0.02)$ & 0.11 & $(0.03)$ & -0.05 & $(0.03)$ \\
\hline & Rumänien & 0.25 & $(0.02)$ & 0.42 & $(0.03)$ & 0.07 & $(0.02)$ & 0.10 & $(0.02)$ & 0.32 & $(0.03)$ & -0.13 & $(0.02)$ & -0.15 & $(0.03)$ & -0.10 & $(0.04)$ & -0.19 & $(0.03)$ \\
\hline & Russ. Föderation & 0.09 & $(0.01)$ & 0.27 & $(0.02)$ & -0.08 & $(0.02)$ & 0.07 & $(0.01)$ & 0.29 & $(0.02)$ & -0.15 & $(0.02)$ & -0.02 & $(0.02)$ & 0.02 & $(0.03)$ & -0.07 & $(0.02)$ \\
\hline & Thailand & 0.17 & $(0.01)$ & 0.25 & $(0.01)$ & 0.05 & $(0.01)$ & 0.54 & $(0.01)$ & 0.67 & $(0.01)$ & 0.36 & $(0.02)$ & 0.37 & $(0.02)$ & 0.42 & $(0.02)$ & 0.31 & $(0.02)$ \\
\hline
\end{tabular}

Anmerkung: Statistisch signifikante Werte sind durch Fettdruck gekennzeichnet (siehe Anhang A3).

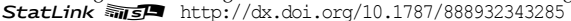


[Teil 1/3]

Prozentsatz der Schüler für verschiedene Items auf dem Index der Freude am Lesen

in PISA 2000 und PISA 2009'

Tabelle V.5.3 Prozentsatz der Schüler, die für folgende Aussagen "Stimmt eher" oder "Stimmt ganz genau" ankreuzten

\begin{tabular}{|c|c|c|c|c|c|c|c|c|c|c|c|c|c|c|c|c|c|c|c|}
\hline & \multicolumn{18}{|c|}{ PISA 2000} \\
\hline & & \multicolumn{2}{|c|}{$\begin{array}{c}\text { Ich lese nur, } \\
\text { wenn ich } \\
\text { muss }\end{array}$} & \multicolumn{2}{|c|}{$\begin{array}{l}\text { Lesen ist } \\
\text { eines meiner } \\
\text { liebsten } \\
\text { Hobbys }\end{array}$} & \multicolumn{2}{|c|}{$\begin{array}{c}\text { Ich spreche } \\
\text { gerne mit } \\
\text { anderen } \\
\text { Leuten über } \\
\text { Bücher }\end{array}$} & \multicolumn{2}{|c|}{$\begin{array}{c}\text { Es fällt mir } \\
\text { schwer, } \\
\text { Bücher zu } \\
\text { Ende zu lesen }\end{array}$} & \multicolumn{2}{|c|}{$\begin{array}{l}\text { Ich freue } \\
\text { mich, wenn } \\
\text { ich ein Buch } \\
\text { geschenkt } \\
\text { bekomme }\end{array}$} & \multicolumn{2}{|c|}{$\begin{array}{l}\text { Für mich ist } \\
\text { Lesen Zeit- } \\
\text { verschwen- } \\
\text { dung }\end{array}$} & \multicolumn{2}{|c|}{$\begin{array}{l}\text { Ich gehe } \\
\text { gerne in } \\
\text { Buchhand- } \\
\text { lungen oder } \\
\text { Büchereien }\end{array}$} & \multicolumn{2}{|c|}{$\begin{array}{c}\text { Ich lese nur, } \\
\text { um Informa- } \\
\text { tionen zu } \\
\text { bekommen, } \\
\text { die ich } \\
\text { brauche }\end{array}$} & \multicolumn{2}{|c|}{$\begin{array}{l}\text { Ich kann } \\
\text { nicht länger } \\
\text { als ein paar } \\
\text { Minuten } \\
\text { stillsitzen } \\
\text { und lesen }\end{array}$} \\
\hline & & $\%$ & S.E. & $\%$ & S.E. & $\%$ & S.E. & $\%$ & S.E. & $\%$ & S.E. & $\%$ & S.E. & $\%$ & S.E. & $\%$ & S.E. & $\%$ & S.E. \\
\hline \multirow{31}{*}{ 仓ે } & Australien & 38.6 & $(1.2)$ & 31.4 & (1.1) & 29.6 & $(1.2)$ & 32.8 & (0.9) & 45.1 & (1.3) & 23.7 & $(0.8)$ & 42.4 & (1.1) & 44.9 & (1.3) & 21.0 & $(0.8)$ \\
\hline & Österreich & 42.9 & (1.1) & 28.6 & $(1.2)$ & 24.5 & (1.0) & 33.6 & $(0.9)$ & 45.4 & (1.3) & 30.0 & (1.1) & 30.1 & $(1.0)$ & 55.1 & (1.3) & 22.0 & (0.9) \\
\hline & Belgien & 45.9 & $(0.8)$ & 26.4 & $(0.8)$ & 29.0 & $(0.8)$ & 33.8 & $(0.7)$ & 36.5 & $(0.7)$ & 33.6 & $(0.7)$ & 39.8 & (0.8) & 50.6 & $(0.7)$ & 29.4 & (0.7) \\
\hline & Kanada & 36.7 & $(0.5)$ & 33.7 & $(0.5)$ & 32.8 & $(0.6)$ & 30.7 & $(0.4)$ & 46.4 & $(0.5)$ & 23.4 & $(0.4)$ & 50.7 & $(0.5)$ & 43.3 & $(0.5)$ & 24.8 & (0.4) \\
\hline & Chile & 50.8 & (1.0) & 36.7 & $(0.9)$ & 57.4 & (1.0) & 45.9 & $(0.8)$ & 45.0 & $(0.9)$ & 18.8 & $(0.8)$ & 48.1 & (1.0) & 57.4 & (1.0) & 37.2 & (1.0) \\
\hline & Tschech. Rep. & 27.0 & $(0.9)$ & 51.6 & $(0.9)$ & 48.6 & $(0.9)$ & 32.3 & $(0.8)$ & 58.6 & $(0.9)$ & 21.1 & $(0.8)$ & 50.7 & $(0.8)$ & 44.4 & (1.0) & 23.3 & $(0.7)$ \\
\hline & Dänemark & 36.6 & $(0.8)$ & 31.0 & $(0.7)$ & 35.8 & (0.9) & 28.3 & $(0.8)$ & 47.6 & $(1.0)$ & 24.3 & $(0.8)$ & 48.3 & $(0.9)$ & 43.8 & $(0.9)$ & 21.3 & $(0.7)$ \\
\hline & Finnland & 26.4 & $(0.7)$ & 41.0 & (0.9) & 33.3 & (0.8) & 24.8 & $(0.7)$ & 54.7 & (1.0) & 23.2 & (0.8) & 59.6 & $(0.8)$ & 34.7 & (0.8) & 13.8 & (0.6) \\
\hline & Frankreich & 36.5 & $(0.9)$ & 32.7 & $(0.8)$ & 38.7 & $(0.9)$ & 33.6 & $(0.8)$ & 42.1 & (1.0) & 22.6 & $(0.8)$ & 50.2 & $(0.9)$ & 47.0 & $(0.8)$ & 30.0 & (0.8) \\
\hline & Deutschland & 39.1 & $(0.8)$ & 29.1 & $(0.7)$ & 25.6 & $(0.9)$ & 33.1 & $(0.9)$ & 45.9 & (1.1) & 30.7 & $(0.9)$ & 35.7 & $(0.9)$ & 52.4 & (1.1) & 20.1 & $(0.7)$ \\
\hline & Griechenland & 33.1 & (1.2) & 37.3 & (1.0) & 49.2 & (1.3) & 35.8 & (0.9) & 44.9 & (1.5) & 15.5 & $(0.9)$ & 56.4 & $(1.2)$ & 46.8 & (1.2) & 35.3 & (1.1) \\
\hline & Ungarn & 29.3 & $(1.0)$ & 29.1 & (0.9) & 36.8 & $(0.9)$ & 24.7 & $(0.7)$ & 56.5 & $(1.2)$ & 15.8 & $(0.7)$ & 46.0 & $(0.9)$ & 47.1 & $(1.1)$ & 23.5 & $(0.9)$ \\
\hline & Island & 33.1 & $(0.8)$ & 27.0 & (0.8) & 25.8 & (0.8) & 22.3 & $(0.6)$ & 59.7 & $(0.9)$ & 20.5 & $(0.7)$ & 43.8 & $(0.8)$ & 37.4 & (0.9) & 24.0 & $(0.7)$ \\
\hline & Irland & 33.5 & $(0.9)$ & 35.7 & (1.0) & 27.8 & $(0.9)$ & 41.7 & (0.9) & 47.7 & (1.0) & 19.3 & $(0.7)$ & 40.9 & (1.2) & 45.7 & (1.1) & 29.8 & (0.8) \\
\hline & Israel & 34.8 & (1.7) & 38.4 & (2.5) & 41.9 & $(2.4)$ & 22.6 & (1.4) & 52.0 & (1.9) & 21.3 & $(1.2)$ & 46.4 & (2.3) & 41.3 & $(2.2)$ & 21.3 & (1.4) \\
\hline & Italien & 26.1 & (1.0) & 44.8 & (1.0) & 43.2 & (1.0) & 40.0 & $(0.7)$ & 46.1 & (1.1) & 17.8 & (0.6) & 41.8 & (1.4) & 50.6 & (1.0) & 31.6 & (0.9) \\
\hline & Japan & 48.3 & (1.0) & 36.4 & (1.0) & 36.5 & $(0.9)$ & 40.6 & $(0.8)$ & 40.9 & (1.0) & 19.7 & $(0.8)$ & 66.8 & (1.1) & 30.3 & $(0.7)$ & 27.4 & (1.0) \\
\hline & Korea & 40.8 & $(0.9)$ & 34.5 & $(0.9)$ & 30.5 & $(0.9)$ & 42.6 & $(0.7)$ & 49.0 & $(1.2)$ & 12.7 & $(0.6)$ & 47.1 & (1.1) & 40.4 & (1.0) & 18.1 & (0.7) \\
\hline & Luxemburg & $\mathrm{m}$ & $\mathrm{m}$ & $\mathrm{m}$ & $\mathrm{m}$ & $\mathrm{m}$ & $\mathrm{m}$ & $\mathrm{m}$ & $\mathrm{m}$ & $\mathrm{m}$ & $\mathrm{m}$ & $\mathrm{m}$ & $\mathrm{m}$ & $\mathrm{m}$ & $\mathrm{m}$ & $\mathrm{m}$ & $\mathrm{m}$ & $\mathrm{m}$ & $\mathrm{m}$ \\
\hline & Mexiko & 24.9 & $(0.9)$ & 61.6 & (1.3) & 65.2 & (1.1) & 41.8 & $(0.8)$ & 72.7 & (1.1) & 9.2 & $(0.5)$ & 65.3 & (1.3) & 52.6 & (0.9) & 20.7 & (0.8) \\
\hline & Niederlande & $\mathrm{m}$ & $\mathrm{m}$ & $\mathrm{m}$ & $\mathrm{m}$ & $\mathrm{m}$ & $\mathrm{m}$ & $\mathrm{m}$ & $\mathrm{m}$ & m & $\mathrm{m}$ & $\mathrm{m}$ & $\mathrm{m}$ & $\mathrm{m}$ & $\mathrm{m}$ & $\mathrm{m}$ & $\mathrm{m}$ & $\mathrm{m}$ & $\mathrm{m}$ \\
\hline & Neuseeland & 36.0 & (1.0) & 33.1 & $(0.9)$ & 33.2 & (1.1) & 36.7 & (1.0) & 50.6 & $(0.9)$ & 19.3 & $(0.8)$ & 51.5 & (1.2) & 43.5 & (1.0) & 21.1 & (0.8) \\
\hline & Norwegen & 42.6 & $(0.8)$ & 24.1 & $(0.8)$ & 21.9 & $(0.7)$ & 29.2 & $(0.7)$ & 39.0 & $(0.9)$ & 30.4 & $(0.9)$ & 39.0 & $(0.9)$ & 49.1 & (0.9) & 24.1 & (1.0) \\
\hline & Polen & 36.3 & (1.4) & 38.6 & (1.1) & 38.3 & $(1.0)$ & 36.3 & (1.1) & 47.9 & $(1.2)$ & 24.9 & (1.1) & 56.0 & (1.1) & 53.6 & (1.4) & 21.2 & (1.0) \\
\hline & Portugal & 24.3 & (1.1) & 54.3 & $(0.9)$ & 57.3 & $(0.9)$ & 27.8 & $(0.8)$ & 64.7 & $(0.9)$ & 13.2 & $(0.8)$ & 69.6 & (1.0) & 35.2 & $(0.9)$ & 24.6 & (1.0) \\
\hline & Spanien & 41.3 & (1.1) & 35.4 & $(0.9)$ & 41.5 & (1.1) & 42.3 & $(0.7)$ & 37.7 & $(0.9)$ & 15.7 & $(0.7)$ & 42.1 & (1.0) & 46.0 & (1.1) & 19.9 & $(0.8)$ \\
\hline & Schweden & 37.4 & $(1.0)$ & 29.4 & $(0.7)$ & 30.0 & $(0.8)$ & 25.7 & $(0.7)$ & 38.0 & $(0.8)$ & 28.9 & $(0.9)$ & 42.0 & $(1.0)$ & 43.4 & $(0.9)$ & 21.9 & $(0.7)$ \\
\hline & Schweiz & 36.1 & $(0.9)$ & 34.9 & $(1.1)$ & 31.8 & $(1.0)$ & 30.6 & $(0.8)$ & 53.4 & (1.1) & 25.5 & $(0.9)$ & 45.6 & $(1.1)$ & 45.6 & (1.1) & 22.3 & $(0.7)$ \\
\hline & Ver. Königreich & $\mathrm{m}$ & $\mathrm{m}$ & $\mathrm{m}$ & $\mathrm{m}$ & $\mathrm{m}$ & $\mathrm{m}$ & $\mathrm{m}$ & $\mathrm{m}$ & $\mathrm{m}$ & $\mathrm{m}$ & $\mathrm{m}$ & $\mathrm{m}$ & $\mathrm{m}$ & $\mathrm{m}$ & $\mathrm{m}$ & $\mathrm{m}$ & $\mathrm{m}$ & $\mathrm{m}$ \\
\hline & Ver. Staaten & 45.4 & (1.5) & 29.8 & $(1.0)$ & 34.7 & (1.5) & 35.8 & (1.0) & 39.9 & (1.4) & 26.0 & (1.4) & 53.0 & (1.5) & 47.5 & $(1.7)$ & 30.6 & (1.4) \\
\hline & OECD-26-Durchschnitt & 36.2 & $(0.2)$ & 36.1 & $(0.2)$ & 37.6 & $(0.2)$ & 33.5 & $(0.2)$ & 48.6 & $(0.2)$ & 21.4 & $(0.2)$ & 49.2 & $(0.2)$ & 45.2 & $(0.2)$ & 24.6 & $(0.2)$ \\
\hline \multirow{12}{*}{ 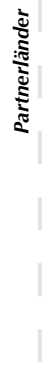 } & Albanien & 22.0 & $(0.8)$ & 64.1 & (1.0) & 78.3 & $(0.8)$ & 23.1 & $(0.8)$ & 84.9 & $(0.6)$ & 11.5 & (0.8) & 83.6 & $(0.7)$ & 33.0 & (1.1) & 17.5 & $(0.9)$ \\
\hline & Argentinien & 47.7 & $(1.7)$ & 39.7 & $(1.1)$ & 42.3 & (1.6) & 49.5 & (1.5) & 42.3 & (1.7) & 19.5 & (1.1) & 43.8 & (1.3) & 58.7 & (1.7) & 34.2 & (1.4) \\
\hline & Brasilien & 42.8 & (1.1) & 48.6 & (1.1) & 52.5 & (1.2) & 35.0 & (1.0) & 63.0 & $(1.2)$ & 10.2 & $(0.5)$ & 60.3 & (1.2) & 54.1 & (1.3) & 26.5 & (1.0) \\
\hline & Bulgarien & 45.7 & $(1.2)$ & 45.8 & (1.4) & 43.6 & (1.7) & 27.0 & (0.9) & 48.5 & (1.1) & 18.9 & $(0.7)$ & 45.2 & (1.1) & 55.9 & (1.1) & 27.8 & (1.0) \\
\hline & Hongkong (China) & 50.4 & $(0.9)$ & 53.0 & $(0.9)$ & 56.7 & $(0.7)$ & 34.6 & $(0.9)$ & 45.7 & $(0.9)$ & 12.6 & $(0.5)$ & 60.4 & $(0.9)$ & 49.1 & $(0.9)$ & 24.6 & (0.7) \\
\hline & Indonesien & 7.9 & $(0.5)$ & 89.8 & $(0.9)$ & 85.0 & $(0.6)$ & 29.2 & $(0.8)$ & 89.0 & $(0.7)$ & 6.8 & $(0.5)$ & 87.7 & $(0.8)$ & 50.6 & (1.1) & 27.4 & (0.9) \\
\hline & Lettland & 35.2 & (1.3) & 37.5 & (1.0) & 37.5 & $(1.2)$ & 35.4 & $(1.2)$ & 48.1 & (1.6) & 23.5 & (1.0) & 45.3 & (1.1) & 55.8 & (1.3) & 20.1 & (1.1) \\
\hline & Liechtenstein & 40.8 & (2.9) & 31.0 & (2.4) & 24.4 & $(2.2)$ & 30.6 & (2.6) & 49.6 & (2.8) & 28.1 & (2.4) & 35.4 & (2.7) & 50.5 & (2.7) & 19.2 & (2.1) \\
\hline & Peru & 19.2 & $(0.9)$ & 65.4 & $(1.0)$ & 70.0 & (1.1) & 55.8 & $(0.8)$ & 69.0 & $(1.1)$ & 16.6 & $(0.6)$ & 72.1 & $(1.2)$ & 49.5 & $(0.9)$ & 47.2 & (1.0) \\
\hline & Rumänien & 45.0 & $(1.0)$ & 59.8 & $(1.1)$ & 57.8 & $(1.0)$ & 37.8 & (1.1) & 70.9 & $(0.8)$ & 19.8 & (1.0) & 66.1 & (0.8) & 49.8 & (1.0) & 29.8 & (1.1) \\
\hline & Russ. Föderation & 33.6 & $(0.8)$ & 42.6 & (1.1) & 43.7 & (1.0) & 31.6 & $(0.6)$ & 47.5 & (1.0) & 16.6 & $(0.7)$ & 49.1 & $(0.8)$ & 59.6 & $(0.8)$ & 12.7 & (0.5) \\
\hline & Thailand & 64.9 & $(0.8)$ & 81.0 & $(0.8)$ & 71.8 & (1.0) & 44.9 & (1.0) & 71.9 & (1.0) & 20.6 & (1.1) & 82.9 & (0.9) & 56.1 & $(1.2)$ & 27.6 & (1.1) \\
\hline
\end{tabular}

Anmerkung: Statistisch signifikante Werte sind durch Fettdruck gekennzeichnet (siehe Anhang A3).

Anmerkung: Statistisch signifikante Werte sind durch Fettdruck gekennzeichnet (siehe Anhang A3).
1. Die Items "Ich sage gerne meine Meinung über Bücher, die ich gelesen habe“ und "Ich tausche gerne Bücher mit meinen Freundinnen und Freunden“ wurden in PISA 2000 nicht verwendet und können deshalb nicht mit PISA 2009 verglichen werden.

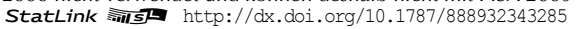


[Teil 2/3]

Prozentsatz der Schüler für verschiedene Items auf dem Index der Freude am Lesen

in PISA 2000 und PISA 2009'

Tabelle V.5.3 Prozentsatz der Schüler, die für folgende Aussagen „Stimmt eher" oder „Stimmt ganz genau“ ankreuzten

\begin{tabular}{|c|c|c|c|c|c|c|c|c|c|c|c|c|c|c|c|c|c|c|c|}
\hline & \multicolumn{18}{|c|}{ PISA 2009} \\
\hline & & \multicolumn{2}{|c|}{$\begin{array}{l}\text { Ich lese nur, } \\
\text { wenn ich } \\
\text { muss }\end{array}$} & \multicolumn{2}{|c|}{$\begin{array}{c}\text { Lesen ist } \\
\text { eines meiner } \\
\text { liebsten } \\
\text { Hobbys }\end{array}$} & \multicolumn{2}{|c|}{$\begin{array}{l}\text { Ich spreche } \\
\text { gerne mit } \\
\text { anderen } \\
\text { Leuten über } \\
\text { Bücher }\end{array}$} & \multicolumn{2}{|c|}{\begin{tabular}{|c|} 
Es fällt mir \\
schwer, \\
Bücher zu \\
Ende zu lesen
\end{tabular}} & \multicolumn{2}{|c|}{$\begin{array}{l}\text { Ich freue } \\
\text { mich, wenn } \\
\text { ich ein Buch } \\
\text { geschenkt } \\
\text { bekomme }\end{array}$} & \multicolumn{2}{|c|}{$\begin{array}{l}\text { Für mich ist } \\
\text { Lesen Zeit- } \\
\text { verschwen- } \\
\quad \text { dung }\end{array}$} & \multicolumn{2}{|c|}{$\begin{array}{l}\text { Ich gehe } \\
\text { gerne in } \\
\text { Buchhand- } \\
\text { lungen oder } \\
\text { Büchereien }\end{array}$} & \multicolumn{2}{|c|}{$\begin{array}{c}\text { Ich lese nur, } \\
\text { um Informa- } \\
\text { tionen zu } \\
\text { bekommen, } \\
\text { die ich } \\
\text { brauche }\end{array}$} & \multicolumn{2}{|c|}{$\begin{array}{l}\text { Ich kann } \\
\text { nicht länger } \\
\text { als ein paar } \\
\text { Minuten } \\
\text { stillsitzen } \\
\text { und lesen }\end{array}$} \\
\hline & & $\%$ & S.E. & $\%$ & S.E. & $\%$ & S.E. & $\%$ & S.E. & $\%$ & S.E. & $\%$ & S.E. & $\%$ & S.E. & $\%$ & S.E. & $\%$ & S.E. \\
\hline \multirow{31}{*}{ 仓ે } & Australien & 40.9 & $(0.6)$ & 35.5 & $(0.8)$ & 38.8 & $(0.7)$ & 32.7 & $(0.5)$ & 50.6 & $(0.6)$ & 25.9 & $(0.5)$ & 46.6 & $(0.7)$ & 42.3 & $(0.8)$ & 23.6 & (0.5) \\
\hline & Österreich & $\mathrm{m}$ & $\mathrm{m}$ & $\mathrm{m}$ & $\mathrm{m}$ & $\mathrm{m}$ & $\mathrm{m}$ & $\mathrm{m}$ & $\mathrm{m}$ & $\mathrm{m}$ & $\mathrm{m}$ & $\mathrm{m}$ & $\mathrm{m}$ & $\mathrm{m}$ & $\mathrm{m}$ & $\mathrm{m}$ & $\mathrm{m}$ & $\mathrm{m}$ & $\mathrm{m}$ \\
\hline & Belgien & 44.5 & $(0.7)$ & 24.1 & (0.6) & 28.9 & $(0.7)$ & 34.5 & $(0.8)$ & 36.9 & $(0.7)$ & 34.6 & $(0.8)$ & 36.7 & $(0.7)$ & 47.3 & $(0.7)$ & 28.3 & $(0.7)$ \\
\hline & Kanada & 37.3 & $(0.5)$ & 38.6 & $(0.5)$ & 43.2 & $(0.5)$ & 27.4 & $(0.5)$ & 49.6 & $(0.7)$ & 22.1 & $(0.5)$ & 54.0 & (0.6) & 38.7 & $(0.6)$ & 22.2 & (0.5) \\
\hline & Chile & 34.8 & $(0.8)$ & 32.1 & $(0.6)$ & 47.4 & (0.8) & 46.5 & $(0.8)$ & 38.3 & $(0.9)$ & 17.8 & $(0.6)$ & 33.7 & (0.8) & 64.8 & $(0.8)$ & 33.0 & (0.8) \\
\hline & Tschech. Rep. & 40.8 & $(0.8)$ & 33.4 & $(0.7)$ & 34.9 & $(0.9)$ & 34.3 & $(0.7)$ & 43.8 & $(0.9)$ & 32.5 & $(0.8)$ & 34.5 & $(0.8)$ & 51.8 & $(0.9)$ & 32.5 & $(0.8)$ \\
\hline & Dänemark & 45.4 & (1.0) & 24.2 & $(0.7)$ & 36.7 & (1.0) & 25.2 & $(0.8)$ & 42.8 & (1.0) & 25.9 & $(0.9)$ & 34.7 & $(0.9)$ & 47.5 & (0.9) & 19.6 & $(0.7)$ \\
\hline & Finnland & 34.7 & $(0.8)$ & 34.0 & (0.8) & 34.1 & $(0.9)$ & 27.7 & $(0.7)$ & 52.1 & $(0.8)$ & 27.3 & $(0.8)$ & 47.6 & $(0.9)$ & 36.3 & $(0.8)$ & 14.5 & (0.6) \\
\hline & Frankreich & 33.9 & (1.0) & 31.2 & (1.0) & 42.6 & (1.2) & 39.2 & (1.0) & 41.5 & (1.0) & 25.8 & (1.0) & 47.2 & (1.1) & 43.5 & $(1.2)$ & 26.5 & (0.9) \\
\hline & Deutschland & 39.1 & $(0.8)$ & 32.6 & $(0.8)$ & 32.2 & $(0.8)$ & 27.5 & $(0.7)$ & 48.5 & $(0.8)$ & 29.5 & $(0.7)$ & 34.5 & $(0.8)$ & 44.6 & $(0.8)$ & 17.0 & (0.5) \\
\hline & Griechenland & 42.8 & (1.1) & 29.1 & $(0.8)$ & 37.7 & (1.0) & 38.6 & $(0.9)$ & 42.7 & (1.0) & 13.0 & $(0.6)$ & 45.5 & (1.1) & 39.2 & $(1.2)$ & 30.9 & (0.8) \\
\hline & Ungarn & 32.5 & (1.1) & 34.8 & (1.0) & 38.8 & (1.0) & 20.9 & $(0.7)$ & 55.4 & (1.1) & 22.2 & $(0.8)$ & 46.5 & (1.0) & 47.4 & $(0.9)$ & 19.5 & $(0.9)$ \\
\hline & Island & 47.8 & $(0.9)$ & 24.0 & $(0.8)$ & 33.0 & $(0.8)$ & 29.8 & $(0.8)$ & 60.8 & $(0.8)$ & 25.0 & $(0.7)$ & 40.5 & (0.8) & 41.6 & (0.8) & 25.7 & (0.7) \\
\hline & Irland & 39.2 & (1.0) & 31.7 & (0.9) & 34.7 & (1.1) & 40.4 & (1.0) & 45.8 & (0.9) & 24.1 & (0.9) & 40.0 & (0.9) & 44.9 & (1.1) & 31.6 & (0.9) \\
\hline & Israel & 38.8 & $(0.9)$ & 40.7 & (1.0) & 41.0 & (0.9) & 31.4 & $(0.7)$ & 47.0 & (1.0) & 23.6 & $(0.8)$ & 42.2 & (1.0) & 47.0 & (1.0) & 25.9 & (0.8) \\
\hline & Italien & 28.8 & $(0.5)$ & 39.8 & $(0.5)$ & 44.2 & $(0.5)$ & 38.4 & $(0.4)$ & 48.8 & $(0.5)$ & 21.5 & $(0.5)$ & 39.4 & $(0.6)$ & 47.8 & $(0.5)$ & 30.4 & (0.5) \\
\hline & Japan & 47.5 & $(0.8)$ & 42.0 & (0.9) & 43.6 & $(0.8)$ & 28.4 & $(0.7)$ & 45.6 & $(0.8)$ & 15.2 & (0.6) & 66.5 & $(0.7)$ & 24.2 & $(0.7)$ & 20.6 & (0.6) \\
\hline & Korea & 54.8 & $(0.9)$ & 39.1 & (0.8) & 38.5 & $(0.8)$ & 32.4 & (0.8) & 55.2 & (1.0) & 9.5 & (0.5) & 42.2 & $(1.0)$ & 31.0 & (0.8) & 15.9 & (0.6) \\
\hline & Luxemburg & 47.7 & $(0.8)$ & 26.2 & $(0.8)$ & 25.6 & $(0.7)$ & 29.9 & $(0.8)$ & 36.9 & $(0.7)$ & 33.4 & (0.8) & 29.4 & $(0.7)$ & 49.0 & $(0.7)$ & 25.9 & (0.6) \\
\hline & Mexiko & 41.1 & (0.6) & 49.3 & (0.5) & 48.2 & $(0.4)$ & 39.2 & $(0.4)$ & 57.3 & $(0.5)$ & 12.2 & $(0.3)$ & 46.9 & $(0.5)$ & 55.4 & $(0.5)$ & 23.9 & (0.4) \\
\hline & Niederlande & 52.7 & (1.4) & 19.1 & $(1.0)$ & 18.9 & $(0.9)$ & 25.6 & $(0.8)$ & 40.4 & $(1.2)$ & 33.9 & (1.3) & 28.6 & $(0.9)$ & 49.3 & (1.4) & 26.7 & (1.1) \\
\hline & Neuseeland & 38.0 & $(0.8)$ & 37.5 & $(0.8)$ & 42.7 & $(0.9)$ & 30.6 & $(0.7)$ & 56.5 & $(0.8)$ & 18.1 & $(0.7)$ & 54.4 & $(0.9)$ & 39.7 & $(0.9)$ & 18.3 & $(0.7)$ \\
\hline & Norwegen & 44.4 & $(0.9)$ & 22.0 & $(0.7)$ & 28.1 & $(0.8)$ & 28.8 & $(0.7)$ & 40.4 & $(0.9)$ & 29.9 & $(0.8)$ & 31.2 & $(0.7)$ & 50.3 & $(0.9)$ & 24.9 & $(0.7)$ \\
\hline & Polen & 44.2 & $(0.8)$ & 37.1 & $(0.9)$ & 38.9 & $(0.7)$ & 34.5 & $(0.7)$ & 41.1 & (0.9) & 26.8 & $(0.8)$ & 42.7 & (1.0) & 53.6 & $(0.7)$ & 29.9 & (0.8) \\
\hline & Portugal & 22.0 & (0.6) & 35.6 & $(0.8)$ & 48.6 & $(0.8)$ & 29.4 & $(0.7)$ & 52.9 & $(0.6)$ & 18.8 & $(0.5)$ & 54.0 & $(0.8)$ & 42.9 & $(0.9)$ & 31.3 & $(0.7)$ \\
\hline & Spanien & 43.7 & $(0.7)$ & 33.7 & $(0.5)$ & 42.4 & $(0.7)$ & 44.2 & (0.6) & 35.3 & $(0.5)$ & 17.7 & $(0.6)$ & 30.4 & (0.6) & 46.2 & $(0.7)$ & 22.8 & (0.5) \\
\hline & Schweden & 39.4 & $(0.7)$ & 27.2 & $(0.7)$ & 33.6 & $(0.9)$ & 23.9 & $(0.7)$ & 35.6 & $(0.9)$ & 27.7 & $(0.7)$ & 35.2 & $(0.9)$ & 41.6 & $(0.9)$ & 20.6 & $(0.7)$ \\
\hline & Schweiz & 43.4 & $(1.0)$ & 29.5 & $(0.6)$ & 31.2 & $(0.8)$ & 31.7 & $(0.7)$ & 45.0 & $(0.8)$ & 31.3 & $(0.8)$ & 39.6 & $(0.9)$ & 45.8 & $(0.8)$ & 21.6 & $(0.6)$ \\
\hline & Ver. Königreich & 41.8 & $(0.8)$ & 27.1 & $(0.6)$ & 35.0 & $(0.9)$ & 37.1 & $(0.8)$ & 49.1 & $(0.7)$ & 23.4 & $(0.7)$ & 34.2 & $(0.8)$ & 48.2 & $(0.8)$ & 28.0 & $(0.7)$ \\
\hline & Ver. Staaten & 49.7 & $(1.0)$ & 30.5 & (1.1) & 40.9 & $(1.0)$ & 31.0 & (1.0) & 37.9 & (1.0) & 25.8 & $(0.9)$ & 53.8 & (1.0) & 47.1 & (1.1) & 28.3 & $(0.9)$ \\
\hline & OECD-26-Durchschnitt & 40.4 & $(0.2)$ & 33.4 & $(0.2)$ & 38.6 & $(0.2)$ & 32.6 & $(0.1)$ & 46.4 & $(0.2)$ & 23.2 & $(0.1)$ & 43.1 & $(0.2)$ & 44.7 & $(0.2)$ & 24.6 & $(0.1)$ \\
\hline \multirow{12}{*}{ 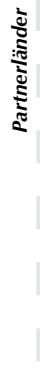 } & Albanien & 36.5 & (1.0) & 67.7 & $(0.9)$ & 72.6 & $(0.8)$ & 22.4 & $(0.8)$ & 84.8 & $(0.7)$ & 6.3 & $(0.5)$ & 81.3 & $(0.7)$ & 37.4 & (1.1) & 21.5 & (0.7) \\
\hline & Argentinien & 57.7 & $(1.0)$ & 32.2 & $(0.9)$ & 38.8 & (1.0) & 55.1 & (1.0) & 46.3 & (1.1) & 19.6 & $(0.8)$ & 36.2 & (1.0) & 66.4 & $(1.0)$ & 35.1 & (1.0) \\
\hline & Brasilien & 15.6 & $(0.5)$ & 48.1 & $(0.8)$ & 51.3 & $(0.7)$ & 35.3 & $(0.6)$ & 51.4 & $(0.6)$ & 7.6 & $(0.3)$ & 53.4 & (0.6) & 40.7 & $(0.8)$ & 30.4 & (0.6) \\
\hline & Bulgarien & 47.6 & (1.3) & 36.3 & (1.3) & 36.3 & (1.4) & 31.0 & $(0.8)$ & 43.0 & (1.4) & 21.6 & $(1.2)$ & 47.6 & (1.3) & 56.8 & (1.4) & 30.9 & (1.0) \\
\hline & Hongkong (China) & 42.9 & $(0.9)$ & 64.9 & $(0.8)$ & 60.6 & $(0.8)$ & 23.2 & $(0.6)$ & 58.5 & $(1.0)$ & 9.2 & $(0.4)$ & 64.8 & $(0.8)$ & 37.7 & $(0.8)$ & 16.4 & (0.5) \\
\hline & Indonesien & 32.4 & $(1.0)$ & 77.3 & $(0.9)$ & 73.6 & $(0.9)$ & 38.2 & (0.9) & 85.4 & $(0.7)$ & 4.3 & $(0.4)$ & 80.1 & $(0.9)$ & 52.8 & (1.0) & 28.4 & $(0.7)$ \\
\hline & Lettland & 42.7 & (1.0) & 29.4 & $(0.9)$ & 38.9 & (1.1) & 31.3 & $(0.8)$ & 41.1 & (1.1) & 26.6 & $(1.0)$ & 43.6 & $(1.2)$ & 54.6 & (1.1) & 23.3 & $(0.8)$ \\
\hline & Liechtenstein & 51.0 & $(2.4)$ & 21.2 & $(2.2)$ & 21.1 & $(2.1)$ & 29.3 & $(2.1)$ & 40.4 & (2.6) & 38.9 & (2.6) & 28.8 & $(2.2)$ & 51.6 & (2.6) & 21.2 & (2.3) \\
\hline & Peru & 40.5 & $(0.8)$ & 62.2 & $(0.9)$ & 64.0 & $(0.9)$ & 38.1 & $(0.8)$ & 70.6 & $(0.9)$ & 9.7 & $(0.5)$ & 58.9 & $(0.9)$ & 50.1 & $(0.9)$ & 23.8 & $(0.7)$ \\
\hline & Rumänien & 31.8 & (1.1) & 40.2 & (1.1) & 47.7 & $(1.0)$ & 39.6 & (1.1) & 65.0 & $(1.1)$ & 23.3 & $(0.8)$ & 55.3 & $(1.2)$ & 60.6 & $(1.2)$ & 28.1 & (0.8) \\
\hline & Russ. Föderation & 38.0 & $(0.9)$ & 37.9 & (1.0) & 41.3 & (1.0) & 28.8 & $(0.7)$ & 43.0 & (1.0) & 18.1 & $(0.7)$ & 44.9 & (1.0) & 59.5 & (0.9) & 13.6 & (0.6) \\
\hline & Thailand & 24.3 & $(0.7)$ & 82.0 & (0.6) & 66.9 & (0.6) & 31.5 & $(0.8)$ & 73.4 & $(0.7)$ & 9.3 & $(0.5)$ & 83.8 & (0.6) & 33.3 & $(0.8)$ & 20.2 & (0.6) \\
\hline
\end{tabular}

Anmerkung: Statistisch signifikante Werte sind durch Fettdruck gekennzeichnet (siehe Anhang A3).

1. Die Items "Ich sage gerne meine Meinung über Bücher, die ich gelesen habe" und "Ich tausche gerne Bücher mit meinen Freundinnen und Freunden“ wurden in PISA 2000 nicht verwendet und können deshalb nicht mit PISA 2009 verglichen werden.

StatLink त्राज़ http://dx.doi.org/10.1787/888932343285 
[Teil 3/3]

Prozentsatz der Schüler für verschiedene Items auf dem Index der Freude am Lesen

in PISA 2000 und PISA 2009'

Tabelle V.5.3 Prozentsatz der Schüler, die für folgende Aussagen "Stimmt eher" oder "Stimmt ganz genau“ ankreuzten

\begin{tabular}{|c|c|c|c|c|c|c|c|c|c|c|c|c|c|c|c|c|c|c|c|}
\hline & \multicolumn{18}{|c|}{ Veränderung zwischen 2000 und 2009 (PISA 2009 - PISA 2000) } \\
\hline & & \multicolumn{2}{|c|}{$\begin{array}{l}\text { Ich lese nur, } \\
\text { wenn ich } \\
\text { muss }\end{array}$} & \multicolumn{2}{|c|}{$\begin{array}{l}\text { Lesen ist } \\
\text { eines meiner } \\
\text { liebsten } \\
\text { Hobbys }\end{array}$} & \multicolumn{2}{|c|}{$\begin{array}{l}\text { Ich spreche } \\
\text { gerne mit } \\
\text { anderen } \\
\text { Leuten über } \\
\text { Bücher }\end{array}$} & \multicolumn{2}{|c|}{$\begin{array}{c}\text { Es fällt mir } \\
\text { schwer, } \\
\text { Bücher zu } \\
\text { Ende zu lesen }\end{array}$} & \multicolumn{2}{|c|}{\begin{tabular}{|} 
Ich freue \\
mich, wenn \\
ich ein Buch \\
geschenkt \\
bekomme
\end{tabular}} & \multicolumn{2}{|c|}{$\begin{array}{l}\text { Für mich ist } \\
\text { Lesen Zeit- } \\
\text { verschwen- } \\
\text { dung }\end{array}$} & \multicolumn{2}{|c|}{$\begin{array}{l}\text { Ich gehe } \\
\text { gerne in } \\
\text { Buchhand- } \\
\text { lungen oder } \\
\text { Büchereien }\end{array}$} & \multicolumn{2}{|c|}{$\begin{array}{c}\text { Ich lese nur, } \\
\text { um Informa- } \\
\text { tionen zu } \\
\text { bekommen, } \\
\text { die ich } \\
\text { brauche }\end{array}$} & \multicolumn{2}{|c|}{$\begin{array}{c}\text { Ich kann } \\
\text { nicht länger } \\
\text { als ein paar } \\
\text { Minuten } \\
\text { stillsitzen und } \\
\text { lesen }\end{array}$} \\
\hline & & Diff. $\%$ & S.E. & Diff. $\%$ & S.E. & Diff. \% & S.E. & Diff. $\%$ & S.E. & Diff. $\%$ & S.E. & Diff. \% & S.E. & Diff. \% & S.E. & Diff. \% & S.E. & Diff. $\%$ & S.E. \\
\hline \multirow{31}{*}{ 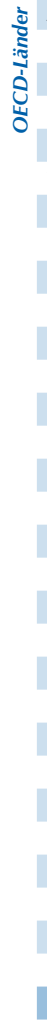 } & Australien & 2.3 & (1.3) & 4.1 & (1.3) & 9.3 & $(1.4)$ & -0.1 & (1.1) & 5.6 & $(1.4)$ & 2.2 & $(1.0)$ & 4.2 & $(1.4)$ & -2.6 & (1.5) & 2.6 & (1.0) \\
\hline & Österreich & $\mathrm{m}$ & $\mathrm{m}$ & $\mathrm{m}$ & $\mathrm{m}$ & $\mathrm{m}$ & $\mathrm{m}$ & $\mathrm{m}$ & $\mathrm{m}$ & $\mathrm{m}$ & $\mathrm{m}$ & $\mathrm{m}$ & $\mathrm{m}$ & $\mathrm{m}$ & $\mathrm{m}$ & $\mathrm{m}$ & $\mathrm{m}$ & $\mathrm{m}$ & $\mathrm{m}$ \\
\hline & Belgien & -1.5 & $(1.1)$ & -2.3 & $(0.9)$ & -0.1 & (1.1) & 0.7 & (1.1) & 0.4 & $(1.0)$ & 1.0 & (1.1) & -3.1 & (1.1) & -3.4 & (1.0) & -1.1 & (1.0) \\
\hline & Kanada & 0.6 & $(0.7)$ & 4.9 & $(0.7)$ & 10.4 & $(0.8)$ & -3.3 & $(0.7)$ & 3.2 & $(0.8)$ & -1.4 & (0.6) & 3.3 & $(0.8)$ & -4.6 & $(0.8)$ & -2.6 & $(0.6)$ \\
\hline & Chile & -16.1 & (1.3) & -4.6 & (1.1) & -10.0 & (1.3) & 0.6 & (1.1) & -6.7 & (1.3) & -1.0 & (1.0) & -14.4 & (1.3) & 7.4 & $(1.2)$ & -4.2 & (1.3) \\
\hline & Tschech. Rep. & 13.8 & $(1.2)$ & -18.2 & (1.1) & -13.7 & (1.3) & 1.9 & (1.1) & -14.8 & (1.3) & 11.4 & (1.1) & -16.2 & (1.1) & 7.4 & (1.3) & 9.2 & (1.0) \\
\hline & Dänemark & 8.8 & (1.3) & -6.8 & (1.0) & 0.8 & (1.3) & -3.1 & (1.1) & -4.8 & (1.4) & 1.7 & (1.2) & -13.6 & (1.3) & 3.7 & (1.3) & -1.7 & (1.0) \\
\hline & Finnland & 8.2 & (1.1) & -7.0 & (1.2) & 0.7 & $(1.2)$ & 2.9 & (1.0) & -2.7 & (1.3) & 4.1 & (1.1) & -12.0 & $(1.2)$ & 1.6 & (1.1) & 0.7 & (0.9) \\
\hline & Frankreich & -2.6 & (1.4) & -1.5 & $(1.2)$ & 3.9 & (1.5) & 5.6 & (1.3) & -0.6 & (1.4) & 3.2 & (1.3) & -2.9 & (1.5) & -3.5 & (1.5) & -3.5 & $(1.2)$ \\
\hline & Deutschland & 0.0 & (1.1) & 3.4 & (1.0) & 6.5 & $(1.2)$ & -5.6 & $(1.2)$ & 2.7 & (1.4) & -1.2 & (1.1) & -1.2 & $(1.2)$ & -7.9 & (1.3) & -3.1 & $(0.8)$ \\
\hline & Griechenland & 9.7 & (1.6) & -8.2 & (1.3) & -11.5 & (1.6) & 2.8 & $(1.2)$ & -2.2 & (1.8) & -2.5 & (1.1) & -10.9 & (1.6) & -7.7 & (1.7) & -4.4 & (1.4) \\
\hline & Ungarn & 3.1 & (1.4) & 5.7 & (1.4) & 1.9 & (1.4) & -3.8 & $(1.0)$ & -1.1 & (1.6) & 6.5 & (1.0) & 0.5 & (1.4) & 0.4 & (1.4) & -3.9 & $(1.2)$ \\
\hline & Island & 14.7 & $(1.2)$ & -3.0 & $(1.1)$ & 7.2 & $(1.2)$ & 7.5 & (1.0) & 1.1 & $(1.2)$ & 4.5 & (1.0) & -3.3 & (1.1) & 4.2 & (1.1) & 1.8 & (1.0) \\
\hline & Irland & 5.6 & (1.4) & -4.0 & (1.4) & 6.9 & (1.4) & -1.3 & (1.4) & -1.9 & (1.4) & 4.7 & (1.1) & -1.0 & (1.5) & -0.8 & (1.5) & 1.7 & (1.2) \\
\hline & Israel & 4.1 & $(2.0)$ & 2.3 & $(2.7)$ & -0.9 & (2.6) & 8.8 & (1.6) & -5.0 & (2.1) & 2.3 & (1.5) & -4.2 & (2.5) & 5.6 & (2.4) & 4.6 & (1.6) \\
\hline & Italien & 2.7 & (1.1) & -5.1 & (1.1) & 1.0 & (1.2) & -1.6 & $(0.9)$ & 2.6 & (1.2) & 3.8 & (0.8) & -2.4 & (1.5) & -2.8 & (1.1) & -1.2 & (1.0) \\
\hline & Japan & -0.8 & (1.3) & 5.5 & (1.3) & 7.1 & $(1.2)$ & -12.2 & (1.1) & 4.7 & (1.3) & -4.5 & (1.0) & -0.3 & (1.3) & -6.2 & $(1.0)$ & -6.7 & (1.1) \\
\hline & Korea & 14.0 & (1.3) & 4.6 & $(1.2)$ & 7.9 & $(1.2)$ & -10.2 & (1.1) & 6.2 & (1.5) & -3.2 & $(0.8)$ & -4.9 & (1.4) & -9.5 & (1.3) & -2.2 & $(0.9)$ \\
\hline & Luxemburg & $\mathrm{m}$ & $\mathrm{m}$ & $\mathrm{m}$ & $\mathrm{m}$ & $\mathrm{m}$ & $\mathrm{m}$ & $\mathrm{m}$ & $\mathrm{m}$ & $\mathrm{m}$ & $\mathrm{m}$ & $\mathrm{m}$ & $\mathrm{m}$ & $\mathrm{m}$ & $\mathrm{m}$ & $\mathrm{m}$ & $\mathrm{m}$ & $\mathrm{m}$ & $\mathrm{m}$ \\
\hline & Mexiko & 16.2 & $(1.0)$ & -12.3 & (1.4) & -17.0 & $(1.2)$ & -2.6 & $(0.9)$ & -15.5 & $(1.2)$ & 3.0 & $(0.6)$ & -18.4 & (1.4) & 2.8 & (1.0) & 3.2 & $(0.9)$ \\
\hline & Niederlande & $\mathrm{m}$ & $\mathrm{m}$ & $\mathrm{m}$ & $\mathrm{m}$ & $\mathrm{m}$ & $\mathrm{m}$ & $\mathrm{m}$ & $\mathrm{m}$ & $\mathrm{m}$ & $\mathrm{m}$ & $\mathrm{m}$ & $\mathrm{m}$ & $\mathrm{m}$ & $\mathrm{m}$ & $\mathrm{m}$ & $\mathrm{m}$ & $\mathrm{m}$ & $\mathrm{m}$ \\
\hline & Neuseeland & 2.0 & (1.3) & 4.4 & $(1.2)$ & 9.5 & (1.4) & -6.0 & $(1.2)$ & 5.9 & $(1.2)$ & -1.2 & (1.1) & 2.9 & (1.5) & -3.9 & (1.4) & -2.9 & (1.0) \\
\hline & Norwegen & 1.8 & $(1.2)$ & -2.1 & (1.1) & 6.3 & $(1.1)$ & -0.4 & $(1.0)$ & 1.4 & (1.3) & -0.6 & $(1.2)$ & -7.8 & $(1.2)$ & 1.2 & (1.3) & 0.7 & (1.2) \\
\hline & Polen & 7.9 & (1.6) & -1.5 & (1.4) & 0.5 & (1.3) & -1.9 & (1.3) & -6.8 & (1.5) & 2.0 & (1.3) & -13.3 & (1.5) & 0.0 & (1.6) & 8.7 & (1.2) \\
\hline & Portugal & -2.3 & $(1.2)$ & -18.8 & $(1.2)$ & -8.7 & (1.3) & 1.6 & (1.0) & -11.8 & (1.1) & 5.6 & $(0.9)$ & -15.5 & (1.3) & 7.7 & (1.3) & 6.7 & $(1.2)$ \\
\hline & Spanien & 2.3 & (1.3) & -1.7 & $(1.1)$ & 0.9 & (1.3) & 1.9 & (1.0) & -2.4 & (1.1) & 2.0 & $(0.9)$ & -11.7 & (1.1) & 0.2 & (1.3) & 2.9 & $(0.9)$ \\
\hline & Schweden & 2.0 & $(1.2)$ & -2.2 & (1.0) & 3.6 & $(1.2)$ & -1.8 & (1.0) & -2.4 & $(1.2)$ & -1.2 & $(1.1)$ & -6.9 & (1.3) & -1.8 & (1.3) & -1.3 & $(1.0)$ \\
\hline & Schweiz & 7.3 & (1.3) & -5.5 & $(1.3)$ & -0.6 & $(1.2)$ & 1.1 & (1.1) & -8.4 & (1.4) & 5.8 & $(1.2)$ & -5.9 & (1.4) & 0.1 & (1.4) & -0.7 & $(0.9)$ \\
\hline & Ver. Königreich & $\mathrm{m}$ & $\mathrm{m}$ & $\mathrm{m}$ & $\mathrm{m}$ & $\mathrm{m}$ & $\mathrm{m}$ & $\mathrm{m}$ & $\mathrm{m}$ & $\mathrm{m}$ & $\mathrm{m}$ & $\mathrm{m}$ & $\mathrm{m}$ & $\mathrm{m}$ & $\mathrm{m}$ & $\mathrm{m}$ & $\mathrm{m}$ & $\mathrm{m}$ & $\mathrm{m}$ \\
\hline & Ver. Staaten & 4.3 & (1.8) & 0.7 & (1.5) & 6.2 & (1.8) & -4.8 & (1.4) & -2.1 & (1.7) & -0.2 & (1.7) & 0.8 & (1.8) & -0.4 & $(2.0)$ & -2.3 & $(1.7)$ \\
\hline & OECD-26-Durchschnitt & 4.2 & $(0.3)$ & -2.7 & $(0.3)$ & 1.1 & $(0.3)$ & -0.9 & $(0.2)$ & -2.1 & $(0.3)$ & 1.8 & $(0.2)$ & -6.1 & $(0.3)$ & -0.5 & $(0.3)$ & 0.0 & $(0.2)$ \\
\hline \multirow{12}{*}{ 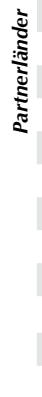 } & Albanien & 14.5 & (1.3) & 3.7 & (1.4) & -5.7 & $(1.2)$ & -0.7 & (1.1) & -0.1 & $(0.9)$ & -5.2 & $(0.9)$ & -2.3 & (1.0) & 4.4 & (1.5) & 4.0 & (1.1) \\
\hline & Argentinien & 9.9 & $(2.0)$ & -7.5 & (1.4) & -3.6 & (1.9) & 5.5 & (1.8) & 4.0 & (2.0) & 0.0 & (1.4) & -7.6 & (1.6) & 7.7 & (1.9) & 1.0 & (1.7) \\
\hline & Brasilien & -27.2 & (1.2) & -0.5 & (1.3) & -1.2 & (1.4) & 0.4 & $(1.2)$ & -11.5 & (1.3) & -2.7 & $(0.6)$ & -6.9 & (1.4) & -13.4 & (1.5) & 3.9 & $(1.2)$ \\
\hline & Bulgarien & 1.9 & (1.8) & -9.5 & (1.9) & -7.3 & $(2.2)$ & 4.0 & (1.3) & -5.5 & (1.8) & 2.7 & (1.4) & 2.4 & (1.7) & 1.0 & (1.8) & 3.0 & (1.4) \\
\hline & Hongkong (China) & -7.5 & (1.3) & 11.9 & $(1.2)$ & 3.9 & (1.1) & -11.4 & (1.1) & 12.8 & (1.4) & -3.4 & $(0.7)$ & 4.4 & $(1.2)$ & -11.4 & $(1.2)$ & -8.2 & $(0.9)$ \\
\hline & Indonesien & 24.4 & $(1.2)$ & -12.5 & (1.3) & -11.5 & $(1.0)$ & 9.0 & $(1.2)$ & -3.6 & $(1.0)$ & -2.5 & $(0.7)$ & -7.5 & $(1.2)$ & 2.2 & (1.5) & 1.1 & (1.2) \\
\hline & Lettland & 7.5 & (1.6) & -8.1 & (1.4) & 1.4 & (1.6) & -4.2 & (1.5) & -7.0 & (1.9) & 3.1 & (1.4) & -1.7 & (1.6) & -1.2 & (1.7) & 3.1 & (1.3) \\
\hline & Liechtenstein & 10.3 & (3.8) & -9.8 & (3.2) & -3.2 & (3.0) & -1.3 & (3.4) & -9.2 & (3.8) & 10.8 & (3.5) & -6.6 & (3.5) & 1.1 & (3.7) & 2.0 & (3.1) \\
\hline & Peru & 21.3 & $(1.2)$ & -3.2 & (1.4) & -6.0 & (1.4) & -17.7 & $(1.2)$ & 1.6 & (1.4) & -6.9 & $(0.8)$ & -13.2 & (1.5) & 0.6 & (1.3) & -23.4 & $(1.2)$ \\
\hline & Rumänien & -13.2 & (1.5) & -19.6 & (1.5) & -10.1 & (1.4) & 1.8 & (1.6) & -5.9 & (1.4) & 3.6 & (1.3) & -10.8 & (1.5) & 10.8 & (1.6) & -1.7 & (1.4) \\
\hline & Russ. Föderation & 4.4 & $(1.2)$ & -4.7 & (1.5) & -2.4 & (1.4) & -2.8 & $(1.0)$ & -4.5 & (1.4) & 1.4 & $(0.9)$ & -4.2 & (1.3) & -0.1 & $(1.2)$ & 0.9 & $(0.7)$ \\
\hline & Thailand & -40.5 & (1.1) & 1.0 & (1.0) & -4.9 & $(1.2)$ & -13.4 & (1.3) & 1.5 & $(1.2)$ & -11.3 & (1.3) & 0.9 & (1.1) & -22.8 & (1.4) & -7.4 & (1.3) \\
\hline
\end{tabular}

Anmerkung: Statistisch signifikante Werte sind durch Fettdruck gekennzeichnet (siehe Anhang A3).

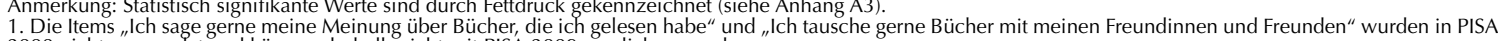
2000 nicht verwendet und können deshalb nicht mit PISÁ 2009 verglichen werden.

StatLink ints http://dx.doi.org/10.1787/888932343285 
[Teil 1/3]

Prozentsatz der Schüler in PISA 2000 und PISA 2009, die zum Vergnügen lesen,

nach sozioökonomischem Hintergrund und Geschlecht

Tabelle V.5.4 Auf Grundlage der Schülerangaben

\begin{tabular}{|c|c|c|c|c|c|c|c|c|c|c|c|c|c|}
\hline & \multicolumn{12}{|c|}{ PISA 2000} \\
\hline & & \multicolumn{4}{|c|}{ Alle Schüler } & \multicolumn{4}{|c|}{ Jungen } & \multicolumn{4}{|c|}{ Mädchen } \\
\hline & & \multicolumn{2}{|c|}{$\begin{array}{c}\text { Unterstes } \\
\text { ESCS-Quartil }\end{array}$} & \multicolumn{2}{|c|}{$\begin{array}{c}\text { Oberstes } \\
\text { ESCS-Quartil }\end{array}$} & \multicolumn{2}{|c|}{$\begin{array}{c}\text { Unterstes } \\
\text { ESCS-Quartil }\end{array}$} & \multicolumn{2}{|c|}{$\begin{array}{c}\text { Oberstes } \\
\text { ESCS-Quartil }\end{array}$} & \multicolumn{2}{|c|}{$\begin{array}{c}\text { Unterstes } \\
\text { ESCS-Quartil }\end{array}$} & \multicolumn{2}{|c|}{$\begin{array}{c}\text { Oberstes } \\
\text { ESCS-Quartil }\end{array}$} \\
\hline & & $\%$ & S.E. & $\%$ & S.E. & $\%$ & S.E. & $\%$ & S.E. & $\%$ & S.E. & $\%$ & S.E. \\
\hline \multirow{31}{*}{ 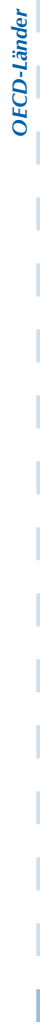 } & Australien & 58.5 & (2.1) & 78.2 & (1.8) & 49.0 & $(2.7)$ & 74.4 & (2.3) & 68.2 & (2.6) & 82.6 & (2.6) \\
\hline & Österreich & 53.0 & (1.8) & 66.1 & (1.8) & 43.6 & (2.3) & 53.8 & (2.0) & 62.6 & (2.3) & 79.4 & (1.9) \\
\hline & Belgien & 55.6 & (2.1) & 67.8 & (1.3) & 46.7 & (2.9) & 59.8 & (2.1) & 64.6 & (1.9) & 77.5 & (1.4) \\
\hline & Kanada & 61.4 & $(0.7)$ & 75.1 & $(0.7)$ & 49.0 & (0.9) & 68.2 & $(1.2)$ & 72.8 & (0.9) & 82.4 & $(0.8)$ \\
\hline & Chile & 73.9 & (1.5) & 77.0 & $(1.2)$ & 69.0 & $(2.2)$ & 69.0 & (1.7) & 78.1 & (1.8) & 83.5 & (1.5) \\
\hline & Tschech. Rep. & 69.7 & (1.4) & 80.7 & $(1.1)$ & 58.9 & (2.3) & 71.3 & (2.0) & 79.5 & (1.3) & 90.4 & (1.2) \\
\hline & Dänemark & 69.2 & (1.4) & 80.7 & (1.5) & 55.9 & (2.3) & 74.4 & (2.1) & 79.8 & $(1.7)$ & 88.2 & (1.5) \\
\hline & Finnland & 75.9 & $(1.2)$ & 81.7 & (1.3) & 63.5 & (2.0) & 71.7 & (2.0) & 86.3 & (1.3) & 92.1 & (1.1) \\
\hline & Frankreich & 65.6 & (1.4) & 78.3 & (1.5) & 56.9 & $(2.0)$ & 71.7 & (2.0) & 73.5 & (1.9) & 85.0 & (1.5) \\
\hline & Deutschland & 56.3 & (1.6) & 70.8 & (1.5) & 47.6 & (3.6) & 58.6 & (2.4) & 64.5 & $(2.1)$ & 84.7 & (1.5) \\
\hline & Griechenland & 74.8 & (1.5) & 80.1 & (1.4) & 70.6 & (2.6) & 79.2 & (2.1) & 78.7 & (1.8) & 81.1 & (1.5) \\
\hline & Ungarn & 70.4 & $(2.2)$ & 82.4 & (1.1) & 63.9 & (2.9) & 76.7 & (1.8) & 76.6 & (2.4) & 88.7 & (1.6) \\
\hline & Island & 67.6 & (1.5) & 75.8 & (1.4) & 59.6 & (2.4) & 70.0 & (2.0) & 74.6 & (1.9) & 81.9 & (1.9) \\
\hline & Irland & 62.1 & (1.6) & 73.7 & (1.6) & 54.2 & $(2.6)$ & 64.6 & (2.3) & 69.0 & (1.9) & 83.1 & (1.9) \\
\hline & Israel & 64.8 & $(4.1)$ & 63.3 & (2.8) & 56.1 & (4.9) & 54.2 & (3.1) & 69.2 & $(4.3)$ & 71.5 & (3.5) \\
\hline & Italien & 65.8 & (1.8) & 76.7 & (1.4) & 58.1 & $(2.7)$ & 70.8 & (1.8) & 72.8 & $(2.0)$ & 82.7 & (1.8) \\
\hline & Japan & $\mathrm{m}$ & $\mathrm{m}$ & $\mathrm{m}$ & $\mathrm{m}$ & $\mathrm{m}$ & $\mathrm{m}$ & $\mathrm{m}$ & $\mathrm{m}$ & $\mathrm{m}$ & $\mathrm{m}$ & $\mathrm{m}$ & $\mathrm{m}$ \\
\hline & Korea & 61.3 & (1.5) & 77.9 & (1.3) & 59.1 & (1.9) & 76.9 & (1.7) & 63.7 & (2.5) & 79.3 & (2.5) \\
\hline & Luxemburg & $\mathrm{m}$ & $\mathrm{m}$ & $\mathrm{m}$ & $\mathrm{m}$ & $\mathrm{m}$ & $\mathrm{m}$ & $\mathrm{m}$ & $\mathrm{m}$ & $\mathrm{m}$ & $\mathrm{m}$ & $\mathrm{m}$ & $\mathrm{m}$ \\
\hline & Mexiko & 89.4 & (1.1) & 83.1 & (2.0) & 85.4 & (1.6) & 77.5 & $(2.7)$ & 93.6 & (1.1) & 89.0 & (1.9) \\
\hline & Niederlande & m & $\mathrm{m}$ & $\mathrm{m}$ & $\mathrm{m}$ & $\mathrm{m}$ & $\mathrm{m}$ & $\mathrm{m}$ & $\mathrm{m}$ & $\mathrm{m}$ & $\mathrm{m}$ & $\mathrm{m}$ & $\mathrm{m}$ \\
\hline & Neuseeland & 67.8 & (1.7) & 76.6 & (1.5) & 62.9 & $(2.0)$ & 70.7 & $(2.1)$ & 72.6 & $(2.3)$ & 82.8 & (2.0) \\
\hline & Norwegen & 62.9 & (1.7) & 72.5 & (1.5) & 50.7 & $(2.7)$ & 64.0 & (1.8) & 73.6 & (2.0) & 83.2 & (2.0) \\
\hline & Polen & 72.0 & (1.9) & 81.5 & $(1.7)$ & 61.0 & $(2.9)$ & 77.5 & $(2.2)$ & 81.6 & (1.9) & 86.0 & (2.3) \\
\hline & Portugal & 79.7 & (1.5) & 87.7 & $(1.0)$ & 68.5 & (2.4) & 80.2 & (1.7) & 88.4 & (1.4) & 94.8 & (1.0) \\
\hline & Spanien & 61.6 & $(2.1)$ & 76.8 & (1.5) & 51.0 & $(2.7)$ & 70.3 & (1.9) & 69.7 & (2.8) & 84.0 & (1.8) \\
\hline & Schweden & 60.1 & (1.8) & 69.6 & (1.6) & 47.5 & (2.4) & 63.2 & (2.0) & 71.4 & (2.0) & 77.5 & (2.2) \\
\hline & Schweiz & 58.6 & (1.9) & 74.8 & (1.4) & 42.8 & (2.8) & 66.0 & (1.8) & 75.3 & (1.9) & 85.2 & (1.5) \\
\hline & Ver. Königreich & $\mathrm{m}$ & $\mathrm{m}$ & $\mathrm{m}$ & $\mathrm{m}$ & $\mathrm{m}$ & $\mathrm{m}$ & $\mathrm{m}$ & $\mathrm{m}$ & $\mathrm{m}$ & $\mathrm{m}$ & $\mathrm{m}$ & $\mathrm{m}$ \\
\hline & Ver. Staaten & 56.7 & $(2.0)$ & 67.9 & $(1.5)$ & 47.4 & (3.5) & 62.9 & (2.3) & 65.1 & (2.8) & 73.4 & $(2.0)$ \\
\hline & OECD-26-Durchschnitt & 66.5 & $(0.4)$ & 76.4 & $(0.3)$ & 57.4 & $(0.5)$ & 69.7 & $(0.4)$ & 74.5 & $(0.4)$ & 83.6 & $(0.4)$ \\
\hline \multirow{12}{*}{ 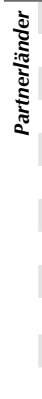 } & Albanien & 93.1 & (1.3) & 92.1 & $(0.8)$ & 92.6 & $(2.2)$ & 88.5 & $(1.4)$ & 93.6 & $(1.1)$ & 95.3 & (1.0) \\
\hline & Argentinien & 70.0 & $(2.7)$ & 73.0 & (1.8) & 63.8 & (3.3) & 64.3 & $(2.7)$ & 75.1 & (3.9) & 80.8 & (2.1) \\
\hline & Brasilien & 80.4 & (1.6) & 81.9 & (1.7) & 72.5 & (2.9) & 74.6 & $(2.8)$ & 86.2 & (1.5) & 89.3 & (1.8) \\
\hline & Bulgarien & 65.1 & (1.9) & 74.9 & $(1.5)$ & 57.4 & (2.4) & 65.4 & (2.0) & 72.8 & (2.3) & 85.4 & (1.6) \\
\hline & Hongkong (China) & 67.3 & $(1.7)$ & 85.6 & (1.1) & 58.3 & (2.4) & 83.0 & (1.7) & 77.1 & (1.9) & 88.1 & (1.4) \\
\hline & Indonesien & 92.4 & $(0.8)$ & 92.7 & $(0.8)$ & 91.6 & (1.1) & 90.8 & (1.4) & 93.1 & (1.1) & 94.5 & (1.1) \\
\hline & Lettland & 79.5 & (1.6) & 84.6 & $(2.1)$ & 69.1 & (2.8) & 75.3 & $(3.7)$ & 88.7 & (1.8) & 94.1 & (1.0) \\
\hline & Liechtenstein & 49.9 & (5.6) & 71.8 & $(5.1)$ & 36.1 & (6.9) & 79.7 & (6.5) & 71.9 & $(8.0)$ & 65.6 & (7.9) \\
\hline & Peru & 92.6 & (0.9) & 89.6 & (1.1) & 91.7 & (1.5) & 87.6 & (1.6) & 93.5 & $(1.2)$ & 91.4 & (1.2) \\
\hline & Rumänien & 79.4 & (1.8) & 86.1 & (1.1) & 78.7 & (3.0) & 82.7 & (2.1) & 80.0 & (2.3) & 89.4 & (1.2) \\
\hline & Russ. Föderation & 76.8 & (1.1) & 84.6 & $(1.2)$ & 69.7 & (1.7) & 78.8 & (1.5) & 82.9 & (1.4) & 90.9 & (1.6) \\
\hline & Thailand & 86.9 & $(1.2)$ & 90.6 & $(1.2)$ & 80.5 & (2.1) & 89.7 & (1.9) & 91.0 & $(2.2)$ & 91.4 & (1.1) \\
\hline
\end{tabular}

Anmerkung: Statistisch signifikante Werte sind durch Fettdruck gekennzeichnet (siehe Anhang A3).

StatLink inst http://dx.doi.org/10.1787/888932343285 
[Teil 2/3]

Prozentsatz der Schüler in PISA 2000 und PISA 2009, die zum Vergnügen lesen,

nach sozioökonomischem Hintergrund und Geschlecht

Tabelle V.5.4 Auf Grundlage der Schülerangaben

\begin{tabular}{|c|c|c|c|c|c|c|c|c|c|c|c|c|c|}
\hline & \multicolumn{12}{|c|}{ PISA 2009} \\
\hline & & \multicolumn{4}{|c|}{ Alle Schüler } & \multicolumn{4}{|c|}{ Jungen } & \multicolumn{4}{|c|}{ Mädchen } \\
\hline & & \multicolumn{2}{|c|}{$\begin{array}{c}\text { Unterstes } \\
\text { ESCS-Quartil }\end{array}$} & \multicolumn{2}{|c|}{$\begin{array}{c}\text { Oberstes } \\
\text { ESCS-Quartil }\end{array}$} & \multicolumn{2}{|c|}{$\begin{array}{c}\text { Unterstes } \\
\text { ESCS-Quartil }\end{array}$} & \multicolumn{2}{|c|}{$\begin{array}{c}\text { Oberstes } \\
\text { ESCS-Quartil }\end{array}$} & \multicolumn{2}{|c|}{$\begin{array}{c}\text { Unterstes } \\
\text { ESCS-Quartil }\end{array}$} & \multicolumn{2}{|c|}{$\begin{array}{c}\text { Oberstes } \\
\text { ESCS-Quartil }\end{array}$} \\
\hline & & $\%$ & S.E. & $\%$ & S.E. & $\%$ & S.E. & $\%$ & S.E. & $\%$ & S.E. & $\%$ & S.E. \\
\hline \multirow{31}{*}{ 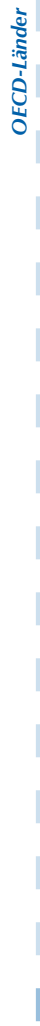 } & Australien & 52.9 & (1.1) & 75.2 & $(0.9)$ & 40.3 & (1.6) & 68.2 & (1.4) & 64.5 & (1.3) & 82.1 & (1.1) \\
\hline & Österreich & $\mathrm{m}$ & $\mathrm{m}$ & $\mathrm{m}$ & $\mathrm{m}$ & $\mathrm{m}$ & $\mathrm{m}$ & $\mathrm{m}$ & $\mathrm{m}$ & $\mathrm{m}$ & $\mathrm{m}$ & $\mathrm{m}$ & $\mathrm{m}$ \\
\hline & Belgien & 45.3 & (1.3) & 68.5 & $(1.0)$ & 34.5 & (1.6) & 60.9 & (1.5) & 55.6 & (1.9) & 76.7 & $(1.2)$ \\
\hline & Kanada & 61.3 & $(1.0)$ & 78.4 & $(0.8)$ & 46.1 & $(1.5)$ & 68.8 & (1.3) & 76.2 & $(1.2)$ & 88.4 & $(0.7)$ \\
\hline & Chile & 62.3 & (1.4) & 62.6 & (1.4) & 55.5 & (2.0) & 52.8 & (1.8) & 69.0 & (1.9) & 72.4 & (1.8) \\
\hline & Tschech. Rep. & 49.1 & (1.6) & 67.5 & (1.4) & 37.4 & (2.5) & 55.4 & (1.9) & 61.1 & (2.6) & 81.4 & (1.3) \\
\hline & Dänemark & 60.1 & (1.5) & 77.7 & (1.3) & 48.9 & $(2.0)$ & 72.6 & (1.8) & 69.7 & (1.9) & 82.7 & (1.6) \\
\hline & Finnland & 59.4 & (1.6) & 75.5 & (1.4) & 44.8 & (2.1) & 64.2 & (2.3) & 75.1 & $(2.0)$ & 87.0 & (1.4) \\
\hline & Frankreich & 48.9 & (1.9) & 74.2 & $(1.6)$ & 38.0 & (2.5) & 65.9 & $(2.2)$ & 58.6 & (2.6) & 82.5 & (1.8) \\
\hline & Deutschland & 45.6 & $(1.8)$ & 73.6 & $(1.4)$ & 31.9 & $(2.2)$ & 63.5 & (2.0) & 58.6 & $(2.1)$ & 84.8 & (1.5) \\
\hline & Griechenland & 79.3 & (1.8) & 86.3 & $(1.2)$ & 73.2 & (2.5) & 80.1 & (1.7) & 85.1 & (1.6) & 93.2 & (1.1) \\
\hline & Ungarn & 67.9 & (1.8) & 84.6 & $(1.2)$ & 57.5 & (2.8) & 77.2 & $(2.0)$ & 76.9 & $(2.0)$ & 92.9 & $(1.2)$ \\
\hline & Island & 57.8 & (1.7) & 69.9 & (1.5) & 42.4 & $(2.7)$ & 62.5 & (2.5) & 71.3 & $(2.3)$ & 77.7 & $(2.2)$ \\
\hline & Irland & 44.3 & $(2.0)$ & 74.1 & (1.5) & 40.7 & $(2.7)$ & 70.5 & $(2.2)$ & 47.9 & (2.5) & 78.0 & (1.8) \\
\hline & Israel & 65.6 & (1.7) & 68.5 & (1.3) & 51.9 & $(2.7)$ & 62.2 & (1.9) & 76.1 & (1.7) & 76.0 & (1.9) \\
\hline & Italien & 58.5 & $(1.0)$ & 74.2 & $(0.8)$ & 45.1 & (1.4) & 63.6 & (1.0) & 71.6 & (1.3) & 85.9 & $(0.8)$ \\
\hline & Japan & 48.8 & (1.4) & 65.0 & (1.6) & 46.7 & $(2.1)$ & 60.6 & (1.6) & 51.3 & (2.0) & 69.4 & (2.6) \\
\hline & Korea & 51.7 & (1.5) & 73.1 & (1.3) & 51.3 & $(2.0)$ & 72.2 & $(1.7)$ & 52.2 & (2.6) & 74.0 & (1.8) \\
\hline & Luxemburg & 41.8 & (1.5) & 65.2 & (1.5) & 26.2 & (1.9) & 52.8 & (2.0) & 55.4 & $(2.0)$ & 79.3 & (1.8) \\
\hline & Mexiko & 83.4 & $(0.6)$ & 72.4 & $(0.9)$ & 78.9 & (1.1) & 64.4 & (1.3) & 87.2 & $(0.8)$ & 81.1 & $(0.7)$ \\
\hline & Niederlande & 44.7 & $(2.2)$ & 63.4 & (1.8) & 27.9 & (2.4) & 49.7 & (2.3) & 60.0 & $(2.7)$ & 77.6 & $(2.2)$ \\
\hline & Neuseeland & 60.4 & (1.7) & 78.7 & (1.3) & 47.6 & (2.4) & 73.0 & (1.7) & 72.3 & (1.9) & 84.6 & (1.9) \\
\hline & Norwegen & 54.6 & (1.6) & 69.0 & (1.7) & 42.4 & $(2.0)$ & 62.1 & $(2.2)$ & 65.9 & $(2.2)$ & 76.6 & $(2.0)$ \\
\hline & Polen & 63.6 & (1.3) & 77.5 & (1.4) & 44.5 & (2.5) & 66.4 & $(2.2)$ & 80.3 & (1.4) & 88.4 & $(1.6)$ \\
\hline & Portugal & 61.4 & (1.5) & 71.1 & (1.5) & 46.5 & $(2.0)$ & 59.6 & (1.9) & 73.6 & (1.7) & 83.2 & (1.9) \\
\hline & Spanien & 52.2 & (1.6) & 68.9 & $(1.1)$ & 42.8 & $(2.0)$ & 59.8 & (1.6) & 61.8 & (2.1) & 78.7 & $(1.3)$ \\
\hline & Schweden & 56.4 & (1.8) & 71.4 & $(1.4)$ & 45.5 & $(2.2)$ & 58.9 & $(2.1)$ & 67.9 & $(2.2)$ & 84.7 & $(1.6)$ \\
\hline & Schweiz & 47.1 & (1.9) & 69.0 & (1.5) & 32.6 & (1.8) & 60.3 & $(2.1)$ & 61.4 & $(2.8)$ & 78.0 & (1.6) \\
\hline & Ver. Königreich & 53.8 & (1.8) & 71.9 & (1.3) & 44.7 & $(2.1)$ & 63.1 & (1.7) & 62.1 & $(2.2)$ & 81.1 & (1.4) \\
\hline & Ver. Staaten & 51.5 & (1.6) & 69.4 & (1.5) & 38.6 & $(1.8)$ & 60.2 & (1.6) & 65.1 & $(2.3)$ & 80.0 & $(2.2)$ \\
\hline & OECD-26-Durchschnitt & 57.3 & $(0.3)$ & 72.9 & $(0.3)$ & 46.4 & $(0.4)$ & 64.8 & $(0.4)$ & 67.6 & $(0.4)$ & 81.6 & $(0.3)$ \\
\hline \multirow{12}{*}{ 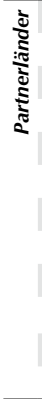 } & Albanien & 92.2 & $(1.2)$ & 93.3 & $(0.8)$ & 88.4 & (1.8) & 89.2 & (1.3) & 95.7 & $(1.2)$ & 97.6 & $(0.6)$ \\
\hline & Argentinien & 63.3 & (1.6) & 58.5 & $(1.7)$ & 55.4 & $(2.3)$ & 50.6 & $(2.3)$ & 69.7 & $(2.1)$ & 66.3 & $(2.4)$ \\
\hline & Brasilien & 80.7 & (1.1) & 78.4 & (1.1) & 73.0 & (1.7) & 68.9 & (1.8) & 85.8 & $(1.2)$ & 88.1 & (1.1) \\
\hline & Bulgarien & 65.6 & $(2.4)$ & 78.9 & (1.6) & 56.2 & $(2.9)$ & 68.3 & (2.5) & 75.7 & $(2.5)$ & 90.3 & (1.3) \\
\hline & Hongkong (China) & 74.3 & (1.3) & 85.2 & $(1.2)$ & 69.1 & (1.9) & 81.4 & (1.5) & 80.3 & (1.8) & 89.8 & (1.4) \\
\hline & Indonesien & 85.2 & $(1.2)$ & 91.6 & $(0.8)$ & 78.2 & (1.9) & 88.9 & $(1.2)$ & 91.8 & $(1.2)$ & 94.6 & $(0.8)$ \\
\hline & Lettland & 64.4 & (1.7) & 78.8 & (1.4) & 44.5 & (2.9) & 66.2 & (2.5) & 80.2 & $(2.4)$ & 91.5 & (1.3) \\
\hline & Liechtenstein & 41.1 & $(5.2)$ & 58.2 & $(5.3)$ & 26.7 & $(7.0)$ & 54.0 & $(6.2)$ & 54.5 & (7.5) & 63.2 & $(8.2)$ \\
\hline & Peru & 90.3 & $(0.9)$ & 83.3 & $(1.1)$ & 89.3 & $(1.3)$ & 79.9 & (1.6) & 91.4 & (1.1) & 86.6 & (1.3) \\
\hline & Rumänien & 75.1 & (1.6) & 75.4 & $(1.4)$ & 67.4 & (1.8) & 65.7 & $(2.1)$ & 82.3 & $(2.1)$ & 85.7 & (1.6) \\
\hline & Russ. Föderation & 76.1 & (1.4) & 83.2 & $(1.0)$ & 65.5 & (2.3) & 77.0 & (1.6) & 86.2 & (1.6) & 89.6 & (1.3) \\
\hline & Thailand & 90.5 & $(1.0)$ & 92.8 & $(0.9)$ & 84.0 & (1.8) & 88.3 & (1.6) & 94.7 & $(0.9)$ & 96.8 & $(0.8)$ \\
\hline
\end{tabular}

Anmerkung: Statistisch signifikante Werte sind durch Fettdruck gekennzeichnet (siehe Anhang A3).

StatLink ins $\mathrm{sttp}$ ://dx.doi.org/10.1787/888932343285 
[Teil 3/3]

Prozentsatz der Schüler in PISA 2000 und PISA 2009, die zum Vergnügen lesen,

nach sozioökonomischem Hintergrund und Geschlecht

Tabelle V.5.4 Auf Grundlage der Schülerangaben

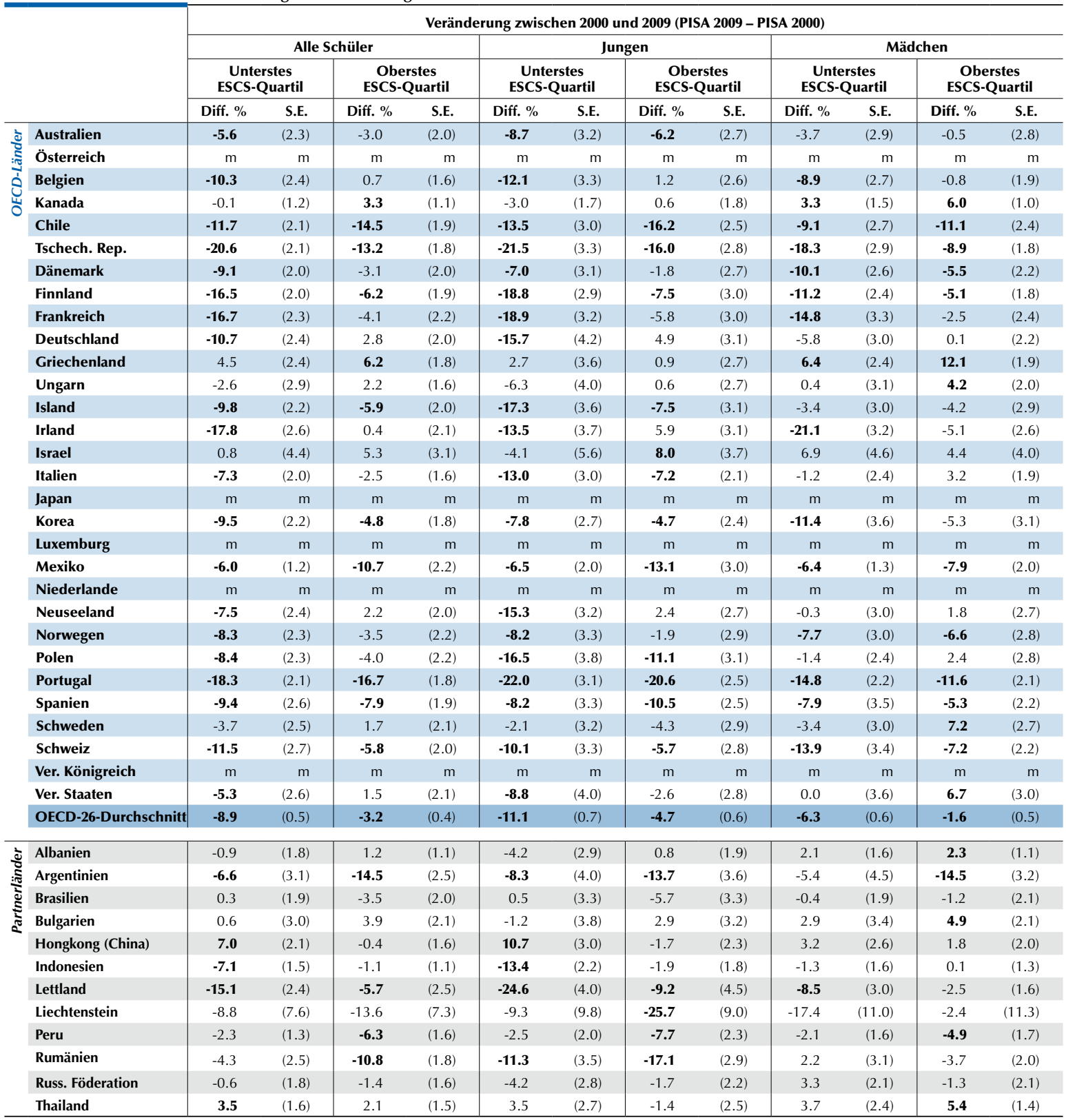

Anmerkung: Statistisch signifikante Werte sind durch Fettdruck gekennzeichnet (siehe Anhang A3).

StatLink त्ताst http://dx.doi.org/10.1787/888932343285 
[Teil 1/3]

Index der Freude am Lesen in PISA 2000 und PISA 2009, nach soziökonomischem Hintergrund und Geschlecht

Tabelle V.5.5 Auf Grundlage der Schülerangaben

\begin{tabular}{|c|c|c|c|c|c|c|c|c|c|c|c|c|c|}
\hline & \multicolumn{12}{|c|}{ PISA 2000} \\
\hline & & \multicolumn{4}{|c|}{ Alle Schüler } & \multicolumn{4}{|c|}{ Jungen } & \multicolumn{4}{|c|}{ Mädchen } \\
\hline & & \multicolumn{2}{|c|}{$\begin{array}{c}\text { Unterstes } \\
\text { ESCS-Quartil }\end{array}$} & \multicolumn{2}{|c|}{$\begin{array}{c}\text { Oberstes } \\
\text { ESCS-Quartil }\end{array}$} & \multicolumn{2}{|c|}{$\begin{array}{c}\text { Unterstes } \\
\text { ESCS-Quartil }\end{array}$} & \multicolumn{2}{|c|}{$\begin{array}{c}\text { Oberstes } \\
\text { ESCS-Quartil }\end{array}$} & \multicolumn{2}{|c|}{$\begin{array}{c}\text { Unterstes } \\
\text { ESCS-Quartil }\end{array}$} & \multicolumn{2}{|c|}{$\begin{array}{c}\text { Oberstes } \\
\text { ESCS-Quartil }\end{array}$} \\
\hline & & $\begin{array}{l}\text { Index- } \\
\text { mittel }\end{array}$ & S.E. & $\begin{array}{l}\text { Index- } \\
\text { mittel }\end{array}$ & S.E. & $\begin{array}{l}\text { Index- } \\
\text { mittel }\end{array}$ & S.E. & $\begin{array}{l}\text { Index- } \\
\text { mittel }\end{array}$ & S.E. & $\begin{array}{l}\text { Index- } \\
\text { mittel }\end{array}$ & S.E. & $\begin{array}{l}\text { Index- } \\
\text { mittel }\end{array}$ & S.E. \\
\hline \multirow{31}{*}{ 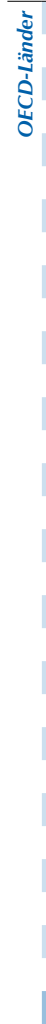 } & Australien & -0.25 & $(0.04)$ & 0.28 & (0.05) & -0.47 & $(0.04)$ & 0.09 & $(0.05)$ & -0.03 & $(0.05)$ & 0.50 & $(0.08)$ \\
\hline & Österreich & -0.29 & $(0.04)$ & 0.24 & $(0.05)$ & -0.68 & $(0.04)$ & -0.22 & $(0.04)$ & 0.11 & $(0.05)$ & 0.75 & $(0.07)$ \\
\hline & Belgien & -0.37 & $(0.03)$ & -0.03 & $(0.03)$ & -0.58 & $(0.04)$ & -0.30 & $(0.04)$ & -0.17 & $(0.04)$ & 0.29 & $(0.04)$ \\
\hline & Kanada & -0.16 & $(0.01)$ & 0.29 & $(0.02)$ & -0.49 & $(0.02)$ & 0.01 & $(0.03)$ & 0.15 & $(0.02)$ & 0.59 & $(0.03)$ \\
\hline & Chile & -0.08 & $(0.03)$ & 0.15 & $(0.04)$ & -0.31 & $(0.05)$ & -0.21 & $(0.04)$ & 0.12 & $(0.04)$ & 0.43 & $(0.05)$ \\
\hline & Tschech. Rep. & 0.03 & $(0.03)$ & 0.39 & $(0.03)$ & -0.39 & $(0.04)$ & 0.01 & $(0.05)$ & 0.37 & $(0.04)$ & 0.78 & $(0.04)$ \\
\hline & Dänemark & -0.12 & $(0.03)$ & 0.29 & $(0.03)$ & -0.45 & $(0.05)$ & -0.01 & $(0.04)$ & 0.14 & $(0.05)$ & 0.66 & $(0.04)$ \\
\hline & Finnland & 0.13 & $(0.03)$ & 0.43 & $(0.04)$ & -0.43 & $(0.04)$ & 0.00 & $(0.04)$ & 0.59 & $(0.04)$ & 0.87 & $(0.05)$ \\
\hline & Frankreich & -0.21 & $(0.03)$ & 0.20 & $(0.04)$ & -0.44 & $(0.04)$ & -0.09 & $(0.05)$ & 0.01 & $(0.04)$ & 0.49 & $(0.05)$ \\
\hline & Deutschland & -0.28 & $(0.03)$ & 0.29 & $(0.04)$ & -0.67 & $(0.05)$ & -0.16 & $(0.06)$ & 0.06 & $(0.05)$ & 0.80 & $(0.05)$ \\
\hline & Griechenland & -0.05 & $(0.03)$ & 0.09 & $(0.04)$ & -0.24 & $(0.03)$ & -0.16 & $(0.05)$ & 0.14 & $(0.03)$ & 0.37 & $(0.04)$ \\
\hline & Ungarn & -0.08 & $(0.03)$ & 0.35 & $(0.04)$ & -0.26 & $(0.05)$ & 0.11 & $(0.05)$ & 0.08 & $(0.04)$ & 0.62 & $(0.04)$ \\
\hline & Island & -0.03 & $(0.03)$ & 0.21 & $(0.03)$ & -0.31 & $(0.04)$ & -0.06 & $(0.04)$ & 0.21 & $(0.04)$ & 0.49 & $(0.04)$ \\
\hline & Irland & -0.20 & $(0.04)$ & 0.20 & $(0.04)$ & -0.50 & $(0.05)$ & -0.10 & $(0.06)$ & 0.05 & $(0.05)$ & 0.50 & $(0.05)$ \\
\hline & Israel & 0.12 & $(0.08)$ & 0.23 & $(0.06)$ & -0.15 & $(0.09)$ & -0.03 & $(0.07)$ & 0.26 & $(0.09)$ & 0.45 & (0.09) \\
\hline & Italien & -0.06 & $(0.03)$ & 0.25 & $(0.04)$ & -0.26 & $(0.05)$ & -0.07 & $(0.05)$ & 0.13 & $(0.04)$ & 0.57 & $(0.04)$ \\
\hline & Japan & m & $\mathrm{m}$ & $\mathrm{m}$ & $\mathrm{m}$ & $\mathrm{m}$ & $\mathrm{m}$ & $\mathrm{m}$ & $\mathrm{m}$ & $\mathrm{m}$ & $\mathrm{m}$ & $\mathrm{m}$ & $\mathrm{m}$ \\
\hline & Korea & -0.14 & $(0.03)$ & 0.29 & $(0.04)$ & -0.24 & $(0.03)$ & 0.20 & $(0.05)$ & -0.03 & $(0.04)$ & 0.42 & $(0.07)$ \\
\hline & Luxemburg & $\mathrm{m}$ & m & $\mathrm{m}$ & m & $\mathrm{m}$ & m & $\mathrm{m}$ & $\mathrm{m}$ & $\mathrm{m}$ & m & $\mathrm{m}$ & $\mathrm{m}$ \\
\hline & Mexiko & 0.39 & $(0.03)$ & 0.25 & $(0.04)$ & 0.28 & $(0.03)$ & 0.07 & $(0.05)$ & 0.51 & $(0.04)$ & 0.44 & (0.05) \\
\hline & Niederlande & $\mathrm{m}$ & $\mathrm{m}$ & $\mathrm{m}$ & $\mathrm{m}$ & $\mathrm{m}$ & $\mathrm{m}$ & $\mathrm{m}$ & $\mathrm{m}$ & $\mathrm{m}$ & $\mathrm{m}$ & $\mathrm{m}$ & $\mathrm{m}$ \\
\hline & Neuseeland & -0.14 & $(0.03)$ & 0.26 & $(0.05)$ & -0.30 & $(0.04)$ & -0.01 & $(0.05)$ & 0.02 & $(0.04)$ & 0.55 & (0.05) \\
\hline & Norwegen & -0.25 & $(0.03)$ & 0.00 & $(0.03)$ & -0.65 & $(0.04)$ & -0.29 & $(0.04)$ & 0.10 & $(0.04)$ & 0.36 & $(0.05)$ \\
\hline & Polen & -0.02 & $(0.04)$ & 0.16 & $(0.06)$ & -0.23 & $(0.04)$ & -0.09 & $(0.05)$ & 0.16 & $(0.05)$ & 0.45 & $(0.06)$ \\
\hline & Portugal & 0.30 & $(0.03)$ & 0.55 & $(0.03)$ & -0.04 & $(0.04)$ & 0.20 & $(0.04)$ & 0.56 & $(0.03)$ & 0.88 & $(0.05)$ \\
\hline & Spanien & -0.19 & $(0.04)$ & 0.21 & $(0.03)$ & -0.44 & $(0.04)$ & -0.03 & $(0.04)$ & 0.00 & $(0.06)$ & 0.46 & $(0.05)$ \\
\hline & Schweden & -0.15 & $(0.04)$ & 0.21 & $(0.03)$ & -0.54 & $(0.05)$ & -0.07 & $(0.05)$ & 0.20 & $(0.05)$ & 0.54 & $(0.04)$ \\
\hline & Schweiz & -0.15 & $(0.04)$ & 0.39 & $(0.05)$ & -0.62 & $(0.05)$ & 0.02 & $(0.05)$ & 0.35 & $(0.04)$ & 0.81 & $(0.06)$ \\
\hline & Ver. Königreich & $\mathrm{m}$ & $\mathrm{m}$ & $\mathrm{m}$ & $\mathrm{m}$ & $\mathrm{m}$ & $\mathrm{m}$ & $\mathrm{m}$ & $\mathrm{m}$ & $\mathrm{m}$ & $\mathrm{m}$ & $\mathrm{m}$ & $\mathrm{m}$ \\
\hline & Ver. Staaten & -0.28 & $(0.04)$ & 0.09 & $(0.05)$ & -0.42 & $(0.05)$ & -0.12 & $(0.07)$ & -0.15 & $(0.06)$ & 0.31 & $(0.07)$ \\
\hline & OECD-26-Durchschnitt & -0.09 & $(0.01)$ & 0.24 & $(0.01)$ & -0.37 & $(0.01)$ & -0.04 & $(0.01)$ & 0.15 & $(0.01)$ & 0.55 & $(0.01)$ \\
\hline \multirow{12}{*}{ 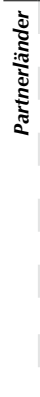 } & Albanien & 0.52 & $(0.03)$ & 0.59 & $(0.02)$ & 0.38 & $(0.05)$ & 0.40 & $(0.04)$ & 0.67 & $(0.04)$ & 0.75 & $(0.03)$ \\
\hline & Argentinien & -0.18 & $(0.05)$ & 0.00 & $(0.05)$ & -0.34 & $(0.07)$ & -0.25 & $(0.06)$ & -0.04 & $(0.07)$ & 0.22 & $(0.05)$ \\
\hline & Brasilien & 0.16 & $(0.03)$ & 0.21 & $(0.05)$ & -0.09 & $(0.04)$ & -0.08 & $(0.04)$ & 0.34 & $(0.03)$ & 0.51 & $(0.06)$ \\
\hline & Bulgarien & -0.08 & $(0.03)$ & 0.20 & $(0.05)$ & -0.24 & $(0.04)$ & -0.06 & $(0.05)$ & 0.09 & $(0.04)$ & 0.48 & $(0.06)$ \\
\hline & Hongkong (China) & -0.02 & $(0.02)$ & 0.30 & $(0.03)$ & -0.16 & $(0.02)$ & 0.20 & $(0.05)$ & 0.13 & $(0.03)$ & 0.40 & $(0.03)$ \\
\hline & Indonesien & 0.59 & $(0.02)$ & 0.57 & $(0.02)$ & 0.48 & $(0.03)$ & 0.43 & $(0.02)$ & 0.69 & $(0.03)$ & 0.70 & $(0.04)$ \\
\hline & Lettland & -0.04 & $(0.04)$ & 0.03 & $(0.03)$ & -0.28 & $(0.07)$ & -0.26 & $(0.04)$ & 0.18 & $(0.04)$ & 0.33 & $(0.03)$ \\
\hline & Liechtenstein & -0.25 & $(0.11)$ & 0.19 & $(0.13)$ & -0.73 & $(0.11)$ & 0.03 & $(0.14)$ & 0.40 & $(0.17)$ & 0.40 & $(0.23)$ \\
\hline & Peru & 0.35 & $(0.03)$ & 0.28 & $(0.04)$ & 0.30 & $(0.04)$ & 0.22 & $(0.05)$ & 0.40 & $(0.04)$ & 0.33 & $(0.05)$ \\
\hline & Rumänien & 0.17 & $(0.04)$ & 0.26 & $(0.04)$ & 0.02 & $(0.04)$ & 0.02 & $(0.05)$ & 0.30 & $(0.05)$ & 0.50 & $(0.04)$ \\
\hline & Russ. Föderation & 0.02 & $(0.03)$ & 0.23 & $(0.03)$ & -0.15 & $(0.03)$ & 0.02 & $(0.03)$ & 0.18 & $(0.03)$ & 0.46 & $(0.04)$ \\
\hline & Thailand & 0.14 & $(0.02)$ & 0.20 & $(0.02)$ & 0.00 & $(0.02)$ & 0.12 & $(0.02)$ & 0.23 & $(0.02)$ & 0.25 & $(0.03)$ \\
\hline
\end{tabular}

Anmerkung: Statistisch signifikante Werte sind durch Fettdruck gekennzeichnet (siehe Anhang A3).

StatLink intst http://dx.doi.org/10.1787/888932343285 
[Teil 2/3]

Index der Freude am Lesen in PISA 2000 und PISA 2009, nach soziökonomischem Hintergrund und Geschlecht

Tabelle V.5.5 Auf Grundlage der Schülerangaben

\begin{tabular}{|c|c|c|c|c|c|c|c|c|c|c|c|c|c|}
\hline & \multicolumn{12}{|c|}{ PISA 2009} \\
\hline & & \multicolumn{4}{|c|}{ Alle Schüler } & \multicolumn{4}{|c|}{ Jungen } & \multicolumn{4}{|c|}{ Mädchen } \\
\hline & & \multicolumn{2}{|c|}{$\begin{array}{c}\text { Unterstes } \\
\text { ESCS-Quartil }\end{array}$} & \multicolumn{2}{|c|}{$\begin{array}{c}\text { Oberstes } \\
\text { ESCS-Quartil }\end{array}$} & \multicolumn{2}{|c|}{$\begin{array}{c}\text { Unterstes } \\
\text { ESCS-Quartil }\end{array}$} & \multicolumn{2}{|c|}{$\begin{array}{c}\text { Oberstes } \\
\text { ESCS-Quartil }\end{array}$} & \multicolumn{2}{|c|}{$\begin{array}{c}\text { Unterstes } \\
\text { ESCS-Quartil }\end{array}$} & \multicolumn{2}{|c|}{$\begin{array}{c}\text { Oberstes } \\
\text { ESCS-Quartil }\end{array}$} \\
\hline & & $\begin{array}{l}\text { Index- } \\
\text { mittel }\end{array}$ & S.E. & $\begin{array}{l}\text { Index- } \\
\text { mittel }\end{array}$ & S.E. & $\begin{array}{l}\text { Index- } \\
\text { mittel }\end{array}$ & S.E. & $\begin{array}{l}\text { Index- } \\
\text { mittel }\end{array}$ & S.E. & $\begin{array}{l}\text { Index- } \\
\text { mittel }\end{array}$ & S.E. & $\begin{array}{l}\text { Index- } \\
\text { mittel }\end{array}$ & S.E. \\
\hline \multirow{31}{*}{ 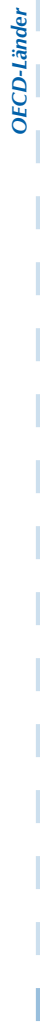 } & Australien & -0.29 & $(0.02)$ & 0.34 & $(0.03)$ & -0.64 & $(0.02)$ & 0.03 & $(0.03)$ & 0.02 & $(0.04)$ & 0.65 & $(0.04)$ \\
\hline & Österreich & m & m & $\mathrm{m}$ & m & m & m & $\mathrm{m}$ & m & $\mathrm{m}$ & m & $\mathrm{m}$ & $\mathrm{m}$ \\
\hline & Belgien & -0.44 & $(0.03)$ & 0.13 & $(0.02)$ & -0.69 & $(0.03)$ & -0.13 & $(0.03)$ & -0.21 & $(0.03)$ & 0.42 & $(0.03)$ \\
\hline & Kanada & -0.07 & $(0.02)$ & 0.39 & $(0.03)$ & -0.50 & $(0.03)$ & -0.02 & $(0.03)$ & 0.34 & $(0.03)$ & 0.80 & $(0.03)$ \\
\hline & Chile & -0.11 & $(0.02)$ & 0.07 & $(0.03)$ & -0.28 & $(0.03)$ & -0.19 & $(0.04)$ & 0.06 & $(0.03)$ & 0.33 & $(0.05)$ \\
\hline & Tschech. Rep. & -0.29 & $(0.03)$ & 0.08 & $(0.03)$ & -0.55 & $(0.03)$ & -0.25 & $(0.03)$ & -0.02 & $(0.04)$ & 0.47 & $(0.04)$ \\
\hline & Dänemark & -0.35 & $(0.02)$ & 0.22 & $(0.02)$ & -0.62 & $(0.03)$ & -0.03 & $(0.03)$ & -0.11 & $(0.03)$ & 0.47 & (0.03) \\
\hline & Finnland & -0.19 & $(0.03)$ & 0.27 & $(0.03)$ & -0.66 & $(0.04)$ & -0.16 & $(0.04)$ & 0.30 & $(0.04)$ & 0.69 & $(0.04)$ \\
\hline & Frankreich & -0.24 & $(0.04)$ & 0.36 & $(0.03)$ & -0.47 & $(0.06)$ & 0.11 & $(0.05)$ & -0.03 & $(0.05)$ & 0.61 & $(0.04)$ \\
\hline & Deutschland & -0.28 & $(0.03)$ & 0.44 & $(0.04)$ & -0.70 & $(0.04)$ & 0.01 & $(0.04)$ & 0.11 & $(0.04)$ & 0.91 & $(0.05)$ \\
\hline & Griechenland & -0.10 & $(0.03)$ & 0.27 & $(0.03)$ & -0.35 & $(0.04)$ & -0.07 & $(0.04)$ & 0.13 & $(0.04)$ & 0.64 & $(0.04)$ \\
\hline & Ungarn & -0.09 & $(0.03)$ & 0.48 & $(0.03)$ & -0.34 & $(0.04)$ & 0.16 & $(0.05)$ & 0.13 & $(0.04)$ & 0.84 & $(0.04)$ \\
\hline & Island & -0.25 & $(0.03)$ & 0.13 & $(0.03)$ & -0.58 & $(0.05)$ & -0.17 & $(0.05)$ & 0.03 & $(0.04)$ & 0.43 & $(0.05)$ \\
\hline & Irland & -0.42 & $(0.04)$ & 0.26 & $(0.04)$ & -0.59 & $(0.05)$ & -0.01 & $(0.05)$ & -0.25 & $(0.05)$ & 0.55 & $(0.04)$ \\
\hline & Israel & -0.04 & $(0.03)$ & 0.21 & $(0.04)$ & -0.41 & $(0.04)$ & -0.04 & $(0.05)$ & 0.25 & $(0.04)$ & 0.49 & $(0.05)$ \\
\hline & Italien & -0.15 & $(0.02)$ & 0.29 & $(0.02)$ & -0.46 & $(0.02)$ & -0.04 & $(0.02)$ & 0.15 & $(0.02)$ & 0.66 & $(0.02)$ \\
\hline & Japan & 0.01 & $(0.03)$ & 0.44 & $(0.03)$ & -0.19 & $(0.04)$ & 0.25 & $(0.05)$ & 0.24 & $(0.04)$ & 0.62 & $(0.05)$ \\
\hline & Korea & -0.07 & $(0.03)$ & 0.36 & $(0.03)$ & -0.16 & $(0.03)$ & 0.25 & $(0.03)$ & 0.05 & $(0.04)$ & 0.47 & $(0.04)$ \\
\hline & Luxemburg & -0.40 & $(0.03)$ & 0.16 & $(0.03)$ & -0.80 & $(0.04)$ & -0.24 & $(0.04)$ & -0.06 & $(0.05)$ & 0.59 & $(0.05)$ \\
\hline & Mexiko & 0.20 & $(0.02)$ & 0.18 & $(0.02)$ & 0.05 & $(0.02)$ & -0.05 & $(0.02)$ & 0.32 & $(0.02)$ & 0.42 & $(0.02)$ \\
\hline & Niederlande & -0.49 & $(0.04)$ & -0.06 & $(0.04)$ & -0.81 & $(0.04)$ & -0.43 & $(0.05)$ & -0.19 & $(0.05)$ & 0.32 & $(0.05)$ \\
\hline & Neuseeland & -0.11 & $(0.03)$ & 0.47 & $(0.04)$ & -0.42 & $(0.04)$ & 0.15 & $(0.04)$ & 0.17 & $(0.04)$ & 0.80 & $(0.05)$ \\
\hline & Norwegen & -0.36 & $(0.03)$ & 0.05 & $(0.04)$ & -0.71 & $(0.04)$ & -0.26 & $(0.04)$ & -0.04 & $(0.04)$ & 0.39 & $(0.05)$ \\
\hline & Polen & -0.15 & $(0.02)$ & 0.39 & $(0.04)$ & -0.52 & $(0.04)$ & -0.05 & $(0.04)$ & 0.16 & $(0.04)$ & 0.81 & $(0.06)$ \\
\hline & Portugal & 0.08 & $(0.02)$ & 0.38 & $(0.03)$ & -0.26 & $(0.03)$ & 0.05 & $(0.03)$ & 0.35 & $(0.03)$ & 0.72 & $(0.04)$ \\
\hline & Spanien & -0.25 & $(0.03)$ & 0.21 & $(0.02)$ & -0.45 & $(0.03)$ & -0.09 & $(0.03)$ & -0.04 & $(0.04)$ & 0.54 & $(0.02)$ \\
\hline & Schweden & -0.30 & $(0.03)$ & 0.14 & $(0.03)$ & -0.61 & $(0.04)$ & -0.26 & $(0.04)$ & 0.02 & $(0.04)$ & 0.55 & $(0.05)$ \\
\hline & Schweiz & -0.32 & $(0.05)$ & 0.36 & $(0.03)$ & -0.74 & $(0.03)$ & -0.03 & $(0.04)$ & 0.09 & $(0.07)$ & 0.75 & $(0.05)$ \\
\hline & Ver. Königreich & -0.35 & $(0.03)$ & 0.20 & $(0.03)$ & -0.57 & $(0.04)$ & -0.06 & $(0.04)$ & -0.15 & $(0.04)$ & 0.47 & $(0.04)$ \\
\hline & Ver. Staaten & -0.24 & $(0.03)$ & 0.28 & $(0.04)$ & -0.56 & $(0.03)$ & -0.05 & $(0.04)$ & 0.09 & $(0.04)$ & 0.65 & $(0.05)$ \\
\hline & OECD-26-Durchschnitt & -0.19 & $(0.01)$ & 0.28 & $(0.01)$ & -0.48 & $(0.01)$ & -0.04 & $(0.01)$ & 0.09 & $(0.01)$ & 0.60 & $(0.01)$ \\
\hline \multirow{12}{*}{ 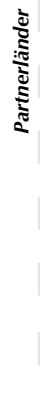 } & Albanien & 0.62 & $(0.03)$ & 0.76 & $(0.02)$ & 0.29 & $(0.04)$ & 0.43 & $(0.03)$ & 0.92 & $(0.03)$ & 1.11 & $(0.03)$ \\
\hline & Argentinien & -0.14 & $(0.03)$ & -0.12 & $(0.03)$ & -0.32 & $(0.04)$ & -0.30 & $(0.04)$ & 0.01 & $(0.04)$ & 0.06 & $(0.04)$ \\
\hline & Brasilien & 0.27 & $(0.02)$ & 0.35 & $(0.03)$ & 0.08 & $(0.02)$ & 0.07 & $(0.04)$ & 0.39 & $(0.02)$ & 0.63 & $(0.03)$ \\
\hline & Bulgarien & -0.16 & $(0.03)$ & 0.24 & $(0.04)$ & -0.36 & $(0.04)$ & -0.08 & $(0.05)$ & 0.04 & $(0.04)$ & 0.57 & $(0.05)$ \\
\hline & Hongkong (China) & 0.17 & $(0.02)$ & 0.48 & $(0.03)$ & 0.00 & $(0.02)$ & 0.32 & $(0.04)$ & 0.36 & $(0.03)$ & 0.67 & $(0.04)$ \\
\hline & Indonesien & 0.41 & $(0.02)$ & 0.43 & $(0.02)$ & 0.31 & $(0.02)$ & 0.30 & $(0.03)$ & 0.51 & $(0.02)$ & 0.58 & $(0.03)$ \\
\hline & Lettland & -0.17 & $(0.03)$ & 0.14 & $(0.03)$ & -0.57 & $(0.03)$ & -0.23 & $(0.04)$ & 0.14 & $(0.04)$ & 0.52 & $(0.03)$ \\
\hline & Liechtenstein & -0.42 & $(0.11)$ & -0.04 & $(0.13)$ & -0.76 & $(0.15)$ & -0.44 & $(0.15)$ & -0.11 & $(0.17)$ & 0.43 & $(0.19)$ \\
\hline & Peru & 0.36 & $(0.02)$ & 0.32 & $(0.02)$ & 0.30 & $(0.03)$ & 0.11 & $(0.03)$ & 0.43 & $(0.03)$ & 0.53 & $(0.03)$ \\
\hline & Rumänien & 0.06 & $(0.03)$ & 0.16 & $(0.03)$ & -0.13 & $(0.03)$ & -0.09 & $(0.03)$ & 0.24 & $(0.04)$ & 0.42 & $(0.04)$ \\
\hline & Russ. Föderation & -0.03 & $(0.02)$ & 0.24 & $(0.03)$ & -0.26 & $(0.03)$ & -0.04 & $(0.04)$ & 0.19 & $(0.03)$ & 0.52 & $(0.03)$ \\
\hline & Thailand & 0.55 & $(0.02)$ & 0.55 & $(0.03)$ & 0.38 & $(0.02)$ & 0.37 & $(0.04)$ & 0.66 & $(0.03)$ & 0.71 & $(0.03)$ \\
\hline
\end{tabular}

Anmerkung: Statistisch signifikante Werte sind durch Fettdruck gekennzeichnet (siehe Anhang A3).

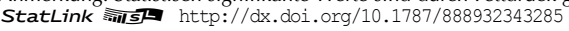


[Teil 3/3]

Index der Freude am Lesen in PISA 2000 und PISA 2009, nach soziökonomischem Hintergrund und Geschlecht

Tabelle V.5.5 Auf Grundlage der Schülerangaben

\begin{tabular}{|c|c|c|c|c|c|c|c|c|c|c|c|c|c|}
\hline & \multicolumn{12}{|c|}{ Veränderung zwischen 2000 und 2009 (PISA 2009 - PISA 2000) } \\
\hline & & \multicolumn{4}{|c|}{ Alle Schüler } & \multicolumn{4}{|c|}{ Jungen } & \multicolumn{4}{|c|}{ Mädchen } \\
\hline & & \multicolumn{2}{|c|}{$\begin{array}{c}\text { Unterstes } \\
\text { ESCS-Quartil }\end{array}$} & \multicolumn{2}{|c|}{$\begin{array}{c}\text { Oberstes } \\
\text { ESCS-Quartil }\end{array}$} & \multicolumn{2}{|c|}{$\begin{array}{c}\text { Unterstes } \\
\text { ESCS-Quartil }\end{array}$} & \multicolumn{2}{|c|}{$\begin{array}{c}\text { Oberstes } \\
\text { ESCS-Quartil }\end{array}$} & \multicolumn{2}{|c|}{$\begin{array}{c}\text { Unterstes } \\
\text { ESCS-Quartil }\end{array}$} & \multicolumn{2}{|c|}{$\begin{array}{c}\text { Oberstes } \\
\text { ESCS-Quartil }\end{array}$} \\
\hline & & Diff. & S.E. & Diff. & S.E. & Diff. & S.E. & Diff. & S.E. & Diff. & S.E. & Diff. & S.E. \\
\hline \multirow{31}{*}{ 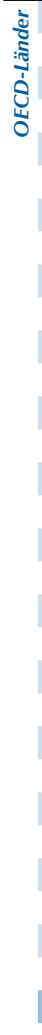 } & Australien & -0.04 & $(0.04)$ & 0.07 & $(0.06)$ & -0.16 & $(0.05)$ & -0.05 & $(0.06)$ & 0.06 & $(0.06)$ & 0.15 & $(0.09)$ \\
\hline & Österreich & $\mathrm{m}$ & $\mathrm{m}$ & $\mathrm{m}$ & $\mathrm{m}$ & $\mathrm{m}$ & $\mathrm{m}$ & $\mathrm{m}$ & $\mathrm{m}$ & $\mathrm{m}$ & $\mathrm{m}$ & $\mathrm{m}$ & $\mathrm{m}$ \\
\hline & Belgien & -0.07 & $(0.04)$ & 0.17 & $(0.04)$ & -0.11 & $(0.05)$ & 0.17 & $(0.05)$ & -0.04 & $(0.05)$ & 0.13 & (0.05) \\
\hline & Kanada & 0.09 & $(0.03)$ & 0.10 & $(0.04)$ & -0.01 & $(0.03)$ & -0.03 & $(0.04)$ & 0.19 & $(0.04)$ & 0.21 & $(0.04)$ \\
\hline & Chile & -0.03 & $(0.04)$ & -0.07 & $(0.05)$ & 0.03 & $(0.05)$ & 0.02 & $(0.06)$ & -0.06 & $(0.05)$ & -0.10 & $(0.07)$ \\
\hline & Tschech. Rep. & -0.32 & $(0.04)$ & -0.31 & $(0.04)$ & -0.16 & $(0.05)$ & -0.26 & $(0.06)$ & -0.39 & $(0.06)$ & -0.32 & $(0.06)$ \\
\hline & Dänemark & -0.22 & $(0.04)$ & -0.07 & $(0.04)$ & -0.17 & $(0.06)$ & -0.01 & $(0.05)$ & -0.25 & $(0.06)$ & -0.19 & $(0.06)$ \\
\hline & Finnland & -0.32 & $(0.05)$ & -0.16 & $(0.05)$ & -0.23 & $(0.06)$ & -0.16 & $(0.06)$ & -0.29 & $(0.06)$ & -0.17 & $(0.06)$ \\
\hline & Frankreich & -0.03 & $(0.05)$ & 0.16 & $(0.05)$ & -0.03 & $(0.07)$ & 0.20 & $(0.07)$ & -0.03 & $(0.06)$ & 0.12 & $(0.06)$ \\
\hline & Deutschland & -0.01 & $(0.05)$ & 0.15 & $(0.06)$ & -0.03 & $(0.06)$ & 0.16 & $(0.07)$ & 0.05 & $(0.06)$ & 0.11 & $(0.08)$ \\
\hline & Griechenland & -0.06 & $(0.04)$ & 0.18 & $(0.05)$ & -0.10 & $(0.05)$ & 0.09 & $(0.06)$ & -0.01 & $(0.05)$ & 0.28 & $(0.05)$ \\
\hline & Ungarn & -0.01 & $(0.04)$ & 0.13 & $(0.05)$ & -0.08 & $(0.06)$ & 0.04 & $(0.07)$ & 0.05 & $(0.05)$ & 0.22 & $(0.06)$ \\
\hline & Island & -0.22 & $(0.05)$ & -0.08 & $(0.05)$ & -0.27 & $(0.06)$ & -0.11 & $(0.06)$ & -0.18 & $(0.06)$ & -0.06 & $(0.07)$ \\
\hline & Irland & -0.22 & $(0.05)$ & 0.06 & $(0.05)$ & -0.09 & $(0.07)$ & 0.09 & $(0.07)$ & -0.31 & $(0.07)$ & 0.05 & $(0.06)$ \\
\hline & Israel & -0.15 & $(0.09)$ & -0.02 & $(0.08)$ & -0.27 & $(0.10)$ & -0.01 & $(0.09)$ & -0.02 & $(0.10)$ & 0.03 & $(0.10)$ \\
\hline & Italien & -0.09 & $(0.04)$ & 0.05 & $(0.04)$ & -0.19 & $(0.06)$ & 0.02 & $(0.05)$ & 0.01 & $(0.04)$ & 0.09 & $(0.05)$ \\
\hline & Japan & $\mathrm{m}$ & $\mathrm{m}$ & $\mathrm{m}$ & $\mathrm{m}$ & $\mathrm{m}$ & $\mathrm{m}$ & $\mathrm{m}$ & $\mathrm{m}$ & $\mathrm{m}$ & $\mathrm{m}$ & $\mathrm{m}$ & $\mathrm{m}$ \\
\hline & Korea & 0.07 & $(0.04)$ & 0.07 & $(0.05)$ & 0.07 & $(0.05)$ & 0.05 & $(0.06)$ & 0.08 & $(0.06)$ & 0.04 & $(0.08)$ \\
\hline & Luxemburg & $\mathrm{m}$ & $\mathrm{m}$ & $\mathrm{m}$ & m & $\mathrm{m}$ & m & $\mathrm{m}$ & m & $\mathrm{m}$ & m & $\mathrm{m}$ & $\mathrm{m}$ \\
\hline & Mexiko & -0.20 & $(0.03)$ & -0.07 & $(0.04)$ & -0.23 & $(0.04)$ & -0.12 & $(0.05)$ & -0.19 & $(0.04)$ & -0.02 & $(0.05)$ \\
\hline & Niederlande & $\mathrm{m}$ & $\mathrm{m}$ & $\mathrm{m}$ & m & $\mathrm{m}$ & m & $\mathrm{m}$ & $\mathrm{m}$ & $\mathrm{m}$ & m & $\mathrm{m}$ & $\mathrm{m}$ \\
\hline & Neuseeland & 0.03 & $(0.04)$ & 0.21 & $(0.06)$ & -0.12 & $(0.06)$ & 0.16 & $(0.06)$ & 0.15 & $(0.06)$ & 0.25 & $(0.07)$ \\
\hline & Norwegen & -0.12 & $(0.05)$ & 0.05 & $(0.05)$ & -0.05 & $(0.06)$ & 0.03 & $(0.06)$ & -0.14 & $(0.06)$ & 0.04 & $(0.07)$ \\
\hline & Polen & -0.13 & $(0.05)$ & 0.23 & $(0.07)$ & -0.29 & $(0.06)$ & 0.05 & $(0.07)$ & 0.00 & $(0.06)$ & 0.36 & (0.09) \\
\hline & Portugal & -0.22 & $(0.04)$ & -0.18 & $(0.04)$ & -0.22 & $(0.05)$ & -0.15 & $(0.05)$ & -0.21 & $(0.05)$ & -0.17 & $(0.06)$ \\
\hline & Spanien & -0.06 & $(0.05)$ & 0.01 & $(0.04)$ & -0.01 & $(0.05)$ & -0.07 & $(0.05)$ & -0.04 & $(0.07)$ & 0.08 & $(0.06)$ \\
\hline & Schweden & -0.15 & $(0.05)$ & -0.07 & $(0.05)$ & -0.08 & $(0.06)$ & -0.19 & $(0.07)$ & -0.18 & $(0.06)$ & 0.01 & $(0.06)$ \\
\hline & Schweiz & -0.17 & $(0.06)$ & -0.03 & $(0.06)$ & -0.12 & $(0.06)$ & -0.05 & $(0.06)$ & -0.25 & $(0.08)$ & -0.06 & $(0.07)$ \\
\hline & Ver. Königreich & $\mathrm{m}$ & $\mathrm{m}$ & $\mathrm{m}$ & $\mathrm{m}$ & $\mathrm{m}$ & $\mathrm{m}$ & $\mathrm{m}$ & m & $\mathrm{m}$ & $\mathrm{m}$ & $\mathrm{m}$ & $\mathrm{m}$ \\
\hline & Ver. Staaten & 0.03 & $(0.05)$ & 0.19 & $(0.06)$ & -0.14 & $(0.06)$ & 0.07 & $(0.08)$ & 0.24 & $(0.07)$ & 0.35 & $(0.08)$ \\
\hline & OECD-26-Durchschnitt & -0.10 & $(0.01)$ & 0.03 & $(0.01)$ & -0.12 & $(0.01)$ & 0.00 & $(0.01)$ & -0.07 & $(0.01)$ & 0.06 & $(0.01)$ \\
\hline \multirow{12}{*}{ 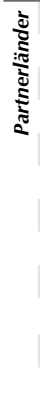 } & Albanien & 0.10 & $(0.04)$ & 0.17 & $(0.04)$ & -0.09 & $(0.06)$ & 0.04 & $(0.05)$ & 0.25 & $(0.05)$ & 0.37 & $(0.05)$ \\
\hline & Argentinien & 0.04 & $(0.06)$ & -0.12 & $(0.06)$ & 0.02 & $(0.09)$ & -0.05 & $(0.07)$ & 0.05 & $(0.08)$ & -0.16 & $(0.07)$ \\
\hline & Brasilien & 0.11 & $(0.03)$ & 0.14 & $(0.05)$ & 0.17 & $(0.04)$ & 0.15 & $(0.06)$ & 0.05 & $(0.04)$ & 0.12 & $(0.06)$ \\
\hline & Bulgarien & -0.08 & $(0.05)$ & 0.04 & $(0.07)$ & -0.12 & $(0.05)$ & -0.03 & $(0.07)$ & -0.05 & $(0.06)$ & 0.10 & $(0.08)$ \\
\hline & Hongkong (China) & 0.19 & $(0.03)$ & 0.18 & $(0.04)$ & 0.15 & $(0.03)$ & 0.12 & $(0.07)$ & 0.23 & $(0.04)$ & 0.27 & $(0.05)$ \\
\hline & Indonesien & -0.18 & $(0.03)$ & -0.14 & $(0.03)$ & -0.17 & $(0.03)$ & -0.13 & $(0.04)$ & -0.19 & $(0.04)$ & -0.11 & $(0.05)$ \\
\hline & Lettland & -0.14 & $(0.05)$ & 0.11 & $(0.04)$ & -0.29 & $(0.07)$ & 0.03 & $(0.05)$ & -0.04 & $(0.06)$ & 0.19 & $(0.04)$ \\
\hline & Liechtenstein & -0.17 & $(0.16)$ & -0.23 & $(0.19)$ & -0.03 & $(0.19)$ & -0.47 & $(0.20)$ & -0.51 & $(0.24)$ & 0.03 & $(0.30)$ \\
\hline & Peru & 0.01 & $(0.04)$ & 0.05 & $(0.04)$ & 0.00 & $(0.05)$ & -0.12 & $(0.06)$ & 0.03 & $(0.05)$ & 0.21 & $(0.06)$ \\
\hline & Rumänien & -0.11 & $(0.05)$ & -0.11 & $(0.05)$ & -0.15 & $(0.05)$ & -0.11 & $(0.06)$ & -0.06 & $(0.07)$ & -0.08 & $(0.06)$ \\
\hline & Russ. Föderation & -0.05 & $(0.03)$ & 0.01 & $(0.04)$ & -0.11 & $(0.04)$ & -0.06 & $(0.05)$ & 0.01 & $(0.04)$ & 0.06 & $(0.05)$ \\
\hline & Thailand & 0.42 & $(0.03)$ & 0.35 & $(0.03)$ & 0.39 & $(0.03)$ & 0.25 & $(0.04)$ & 0.44 & $(0.04)$ & 0.45 & $(0.04)$ \\
\hline
\end{tabular}

Anmerkung: Statistisch signifikante Werte sind durch Fettdruck gekennzeichnet (siehe Anhang A3).

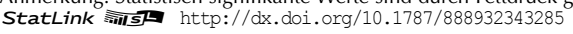


[Teil 1/2]

Prozentsatz der Schüler, die verschiedene Arten von Texten lesen, in PISA 2000 und PISA 2009

Prozentsatz der Schüler, die angaben, dass sie aus eigenem Antrieb "mehrmals im Monat" oder "mehrmals in der Tabelle V.5.6 Woche" die folgenden Arten von Texten lesen

\begin{tabular}{|c|c|c|c|c|c|c|c|c|c|c|c|c|c|c|c|c|c|c|c|c|c|}
\hline & \multicolumn{10}{|c|}{ PISA 2000} & \multicolumn{10}{|c|}{ PISA 2009} \\
\hline & & \multicolumn{2}{|c|}{$\begin{array}{c}\text { Zeitschriften/ } \\
\text { Magazine }\end{array}$} & \multicolumn{2}{|c|}{$\begin{array}{c}\text { Comic-Hefte/ } \\
\text { Comics }\end{array}$} & \multicolumn{2}{|c|}{$\begin{array}{c}\text { Romane, } \\
\text { Erzählungen, } \\
\text { Geschichten }\end{array}$} & \multicolumn{2}{|c|}{ Sachbücher } & \multicolumn{2}{|c|}{$\begin{array}{c}\text { Tages- } \\
\text { zeitungen }\end{array}$} & \multicolumn{2}{|c|}{$\begin{array}{c}\text { Zeitschriften/ } \\
\text { Magazine }\end{array}$} & \multicolumn{2}{|c|}{$\begin{array}{c}\text { Comic-Hefte/ } \\
\text { Comics }\end{array}$} & \multicolumn{2}{|c|}{$\begin{array}{c}\text { Romane, } \\
\text { Erzählungen, } \\
\text { Geschichten }\end{array}$} & \multicolumn{2}{|c|}{ Sachbücher } & \multicolumn{2}{|c|}{$\begin{array}{c}\text { Tages- } \\
\text { zeitungen }\end{array}$} \\
\hline & & $\%$ & S.E. & $\%$ & S.E. & $\%$ & S.E. & $\%$ & S.E. & $\%$ & S.E. & $\%$ & S.E. & $\%$ & S.E. & $\%$ & S.E. & $\%$ & S.E. & $\%$ & S.E. \\
\hline \multirow{31}{*}{ 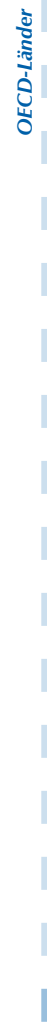 } & Australien & 63.2 & (0.9) & 10.4 & (0.6) & 31.3 & (1.2) & 19.6 & (0.9) & 65.9 & (1.2) & 50.0 & (0.6) & 9.0 & $(0.4)$ & 38.3 & $(0.6)$ & 20.0 & $(0.4)$ & 53.7 & $(0.7)$ \\
\hline & Österreich & 71.0 & $(0.7)$ & 16.5 & (0.6) & 24.1 & (0.9) & 18.6 & $(0.7)$ & 72.0 & (1.0) & $\mathrm{m}$ & $\mathrm{m}$ & $\mathrm{m}$ & $\mathrm{m}$ & $\mathrm{m}$ & $\mathrm{m}$ & $\mathrm{m}$ & $\mathrm{m}$ & $\mathrm{m}$ & $\mathrm{m}$ \\
\hline & Belgien & 68.1 & (0.9) & 38.3 & (0.8) & 20.8 & $(0.8)$ & 16.2 & (0.6) & 46.2 & (0.6) & 65.8 & $(0.7)$ & 31.8 & $(0.7)$ & 22.5 & $(0.7)$ & 13.7 & $(0.6)$ & 50.4 & (0.9) \\
\hline & Kanada & 67.0 & $(0.4)$ & 16.3 & $(0.4)$ & 30.9 & $(0.5)$ & 16.7 & $(0.3)$ & 57.6 & (0.5) & 48.1 & $(0.5)$ & 14.4 & $(0.4)$ & 42.0 & (0.6) & 20.0 & $(0.4)$ & 47.9 & $(0.8)$ \\
\hline & Chile & 55.6 & $(0.8)$ & 29.8 & $(0.8)$ & 28.8 & (0.9) & 24.8 & $(0.7)$ & 58.0 & (1.0) & 49.6 & (0.8) & 20.9 & (0.6) & 30.6 & (0.8) & 16.4 & $(0.6)$ & 58.5 & (0.9) \\
\hline & Tschech. Rep. & 79.3 & $(0.6)$ & 17.0 & $(0.7)$ & 29.0 & $(0.8)$ & 13.2 & $(0.6)$ & 62.1 & $(0.9)$ & 68.3 & $(0.8)$ & 15.4 & $(0.6)$ & 17.5 & $(0.8)$ & 12.0 & $(0.6)$ & 66.0 & $(0.8)$ \\
\hline & Dänemark & 74.1 & $(0.8)$ & 61.8 & (1.0) & 31.9 & $(0.9)$ & 27.8 & (1.0) & 64.3 & (1.0) & 65.5 & $(0.8)$ & 20.6 & $(0.7)$ & 30.5 & $(0.8)$ & 27.1 & $(0.7)$ & 51.7 & $(0.9)$ \\
\hline & Finnland & 75.1 & $(0.7)$ & 65.9 & (0.9) & 27.2 & $(0.7)$ & 14.5 & (0.6) & 85.1 & $(0.7)$ & 64.9 & (0.8) & 60.1 & $(0.9)$ & 26.1 & (0.8) & 15.5 & $(0.5)$ & 75.4 & $(0.8)$ \\
\hline & Frankreich & 62.3 & $(0.9)$ & 32.6 & $(0.9)$ & 23.4 & $(0.7)$ & 20.6 & $(0.6)$ & 47.3 & (1.0) & 62.5 & $(0.8)$ & 30.4 & $(0.8)$ & 28.9 & $(1.0)$ & 12.0 & $(0.5)$ & 46.7 & (1.1) \\
\hline & Deutschland & 65.7 & $(1.0)$ & 11.8 & $(0.5)$ & 26.8 & $(0.7)$ & 16.8 & (0.6) & 62.9 & (1.0) & 54.9 & $(0.8)$ & 11.3 & $(0.5)$ & 32.8 & $(0.8)$ & 17.2 & $(0.8)$ & 61.8 & (1.0) \\
\hline & Griechenland & 60.8 & $(0.9)$ & 23.1 & $(0.8)$ & 23.6 & $(0.8)$ & 26.4 & (1.0) & 46.2 & $(0.9)$ & 60.5 & $(0.9)$ & 24.7 & $(0.7)$ & 21.5 & $(0.7)$ & 7.2 & $(0.4)$ & 42.8 & (0.9) \\
\hline & Ungarn & 71.8 & (0.9) & 18.0 & $(0.7)$ & 25.1 & (1.1) & 31.6 & (0.8) & 60.8 & $(1.2)$ & 60.7 & (1.0) & 26.6 & (0.8) & 31.5 & $(1.0)$ & 34.9 & $(0.8)$ & 71.8 & (0.9) \\
\hline & Island & 79.1 & (0.6) & 49.5 & $(0.7)$ & 22.0 & $(0.7)$ & 18.0 & (0.6) & 89.2 & $(0.6)$ & 58.2 & (0.9) & 30.9 & $(0.8)$ & 27.3 & $(0.7)$ & 17.1 & $(0.7)$ & 82.0 & $(0.7)$ \\
\hline & Irland & 61.5 & $(0.9)$ & 8.6 & $(0.6)$ & 26.2 & (0.9) & 14.8 & $(0.7)$ & 75.2 & $(0.8)$ & 57.1 & (0.9) & 7.5 & $(0.5)$ & 30.3 & (1.0) & 16.0 & $(0.7)$ & 67.5 & (0.9) \\
\hline & Israel & 48.9 & $(1.2)$ & 28.1 & (1.5) & 33.9 & (2.1) & 26.5 & (1.8) & 79.6 & (1.0) & 38.8 & $(0.9)$ & 17.5 & $(0.6)$ & 29.7 & $(0.7)$ & 26.5 & (1.0) & 74.7 & (0.8) \\
\hline & Italien & 66.1 & (0.9) & 27.7 & (0.9) & 31.7 & (0.9) & 14.1 & (0.6) & 61.1 & $(0.8)$ & 48.8 & $(0.5)$ & 17.4 & $(0.3)$ & 35.0 & $(0.5)$ & 4.9 & $(0.2)$ & 53.4 & (0.5) \\
\hline & Japan & 81.8 & $(0.7)$ & 83.8 & $(0.7)$ & 27.5 & $(0.8)$ & 9.8 & $(0.5)$ & 69.9 & (1.1) & 64.5 & $(0.8)$ & 72.4 & $(0.8)$ & 42.0 & (1.1) & 11.1 & $(0.4)$ & 57.6 & $(0.9)$ \\
\hline & Korea & 39.3 & (0.8) & 62.7 & (1.2) & 34.9 & (0.8) & 23.1 & (0.6) & 69.7 & $(0.9)$ & 21.2 & (0.6) & 40.5 & (1.0) & 46.6 & (0.8) & 30.0 & $(0.9)$ & 45.1 & (1.2) \\
\hline & Luxemburg & $\mathrm{m}$ & $\mathrm{m}$ & $\mathrm{m}$ & $\mathrm{m}$ & $\mathrm{m}$ & $\mathrm{m}$ & $\mathrm{m}$ & $\mathrm{m}$ & $\mathrm{m}$ & $\mathrm{m}$ & 68.7 & $(0.8)$ & 20.3 & $(0.7)$ & 28.8 & $(0.7)$ & 19.3 & $(0.5)$ & 70.7 & $(0.6)$ \\
\hline & Mexiko & 46.3 & (1.3) & 24.8 & $(0.8)$ & 36.4 & $(0.9)$ & 22.2 & $(0.7)$ & 46.1 & (1.6) & 46.9 & $(0.5)$ & 27.1 & $(0.4)$ & 37.9 & $(0.4)$ & 18.7 & $(0.3)$ & 47.8 & $(0$ \\
\hline & Niederlande & $\mathrm{m}$ & $\mathrm{m}$ & $\mathrm{m}$ & $\mathrm{m}$ & $\mathrm{m}$ & $\mathrm{m}$ & $\mathrm{m}$ & $\mathrm{m}$ & $\mathrm{m}$ & $\mathrm{m}$ & 57.2 & (1.4) & 23.1 & $(0.9)$ & 21.5 & (1.0) & 12.6 & $(0.6)$ & 48.5 & (1.5) \\
\hline & Neuseeland & 70.3 & $(0.8)$ & 11.6 & (0.6) & 34.8 & $(0.8)$ & 25.2 & $(0.8)$ & 66.4 & $(0.9)$ & 53.1 & (1.0) & 11.6 & $(0.5)$ & 44.3 & $(0.8)$ & 25.4 & $(0.7)$ & 53.1 & $(0.9)$ \\
\hline & Norwegen & 69.2 & $(0.9)$ & 57.7 & $(0.9)$ & 24.3 & $(0.8)$ & 21.2 & (0.6) & 84.4 & $(0.7)$ & 60.6 & (0.9) & 42.2 & (0.9) & 26.3 & $(0.8)$ & 27.2 & $(0.8)$ & 73.4 & $(0.8)$ \\
\hline & Polen & 70.8 & $(1.0)$ & 10.7 & $(0.7)$ & 18.7 & (1.0) & 17.2 & $(0.7)$ & 74.2 & (1.0) & 65.8 & $(0.7)$ & 11.3 & $(0.5)$ & 20.1 & $(0.7)$ & 20.8 & (0.8) & 79.1 & (0.6) \\
\hline & Portugal & 71.5 & $(0.9)$ & 24.4 & $(0.7)$ & 32.0 & $(0.7)$ & 13.1 & $(0.7)$ & 52.8 & $(1.0)$ & 63.8 & $(0.7)$ & 18.6 & $(0.5)$ & 28.0 & $(0.6)$ & 14.2 & $(0.5)$ & 51.5 & $(0.7)$ \\
\hline & Spanien & 61.3 & $(0.7)$ & 18.7 & $(0.7)$ & 25.1 & $(0.7)$ & 23.0 & $(0.8)$ & 46.4 & (1.1) & 51.3 & $(0.7)$ & 12.0 & $(0.4)$ & 30.1 & $(0.5)$ & 18.3 & $(0.4)$ & 45.1 & $(0.7)$ \\
\hline & Schweden & 68.3 & $(0.8)$ & 35.9 & $(0.8)$ & 33.6 & $(0.8)$ & 12.7 & $(0.6)$ & 82.6 & $(0.6)$ & 58.2 & $(0.9)$ & 22.5 & $(0.7)$ & 32.4 & $(0.9)$ & 9.9 & $(0.6)$ & 71.6 & $(0.9)$ \\
\hline & Schweiz & 66.5 & $(0.7)$ & 26.2 & (0.8) & 30.3 & $(0.9)$ & 16.8 & (0.6) & 66.1 & $(1.0)$ & 66.8 & $(0.7)$ & 24.1 & $(0.6)$ & 30.1 & $(0.7)$ & 15.0 & $(0.5)$ & 79.5 & $(0.8)$ \\
\hline & Ver. Königreich & $\mathrm{m}$ & $\mathrm{m}$ & $\mathrm{m}$ & $\mathrm{m}$ & $\mathrm{m}$ & $\mathrm{m}$ & $\mathrm{m}$ & $\mathrm{m}$ & $\mathrm{m}$ & $\mathrm{m}$ & 59.6 & $(0.8)$ & 7.8 & $(0.4)$ & 31.5 & $(0.7)$ & 19.5 & $(0.5)$ & 61.2 & $(0.8)$ \\
\hline & Ver. Staaten & 68.1 & (1.4) & 11.4 & (1.1) & 28.2 & (1.1) & 18.4 & (1.0) & 56.0 & (1.4) & 46.8 & $(0.7)$ & 10.4 & $(0.6)$ & 36.6 & (1.1) & 20.5 & $(0.8)$ & 37.0 & (1.0) \\
\hline & OECD-26-Durchschnitt & 65.8 & $(0.2)$ & 31.0 & $(0.2)$ & 28.4 & $(0.2)$ & 19.4 & $(0.2)$ & 64.5 & $(0.2)$ & 55.9 & $(0.2)$ & 24.3 & $(0.1)$ & 31.5 & $(0.2)$ & 18.1 & $(0.1)$ & 59.4 & $(0.2)$ \\
\hline \multirow{12}{*}{ 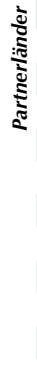 } & Albanien & 54.2 & $(1.2)$ & 31.9 & (1.4) & 51.9 & (1.1) & 18.4 & $(0.7)$ & 52.7 & $(0.9)$ & 44.6 & (1.1) & 43.7 & $(1.2)$ & 53.7 & (1.1) & 22.1 & $(0.8)$ & 60.2 & $(1.2)$ \\
\hline & Argentinien & 62.5 & $(2.0)$ & 29.9 & (1.6) & 28.5 & (1.3) & 27.3 & $(1.7)$ & 58.9 & (1.9) & 52.6 & $(0.9)$ & 29.3 & $(0.9)$ & 26.9 & $(0.9)$ & 28.5 & $(0.9)$ & 49.0 & (1.1) \\
\hline & Brasilien & 60.0 & $(0.9)$ & 29.9 & (1.1) & 32.3 & $(1.0)$ & 36.3 & $(1.0)$ & 50.8 & (1.4) & 48.9 & $(0.6)$ & 33.5 & $(0.7)$ & 35.7 & $(0.6)$ & 15.1 & $(0.4)$ & 44.3 & (0.8) \\
\hline & Bulgarien & 70.5 & $(1.0)$ & 18.3 & $(0.8)$ & 32.9 & $(1.0)$ & 34.4 & $(0.9)$ & 82.8 & $(0.9)$ & 61.8 & (1.1) & 17.7 & $(0.8)$ & 34.3 & (1.5) & 29.6 & (1.0) & 57.2 & (1.1) \\
\hline & Hongkong (China) & 68.7 & $(0.6)$ & 42.2 & (1.0) & 35.1 & (1.0) & 36.3 & $(0.8)$ & 88.0 & $(0.6)$ & 48.5 & (0.9) & 30.4 & $(0.8)$ & 48.5 & $(0.8)$ & 35.0 & $(0.9)$ & 84.1 & $(0.7)$ \\
\hline & Indonesien & 49.3 & (1.2) & 40.0 & $(1.2)$ & 36.6 & (1.1) & 21.9 & $(0.9)$ & 67.1 & $(1.0)$ & 55.2 & $(1.2)$ & 52.3 & $(0.9)$ & 59.3 & $(1.0)$ & 36.1 & $(1.2)$ & 65.4 & (1.1) \\
\hline & Lettland & 76.4 & $(0.9)$ & 14.0 & $(0.9)$ & 31.3 & $(1.0)$ & 18.1 & (1.5) & 76.5 & (1.0) & 71.1 & (1.1) & 9.5 & $(0.6)$ & 32.8 & (1.1) & 25.9 & $(0.8)$ & 65.2 & (1.0) \\
\hline & Liechtenstein & 70.5 & (2.5) & 14.9 & $(2.1)$ & 25.9 & $(2.5)$ & 15.2 & $(2.0)$ & 67.3 & $(2.7)$ & 64.8 & (2.6) & 20.9 & $(2.1)$ & 28.3 & (2.3) & 14.9 & $(2.0)$ & 72.1 & (2.4) \\
\hline & Peru & 38.2 & $(1.2)$ & 36.8 & (1.0) & 41.5 & $(0.9)$ & 39.2 & $(0.9)$ & 64.0 & (1.0) & 47.9 & $(0.9)$ & 48.6 & $(0.7)$ & 55.4 & $(0.9)$ & 39.9 & $(0.8)$ & 73.9 & (1.0) \\
\hline & Rumänien & 63.0 & $(1.2)$ & 11.5 & (0.6) & 43.9 & (0.9) & 27.4 & (1.0) & 52.0 & (1.0) & 62.7 & (1.0) & 11.9 & $(0.5)$ & 31.8 & (1.0) & 22.8 & $(0.6)$ & 50.3 & (1.4) \\
\hline & Russ. Föderation & 74.8 & $(0.8)$ & 24.8 & (1.3) & 53.4 & (1.0) & 48.6 & $(0.8)$ & 76.1 & $(0.9)$ & 67.3 & (0.9) & 17.3 & $(0.9)$ & 58.7 & (1.0) & 26.3 & $(0.7)$ & 64.1 & (1.1) \\
\hline & Thailand & 37.9 & $(1.0)$ & 60.6 & (1.1) & 50.5 & $(1.0)$ & 46.3 & (1.1) & 73.5 & (1.4) & 53.1 & $(1.0)$ & 72.2 & $(0.8)$ & 65.1 & $(0.8)$ & 50.5 & $(0.7)$ & 72.0 & (0.9) \\
\hline
\end{tabular}

Anmerkung: Statistisch signifikante Werte sind durch Fettdruck gekennzeichnet (siehe Anhang A3).

StatLink הत्ञाs http://dx.doi.org/10.1787/888932343285 
[Teil 2/2]

Prozentsatz der Schüler, die verschiedene Arten von Texten lesen, in PISA 2000 und PISA 2009

Prozentsatz der Schüler, die angaben, dass sie aus eigenem Antrieb "mehrmals im Monat" oder "mehrmals in der Tabelle V.5.6 Woche" die folgenden Arten von Texten lesen

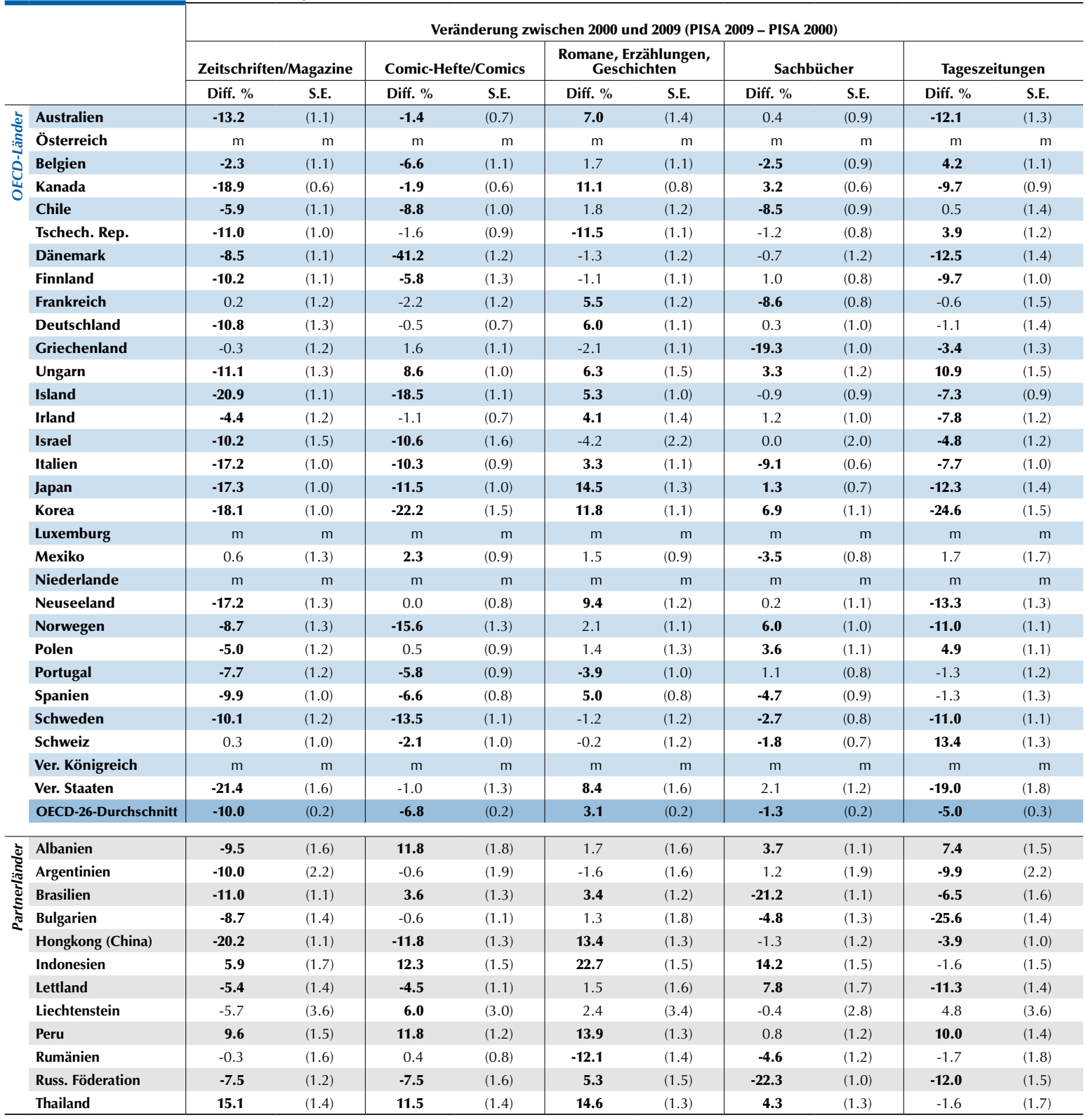

Anmerkung: Statistisch signifikante Werte sind durch Fettdruck gekennzeichnet (siehe Anhang A3).

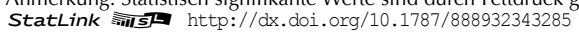


[Teil 1/4]

Prozentsatz der Schüler, die verschiedene Arten von Texten lesen, in PISA 2000 und PISA 2009, nach Geschlecht

Prozentsatz der Schüler, die angaben, dass sie aus eigenem Antrieb „,mehrmals im Monat" oder „mehrmals in der Tabelle V.5.7 Woche" die folgenden Arten von Texten lesen

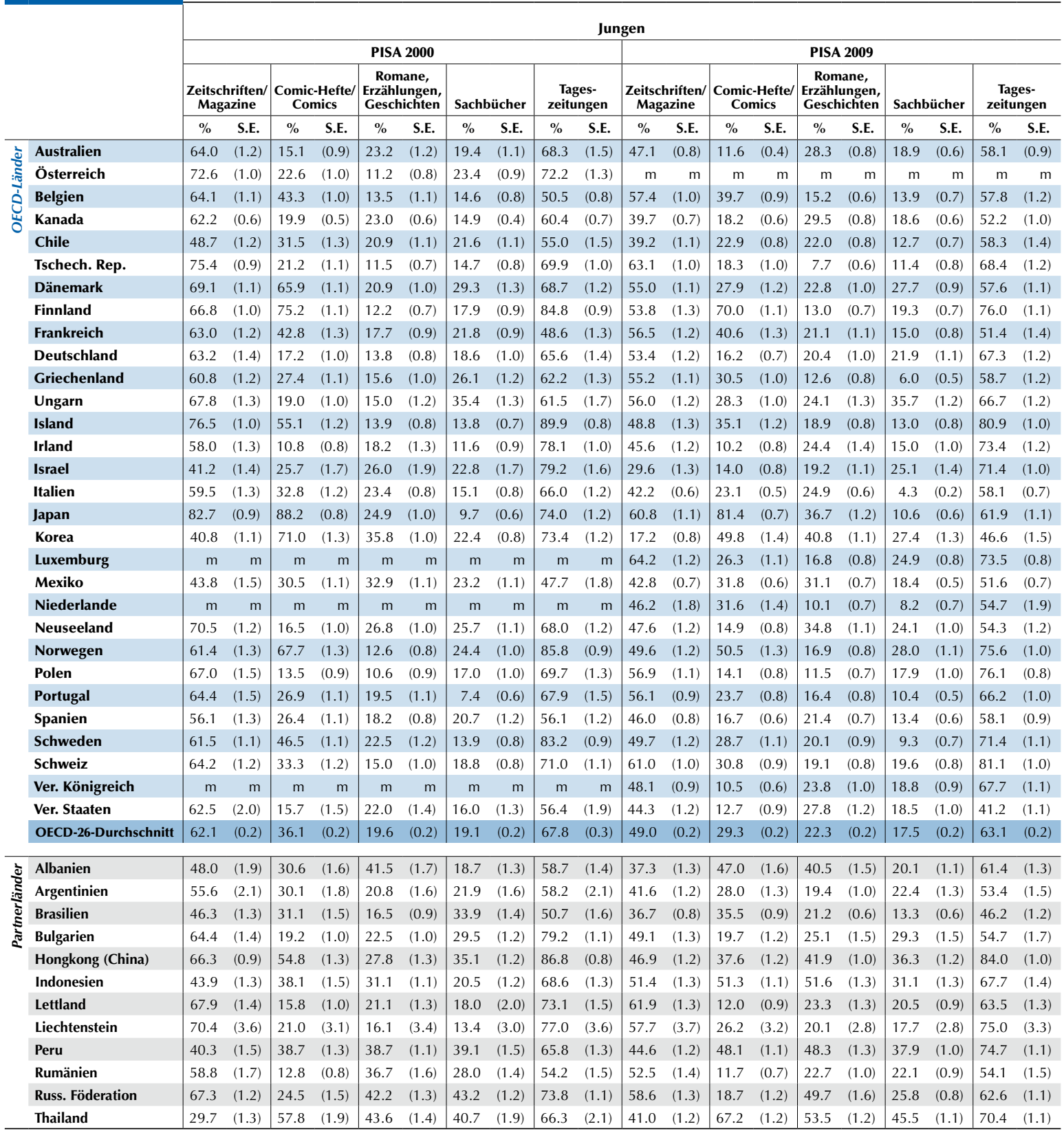

Anmerkung: Statistisch signifikante Werte sind durch Fettdruck gekennzeichnet (siehe Anhang A3).

StatLink inist http://dx.doi.org/10.1787/888932343285 
[Teil 2/4]

Prozentsatz der Schüler, die verschiedene Arten von Texten lesen, in PISA 2000 und PISA 2009, nach Geschlecht

Prozentsatz der Schüler, die angaben, dass sie aus eigenem Antrieb „mehrmals im Monat“ oder „mehrmals in der Tabelle V.5.7 Woche" die folgenden Arten von Texten lesen

\begin{tabular}{|c|c|c|c|c|c|c|c|c|c|c|c|}
\hline & \multicolumn{10}{|c|}{ Jungen } \\
\hline & & \multicolumn{10}{|c|}{ Veränderung zwischen 2000 und 2009 (PISA 2009 - PISA 2000) } \\
\hline & & \multicolumn{2}{|c|}{ Zeitschriften/Magazine } & \multicolumn{2}{|c|}{ Comic-Hefte/Comics } & \multicolumn{2}{|c|}{$\begin{array}{l}\text { Romane, Erzählungen, } \\
\text { Geschichten }\end{array}$} & \multicolumn{2}{|c|}{ Sachbücher } & \multicolumn{2}{|c|}{ Tageszeitungen } \\
\hline & & Diff. \% & S.E. & Diff. $\%$ & S.E. & Diff. \% & S.E. & Diff. \% & S.E. & Diff. \% & S.E. \\
\hline \multirow{31}{*}{ 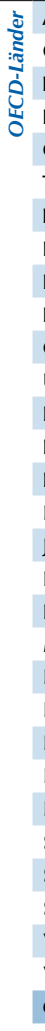 } & Australien & -16.9 & (1.5) & -3.5 & $(1.0)$ & 5.1 & (1.4) & -0.6 & $(1.3)$ & -10.1 & $(1.8)$ \\
\hline & Österreich & $\mathrm{m}$ & $\mathrm{m}$ & $\mathrm{m}$ & $\mathrm{m}$ & $\mathrm{m}$ & $\mathrm{m}$ & $\mathrm{m}$ & $\mathrm{m}$ & m & $\mathrm{m}$ \\
\hline & Belgien & -6.8 & (1.4) & -3.6 & (1.3) & 1.8 & (1.3) & -0.7 & (1.1) & 7.3 & (1.5) \\
\hline & Kanada & -22.5 & (1.0) & -1.7 & $(0.8)$ & 6.5 & (1.0) & 3.7 & $(0.7)$ & -8.2 & (1.2) \\
\hline & Chile & -9.6 & (1.6) & -8.7 & (1.5) & 1.1 & (1.3) & -8.8 & (1.3) & 3.3 & (2.1) \\
\hline & Tschech. Rep. & -12.3 & (1.3) & -2.9 & (1.5) & -3.8 & (0.9) & -3.4 & (1.1) & -1.5 & (1.6) \\
\hline & Dänemark & -14.2 & (1.5) & -38.0 & (1.7) & 2.0 & (1.4) & -1.6 & (1.6) & -11.1 & (1.7) \\
\hline & Finnland & -13.0 & (1.6) & -5.2 & (1.6) & 0.8 & (1.0) & 1.4 & (1.1) & -8.8 & (1.4) \\
\hline & Frankreich & -6.6 & (1.7) & -2.1 & (1.8) & 3.4 & (1.4) & -6.8 & (1.2) & 2.8 & (2.0) \\
\hline & Deutschland & -9.7 & (1.9) & -1.0 & (1.3) & 6.7 & $(1.2)$ & 3.4 & (1.5) & 1.7 & (1.8) \\
\hline & Griechenland & -5.6 & (1.6) & 3.1 & (1.5) & -2.9 & $(1.2)$ & -20.2 & (1.3) & -3.5 & (1.8) \\
\hline & Ungarn & -11.8 & (1.8) & 9.3 & (1.5) & 9.2 & (1.8) & 0.3 & (1.8) & 5.2 & (2.1) \\
\hline & Island & -27.7 & (1.6) & -20.0 & (1.7) & 4.9 & (1.2) & -0.8 & (1.0) & -8.9 & (1.3) \\
\hline & Irland & -12.4 & (1.8) & -0.6 & (1.1) & 6.2 & (1.9) & 3.3 & (1.3) & -4.6 & (1.6) \\
\hline & Israel & -11.6 & (1.9) & -11.7 & (1.9) & -6.8 & $(2.2)$ & 2.3 & (2.2) & -7.8 & (1.9) \\
\hline & Italien & -17.4 & (1.4) & -9.7 & (1.3) & 1.5 & (1.0) & -10.8 & (0.8) & -7.9 & (1.4) \\
\hline & Japan & -21.9 & (1.5) & -6.7 & (1.1) & 11.8 & (1.6) & 0.9 & (0.9) & -12.1 & (1.6) \\
\hline & Korea & -23.6 & (1.4) & -21.2 & (1.9) & 5.0 & (1.5) & 5.0 & (1.5) & -26.8 & (1.9) \\
\hline & Luxemburg & $\mathrm{m}$ & $\mathrm{m}$ & $\mathrm{m}$ & $\mathrm{m}$ & $\mathrm{m}$ & $\mathrm{m}$ & $\mathrm{m}$ & $\mathrm{m}$ & $\mathrm{m}$ & $\mathrm{m}$ \\
\hline & Mexiko & -0.9 & (1.7) & 1.3 & (1.3) & -1.8 & (1.3) & -4.8 & (1.2) & 3.9 & (2.0) \\
\hline & Niederlande & $\mathrm{m}$ & $\mathrm{m}$ & $\mathrm{m}$ & $\mathrm{m}$ & $\mathrm{m}$ & $\mathrm{m}$ & $\mathrm{m}$ & $\mathrm{m}$ & $\mathrm{m}$ & $\mathrm{m}$ \\
\hline & Neuseeland & -22.9 & (1.7) & -1.6 & (1.3) & 8.0 & (1.5) & -1.6 & (1.5) & -13.7 & (1.7) \\
\hline & Norwegen & -11.7 & (1.8) & -17.1 & (1.8) & 4.3 & (1.1) & 3.6 & (1.4) & -10.2 & (1.3) \\
\hline & Polen & -10.1 & (1.9) & 0.7 & (1.2) & 0.9 & (1.2) & 0.9 & (1.4) & 6.4 & (1.6) \\
\hline & Portugal & -8.3 & (1.7) & -3.2 & (1.4) & -3.1 & (1.3) & 3.0 & $(0.8)$ & -1.8 & (1.8) \\
\hline & Spanien & -10.1 & (1.5) & -9.7 & $(1.2)$ & 3.2 & (1.1) & -7.3 & (1.3) & 2.0 & (1.5) \\
\hline & Schweden & -11.9 & (1.6) & -17.8 & (1.6) & -2.3 & (1.5) & -4.6 & (1.1) & -11.9 & (1.4) \\
\hline & Schweiz & -3.2 & (1.5) & -2.5 & (1.5) & 4.1 & (1.3) & 0.9 & (1.1) & 10.1 & (1.5) \\
\hline & Ver. Königreich & $\mathrm{m}$ & $\mathrm{m}$ & $\mathrm{m}$ & $\mathrm{m}$ & $\mathrm{m}$ & $\mathrm{m}$ & $\mathrm{m}$ & $\mathrm{m}$ & $\mathrm{m}$ & $\mathrm{m}$ \\
\hline & Ver. Staaten & -18.2 & (2.3) & -3.1 & (1.7) & 5.8 & (1.9) & 2.4 & (1.6) & -15.2 & (2.2) \\
\hline & OECD-26-Durchschnitt & -13.1 & $(0.3)$ & -6.8 & $(0.3)$ & 2.8 & $(0.3)$ & -1.6 & $(0.3)$ & -4.7 & $(0.3)$ \\
\hline \multirow{12}{*}{ 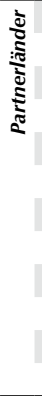 } & Albanien & -10.7 & (2.3) & 16.5 & (2.3) & -0.9 & (2.3) & 1.4 & (1.7) & 2.8 & (1.9) \\
\hline & Argentinien & -14.1 & (2.4) & -2.2 & $(2.2)$ & -1.4 & (1.9) & 0.5 & (2.1) & -4.8 & (2.6) \\
\hline & Brasilien & -9.7 & (1.5) & 4.4 & (1.7) & 4.6 & (1.1) & -20.6 & (1.5) & -4.5 & (2.0) \\
\hline & Bulgarien & -15.3 & (2.0) & 0.4 & (1.5) & 2.6 & (1.8) & -0.2 & (1.9) & -24.5 & (2.0) \\
\hline & Hongkong (China) & -19.4 & (1.5) & -17.2 & (1.8) & 14.1 & (1.7) & 1.1 & (1.7) & -2.8 & (1.2) \\
\hline & Indonesien & 7.5 & (1.8) & 13.1 & (1.9) & 20.5 & (1.7) & 10.6 & (1.7) & -0.9 & (1.9) \\
\hline & Lettland & -6.0 & (1.9) & -3.8 & (1.4) & 2.3 & (1.8) & 2.5 & (2.2) & -9.6 & (2.0) \\
\hline & Liechtenstein & -12.7 & (5.2) & 5.2 & (4.5) & 4.1 & (4.4) & 4.3 & (4.1) & -2.0 & (4.9) \\
\hline & Peru & 4.3 & (1.9) & 9.5 & (1.6) & 9.7 & (1.7) & -1.2 & (1.8) & 8.9 & (1.7) \\
\hline & Rumänien & -6.3 & (2.2) & -1.0 & (1.1) & -14.0 & (1.9) & -6.0 & (1.7) & -0.1 & (2.2) \\
\hline & Russ. Föderation & -8.7 & (1.7) & -5.9 & (1.9) & 7.5 & (2.0) & -17.5 & (1.4) & -11.2 & (1.5) \\
\hline & Thailand & 11.2 & (1.7) & 9.4 & (2.2) & 10.0 & (1.8) & 4.8 & $(2.2)$ & 4.1 & (2.4) \\
\hline
\end{tabular}

Anmerkung: Statistisch signifikante Werte sind durch Fettdruck gekennzeichnet (siehe Anhang A3).

StatLink ints http://dx.doi.org/10.1787/888932343285 
[Teil 3/4]

Prozentsatz der Schüler, die verschiedene Arten von Texten lesen, in PISA 2000 und PISA 2009, nach Geschlecht

Prozentsatz der Schüler, die angaben, dass sie aus eigenem Antrieb „mehrmals im Monat" oder „mehrmals in der Tabelle V.5.7 Woche" die folgenden Arten von Texten lesen

\begin{tabular}{|c|c|c|c|c|c|c|c|c|c|c|c|c|c|c|c|c|c|c|c|c|c|}
\hline & & \multicolumn{20}{|c|}{ Mädchen } \\
\hline & & \multicolumn{10}{|c|}{ PISA 2000} & \multicolumn{10}{|c|}{ PISA 2009} \\
\hline & & \multicolumn{2}{|c|}{$\begin{array}{c}\text { Zeitschriften/ } \\
\text { Magazine }\end{array}$} & \multicolumn{2}{|c|}{$\begin{array}{c}\text { Comic-Hefte/ } \\
\text { Comics }\end{array}$} & \multicolumn{2}{|c|}{$\begin{array}{c}\text { Romane, } \\
\text { Erzählungen, } \\
\text { Geschichten }\end{array}$} & \multicolumn{2}{|c|}{ Sachbücher } & \multicolumn{2}{|c|}{$\begin{array}{c}\text { Tages- } \\
\text { zeitungen }\end{array}$} & \multicolumn{2}{|c|}{$\begin{array}{c}\text { Zeitschriften/ } \\
\text { Magazine }\end{array}$} & \multicolumn{2}{|c|}{$\begin{array}{c}\text { Comic-Hefte/ } \\
\text { Comics }\end{array}$} & \multicolumn{2}{|c|}{$\begin{array}{c}\text { Romane, } \\
\text { Erzählungen, } \\
\text { Geschichten }\end{array}$} & \multicolumn{2}{|c|}{ Sachbücher } & \multicolumn{2}{|c|}{$\begin{array}{c}\text { Tages- } \\
\text { zeitungen }\end{array}$} \\
\hline & & $\%$ & S.E. & $\%$ & S.E. & $\%$ & S.E. & $\%$ & S.E. & $\%$ & S.E. & $\%$ & S.E. & $\%$ & S.E. & $\%$ & S.E. & $\%$ & S.E. & $\%$ & S.E. \\
\hline \multirow{31}{*}{ 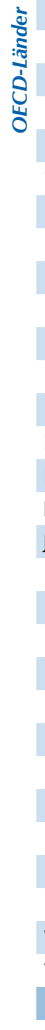 } & Australien & 62.2 & $(1.2)$ & 5.3 & $(0.6)$ & 40.2 & (1.6) & 19.7 & (1.1) & 63.2 & (1.3) & 52.7 & $(0.7)$ & 6.6 & $(0.5)$ & 47.8 & $(0.8)$ & 21.1 & $(0.5)$ & 49.5 & (0.9) \\
\hline & Österreich & 69.3 & (1.1) & 10.1 & $(0.6)$ & 37.6 & (1.1) & 13.5 & $(0.7)$ & 71.7 & (1.3) & $\mathrm{m}$ & $\mathrm{m}$ & $\mathrm{m}$ & $\mathrm{m}$ & $\mathrm{m}$ & $\mathrm{m}$ & $\mathrm{m}$ & $\mathrm{m}$ & $\mathrm{m}$ & $\mathrm{m}$ \\
\hline & Belgien & 72.3 & $(1.2)$ & 33.0 & (1.0) & 28.8 & (1.0) & 17.8 & (0.8) & 41.4 & (1.0) & 74.4 & $(0.8)$ & 23.7 & $(0.9)$ & 29.9 & (1.0) & 13.5 & $(0.7)$ & 42.8 & (1.0) \\
\hline & Kanada & 71.8 & $(0.6)$ & 12.7 & $(0.5)$ & 38.8 & $(0.7)$ & 18.5 & (0.5) & 54.8 & $(0.6)$ & 56.5 & $(0.8)$ & 10.6 & $(0.5)$ & 54.5 & $(0.8)$ & 21.3 & $(0.6)$ & 43.7 & (0.9) \\
\hline & Chile & 61.5 & (1.0) & 28.2 & $(0.9)$ & 35.8 & (1.1) & 27.7 & $(0.9)$ & 60.7 & (1.3) & 60.4 & $(0.9)$ & 18.9 & $(0.7)$ & 39.6 & (1.1) & 20.1 & $(0.9)$ & 58.7 & (1.0) \\
\hline & Tschech. Rep. & 82.8 & $(0.8)$ & 13.3 & $(0.7)$ & 44.6 & (1.3) & 11.8 & $(0.7)$ & 55.3 & (1.1) & 74.2 & (1.0) & 12.2 & $(0.7)$ & 28.5 & $(1.2)$ & 12.7 & $(0.7)$ & 63.4 & (1.0) \\
\hline & Dänemark & 79.1 & (1.1) & 57.8 & $(1.2)$ & 43.0 & (1.3) & 26.2 & $(1.2)$ & 59.7 & (1.4) & 75.9 & $(1.0)$ & 13.5 & $(0.8)$ & 38.1 & (1.1) & 26.6 & $(1.0)$ & 45.9 & (1.2) \\
\hline & Finnland & 83.0 & $(0.8)$ & 57.2 & $(1.0)$ & 41.2 & (1.1) & 11.2 & $(0.7)$ & 85.3 & $(0.8)$ & 76.0 & $(0.9)$ & 50.3 & $(1.2)$ & 39.2 & $(1.2)$ & 11.7 & $(0.6)$ & 74.8 & $(0.9)$ \\
\hline & Frankreich & 61.5 & (1.1) & 23.1 & $(0.9)$ & 28.7 & $(0.9)$ & 19.5 & $(0.8)$ & 46.1 & (1.3) & 68.2 & (1.1) & 20.7 & $(0.9)$ & 36.2 & (1.3) & 9.1 & $(0.7)$ & 42.3 & (1.3) \\
\hline & Deutschland & 68.3 & $(1.2)$ & 6.7 & $(0.6)$ & 39.7 & (1.1) & 15.3 & $(0.7)$ & 60.3 & (1.1) & 56.3 & (1.1) & 6.5 & $(0.5)$ & 45.1 & (1.1) & 12.4 & $(0.8)$ & 56.3 & (1.3) \\
\hline & Griechenland & 60.8 & (1.4) & 18.8 & (1.0) & 31.6 & (1.1) & 26.7 & (1.2) & 30.2 & (1.3) & 65.6 & (1.1) & 19.1 & $(0.9)$ & 30.1 & $(0.9)$ & 8.3 & $(0.7)$ & 27.6 & (1.3) \\
\hline & Ungarn & 75.9 & (1.1) & 16.9 & (1.0) & 35.4 & (1.5) & 27.7 & (1.0) & 60.4 & (1.4) & 65.4 & (1.3) & 24.9 & (1.1) & 38.9 & (1.3) & 34.1 & (1.1) & 76.9 & (1.2) \\
\hline & Island & 81.5 & (1.0) & 43.9 & (1.0) & 29.9 & (1.0) & 21.9 & $(0.9)$ & 88.6 & $(0.8)$ & 67.5 & (1.1) & 26.8 & (1.1) & 35.7 & (1.2) & 21.2 & (1.1) & 83.0 & (0.8) \\
\hline & Irland & 64.9 & (1.1) & 6.6 & $(0.7)$ & 34.0 & (1.1) & 17.9 & (1.0) & 72.5 & (1.1) & 68.8 & (1.3) & 4.8 & $(0.5)$ & 36.3 & (1.3) & 17.1 & $(0.8)$ & 1.4 & (1.3) \\
\hline & Israel & 54.4 & (1.7) & 29.7 & (1.8) & 39.3 & (2.4) & 29.0 & (2.1) & 79.8 & (1.5) & 47.4 & $(1.2)$ & 20.8 & (0.9) & 39.5 & (1.0) & 27.8 & $(1.2)$ & 77.8 & (1.0) \\
\hline & Italien & 72.5 & $(0.9)$ & 22.6 & (0.9) & 40.1 & (1.2) & 13.1 & (0.8) & 56.2 & (1.4) & 55.8 & $(0.6)$ & 11.3 & $(0.3)$ & 45.7 & (0.6) & 5.7 & $(0.3)$ & 48.5 & (0.7) \\
\hline & Japan & 81.0 & $(0.9)$ & 79.7 & (1.0) & 30.1 & (1.2) & 9.9 & (0.6) & 65.9 & (1.4) & 68.5 & $(0.8)$ & 62.7 & (1.1) & 47.8 & $(1.2)$ & 11.7 & $(0.6)$ & 53.0 & (1.2) \\
\hline & Korea & 37.4 & (1.0) & 52.1 & (1.4) & 33.8 & (1.5) & 23.9 & $(1.0)$ & 65.0 & (1.5) & 25.6 & $(1.0)$ & 30.2 & (1.0) & 53.2 & $(1.2)$ & 33.0 & $(1.2)$ & 43.4 & (1.8) \\
\hline & Luxemburg & $\mathrm{m}$ & $\mathrm{m}$ & $\mathrm{m}$ & $\mathrm{m}$ & $\mathrm{m}$ & $\mathrm{m}$ & $\mathrm{m}$ & $\mathrm{m}$ & $\mathrm{m}$ & $\mathrm{m}$ & 73.2 & (1.0) & 14.2 & $(0.8)$ & 41.0 & (1.1) & 13.6 & $(0.7)$ & 67.8 & (1.0) \\
\hline & Mexiko & 48.9 & (1.5) & 19.1 & $(0.9)$ & 39.9 & $(1.0)$ & 21.2 & $(1.0)$ & 44.5 & (1.9) & 50.9 & $(0.5)$ & 22.5 & $(0.5)$ & 44.4 & $(0.5)$ & 18.9 & $(0.5)$ & 44.1 & $(0$ \\
\hline & Niederlande & $\mathrm{m}$ & $\mathrm{m}$ & $\mathrm{m}$ & $\mathrm{m}$ & $\mathrm{m}$ & $\mathrm{m}$ & $\mathrm{m}$ & $\mathrm{m}$ & $\mathrm{m}$ & $\mathrm{m}$ & 67.9 & (1.4) & 14.9 & (1.1) & 32.7 & (1.5) & 16.8 & $(1.1)$ & 42.4 & (1.6) \\
\hline & Neuseeland & 70.1 & (1.1) & 6.7 & $(0.7)$ & 42.7 & (1.1) & 24.6 & $(1.2)$ & 65.0 & (1.3) & 58.8 & (1.4) & 8.2 & $(0.6)$ & 53.9 & $(1.0)$ & 26.7 & $(1.0)$ & 51.9 & (1.4) \\
\hline & Norwegen & 77.6 & (1.1) & 47.6 & (1.3) & 36.3 & $(1.2)$ & 17.9 & $(0.9)$ & 83.1 & (1.0) & 72.0 & $(1.2)$ & 33.4 & (1.1) & 36.2 & (1.4) & 26.5 & (1.1) & 71.1 & (1.2) \\
\hline & Polen & 74.7 & (1.3) & 8.0 & (0.9) & 26.8 & (1.5) & 17.3 & (1.0) & 78.8 & (1.1) & 74.7 & $(0.9)$ & 8.4 & $(0.6)$ & 28.6 & (1.1) & 23.6 & $(0.9)$ & 82.1 & $(0.7)$ \\
\hline & Portugal & 78.1 & $(0.8)$ & 22.0 & (1.0) & 43.4 & $(0.9)$ & 18.3 & (1.1) & 38.9 & $(1.2)$ & 71.3 & (1.0) & 13.7 & $(0.8)$ & 39.2 & (1.1) & 17.8 & $(0.7)$ & 37.5 & (1.0) \\
\hline & Spanien & 66.5 & (1.1) & 11.2 & $(0.7)$ & 31.7 & (1.1) & 25.2 & $(0.9)$ & 37.1 & (1.4) & 56.8 & $(0.9)$ & 7.2 & $(0.5)$ & 39.0 & $(0.7)$ & 23.3 & $(0.7)$ & 31.8 & (0.9) \\
\hline & Schweden & 75.4 & (1.0) & 25.2 & (1.0) & 45.1 & (1.1) & 11.5 & $(0.9)$ & 82.0 & $(0.9)$ & 66.9 & (1.1) & 16.1 & $(0.9)$ & 45.1 & (1.3) & 10.6 & $(0.8)$ & 71.8 & (1.0) \\
\hline & Schweiz & 68.7 & $(1.0)$ & 19.3 & $(0.8)$ & 45.6 & $(1.2)$ & 14.8 & $(0.7)$ & 61.4 & (1.3) & 72.8 & $(0.9)$ & 17.3 & $(0.8)$ & 41.5 & (1.1) & 10.3 & $(0.5)$ & 77.9 & (0.9) \\
\hline & Ver. Königreich & $\mathrm{m}$ & & $\mathrm{m}$ & $\mathrm{m}$ & $\mathrm{m}$ & $\mathrm{m}$ & $\mathrm{m}$ & $\mathrm{m}$ & $\mathrm{m}$ & $\mathrm{m}$ & 70.7 & $(1.0)$ & 5.2 & $(0.4)$ & 38.9 & $(1.0)$ & 20.1 & $(0.6)$ & 54.9 & (0.9) \\
\hline & Ver. Staaten & 73.3 & (1.5) & 7.4 & (1.1) & 33.9 & (1.5) & 20.7 & (1.3) & 55.6 & $(1.8)$ & 49.3 & $(1.0)$ & 7.9 & $(0.6)$ & 45.8 & (1.3) & 22.7 & (1.1) & 32.6 & (1.5) \\
\hline & OECD-26-Durchschnitt & 69.4 & $(0.2)$ & 25.9 & $(0.2)$ & 36.9 & $(0.2)$ & 19.6 & $(0.2)$ & 61.2 & $(0.2)$ & 62.8 & $(0.2)$ & 19.2 & $(0.2)$ & 40.8 & $(0.2)$ & 18.8 & $(0.2)$ & 55.8 & $(0.2)$ \\
\hline \multirow{12}{*}{ 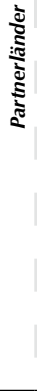 } & Albanien & 59.7 & $(1.2)$ & 33.0 & (1.5) & 61.1 & (1.4) & 18.2 & $(0.8)$ & 47.5 & (1.4) & 52.3 & (1.4) & 40.2 & (1.3) & 67.1 & (1.5) & 24.3 & $(1.1)$ & 58.9 & (1.6) \\
\hline & Argentinien & 67.9 & $(2.0)$ & 29.7 & $(2.2)$ & 34.6 & (1.6) & 31.5 & (2.5) & 59.4 & $(2.2)$ & 61.8 & (1.1) & 30.4 & $(1.2)$ & 33.4 & (1.3) & 33.6 & $(1.2)$ & 45.3 & (1.3) \\
\hline & Brasilien & 71.5 & $(1.1)$ & 28.9 & (1.5) & 45.7 & (1.4) & 38.3 & $(1.2)$ & 50.9 & (1.7) & 59.7 & $(0.9)$ & 31.7 & $(0.8)$ & 48.4 & $(0.9)$ & 16.6 & $(0.6)$ & 42.6 & (1.0) \\
\hline & Bulgarien & 76.8 & $(1.2)$ & 17.3 & $(0.9)$ & 43.5 & $(1.2)$ & 39.4 & $(1.2)$ & 86.5 & $(0.9)$ & 75.3 & $(1.2)$ & 15.6 & $(1.0)$ & 43.9 & (1.7) & 29.9 & $(1.2)$ & 59.7 & (1.1) \\
\hline & Hongkong (China) & 71.2 & $(0.9)$ & 29.6 & (1.0) & 42.4 & (1.3) & 37.5 & $(1.2)$ & 89.3 & $(0.9)$ & 50.3 & $(1.2)$ & 22.4 & $(1.0)$ & 55.8 & (1.2) & 33.6 & $(1.0)$ & 84.3 & (0.9) \\
\hline & Indonesien & 54.4 & (1.6) & 41.8 & (1.5) & 41.8 & (1.5) & 23.2 & (1.1) & 65.6 & (1.3) & 59.0 & (1.6) & 53.3 & $(1.2)$ & 66.8 & $(1.3)$ & 41.0 & (1.4) & 63.2 & (1.3) \\
\hline & Lettland & 84.5 & $(1.0)$ & 12.3 & (1.4) & 41.0 & (1.5) & 18.2 & (1.5) & 79.7 & (1.1) & 80.0 & $(1.2)$ & 7.1 & $(0.8)$ & 42.0 & (1.7) & 31.2 & (1.3) & 66.8 & (1.3) \\
\hline & Liechtenstein & 71.9 & $(3.1)$ & 8.5 & (2.4) & 36.1 & (3.6) & 17.3 & (2.8) & 58.8 & (4.4) & 72.7 & (3.4) & 15.0 & (2.8) & 37.2 & (3.9) & 11.7 & (2.4) & 68.8 & (3.4) \\
\hline & Peru & 36.1 & (1.7) & 34.9 & (1.3) & 44.3 & (1.3) & 39.3 & $(1.2)$ & 62.1 & (1.5) & 51.2 & $(1.2)$ & 49.0 & $(1.2)$ & 62.5 & (1.0) & 42.1 & $(1.2)$ & 73.2 & (1.3) \\
\hline & Rumänien & 66.7 & (1.5) & 10.4 & $(0.8)$ & 50.3 & (1.3) & 26.8 & (1.4) & 50.0 & $(1.2)$ & 72.4 & $(1.2)$ & 12.1 & $(0.7)$ & 40.5 & (1.4) & 23.5 & (1.0) & 46.7 & (1.7) \\
\hline & Russ. Föderation & 82.1 & $(0.8)$ & 25.1 & (1.4) & 64.3 & (1.0) & 53.9 & $(0.8)$ & 78.4 & (1.1) & 75.8 & $(0.9)$ & 16.0 & (1.0) & 67.5 & $(1.0)$ & 26.8 & $(1.0)$ & 65.6 & (1.5) \\
\hline & Thailand & 43.6 & $(1.3)$ & 62.6 & $(1.2)$ & 55.3 & $(1.1)$ & 50.1 & $(1.2)$ & 78.5 & $(1.3)$ & 62.2 & $(1.0)$ & 75.9 & $(0.7)$ & 73.9 & $(0.8)$ & 54.4 & $(1.0)$ & 73.1 & $(1.1)$ \\
\hline
\end{tabular}

Anmerkung: Statistisch signifikante Werte sind durch Fettdruck gekennzeichnet (siehe Anhang A3).

StatLink inist http://dx.doi.org/10.1787/888932343285 
[Teil 4/4]

Prozentsatz der Schüler, die verschiedene Arten von Texten lesen, in PISA 2000 und PISA 2009, nach Geschlecht

Prozentsatz der Schüler, die angaben, dass sie aus eigenem Antrieb „mehrmals im Monat" oder "mehrmals in der Tabelle V.5.7 Woche" die folgenden Arten von Texten lesen

\begin{tabular}{|c|c|c|c|c|c|c|c|c|c|c|c|}
\hline & & \multicolumn{10}{|c|}{ Mädchen } \\
\hline & & \multicolumn{10}{|c|}{ Veränderung zwischen 2000 und 2009 (PISA 2009 - PISA 2000) } \\
\hline & & \multicolumn{2}{|c|}{ Zeitschriften/Magazine } & \multicolumn{2}{|c|}{ Comic-Hefte/Comics } & \multicolumn{2}{|c|}{$\begin{array}{c}\text { Romane, Erzählungen, } \\
\text { Geschichten }\end{array}$} & \multicolumn{2}{|c|}{ Sachbücher } & \multicolumn{2}{|c|}{ Tageszeitungen } \\
\hline & & Diff. \% & S.E. & Diff. \% & S.E. & Diff. \% & S.E. & Diff. $\%$ & S.E. & Diff. $\%$ & S.E. \\
\hline \multirow{31}{*}{ 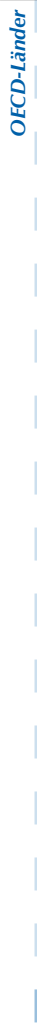 } & Australien & -9.5 & $(1.4)$ & 1.3 & $(0.8)$ & 7.6 & $(1.8)$ & 1.4 & $(1.2)$ & -13.7 & $(1.6)$ \\
\hline & Österreich & $\mathrm{m}$ & $\mathrm{m}$ & $\mathrm{m}$ & $\mathrm{m}$ & $\mathrm{m}$ & $\mathrm{m}$ & $\mathrm{m}$ & $\mathrm{m}$ & $\mathrm{m}$ & $\mathrm{m}$ \\
\hline & Belgien & 2.1 & (1.4) & -9.3 & (1.3) & 1.1 & (1.4) & -4.3 & (1.0) & 1.4 & (1.5) \\
\hline & Kanada & -15.3 & $(1.0)$ & -2.1 & $(0.7)$ & 15.7 & (1.1) & 2.7 & $(0.8)$ & -11.1 & (1.0) \\
\hline & Chile & -1.1 & (1.3) & -9.3 & $(1.2)$ & 3.8 & (1.5) & -7.6 & $(1.2)$ & -2.0 & (1.6) \\
\hline & Tschech. Rep. & -8.6 & $(1.2)$ & -1.0 & $(1.0)$ & -16.1 & $(1.7)$ & 0.9 & $(1.0)$ & 8.1 & (1.5) \\
\hline & Dänemark & -3.2 & (1.4) & -44.2 & (1.5) & -4.9 & (1.7) & 0.4 & (1.5) & -13.8 & (1.9) \\
\hline & Finnland & -7.0 & $(1.2)$ & -6.9 & (1.6) & -2.1 & (1.6) & 0.5 & $(1.0)$ & -10.5 & (1.2) \\
\hline & Frankreich & 6.6 & (1.5) & -2.4 & (1.3) & 7.5 & (1.6) & -10.4 & (1.1) & -3.8 & (1.8) \\
\hline & Deutschland & -11.9 & (1.6) & -0.1 & $(0.8)$ & 5.4 & (1.6) & -2.9 & $(1.1)$ & -4.0 & (1.7) \\
\hline & Griechenland & 4.8 & (1.7) & 0.2 & (1.4) & -1.5 & (1.5) & -18.4 & (1.4) & -2.6 & (1.8) \\
\hline & Ungarn & -10.5 & (1.7) & 8.0 & (1.5) & 3.6 & (2.0) & 6.4 & (1.4) & 16.5 & (1.8) \\
\hline & Island & -14.1 & (1.5) & -17.1 & (1.5) & 5.8 & (1.6) & -0.7 & (1.4) & -5.6 & (1.1) \\
\hline & Irland & 3.9 & $(1.7)$ & -1.8 & $(0.8)$ & 2.3 & $(1.7)$ & -0.8 & (1.3) & -11.1 & (1.7) \\
\hline & Israel & -7.0 & $(2.1)$ & -9.0 & (2.0) & 0.2 & (2.6) & -1.2 & (2.4) & -2.0 & (1.8) \\
\hline & Italien & -16.8 & $(1.1)$ & -11.3 & $(1.0)$ & 5.6 & (1.3) & -7.4 & $(0.9)$ & -7.7 & (1.5) \\
\hline & Japan & -12.4 & $(1.2)$ & -17.0 & (1.5) & 17.7 & $(1.7)$ & 1.8 & $(0.9)$ & -13.0 & (1.9) \\
\hline & Korea & -11.8 & (1.4) & -22.0 & $(1.8)$ & 19.4 & (1.9) & 9.0 & (1.6) & -21.6 & (2.4) \\
\hline & Luxemburg & m & $\mathrm{m}$ & m & $\mathrm{m}$ & m & $\mathrm{m}$ & $\mathrm{m}$ & $\mathrm{m}$ & m & m \\
\hline & Mexiko & 2.0 & (1.6) & 3.4 & $(1.0)$ & 4.6 & $(1.2)$ & -2.3 & (1.1) & -0.4 & $(2.0)$ \\
\hline & Niederlande & $\mathrm{m}$ & $\mathrm{m}$ & $\mathrm{m}$ & $\mathrm{m}$ & $\mathrm{m}$ & $\mathrm{m}$ & m & $\mathrm{m}$ & m & m \\
\hline & Neuseeland & -11.3 & (1.8) & 1.5 & $(0.9)$ & 11.3 & (1.5) & 2.1 & (1.5) & -13.0 & (1.9) \\
\hline & Norwegen & -5.6 & (1.6) & -14.2 & (1.7) & -0.1 & (1.8) & 8.6 & (1.4) & -11.9 & (1.6) \\
\hline & Polen & 0.0 & (1.6) & 0.5 & $(1.1)$ & 1.7 & (1.9) & 6.3 & (1.3) & 3.2 & (1.4) \\
\hline & Portugal & -6.8 & $(1.3)$ & -8.2 & $(1.2)$ & -4.2 & (1.4) & -0.5 & (1.3) & -1.4 & (1.5) \\
\hline & Spanien & -9.7 & (1.4) & -4.1 & $(0.8)$ & 7.2 & (1.3) & -1.9 & $(1.1)$ & -5.4 & (1.7) \\
\hline & Schweden & -8.5 & $(1.5)$ & -9.1 & (1.4) & 0.0 & (1.7) & -0.9 & (1.1) & -10.2 & (1.4) \\
\hline & Schweiz & 4.1 & $(1.3)$ & -1.9 & $(1.1)$ & -4.1 & (1.6) & -4.5 & $(0.9)$ & 16.5 & (1.6) \\
\hline & Ver. Königreich & $\mathrm{m}$ & $\mathrm{m}$ & $\mathrm{m}$ & $\mathrm{m}$ & $\mathrm{m}$ & $\mathrm{m}$ & $\mathrm{m}$ & $\mathrm{m}$ & $\mathrm{m}$ & m \\
\hline & Ver. Staaten & -23.9 & $(1.8)$ & 0.5 & $(1.3)$ & 12.0 & $(2.0)$ & 2.1 & $(1.7)$ & -23.0 & $(2.3)$ \\
\hline & OECD-26-Durchschnitt & -6.6 & $(0.3)$ & -6.7 & $(0.3)$ & 3.8 & $(0.3)$ & -0.8 & $(0.3)$ & -5.5 & $(0.3)$ \\
\hline \multirow{12}{*}{ 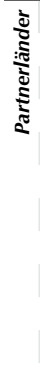 } & Albanien & -7.4 & (1.9) & 7.2 & (2.0) & 6.0 & (2.0) & 6.0 & (1.4) & 11.5 & $(2.1)$ \\
\hline & Argentinien & -6.0 & (2.3) & 0.8 & (2.5) & -1.3 & (2.1) & 2.1 & (2.8) & -14.1 & (2.6) \\
\hline & Brasilien & -11.8 & (1.4) & 2.8 & (1.7) & 2.8 & (1.7) & -21.7 & (1.3) & -8.3 & $(2.0)$ \\
\hline & Bulgarien & -1.4 & $(1.7)$ & -1.7 & (1.4) & 0.4 & $(2.1)$ & -9.5 & $(1.7)$ & -26.7 & (1.5) \\
\hline & Hongkong (China) & -20.8 & (1.5) & -7.2 & (1.5) & 13.4 & (1.8) & -3.9 & (1.6) & -5.0 & (1.3) \\
\hline & Indonesien & 4.6 & $(2.2)$ & 11.5 & (1.9) & 25.0 & (2.0) & 17.7 & (1.8) & -2.5 & (1.8) \\
\hline & Lettland & -4.5 & (1.6) & -5.2 & (1.6) & 1.0 & (2.3) & 13.0 & (2.0) & -12.8 & (1.7) \\
\hline & Liechtenstein & 0.8 & (4.6) & 6.6 & (3.7) & 1.2 & (5.3) & -5.6 & (3.7) & 10.0 & (5.6) \\
\hline & Peru & 15.0 & $(2.1)$ & 14.1 & (1.8) & 18.2 & (1.6) & 2.7 & (1.7) & 11.1 & (2.0) \\
\hline & Rumänien & 5.7 & (1.9) & 1.7 & $(1.0)$ & -9.8 & (1.9) & -3.3 & (1.7) & -3.4 & (2.1) \\
\hline & Russ. Föderation & -6.4 & $(1.2)$ & -9.1 & $(1.7)$ & 3.2 & (1.4) & -27.1 & (1.3) & -12.8 & (1.9) \\
\hline & Thailand & 18.6 & $(1.6)$ & 13.3 & (1.4) & 18.6 & (1.4) & 4.2 & $(1.5)$ & -5.4 & $(1.7)$ \\
\hline
\end{tabular}

Anmerkung: Statistisch signifikante Werte sind durch Fettdruck gekennzeichnet (siehe Anhang A3).

StatLink *illst http://dx.doi.org/10.1787/888932343285 
[Teil 1/1]

Leistung auf der Gesamtskala Lesekompetenz von Schülern, die fiktionale Texte lesen,

in PISA 2000 und PISA 2009

Tabelle V.5.8 Auf Grundlage der Schülerangaben

\begin{tabular}{|c|c|c|c|c|c|c|c|c|c|c|c|c|c|c|c|c|c|c|c|}
\hline & \multicolumn{6}{|c|}{ PISA 2000} & \multicolumn{6}{|c|}{ PISA 2009} & \multicolumn{6}{|c|}{$\begin{array}{c}\text { Veränderung zwischen } 2000 \text { und } 2009 \\
\text { (PISA 2009 - PISA 2000) }\end{array}$} \\
\hline & & \multicolumn{2}{|c|}{$\begin{array}{c}\text { Leser } \\
\text { fiktionaler } \\
\text { Texte }\end{array}$} & \multicolumn{2}{|c|}{$\begin{array}{c}\text { Nichtleser } \\
\text { fiktionaler } \\
\text { Texte }\end{array}$} & \multicolumn{2}{|c|}{ Differenz } & \multicolumn{2}{|c|}{$\begin{array}{c}\text { Leser } \\
\text { fiktionaler } \\
\text { Texte }\end{array}$} & \multicolumn{2}{|c|}{$\begin{array}{c}\text { Nichtleser } \\
\text { fiktionaler } \\
\text { Texte }\end{array}$} & \multicolumn{2}{|c|}{ Differenz } & \multicolumn{2}{|c|}{$\begin{array}{c}\text { Leser } \\
\text { fiktionaler } \\
\text { Texte }\end{array}$} & \multicolumn{2}{|c|}{$\begin{array}{c}\text { Nichtleser } \\
\text { fiktionaler } \\
\text { Texte }\end{array}$} & \multicolumn{2}{|c|}{ Differenz } \\
\hline & & $\begin{array}{c}\text { Mittel- } \\
\text { wert }\end{array}$ & S.E. & $\begin{array}{c}\text { Mittel- } \\
\text { wert }\end{array}$ & S.E. & $\begin{array}{c}\text { Punkt- } \\
\text { diff. }\end{array}$ & S.E. & $\begin{array}{c}\text { Mittel- } \\
\text { wert }\end{array}$ & S.E. & $\begin{array}{c}\text { Mittel- } \\
\text { wert }\end{array}$ & S.E. & $\begin{array}{l}\text { Punkt- } \\
\text { diff. }\end{array}$ & S.E. & $\begin{array}{c}\text { Punkt- } \\
\text { diff. }\end{array}$ & S.E. & $\begin{array}{l}\text { Punkt- } \\
\text { diff. }\end{array}$ & S.E. & $\begin{array}{l}\text { Punkt- } \\
\text { diff. }\end{array}$ & S.E. \\
\hline \multirow{31}{*}{ 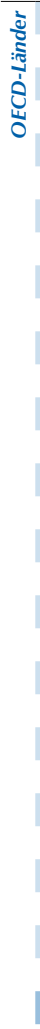 } & Australien & 573 & $(4.7)$ & 510 & (3.2) & 63 & $(4.3)$ & 564 & (2.8) & 488 & (2.0) & 75 & (2.3) & -10 & $(7.3)$ & -22 & $(6.2)$ & 12 & (4.9) \\
\hline & Österreich & 532 & $(4.2)$ & 482 & (2.5) & 50 & $(4.2)$ & $\mathrm{m}$ & $\mathrm{m}$ & $\mathrm{m}$ & $\mathrm{m}$ & $\mathrm{m}$ & $\mathrm{m}$ & $\mathrm{m}$ & $\mathrm{m}$ & $\mathrm{m}$ & $\mathrm{m}$ & $\mathrm{m}$ & $\mathrm{m}$ \\
\hline & Belgien & 551 & (7.9) & 503 & (3.3) & 48 & (7.3) & 561 & (3.1) & 499 & $(2.4)$ & 62 & (3.5) & 10 & (9.8) & -4 & (6.4) & 15 & (8.1) \\
\hline & Kanada & 572 & $(2.0)$ & 519 & (1.6) & 53 & $(2.0)$ & 558 & (1.7) & 502 & (1.6) & 56 & (1.8) & -14 & (5.6) & -17 & (5.4) & 3 & (2.7) \\
\hline & Chile & 422 & $(4.7)$ & 408 & (3.5) & 14 & (3.6) & 462 & (3.8) & 446 & (3.1) & 16 & (2.7) & 40 & (7.8) & 38 & (6.8) & 2 & (4.6) \\
\hline & Tschech. Rep. & 532 & (3.1) & 490 & (2.4) & 43 & $(3.2)$ & 541 & $(4.1)$ & 470 & (2.9) & 71 & $(4.1)$ & 9 & (7.1) & -19 & $(6.2)$ & 28 & (5.2) \\
\hline & Dänemark & 535 & (3.1) & 484 & (2.5) & 52 & (3.4) & 525 & $(2.7)$ & 483 & (2.3) & 42 & (2.8) & -10 & (6.4) & 0 & (6.0) & -10 & (4.4) \\
\hline & Finnland & 595 & $(2.7)$ & 530 & (2.9) & 66 & (3.3) & 590 & (2.8) & 517 & $(2.2)$ & 73 & (2.9) & -5 & (6.3) & -12 & $(6.2)$ & 7 & (4.4) \\
\hline & Frankreich & 536 & (3.3) & 502 & (2.8) & 34 & (3.4) & 549 & (3.9) & 477 & (3.6) & 72 & (4.8) & 13 & (7.1) & -25 & (6.7) & 38 & (5.9) \\
\hline & Deutschland & 539 & (3.2) & 479 & (2.5) & 60 & (3.1) & 551 & (2.9) & 483 & (3.0) & 69 & (3.3) & 12 & (6.6) & 3 & (6.3) & 9 & (4.5) \\
\hline & Griechenland & 499 & $(6.0)$ & 470 & (4.9) & 29 & $(4.5)$ & 523 & (3.5) & 472 & (4.9) & 50 & $(4.7)$ & 23 & (8.5) & 2 & (8.5) & 21 & (6.5) \\
\hline & Ungarn & 514 & $(5.2)$ & 471 & (3.9) & 43 & (4.9) & 519 & (4.6) & 484 & $(3.1)$ & 35 & $(4.2)$ & 5 & (8.5) & 12 & $(7.0)$ & -8 & (6.5) \\
\hline & Island & 553 & (3.5) & 497 & (1.7) & 56 & $(4.1)$ & 549 & (2.8) & 484 & (1.7) & 65 & (3.3) & -4 & (6.7) & -13 & (5.5) & 9 & (5.2) \\
\hline & Irland & 567 & (4.1) & 515 & (3.2) & 52 & (4.1) & 542 & (3.5) & 480 & (3.1) & 62 & (3.6) & -25 & (7.3) & -35 & (6.6) & 10 & (5.4) \\
\hline & Israel & 474 & (10.2) & 455 & (9.1) & 19 & $(7.1)$ & 500 & $(4.2)$ & 471 & (3.6) & 30 & $(4.3)$ & 26 & (12.1) & 15 & (11.0) & 11 & (8.3) \\
\hline & Italien & 505 & (3.1) & 482 & (3.1) & 23 & (3.1) & 517 & (1.9) & 471 & (1.8) & 47 & (2.3) & 12 & (6.1) & -12 & (6.1) & 24 & (3.9) \\
\hline & Japan & 550 & (5.4) & 515 & (5.1) & 36 & (3.0) & 548 & (3.3) & 501 & $(4.0)$ & 47 & (3.8) & -2 & (8.0) & -14 & $(8.2)$ & 12 & (4.8) \\
\hline & Korea & 545 & (2.5) & 514 & (2.7) & 31 & (2.3) & 556 & (3.1) & 526 & $(4.0)$ & 30 & (2.8) & 11 & (6.4) & 11 & (6.9) & 0 & (3.6) \\
\hline & Luxemburg & $\mathrm{m}$ & $\mathrm{m}$ & $\mathrm{m}$ & $\mathrm{m}$ & $\mathrm{m}$ & $\mathrm{m}$ & 527 & (2.6) & 452 & (1.4) & 75 & (3.1) & $\mathrm{m}$ & $\mathrm{m}$ & $\mathrm{m}$ & $\mathrm{m}$ & $\mathrm{m}$ & $\mathrm{m}$ \\
\hline & Mexiko & 430 & (3.9) & 419 & (3.6) & 11 & (3.1) & 424 & $(2.2)$ & 429 & (2.0) & -5 & (1.6) & -7 & (6.7) & 10 & (6.4) & -16 & (3.5) \\
\hline & Niederlande & $\mathrm{m}$ & $\mathrm{m}$ & $\mathrm{m}$ & $\mathrm{m}$ & $\mathrm{m}$ & $\mathrm{m}$ & 552 & (5.1) & 501 & (5.5) & 52 & $(4.8)$ & $\mathrm{m}$ & $\mathrm{m}$ & $\mathrm{m}$ & $\mathrm{m}$ & $\mathrm{m}$ & $\mathrm{m}$ \\
\hline & Neuseeland & 565 & (3.8) & 516 & (2.8) & 48 & (3.8) & 559 & (3.0) & 494 & (2.6) & 65 & (3.6) & -6 & (6.9) & -22 & (6.3) & 16 & (5.2) \\
\hline & Norwegen & 557 & (3.3) & 494 & (3.2) & 63 & $(4.0)$ & 551 & (3.4) & 487 & $(2.5)$ & 63 & (3.3) & -7 & (6.8) & -6 & (6.4) & 0 & (5.2) \\
\hline & Polen & 509 & $(7.4)$ & 479 & (4.3) & 30 & (6.6) & 544 & $(4.0)$ & 491 & (2.5) & 53 & (3.7) & 35 & (9.7) & 12 & $(7.0)$ & 23 & (7.5) \\
\hline & Portugal & 485 & (5.8) & 467 & (4.4) & 19 & $(4.2)$ & 518 & (3.8) & 479 & (3.0) & 39 & (3.1) & 33 & (8.5) & 12 & (7.3) & 21 & (5.2) \\
\hline & Spanien & 521 & (3.2) & 486 & (2.9) & 35 & (3.5) & 519 & $(2.2)$ & 466 & (2.1) & 54 & (2.0) & -2 & (6.3) & -20 & (6.1) & 18 & (4.0) \\
\hline & Schweden & 557 & (3.1) & 497 & (2.3) & 60 & $(3.2)$ & 549 & (3.3) & 475 & $(2.7)$ & 74 & $(3.1)$ & -7 & $(6.7)$ & -22 & $(6.0)$ & 15 & (4.5) \\
\hline & Schweiz & 532 & (5.8) & 483 & (3.6) & 49 & $(4.5)$ & 550 & (3.3) & 480 & $(2.4)$ & 70 & $(2.9)$ & 18 & (8.3) & -2 & (6.6) & 20 & (5.4) \\
\hline & Ver. Königreich & $\mathrm{m}$ & $\mathrm{m}$ & $\mathrm{m}$ & $\mathrm{m}$ & $\mathrm{m}$ & $\mathrm{m}$ & 542 & (3.0) & 475 & $(2.3)$ & 67 & $(3.1)$ & $\mathrm{m}$ & $\mathrm{m}$ & $\mathrm{m}$ & $\mathrm{m}$ & $\mathrm{m}$ & $\mathrm{m}$ \\
\hline & Ver. Staaten & 539 & $(7.2)$ & 498 & $(7.0)$ & 41 & $(4.7)$ & 532 & $(4.8)$ & 483 & $(3.1)$ & 50 & (3.6) & -7 & (9.9) & -15 & $(9.1)$ & 8 & (6.0) \\
\hline & OECD-26-Durchschnitt & 529 & (1.0) & 488 & $(0.8)$ & 41 & $(0.8)$ & 535 & $(0.7)$ & 482 & $(0.6)$ & 53 & $(0.7)$ & 5 & (5.1) & -6 & $(5.0)$ & 11 & (1.1) \\
\hline \multirow{12}{*}{ 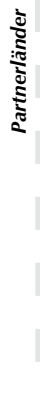 } & Albanien & 361 & $(4.2)$ & 353 & (3.4) & 8 & $(4.2)$ & 400 & $(4.2)$ & 375 & $(4.7)$ & 25 & $(4.3)$ & 39 & $(7.7)$ & 22 & (7.6) & 17 & (6.0) \\
\hline & Argentinien & 443 & $(11.2)$ & 421 & (8.3) & 22 & $(5.2)$ & 406 & (5.8) & 402 & $(4.7)$ & 4 & $(4.8)$ & -37 & $(13.6)$ & -18 & $(10.7)$ & -19 & (7.1) \\
\hline & Brasilien & 402 & (3.5) & 399 & (3.5) & 3 & (3.6) & 416 & (3.5) & 414 & (2.8) & 1 & (2.8) & 14 & $(7.0)$ & 15 & $(6.7)$ & -1 & (4.6) \\
\hline & Bulgarien & 466 & (5.9) & 425 & (4.7) & 41 & $(4.4)$ & 461 & $(8.2)$ & 420 & $(6.0)$ & 41 & $(6.2)$ & -4 & $(11.2)$ & -4 & $(9.0)$ & 0 & (7.5) \\
\hline & Hongkong (China) & 551 & (3.5) & 513 & (3.0) & 38 & (3.6) & 552 & (2.5) & 516 & (2.4) & 36 & (2.5) & 1 & $(6.5)$ & 3 & $(6.2)$ & -2 & (4.4) \\
\hline & Indonesien & 377 & (4.8) & 371 & (3.8) & 6 & (3.6) & 408 & (3.9) & 394 & $(4.0)$ & 14 & $(2.5)$ & 31 & $(7.9)$ & 23 & (7.4) & 8 & (4.4) \\
\hline & Lettland & 491 & (5.8) & 448 & (5.7) & 43 & $(4.8)$ & 500 & $(4.0)$ & 477 & (3.0) & 23 & (3.5) & 9 & (8.6) & 29 & (8.1) & -20 & (5.9) \\
\hline & Liechtenstein & 527 & (10.8) & 471 & (5.7) & 56 & (13.6) & 543 & (7.5) & 484 & $(4.0)$ & 59 & (9.6) & 16 & (14.0) & 13 & (8.5) & 3 & (16.7) \\
\hline & Peru & 338 & (4.8) & 333 & (4.6) & 5 & (3.9) & 372 & (3.6) & 372 & (5.0) & 0 & (3.5) & 34 & (7.7) & 39 & (8.4) & -5 & (5.2) \\
\hline & Rumänien & 432 & (3.5) & 428 & (4.7) & 3 & (4.6) & 437 & $(4.7)$ & 421 & $(4.2)$ & 16 & (3.8) & 6 & (7.6) & -8 & (8.0) & 13 & (6.0) \\
\hline & Russ. Föderation & 482 & (4.1) & 445 & (4.5) & 38 & (2.6) & 477 & (3.3) & 439 & (3.9) & 38 & (3.4) & -5 & $(7.2)$ & -5 & $(7.7)$ & 0 & (4.3) \\
\hline & Thailand & 440 & (3.5) & 422 & (3.3) & 18 & $(2.7)$ & 428 & (2.8) & 410 & (2.9) & 18 & (2.4) & -12 & (6.7) & -12 & (6.6) & 0 & (3.6) \\
\hline
\end{tabular}

Anmerkung: Statistisch signifikante Werte sind durch Fettdruck gekennzeichnet (siehe Anhang A3).

StatLink : 
[Teil 1/2]

Leistung auf der Gesamtskala Lesekompetenz von Schülern, die fiktionale Texte lesen, in PISA 2000 und PISA 2009, nach Geschlecht

Tabelle V.5.9 Auf Grundlage der Schülerangaben

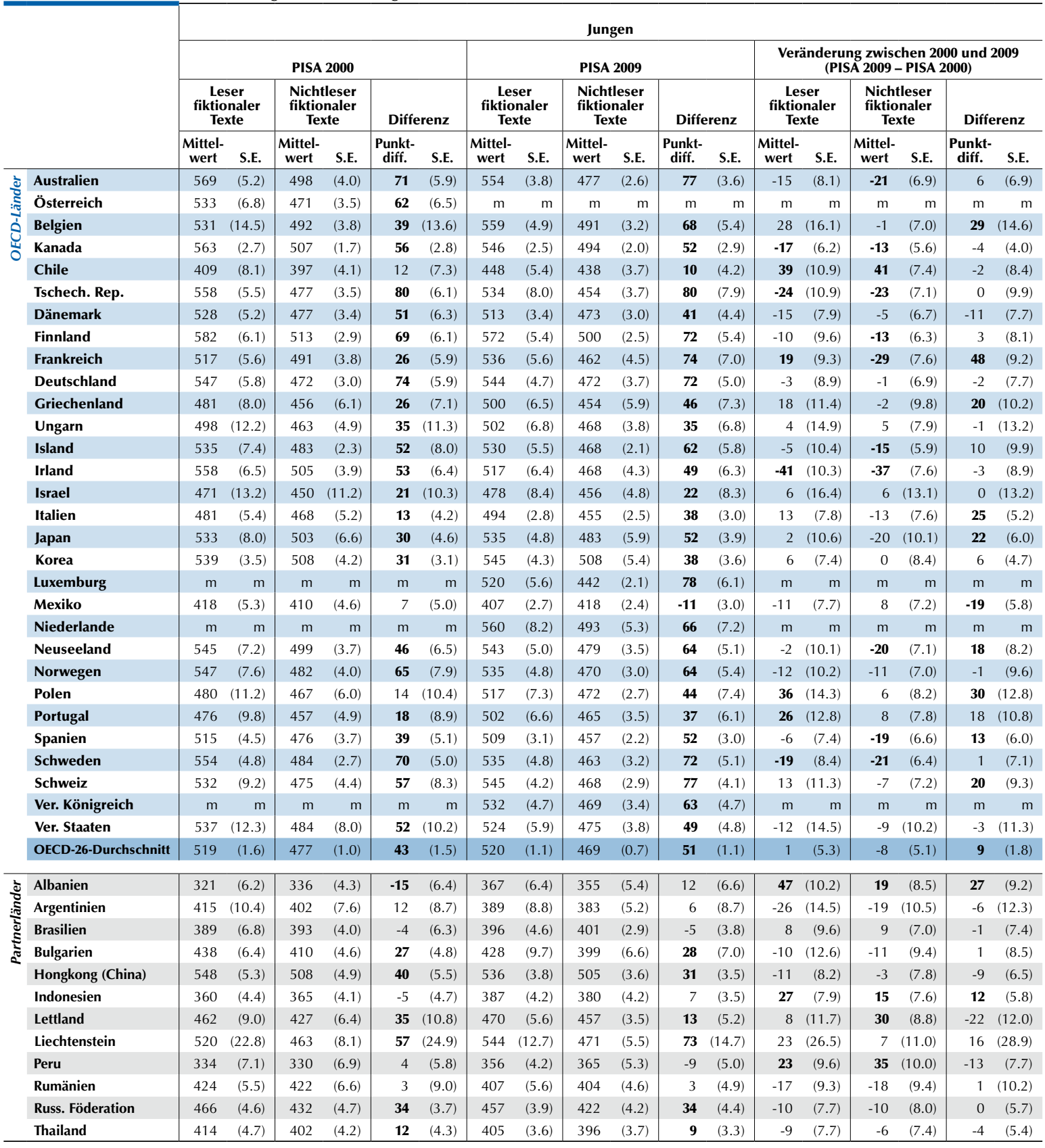

Anmerkung: Statistisch signifikante Werte sind durch Fettdruck gekennzeichnet (siehe Anhang A3).

StatLink : ints http://dx.doi.org/10.1787/888932343285 
[Teil 2/2]

Leistung auf der Gesamtskala Lesekompetenz von Schülern, die fiktionale Texte lesen, in PISA 2000 und PISA 2009, nach Geschlecht

Tabelle V.5.9 Auf Grundlage der Schülerangaben

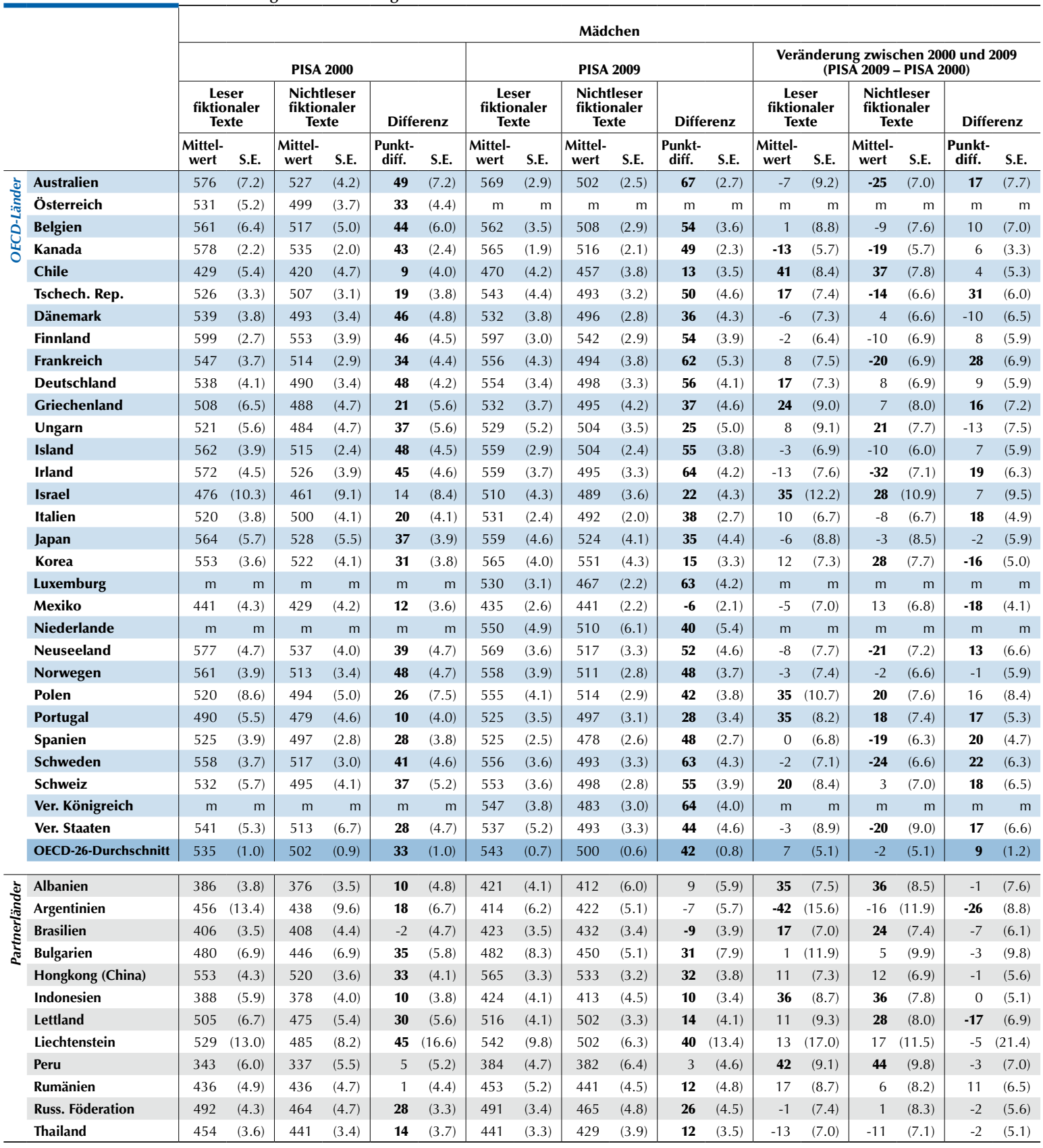

Anmerkung: Statistisch signifikante Werte sind durch Fettdruck gekennzeichnet (siehe Anhang A3).

StatLink त्राज ht http://dx.doi.org/10.1787/888932343285 
[Teil 1/1]

Vielseitigkeit des Lesestoffs in PISA 2000 und PISA 2009, nach Geschlecht Tabelle V.5.10 Auf Grundlage der Schülerangaben

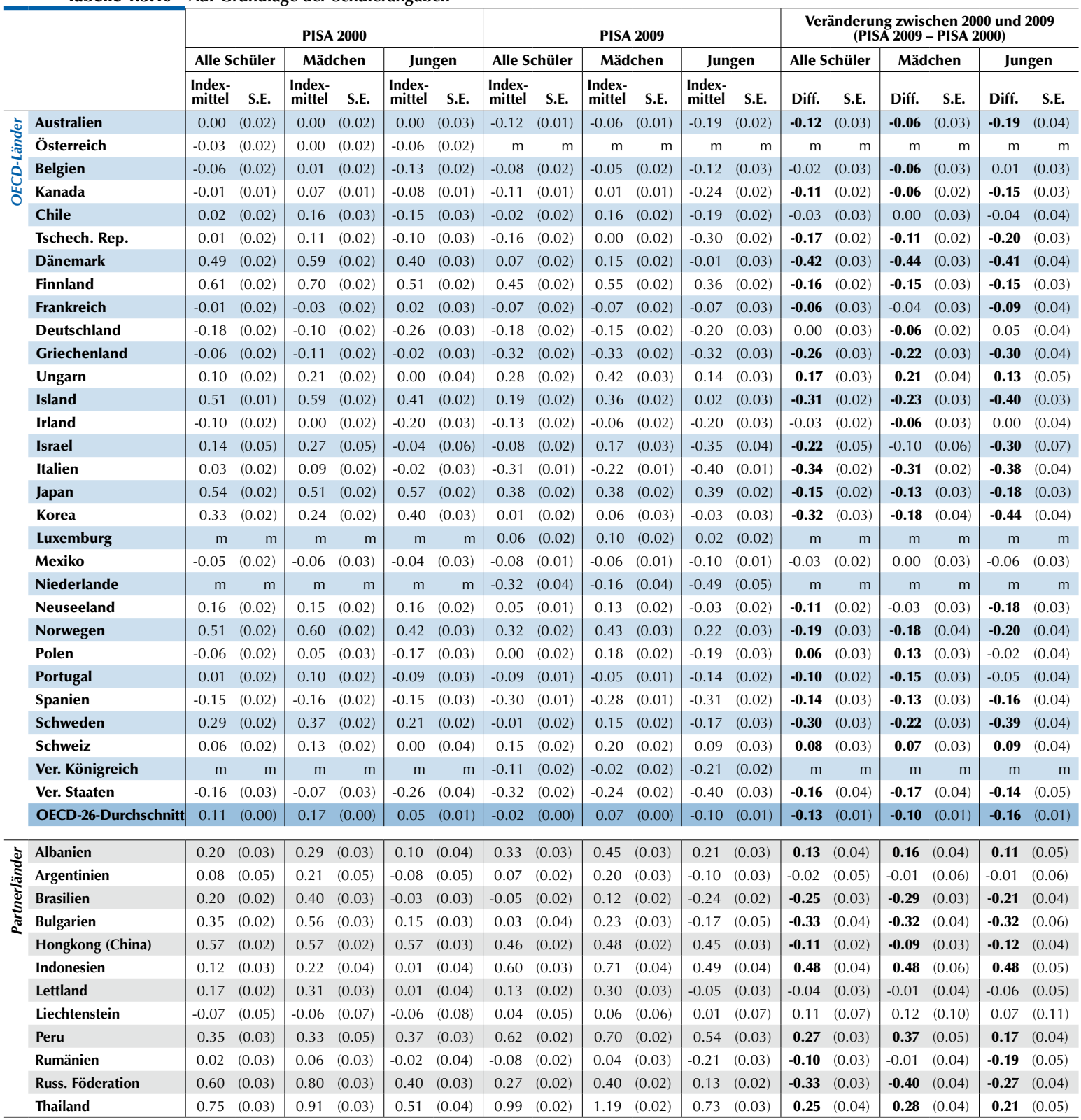

Anmerkung: Statistisch signifikante Werte sind durch Fettdruck gekennzeichnet (siehe Anhang A3).

StatLink inst http://dx.doi.org/10.1787/888932343285 
[Teil 1/1]

Lehrer-Schüler-Beziehungen in PISA 2000 und PISA 2009

Tabelle V.5.11 Prozentsatz der Schüler, die folgenden Aussagen „eher" oder "ganz" zustimmten

\begin{tabular}{|c|c|c|c|c|c|c|c|c|c|c|c|c|c|c|c|c|c|c|c|}
\hline & \multicolumn{6}{|c|}{ PISA 2000} & \multicolumn{6}{|c|}{ PISA 2009} & \multicolumn{6}{|c|}{$\begin{array}{c}\text { Veränderung zwischen } 2000 \text { und } 2009 \\
\text { (PISA 2009 - PISA 2000) }\end{array}$} \\
\hline & & \multicolumn{2}{|c|}{$\begin{array}{c}\text { Die meisten } \\
\text { meiner Lehrer } \\
\text { interessieren } \\
\text { sich für das, } \\
\text { was ich zu } \\
\text { sagen habe }\end{array}$} & \multicolumn{2}{|c|}{$\begin{array}{c}\text { Wenn ich } \\
\text { zusätzlich } \\
\text { Hilfe brauche, } \\
\text { bekomme } \\
\text { ich sie von } \\
\text { meinen } \\
\text { Lehrern }\end{array}$} & \multicolumn{2}{|c|}{$\begin{array}{c}\text { Die meisten } \\
\text { meiner Lehrer } \\
\text { behandeln } \\
\text { mich fair }\end{array}$} & \multicolumn{2}{|c|}{$\begin{array}{c}\text { Die meisten } \\
\text { meiner Lehrer } \\
\text { interessieren } \\
\text { sich für das, } \\
\text { was ich zu } \\
\text { sagen habe }\end{array}$} & \multicolumn{2}{|c|}{\begin{tabular}{|c|} 
Wenn ich \\
zusätzlich \\
Hilfe brauche, \\
bekomme \\
ich sie von \\
meinen \\
Lehrern
\end{tabular}} & \multicolumn{2}{|c|}{$\begin{array}{c}\text { Die meisten } \\
\text { meiner Lehrer } \\
\text { behandeln } \\
\text { mich fair }\end{array}$} & \multicolumn{2}{|c|}{$\begin{array}{c}\text { Die meisten } \\
\text { meiner Lehrer } \\
\text { interessieren } \\
\text { sich für das, } \\
\text { was ich zu } \\
\text { sagen habe }\end{array}$} & \multicolumn{2}{|c|}{$\begin{array}{c}\text { Wenn ich } \\
\text { zusätzlich } \\
\text { Hilfe brauche, } \\
\text { bekomme } \\
\text { ich sie von } \\
\text { meinen } \\
\text { Lehrern }\end{array}$} & \multicolumn{2}{|c|}{$\begin{array}{c}\text { Die meisten } \\
\text { meiner Lehrer } \\
\text { behandeln } \\
\text { mich fair }\end{array}$} \\
\hline & & $\%$ & S.E. & $\%$ & S.E. & $\%$ & S.E. & $\%$ & S.E. & $\%$ & S.E. & $\%$ & S.E. & Diff. \% & S.E. & Diff. \% & S.E. & Diff. \% & S.E. \\
\hline \multirow{31}{*}{ 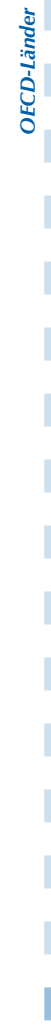 } & Australien & 71.7 & $(1.0)$ & 84.3 & $(0.8)$ & 82.7 & $(0.8)$ & 71.4 & $(0.5)$ & 84.2 & $(0.4)$ & 84.6 & $(0.4)$ & -0.4 & (1.1) & 0.0 & $(0.9)$ & 1.9 & $(0.8)$ \\
\hline & Österreich & 55.5 & $(1.0)$ & 59.6 & (1.1) & 75.0 & $(0.8)$ & $\mathrm{m}$ & $\mathrm{m}$ & $\mathrm{m}$ & $\mathrm{m}$ & $\mathrm{m}$ & $\mathrm{m}$ & $\mathrm{m}$ & $\mathrm{m}$ & $\mathrm{m}$ & $\mathrm{m}$ & $\mathrm{m}$ & $\mathrm{m}$ \\
\hline & Belgien & 68.3 & $(0.8)$ & 77.7 & $(0.7)$ & 81.8 & $(0.7)$ & 67.3 & $(0.7)$ & 84.1 & $(0.5)$ & 85.8 & $(0.5)$ & -1.0 & $(1.0)$ & 6.4 & $(0.9)$ & 4.0 & $(0.9)$ \\
\hline & Kanada & 70.5 & $(0.5)$ & 87.4 & $(0.3)$ & 80.8 & $(0.4)$ & 74.0 & $(0.4)$ & 89.5 & $(0.3)$ & 88.0 & $(0.3)$ & 3.5 & $(0.6)$ & 2.1 & $(0.4)$ & 7.2 & $(0.5)$ \\
\hline & Chile & 66.0 & (1.1) & 70.1 & $(0.9)$ & 63.7 & $(1.0)$ & 72.4 & $(0.8)$ & 77.4 & $(0.8)$ & 70.9 & (0.9) & 6.5 & (1.4) & 7.3 & $(1.2)$ & 7.2 & (1.3) \\
\hline & Tschech. Rep. & 56.6 & (1.1) & 72.4 & (1.1) & 68.3 & $(1.0)$ & 56.9 & $(0.9)$ & 78.3 & $(0.7)$ & 72.2 & $(0.7)$ & 0.3 & (1.4) & 5.9 & (1.3) & 3.9 & $(1.2)$ \\
\hline & Dänemark & 71.7 & $(0.9)$ & 78.7 & $(0.8)$ & 87.5 & $(0.7)$ & 71.1 & $(0.8)$ & 79.2 & $(0.8)$ & 85.2 & $(0.6)$ & -0.6 & (1.2) & 0.5 & (1.1) & -2.3 & $(0.9)$ \\
\hline & Finnland & 64.7 & $(0.9)$ & 82.5 & $(0.7)$ & 75.2 & $(0.8)$ & 62.7 & $(0.8)$ & 84.2 & $(0.7)$ & 79.8 & $(0.7)$ & -2.0 & $(1.2)$ & 1.7 & $(1.0)$ & 4.6 & $(1.0)$ \\
\hline & Frankreich & 61.7 & (1.0) & 75.9 & $(0.7)$ & 72.7 & $(0.7)$ & 61.7 & (1.0) & 79.8 & $(0.7)$ & 88.3 & $(0.7)$ & 0.0 & (1.5) & 3.9 & (1.0) & 15.6 & (1.0) \\
\hline & Deutschland & 50.9 & (1.0) & 58.6 & (1.0) & 72.7 & $(0.8)$ & 68.8 & $(0.8)$ & 70.5 & (0.9) & 77.4 & $(0.8)$ & 17.9 & (1.3) & 11.9 & (1.4) & 4.7 & (1.1) \\
\hline & Griechenland & 64.8 & $(1.0)$ & 61.5 & $(1.2)$ & 64.7 & (1.0) & 62.2 & $(0.8)$ & 63.0 & $(0.9)$ & 65.0 & $(1.0)$ & -2.6 & $(1.2)$ & 1.6 & (1.5) & 0.2 & (1.4) \\
\hline & Ungarn & 79.1 & $(0.7)$ & 73.3 & $(0.9)$ & 68.6 & (0.9) & 79.1 & $(0.9)$ & 77.4 & $(0.8)$ & 74.1 & (0.9) & 0.0 & (1.2) & 4.1 & $(1.2)$ & 5.6 & (1.2) \\
\hline & Island & 62.6 & $(0.7)$ & 73.4 & $(0.8)$ & 71.9 & $(0.8)$ & 73.9 & $(0.8)$ & 82.0 & $(0.7)$ & 80.3 & $(0.8)$ & 11.3 & (1.1) & 8.6 & (1.0) & 8.4 & (1.1) \\
\hline & Irland & 57.3 & $(1.0)$ & 73.2 & $(0.8)$ & 77.9 & $(0.8)$ & 62.8 & $(1.0)$ & 77.4 & $(0.9)$ & 81.1 & $(0.8)$ & 5.5 & (1.4) & 4.2 & $(1.2)$ & 3.1 & (1.1) \\
\hline & Israel & 62.2 & (1.4) & 71.4 & (1.8) & 72.2 & $(1.2)$ & 68.0 & $(0.9)$ & 70.2 & $(0.9)$ & 79.6 & $(0.6)$ & 5.8 & (1.7) & -1.2 & (2.0) & 7.5 & (1.4) \\
\hline & Italien & 70.9 & $(1.2)$ & 73.1 & (1.0) & 63.6 & (0.9) & 62.3 & $(0.5)$ & 76.7 & $(0.4)$ & 79.1 & $(0.4)$ & -8.5 & (1.3) & 3.6 & (1.1) & 15.5 & (1.0) \\
\hline & Japan & 49.8 & $(1.2)$ & 64.2 & $(1.2)$ & 69.3 & (1.1) & 63.0 & $(0.8)$ & 63.5 & $(0.7)$ & 74.4 & $(0.8)$ & 13.2 & (1.4) & -0.7 & (1.4) & 5.0 & (1.3) \\
\hline & Korea & 40.7 & (0.9) & 76.4 & (0.9) & 66.4 & (0.9) & 57.2 & (0.9) & 83.3 & $(0.7)$ & 75.3 & $(0.7)$ & 16.4 & (1.3) & 6.9 & (1.1) & 8.8 & (1.1) \\
\hline & Luxemburg & $\mathrm{m}$ & $\mathrm{m}$ & $\mathrm{m}$ & $\mathrm{m}$ & $\mathrm{m}$ & $\mathrm{m}$ & 63.2 & (0.8) & 72.2 & $(0.7)$ & 78.0 & $(0.7)$ & $\mathrm{m}$ & $\mathrm{m}$ & $\mathrm{m}$ & $\mathrm{m}$ & $\mathrm{m}$ & $\mathrm{m}$ \\
\hline & Mexiko & 85.1 & (0.6) & 78.8 & $(0.7)$ & 65.3 & $(0.8)$ & 76.9 & $(0.4)$ & 78.4 & $(0.4)$ & 74.7 & $(0.4)$ & -8.2 & $(0.7)$ & -0.4 & $(0.8)$ & 9.3 & $(0.9)$ \\
\hline & Niederlande & $\mathrm{m}$ & $\mathrm{m}$ & $\mathrm{m}$ & $\mathrm{m}$ & $\mathrm{m}$ & $\mathrm{m}$ & 65.9 & $(0.9)$ & 85.0 & $(0.8)$ & 85.1 & $(0.8)$ & $\mathrm{m}$ & $\mathrm{m}$ & $\mathrm{m}$ & $\mathrm{m}$ & $\mathrm{m}$ & $\mathrm{m}$ \\
\hline & Neuseeland & 67.7 & $(0.9)$ & 82.2 & $(0.7)$ & 82.2 & $(0.8)$ & 72.6 & $(0.7)$ & 87.4 & $(0.6)$ & 86.3 & $(0.5)$ & 4.9 & $(1.1)$ & 5.3 & $(0.9)$ & 4.1 & (1.0) \\
\hline & Norwegen & 55.8 & (1.3) & 71.7 & $(0.9)$ & 69.8 & $(1.0)$ & 54.9 & $(0.9)$ & 74.2 & $(0.9)$ & 73.6 & $(0.7)$ & -0.9 & (1.6) & 2.5 & (1.3) & 3.8 & $(1.2)$ \\
\hline & Polen & 64.5 & (1.0) & 56.7 & (1.0) & 57.0 & $(1.2)$ & 59.5 & $(0.8)$ & 73.4 & $(0.8)$ & 70.6 & $(0.8)$ & -4.9 & (1.3) & 16.7 & (1.3) & 13.6 & (1.5) \\
\hline & Portugal & 75.4 & $(0.8)$ & 76.9 & $(0.8)$ & 83.8 & $(0.6)$ & 81.9 & $(0.6)$ & 89.8 & $(0.6)$ & 81.5 & $(0.6)$ & 6.5 & $(1.0)$ & 12.9 & $(1.0)$ & -2.3 & (0.9) \\
\hline & Spanien & 63.4 & $(1.0)$ & 67.5 & $(1.0)$ & 83.9 & $(0.6)$ & 67.5 & $(0.6)$ & 68.5 & (0.6) & 79.3 & $(0.5)$ & 4.1 & $(1.2)$ & 1.0 & (1.2) & -4.6 & $(0.8)$ \\
\hline & Schweden & 68.3 & (1.1) & 78.1 & $(0.9)$ & 80.2 & $(0.8)$ & 71.5 & $(0.9)$ & 81.8 & $(0.9)$ & 82.4 & $(0.6)$ & 3.2 & (1.4) & 3.7 & (1.3) & 2.2 & $(1.0)$ \\
\hline & Schweiz & 67.0 & (1.1) & 77.7 & $(0.9)$ & 78.7 & $(0.8)$ & 70.4 & $(0.7)$ & 82.3 & $(0.7)$ & 83.0 & $(0.6)$ & 3.4 & (1.3) & 4.5 & $(1.2)$ & 4.3 & $(1.0)$ \\
\hline & Ver. Königreich & $\mathrm{m}$ & $\mathrm{m}$ & $\mathrm{m}$ & $\mathrm{m}$ & $\mathrm{m}$ & $\mathrm{m}$ & 69.3 & $(0.8)$ & 88.5 & $(0.6)$ & 83.4 & $(0.7)$ & $\mathrm{m}$ & $\mathrm{m}$ & $\mathrm{m}$ & $\mathrm{m}$ & $\mathrm{m}$ & $\mathrm{m}$ \\
\hline & Ver. Staaten & 70.8 & $(1.1)$ & 82.2 & $(0.9)$ & 82.2 & $(1.1)$ & 73.6 & $(1.0)$ & 88.4 & $(0.5)$ & 88.6 & $(0.4)$ & 2.8 & (1.5) & 6.2 & $(1.0)$ & 6.4 & $(1.2)$ \\
\hline & OECD-26-Durchschnitt & 64.9 & $(0.2)$ & 74.1 & $(0.2)$ & 74.0 & $(0.2)$ & 67.8 & $(0.2)$ & 78.7 & $(0.1)$ & 79.3 & $(0.1)$ & 2.9 & $(0.2)$ & 4.6 & $(0.2)$ & 5.3 & $(0.2)$ \\
\hline \multirow{12}{*}{ } & Albanien & 73.7 & $(0.9)$ & 76.1 & $(1.0)$ & 85.0 & $(0.9)$ & 89.5 & $(0.5)$ & 91.9 & $(0.5)$ & 94.0 & $(0.4)$ & 15.8 & (1.1) & 15.9 & $(1.1)$ & 8.9 & $(0.9)$ \\
\hline & Argentinien & 65.3 & (1.4) & 69.4 & (1.7) & 73.7 & (1.6) & 73.0 & $(1.0)$ & 67.7 & $(1.2)$ & 79.5 & $(0.8)$ & 7.7 & $(1.7)$ & -1.7 & $(2.1)$ & 5.8 & $(1.7)$ \\
\hline & Brasilien & 74.1 & $(1.0)$ & 88.0 & $(0.7)$ & 85.6 & $(0.7)$ & 74.4 & $(0.8)$ & 77.8 & $(0.7)$ & 83.0 & $(0.6)$ & 0.3 & $(1.2)$ & -10.2 & $(1.0)$ & -2.6 & $(0.9)$ \\
\hline & Bulgarien & 74.4 & $(0.8)$ & 75.8 & $(1.0)$ & 71.8 & $(0.9)$ & 70.9 & $(1.1)$ & 79.6 & $(0.7)$ & 73.3 & $(1.1)$ & -3.5 & (1.4) & 3.8 & $(1.2)$ & 1.5 & (1.4) \\
\hline & Hongkong (China) & 66.7 & $(0.9)$ & 85.4 & $(0.6)$ & 69.4 & $(0.8)$ & 66.8 & $(0.9)$ & 88.7 & $(0.5)$ & 81.8 & $(0.6)$ & 0.1 & (1.3) & 3.3 & $(0.7)$ & 12.3 & $(1.0)$ \\
\hline & Indonesien & 67.6 & (1.1) & 78.8 & $(0.7)$ & 91.0 & $(0.6)$ & 63.2 & $(0.9)$ & 84.8 & $(0.7)$ & 91.0 & $(0.4)$ & -4.4 & (1.4) & 6.0 & $(1.0)$ & -0.1 & $(0.7)$ \\
\hline & Lettland & 62.1 & (1.5) & 72.0 & $(1.2)$ & 73.6 & $(1.2)$ & 69.3 & $(0.9)$ & 85.3 & $(0.7)$ & 81.8 & $(0.8)$ & 7.3 & $(1.7)$ & 13.4 & (1.4) & 8.2 & (1.5) \\
\hline & Liechtenstein & 61.3 & (3.0) & 74.6 & (2.4) & 81.1 & $(2.2)$ & 66.4 & (2.6) & 77.7 & (2.4) & 74.6 & $(2.2)$ & 5.1 & $(4.0)$ & 3.1 & (3.4) & -6.5 & (3.1) \\
\hline & Peru & 78.6 & $(1.0)$ & 78.1 & $(0.7)$ & 79.8 & (0.9) & 82.1 & $(0.7)$ & 84.7 & $(0.6)$ & 82.7 & $(0.7)$ & 3.5 & $(1.2)$ & 6.7 & (0.9) & 2.9 & (1.1) \\
\hline & Rumänien & 71.3 & (1.1) & 72.4 & (1.0) & 79.4 & $(0.9)$ & 76.8 & (0.9) & 74.0 & $(0.9)$ & 83.6 & $(0.7)$ & 5.5 & (1.4) & 1.6 & (1.3) & 4.2 & (1.1) \\
\hline & Russ. Föderation & 69.6 & $(0.8)$ & 77.5 & $(1.0)$ & 73.9 & (1.0) & 72.6 & $(0.7)$ & 82.1 & $(0.7)$ & 80.2 & $(0.8)$ & 2.9 & $(1.0)$ & 4.6 & $(1.2)$ & 6.3 & (1.3) \\
\hline & Thailand & 78.6 & $(0.7)$ & 84.2 & $(0.5)$ & 85.6 & $(0.9)$ & 82.1 & $(0.6)$ & 83.0 & $(0.5)$ & 87.4 & $(0.5)$ & 3.5 & (1.0) & -1.2 & $(0.7)$ & 1.8 & $(1.0)$ \\
\hline
\end{tabular}

Anmerkung: Statistisch signifikante Werte sind durch Fettdruck gekennzeichnet (siehe Anhang A3).

StatLink ints http://dx.doi.org/10.1787/888932343285 
[Teil 1/2]

Schuldisziplin in PISA 2000 und PISA 2009

Tabelle V.5.12 Prozentsatz der Schüler, die angaben, dass Folgendes „,nie" oder "nur in einigen Stunden" vorkommt

\begin{tabular}{|c|c|c|c|c|c|c|c|c|c|c|c|c|c|c|c|c|c|c|c|c|c|}
\hline & \multicolumn{10}{|c|}{ PISA 2000} & \multicolumn{10}{|c|}{ PISA 2009} \\
\hline & & \multicolumn{2}{|c|}{$\begin{array}{c}\text { Wir hören } \\
\text { nicht auf } \\
\text { das, was der } \\
\text { Lehrer sagt }\end{array}$} & \multicolumn{2}{|c|}{$\begin{array}{c}\text { Es ist laut } \\
\text { und alles } \\
\text { geht durch- } \\
\text { einander }\end{array}$} & \multicolumn{2}{|c|}{\begin{tabular}{|c|} 
Lehrer \\
muss lange \\
warten, bis \\
Ruhe eintritt
\end{tabular}} & \multicolumn{2}{|c|}{$\begin{array}{c}\text { Wir können } \\
\text { nicht } \\
\text { ungestört } \\
\text { arbeiten }\end{array}$} & \multicolumn{2}{|c|}{$\begin{array}{c}\text { Wir fangen } \\
\text { erst lange } \\
\text { nach dem } \\
\text { Beginn der } \\
\text { Stunde an } \\
\text { zu arbeiten }\end{array}$} & \multicolumn{2}{|c|}{$\begin{array}{c}\text { Wir hören } \\
\text { nicht auf } \\
\text { das, was der } \\
\text { Lehrer sagt }\end{array}$} & \multicolumn{2}{|c|}{$\begin{array}{c}\text { Es ist laut } \\
\text { und alles } \\
\text { geht durch- } \\
\text { einander }\end{array}$} & \multicolumn{2}{|c|}{\begin{tabular}{|c|} 
Lehrer \\
muss lange \\
warten, bis \\
Ruhe eintritt
\end{tabular}} & \multicolumn{2}{|c|}{$\begin{array}{c}\text { Wir können } \\
\text { nicht } \\
\text { ungestört } \\
\text { arbeiten }\end{array}$} & \multicolumn{2}{|c|}{$\begin{array}{l}\text { Wir fangen } \\
\text { erst lange } \\
\text { nach dem } \\
\text { Beginn der } \\
\text { Stunde an } \\
\text { zu arbeiten }\end{array}$} \\
\hline & & $\%$ & S.E. & $\%$ & S.E. & $\%$ & S.E. & $\%$ & S.E. & $\%$ & S.E. & $\%$ & S.E. & $\%$ & S.E. & $\%$ & S.E. & $\%$ & S.E. & $\%$ & S.E. \\
\hline \multirow{5}{*}{ 离 } & Australien & 78.7 & (0.9) & 67.7 & (1.1) & 68.6 & (1.0) & 81.6 & (1.0) & 74.1 & (0.9) & 67.7 & $(0.7)$ & 60.8 & $(0.7)$ & 70.9 & $(0.7)$ & 82.0 & (0.5) & 76.4 & (0.6) \\
\hline & Österreich & 78.1 & (0.8) & 81.1 & (1.0) & 68.5 & (1.2) & 78.8 & (0.9) & 70.8 & (1.1) & $\mathrm{m}$ & $\mathrm{m}$ & $\mathrm{m}$ & $\mathrm{m}$ & $\mathrm{m}$ & $\mathrm{m}$ & $\mathrm{m}$ & $\mathrm{m}$ & $\mathrm{m}$ & $\mathrm{m}$ \\
\hline & Belgien & 75.9 & (0.9) & 62.9 & (1.0) & 64.6 & (1.3) & 85.0 & $(0.7)$ & 68.8 & (0.9) & 71.7 & (0.9) & 62.9 & (1.0) & 67.7 & (0.9) & 84.6 & (0.6) & 71.3 & $(0.8)$ \\
\hline & Kanada & 77.1 & (0.4) & 66.1 & (0.5) & 64.8 & (0.6) & 83.3 & $(0.3)$ & 70.4 & $(0.5)$ & 70.6 & (0.6) & 61.1 & (0.6) & 72.3 & (0.6) & 82.2 & $(0.5)$ & 72.7 & (0.6) \\
\hline & Chile & 73.0 & (0.8) & 51.0 & (1.2) & 59.4 & (1.0) & 77.1 & (0.8) & 66.3 & (0.8) & 73.6 & (0.8) & 62.9 & (1.0) & 65.0 & (1.1) & 81.5 & $(0.7)$ & 70.1 & (0.9) \\
\hline & Tschech. Rep. & 73.9 & (1.0) & 74.0 & $(1.2)$ & 67.7 & (1.4) & 82.7 & $(0.7)$ & 78.8 & $(0.8)$ & 63.2 & (1.4) & 66.0 & (1.4) & 67.6 & (1.2) & 75.3 & (1.1) & 70.2 & $(1.2)$ \\
\hline & Dänemark & 80.3 & $(0.9)$ & 65.6 & (1.2) & 72.3 & (1.2) & 82.8 & (0.8) & 77.2 & (1.0) & 71.7 & $(0.9)$ & 65.2 & (1.1) & 78.1 & (1.0) & 88.1 & $(0.7)$ & 81.9 & $(0.9)$ \\
\hline & Finnland & 70.0 & $(0.9)$ & 56.9 & (1.1) & 60.5 & $(1.2)$ & 84.8 & (0.6) & 78.2 & (0.8) & 60.4 & (1.0) & 52.1 & (1.1) & 63.1 & (1.0) & 79.9 & $(0.8)$ & 67.9 & (1.0) \\
\hline & Frankreich & 72.1 & (0.9) & 56.8 & (1.3) & 64.4 & (1.1) & 84.8 & (0.6) & 62.6 & (0.9) & 64.3 & (1.1) & 56.2 & (1.3) & 64.2 & (1.3) & 76.3 & (1.1) & 62.5 & (1.1) \\
\hline & Deutschland & 75.9 & $(0.8)$ & 77.5 & (1.1) & 63.8 & (1.1) & 76.3 & $(0.7)$ & 72.4 & $(0.8)$ & 84.6 & $(0.7)$ & 83.5 & $(0.8)$ & 77.5 & (0.9) & 82.2 & $(0.8)$ & 81.0 & $(0.7)$ \\
\hline & Griechenland & 70.3 & (1.0) & 53.1 & (1.2) & 56.8 & $(1.2)$ & 59.9 & (1.0) & 65.2 & (0.8) & 55.2 & (1.2) & 58.4 & (1.4) & 62.5 & (1.2) & 56.3 & (1.0) & 65.4 & $(0.7)$ \\
\hline & Ungarn & 77.5 & (1.1) & 76.4 & (1.2) & 65.7 & (1.5) & 74.3 & (1.1) & 83.3 & (0.9) & 70.5 & (1.4) & 70.9 & (1.3) & 68.9 & (1.3) & 79.5 & (1.1) & 78.1 & (1.0) \\
\hline & Island & 80.0 & $(0.7)$ & 71.5 & $(0.7)$ & 66.2 & $(0.7)$ & 83.6 & $(0.7)$ & 80.0 & $(0.7)$ & 74.1 & $(0.6)$ & 66.9 & $(0.7)$ & 73.1 & $(0.7)$ & 83.6 & (0.6) & 80.8 & (0.6) \\
\hline & Irland & 74.9 & (0.9) & 73.7 & (1.0) & 70.8 & $(1.2)$ & 83.4 & (0.9) & 74.8 & $(0.9)$ & 63.7 & (1.1) & 64.6 & $(1.2)$ & 69.9 & (1.2) & 80.8 & $(0.9)$ & 75.1 & (1.0) \\
\hline & Israel & 70.6 & (1.9) & 70.9 & (2.0) & 60.0 & (2.3) & 68.8 & (1.2) & 68.4 & (2.1) & 77.8 & $(0.8)$ & 75.2 & (0.9) & 72.6 & (0.9) & 77.3 & $(0.8)$ & 74.3 & $(0.8)$ \\
\hline & Italien & 64.5 & (1.1) & 53.9 & (1.2) & 51.4 & (1.3) & 77.8 & (0.8) & 70.7 & (0.9) & 66.0 & $(0.5)$ & 68.0 & $(0.7)$ & 69.8 & (0.6) & 81.2 & $(0.5)$ & 73.7 & $(0.6)$ \\
\hline & Japan & 82.6 & (1.2) & 82.3 & (1.6) & 90.5 & (0.9) & 78.6 & (1.1) & 82.1 & (1.2) & 91.6 & $(0.5)$ & 90.3 & $(0.7)$ & 92.8 & $(0.5)$ & 87.0 & $(0.5)$ & 91.4 & $(0.6)$ \\
\hline & Korea & 67.9 & (1.1) & 70.6 & $(1.2)$ & 82.5 & $(0.9)$ & 78.7 & $(0.9)$ & 77.1 & (0.9) & 89.9 & $(0.7)$ & 77.2 & $(0.8)$ & 87.8 & (0.6) & 90.3 & $(0.7)$ & 87.4 & $(0.8)$ \\
\hline & Luxemburg & $\mathrm{m}$ & $\mathrm{m}$ & $\mathrm{m}$ & $\mathrm{m}$ & $\mathrm{m}$ & $\mathrm{m}$ & $\mathrm{m}$ & $\mathrm{m}$ & $\mathrm{m}$ & $\mathrm{m}$ & 59.6 & (0.8) & 65.3 & $(0.7)$ & 64.2 & (0.6) & 71.4 & $(0.7)$ & 64.2 & $(0.6)$ \\
\hline & Mexiko & 80.4 & $(0.8)$ & 74.9 & (1.0) & 71.0 & (1.1) & 82.4 & $(0.7)$ & 80.5 & $(0.8)$ & 79.1 & $(0.4)$ & 73.3 & $(0.5)$ & 79.1 & $(0.5)$ & 83.2 & $(0.4)$ & 76.5 & $(0.4)$ \\
\hline & Niederlande & $\mathrm{m}$ & $\mathrm{m}$ & $\mathrm{m}$ & $\mathrm{m}$ & $\mathrm{m}$ & $\mathrm{m}$ & $\mathrm{m}$ & $\mathrm{m}$ & $\mathrm{m}$ & $\mathrm{m}$ & 68.1 & (0.9) & 58.9 & (1.1) & 63.2 & (1.0) & 80.5 & (1.0) & 55.5 & (1.1) \\
\hline & Neuseeland & 76.4 & $(0.9)$ & 67.5 & (1.0) & 66.5 & (1.0) & 77.8 & $(0.8)$ & 73.6 & (0.9) & 67.6 & $(0.8)$ & 61.3 & (0.8) & 68.3 & $(0.9)$ & 82.1 & $(0.7)$ & 74.1 & $(0.8)$ \\
\hline & Norwegen & 72.4 & (1.0) & 59.8 & (1.3) & 57.8 & (1.6) & 76.7 & (0.9) & 66.5 & (1.2) & 66.8 & (0.8) & 61.4 & (1.1) & 65.5 & (0.9) & 76.6 & $(0.9)$ & 66.7 & $(1.0)$ \\
\hline & Polen & 79.8 & (1.0) & 81.0 & (1.2) & 73.3 & (1.4) & 86.1 & $(0.8)$ & 79.8 & (1.1) & 67.4 & (1.0) & 74.1 & (1.0) & 73.7 & (1.1) & 79.4 & $(0.8)$ & 79.5 & $(0.9)$ \\
\hline & Portugal & 79.4 & $(0.7)$ & 75.6 & (0.9) & 74.8 & (0.9) & 80.1 & $(0.8)$ & 75.1 & (0.8) & 78.3 & $(0.8)$ & 75.5 & (0.9) & 79.6 & (0.9) & 86.5 & (0.6) & 79.4 & (0.9) \\
\hline & Spanien & 75.0 & $(0.9)$ & 65.6 & (1.3) & 59.2 & (1.5) & 81.4 & $(0.8)$ & 64.6 & (1.0) & 73.5 & $(0.7)$ & 74.1 & $(0.7)$ & 72.5 & (0.6) & 83.1 & $(0.5)$ & 72.7 & $(0.7)$ \\
\hline & Schweden & 70.8 & $(0.9)$ & 61.0 & (1.3) & 56.6 & (1.3) & 77.1 & (0.9) & 68.5 & (1.1) & 75.1 & (1.0) & 67.2 & (1.1) & 71.1 & (1.1) & 82.7 & $(0.7)$ & 76.5 & (1.0) \\
\hline & Schweiz & 81.6 & $(0.8)$ & 81.5 & (0.9) & 72.3 & (1.1) & 81.3 & $(0.7)$ & 76.8 & (1.0) & 72.4 & (0.9) & 73.8 & (1.0) & 74.2 & $(0.9)$ & 81.0 & (0.6) & 75.8 & $(0.7)$ \\
\hline & Ver. Königreich & $\mathrm{m}$ & $\mathrm{m}$ & $\mathrm{m}$ & $\mathrm{m}$ & $\mathrm{m}$ & $\mathrm{m}$ & $\mathrm{m}$ & $\mathrm{m}$ & $\mathrm{m}$ & $\mathrm{m}$ & 73.0 & $(0.9)$ & 68.4 & (1.0) & 73.8 & $(0.9)$ & 85.6 & $(0.7)$ & 81.2 & $(0.8)$ \\
\hline & Ver. Staaten & 73.8 & (1.1) & 70.2 & (1.3) & 72.5 & (1.3) & 81.5 & (1.0) & 74.9 & (1.0) & 75.5 & $(0.8)$ & 72.0 & $(0.8)$ & 78.9 & $(0.8)$ & 87.1 & $(0.6)$ & 81.6 & $(0.8)$ \\
\hline & OECD-26-Durchschnitt & 75.2 & $(0.2)$ & 68.0 & $(0.2)$ & 66.7 & $(0.2)$ & 79.5 & $(0.2)$ & 73.5 & $(0.2)$ & 72.0 & $(0.2)$ & 68.3 & $(0.2)$ & 72.6 & $(0.2)$ & 81.2 & $(0.1)$ & 75.5 & $(0.2)$ \\
\hline
\end{tabular}

\begin{tabular}{|c|c|c|c|c|c|c|c|c|c|c|c|c|c|c|c|c|c|c|c|c|c|}
\hline \multirow{12}{*}{ 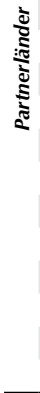 } & Albanien & 89.6 & $(0.6)$ & 92.4 & $(0.4)$ & 87.6 & $(0.7)$ & 84.8 & $(0.8)$ & 85.0 & $(0.7)$ & 88.7 & $(1.0)$ & 88.0 & $(0.8)$ & 86.1 & $(0.9)$ & 86.7 & $(0.8)$ & 88.2 & $(0.7)$ \\
\hline & Argentinien & 66.0 & (2.8) & 51.5 & $(2.7)$ & 55.5 & (3.5) & 75.0 & $(2.0)$ & 61.6 & (1.0) & 66.9 & (1.3) & 57.0 & (1.3) & 61.9 & $(1.2)$ & 73.9 & (1.1) & 65.5 & (1.1) \\
\hline & Brasilien & 70.5 & $(0.9)$ & 58.2 & (1.1) & 63.2 & (1.4) & 75.2 & $(0.8)$ & 60.3 & $(1.2)$ & 75.3 & $(0.7)$ & 59.8 & $(0.9)$ & 66.6 & $(0.7)$ & 75.9 & $(0.8)$ & 62.9 & (0.6) \\
\hline & Bulgarien & 70.3 & (1.0) & 74.9 & (1.2) & 65.6 & (1.4) & 79.8 & (0.9) & 74.1 & (1.1) & 69.0 & (1.2) & 72.2 & (1.3) & 73.4 & (1.5) & 75.5 & $(1.2)$ & 76.5 & (1.3) \\
\hline & Hongkong (China) & 72.2 & $(1.0)$ & 78.9 & $(0.9)$ & 79.0 & $(0.8)$ & 71.0 & $(0.9)$ & 66.0 & $(0.9)$ & 87.2 & $(0.6)$ & 87.9 & (0.6) & 89.4 & $(0.5)$ & 88.0 & $(0.6)$ & 86.2 & $(0.7)$ \\
\hline & Indonesien & 84.3 & $(0.9)$ & 65.6 & $(1.2)$ & 48.7 & (1.1) & 85.7 & $(0.8)$ & 79.5 & $(0.9)$ & 84.3 & $(0.8)$ & 74.8 & $(0.9)$ & 79.2 & $(0.9)$ & 84.0 & $(0.8)$ & 84.1 & $(0.8)$ \\
\hline & Lettland & 81.0 & $(1.0)$ & 83.1 & (1.0) & 80.6 & (1.1) & 83.0 & $(0.9)$ & 83.7 & (1.0) & 77.8 & $(1.0)$ & 78.1 & (1.2) & 79.1 & (1.1) & 85.6 & $(0.8)$ & 86.1 & (0.8) \\
\hline & Liechtenstein & 85.1 & $(2.0)$ & 89.9 & (1.8) & 74.6 & (1.9) & 78.9 & (2.3) & 84.8 & (1.9) & 70.8 & (2.6) & 81.1 & $(2.0)$ & 75.8 & (2.5) & 79.4 & $(2.2)$ & 80.2 & (2.2) \\
\hline & Peru & 74.0 & $(0.9)$ & 66.0 & (1.1) & 77.1 & (1.0) & 71.7 & $(0.9)$ & 66.8 & (1.1) & 83.2 & $(0.7)$ & 77.2 & $(0.8)$ & 84.6 & $(0.7)$ & 85.4 & $(0.6)$ & 81.8 & $(0.8)$ \\
\hline & Rumänien & 80.6 & (1.0) & 83.9 & $(0.9)$ & 80.3 & (1.1) & 79.1 & (1.0) & 79.0 & (1.0) & 88.6 & $(0.7)$ & 89.3 & $(0.7)$ & 88.5 & $(0.7)$ & 88.5 & $(0.7)$ & 86.6 & (0.6) \\
\hline & Russ. Föderation & 83.7 & $(0.6)$ & 87.2 & $(0.5)$ & 80.8 & $(0.9)$ & 83.0 & $(0.7)$ & 86.3 & (0.8) & 81.0 & $(0.7)$ & 86.0 & $(0.7)$ & 85.3 & $(0.7)$ & 84.8 & $(0.7)$ & 88.6 & (0.6) \\
\hline & Thailand & 87.2 & $(0.6)$ & 68.5 & $(0.9)$ & 80.5 & $(0.9)$ & 85.1 & $(0.8)$ & 89.2 & $(0.8)$ & 90.5 & $(0.5)$ & 85.3 & $(0.5)$ & 86.4 & $(0.6)$ & 91.3 & $(0.5)$ & 91.4 & $(0.5)$ \\
\hline
\end{tabular}

Anmerkung: Statistisch signifikante Werte sind durch Fettdruck gekennzeichnet (siehe Anhang A3).

StatLink ज्ञाज http://dx.doi.org/10.1787/888932343285 
[Teil 2/2]

Schuldisziplin in PISA 2000 und PISA 2009

Tabelle V.5.12 Prozentsatz der Schüler, die angaben, dass Folgendes "nie" oder "nur in einigen Stunden" vorkommt

\begin{tabular}{|c|c|c|c|c|c|c|c|c|c|c|c|}
\hline & & \multicolumn{10}{|c|}{ Veränderung zwischen 2000 und 2009 (PISA 2009 - PISA 2000) } \\
\hline & & \multicolumn{2}{|c|}{$\begin{array}{c}\text { Wir hören nicht auf das, } \\
\text { was der Lehrer sagt }\end{array}$} & \multicolumn{2}{|c|}{$\begin{array}{c}\text { Es ist laut und alles geht } \\
\text { durcheinander }\end{array}$} & \multicolumn{2}{|c|}{$\begin{array}{c}\text { Lehrer muss lange } \\
\text { warten, bis Ruhe eintritt }\end{array}$} & \multicolumn{2}{|c|}{$\begin{array}{l}\text { Wir können nicht } \\
\text { ungestört arbeiten }\end{array}$} & \multicolumn{2}{|c|}{$\begin{array}{l}\text { Wir fangen erst lange } \\
\text { nach dem Beginn der } \\
\text { Stunde an zu arbeiten }\end{array}$} \\
\hline & & Diff. \% & S.E. & Diff. \% & S.E. & Diff. \% & S.E. & Diff. \% & S.E. & Diff. \% & S.E. \\
\hline \multirow{31}{*}{ 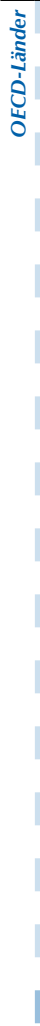 } & Australien & -10.9 & $(1.2)$ & -6.9 & $(1.3)$ & 2.3 & $(1.2)$ & 0.4 & $(1.1)$ & 2.3 & $(1.1)$ \\
\hline & Österreich & $\mathrm{m}$ & $\mathrm{m}$ & $\mathrm{m}$ & $\mathrm{m}$ & $\mathrm{m}$ & $\mathrm{m}$ & $\mathrm{m}$ & $\mathrm{m}$ & $\mathrm{m}$ & $\mathrm{m}$ \\
\hline & Belgien & -4.2 & (1.3) & 0.0 & (1.4) & 3.2 & (1.6) & -0.4 & $(0.9)$ & 2.5 & $(1.2)$ \\
\hline & Kanada & -6.5 & $(0.7)$ & -4.9 & $(0.8)$ & 7.4 & $(0.8)$ & -1.1 & $(0.6)$ & 2.2 & $(0.8)$ \\
\hline & Chile & 0.5 & $(1.2)$ & 11.9 & (1.5) & 5.7 & (1.5) & 4.4 & (1.0) & 3.8 & $(1.2)$ \\
\hline & Tschech. Rep. & -10.7 & $(1.7)$ & -7.9 & (1.8) & -0.1 & (1.8) & -7.3 & (1.3) & -8.6 & (1.4) \\
\hline & Dänemark & -8.6 & (1.3) & -0.5 & (1.6) & 5.7 & (1.6) & 5.3 & (1.0) & 4.7 & (1.3) \\
\hline & Finnland & -9.6 & (1.4) & -4.8 & (1.6) & 2.7 & (1.5) & -4.9 & (1.0) & -10.3 & (1.3) \\
\hline & Frankreich & -7.7 & (1.4) & -0.6 & (1.9) & -0.2 & $(1.7)$ & -8.5 & (1.3) & -0.1 & (1.4) \\
\hline & Deutschland & 8.7 & (1.1) & 6.0 & (1.4) & 13.7 & (1.4) & 5.9 & $(1.0)$ & 8.6 & (1.1) \\
\hline & Griechenland & -15.1 & (1.6) & 5.3 & (1.9) & 5.6 & (1.7) & -3.7 & (1.4) & 0.2 & (1.1) \\
\hline & Ungarn & -7.0 & (1.8) & -5.5 & (1.8) & 3.2 & (1.9) & 5.2 & (1.6) & -5.2 & (1.3) \\
\hline & Island & -6.0 & (0.9) & -4.6 & (1.0) & 6.9 & (1.0) & -0.1 & (0.9) & 0.8 & $(0.9)$ \\
\hline & Irland & -11.2 & (1.4) & -9.1 & (1.6) & -1.0 & (1.6) & -2.5 & $(1.2)$ & 0.3 & (1.4) \\
\hline & Israel & 7.3 & $(2.1)$ & 4.3 & (2.2) & 12.6 & (2.4) & 8.5 & (1.4) & 5.9 & $(2.2)$ \\
\hline & Italien & 1.5 & $(1.2)$ & 14.1 & (1.4) & 18.4 & (1.4) & 3.4 & $(0.9)$ & 3.0 & (1.0) \\
\hline & Japan & 9.0 & (1.3) & 7.9 & (1.8) & 2.3 & $(1.0)$ & 8.5 & $(1.2)$ & 9.3 & (1.4) \\
\hline & Korea & 22.0 & (1.3) & 6.7 & (1.4) & 5.3 & (1.1) & 11.6 & $(1.2)$ & 10.3 & $(1.2)$ \\
\hline & Luxemburg & $\mathrm{m}$ & $\mathrm{m}$ & $\mathrm{m}$ & $\mathrm{m}$ & $\mathrm{m}$ & $\mathrm{m}$ & $\mathrm{m}$ & $\mathrm{m}$ & $\mathrm{m}$ & $\mathrm{m}$ \\
\hline & Mexiko & -1.3 & $(0.9)$ & -1.6 & $(1.2)$ & 8.1 & $(1.2)$ & 0.8 & $(0.8)$ & -4.0 & $(0.9)$ \\
\hline & Niederlande & $\mathrm{m}$ & $\mathrm{m}$ & $\mathrm{m}$ & $\mathrm{m}$ & $\mathrm{m}$ & $\mathrm{m}$ & $\mathrm{m}$ & $\mathrm{m}$ & $\mathrm{m}$ & $\mathrm{m}$ \\
\hline & Neuseeland & -8.8 & $(1.2)$ & -6.2 & (1.3) & 1.8 & (1.4) & 4.3 & (1.1) & 0.5 & $(1.2)$ \\
\hline & Norwegen & -5.6 & (1.3) & 1.6 & (1.7) & 7.7 & (1.8) & 0.0 & (1.2) & 0.2 & (1.5) \\
\hline & Polen & -12.3 & (1.4) & -6.9 & (1.6) & 0.4 & (1.8) & -6.7 & (1.1) & -0.3 & (1.4) \\
\hline & Portugal & -1.2 & (1.1) & 0.0 & $(1.2)$ & 4.8 & (1.3) & 6.4 & (1.0) & 4.3 & $(1.2)$ \\
\hline & Spanien & -1.5 & $(1.1)$ & 8.5 & (1.4) & 13.4 & (1.6) & 1.7 & $(0.9)$ & 8.0 & (1.3) \\
\hline & Schweden & 4.3 & $(1.3)$ & 6.2 & $(1.7)$ & 14.5 & $(1.7)$ & 5.7 & $(1.2)$ & 7.9 & (1.5) \\
\hline & Schweiz & -9.2 & $(1.2)$ & -7.7 & (1.3) & 1.9 & (1.4) & -0.3 & $(0.9)$ & -1.0 & $(1.2)$ \\
\hline & Ver. Königreich & $\mathrm{m}$ & $\mathrm{m}$ & $\mathrm{m}$ & $\mathrm{m}$ & $\mathrm{m}$ & $\mathrm{m}$ & $\mathrm{m}$ & $\mathrm{m}$ & $\mathrm{m}$ & $\mathrm{m}$ \\
\hline & Ver. Staaten & 1.7 & $(1.3)$ & 1.8 & (1.6) & 6.4 & (1.5) & 5.7 & (1.1) & 6.7 & $(1.3)$ \\
\hline & OECD-26-Durchschnitt & -3.2 & $(0.3)$ & 0.3 & $(0.3)$ & 5.9 & $(0.3)$ & 1.6 & $(0.2)$ & 2.0 & $(0.3)$ \\
\hline \multirow{12}{*}{ 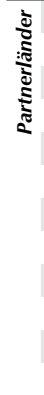 } & Albanien & -0.9 & $(1.1)$ & -4.4 & (0.9) & -1.5 & $(1.2)$ & 1.9 & (1.1) & 3.3 & (1.0) \\
\hline & Argentinien & 0.9 & $(3.1)$ & 5.5 & $(3.0)$ & 6.4 & $(3.7)$ & -1.1 & (2.3) & 4.0 & (1.5) \\
\hline & Brasilien & 4.7 & $(1.2)$ & 1.6 & (1.5) & 3.4 & (1.6) & 0.6 & $(1.2)$ & 2.5 & (1.3) \\
\hline & Bulgarien & -1.4 & (1.5) & -2.7 & (1.8) & 7.8 & $(2.0)$ & -4.4 & (1.5) & 2.5 & $(1.7)$ \\
\hline & Hongkong (China) & 15.0 & $(1.2)$ & 9.1 & (1.1) & 10.4 & (1.0) & 17.0 & (1.1) & 20.2 & (1.1) \\
\hline & Indonesien & 0.0 & $(1.2)$ & 9.2 & (1.5) & 30.5 & (1.5) & -1.7 & $(1.1)$ & 4.5 & $(1.2)$ \\
\hline & Lettland & -3.1 & (1.4) & -5.1 & (1.5) & -1.5 & (1.6) & 2.6 & $(1.2)$ & 2.4 & (1.3) \\
\hline & Liechtenstein & -14.3 & (3.3) & -8.9 & $(2.7)$ & 1.2 & $(3.2)$ & 0.5 & (3.1) & -4.6 & (2.9) \\
\hline & Peru & 9.2 & $(1.2)$ & 11.3 & (1.3) & 7.6 & $(1.2)$ & 13.7 & (1.0) & 15.0 & (1.4) \\
\hline & Rumänien & 8.0 & $(1.2)$ & 5.4 & (1.1) & 8.2 & (1.3) & 9.4 & (1.3) & 7.6 & (1.2) \\
\hline & Russ. Föderation & -2.7 & $(0.9)$ & -1.2 & $(0.9)$ & 4.5 & $(1.2)$ & 1.8 & $(1.0)$ & 2.3 & $(1.0)$ \\
\hline & Thailand & 3.3 & $(0.8)$ & 16.8 & $(1.1)$ & 5.8 & $(1.0)$ & 6.2 & $(0.9)$ & 2.2 & $(0.9)$ \\
\hline
\end{tabular}

Anmerkung: Statistisch signifikante Werte sind durch Fettdruck gekennzeichnet (siehe Anhang A3).

StatLink intsh http://dx.doi.org/10.1787/888932343285 



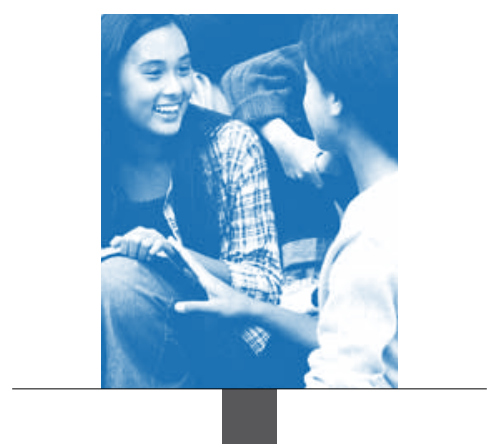

Anhang C

ENTWICKLUNG UND UMSETZUNG VON PISA EIN KOOPERATIONSPROJEKT 


\section{EINFÜHRUNG}

PISA ist ein Kooperationsprojekt, das wissenschaftliches Fachwissen der Teilnehmerländer zusammenführt und in dem Entscheidungen gemeinschaftlich auf der Basis politischer Interessen der teilnehmenden Länder getroffen werden.

Der PISA-Verwaltungsrat, in dem jedes Land vertreten ist, definiert im Rahmen der OECD-Zielsetzungen die Politikprioritäten für PISA und überwacht die Einhaltung dieser Prioritäten während der Programmumsetzung. Diese Aufgabe umfasst die Festlegung von Prioritäten für die Ausarbeitung der Indikatoren, die Erstellung der Instrumente zur Leistungsmessung und die Berichterstattung über die Ergebnisse.

Es wurden Arbeitsgruppen mit Experten aus den Teilnehmerstaaten gebildet, die gewährleisten sollen, dass die politischen Zielsetzungen mit der größtmöglichen international verfügbaren verfahrenstechnischen Kompetenz verknüpft werden. Durch ihre Beteiligung an diesen Expertengruppen stellen die Länder sicher, dass die eingesetzten Instrumente international valide sind und zugleich dem kulturellen und curricularen Kontext der OECD-Mitgliedsländer Rechnung tragen, die eingesetzten Beurteilungsinstrumente über sehr gute messtechnische Eigenschaften verfügen und diese Instrumente sowohl authentisch als auch bildungspolitisch relevant sind.

Über die nationalen Projektmanager setzen die Teilnehmer das PISA-Programm gemäß den vereinbarten administrativen Verfahren auf nationaler Ebene um. Die nationalen Projektmanager spielen eine entscheidende Rolle, indem sie gewährleisten, dass die Umsetzung hohen qualitativen Ansprüchen genügt, und indem sie die Ergebnisse, Analysen, Berichte und Veröffentlichungen überprüfen und evaluieren.

Zuständig für Design und Implementierung der Erhebungen innerhalb des vom PISA-Verwaltungsrat festgelegten Rahmens sind externe Vertragspartner. Für PISA 2009 wurde der Fragebogen von einem Konsortium unter der Leitung von Cito International in Partnerschaft mit der Universität Twente ausgearbeitet. Die Ausarbeitung und Umsetzung der kognitiven Beurteilung und der internationalen Optionen wurde von einem Konsortium unter der Leitung des Australian Council for Educational Research (ACER) durchgeführt. Zu den weiteren Partnern in diesem Konsortium zählen: cApStAn Linguistic Quality Control in Belgien, das Deutsche Institut für Internationale Pädagogische Forschung (DIPF) in Deutschland, das National Institute for Educational Policy Research (NIER) in Japan, die Unité d'analyse des systèmes et des pratiques d'enseignement (aSPe) in Belgien und WESTAT in den Vereinigten Staaten.

Das OECD-Sekretariat hat die Gesamtmanagementverantwortung für das Programm, verfolgt dessen praktische Umsetzung, fungiert als Sekretariat für den PISA-Verwaltungsrat, bemüht sich um Konsensbildung zwischen den Ländern und dient als Ansprechpartner zwischen dem PISA-Verwaltungsrat und dem internationalen Konsortium, das mit der Implementierung der Aktivitäten beauftragt ist. Das OECD-Sekretariat erstellt auch die Indikatoren, analysiert und arbeitet die internationalen Berichte und Veröffentlichungen in Zusammenarbeit mit dem PISA-Konsortium und in enger Konsultation mit den Mitgliedsländern sowohl auf politischer Ebene (PISAVerwaltungsrat) als auch auf Implementierungsebene (nationale Projektmanager) aus.

Nachstehend sind die Mitglieder der verschiedenen PISA-Organe wie auch die einzelnen Fachleute und Consultants, die an PISA mitgewirkt haben, aufgeführt.

\section{Mitglieder des PISA-Verwaltungsrats}

Vorsitzende: Lorna Bertrand

\section{OECD-Länder}

Australien: Tony Zanderigo

Belgien: Christiane Blondin, Isabelle Erauw und Micheline Scheys

Chile: Leonor Cariola

Dänemark: Tine Bak

Deutschland: Annemarie Klemm, Maximilian Müller-Härlin und Elfriede Ohrnberger

Estland: Maie Kitsing

Finnland: Jari Rajanen

Frankreich: Bruno Trosseille

Griechenland: Panagiotis Kazantzis (01.07.05 - 31.03.10), Vassilia Hatzinikita (ab 31.03.10)

Irland: Jude Cosgrove

Island: Júlíus K. Björnsson

Israel: Michal Beller

Italien: Piero Cipollone

Japan: Ryo Watanabe
Kanada: Pierre Brochu, Patrick Bussière und Tomasz Gluszynski

Korea: Whan Sik Kim

Luxemburg: Michel Lanners

Mexiko: Francisco Ciscomani

Neuseeland: Lynne Whitney

Niederlande: Paul van Oijen

Norwegen: Anne-Berit Kavli

Österreich: Mark Német

Polen: Stanislaw Drzazdzewski

Portugal: Carlos Pinto Ferreira

Schweden: Anita Wester

Schweiz: Ariane Baechler Söderström und Heinz Rhyn

Slowakische Republik: Julius Hauser, Romana Kanovska und Paulina Korsnakova

Slowenien: Andreja Barle Lakota

Spanien: Carme Amorós Basté und Enrique Roca Cobo

Tschechische Republik: Jana Strakova

Türkei: Meral Alkan

Ungarn: Benö Csapó

Vereinigtes Königreich: Lorna Bertrand und Mal Cooke

Vereinigte Staaten: Daniel McGrath und Eugene Owen 


\section{Beobachter}

Albanien: Ndricim Mehmeti

Argentinien: Liliana Pascual

Aserbaidschan: Talib Sharifov

Brasilien: Joaquim José Soares Neto

Bulgarien: Neda Kristanova

Chinesisch Taipeh: Chih-Wei Hue und Fou-Lai Lin

Dubai (VAE): Mariam Al Ali

Hongkong (China): Esther Sui-chu Ho

Indonesien: Mansyur Ramli

Jordanien: Khattab Mohammad Abulibdeh

Kasachstan: Yermekov Nurmukhammed Turlynovich

Katar: Adel Sayed

Kirgisistan: Inna Valkova

Kolumbien: Margarita Peña

Kroatien: Michelle Braš-Roth

Lettland: Andris Kangro

Liechtenstein: Christian Nidegger

Litauen: Rita Dukynaite

Macau (China): Kwok-cheung Cheung

Montenegro: Zeljko Jacimovic

Panama: Arturo Rivera

Peru: Liliana Miranda Molina

Rumänien: Roxana Mihail

Russische Föderation: Galina Kovalyova

Serbien: Dragica Pavlovic Babic

Shanghai (China): Minxuan Zhang

Singapur: Low Khah Gek

Thailand: Precharn Dechsri

Trinidad und Tobago: Harrilal Seecharan

Tunesien: Kameleddine Gaha

Uruguay: Andrés Peri

Nationale Projektmanager für PISA 2009

Albanien: Alfonso Harizaj

Argentinen: Antonio Gutiérrez

Aserbaidschan: Emin Meherremov

Australien: Sue Thomson

Belgien: Ariane Baye und Inge De Meyer

Brasilien: Sheyla Carvalho Lira

Bulgarien: Svetla Petrova

Chile: Ema Lagos

Chinesisch Taipeh: Pi-Hsia Hung

Dänemark: Niels Egelund

Deutschland: Nina Jude und Eckhard Klieme

Dubai (VAE): Mariam Al Ali

Estland: Gunda Tire

Finnland: Jouni Välijärvi

Frankreich: Sylvie Fumel

Griechenland: Panagiotis Kazantzis (vom 01.07.05 bis 18.11.08), Chryssa Sofianopoulou (ab 18.11.08)

Hongkong (China): Esther Sui-chu Ho
Indonesien: Burhanuddin Tola

Irland: Rachel Perkins

Island: Almar Midvik Halldorsson

Israel: Inbal Ron Kaplan und Joel Rapp

Italien: Laura Palmerio

Japan: Ryo Watanabe

Jordanien: Khattab Mohammad Abulibdeh

Kanada: Pierre Brochu und Tamara Knighton

Kasachstan: Damitov Bazar Kabdoshevich

Katar: Asaad Tounakti

Kirgisistan: Inna Valkova

Kolumbien: Francisco Ernesto Reyes

Korea: Kyung-Hee Kim

Kroatien: Michelle Braš Roth

Lettland: Andris Kangro

Liechtenstein: Christian Nidegger

Litauen: Jolita Dudaité

Luxemburg: Bettina Boehm

Macau (China): Kwok-cheung Cheung

Mexiko: María-Antonieta Díaz-Gutiérrez

Montenegro: Verica Ivanovic

Neuseeland: Maree Telford

Niederlande: Erna Gille

Norwegen: Marit Kjaernsli

Österreich: Ursula Schwantner

Panama: Zoila Castillo

Peru: Liliana Miranda Molina

Polen: Michal Federowicz

Portugal: Anabela Serrão

Rumänien: Silviu Cristian Mirescu

Russische Föderation: Galina Kovalyova

Schweden: Karl-Göran Karlsson

Schweiz: Christian Nidegger

Serbien: Dragica Pavlovic Babic

Shanghai (China): Jing Lu und MinXuan Zhang

Singapur: Chia Siang Hwa und Poon Chew Leng

Slowakische Republik: Paulina Korsnakova

Slowenien: Mojca Straus

Spanien: Lis Cercadillo

Thailand: Sunee Klainin

Trinidad und Tobago: Harrilal Seecharan

Tschechische Republik: Jana Paleckova

Tunesien: Kameleddine Gaha

Türkei: Müfide Çaliskan

Ungarn: Ildikó Balázsi

Uruguay: María Sánchez

Vereinigtes Königreich: Jenny Bradshaw und Mal Cooke Vereinigte Staaten: Dana Kelly und Holly Xie

\section{OECD-Sekretariat}

Andreas Schleicher (PISA-Gesamtkoordination und Kontakte zu den Partnerländern/-volkswirtschaften) 
Marilyn Achiron (Redaktionelle Unterstützung)

Marika Boiron (Redaktionelle Unterstützung)

Simone Bloem (Analytische Unterstützung)

Francesca Borgonovi (Analytische Unterstützung)

Niccolina Clements (Redaktionelle Unterstützung)

Michael Davidson (Projektmanagement und analytische Unterstützung)

Juliet Evans (Administrative Unterstützung und Kontakte zu den Partnerländern/-volkswirtschaften)

Miyako Ikeda (Analytische Unterstützung)

Maciej Jakubowski (Analytische Unterstützung)

Guillermo Montt (Analytische Unterstützung)

Diana Morales (Administrative Unterstützung)

Soojin Park (Analytische Unterstützung)

Mebrak Tareke (Redaktionelle Unterstützung)

Sophie Vayssettes (Analytische Unterstützung)

Elisabeth Villoutreix (Redaktionelle Unterstützung)

Karin Zimmer (Projektmanagement)

Pablo Zoido (Analytische Unterstützung)

\section{Deutsche Übersetzung}

Thomas Krischer (Revision)

Ira Haugk (Revision)

Martina Dzierzawski (Übersetzung)

Susanne Hägele (Übersetzung)

Daniela Herzog (Übersetzung)

Carola Noubani (Übersetzung)

Bettina Peche (Übersetzung)

Susanne Gniech (Terminologische Unterstützung)

Geert Gruben (Terminologische Unterstützung)

Gabriele Gwinner (Redaktionelle Unterstützung, Layout)

Gabriele Speer (Administrative Unterstützung)

Ilse Ferrario (Administrative Unterstützung)

\section{PISA-Expertengruppen für PISA 2009}

\section{Expertengruppe Lesen}

Irwin Kirsch (Education Testing Service, New Jersey, USA)

Sachiko Adachi (Nigata University, Japan)

Charles Alderson (Lancaster University, Vereinigtes Königreich)

John de Jong (Language Testing Services, Niederlande)

John Guthrie (University of Maryland, USA)

Dominique Lafontaine (Université de Liège, Belgien)

Minwoo Nam (Korea Institute of Curriculum and Evaluation)

Jean-François Rouet (Université de Poitiers, Frankreich)

Wolfgang Schnotz (Universität Koblenz-Landau, Deutschland)

Eduardo Vidal-Abarca (Universität Valencia, Spanien)

\section{Expertengruppe Mathematik}

Jan de Lange (Vorsitzender) (Utrecht University, Niederlande)

Werner Blum (Universität Kassel, Deutschland)

John Dossey (Illinois State University, USA)

Zbigniew Marciniak (Universität Warschau, Polen)

Mogens Niss (Universität Roskilde, Dänemark)
Yoshinori Shimizu (University of Tsukuba, Japan)

\section{Expertengruppe Naturwissenschaften}

Rodger Bybee (Vorsitzender) (BSCS, Colorado Springs, USA)

Peter Fensham (Queensland University of Technology, Australien)

Svein Lie (Universität Oslo, Norwegen)

Yasushi Ogura (National Institute for Educational Policy

Research, Japan)

Manfred Prenzel (Universität Kiel, Deutschland)

Andrée Tiberghien (Université de Lyon, Frankreich)

\section{Expertengruppe Fragebogen}

Jaap Scheerens (Vorsitzender) (Universität Twente, Niederlande) Pascal Bressoux (Université Pierre Mendès, Frankreich)

Yin Cheong Cheng (Hong Kong Institute of Education, Hongkong-China)

David Kaplan (University of Wisconsin - Madison, USA)

Eckhard Klieme (DIPF, Deutschland)

Henry Levin (Columbia University, USA)

Pirjo Linnakylä (Universität Jyväskylä, Finnland)

Ludger Wößmann (Universität München, Deutschland)

\section{PISA Technische Beratergruppe}

Keith Rust (Vorsitzender) (Westat, USA)

Ray Adams (ACER)

John de Jong (Language Testing Services, Niederlande)

Cees Glas (Universität Twente, Niederlande)

Aletta Grisay (Consultant, Saint-Maurice, Frankreich)

David Kaplan (University of Wisconsin - Madison, USA)

Christian Monseur (Université de Liège, Belgien)

Sophia Rabe-Hesketh (University of California - Berkeley, USA)

Thierry Rocher (Ministère de l'éducation nationale, Frankreich)

Norman Verhelst (CITO, Niederlande)

Kentaro Yamamoto (ETS, New Jersey, USA)

Rebecca Zwick (University of California - Santa Barbara, USA)

\section{PISA-Konsortium 2009 Fragebogenausarbeitung}

\section{Cito International}

Johanna Kordes

Hans Kuhlemeier

Astrid Mols

Henk Moelands

José Noijons

\section{Universität Twente}

Cees Glas

Khurrem Jehangir

Jaap Scheerens

PISA-Konsortium 2009 Entwicklung und Umsetzung der kognitiven Beurteilung und der internationalen Optionen Australian Council for Educational Research

Ray Adams (Direktor des PISA-Konsortiums 2009)

Susan Bates (Projektverwaltung)

Alla Berezner (Datenmanagement und -analyse) 
Yan Bibby (Datenverarbeitung und -analyse)

Esther Brakey (Administrative Unterstützung)

Wei Buttress (Projektverwaltung, Qualitätskontrolle)

Renee Chow (Datenverarbeitung und -analyse)

Judith Cosgrove (Datenverarbeitung und -analyse, Unterstützung der nationalen Zentren)

John Cresswell (Berichterstattung und Verbreitung)

Alex Daraganov (Datenverarbeitung und -analyse)

Daniel Duckworth (Erhebungsinstrumente Lesekompetenz und Testausarbeitung)

Kate Fitzgerald (Datenverarbeitung und Stichprobenauswahl)

Daniel Fullarton (IT-Dienste)

Eveline Gebhardt (Datenmanagement und -analyse)

Mee-Young Handayani (Datenmanagement und -analyse)

Elizabeth Hersbach (Qualitätssicherung)

Sam Haldane (IT-Dienste und computergestützte Evaluierung)

Karin Hohlfield (Erhebungsinstrumente Lesekompetenz und Testausarbeitung)

Jennifer Hong (Datenverarbeitung und Stichprobenauswahl)

Tony Huang (Projektverwaltung und IT-Dienste)

Madelaine Imber (Erhebungsinstrumente Lesekompetenz und administrative Unterstützung)

Nora Kovarcikova (Erhebungsverfahren)

Winson Lam (IT-Dienste)

Tom Lumley (Erhebungsinstrumente Lesekompetenz gedruckter und elektronischer Texte und Testausarbeitung)

Greg Macaskill (Datenmanagement und -verarbeitung, Stichprobenauswahl)

Ron Martin (Erhebungsinstrumente Naturwissenschaften und Testausarbeitung)

Barry McCrae (Manager Beurteilung Lesekompetenz elektronischer Texte, Erhebungsinstrumente

Naturwissenschaften und Testausarbeitung)

Juliette Mendelovits (Erhebungsinstrumente Lesekompetenz gedruckter und elektronischer Texte und Testausarbeitung)

Martin Murphy (Feldoperationen und Stichprobenauswahl)

Thoa Nguyen (Datenmanagement und -analyse)

Penny Pearson (Administrative Unterstützung)

Anna Plotka (Grafische Gestaltung)

Alla Routitsky (Datenmanagement und -verarbeitung)

Wolfram Schulz (Datenmanagement und -verarbeitung)

Dara Searle (Erhebungsinstrumente Lesekompetenz gedruckter und elektronischer Texte und Testausarbeitung)

Naoko Tabata (Erhebungsverfahren)

Ross Turner (Management, Erhebungsinstrumente Mathematik und Testausarbeitung)

Daniel Urbach (Datenmanagement und -analyse)

Eva Van de gaer (Datenanalyse)

Charlotte Waters (Projektverwaltung, Datenverarbeitung und -analyse)

Maurice Walker (Beurteilung Lesekompetenz elektronischer Texte und Stichprobenauswahl)

Wahyu Wardono (Projektverwaltung und IT-Dienste)

Louise Wenn (Datenmanagement und -analyse)
Yan Wiwecka (IT-Dienste)

\section{Westat}

Eugene Brown (Gewichtung)

Fran Cohen (Gewichtung)

Susan Fuss (Stichprobenauswahl und Gewichtung)

Amita Gopinath (Gewichtung)

Sheila Krawchuk (Stichprobenauswahl, Gewichtung und Qualitätskontrolle)

Thanh Le (Stichprobenauswahl, Gewichtung und Qualitätskontrolle)

Jane Li (Stichprobenauswahl und Gewichtung)

John Lopdell (Stichprobenauswahl und Gewichtung)

Shawn Lu (Gewichtung)

Keith Rust (Leiter des PISA-Konsortiums für

Stichprobenauswahl und Gewichtung)

William Wall (Gewichtung)

Erin Wilson (Stichprobenauswahl und Gewichtung)

Marianne Winglee (Gewichtung)

Sergey Yagodin (Gewichtung)

\section{The National Institute for Educational Research in Japan}

Hidefumi Arimoto (Erhebungsinstrumente Lesekompetenz und Testausarbeitung)

Hisashi Kawai (Erhebungsinstrumente Lesekompetenz und Testausarbeitung)

\section{cApStAn Linguistic Quality Control}

Steve Dept (Übersetzungs- und Überprüfungsdienste)

Andrea Ferrari (Übersetzungs- und Überprüfungsmethoden)

Laura Wäyrynen (Überprüfungsmanagement )

\section{Unité d'analyse des systèmes et des pratiques d'enseignement (aSPe)}

Ariane Baye (Erhebungsinstrumente Lesekompetenz gedruckter und elektronischer Texte und Testausarbeitung) Casto Grana-Monteirin (Übersetzungs- und Überprüfungsdienste)

Dominique Lafontaine (Mitglied der Expertengruppe Lesen) Christian Monseur (Datenanalyse und Mitglied der technischen Beratergruppe)

Anne Matoul (Übersetzungs- und Überprüfungsdienste) Patricia Schillings (Erhebungsinstrumente Lesekompetenz gedruckter und elektronischer Texte und Testausarbeitung)

\section{Deutsches Institut für Internationale Pädagogische Forschung (DIPF)}

Cordula Artelt (Universität Bamberg) (Erhebungsinstrumente Lesekompetenz und Rahmenkonzept)

Michel Dorochevsky (Softcon) (Softwareentwicklung)

Frank Goldhammer (Erhebungsinstrumente Lesekompetenz elektronischer Texte und Testausarbeitung)

Dieter Heyer (Softcon) (Softwareentwicklung)

Nina Jude (Projektkoordinatorin am DIPF)

Eckhard Klieme (Projektleiter am DIPF)

Holger Martin (Softcon) (Softwareentwicklung)

Johannes Naumann (Erhebungsinstrumente Lesekompetenz elektronischer Texte und Testausarbeitung) 
Jean-Paul Reeff (Selbstständiger Berater)

Heiko Rölke (Technischer Projektleiter am DIPF)

Wolfgang Schneider (Universität Würzburg)

(Erhebungsinstrumente Lesekompetenz und Rahmenkonzept)

Petra Stanat (Humboldt Universität, Berlin)

(Erhebungsinstrumente Lesekompetenz und Testausarbeitung)

Britta Upsing (Erhebungsinstrumente Lesekompetenz

elektronischer Texte und Testausarbeitung)

\section{Sonstige Experten}

Tobias Dörfler (Universität Bamberg) (Ausarbeitung

Erhebungsinstrumente Lesekompetenz)

Tove Stjern Frønes (ILS, Universität Oslo) (Ausarbeitung

Erhebungsinstrumente Lesekompetenz)

Béatrice Halleux (Consultant, HallStat SPRL) (Übersetzung/

Überprüfung, Ausarbeitung französische Quellen)

Øystein Jetne (ILS, Universität Oslo) (Erhebungsinstrumente

Lesekompetenz gedruckter und elektronischer Texte und Testausarbeitung)

Kees Lagerwaard (Institute for Educational Measurement of Netherlands) (Ausarbeitung Erhebungsinstrumente Mathematik)

Pirjo Linnakylä (Universität Jyväskylä) (Ausarbeitung Erhebungsinstrumente Lesekompetenz)

Anne-Laure Monnier (Consultant, Frankreich) (Ausarbeitung französische Quellen)

Jan Mejding (Danish Schoool of Education, Universität

Aarhus) (Ausarbeitung Erhebungsinstrumente Lesekompetenz gedruckter und elektronischer Texte)

Eva Kristin Narvhus (ILS, Universität Oslo)

(Erhebungsinstrumente Lesekompetenz gedruckter und elektronischer Texte, Testinstrumente und Testausarbeitung)

Rolf V. Olsen (ILS, Universität Oslo) (Ausarbeitung

Erhebungsinstrumente Naturwissenschaften)

Robert Laurie (New Brunswick Department of Education, Kanada) (Ausarbeitung Erhebungsinstrumente Naturwissenschaften)

Astrid Roe (ILS, Universität Oslo) (Erhebungsinstrumente Lesekompetenz gedruckter und elektronischer Texte und Testausarbeitung)

Hanako Senuma (Universität Tamagawa, Japan) (Ausarbeitung Erhebungsinstrumente Mathematik)

\section{Sonstige Beiträge zur Publikation}

Kai von Ahlefeld (Layout) 


\section{ORGANISATION FÜR WIRTSCHAFTLICHE ZUSAMMENARBEIT UND ENTWICKLUNG}

Die OECD ist ein in seiner Art einzigartiges Forum, in dem Regierungen gemeinsam an der Bewältigung von Herausforderungen der Globalisierung im Wirtschafts-, Sozial- und Umweltbereich arbeiten. Die OECD steht auch in vorderster Linie bei den Bemühungen um ein besseres Verständnis der neuen Entwicklungen und durch sie ausgelöster Befürchtungen, indem sie Untersuchungen zu Themen wie Corporate Governance, Informationswirtschaft oder Bevölkerungsalterung durchführt. Die Organisation bietet den Regierungen einen Rahmen, der es ihnen ermöglicht, ihre Politikerfahrungen auszutauschen, nach Lösungsansätzen für gemeinsame Probleme zu suchen, empfehlenswerte Praktiken aufzuzeigen und auf eine Koordinierung nationaler und internationaler Politiken hinzuarbeiten.

Die OECD-Mitgliedstaaten sind: Australien, Belgien, Chile, Dänemark, Estland, Deutschland, Finnland, Frankreich, Griechenland, Irland, Island, Israel, Italien, Japan, Kanada, Korea, Luxemburg, Mexiko, Neuseeland, die Niederlande, Norwegen, Österreich, Polen, Portugal, Schweden, Schweiz, die Slowakische Republik, Slowenien, Spanien, die Tschechische Republik, Türkei, Ungarn, das Vereinigte Königreich und die Vereinigten Staaten. Die Europäische Kommission nimmt an den Arbeiten der OECD teil.

OECD Publishing sorgt dafür, dass die Ergebnisse der statistischen Analysen und der Untersuchungen der Organisation zu wirtschaftlichen, sozialen und umweltpolitischen Themen sowie die von den Mitgliedstaaten vereinbarten Übereinkommen, Leitlinien und Standards weite Verbreitung finden. 


\section{BAND V}

Sind die Schülerinnen und Schüler gut auf die Herausforderungen der Zukunft vorbereitet? Können sie analysieren, logisch denken und ihre Ideen effektiv kommunizieren? Haben sie Interessen entwickelt, die sie ihr ganzes Leben hindurch als produktive Mitglieder von Wirtschaft und Gesellschaft weiterverfolgen können? Die Internationale Schulleistungsstudie der OECD (PISA) sucht durch die umfassendste und weitreichendste internationale Erhebung der Kenntnisse und Fähigkeiten von Schülerinnen und Schülern Antworten auf diese Fragen zu liefern. Die Gruppe der an PISA teilnehmenden Länder und Volkswirtschaften macht insgesamt nahezu $90 \%$ der Weltwirtschaft aus.

PISA 2009 Ergebnisse stellt die Erkenntnisse vor, die bei der jüngsten PISA-Erhebung gewonnen wurden. Schwerpunkt war diesmal wieder die Lesekompetenz, evaluiert wurden aber auch die Schülerleistungen in Mathematik und Naturwissenschaften.

- Band I, Was Schülerinnen und Schüler wissen und können: Schülerleistungen in Lesekompetenz, Mathematik und Naturwissenschaften, vergleicht die Kenntnisse und Fähigkeiten der Schüler in den verschiedenen Ländern.

- Band II, Potenziale nutzen und Chancengerechtigkeit sichern: Sozialer Hintergrund und Schülerleistungen, befasst sich mit der Frage, wie gut es den Bildungssystemen gelingt, den Effekt des sozioökonomischen Hintergrunds und des Migrationsstatus auf die Schüler- und Schulleistungen einzudämmen.

- Band III, Learning to Learn: Student Engagement, Strategies and Practices (nur in Englisch verfügbar), setzt sich mit der Motivation, dem Leseengagement und dem Einsatz effektiver Lernstrategien bei 15-Jährigen auseinander.

- Band IV, Was macht eine Schule erfolgreich? Lernumfeld und schulische Organisation in PISA, untersucht, wie sich die personellen, finanziellen und materiellen Ressourcen sowie die Politik und Praxis der Schulen auf die Lernergebnisse der Schüler auswirken.

- Band V, Lernfortschritte im globalen Wettbewerb: Veränderungen bei den Schülerleistungen seit 2000, befasst sich mit der Frage, welche Fortschritte die Länder bei der Anhebung des Leistungsniveaus und der Verbesserung der Chancengerechtigkeit in der Bildung erzielt haben.

- Band VI, Students On Line: Reading and Using Digital Information (nur in Englisch verfügbar), untersucht, wie gut die Schülerinnen und Schüler Informationstechnologien für Lernzwecke nutzen können.

PISA 2009 markiert den Beginn des zweiten Erhebungszyklus, in dessen Rahmen 2012 schwerpunktmäßig die Mathematikleistungen und 2015 die Leistungen in Naturwissenschaften untersucht werden sollen.

\section{Die Internationale Schulleistungsstudie der OECD (PISA)}

PISA richtet das Augenmerk auf die Fähigkeit der Jugendlichen, ihre Kenntnisse und Fertigkeiten zur Bewältigung alltäglicher Herausforderungen einzusetzen. Diese Orientierung spiegelt eine Veränderung in den Zielen der Lehrpläne wider, in denen es zunehmend darum geht, wie die Schülerinnen und Schüler ihr Schulwissen konkret nutzen können, und nicht mehr nur um die Fähigkeit zur Wiedergabe des Gelernten. Zu den Besonderheiten von PISA gehören:

- Politikorientierung: Daten über die Lernergebnisse der Schülerinnen und Schüler werden mit Informationen über deren Hintergrundmerkmale und über wichtige Faktoren, die ihr Lernen innerhalb und außerhalb der Schule beeinflussen, verknüpft, um Unterschiede bei den Leistungsstrukturen hervorzuheben und zu untersuchen, wodurch sich Schüler, Schulen und Bildungssysteme, die hohe Leistungen erbringen, auszeichnen.

- Innovatives Konzept der Grundbildung: Dabei geht es um die Kapazität der Schülerinnen und Schüler, Kenntnisse und Fertigkeiten in wichtigen Fächern zur Definition, Interpretation und Lösung von Problemen in einer Vielzahl von Situationen zu nutzen, sowie ihre Fähigkeit, zu analysieren, logisch zu denken und effektiv zu kommunizieren.

- Relevanz für das lebenslange Lernen: Bei PISA werden nicht nur die Kompetenzen der Schülerinnen und Schüler in den Schulfächern erfasst, sondern auch Informationen über ihre Lernmotivation, Selbsteinschätzung und Lernstrategien erhoben.

- Regelmäßigkeit: Die Länder haben die Möglichkeit, ihre Fortschritte bei der Verwirklichung entscheidender Lernziele im Zeitverlauf zu beobachten.

- Große geografische Reichweite und kooperativer Charakter: An PISA 2009 nahmen alle 34 OECD-Mitgliedstaaten sowie 41 Partnerländer und -volkswirtschaften teil. 